\title{
Synthesis And Fluorescence Properties of Anthracene Derivatives and their Metal COMPLEXES
}

\author{
Dissertation zur Erlangung des \\ mathematisch-naturwissenschaftlichen Doktorgrades \\ "Doctor rerum naturalium" \\ der Georg-August-Universität Göttingen
}

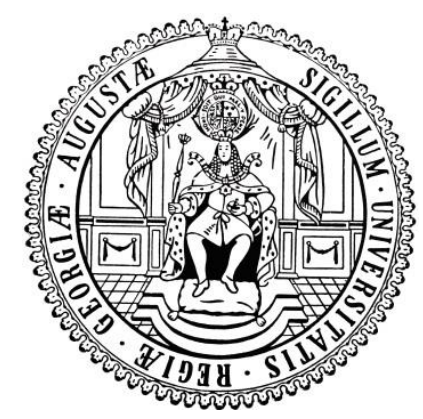

vorgelegt von

Dipl.-Chem. Nils Finkelmeier aus Holzminden

Göttingen 2013 

Referent: Prof. Dr. D. Stalke

Korreferent: Prof. Dr. F. Meyer

Tag der mündlichen Prüfung: 20.06.2013 





\section{CONTENTS}

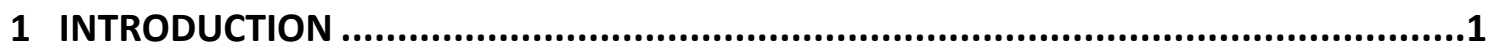

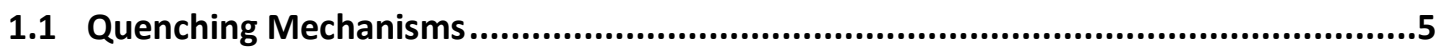

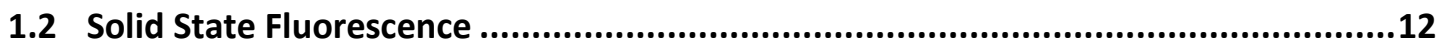

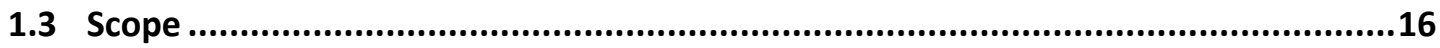

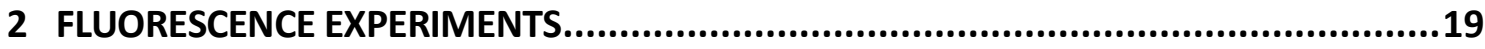

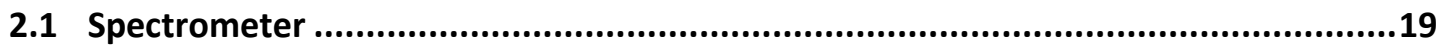

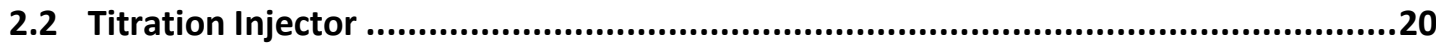

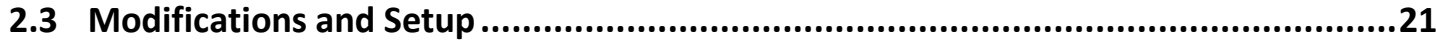

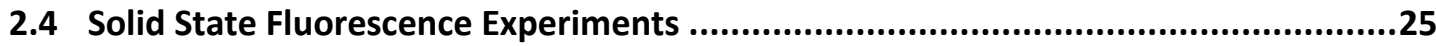

3 ANTHRACENE DERIVATIVES WITHOUT SPACERS .............................................27

3.1 Fluorescence of Asymmetric Phosphanyl and Phosphorylanthracenes ...................28

3.2 9,10-Bis(diphenylthiophosphoryl)anthracene (SPAnPS) ........................................49

3.3 9-(Bis(diethylamino)phosphoryl)-10-methylanthracenes and

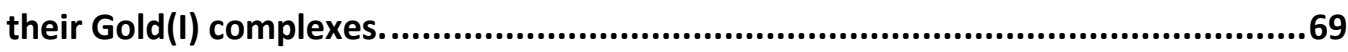

3.4 Synthesis of new Phosphoryl Anthracenes and Fluorescence Characterizations .......93

3.5 Metal Complexes of Phosphanyl and Phosphorylanthracenes .............................126

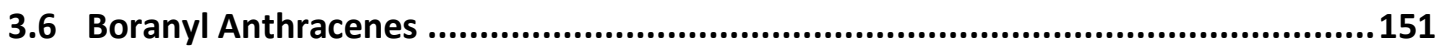

4 ANTHRACENE DERIVATIVES CONTAINING SPACERS .....................................171

4.1 Synthesis of Precursor Molecules ...........................................................................172

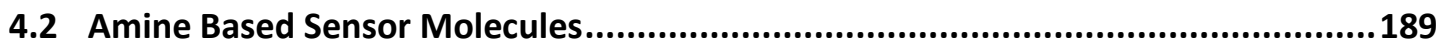

4.3 Phosphane Derivatives Containing Spacers.........................................................218

4.4 Unification of Phosphanes and Sensor Compounds.............................................233

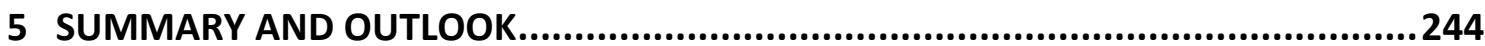




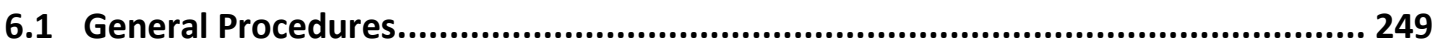

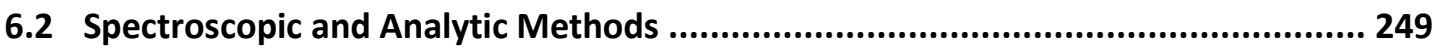

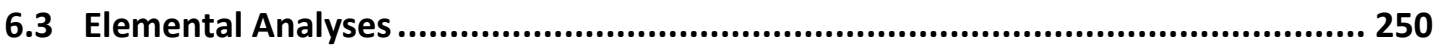

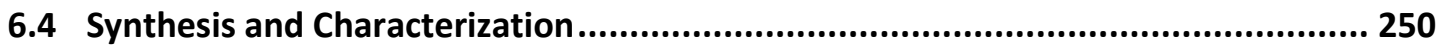

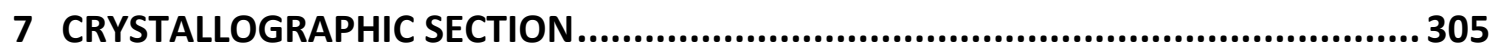

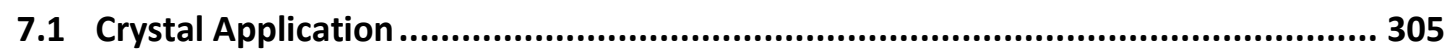

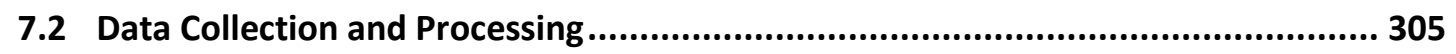

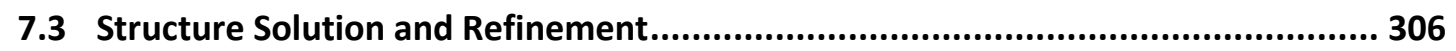

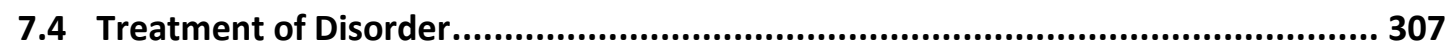

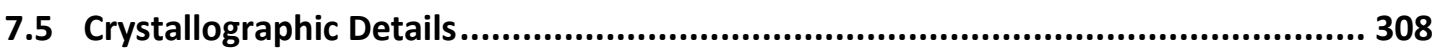

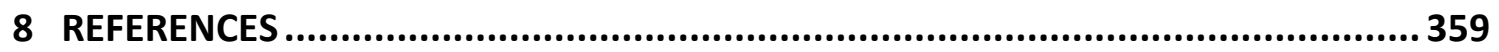




\section{AbBreviations}

\begin{tabular}{|c|c|}
\hline$\AA$ & ÅNGSTROM \\
\hline ADP & anisotropic displacement parameters \\
\hline ADS & anthracenedisulfunic acid \\
\hline AlE & aggregation induced emission \\
\hline $\mathrm{Bu}$ & butyl \\
\hline calcd & calculated \\
\hline c.f. & confer; compare \\
\hline CHEF & chelation enhanced emission \\
\hline $\mathrm{Cp}$ & cyclopentadienyl \\
\hline CSD & Cambridge Structural Database \\
\hline Cy & cyclohexyl \\
\hline DCM & dichloromethane \\
\hline DME & dimethoxyethane \\
\hline e & electron \\
\hline e.g. & exempli gratia; for example \\
\hline Eq. & Equation \\
\hline eq. & equivalents \\
\hline Et & ethyl \\
\hline et al. & et alii; and others \\
\hline EtOAc & ethyl acetate \\
\hline GoF & goodness of fit \\
\hline HOMO & highest occupied molecular orbital \\
\hline$i$ & iso \\
\hline i.e. & id est; that is \\
\hline IPC & isopinocampheyl \\
\hline LP & lone pair \\
\hline LUMO & lowest unoccupied molecular orbital \\
\hline M & metal atom \\
\hline $\max$ & maximal \\
\hline M & molar \\
\hline $\mathrm{Me}$ & methyl \\
\hline $\mathrm{MeCN}$ & acetonitrile \\
\hline $\mathrm{MeOH}$ & methanol \\
\hline Mes & mesityl \\
\hline $\min$. & minimal \\
\hline MS & mass spectrometry \\
\hline$n$ & normal \\
\hline NMR & nuclear magnetic resonance \\
\hline PET & photoinduced electron transfer \\
\hline
\end{tabular}




$\begin{array}{ll}\text { Ph } & \text { phenyl } \\ \text { Pic } & \text { picolyl (2-methylpyridyl) } \\ \text { pm } & \text { picometer } \\ \text { PMDETA } & N, N, N^{\prime}, N^{\prime}, N^{\prime \prime} \text {-pentamethyldiethylenetriamine } \\ \text { ppm } & \text { parts per million } \\ \text { Pr } & \text { propyl } \\ \text { Py } & \text { pyridyl } \\ \text { R } & \text { hydrogen atom or alkyl or aryl group } \\ \text { rt } & \text { room temperature } \\ t & \text { tertiary } \\ \text { THF } & \text { tetrahydrofuran } \\ \text { TMEDA } & N, N, N^{\prime}, N^{\prime} \text {-tetramethylethylenediamine } \\ \text { TMS } & \text { trimethylsilyl }\end{array}$






\section{INTRODUCTION}

Ever since the first observations of fluorescence by the Irish mathematician and physicist Sir George Gabriel Stokes in the middle of the $19^{\text {th }}$ century, $^{[1]}$ this phenomenon has been an undiminished source of fascination for both scientists and common spectators. Stokes had observed the strong emission of deep blue light from fluorite crystals upon irradiation with ultraviolet light and called this behaviour fluorescence. Stokes was also the first to formulate general principles of this phenomenon, e.g. the fact that emitted light is always of longer wavelength than the irradiated light is still known under the term Stokes shift. ${ }^{[2]}$

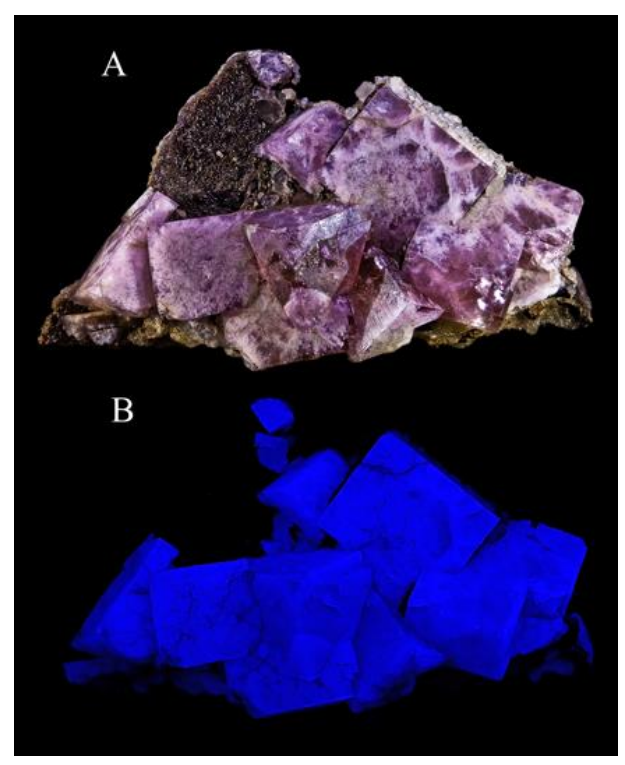

Figure 1-1: Fluorescence of fluorite crystals; A: in daylight, B: in UV light.

Around the same time Herschel described the fluorescence of dissolved molecules for the first time in his studies on quinine. ${ }^{[3]}$ Not until approximately eighty years later, the fluorescence of fluorite crystals could be scientifically explained by dotation of the mineral with europium ions. ${ }^{[4]}$ The theoretical understanding of fluorescence was delayed to a similar degree. Only in the course of the rise of the quantum theory in the early $20^{\text {th }}$ century and the subsequent advances in physics and physical chemistry, a profound understanding of the electronic processes on which luminescence phenomena are founded was developed. Step by step the empirical observations made by Stokes and following researchers could be explained. 
Today the electronic transitions and processes involved in luminescence have been deciphered in the minutest details and can - in their main features - be considered as basic knowledge of physical chemistry. Generally spoken, luminescence is the transition of a compound or molecule from an electronically excited state to an energetically lower state - usually the electronic ground state - under emission of radiation. ${ }^{[5]}$ The population of the excited state can be facilitated via different routes. Although mechanical force (triboluminescence) as well as chemical reactions (chemoluminescence) $^{[2]}$ have been shown to induce excitation of luminescent compounds, the most renowned and established process is based on the absorption of light and is therefore called photoluminescence. The precondition of photoluminescence is the presence of suitable energetic states within the respective compound which exhibit an energetic gap in the range of UV- or visible light photons. Only then the energy of a photon is sufficient for excitation of the compound.
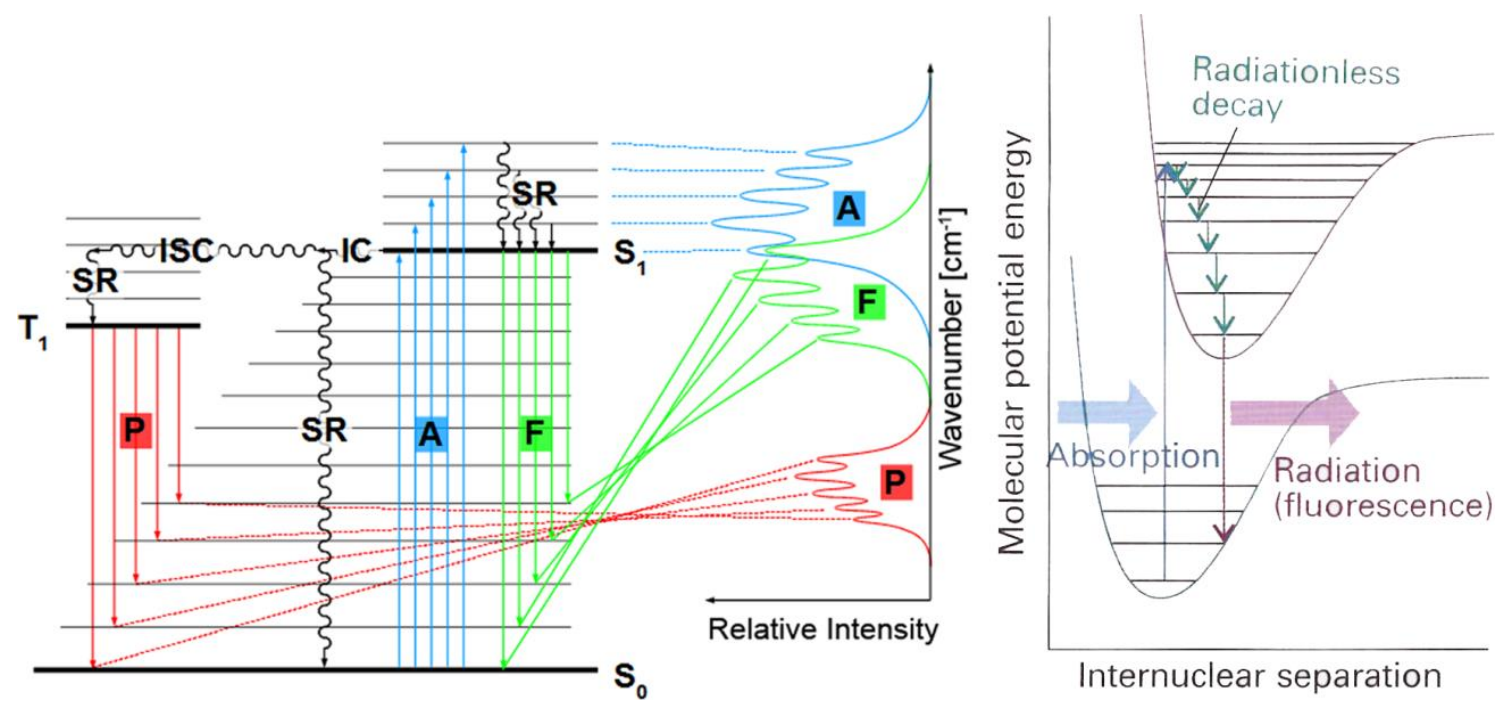

Figure 1-2: Left Jablonski diagram: right: fluorescence diagram. ${ }^{[5]}$

The general mechanisms of luminescence processes are depicted in Figure 1-2. When describing molecules according to the molecular orbital (MO) theory, the population of energetic states is determined by the Boltzmann statistic, which defines that the probability of occupation of excited states declines exponentially with rising energy. ${ }^{[5]}$ Hence, at standard conditions virtually only the electronic ground state is populated. The same distribution applies to the vibrational state of molecules. By absorption of a photon of suitable wavelength, an electron can be transferred from the ground state to an excited electronic state. 
Generally an excited vibrational state of the excited electronic state is populated by excitation of an electron, which will be explained in the following. The excitation is followed by a non-radiative decay process to the vibrational ground state, which proceeds very fast, within ca. $10^{-12} \mathrm{~s} .{ }^{[6]}$ This relaxation is induced e.g. by collisions of the excited molecule with solvent molecules. By overlap with energetic states of similar potential energy, the excited electron can be transferred to states of different spin multiplicity. This process is called inter system crossing (ISC) and is formally spin forbidden due to the change of the overall spin angular momentum, which makes this transition unlikely. This principle can be loosened by the presence of heavy atoms in the structure whose relativistic effects can benefit ISC. ${ }^{[7]}$ The radiative processes which follow from ISC (e.g. phosphorescence) will not be further reviewed.

If ISC does not occur, the electron undergoes transition back to the electronic ground state under emission of radiation, which is defined as fluorescence. Because during this process the spin angular momentum remains unchanged $(\Delta S=0)$, it is nonforbidden and therefore a fast process compared to e.g. phosphorescence. The lifetime of fluorescence excited states ranges form $10^{-3}$ to $10^{-9} \mathrm{~s}^{[8]}$ In the diagram in Figure 1-3 (right) two general principles of fluorescence can be visualized. First of all, the non-radiative decay within the excited electronic state $s_{1}$ induces a loss of energy. Therefore the energy of the emitted photon must always be equal to or lower than that of the absorbed photon, and therewith of longer wavelength. This explains the red-shift of emission observed by Stokes in the investigation of fluorite, known as the Stokes shift. Secondly, the fact that fluorescence emission exclusively occurs from the vibrational ground state of the electronically excited state $s_{1}$ makes the emission spectrum of every molecule independent of the excitation wavelength. Hence, no matter which wavelength is irradiated or which vibrational state of $s_{1}$ is populated in the excitation process, the wavelength and shape of the emission spectrum always remains identical, which is known as the Kasha rule. ${ }^{[9]}$

Because excitation can populate several different vibrational states of $\mathrm{s}_{1}$, molecules usually do not only possess a single excitation wavelength, but several possible wavelengths which lead to population of $s_{1}$. These are known as excitation bands and their intensity - and therewith the shape of the excitation spectrum - is defined by the probability of the respective transition. The probabilities of these transitions are described by the Franck-Condon principle, which is illustrated in Figure 1-3 (left). ${ }^{[5]}$ 

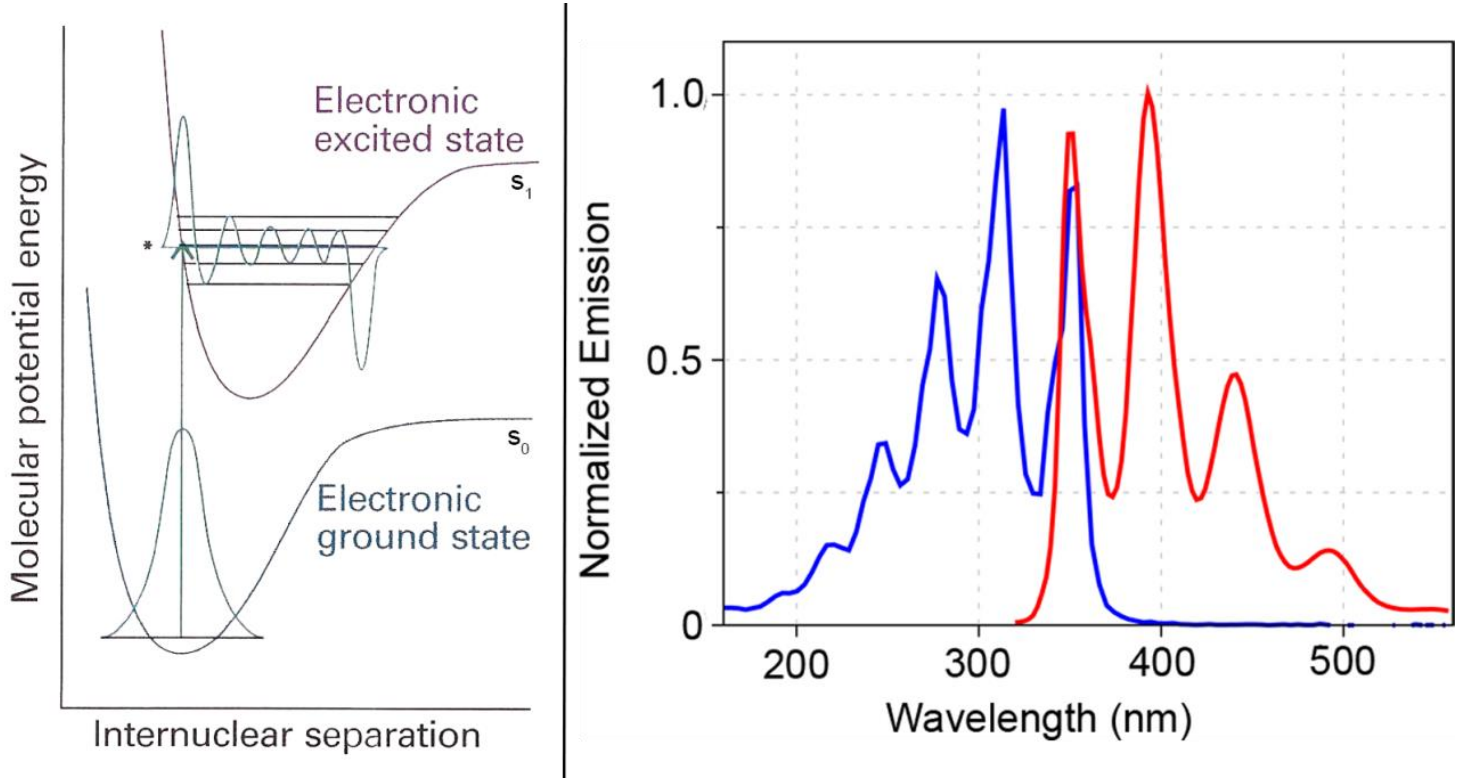

Figure 1-3: Left: electronic excitation between regions of maximum population probability according to the Franck-Condon principle; right: excitation and emission spectrum of anthracene. ${ }^{[10]}$

As stated earlier, virtually only the vibrational ground state of the electronic ground state $s_{0}$ is populated at standard conditions, as defined by the Boltzmann statistic. The wave function of the vibrational ground state of $s_{0}$ exhibits a symmetrical maximum at the center of the potential well, as depicted in Figure 1-3 (left). Because excitation is a fast process, the Franck-Condon principle presumes a fixed internuclear separation during the excitation process, which leads to a vertical transition of the electron (indicated in Figure 1-3 by a vertical arrow). Figuratively, every intersection of this transition arrow with a vibrational state of $s_{1}$ is a potential end point of the excitation process. Whether the transition into the respective energy state actually occurs is dictated by the wave function. Transitions preferentially occur between maxima of the wave functions of ground- and excited state, which produce a correspondingly high probability. ${ }^{[5,11]}$ Regions in which the wave function assumes low values lead to a low transition probability.

In the same way that the excitation process can populate different vibrational states of $s_{1}$, the emission from the ground state of $s_{1}$ can also proceed into different vibrational states of $s_{0}$, and the transition probabilities are again dictated by the wave functions. ${ }^{[6]}$ Therefore emission spectra also usually exhibit a vibrational band structure originating from different transitions, and often the vibrational structures of excitation and emission act like two mirrored images (Figure 1-3, right). Besides the emission of fluorescence, electrons can also return to the electronic ground state by 
non-radiative processes. Besides the already mentioned ISC, one other option is internal conversion (IC), ${ }^{[5]}$ in which overlap of high vibrational states of $s_{0}$ with the vibrational ground state of $s_{1}$ enables the transition of the electron. Another possibility is the collision of excited molecules, which may also lead to a non-radiative decay of the excited state, known as dynamic quenching. ${ }^{[7]}$ The effectiveness of the fluorescence process is described by the quantum yield. It is defined as the quotient of emitted (fluorescence) photons and previously absorbed photons and is a compound specific property. ${ }^{[7 \mathrm{~d}]}$ An ideal quantum yield would be 1.0 , where every absorbed photon produces an equivalent fluorescence photon, which has not been observed for any compound. Quantum yields above 0.5 can be considered high.

While nature has created materials and molecules which exhibit fluorescence and even living organisms have adopted and developed luminescence phenomena in the course of evolution, mankind has taken possession of this process by understanding its mechanisms and has utilized it for its own benefit. Today fluorescent compounds are omnipresent in every-day life, whether in the coating of neon tubes and energy saving lamps or in color pigments.

Apart from the preparation of fluorescent materials, the possibility of taking influence on the fluorescence mechanism has become increasingly important in the past decades. The ability of intentional on/off switching of fluorescence has made fluorescence based molecular switches and sensing devices accessible and has therewith created a whole new academic field. ${ }^{[12]}$ The different strategies pursued for the modification of the fluorescence mechanism will be presented in the following.

\subsection{Quenching Mechanisms}

In order to take influence on the fluorescence of compounds, they must be structurally altered by chemical synthesis. Furthermore, many strategies of manipulating fluorescence mechanisms are dependent on free movement and flexibility of the compound, which is only given in solution. ${ }^{[13]}$ These two criteria are only fulfilled by organic molecules, which is why they have by far overtaken inorganic materials in the context of fluorescence. Luckily, the large conjugated $\pi$ systems of many aromatic compounds are well suitable for the emission of fluorescence, as their HOMO/LUMO energy gaps are in the energetic range of visible light. ${ }^{[6]}$ In combination 
with multiple possibilities of derivitization, these aromatic compounds (often referred to as fluorophores) are ideal for designing fluorescent molecules. One of these fluorophores is anthracene, which plays a key role throughout this entire thesis. Its three aromatic rings offer ideal conditions for absorption of photons and its high quantum yield, moderate price and well accessible possibilities of derivitization further underline its attractiveness in this context. Its fluorescence was first described by Wiedemann and Schmidt who observed its strong blue emission in the gas phase. ${ }^{[14]}$

The majority of mechanisms which take influence on fluorescence properties are directed at on/off switching of emission. The "off-switching" of emission is mostly referred to as quenching and can be induced by molecules of eligible redox potential. The most established quenching mechanism is photoinduced electron transfer (PET). ${ }^{[12-13,15]}$ It has been utilized in countless sensing devices and molecular switches and is one of the best known processes in fluorescence research. ${ }^{[16]}$ High performance time resolved spectroscopy has even enabled the determination of lifetimes of involved intermediates. ${ }^{[17]}$ It is based on an electron transfer between a quencher and a fluorophore which leads to an interruption of the fluorescence mechanism. A simplified scheme of the PET process is depicted in Scheme 1-1.

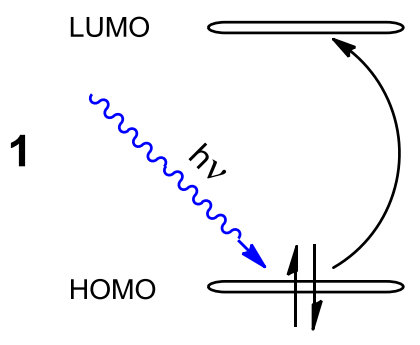

A

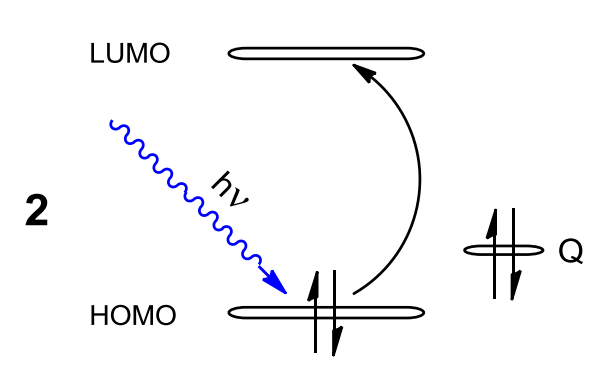

A

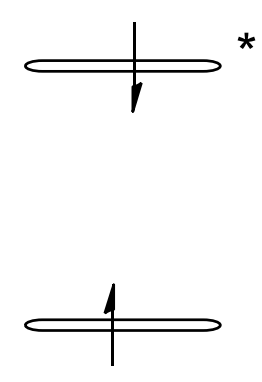

B

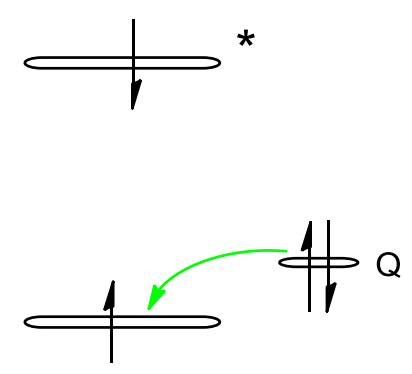

B

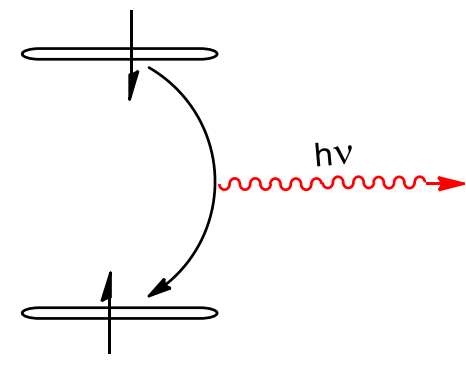

C

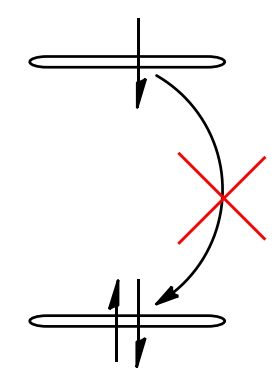

C

Scheme 1-1: 1 (top): regular fluorescence mechanism; 2 (bottom): quenching by photoinduced electron transfer (PET). 
The usual fluorescence pattern (Scheme 1-1, mechanism 1) of excitation of an electron to an excited electronic state (1B), followed by the emission of radiation and returning of the electron to the initial ground state $(\mathbf{1 C})$ produces a vacancy in the ground state orbital from which this electron was excited (Scheme 1-1, 1B). Although this vacancy only exists for a very short period of time (the lifetime of the excited state), it is sufficiently long to transfer an electron into this orbital (Scheme 1-1, 2B). The orbital is then fully occupied, which hinders the excited electron in returning to its ground state, and therewith prevents fluorescence emission. ${ }^{[13]}$ The transferred electron is supplied by a quencher molecule, which makes the quencher the electron donator and the excited fluorophore the electron acceptor in the described model. ${ }^{[18]}$ Although there are also examples in which the excited fluorophore is the electron donator which transfers the excited electron to a different acceptor, ${ }^{[13,19]}$ the model presented above is far more common, especially in regard to the anthracene fluorophore.

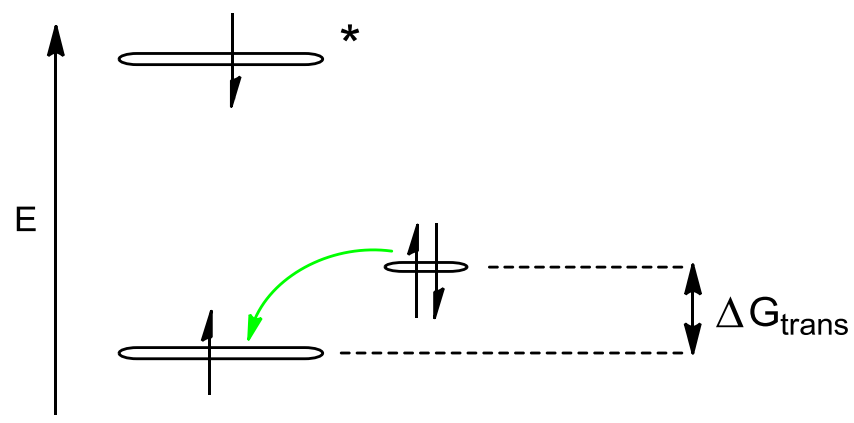

A

Scheme 1-2: Energetic contribution of the electron transfer from donor (D) to acceptor (A).

The driving force behind the electron transfer process is the redox potential of the involved compounds. Therefore an effective quencher must exhibit a slightly higher potential energy than the excited fluorophore in order to quench its emission. By transferring an electron, the energy difference between both states is released, making the transfer energetically favoured (Scheme 1-2). ${ }^{[13]}$ The possibility of calculating the redox potentials and HOMO/LUMO energies of fluorophores and quenchers has made the theoretical prediction of quenching and its effectiveness possible. ${ }^{[20]}$ The effectiveness of quenching can also be influenced synthetically, e.g. by introduction of cyano substituents to anthracene fluorophores which increases the electron affinity of the excited fluorophore, which subsequently improves its electron 
acceptor qualities. ${ }^{[7 d]}$ This in turn makes the electron transfer energetically more favourable and therefore higher transfer rates are achieved. For many aromatic fluorophores - and especially for anthracene - amines have been identified as effective quenchers. Redox potentials of several fluorophores and quenchers are compiled in Table 1-1.

Table 1-1: Redox potentials of selected fluorophores and quenchers. ${ }^{[13]}$

\begin{tabular}{cc|cc}
\hline \multicolumn{2}{c|}{ Fluorophores } & \multicolumn{2}{c}{ Quenchers } \\
\hline Compound & $E^{0}\left(\mathrm{~A} / \mathrm{A}^{-}\right)[\mathrm{V}]$ & Compound & $E^{0}\left(\mathrm{D}^{+} / \mathrm{D}\right)[\mathrm{V}]$ \\
Anthracene & -1.93 & Triethylamine & 0.96 \\
$p$-Benzoquinone & -0.54 & Indene & 1.52 \\
9,10-Dicaynoanthracene & -0.89 & N,N-Diethylaniline & 0.76 \\
1-Cyanonaphthalene & -1.98 & N,N-Dimethylaniline & 0.81 \\
Nitrobenzene & -1.76 & 1,3,5-Trimethoxybenzene & 1.49 \\
\hline
\end{tabular}

Besides suitable redox potentials, also the speed of the transfer process is crucial for effective quenching. In sight of the very short excited state lifetime, the electron transfer must be very fast. Fast transfer is best actualized by direct orbital overlap between electron donor and acceptor. ${ }^{[13]}$ This requires an optimal geometrical arrangement of both quencher and fluorophore. While quencher and fluorophore can be separate and independent compounds which achieve this overlap by approximation of two freely moving molecules in solution, ${ }^{[13]}$ a linkage between quencher and fluorophore has been shown to be far more effective. This way both moieties can be bonded in a pre-organized arrangement which benefits orbital overlap. These links between quenchers and fluorophores are called spacers.

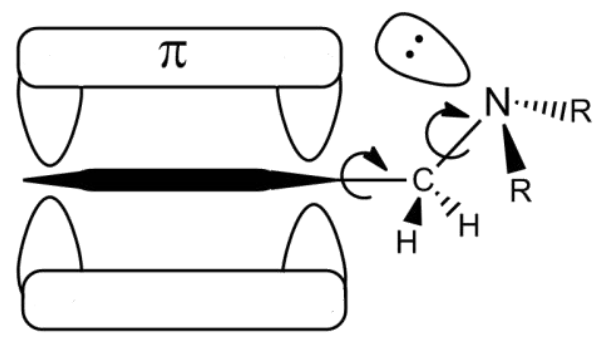

Scheme 1-3: Rotation of the quencher moiety around the single bonds of a methylene spacer for orbital overlap with the fluorophore $\pi$ system. 
Though different spacer concepts are known, including rigid spacers in which electron transfer is accomplished through the molecular orbitals of the spacer unit, ${ }^{[13]}$ most spacers are flexible alkyl chains. These may vary in length, but the most widely spread species is the simple methylene spacer. ${ }^{[15 d, 21]}$ By rotation of the quencher about the single bonds of the spacer, a high probability of orbital overlap with the fluorophore is produced, which leads to effective quenching (Scheme 1-1). The rotational rates around single bonds in solution are very high and range around $10^{12} \mathrm{~s}^{-1}$ which explains the high probability of transfer. The transfer itself is not irrevocable, the transferred electron can return to its origin. ${ }^{[7 \mathrm{~d}]}$ Even if the transfer to the fluorophore and back to the quencher both occurs within the excited state lifetime, it is still sufficient to induce effective quenching, as emission is statistically still largely suppressed. Especially for amine quenchers, methylene spacers have been shown to offer the most ideal geometry for electron transfer, which was discovered by direct comparison of quenching rates to those of analogous compounds with ethylene and propylene spacers. ${ }^{[22]}$ Besides supplying the desired flexibility and geometry, spacers also limit the interaction of the quencher substituent with the fluorophore to the mere electron transfer process. Independent of the quencher substituent introduced at the other end of the spacer, the emission wavelength of the fluorophore is not affected. Therefore the emission spectra of all anthracene derivatives containing alkyl spacers are virtually identical in shape and wavelength to that of 9-methylanthracene.

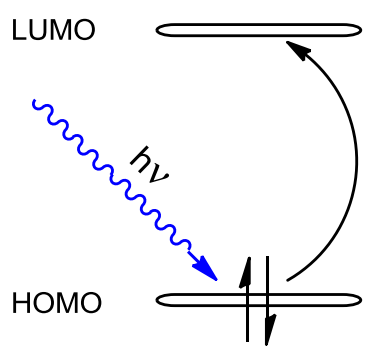

A
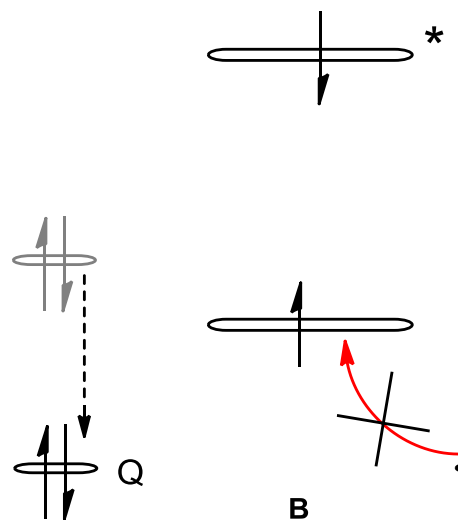

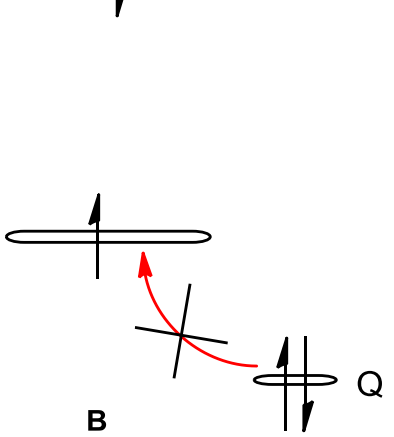

B

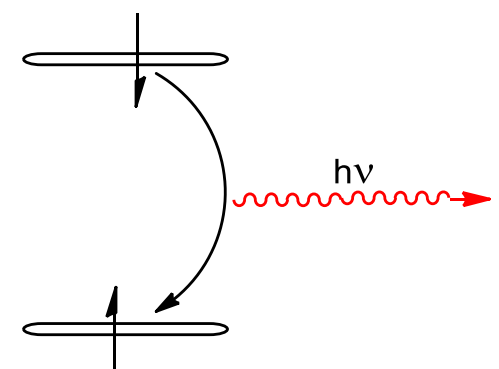

C

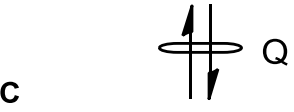

Scheme 1-4: Inhibition of the PET mechanism by bonding induced lowering the quencher's redox potential.

Now that quencher and fluorophore have been connected and arranged in an ideal geometry for quenching, a formerly fluorescent compound is now non-fluorescent. To benefit from the tediously developed mechanism, the PET must be switched off under 
defined conditions in order to recover the fluorescence of the compound. This can be achieved by lowering the redox potential of the quencher below that of the excited fluorophore (Scheme 1-4). Once this is accomplished, the electron transfer process is no longer energetically favoured and ceases immediately. ${ }^{[13,18]}$ For amines, the lone pair of the nitrogen atom acts as the electron donating quencher. By incorporating this lone pair into a bonding situation, which is achieved by interaction with an analyte, its redox potential is substantially lowered (Scheme 1-4, A). This inhibits the PET between amine and fluorophore and drastically enhances fluorescence emission. The synthesized compound is now a PET sensor molecule according to the quencherspacer-fluorophore concept (Figure 1-4). ${ }^{[23]}$

The first sensors of this kind carried simple tertiary amines and were used for the smallest and simplest of all analytes: the proton. ${ }^{[15 b]}$ Protonation of amines has the strongest lowering effect on their redox potentials and leads to the strongest enhancement of emission at low pH values.

By variation of the amine bound substituents the basicity of the quenchers were altered, which produced sensor molecules with different sensitivities for proton detection in different $\mathrm{pH}$ intervals. ${ }^{[21]}$ Besides protonation, also the coordination of metal ions can sufficiently lower the redox potential of amine quenchers. ${ }^{\text {[23a] }}$ The observed emission enhancements are further increased by the formation of chelate complexes which induce even stronger interactions between quenchers and cations. This phenomenon is referred to as chelation enhanced fluorescence (CHEF). ${ }^{[24]} \mathrm{A}$ sensor is not only defined by a clearly measurable yes/no signal (with a high on/off intensity ratio), the selectivity towards a single defined analyte is equally important.

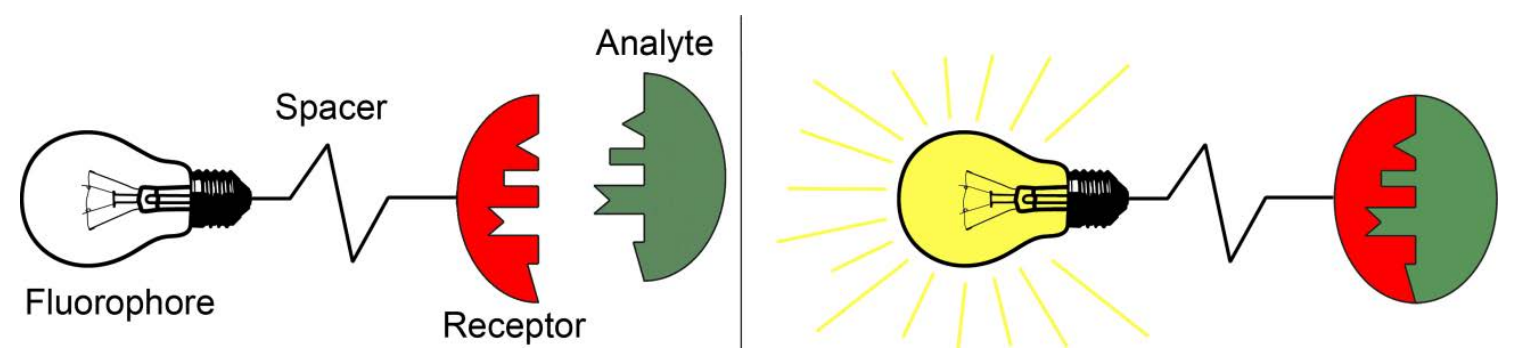

Figure 1-4: Chemical PET sensor according to the fluorophore-spacer-receptor concept; left: "off" mode, PET is active; right: "on" mode, PET is inhibited.

To improve the selectivity of quencher moieties towards particular cations, the synthesized quenchers became more and more complex and were thus referred to as 
receptor units which ideally function according to a lock/key principle while retaining the characteristics of a quencher.

With the fulfilment of selectivity in addition to the on/off switching of fluorescence, a compound can be classified as a fully functioning fluorescence chemosensor (Figure 1-4). The requirements for a chemical sensor are formulated in the corresponding IUPAC definition: ${ }^{[25]}$

A chemical sensor is a device that transforms chemical information, ranging from the concentration of a specific sample component to total composition analysis, into an analytically useful signal.

As emission of light is one of the fastest and most precisely detectable signals of all, this is also acknowledged by the IUPAC in a classification of sensors:

Optical devices transform changes of optical phenomena, which are the result of an interaction of the analyte with the receptor part. This group may be further subdivided according to the type of optical properties which have been applied in chemical sensors:

$[\ldots]$

d) fluorescence, measured as the positive emission effect caused by irradiation. Also, selective quenching of fluorescence may be the basis of such devices.

To date, countless sensor compounds have been developed on the basis of PET. Receptor units have become highly complex, incorporating crown ethers and cryptands into their structures for maximum selectivity towards cations. ${ }^{[23 c, 26]}$ But also PET sensors for various anions have been prepared ${ }^{[16 a, 27]}$ as well as sensing devices for larger molecules like sugars or even certain proteins. Also combinations of different receptors have been utilized, producing logic devices and more complex molecular switches. ${ }^{[28]}$ Due to the high sensitivity, even detection of single molecules is possible and sensors have been used in diverse environments. ${ }^{[29]}$ Even the introduction of sensor molecules into living cells has been realized e.g. to measure the dependency of certain biological processes on the concentrations of metal cations or $\mathrm{pH} .{ }^{[30]}$

In addition to the established PET mechanism, several new detection processes have been reported in the past years. ${ }^{[31]}$ Though they differ in their mechanistic details, they are - in the bottom line - all based on electron transfer phenomena. Also 
well-known and elaborately reviewed in literature is detection via charge transfer (CT). A distinction is made between intramolecular charge transfer (ICT), ${ }^{[32]}$ which mostly produces detectable shifts of emitted radiation and charge transfers processes between receptor ligand and analyte (LMCT or MLCT). ${ }^{[15 c, 33]}$ Transitions like these are known from various metal complexes, where these transitions often lead to intense coloring of the respective complexes, but they can also be applied in chemical sensing.

Lately, the mechanism of aggregation induced emission (AIE) has attracted attention. ${ }^{[34]}$ This is a process which is observed for molecules which are nonfluorescent in diluted solutions. By free rotation of their substituents radiationless relaxation of excited states is achieved. Only at high concentrations (which usually lead to quenching by increasing collision rates rather than emission enhancement ${ }^{[35]}$ ) a strong increase of emission is observed. ${ }^{[36]}$ By formation of aggregates, the free rotation of substituents is hindered and the non-radiative decay is interrupted, which leads to recovery of fluorescence emission. ${ }^{[37]}$ This effect is not limited to high concentrations of solutions, also the targeted formation of aggregates between sensor compounds and analyte molecules can produce this effect. This way not only cations $\left(\mathrm{Hg}^{2+}, \mathrm{Ag}^{+}\right)^{[38]}$ but also large biomolecules (ATP, DNA fragments) ${ }^{[39]}$ have been successfully detected. Also fluorescence altering mechanisms based on isomerization of $\mathrm{C}=\mathrm{N}$ double bonds have been reported, ${ }^{[40]}$ in addition to several others which have been sporadically applied.

\subsection{Solid State Fluorescence}

In identical manner as organic compounds have overpowered inorganic materials in the field of in-solution fluorescence, a development from strictly mineral inorganic luminescent materials towards organic luminophores could be observed in the past decades. $^{[41]}$ The growing ambition of developing organic materials with tuneable emission properties has led to numerous publications in this field of research.

While the dynamic processes and electronic effects which dominate luminescence properties in solution are comparatively well known, ${ }^{[12-13,23 c, 31]}$ the knowledge of mechanisms and parameters which influence solid state fluorescence of organic materials are for the most part diffuse. Assumptions regarding the influence of molecular interactions on fluorescence properties differ significantly depending on the 
described systems and are in some cases even contradictory. ${ }^{[42]}$ Most of the quenching processes which strongly influence the emission intensities of dissolved fluorescent compounds (as utilized in most sensor molecules) are dependent on dynamic rotation around bonds in solution to achieve the required orbital overlap for electron transfer to the excited fluorophore. ${ }^{[13]}$ In the solid state, these mechanisms no longer apply due to the rigidness of the molecules. Although electron transfer is also possible in the solid state, it is exceedingly rare and demands highly specific structural requirements. ${ }^{[13]}$ Hence, other parameters such as formation of excited dimers (excimers) $^{[43]}$ or exciplexes, ${ }^{[44]}$ packing effects, $\left.{ }^{[42 a, 4} 42 c, 45\right]$ and intermolecular interactions $^{[46]}$ often predominantly influence fluorescence properties of organic compounds in the solid state.

While fluorescence of organic compounds in solution is a common phenomenon, the occurrence of solid state fluorescence of organic compounds is generally considered as rare. ${ }^{[42 b, 42 c, 47]}$ First systematic investigations of the influence of packing on solid state fluorescence were performed by Langhals et al. on pigment dyes. ${ }^{[22 a, 45 a]}$ These led to several basic assumptions, in particular regarding the effect of $\pi-\pi$ interactions on emission intensities and quantum yields. Langhals and co-workers found that two modifications of one dye, which do not differ in their fluorescence properties in solution, differed significantly in the crystalline state in terms of emission intensity and position of the absorption maximum. They ascribed these deviations to differences in the interactions of the fluorophores. The modification which showed the shorter $\pi-\pi$ distance was weaker in its emission intensity and also exhibited the bathochromic shift stated above. Hence, these phenomena were ascribed to an emission supressing effect of strong $\pi-\pi$ interaction. The Diketopyrrolopyrrole pigment used for these experiments exhibits a fairly small $\pi$ system (Scheme 1-5).

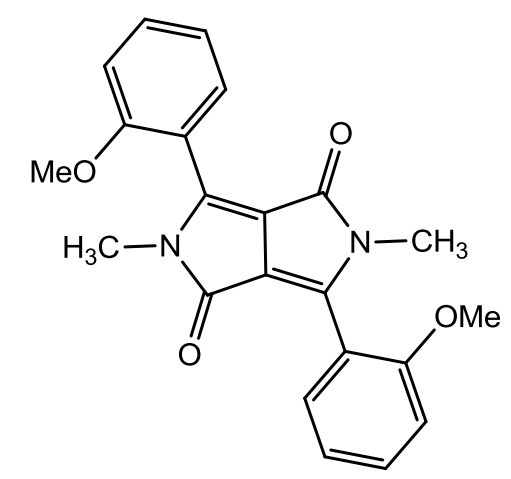

Scheme 1-5: Diketopyrrolopyrrole pigment used by Langhals et al. for the investigation of packing effects on solid state fluorescence properties. ${ }^{[42 a, 45 a]}$ 
Although this system is quite extraordinary compared to "simple" aromatic fluorophores like naphthalene of anthracene, these assumptions have become the basis of argumentation in the majority of subsequent publications. Hence, the conclusions drawn from research on these pigment dyes were afterwards applied to numerous other systems and fluorophores. Since then also contradictory effects of $\pi-\pi$ overlap have been reported. For example Dreuw et al. reported on a naphthalene derivative which exhibits strong solid state fluorescence despite short $\pi-\pi$ distances and large $\pi-\pi$ overlap of fluorophores in 2005. ${ }^{[42 b]}$ Though contradictory results have been repeatedly reported, the majority of publications follow the thesis of solid state fluorescence quenching by $\pi-\pi$ interaction. ${ }^{[48]}$

Of numerous utilized organic fluorophores, especially anthracene moieties have proven of value in countless fluorescent compounds. While the in-solutionfluorescence properties of many anthracene derivatives have been thoroughly described in literature (c.f. 1.1), a quite manageable number of publications address corresponding solid state fluorescence phenomena. From 2005 onward, especially the workgroup around Miyata and Tohnai has contributed several publications to this research topic. By synthesizing alkyl ammonium salts from 2,6-anthracenedisulfonic acid (2,6-ADS) and primary amines, they succeeded in altering the packing motifs of their fluorophores depending on the length of the ammonium alkyl-chain. ${ }^{[49]}$ By these different packing motifs, the luminescence properties of their compounds in the solid state were also significantly affected. Two different packing forms were observed: a strongly fluorescent two dimensional motif and a one-dimensional motif which was weakly fluorescent. The differences in the observed emission intensities were ascribed to weaker distortion of the anthracene fluorophore, which was determined by IR spectroscopy. Their research in this field was further expanded by alteration of the alkyl amines, leading to corresponding ammonium salts with varying steric demand. ${ }^{[50]}$ Also aromatic amines were used, which led to strongly fluorescent solids. ${ }^{[51]}$ Differences in quantum yields and emission wavelengths of the obtained structures were again assigned to fluorophore distortion and intermolecular distance of the fluorescent molecules within the respective packing motif.

Additionally, chiral amines were reacted with 2,6-anthracenedisulfonic acid. ${ }^{[52]}$ By addition of host molecules, ternary intercalation structures were formed with varying host-dependent fluorescence properties. Though in this case quenching effects 
induced by distortion or molecular contact were not addressed, the red-shifted solid state emission of one molecular arrangement was attributed to formation of an excimer complex. In a recent publication, the 2,6-ADS fluorophore was replaced by $1,8-A D S$, which made more complex structures with larger cavities accessible. ${ }^{[53]}$
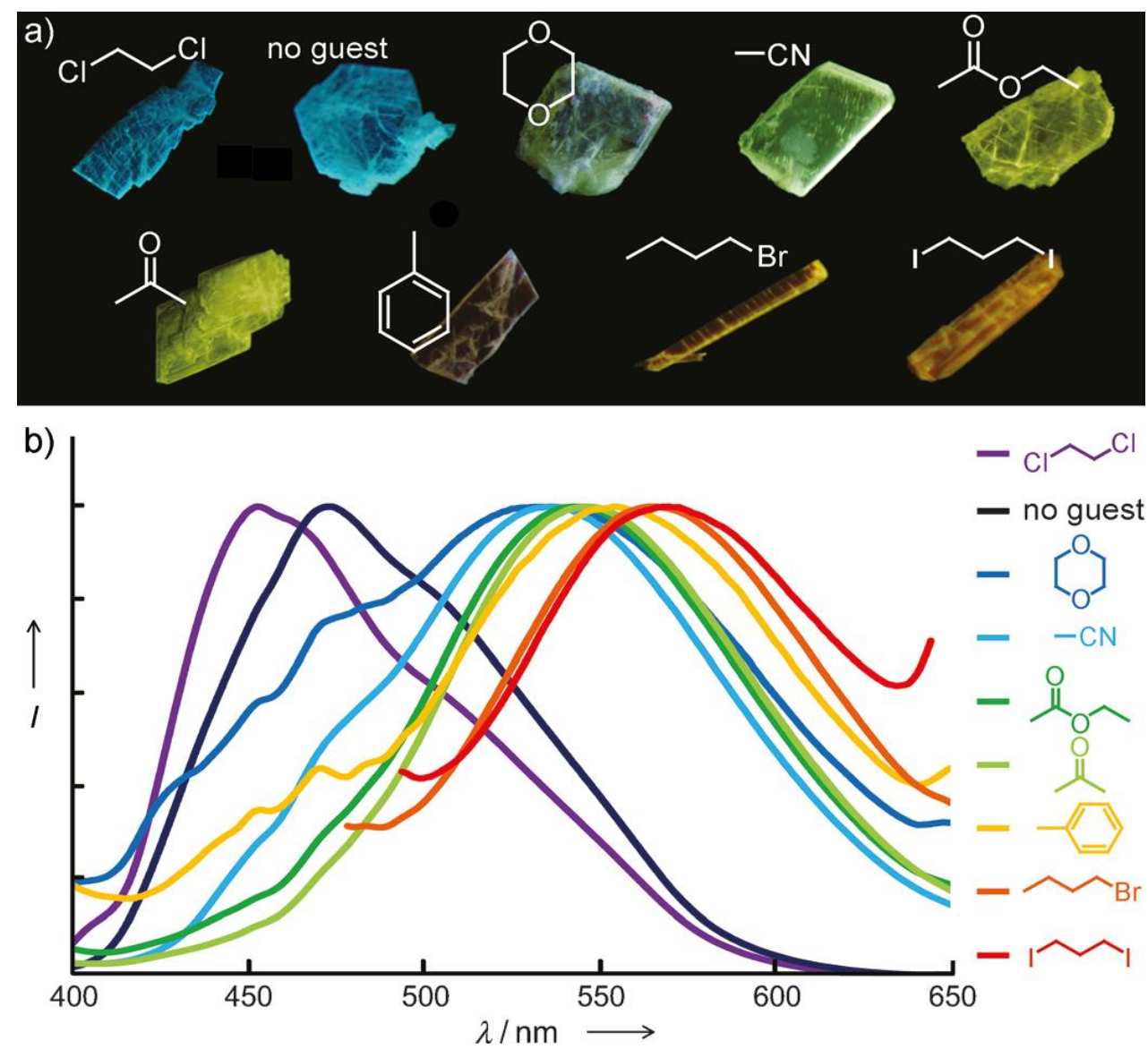

Figure 1-5: Host dependent shift of emission in the intercalation structure of 1,8-ADS and triphenylmethylamine (TPMA) by Hinoue et al. ${ }^{[53]}$

This again made the intercalation of various host molecules possible. Depending on the host, remarkable shifts of the emission maxima of up to $120 \mathrm{~nm}$ were observed (Figure 1-5), which were shown to correlate with the degree of $\pi-\pi$ overlap and the $\pi-\pi$ distances. Similar phenomena have also been reported in other publications. ${ }^{[50,54]}$

Furthermore, although mainly addressing photodimerization of anthracene derivatives in the solid state, the workgroup around Kohmoto also reported on several anthracene derivatives with remarkable solid state fluorescence properties resulting from packing induced intermolecular interactions. ${ }^{[55]}$ The strong emission and also notable red-shift of emission were reasoned to be caused by excimer emission. Finally, Fei et al. reported on a strongly fluorescent host/guest complex between 9,10- 
bis(diphenylthiophosphoryl)anthracene and toluene molecules. The strong fluorescence was ascribed to the formation of a T-shaped excimer between the guest molecules and the fluorophore. $\mathrm{C}-\mathrm{H} \cdots \pi$ bonding between host and guest was highlighted as one on the crucial factors for the formation of the fluorescent arrangement (for a detailed explanation of $\mathrm{C}-\mathrm{H}^{\cdots} \pi \pi$ bonding please see 3.2). ${ }^{[44 \mathrm{~b}, 44 \mathrm{c}]}$

Although argumentation and the resulting assumptions are conclusive in themselves in all publications described above, some results clearly contradict one another, especially in terms of the effects of $\pi$ - $\pi$-interaction on emission properties. Other factors which have repeatedly been referred to in this context, such as quenching due to distortion of aromatic fluorophores, or the role of $\mathrm{C}-\mathrm{H}^{\cdots}{ }^{\prime} \pi$ bonding are not considered in all publications. The alignment of all these points of argumentation is clearly an issue within the scientific field of solid state fluorescence and the contradictions and uncertainties require clarification.

\subsection{Scope}

In the light of the inconsistencies among the various hypotheses on the effects of structural properties on solid state fluorescence of organic compounds stated in 1.2, the investigation of the interdependencies of structural alterations and solid state fluorescence was a key aspect of this thesis. The development of a system for quantification of structural properties as a basis of the comparison of fluorescent compounds was targeted. Furthermore, suitable compounds for this comparison were to be synthesized and crystallized for the acquisition of their crystal structures. By alignment of the structural features with the acquired solid state fluorescence data, the derivation of requirements for - and principles of - solid state fluorescence was aimed for. This research was to be founded on phosphanyl- and phosphorylanthracenes, which have been a major exploratory focus of the Stalke group in the past years.

Furthermore, synthesis of metal complexes from phosphanyl and phosphorylanthracenes and transition metal cations was of interest. The investigation of the coordination modes of these compounds towards varying cations and monitoring the influence of complex formation on fluorescence properties in the solid state and in solution were major goals of this thesis. The exploration of pathways for 
the synthesis of functionalized phosphanylanthracenes for the development of chelating phosphanylanthracenes was also sighted.

Moreover, investigation of the photoinduced electron transfer (PET) mechanism was focused on. The synthesis of new receptor units and corresponding sensor molecules according to the receptor-spacer-fluorophore principle as well as monitoring their sensitivity towards different analytes was aimed for. By introduction of substituents to the fluorophore, the influence of secondary substituents on quenching mechanisms was to be explored. The possibility of uniting a sensor molecule with an emission altering second substituent was set as the ultimate goal in this context. Finally the feasibility of transferring the PET mechanism from amines to other quencher systems was to be verified.

Because phosphanylanthracenes, which are primarily addressed in the context of solid state fluorescence, all feature substituents which are directly bound to the fluorophore, and molecules which will be described with regard to the PET mechanism all bear spacers between the fluorophore and the main functional group, the structure of this thesis is will also be divided into two main parts. One chapter will be dedicated to molecules without spacers and one chapter will focus on molecules containing spacers in their structures. 



\section{FLUORESCENCE EXPERIMENTS}

For the detailed investigation of fluorescence properties of the synthesized compounds a versatile experimental setup which meets the requirements of the individual compound is essential. A potent fluorescence spectrometer equipped with compatible accessory devices is a requirement for the acquisition of reliable and high quality data. But the understanding of the functioning modes of these technical devices and the possibility of making adjustments to stock equipment are of equal importance for developing experimental conditions suitable for the investigation of heterogeneous compounds. Also recognizing and understanding the limits of the devices used is necessary. In the following the fluorescence spectrometer as well as the modifications made and the resulting experimental setup will be illustrated.

\subsection{Spectrometer}

The fluorescence spectrometer used was a HORIBA FluoroMax 4. HORIBA stands in a long tradition of building high quality optical devices and spectrometers. The FluoroMax 4 is equipped with a $150 \mathrm{~W}$ xenon arc lamp which emits a continuous spectrum of light. This light is focused and passes through an adjustable excitation entry slit which regulates the input intensity of the excitation beam. The beam is then focused onto a Czerny-Turner-monochromator for wavelength selection. ${ }^{\text {[56] }}$

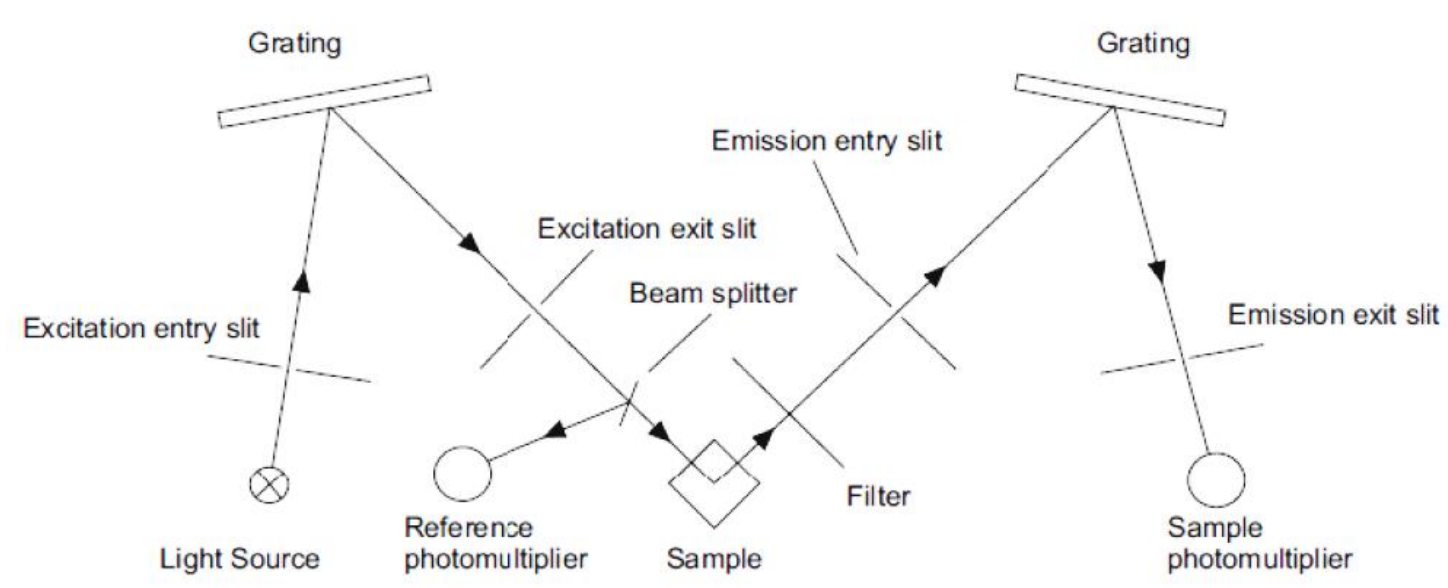

Figure 2-1: Simplified schematic beam path of a fluorescence spectrometer. 
This optical device is capable of accurate wavelength selection, in which high wavelength selectivity is achieved at the cost of light intensity. Accuracy of the selected wavelength and intensity of the excitation beam therefore stand in inverse proportion to one another. The approximately monochrome light is then directed to a beam splitter which directs a small partition of the excitation beam to a reference detector. Thereby the variability of light intensity emitted by the xenon lamp in the spectral range from $200-700 \mathrm{~nm}$ is compensated. The excitation beam is then directed to the experimental chamber where it passes through the fluorescence sample. In orthogonal orientation the emitted fluorescence light is detected. It passes through an adjustable emission slit and is focused on the emission monochromator. From there it is finally directed to the detection device which is a photomultiplier. A simplified scheme of the beam path is depicted in Figure 2-1. As the scheme shows, signal detection is only possible in orthogonal orientation to the primary beam. This allows no UV/VIS absorption measurements or related experiments which would require the primary beam to pass through the sample cell and also require a detection device in the direction of the primary beam.

The excitation- and emission slits of the spectrometer can be adjusted depending on the emission intensity of the respective sample to execute the measurement in the optimum operating range of intensity of the photomultiplier. This way overflow of the detector can be prevented and a suitable signal/noise ratio can be achieved even for weakly fluorescent samples. For comparability of emission intensities, the respective measurements must of course be carried out at identical settings of the excitation and emission slits.

\subsection{Titration Injector}

The titration injector is a device which can add preset volumes of solvents or analyte solutions to sample solutions or remove preset volumes from sample solutions. Both options can also be combined. It is driven by the spectrometer software and can be programmed to operate in an individually adjustable number of cycles in sync with the experimental strategy of the respective fluorescence experiment. 


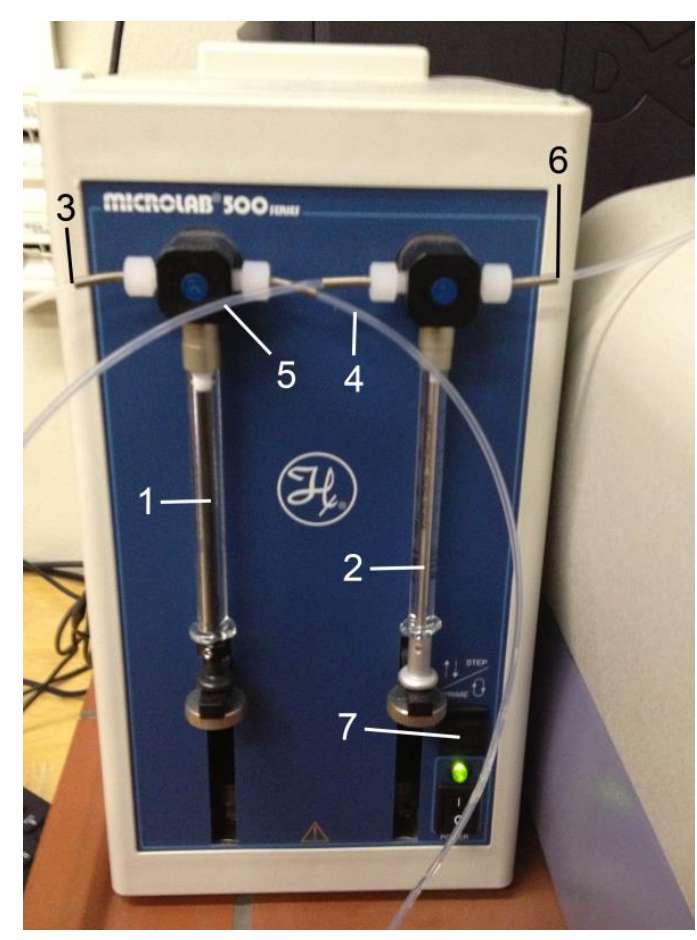

Figure 2-2: Hamilton Microlab 500 titration injector: 1: dispenser syringe, 2: aspirator syringe, 3 : dispenser syringe filling tube, 4: dispenser syringe dispenser tube, 5: aspirator syringe aspiration tube, 6: aspirator syringe dispenser tube, 7: manual control for "prime mode".

It has one $1000 \mu \mathrm{L}$ dispenser syringe and one $250 \mu \mathrm{L}$ aspirator syringe which are both operated by high precision stepping motors (Figure 2-2). This ensures a volume accuracy of $\pm 1 \mu \mathrm{L}$ for both syringes. ${ }^{[57]}$ The tubing consists of four Teflon tubes which can be connected to solvent or analyte supplying flasks or to the fluorescence cuvette. The dispenser syringe filling tube (3) is usually connected to a flask filled with solvent or analyte solution, the dispenser syringe dispenser tube (4) is connected to the cuvette to which this solution is added - usually through a septum (c.f. 2.3). The aspirator syringe aspiration tube (5) is also connected to the cuvette for removal of sample solution (c.f. 2.3), the aspirator syringe dispenser tube (6) is mostly used to discharged removed sample solution for disposal.

\subsection{Modifications and Setup}

Although several accessory devices - for example for solid state or low temperature experiments - are accessible for fluorescence spectrometers, virtually no equipment is available which allows experiments under inert conditions. Because several compounds which were synthesized and subjected to fluorescence investigations in 
the course of this work are air and moisture sensitive, accordant modifications of the stock equipment had to be carried out. Especially the low concentrations of the solutions used would lead to complete oxidation or decomposition of the parent compound, even in presence of small amounts of aerial oxygen. Therefore the development of technical solutions which are suitable for inert gas or Schlenk conditions ${ }^{[58]}$ were indispensable.

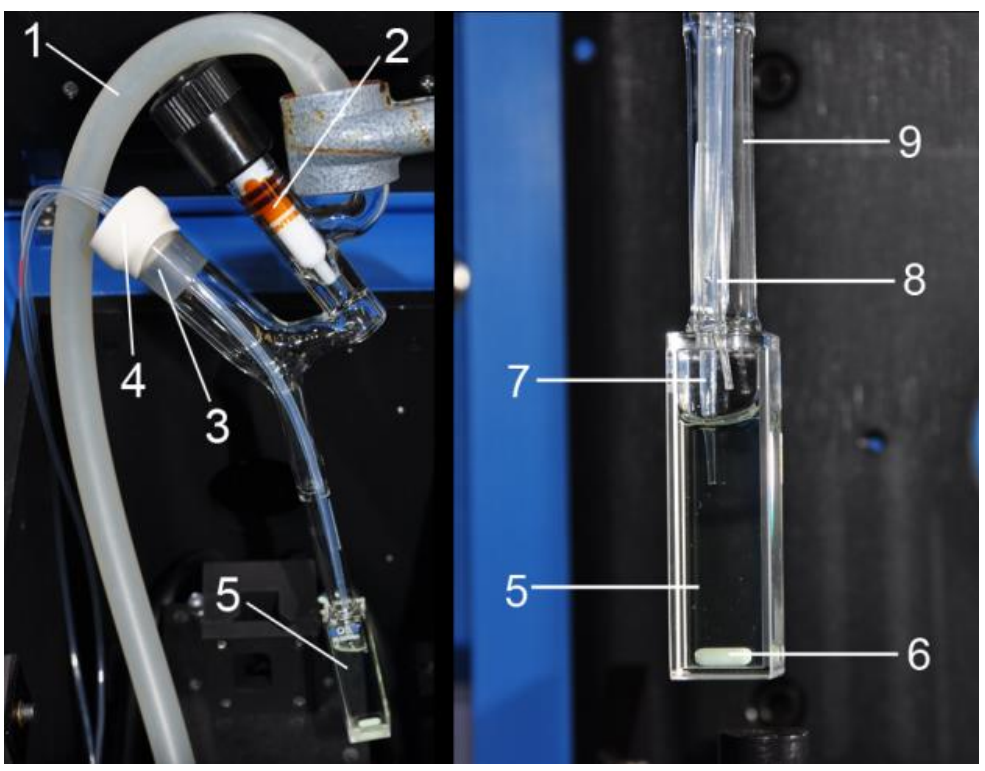

Figure 2-2: Schlenk-cuvette: Schlenk top piece (left) and sample cell (right). 1: silicone tube (for argon supply), 2: Teflon stopcock, 3: glass joint, 4: septum carrying titration injector tubing, 5: sample cell, 6: magnetic stirring bar, 7: aspirator tube, 8: dispenser tube, 9: shaft connecting to Schlenk top piece.

Regular fluorescence cuvettes have screw-on lids and are filled with sample solutions under ambient conditions, which makes exposure of the sample solutions to aerial oxygen inevitable. The main challenge was not only to develop a Schlenk-cuvette but also to ensure inert conditions when using titration injector tubing in dilution or titration experiments, sometimes over several hours. At the same time the exclusion even of diffuse light from the experimental chamber had to be retained at all times. Consequently, a Schlenk-cuvette was developed (Figure 2-3), which allows the infilling of sample solutions via syringe through a septum. The Teflon stopcock prevents the contamination of the sample with joint grease. After filling with sample solution the cuvette can be closed with a plug for regular fluorescence experiments or equipped with a septum carrying the dispenser tubing for titration experiments. As Figure 2-3 (right) shows, the aspirator tube (7) reaches into the sample solution in order to remove a preset volume from the sample cell which can subsequently be replaced by an equal volume of solvent or analyte solution through the dispenser tube (8). Because 
a septum may be porous and permeable towards air, a slight over pressure of inert gas must be present at all times during the experiment. Consequently, a Schlenk-line was installed as shown in Figure 2-5. To supply the required over pressure during titration experiments, inert gas has to accessible within the experimental chamber.

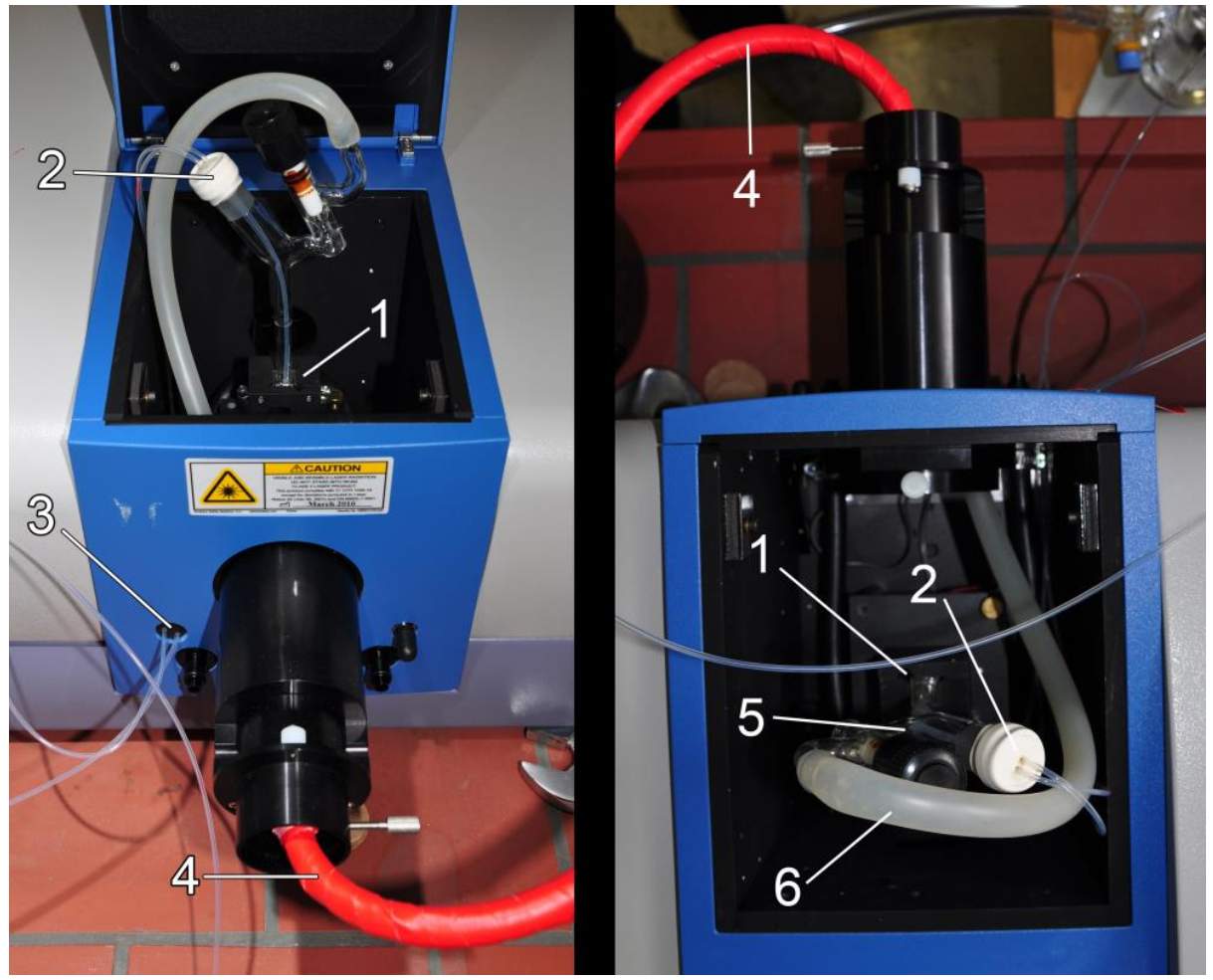

Figure 2-3: Experimental chamber front view (left) and top view (right): 1: sample holder (including magnetic stirring device), 2: septum carrying titration injector tubing, 3: introduction holes for titration injector tubing, 4: light shielding of the silicone tube, 5: Schlenk top piece, 6: silicone tube (for argon supply.

Constant pressure was actualized by using highly flexible silicone tubing which was put through already existing openings on the casing of the spectrometer, which were originally designed to carry optional additional equipment (Figure 2-5). The tubing was also wrapped in dark tape to prevent intrusion of light. The problems resulting from the distinctly larger dimensions of the Schlenk-cuvette compared to regular fluorescence cells were met by using a top piece on the experimental chamber which was originally built for a low temperature experimental setup. 


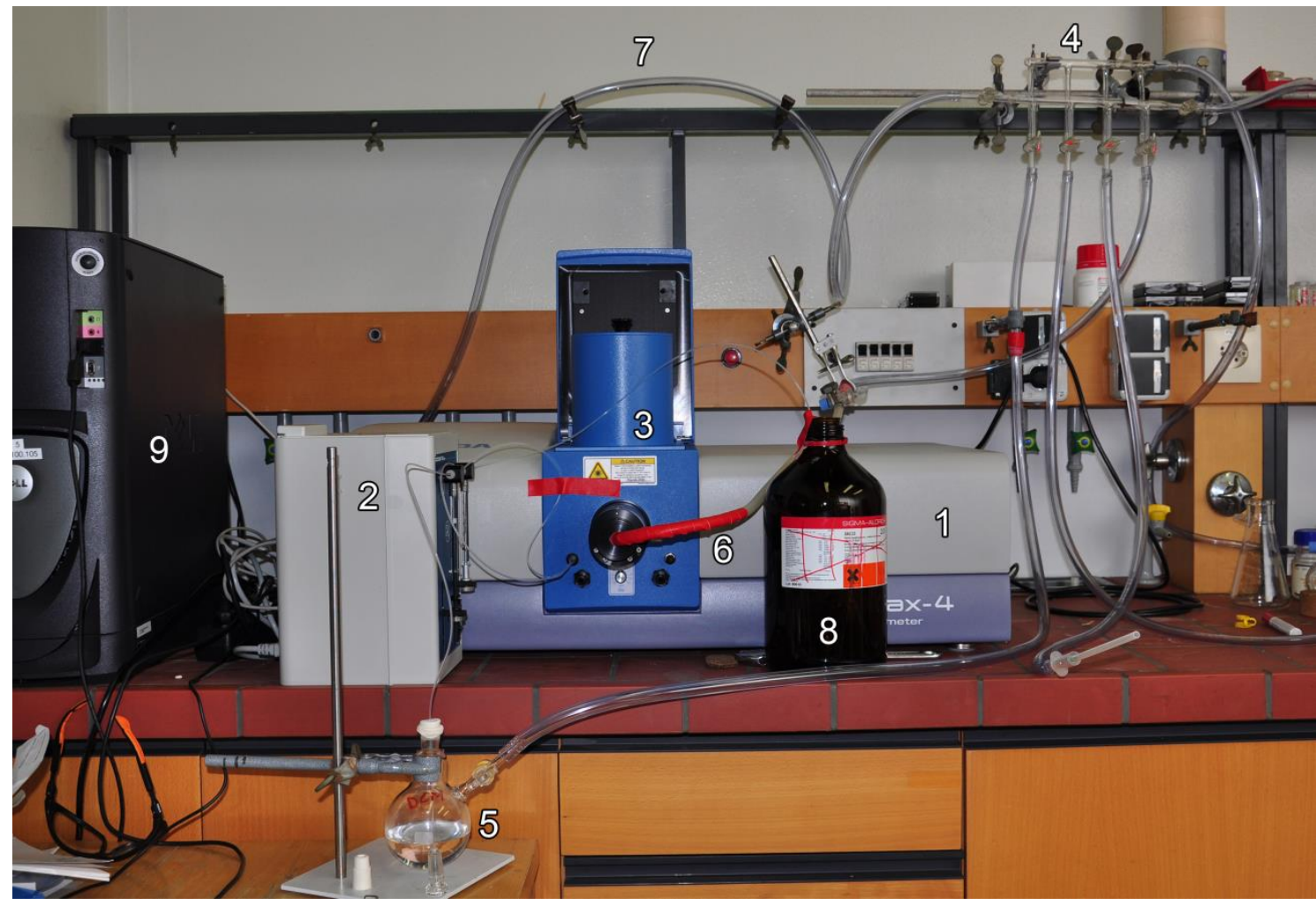

Figure 2-4: Experimental setup. 1: fluorescence spectrometer, 2: titration injector, 3: experimental chamber (with top piece) 4: Schlenk-line, 5: Schlenk-flask containing solvent or analyte solution, 6: light shielded silicone tubing, 7: argon supply, 8: solvent disposal, 9: computer for data collection.

The complete experimental setup as shown in Figure 2-5 allows the execution of all experiments under inert conditions. Dry and degassed solvents as well as solutions in Schlenk-flasks can be added to fluorescence samples for dilution and titration experiments and the constant over pressure of argon inside the sample cell prevents contamination with air at all times.

To remove aerial oxygen from the titration injector tubing and syringes, the entire unit was purged with argon for several minutes prior to each experiment by running several cycles of aspiring and discharging argon from a Schlenk-flask connected to the Schlenk-line ("prime mode"). This was followed by purging of the titration injector with the dry solvent or analyte solution used in the respective titration experiment. 


\subsection{Solid State Fluorescence Experiments}

The acquisition of solid state fluorescence data requires a completely different setup than the in-solution experiments described before. Because the excitation beam cannot pass through a solid sample, a so-called "front-face" setup is used, in which a solid sample is irradiated by the excitation beam. The fluorescence light emitted from the surface of this sample is then focused by a lens to a narrow beam, which is then directed to the emission slit of the spectrometer via mirror optics (Figure 2-6, right).

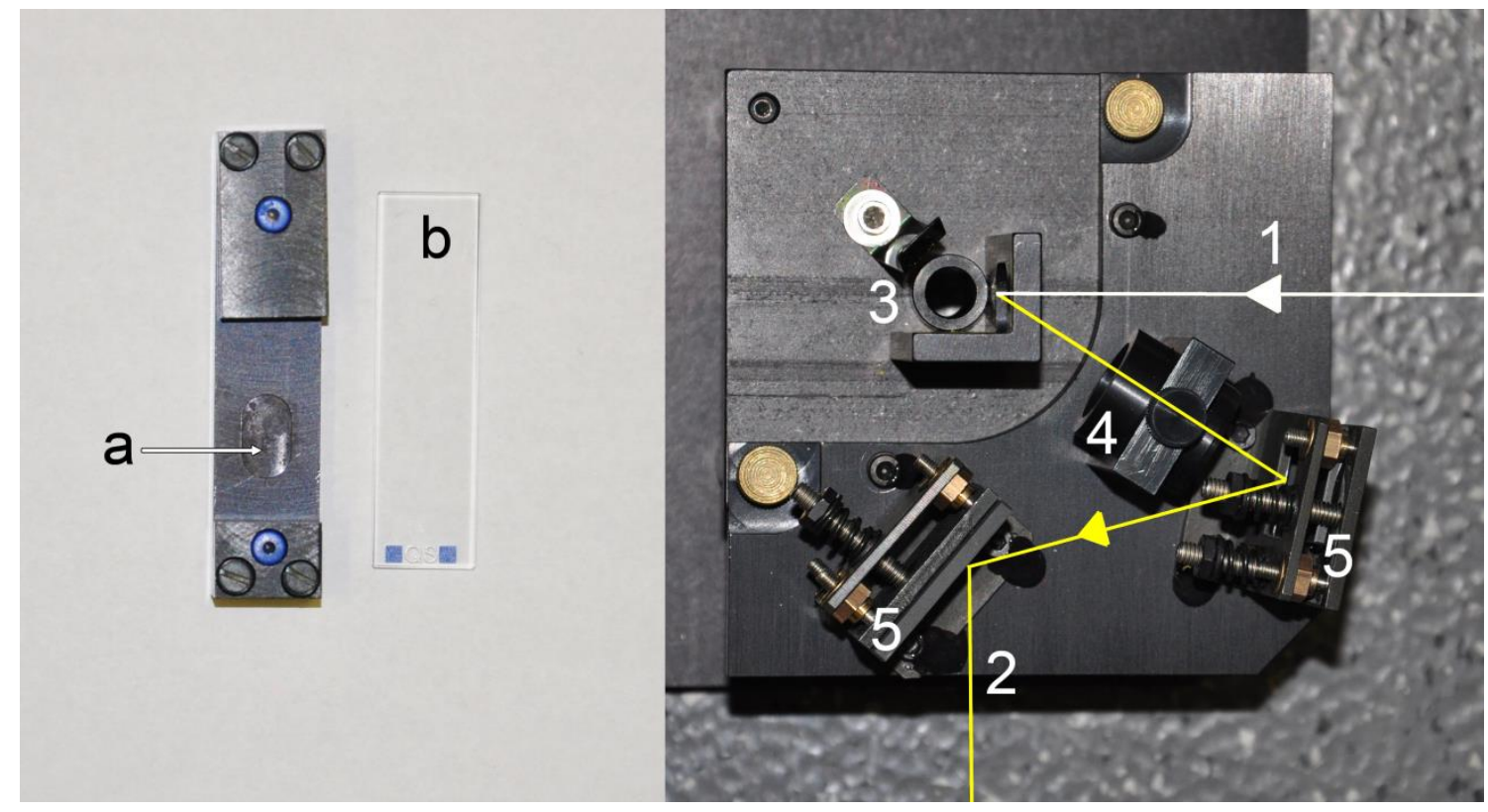

Figure 2-6: Left: solid state fluorescence sample cell; cavity (a), quartz glass cover (b); right: "front-face" experimental setup (top view): excitation beam (1), fluorescence light (2), sample holder (3), lens (4), mirrors (5).

The sample cell itself is a solid metal block with a small cavity into which the powder or microcrystalline sample is filled (Figure 2-6, left). The filled cavity is then covered with a quartz glass window to prevent the sample from falling out of the cavity when the sample cell is inserted into the sample holder in an upright position. Though the quartz glass window is pressed onto the metal surrounding the cavity, it is not air tight and air sensitive samples need to be prepared in an argon glove box and measured quickly after to prevent acquisition data which is falsified by decomposition.

Although this setup does not enable the acquisition of absolute emission values in the form of quantum yields, it does deliver very consistent data which are well suitable for comparison of relative emission intensities at identical experimental conditions. 
Because the determination of quantum yields e.g. using an Ulbricht sphere is often defective and may exhibit inaccuracies of up to $20 \%$, the emission intensities measured using the "front-face" setup can be considered at least equally accurate when comparing samples among one another. 


\section{Anthracene Derivatives Without Spacers}

As stated in the introduction, all compounds described in this thesis can be separated into two categories - molecules containing an alkyl spacer between the anthracene moiety and the main functional group or substituent, and those without spacers, with substituents bound directly to the aromatic ring system. In the following subchapters, the latter species of compounds will be addressed - most of them carrying phosphanyl or phoshoryl substituents.

All previous research in the Stalke group in this sector is founded on the work of Fei et al. who first recognized the potential of phosphoryl anthracenes for the development of fluorescent materials and solid state host/guest based sensing devices in 2003 . ${ }^{[46 \text {, }}$ 44c]<smiles>[R][PH]([R])(F)c1c2ccccc2c([PH]([R])([R])F)c2ccccc12</smiles>

a<smiles>[R][R]([R])(F)c1c2ccccc2c([PH]([R])([R])F)c2ccccc12</smiles>

b<smiles>[R]c1c2ccccc2c([PH]([R])([R])F)c2ccccc12</smiles>

c

$$
\begin{array}{lll}
\mathrm{E}=\text { lone pair, } \mathrm{O}, \mathrm{S}, \mathrm{Se} & \mathrm{E}=\text { lone pair, } \mathrm{S} & \mathrm{E}=\text { lone pair, } \mathrm{O}, \mathrm{S} \mathrm{Se} \\
\mathrm{R}={ }^{i} \mathrm{Pr}, \mathrm{Ph}, \mathrm{NMe}_{2}, \mathrm{NEt}_{2} & \mathrm{R}=\mathrm{Ph}, & \mathrm{R}={ }^{i} \mathrm{Pr}, \mathrm{Ph}, \mathrm{NMe}_{2}, \mathrm{NEt}_{2} \\
& \mathrm{R}^{\prime}={ }^{i} \mathrm{Pr}, \mathrm{Cy}, \mathrm{Ph} & \mathrm{R}^{\prime}=\mathrm{CH}_{3}, \mathrm{Br}, \mathrm{Cl}, \mathrm{NH}_{2}, \mathrm{H}
\end{array}
$$

Scheme 3-1: General structures of phosphoryl anthracenes.

Since then, numerous related compounds with varying substituents and of symmetric (Scheme 3-1, a) or asymmetric constitution (Scheme 3-1, b, c) have been synthesized and described. ${ }^{[59]}$ The initial aims of understanding requirements for - and mechanisms of - solid state and in-solution fluorescence of this compound class, as well as deriving findings and concepts for the development of sensor systems, had not yet been fulfilled. Therefore a thorough and systematic investigation of synthesis and fluorescence properties of new anthracene derivatives as well as of several previously 
published compounds were performed and will be documented in the following chapters with the aim of bringing light and insight into this vast and dark array of compounds and hypotheses.

\subsection{Fluorescence of Asymmetric Phosphanyl and Phosphorylanthracenes}

This first subchapter focusses on a series of compounds whose syntheses and crystal structures have - with few exceptions - been previously described in publications or PhD and diploma theses. These compounds were re-synthesized and subsequently subjected to fluorescence investigations. The goals of these investigations were not only the characterization and comparison of the fluorescence properties of these compounds, but also finding suitable experimental conditions for this whole compound class to ensure consistent quality and comparability of fluorescence data throughout this entire thesis. Understanding the influence of sample concentrations and compound specific properties as e.g. varying phosphane substituents, the chalcogens used for phosphane oxidation, and $+1 /-1$ substituents in 10-position is of great importance for choosing the optimal experimental conditions for each compound. Because to date very little is known about the fluorescence properties of phosphanyl and phosphoryl anthracenes, and existing reports are rather vague, a fairly basic and general approach was chosen to develop a basis of knowledge to found the following chapters on.

\subsubsection{Synthesis}

All following compounds were prepared according to literature procedures. ${ }^{\text {[59a] }}$ Introduction of substituents was achieved by replacement of the halogen substituents (Scheme 3-2). This was facilitated by selective mono-lithiation ${ }^{[60]}$ of the respective bromo anthracene in diethyl ether at $-15^{\circ} \mathrm{C}$ and subsequent reaction with the respective electrophile. Compounds $1-6$ and 13, 14 have been described by Stern, ${ }^{[59 c,}$ ${ }^{61]}$ and compounds 7-10 have been published by Schwab ${ }^{[59 a, 59 b]}$. Thus, only $\mathbf{1 1}$ and $\mathbf{1 2}$ are new compounds which have not yet been previously synthesized. 


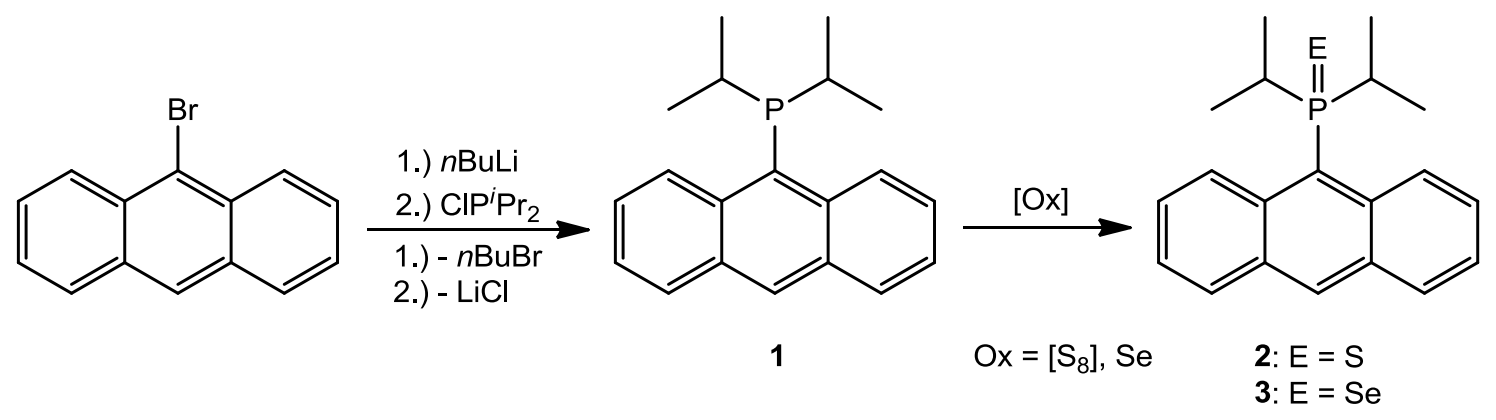

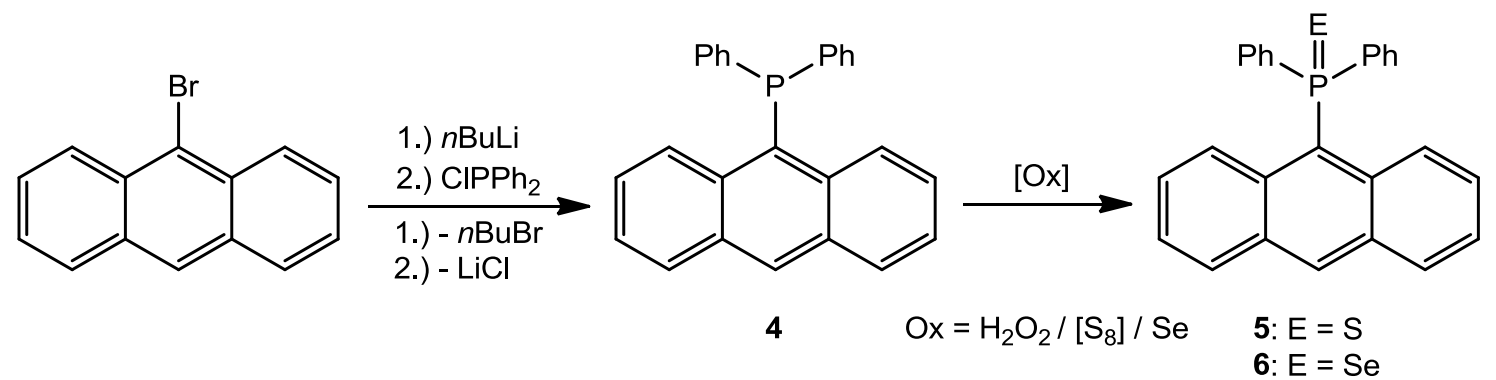

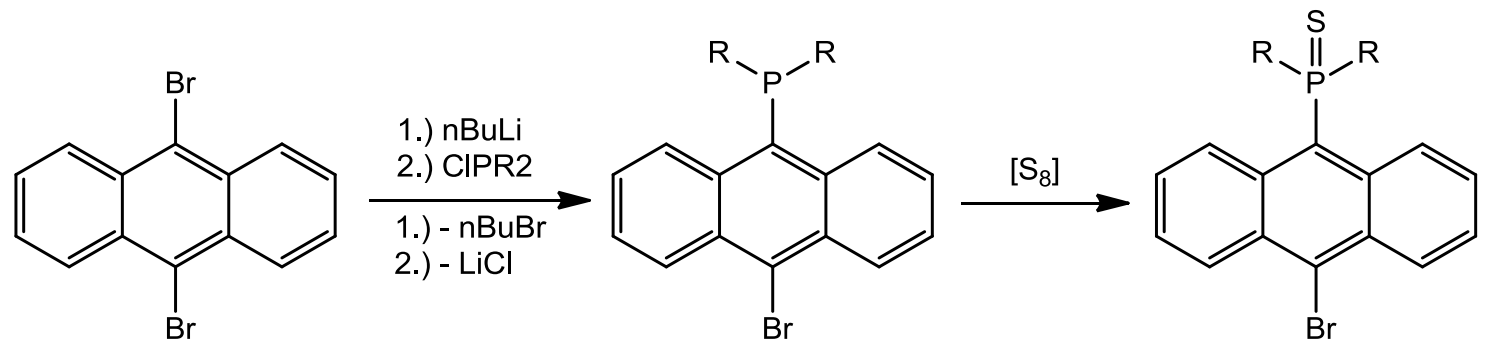

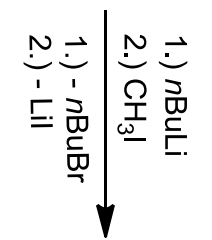<smiles>Cc1c2ccccc2c(Br)c2ccccc12</smiles>

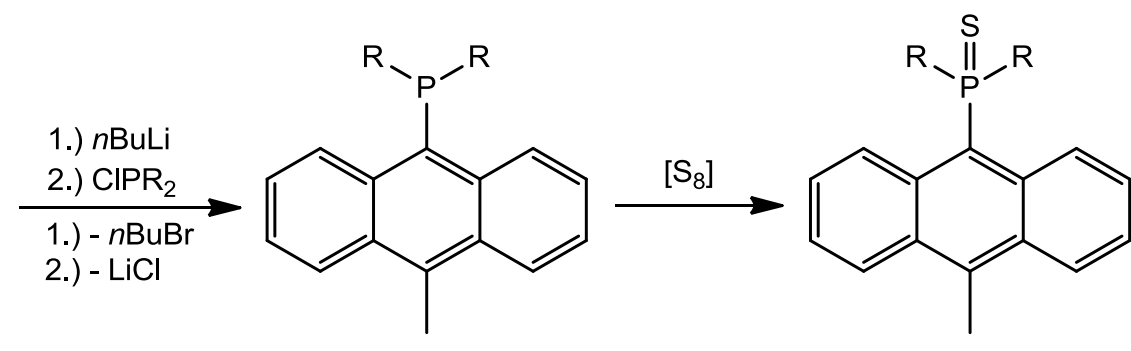

11: $\mathrm{R}={ }^{i} \mathrm{Pr}$

12: $\mathrm{R}={ }^{i} \mathrm{Pr}$

13: $\mathrm{R}=\mathrm{Ph}$

14: $\mathrm{R}=\mathrm{Ph}$

Scheme 3-2: Synthesis of 1-14.

Accruing lithium salts were removed by aqueous work-up (introduction of methyl groups in 10-position) or filtration (introduction of phosphane substituents). 
Oxidations generating phosphorus(V) compounds were carried out by refluxing the unoxidized compounds with elemental sulfur or selenium in toluene over $6 \mathrm{~h}$.

All compounds were recrystallized for purification as even small impurities can strongly affect the resulting fluorescence spectra. Because possible byproducts or unreacted starting materials are most likely also anthracene derivatives, their fluorescence phenomena may overlay or interfere with those of the actual product, causing major alterations and - in consequence - deficient results. Therefore high purity of the investigated compounds had top priority. All compounds were recrystallized from toluene, except for $\mathbf{1}, \mathbf{4}, \mathbf{7}, \mathbf{9}$, and $\mathbf{1 1}$, which were recrystallized from DCM. The crystals or precipitates were isolated by filtration and dried under reduced pressure. Purity of the products was monitored by ${ }^{31} \mathrm{P}$ - and ${ }^{1} \mathrm{H} N M R$ experiments. Unfortunately, $\mathbf{8}$ and $\mathbf{1 2}$ could not be obtained in sufficient purity for fluorescence experiments.

\subsubsection{Fluorescence Properties and Substituent Effects}

\section{a) Sample Concentrations}

Every fluorescent compound shows a more or less characteristic dependency of observed fluorescence emission on the concentration of the particular sample. While changes in the wavelength of emitted radiation are quite rare, the influence of sample concentration on emission intensity is striking. In general there is a long list of factors which may influence the fluorescence properties of a molecule in solution, starting with the solvent itself. Polarity ${ }^{[62]}$ and viscosity ${ }^{[63]}$ of the solvent, as well as its redox potential $^{[13]}$ and even ambient temperature ${ }^{[64]}$ can affect the fluorescence of the dissolved compound. Though factors like these should be taken into account when interpreting observed phenomena, the properties of each individual compound analyzed usually outnumber the effects induced by solvent properties.

The most elementary precondition for the acquisition of fluorescence data is the solubility of the analyte in the used solvent. Though sample concentrations are mostly very low, the analyte must be completely dissolved to generate representative data. Because solubility is dependent on factors as polarity of analyte and solvent, a suitable solvent has to be determined in advance of the fluorescence experiment. Comparability between different compounds without limitations is only possible if the 
solvent used for each of the compared compounds is identical. In this chapter all compounds are of aromatic nature and showed good solubility in DCM, which made the choice of the solvent obvious. Nevertheless, solubility is an important factor in the relation between sample concentration and observed fluorescence. Furthermore, factors as for example color (and therewith absorption) of the analyte are very important when describing the dependency of emission intensity on sample concentration. Though one would intuitionally assume a proportional increase of observed emission intensity with rising sample concentration, the characteristic absorption of the particular analyte leads to a contrary effect with increasing sample concentrations. As illustrated in Figure 3-1 (right), a low sample concentration allows the primary excitation beam to pass through the sample without notable absorption.
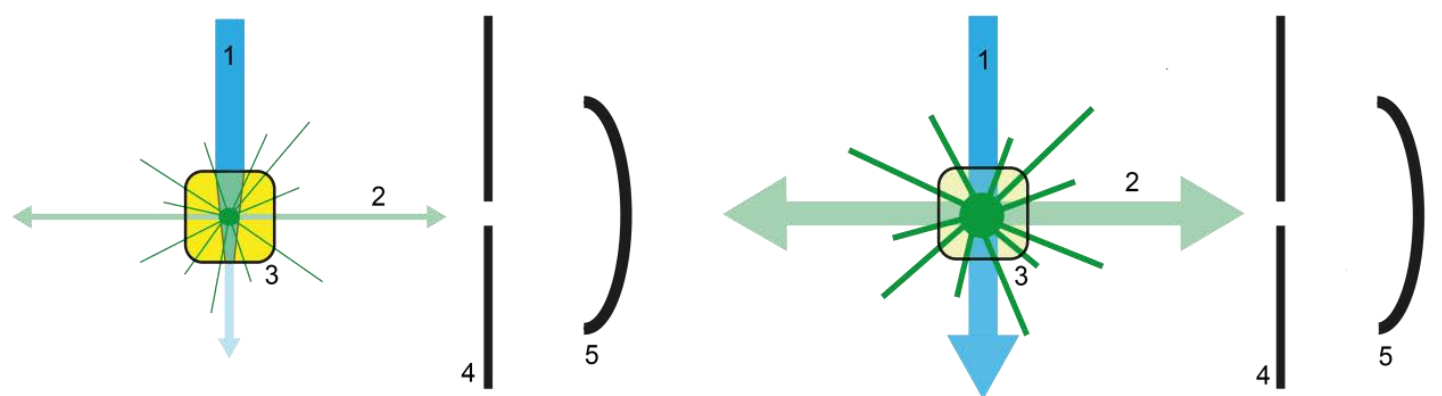

Figure 3-1: Diagram of the excitation beam passing through samples of high concentration (left) and low concentration (right); 1: excitation beam, 2: emitted light, 3: sample cell, 4 emission slit, 5: detector.

This ensures homogeneous excitation of dissolved molecules within the path of the excitation beam throughout the sample cell. This is important, because the emission of the resulting fluorescence light is also homogeneous and the detected emission is representative of the entire sample. In contrast, a sample of high concentration or a solution of a strongly absorbing compound of moderate concentration leads to inhomogeneous excitation of molecules along the path of the excitation beam. As a result the majority of photons are absorbed by the sample solution after passing through only the first few millimeters of the sample (Figure 3-1, left). Hence, only molecules in in this peripheral region of the sample cell are excited, while the molecules in the central regions of the cell are not, due to the small number of remaining photons passing through the entire sample. Because the emitted light is in fact detected at the center of the sample cell, the measured intensity is not representative of the sample and is distinctly weaker than the intensity observed for lower concentrated samples. This explains the observation that the measured emission 
of highly concentrated samples is weaker than of diluted samples of the same compound, despite the larger number of fluorescent molecules contained in samples of high concentration.

The second effect which also links sample concentration and fluorescence emission is the collision of excited fluorophores. When excited fluorophores collide with one another or with ground state fluorophores, the excited molecules mostly return to their ground state via non-radiative decay pathways. ${ }^{[7 d]}$ With rising sample concentration the probability of collision within the sample increases. This may lead to notable fluorescence quenching, especially when the collision factor reaches values in the range of the reciprocal value of the excited state lifetime. ${ }^{[11]}$ This effect contributes to fluorescence suppression at high sample concentrations in addition to the absorption effects mentioned before. When the concentration of a sample is low enough, absorption and quenching via collision become negligible and the measured intensity is only dependent on the quantum yield of the compound and the number of molecules in the path of the excitation beam which results in a linear relation between measured intensity and sample concentration.

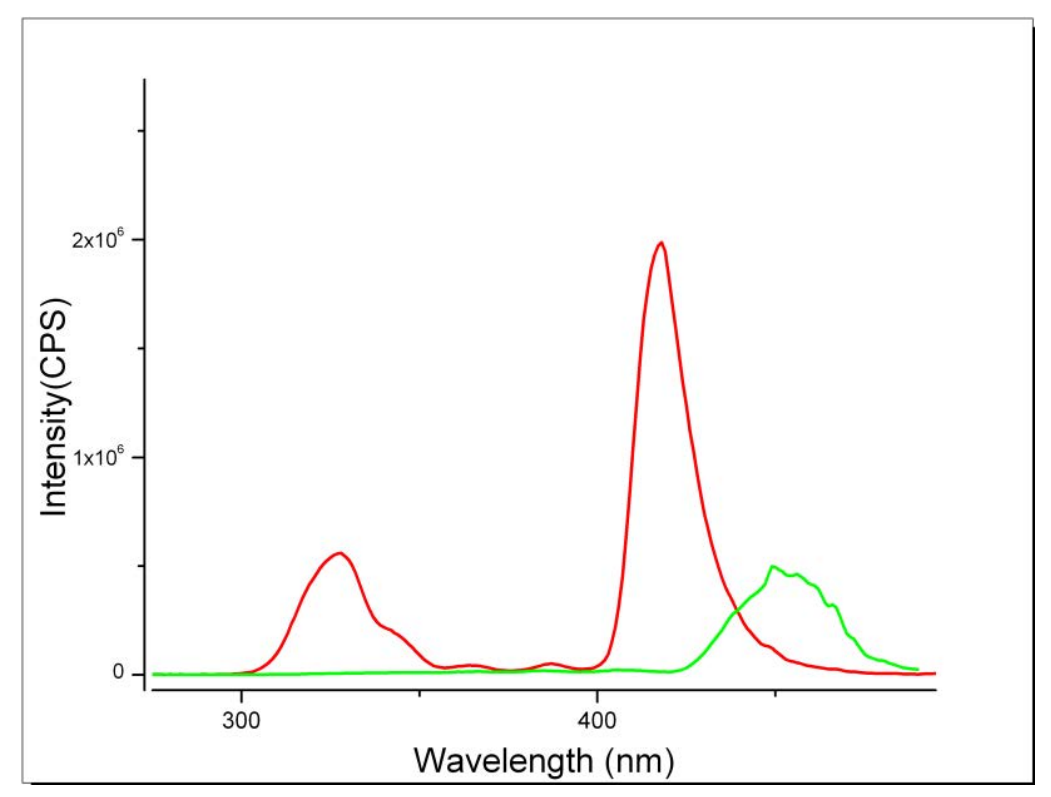

Figure 3-2: Excitation spectrum of HAnPPh2 (4) at $10^{-2} \mathrm{M}$ (green) and $10^{-3} \mathrm{M}($ red).

In practice it is useful to experimentally determine the range in which sample concentration and emission intensity decrease in approximately linear proportion by dilution experiments for each group of related compounds. Later experiments can then be carried out at suitable concentrations which make effects e.g. of sample 
dilution on observed emission assessable. The dilution experiments were conducted using the titration injector. A sample solution with a defined concentration was filled into the sample cell and the injector tubing was attached so the aspirator tube reached into the sample solution, as shown in Figure 2-3. The cell was equipped with a magnetic stirring bar to assure homogeneous mixing of the sample after every dilution cycle. By acquisition of an excitation fluorescence spectrum, a suitable excitation wavelength was chosen. After the measurement of an emission spectrum at the starting concentration, the injector was programmed to remove a volume of $150 \mu \mathrm{L}$ of sample solution per cycle using the aspirator syringe and replace it with the identical volume of pure solvent through the dispenser syringe, retaining a constant volume. After a preset delay time of $15 \mathrm{~s}$ for mixing of the sample, the next emission spectrum was recorded. This cycle was repeated up to 300 times, generating datasets of concentration dependent emission spectra.

This experiment was inter alia performed with $\mathrm{HAnPPh}_{2}(4)$ to represent the unoxidized compounds, $\mathrm{HAnPSPh}_{2}$ (5) as a representative of the sulfur oxidized compounds, and $\mathrm{HAnPSePh}_{2}(6)$ to represent selenium oxidized compounds.

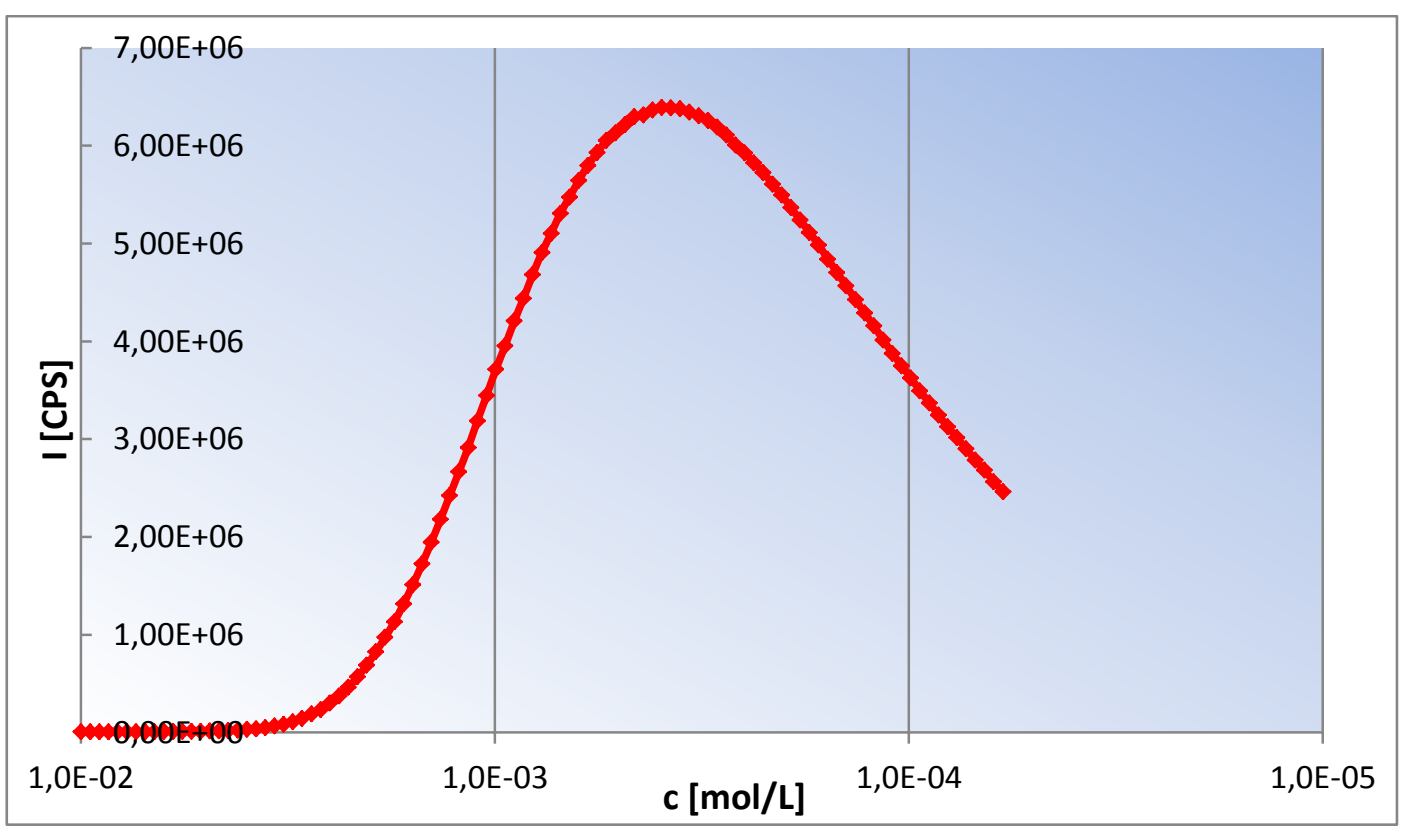

Figure 3-3: Concentration dependent emission of $\mathrm{HAnPPh}_{2}(4)$ at emission maximum (462 nm).

As figures 3-3 to 3-5 show, the experimental results comply very well with the theoretical assumptions made before. All compounds investigated exhibit very weak measured emission intensities at high concentrations. At $10^{-2} \mathrm{~mol} / \mathrm{L}$ fluorescence is 
nearly completely quenched in all cases, mainly due to absorption of the excitation beam within the peripheral layers of the sample. As the sample becomes more diluted, the measured intensity rises rapidly as absorption rate and collision factor within the sample fall. The emission intensity then eventually reaches a maximum, which is often plateau-shaped, with nearly constant emission intensities. The fact that in this range of concentration the observed emission intensity remains constant can be explained by two competing effects: the emission enhancing consequences of decreasing collision factor and absorption are still present, but not as significant as at higher sample concentrations.

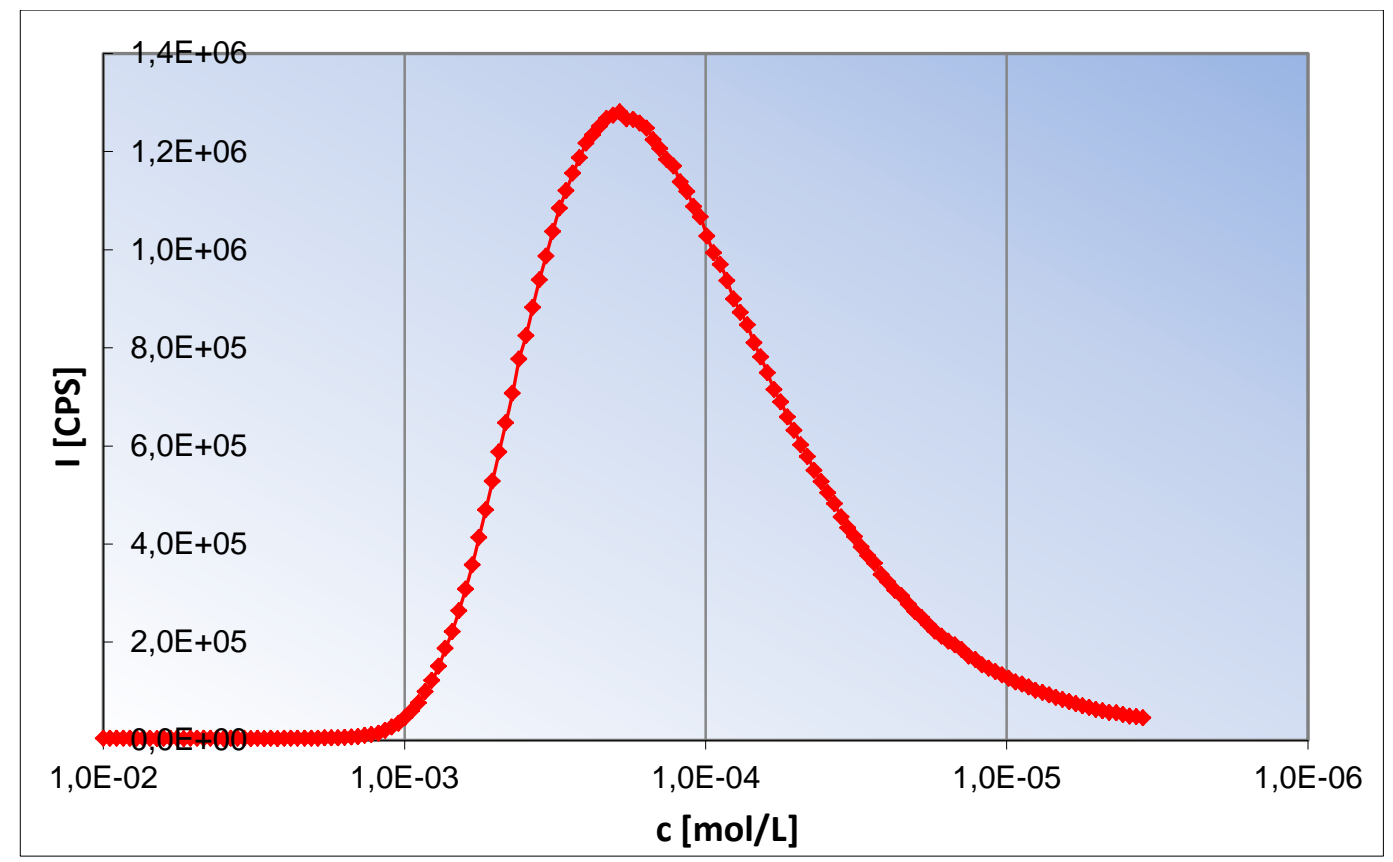

Figure 3-4: Concentration dependent emission of $\mathrm{HAnPSPh}_{2}(5)$ at emission maximum (465 nm).

At the same time the number of fluorescent molecules in the beam path is continuously decreasing with every dilution cycle, which again diminishes the number of total emitted photons. When the sample has reached a concentration at which absorption and collision of excited molecules become negligible, the detected emission intensity falls in proportion to the sample concentration (and therewith in proportion to the number of fluorescent molecules within the path of the excitation beam). This is the desired range of concentration for fluorescence experiments, because changes in emission intensity caused by dilution can be extrapolated and other influences are insignificantly small. 


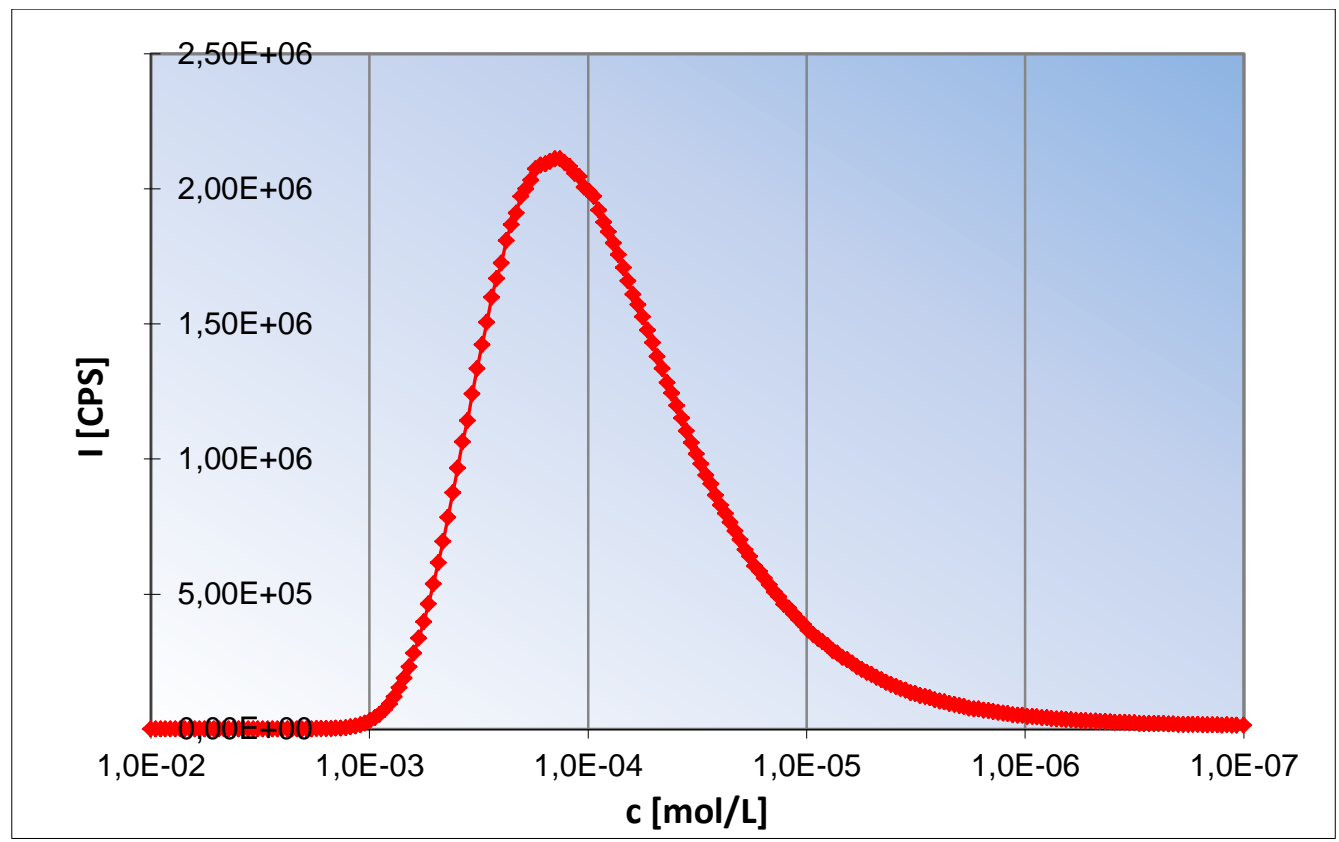

Figure 3-5: Concentration dependent emission of $\mathrm{HAnPSePh}_{2}(6)$ at emission maximum (466 nm).

Table 3-1: concentrations of maximum emission $\left(C_{\max }\right)$ and starting concentrations of linear decay $\left(C_{\text {linear }}\right)$.

\begin{tabular}{lll}
\hline & $\mathrm{C}_{\text {max }}[\mathrm{mol} / \mathrm{L}]$ & $\mathrm{C}_{\text {linear }}[\mathrm{mol} / \mathrm{L}]$ \\
\hline $\mathrm{HAnPPh}_{2}(4)$ & $4.2 \cdot 10^{-4}$ & $3.1 \cdot 10^{-4}$ \\
$\mathrm{HAnPSPh}_{2}(5)$ & $2.0 \cdot 10^{-4}$ & $1.5 \cdot 10^{-4}$ \\
$\mathrm{HAnPSePh}_{2}(6)$ & $1.5 \cdot 10^{-4}$ & $1.0 \cdot 10^{-4}$ \\
\hline
\end{tabular}

Figures 3-3 to 3-5 also underline that the concentration from which onward the observed emission decreases linearly with declining sample concentration differs strongly depending on the investigated compound. While for $\mathrm{HAnPPh}_{2}$ (4) this point is already reached at a dilution of $\sim 3 \cdot 10^{-4} \mathrm{~mol} / \mathrm{L}$,

$\mathrm{HAnPSPh}_{2}(5)$ requires a significantly stronger dilution of $1,5 \cdot 10^{-4} \mathrm{~mol} / \mathrm{L}$ to reach this point. HAnPSePh 2 (6) even requires a dilution of $1 \cdot 10^{-4} \mathrm{~mol} / \mathrm{L}$ (Table 1-1). As stated before, this can be ascribed to the characteristic absorption of the sample. While a solution of $\mathbf{4}$ is of very light yellow color, a solution of $\mathbf{5}$ or $\mathbf{6}$ is intensely yellow at identical concentration. Except for strongly absorbing compounds, sample concentrations of $10^{-4} \mathrm{~mol} / \mathrm{L}$ or less are suitable for the majority of compounds, which will be used as a reference value throughout this thesis.

\section{b) Substituent Effects}

By introducing substituents to the anthracene fluorophore, a multitude of the resulting compound's properties can be influenced. The molecule's polarity and solubility can be adjusted to allow applications ranging from lipophilic to hydrophilic 
environments, depending on the intended utilization of the fluorescent compound. Chelating receptor units and quencher moieties can be introduced, generating sensor molecules or switching devices. Apart from these countless possibilities, the alteration of electronic properties of the fluorophore via anthracene bound substituents is of great interest for designing of fluorescent compounds. The possibility of influencing the redox potential (and therewith electron acceptor properties) as well as shifting the excitation and emission wavelengths of the anthracene derivative are primary research targets - in solid state and in solution. The effects of substituent alterations on fluorescence properties will be investigated by comparison of 1-14 among one another and to the respective precursor molecules.

To gain a general understanding of the influence of substituents in 9- and 10position of the anthracene fluorophore on emission properties, the various starting materials used in this thesis were subjected to fluorescence investigations. The monoand di-substituted anthracene derivatives carry simple halogen or methyl substituents, as shown in Scheme 3-3. The substituents can be classified as electron pushing $+1-$ substituents (methyl group) or electron withdrawing -I-substituents (halogen atom).<smiles>c1ccc2cc3ccccc3cc2c1</smiles>

HAnH<smiles>Cc1c2ccccc2c(Br)c2ccccc12</smiles>

$\mathrm{MeAnBr}$<smiles>Cc1c2ccccc2cc2ccccc12</smiles>

HAnMe<smiles>Brc1c2ccccc2c(Br)c2ccccc12</smiles>

$\mathrm{BrAnBr}$<smiles>Brc1c2ccccc2cc2ccccc12</smiles>

$\mathrm{HAnBr}$<smiles>BrCc1c2ccccc2c(Br)c2ccccc12</smiles>

$\mathrm{BrAnCH} \mathrm{H}_{2} \mathrm{Br}$

Scheme 3-3: starting materials and corresponding abbreviations.

The starting materials were divided into mono-substituted compounds carrying a hydrogen atom in 10-position opposite of the substituent (HAnH, HAnMe, HAnBr), and compounds carrying a bromine substituent in 9-position, representing the disubstituted species ( $\mathrm{HAnBr}, \mathrm{MeAnBr}, \mathrm{BrAnBr}, \mathrm{BrAnCH} \mathrm{CH}_{2} \mathrm{Br}$ ). 


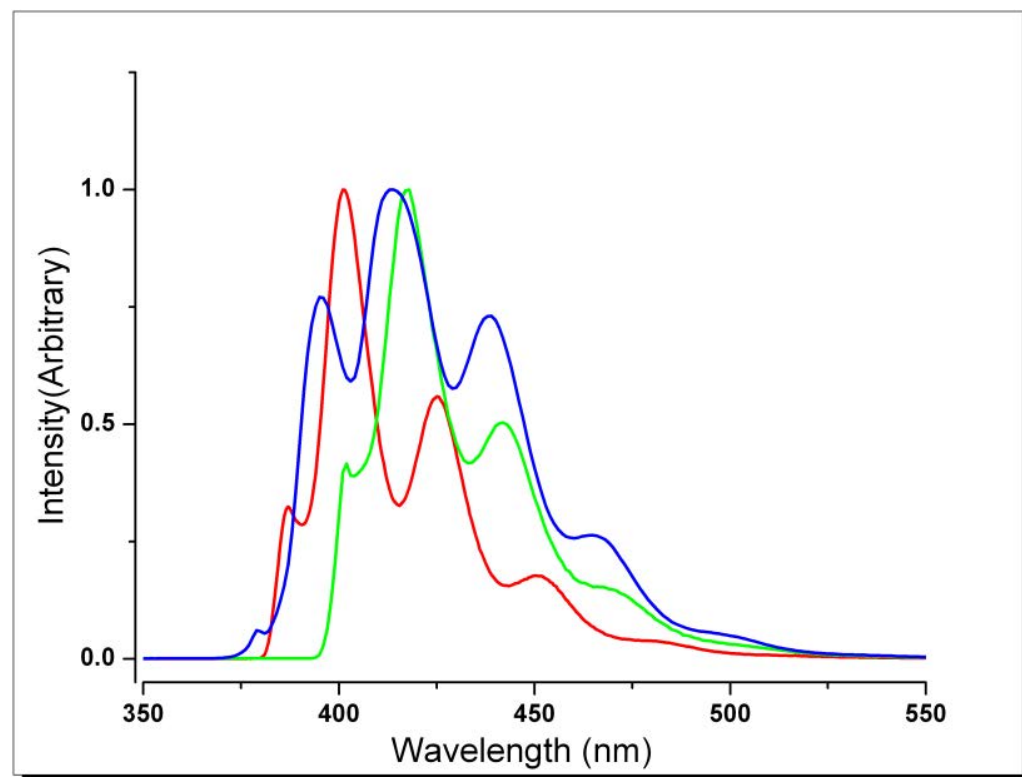

\begin{tabular}{lc}
\hline & $\lambda_{\max }[\mathrm{nm}]$ \\
\hline $\mathrm{HAnH}$ & 401 \\
HAnMe & 414 \\
$\mathrm{HAnBr}$ & 418 \\
\hline
\end{tabular}

Figure 3-6: normalized emission spectra of HAnH (red), HAnMe (blue), and

$\mathrm{HAnBr}$ (green).

Figure 3-6 (red) shows the emission spectrum of un-substituted anthracene with its characteristic vibrational structure consisting of four emission bands. By introduction of a substituent the intensity ratio of the four emission bands is altered, leading to a slightly different shape of the spectrum. Additionally, a red shift of emission for both the $+\mathrm{I}(13 \mathrm{~nm})$ and -I-substituent $(17 \mathrm{~nm})$ can be observed.

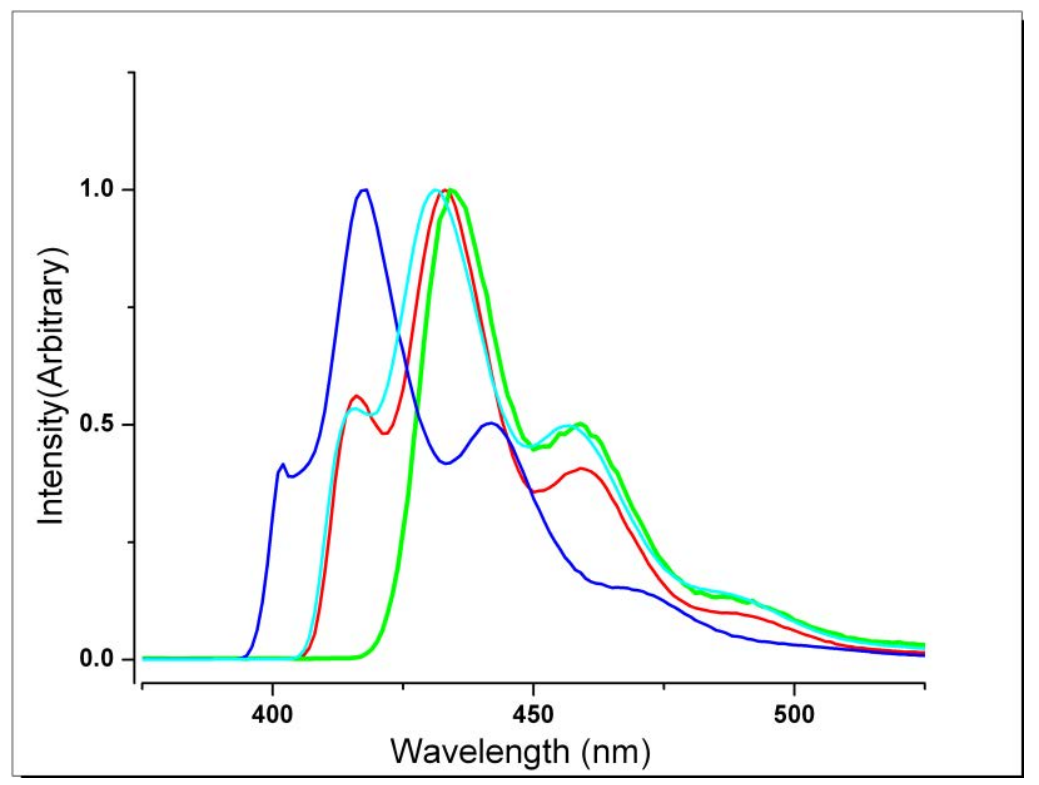

\begin{tabular}{lc}
\hline & $\lambda_{\max }[\mathrm{nm}]$ \\
\hline $\mathrm{HAnBr}$ & 418 \\
$\mathrm{MeAnBr}$ & 431 \\
$\mathrm{BrAnBr}$ & 433 \\
$\mathrm{BrAnCH}_{2} \mathrm{Br}$ & 434 \\
\hline
\end{tabular}

Figure 3-7: Normalized emission spectra of $\mathrm{HAnBr}$ (blue), $\mathrm{MeAnBr}$ (cyan), $\mathrm{BrAnBr}$ (red), and $\mathrm{BrAnCH}_{2} \mathrm{Br}$ (green). 
Similar tendencies are found for the second group of starting materials. The introduction of a second substituent to $\mathrm{HAnBr}$ again leads to a red shift of the emission maximum of $\sim 15 \mathrm{~nm}$ accompanied by minor alterations of the shape of the emission spectrum. Since all di-substituted compounds show nearly identical emission wavelengths, no clear tendencies regarding the influence of $+/-$ l effects can be derived. While the marginal difference in maximum emission wavelengths observed for the mono-substituted $\mathrm{HAnMe}$ and $\mathrm{HAnBr}$ is also found for the di-substituted $\mathrm{MeAnBr}$ and $\mathrm{BrAnBr}$ (the compounds bearing more -I substituents showing the stronger bathochromic shift), this tendency is contradicted by the emission maximum observed for $\mathrm{BrAnCH}_{2} \mathrm{Br}$ (Figure 3-7). Though the -I effect of the bromomethyl substituent can be considered clearly weaker than of a bromo substituent bound directly to the fluorophore, the observed emission maximum of $\mathbf{B r A n C H}_{2} \mathbf{B r}$ is red-shifted even stronger than that of $\mathrm{BrAnBr}$. Hence, the only trends recognizable are increasing bathochromic shift with rising number of substituents (un-substituted < monosubstituted < di-substituted) and increasing bathochromic shift with increasing molecular weight of the compounds. Compared to the consequences of substituent introduction, the latter effect is weak. As the diagram in Figure 3-8 (right) underlines, there is no linear dependency of emission wavelength on molecular weight of the investigated compounds because the leaps triggered by substituent introduction (indicated by arrows in the diagram) clearly outnumber the red-shift of emission induced by increasing molecular mass (indicated by line plots in the diagram).
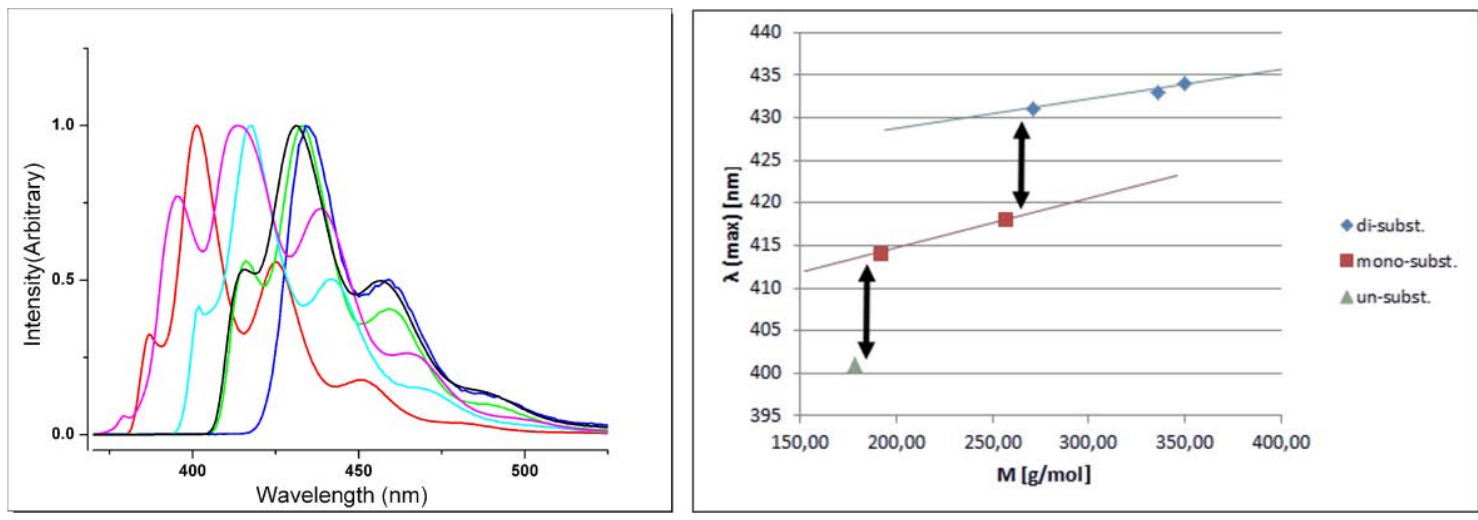

Figure 3-8: Left: Normalized emission spectra of all starting materials; right: lot of molecular weight vs. maximum emission wavelengths.

Though heavy atoms in fluorescent compounds are known to induce red-shifts of emission, this does not appear to be the cause of the observed phenomena, since 
$\mathrm{MeAnBr}$ and $\mathrm{BrAnBr}$ show very similar maximum emission wavelengths, while the latter compound contains the double number of heavy bromine atoms. The consequences of general substituent introduction are however noticeable. The observed red-shifts of emission indicate the sensitivity of the fluorophore's electronic properties towards substituents in 9,10-position, which in consequence lower the HOMO-LUMO gap. The smaller energy difference between these orbitals leads to emission of lower energetic photons, which is again reflected by the longer emission wavelength. Though an emission-shift of $\sim 30 \mathrm{~nm}$ is not very strong, it was achieved in spite of the simple substituents used so far. This denotes that the introduction of a suitable substituent can very well alter the emission properties of anthracene derivative distinctly, leading to tunable emission wavelengths.

To understand the influence of more complex substituents, the phosphanyl and phosphoryl anthracenes whose synthesis was described in 3.1.1 were examined.

From this point onward, the compounds will be referred to as

\begin{tabular}{|c|c|c|}
\hline $\mathrm{HAnP}^{i} \mathrm{Pr}_{2}(\mathbf{1})$ & $\mathrm{HAnPSePh}_{2}$ (6) & $\operatorname{MeAnP} P^{i} \operatorname{Pr}_{2}$ (11) \\
\hline $\mathrm{HAnPS}^{i} \mathrm{Pr}_{2}(\mathbf{2})$ & $\mathrm{BrAnP}^{i} \mathrm{Pr}_{2}(7)$ & $\mathrm{MeAnPS}^{i} \mathrm{Pr}_{2}$ (12) \\
\hline $\mathrm{HAnPSe}^{i} \mathrm{Pr}_{2}$ (3) & $\mathrm{BrAnPS}^{i} \mathrm{Pr}_{2}(\mathbf{8})$ & $\mathrm{MeAnPPh}_{2}(\mathbf{1 3})$ \\
\hline $\mathrm{HAnPPh}_{2}(4)$ & $\mathrm{BrAnPPh}_{2}(9)$ & $\mathrm{MeAnPSPh}_{2}$ (14) \\
\hline $\mathrm{HAnPSPh}_{2}(\mathbf{5})$ & $\mathrm{BrAnPSPh}_{2}(\mathbf{1 0})$ & \\
\hline
\end{tabular}

Like the starting materials before, these compounds can also be separated into monosubstituted and disubstituted species. This way the effects of the second substituent can be monitored. Additionally, phosphanes carrying aromatic (phenyl) or aliphatic (iso-propyl) substituents were used, as well as different chalcogens. This generates a large number of substituent combinations which should facilitate the derivation of tendencies and interdependencies regarding fluorescence properties.

First, the mono-substituted compounds 1-6 will be discussed. $\mathrm{HAnP}^{i} \mathrm{Pr}_{2}$ (1) and $\mathrm{HAnPPh}_{2}$ (4) both bear un-oxidized phosphorus atoms bound directly to the fluorophore and differ only in the iso-propyl (1) and phenyl (4) moieties of the posphanyl substituents. 


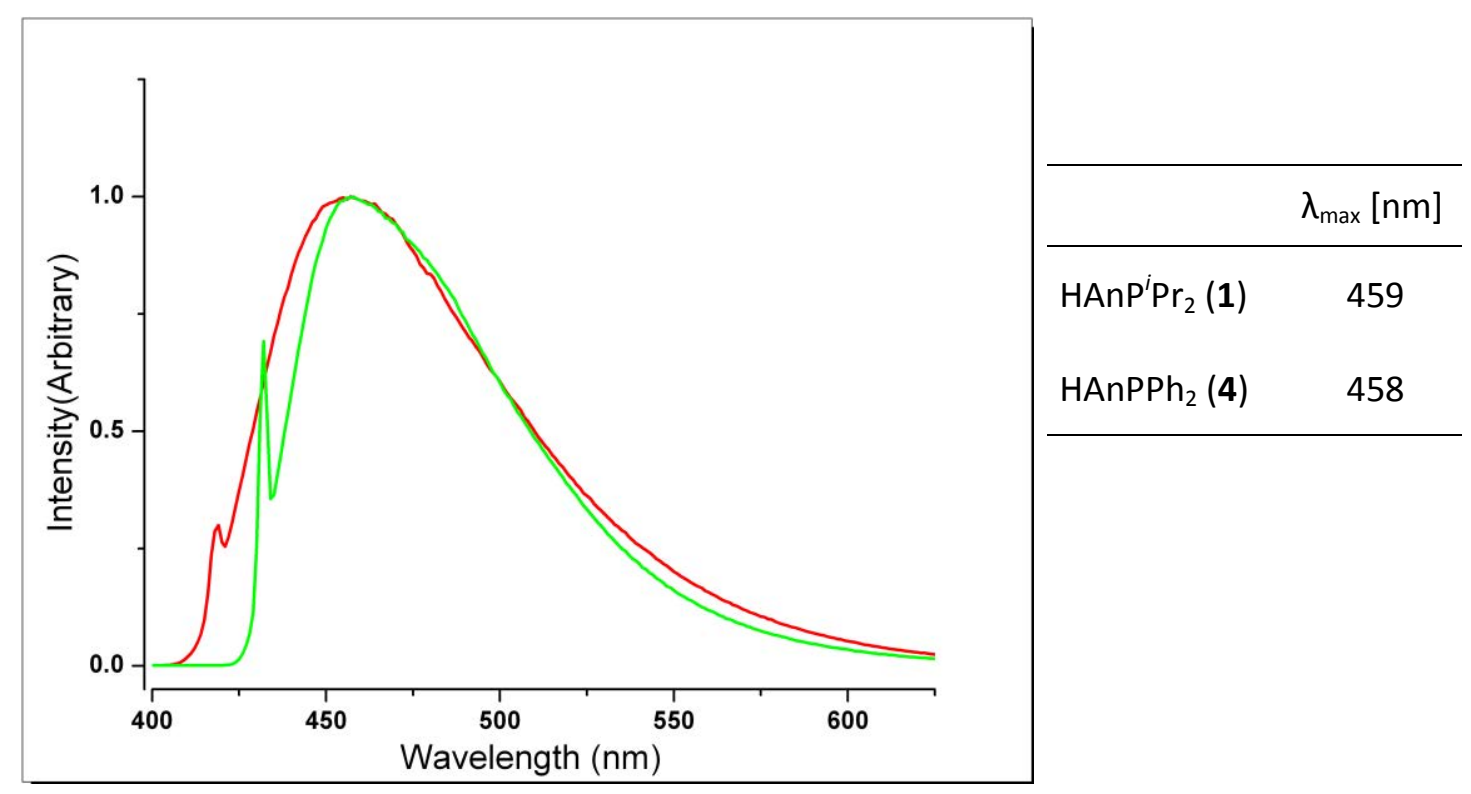

Figure 3-9: normalized emission spectra of $\mathrm{HAnP}^{i} \operatorname{Pr}_{2}$ (1) (green) and $\mathrm{HAnPPh}_{2}$ (4) (red)

When viewing their emission spectra, the most striking feature is the complete absence of the band structure which was previously observed for all starting materials (Figure 3-9). The four characteristic vibrational bands of the anthracene moiety are fused to a single broad maximum by introduction of a phosphanyl substituent. The loss of a band structure for hydrocarbon fluorophores is often ascribed to exciplex emission. Here an excited complex between the fluorophore and a second molecule is formed which exhibits altered energetic states which leads to a notable shift of the emission maximum. Many compounds are capable of forming exciplexes with themselves and do not require the presence of a second compound. While exciplex formation may also have a quenching effect, some of these arrangements are also strongly fluorescent. ${ }^{[7 \mathrm{~d}]}$ In the case of $\mathbf{1}$ and $\mathbf{4}$, both compounds show nearly identical emission maxima, which are red-shifted by over $40 \mathrm{~nm}$ compared to the monosubstituted starting materials $\mathrm{HAnMe}$ and $\mathrm{HAnBr}$. The small sharp peaks in both emission spectra are caused by scattering of the excitation beam and are no features of 1 and 4. While the emission spectra depicted in Figure 3-9 suggest that the phosphane bound iso-propyl/phenyl groups have virtually no influence on the emission properties of $\mathbf{1}$ and $\mathbf{4}$ due to nearly identical shape and maximum wavelengths, a look at the corresponding oxidation products revises this impression. 

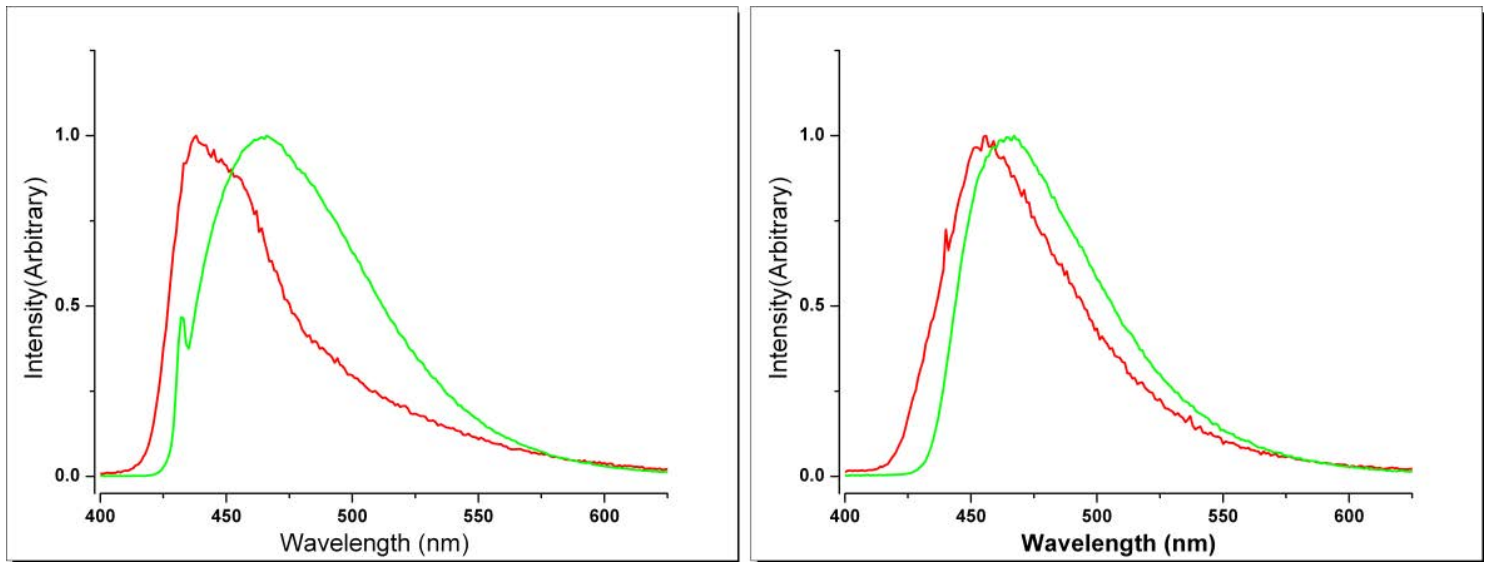

Figure 3-10: left: normalized emission spectra of $\mathrm{HAnPS}^{i} \mathrm{Pr}_{2}$ (2) (red) and $\mathrm{HAnPSPh}_{2}$ (5) (green); right: normalized emission spectra of $\mathrm{HAnPSe}^{i} \mathrm{Pr}_{2}$ (3) (red) and $\mathrm{HAnPSePh}_{2}$ (6) (green).

Table 3-2: Emission maxima of $2,3,5,6$.

\begin{tabular}{|c|c|}
\hline & $\lambda_{\max }[\mathrm{nm}]$ \\
\hline $\mathrm{HAnPS} \underline{S}^{i} \mathrm{Pr}_{2}(2)$ & 438 \\
\hline $\mathrm{HAnPSPh}_{2}(5)$ & 465 \\
\hline HAnPSe ${ }^{i} \operatorname{Pr}_{2}$ (3) & 455 \\
\hline $\mathrm{HAnPSePh}$ (6) & 465 \\
\hline
\end{tabular}

The sulfur oxidized compounds $\mathrm{HAnPS}^{i} \mathrm{Pr}_{2}$ (2) and $\mathrm{HAnPSPh}_{2}$ (5) differ significantly (by $27 \mathrm{~nm}$ ) in their maximum emission wavelengths, while the selenium oxidized HAnPSe'Pr $\mathrm{Pr}_{2}$ (3) and $\mathrm{HAnPSePh}_{2}$ (6) show a difference of $10 \mathrm{~nm}$. This proves that the phosphane-bound substituents indeed affect the position of the emission maximum. In both cases the phenyl substituents carried by the phosphane induce the stronger bathochromic shift. A possible explanation for the observed tendencies in monosubstituted compounds is that the P-bound substituents do not affect the maximum emission wavelength in the case of phosphorous(III) compounds. By oxidation, electrons are withdrawn from the phosphorous atom. Because the phosphorous atom is bound directly to the fluorophore, this electron withdrawal is transferred into the aromatic system. Now the P-bound moieties become relevant, as aliphatic iso-propyl groups are able to supply more electron density to the phosphorous atom than aromatic phenyl groups. The stronger the electron depletion at the phosphorous atom becomes, the stronger the iso-propyl and phenyl substituted derivatives differ. Because of the larger electronegativity of sulfur compared to selenium, and the stronger polarizability of selenium, a stronger electron depletion at the phosphorous atom can be postulated for the S-oxidized compounds. Hence, the observed differences are biggest for the S-oxidized $\mathbf{2}$ and $\mathbf{5}$, smaller for Seoxidized $\mathbf{3}$ and $\mathbf{6}$ and virtually non-existing for the un-oxidized $\mathbf{1}$ and $\mathbf{4}$. 
It is also noticeable that $\mathrm{HAnPS}^{i} \operatorname{Pr}_{2}$ (2) shows a fairly small maximum emission wavelength and a narrow shaped emission band compared to the other monosubstituted compounds, which will be addressed in more detail in 3.1.3.

In the case of the di-substituted compounds, only un-oxidized and sulfur-oxidized species were compared because these two compound classes had differed the most regarding their emission properties in the investigation of mono-substituted compounds. Selenium-oxidized derivatives were not synthesized.

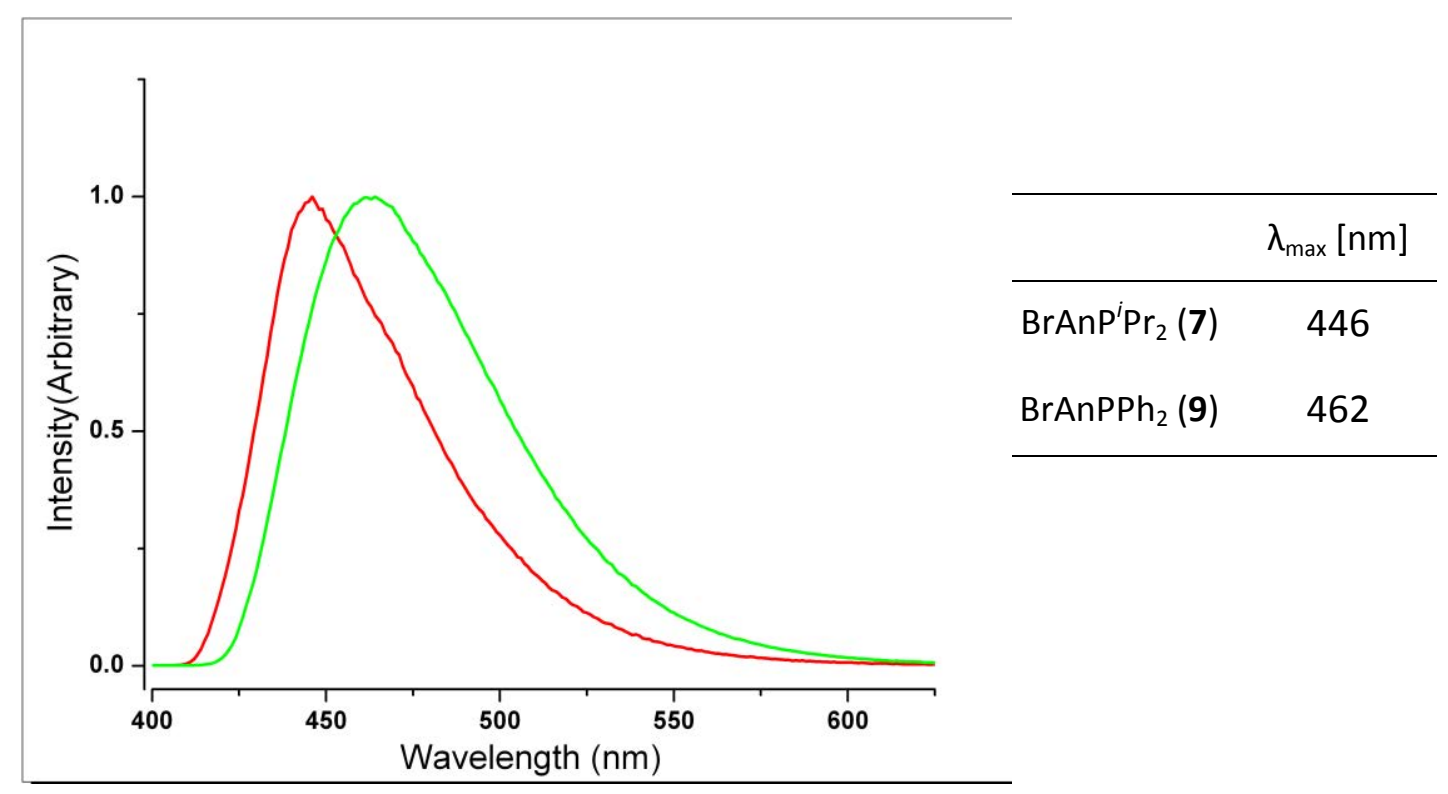

Figure 3-11: Normalized emission spectra of $\operatorname{BrAnP}^{i} \operatorname{Pr}_{2}$ (7) (red), and $\mathrm{BrAnPPh}_{2}$ (9) (green).

Unlike the corresponding mono-substituted compounds 1 and 4, $\operatorname{BrAnP}^{\mathrm{i}} \operatorname{Pr}_{2}$ (7) and $\mathrm{BrAnPPh}_{2}$ (9) do not exhibit identical maximum emission wavelengths (Figure 3-11). They differ by $16 \mathrm{~nm}$ in their emission maxima. The replacement of one bromo substituent in the starting material $\mathrm{BrAnBr}$ with phosphanyl substituents produces a red-shift of $13 \mathrm{~nm}$ (7) and $29 \mathrm{~nm}$ (9), respectively. This is a smaller shift than found for the related mono-substituted compounds 1 and 4, especially in the case of $\operatorname{BrAnP}^{\mathrm{i}} \mathrm{Pr}_{2}$ (7). Again the molecule carrying P-bound phenyl groups shows the stronger bathochromic shift. This finding follows the previous assumptions on supply of electron density towards the phosphorous atom. Though $\mathbf{7}$ and $\mathbf{9}$ are both un-oxidized (which lead to identical emission maxima for the mono-substituted $\mathbf{1}$ and $\mathbf{4}$ ), the faculty of supplying electron density becomes relevant for the di-substituted $\mathbf{7}$ and $\mathbf{9}$ : Because the 9,10-positions of the anthracene moiety strongly influence one another, 
the -l-effect of the bromo substituent is transmitted to the phosphanyl substituent in the opposing 10-position. This way the electron density supplied by the P-bound moieties takes influence on the fluorophores's electronic properties.

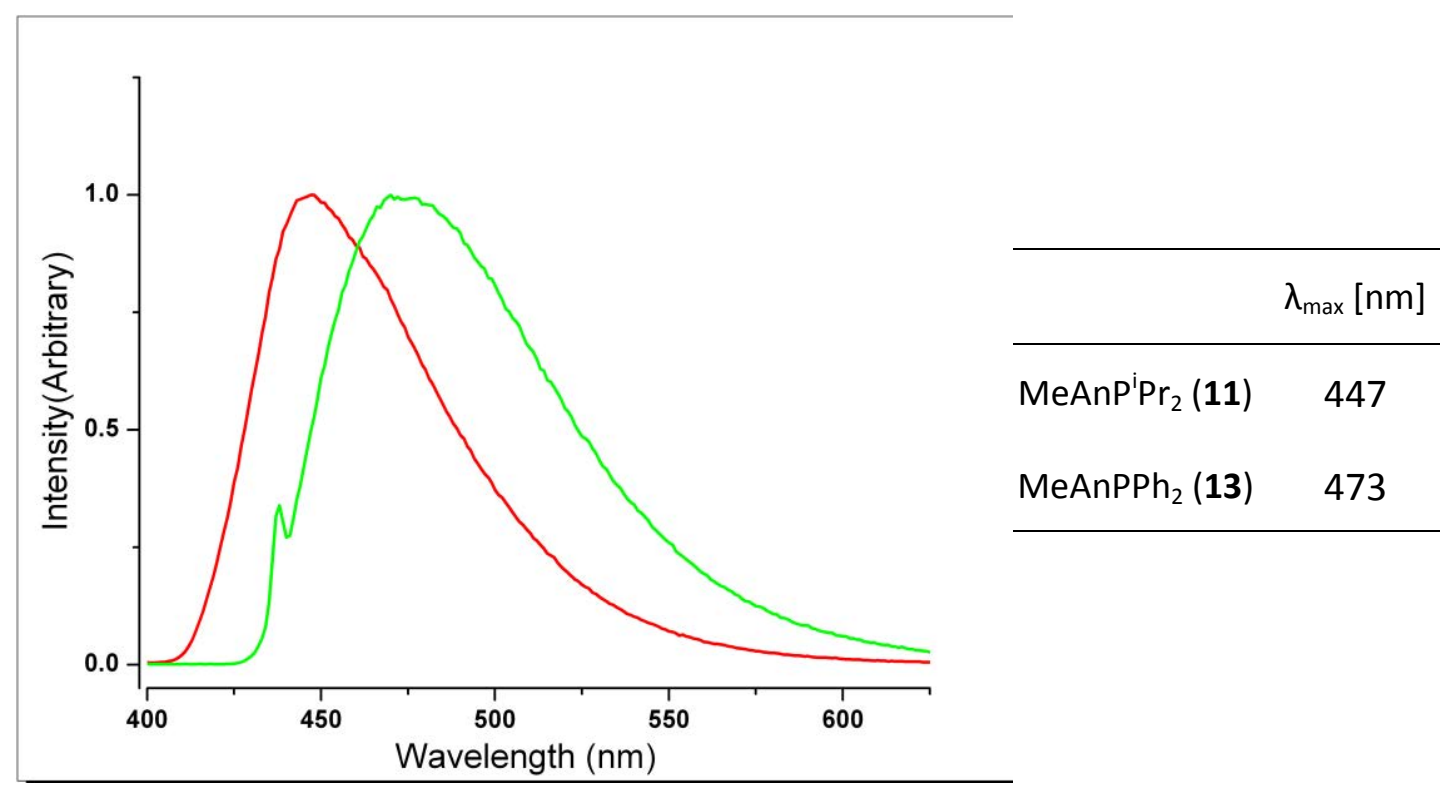

Figure 3-12: Normalized emission spectra of $\operatorname{MeAnP}^{\mathrm{i}} \mathrm{Pr}_{2}$ (11) (red) and $\mathrm{MeAnPPh}_{2}$ (13) (green).

For $\mathrm{MeAnP}^{\mathrm{i}} \mathrm{Pr}_{2}$ (11) and $\mathrm{MeAnPPh}_{2}$ (13) which both carry a methyl substituent in 10position the difference in maximum emission wavelengths becomes even more pronounced (Figure 3-12).

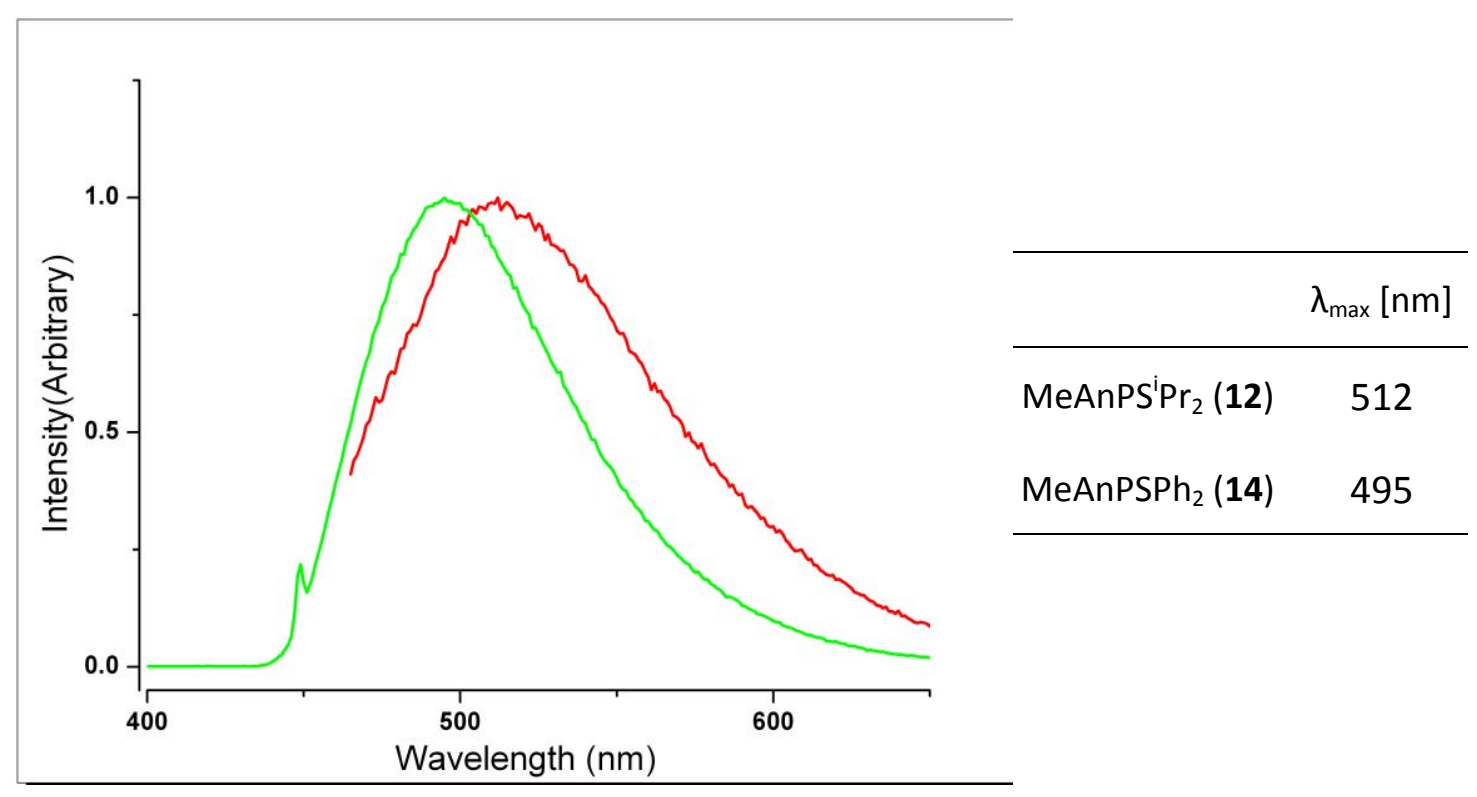

Figure 3-13: Normalized emission spectra of $\mathrm{MeAnPS}^{\mathrm{i}} \mathrm{Pr}_{2}$ (12) (red) and $\mathrm{MeAnPSPh}_{2}$ (12) (green). 
It amounts to $26 \mathrm{~nm}$, which is $10 \mathrm{~nm}$ more than found for $\mathbf{7}$ and $\mathbf{9}$. Also the difference to the starting material MeAnBr adds up to $16 \mathrm{~nm}$ (11) and $42 \mathrm{~nm}(13)$, which is - for the latter - quite large. The sulfur oxidized 12 and 14 exhibit strongest red-shifts of their emission maxima. The combination of an electron withdrawing oxidized phosphoryl substituent and an electron pushing methyl group in 10-position appears to be the optimum arrangement for achievement of bathochromic emission shifts (Figure 3-13).
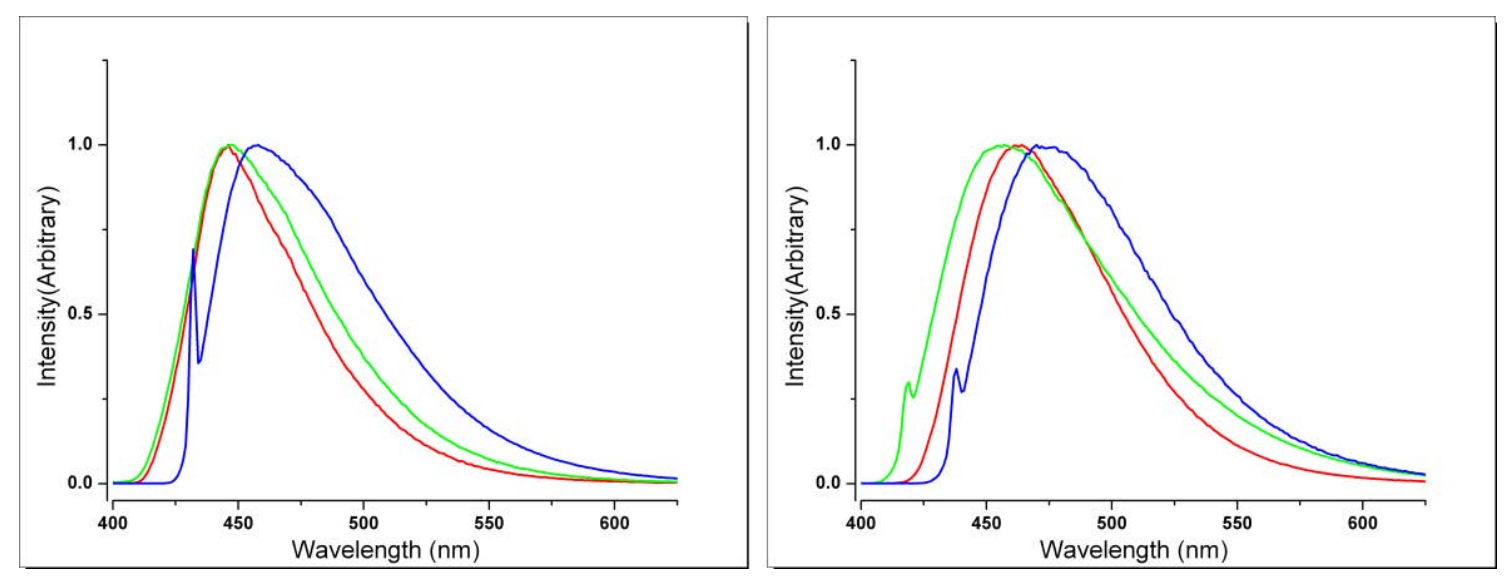

Figure 3-14: Left: normalized emission spectra of $\mathrm{HAnP}^{i} \mathrm{Pr}_{2}$ (1, blue), $\operatorname{BrAnP}^{i} \mathrm{Pr}_{2}$ (7, red), and $\mathrm{MeAnP}^{i} \mathrm{Pr}_{2}$ (11, green); right: normalized emission spectra of $\mathrm{HAnPPh}_{2}$ (4, green), $\mathrm{BrAnPPh}_{2}$ (9, red), and $\mathrm{MeAnPPh}_{2}$ (13, blue).

Comparing the isopropyl substituted with the phenyl substituted compounds, quite contrary behavior is found (all un-oxidized phosphanes). While for the isopropyl substituted compounds, the monosubstitueted derivative surprisingly exhibits the longest maximum emission wavelength, the exact opposite is observed for the phenyl substituted counterparts. Here nearly equidistant red-shifts of the emission maxima are found. The monosubstituted compound exhibits the shortest maximum emission wavelength, followed by the 9-bromo substituted compound. The methyl substituted 12 shows the longest maximum emission wavelength. This order rather complies with the previous findings than that of the isopropyl derivatives. All emission maxima are compiled in Table 3-3.

Having compared the influence of various substituents on the maximum emission wavelengths of $\mathbf{1 - 1 4}$, it is important to also comment on the observed emission intensities. 
Table 3-3: Summary of maximum emission wavelengths of 1-14 and starting materials.

\begin{tabular}{|c|c|c|c|c|c|}
\hline HAnX & $\lambda_{\max }[\mathrm{nm}]$ & BrAnX & $\lambda_{\max }[\mathrm{nm}]$ & MeAnX & $\lambda_{\max }[\mathrm{nm}]$ \\
\hline $\mathrm{HAnBr}$ & 418 & $\mathrm{BrAnBr}$ & 433 & $\mathrm{MeAnBr}$ & 431 \\
\hline $\mathrm{HAnP}^{i} \mathrm{Pr}_{2}(\mathbf{1})$ & 458 & $\mathrm{BrAnP}^{i} \mathrm{Pr}_{2}(7)$ & 446 & $M e A n P^{i} \operatorname{Pr}_{2}(\mathbf{1 1})$ & 447 \\
\hline $\mathrm{HAnPS}^{i} \mathrm{Pr}_{2}(2)$ & 438 & $\mathrm{BrAnPS}^{i} \mathrm{Pr}_{2}$ (8) & $\mathrm{n} / \mathrm{A}$ & $\mathrm{MeAnPS}^{\prime} \mathrm{Pr}_{2}(\mathbf{1 2})$ & 512 \\
\hline $\mathrm{HAnPSe}^{i} \mathrm{Pr}_{2}$ (3) & 455 & & & & \\
\hline $\mathrm{HAnPPh}_{2}(4)$ & 459 & $\mathrm{BrAnPPh}_{2}$ (9) & 462 & $\mathrm{MeAnPPh}_{2}$ (13) & 473 \\
\hline $\mathrm{HAnPSPh}_{2}(5)$ & 465 & $\mathrm{BrAnPSPh}_{2}(\mathbf{1 0})$ & $\mathrm{n} / \mathrm{A}$ & $\mathrm{MeAnPSPh}_{2}(\mathbf{1 4})$ & 495 \\
\hline $\mathrm{HAnPSePh}_{2}(\mathbf{6})$ & 465 & & & & \\
\hline
\end{tabular}

These varied strongly among 1-14, and several of these compounds can be considered weakly fluorescent. Especially the selenium oxidized $\mathbf{3}$ and $\mathbf{6}$, as well as generally the iso-propyl substituted compounds can be subsumed in this category. Therefore these substituents appear less suitable for the future preparation of fluorescent compounds for in-solution applications.

Summing up the gathered results, two main tendencies can be derived. The introduction of substituents in both 9- and 10-position induces a substantial bathochromic shift of the emission maximum compared to monosubstituted derivatives. Furthermore the combination of an electron withdrawing (e.g. oxidized) phosphoryl substituent in 9-position with an electron donating + l substituent (methyl group) in 10-position of the anthracene moiety appears to produce the strongest redshifts of emission. This is demonstrated by the emission maxima of $M e A n \operatorname{PS}^{i} \operatorname{Pr}_{2}$ (12) and $\mathrm{MeAnPSPh}_{2}$ (14) which are the two compounds with the longest emission wavelengths. Possibly this effect could be further enhanced by utilization of stronger +1 substituents in 10-position (e.g. silyl substituents) in combination with phosphoryl substituents in 9-position. 


\subsubsection{Titration Experiments}

To fathom whether the presence of metal cations affects the fluorescence properties of phosphanyl- and phosphorylanthracenes in solution, selected compounds of 1-14 were subjected to titration experiments in which the emission of the respective compound was monitored as a function of the applied cation concentration. To account for the low sample concentrations and the comparatively weak donor strength of the monodentate 1-14, a non-donating solvent was chosen to assure interaction between the investigated compounds and the metal ions. Donating solvents would most likely exclusively coordinate the cations due to the enormous excess of solvent molecules compared to dissolved phosphoryl anthracenes. Therefore again DCM was chosen as a solvent. The poor solubility of most metal salts was of course a limiting factor, which was overcome by the discovery that zinc bromide is well soluble in DCM. Hence, this solvent/cation combination was chosen for the following experiments.

$10^{-4} \mathrm{M}$ solutions of $\mathrm{HAnPS}^{i} \mathrm{Pr}_{2}$ (2), $\mathrm{HAnPPh}_{2}$ (4), $\mathrm{HAnPSPh}_{2}$ (5), HAnPSePh 2 (6), $\mathrm{MeAnPiPr}_{2}$ (11), and $\mathrm{MeAnPPh}_{2}$ (13) in DCM were prepared and each was titrated with $1 \mathrm{mM} \mathrm{ZnBr}$ solution until a threefold excess of $\mathrm{ZnBr}_{2}$ was reached.
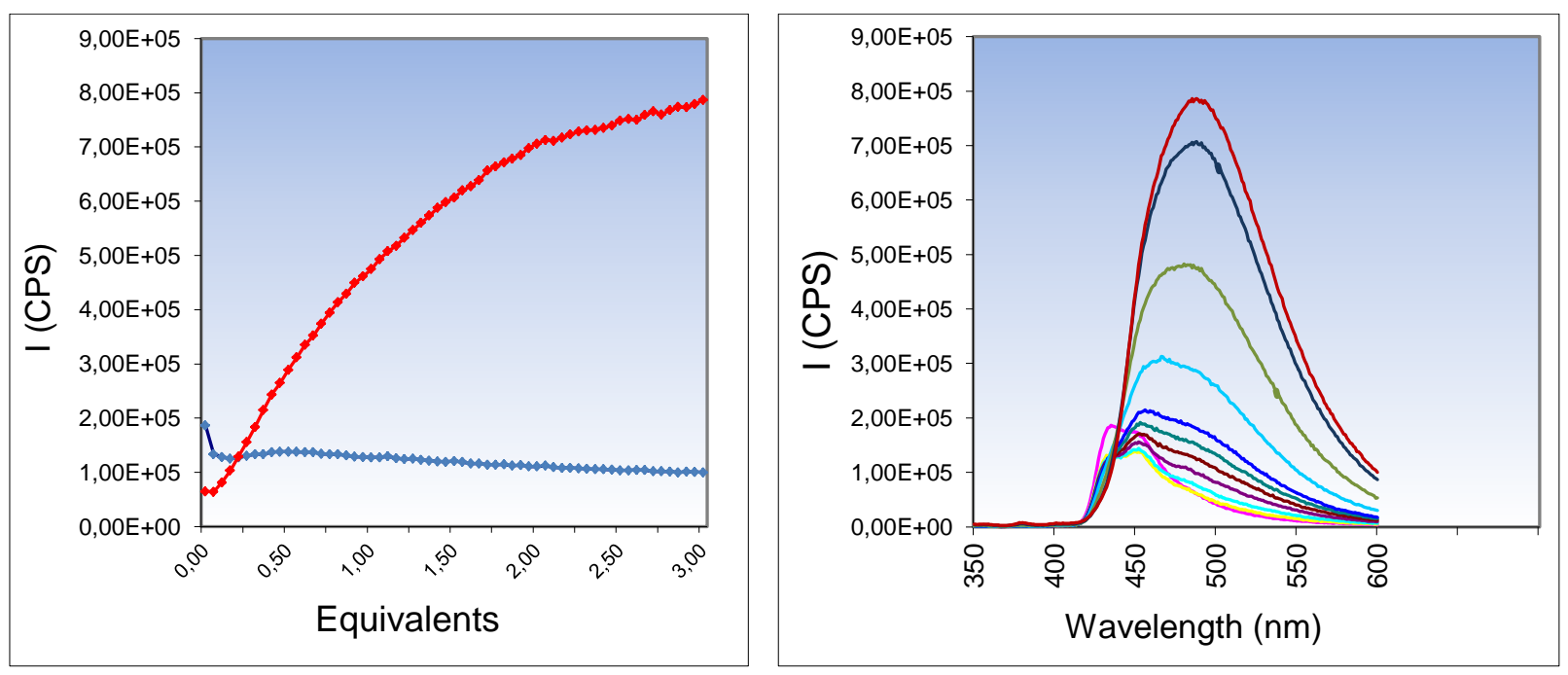

Figure 3-15: Left: emission of 2 upon titration with $\mathrm{ZnBr}_{2}$ at $\lambda_{\mathrm{Em}}=438 \mathrm{~nm}$ (blue) and $\lambda_{\mathrm{Em}}=485 \mathrm{~nm}$ (red); right: stacked emission spectra of 2 upon titration with $\mathrm{ZnBr}_{2}$.

For $\mathrm{HAnPS}^{i} \mathrm{Pr}_{2}$ (2), which had shown a fairly narrow emission maximum ant short wavelength compared to other sulfur oxidized compounds, the addition of $\mathrm{Zn}^{2+}$ leads to a broadening of the emission maximum and a notable increase of the formerly weak 
emission (Figure 3-15). The increase of emission is strong up to and addition of ca. 1.5 equivalents of $\mathrm{ZnBr}_{2}$ and then gradually weakens. The red-shift of the emission maximum from $438 \mathrm{~nm}$ to $485 \mathrm{~nm}$ is also remarkable, which is illustrated by the run of the curves in Figure 3-15 (left) which show the change of emission intensity for two different emission wavelengths which correspond to the maximum emission wavelengths before and after the titration. Though overall still comparatively weakly fluorescent, the addition of $\mathrm{Zn}^{2+}$ appears to inhibit a fluorescence quenching mechanism and also appears to alter the electronic properties of the compound.

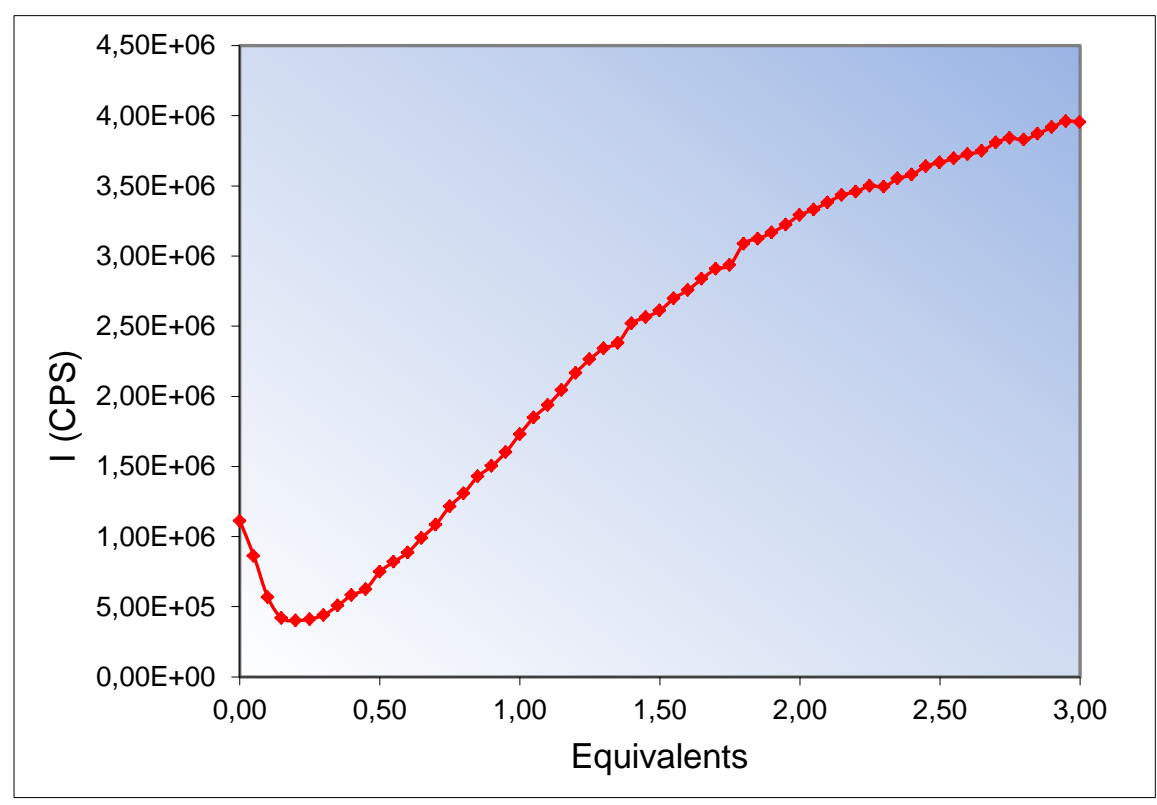

Figure 3-16: Titration of $\mathrm{HAnPPh}_{2}(4)\left(10^{-4} \mathrm{M}\right.$ in DCM) with $\mathrm{ZnBr}_{2}, \lambda_{\mathrm{Em}}=456 \mathrm{~nm}$.

The titration of $\mathrm{HAnPPh}_{2}$ (4) with $\mathrm{ZnBr}_{2}$ triggers a similar increase of emission intensity as observed for 2 (Figure 3-16). This increase of emission is in accordance with the reports of Yip et al. who observed an increase of emission of their closely related 9,10-bis(diphenylphosphanyl)anthracene upon complexion of gold(I), ${ }^{[65]}$ which they assigned to an inhibition of electron transfer between the phosphane and the fluorophore by binding of gold(I). A minor shift of the emission maximum by $5 \mathrm{~nm}$ is also found. The slight decrease of emission intensity at the beginning of the titration can be explained by the formation of local concentration maxima around the metal ions which are present at a much lower concentration than the phosphorylanthracene molecules at the beginning of the experiment. The electrostatic attraction of cations and donors leads to accumulation of molecules around the ions. In these areas of 
higher concentration the collision factor is therewith also higher, which probably leads to the observed quenching.

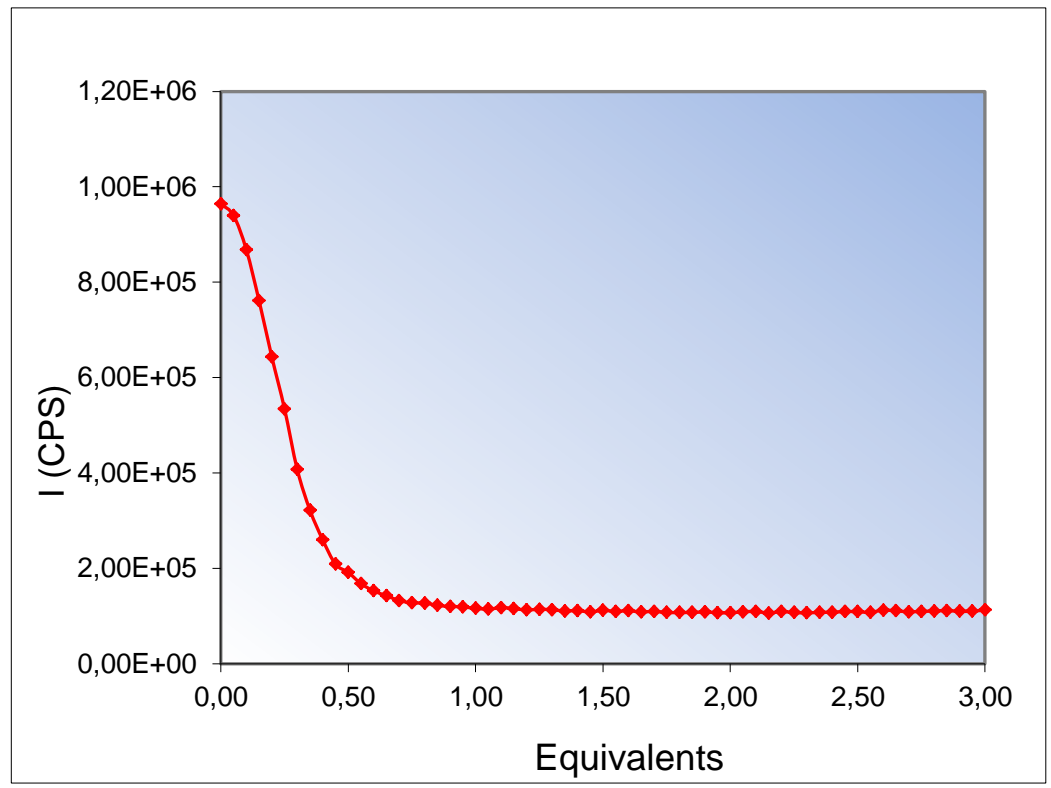

Figure 3-17: Titration of $\mathrm{MeAnP}^{i} \mathrm{Pr}_{2}$ (11) with $\mathrm{ZnBr}_{2}$.

Surprisingly, MeAnP'Pr${ }_{2}$ (11) showed completely different behavior upon titration than the previously investigated compounds. The initially fairly strongly fluorescent compound exhibits a strong decrease of emission intensity up to an addition of ca. 0.5 equivalents of $\mathrm{Znr}_{2}$ (Figure 3-17). From that point onward the emission stays at a constantly low rate.

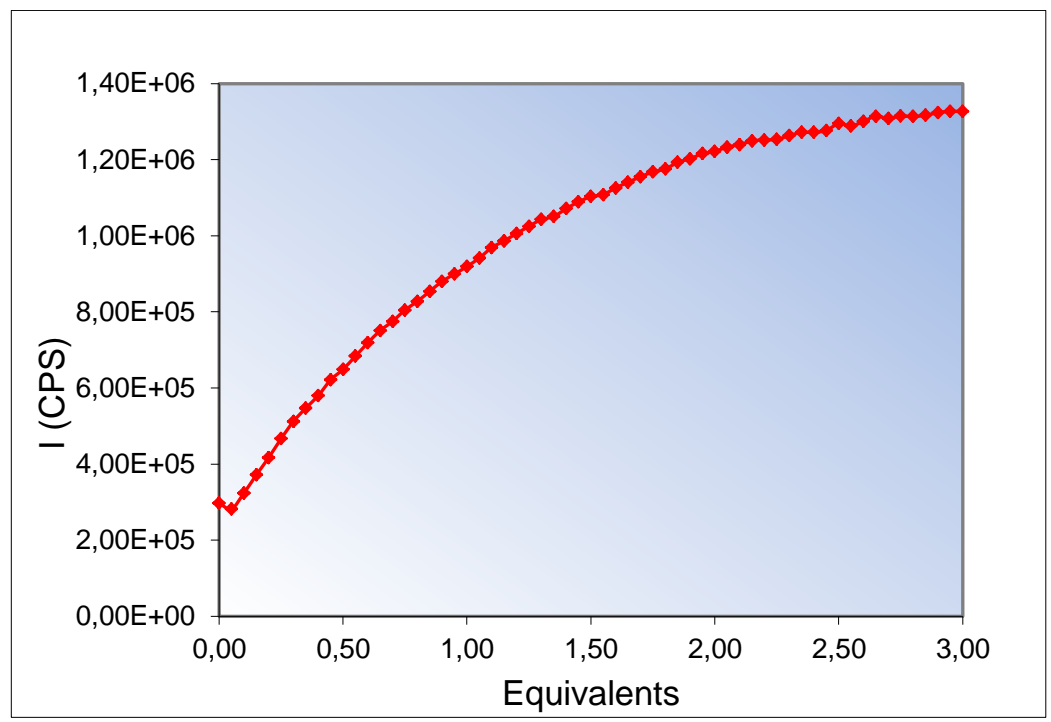

Figure 3-18: Titration of $\mathrm{HAnPSPh}_{2}$ (5) with $\mathrm{ZnBr}_{2}$. 
The behavior of $\mathrm{HAnPSPh}_{2}$ upon titration with $\mathrm{ZnBr}_{2}$ is again very similar to that of HAnPS ${ }^{i} r_{2}(2)$, showing a strong emission increase (Figure 3-18). The observed red-shift of emission is on the other hand much weaker than for $\mathbf{2}$.

The observed phenomena differed strongly among one another, which makes the deduction of a clear tendency tedious. While both sulfur oxidized compounds showed an increase of emission intensity, the un-oxidized compounds exhibited contrary behavior. The increase of the emission of $\mathrm{HAnPPh}_{2}(4)$ confirms the reports of Yip et al., while the strong quenching of $\mathrm{MeAnP}^{i} \mathrm{Pr}_{2}$ (11) does not appear conclusive. Nevertheless these experiments have shown that phosphanyl- and phosphorylanthracenes show sensitivity towards the presence of metal ions which is a finding which will find further application in the course of this thesis.

\subsection{9,10-Bis(diphenylthiophosphoryl)anthracene (SPAnPS)}

One of only few known anthracene based materials which exhibit strong solid state fluorescent was reported by Fei et al. in 2003. ${ }^{[44 b, 44 c]}$ Their 9,10-bis(diphenylthiophosphoryl)anthracene (SPAnPS) was the first reported anthracene derivative which showed sensory properties in the solid state. By intercalation of toluene into the solid state structure in a host/guest complex, a nearly T-shaped exciplex between cocrystallized toluene molecules and the anthracene fluorophore is formed, which exhibits intense solid state fluorescence. When the lattice solvent is removed under heating and reduced pressure, the observed emission is virtually completely quenched. This way, Fei et al. were able to show the importance of the lattice solvent as a crucial factor for the occurrence of solid state fluorescence.

The work on this compound and its intercalation structures was continued by Schwab, ${ }^{[59 b]}$ who succeeded in crystallizing SPAnPS from various aromatic solvents. The resulting intercalation structures were also characterized structurally, but solid state fluorescence was only vaguely monitored by visual examination under exposure of the compounds to UV-light at a fixed wavelength of $366 \mathrm{~nm}$. Accurate fluorescence experiments were not conducted. Also, neither Fei nor Schwab have quantified structural properties of their compounds such as deformation of the fluorophore or intermolecular interactions. Hence, the alignment of structural features and observed solid state fluorescence was for the most part not possible. 
Though Fei and Schwab recognized the presence of the lattice solvent within the structure as a requirement for solid state fluorescence, they were not able to identify whether the direct interaction of solvent molecules with the fluorophore or the conformation of the SPAnPS molecule resulting from the intercalation of solvent were the vital factors for strong emission. Fei et al. came to the conclusion that the excited dimer which is formed by $\mathrm{C}-\mathrm{H}^{\cdots} \pi \mathrm{r}$ bonding between the $\alpha$-hydrogen atom of the intercalated toluene molecule and the fluorophore is crucial (Figure 3-19) ${ }^{[44 b, 44 c]}$, while Schwab concluded that the transoid orientation of the P=S-bonds in the SPAnPS molecule is the predominant factor. ${ }^{[59 b]}$ Moreover, the method used by Fei et al. to prove the dependency of solid state fluorescence on presence of toluene lattice solvent is problematic. By evaporating the solvent from the crystals under reduced pressure, the entire crystalline arrangement and packing motif is destroyed. Crystallization of the SPAnPS molecule without lattice solvent was not achieved, which would provide a valid comparison of an equally ordered crystalline arrangement.

The preparation of new intercalation structures of SPAnPS, determination of the resulting solid state structures, quantification of structural features and assignment of measured solid state emission are therefore important steps in understanding of solid state fluorescence of SPAnPS.

\subsubsection{Quantification of Structural Properties}

As illustrated in the introduction 1.2, steric strain on the fluorophore and structural distortion resulting from it have been introduced as factors of fluorescence quenching by Mizobe et al. ${ }^{[49,51]}$ Because their anthracene derivatives were close to perfectly planar, they quantified steric strain by IR spectroscopy. In contrast, the anthracene derivatives described here show tangible deformations induced by steric strain, which can be quantified by geometrical operations.

The crystal structure of SPAnPS@tol is depicted in Figure 3-19. It shows the SPAnPS molecule and two co-crystallized lattice solvent molecules in interaction with the aromatic $\pi$-system. This figure will be used to visualize the structural properties of anthracene derivatives which shall be quantified. 


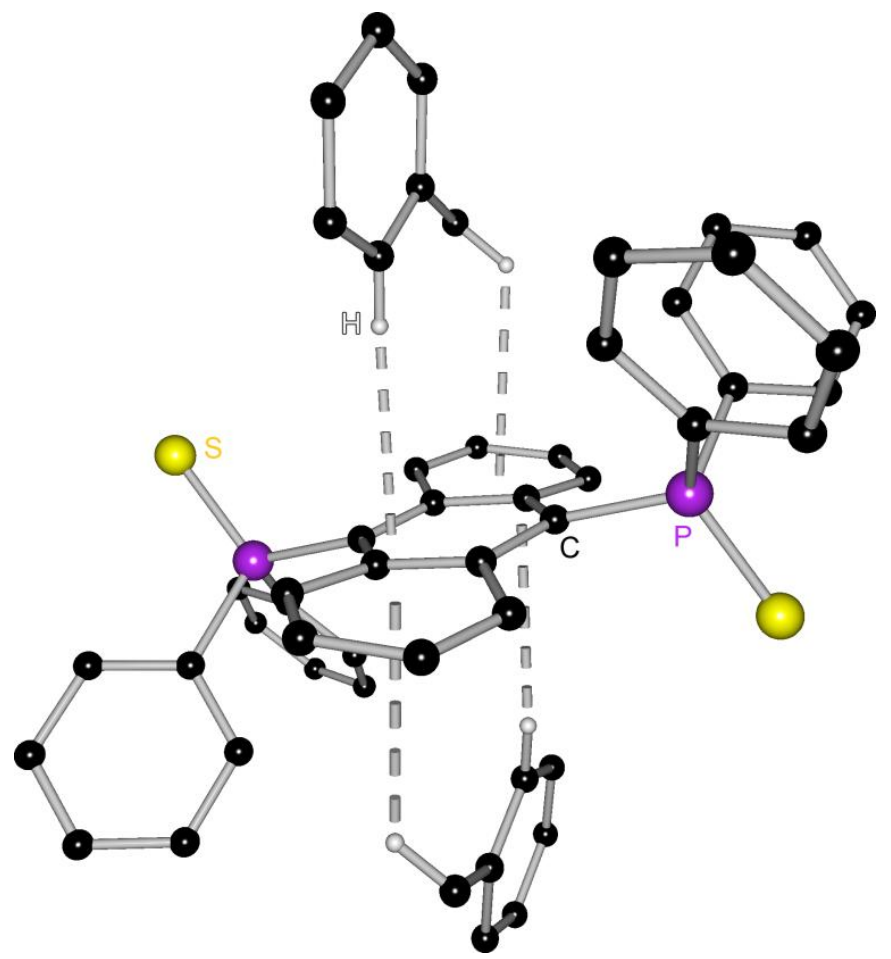

Figure 3-19: Crystal structure of SPAnPS@tol.

It exhibits one of two types of structural deformation of the fluorophore observed for anthracene derivatives. The anthracene moiety can undergo "folding", whereupon both peripheral $\mathrm{C}_{6}$-perimeters are bent symmetrically away from the mean plane towards one another. This type of deformation was quantified by generating planes through the carbon atoms in 1,2,3,4-position and 5,6,7,8-position (Figure 3-20, bottom) and determining their intersecting angle. The atoms in these positions were chosen because they represent the outermost array of the anthracene moiety, at which the impact of folding is maximal and the largest angle relative to the former mean plane is spanned.

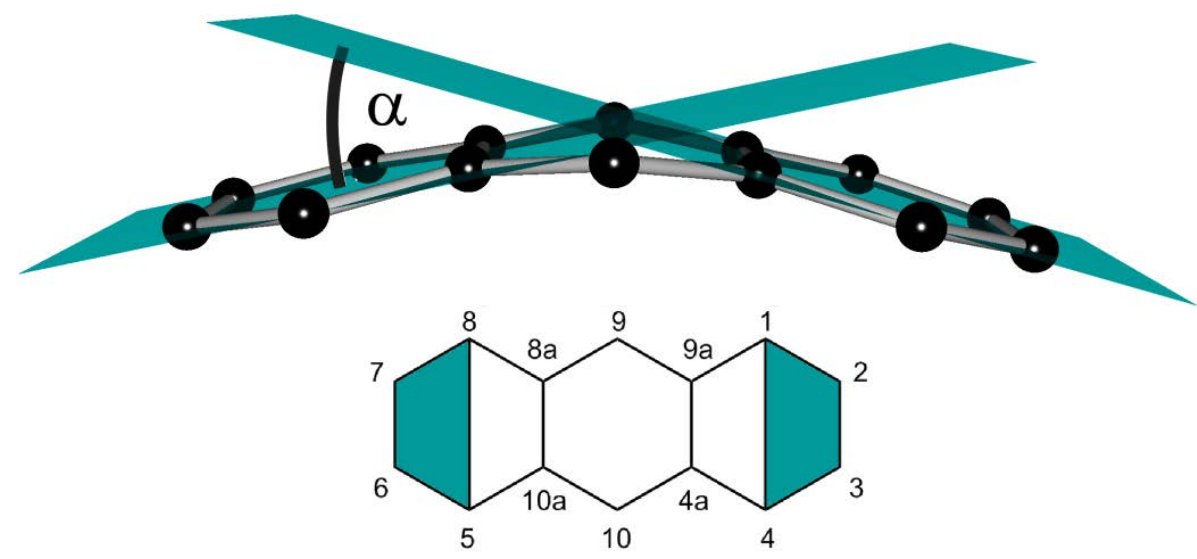

Figure 3-20: Planes generated for determination of the "folding angle" $\boldsymbol{\alpha}$ of the anthracene moiety. 
The second form of deformation is "twisting" of the anthracene moiety. In this case the peripheral $\mathrm{C}_{6}$-perimeters are twisted relative to the central ring (Figure 3-21). This can happen in symmetrical manner, when both outer rings are twisted in the same direction relative to the central ring, or in asymmetrical manner, when they are twisted in opposite directions.

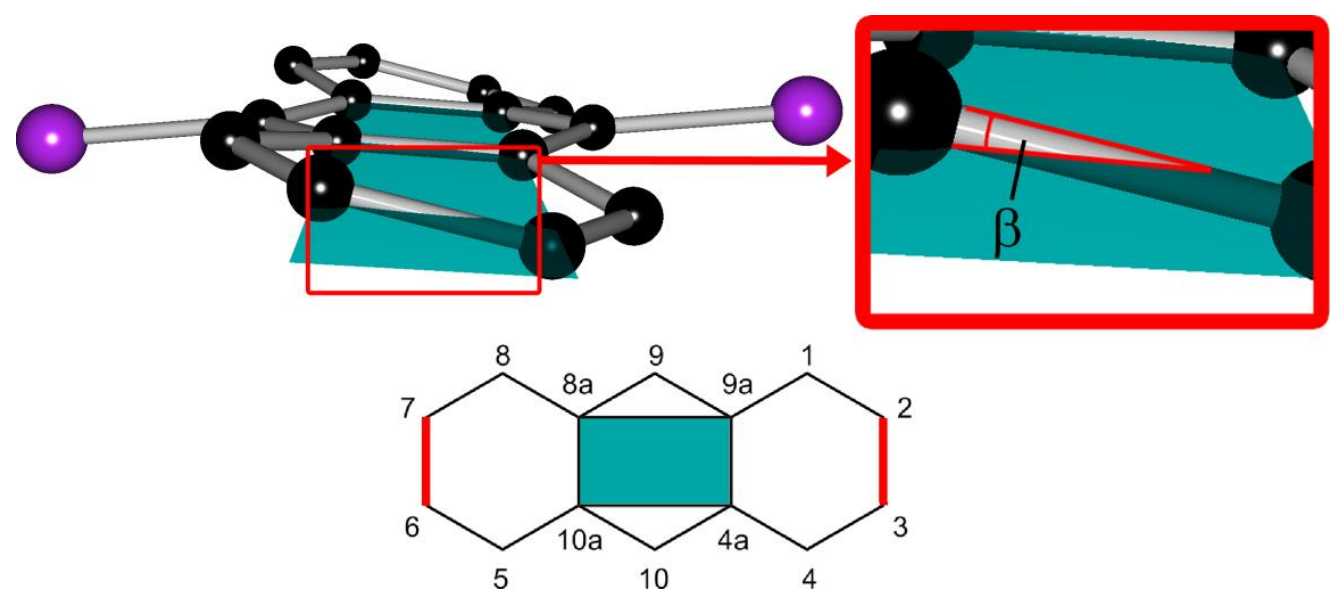

Figure 3-21: Determination of the "twist angle" $\phi$ of the anthracene moiety: $\phi=\left|\beta_{1}\right|+\left|\beta_{2}\right|$.

For quantification of this phenomenon a plane through the $8 a, 9 a, 4 a, 10 a$ carbon atoms of the central ring of the anthracene moiety was generated. Then lines were plotted through the carbon atoms in 2,3-position and 6,7-position and the angles $\beta_{1}$ and $\beta_{2}$ which these enclosed with the plane of the central ring were determined (Figure 3-21, bottom). In un-substituted anthracene these lines are parallel to the plane of the central ring, only the twisting of the molecule leads to an intersection of line and plane. To acquire the total twist angle of the anthracene moiety, the angles of both lines were summed up to the total twist angle $\phi=\left|\beta_{1}\right|+\left|\beta_{2}\right|$. The carbon atoms used for the line plots were again chosen because they represent the most peripheral bond in the anthracene molecule, at which the effect of twist deformation is strongest. Both forms of deformation regularly occur in combination.

By this method a folding angle of $0^{\circ}$ is found for SPAnPS@tol. This can be attributed to the symmetry of the compound. Because the asymmetric unit contains only one half of the SPAnPS molecule, the other half is generated by symmetry operations. This leads to the complete absence of folding. A twisting of the anthracene moiety however is observed. A symmetrical twisting of the outer rings - again induced by the symmetry of the compound - is found, producing two identical twist angles of $10.0^{\circ}$, adding up to a total twist angle of $20.0^{\circ}$. 
The second important factor which can be derived from solid state structures and subsequently quantified is information on intermolecular interactions. While deformation is a property of an isolated molecule, intermolecular interactions result from the packing of molecules in the solid state, which makes the utilization of packing-plots inevitable. As pointed out in the introduction, the most prominent interaction of aromatic compounds like anthracene derivatives is $\pi-\pi$ overlap, often also referred to as $\pi$ stacking. This interaction has often been quantified by measurement of $\pi-\pi$ distances and percentage of $\pi-\pi$ overlap as indicators for the strength of the respective interaction. ${ }^{[47-48,48 e, 50,53-54]}$ Though the interpretation of such interactions regarding their influence on solid state fluorescence has been contradictory in several points (c.f. 1.2), this method was also applied to the anthracene derivatives on hand.

Though sometimes reported, the second type of interaction has seldom been quantified in past publications. ${ }^{[49]} \mathrm{C}-\mathrm{H}$ bonded hydrogen atoms in close proximity to aromatic $\pi$-systems have the ability of forming $\mathrm{C}-\mathrm{H}^{\cdots \cdots} \pi$ bonds. The importance of the energetic contribution of $\mathrm{C}-\mathrm{H} \cdots \pi$ interactions to packing in solid state has been illustrated by Nishio. ${ }^{[66]}$ Some arrangements are so stable that the conformation of the corresponding organic molecules even in solution is influenced by $\mathrm{C}-\mathrm{H}^{\cdots}{ }^{\cdots} \pi$ bonds. Furthermore, recent calculations have verified the importance of this type of interaction despite the weak polarity of the $\mathrm{C}-\mathrm{H}$ bond. The strength of the $\mathrm{C}-\mathrm{H}^{\cdots}{ }^{\prime \prime} \pi$ bond is not only determined by its length, also the angle of the $\mathrm{C}-\mathrm{H}$-bond to the $\pi$-system is crucial - an orthogonal orientation is regarded as the most stable arrangement generating the strongest interaction. ${ }^{[67]}$ Additionally, the polarity of the $\mathrm{C}-\mathrm{H}$-bond is an important factor in classifying the strength of a $\mathrm{C}-\mathrm{H}^{\cdots} \pi$ interaction. The strength of the interaction increases with rising polarity of the $\mathrm{C}-\mathrm{H}$-bond. Therefore aliphatic $\mathrm{sp}^{3} \mathrm{C}-\mathrm{H}$ bonds produce weaker $\mathrm{C}-\mathrm{H}^{\cdots} \pi$ bonds than aromatic $\mathrm{sp}^{2} \mathrm{C}-\mathrm{H}$-bonds. ${ }^{[66]}$

To quantify the observed $\mathrm{C}-\mathrm{H}^{\prime \prime} \pi$ interactions, the distance from the hydrogen atom to the plane of the participating aromatic ring was determined, as well as the angle of the $\mathrm{C}-\mathrm{H}$ bond to this plane (Scheme 3-4). 


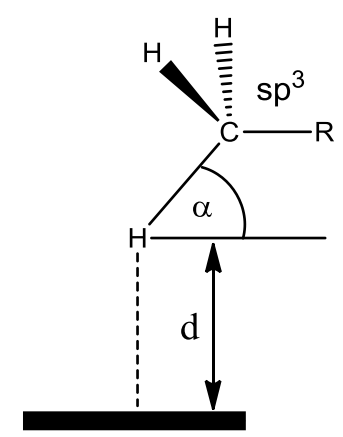

central ring

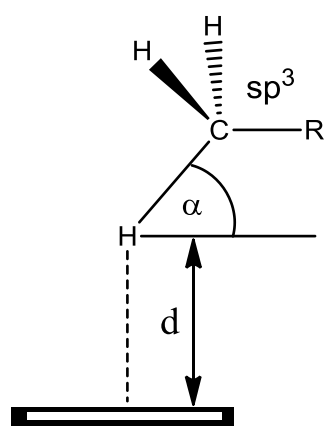

periferal ring

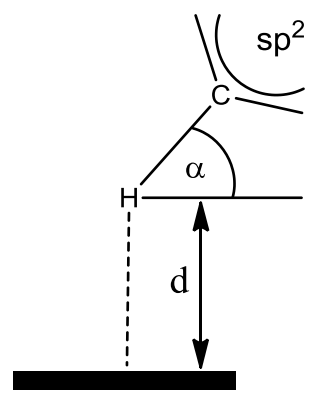

central ring

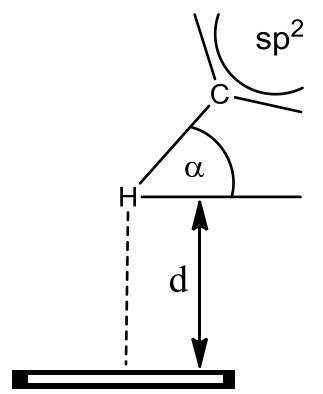

periferal ring

Scheme 3-4: Quantification of $C-H^{\cdots}{ }^{\prime \prime} \pi$ interactions: $d=$ Hydrogen- $\pi$ distance, $\alpha=$ angle of C-H bond to the ring plane.

The importance of $\mathrm{C}-\mathrm{H} \cdots \pi$ bonding in the structure of SPAnPS@tol was recognized by Fei et al. and the contribution of this interaction to the formation of the T-shaped excimer complex was stated. ${ }^{[44 b, 44 c]}$ Figure 3-19 shows the $\mathrm{sp}^{2} \mathrm{C}-\mathrm{H}^{\cdots} \pi$ bond from the toluene molecule to the $\pi$-system of the anthracene moiety. It encloses an angle of $76.8^{\circ}$ with the $\pi$-system, which is fairly close to the optimum orthogonal orientation. Though clearly weaker, there is a second $\mathrm{C}-\mathrm{H}^{\cdots} \pi$ interaction found between a methyl C$\mathrm{H}$ of the toluene molecule and the anthracene $\pi$-system. This was not documented by Fei et al., presumably due to the slightly longer distance, the smaller angle of only $35.0^{\circ}$ and the weaker polarity of the $\mathrm{sp}^{3} \mathrm{C}-\mathrm{H}$ bond. In the following all $\mathrm{C}-\mathrm{H}^{\cdots} \pi$ interactions will be classified this way when a noteworthy contribution of this type of interaction is present.

\subsubsection{Synthesis and Solid State Structures}

The main objective was the preparation of new intercalation structures of SPAnPS for structural and solid state fluorescence comparison to determine the influence of intermolecular interactions and fluorophore deformation on emission properties. In this context the preparation of lattice solvent free crystals - which had not been previously achieved - was of particular interest.

9,10-bis(diphenylthiophosphoryl)anthracene (SPAnPS) was prepared according to literature procedures. ${ }^{[44 b, 44 c]}$ 9,10-Dibromoanthracene was di-lithiated using $n B u L i$ at $15^{\circ} \mathrm{C}$ and then reacted with two equivalents of chlorodiphenylphosphane. Removal of lithium chloride by filtration and oxidation with elemental sulfur in toluene at $110^{\circ} \mathrm{C}$ gave SPAnPS at high yields (Scheme 3-5). Crystallization of SPAnPS from toluene 
yielded the host/guest complex containing two toluene molecules per SPAnPS molecule. This complex will be referred to as SPAnPS@tol (15) from here on.<smiles></smiles>

Scheme 3-5: Synthesis of SPAnPS.

SPAnPS@tol (15) was dissolved in - and crystallized from - each of the following solvents/solvent mixtures: EtOAc, MeCN, Acetone/hexane 2:1, DCM/MeOH 3:1, and toluene $d_{8}$. Hot saturated solutions were prepared and slowly cooled to ambient temperature, $-3^{\circ} \mathrm{C}$, or $-30^{\circ} \mathrm{C}$, respectively. Crystallization conditions for each solvent/solvent mixture are listed in Table 3-4.

\begin{tabular}{ccc}
\hline \multicolumn{3}{c}{ Table 3-4: Crystallization conditions and compound } \\
nomenclature. \\
\hline Solvent & $\mathrm{T}\left[{ }^{\circ} \mathrm{C}\right]$ & Compound name \\
\hline toluene & 22 & SPAnPS@tol (15) \\
MeCN & 22 & SPAnPS@MeCN (16) \\
acetone/hexane & -3 & SPAnPS@Ace (17) \\
DCM/MeOH 3:1 & -30 & SPAnPS@DCM (18) \\
EtOAc & 22 & SPAnPS_pure (19) \\
toluene $d_{8}$ & 22 & SPAnPS@tol_d (20)
\end{tabular}

The previously published structures of SPAnPS all revealed that the high steric demand of two anthracene bound diphenylthiophosphoryl substituents is the driving force for the formation of intercalation structures. The steric demand prevents the anthracene moieties from getting close enough to one another to form $\pi$ stacked arrangements in the solid state. Hence, the observed $\pi-\pi$ distances are regularly between 8 and $10 \AA$, which can be considered negligible in terms of $\pi-\pi$ interaction. Interactions between SPAnPS molecules are therefore generally rare, interactions mostly occur between intercalated molecules and SPAnPS. Due to these large intermolecular distances, cavities or "pockets" within the structure are formed which are suitable in size and shape for the intercalation of aromatic solvents. The conformation of the SPAnPS molecules in these structures is very similar, with a transoid orientation of the $\mathrm{P}=\mathrm{S}$ bonds and a small folding angle and a notable twist 
deformation of the fluorophore. ${ }^{[44 b, 44 c, 59 b]}$ If the intercalated solvent is not aromatic $(\rightarrow$ non-planar) or carries too large substituents to fit into the cavities of the structure, a cisoid conformation is formed with a large folding angle and smaller twist angle. ${ }^{[59 b]}$ Also the transoid conformation mostly leads to intercalation of two guest molecules per host molecule, while the cisoid conformation may intercalate one or two guest molecules.

When SPAnPS is crystallized from acetonitrile, SPAnPS@MeCN (16) is formed. Despite the linear geometry and the low steric demand of the acetonitrile molecule, the cisoid conformation is generated. One guest molecule of acetonitrile is cocrystallized in the structure of $\mathbf{1 6}$ (Figure 3-22).

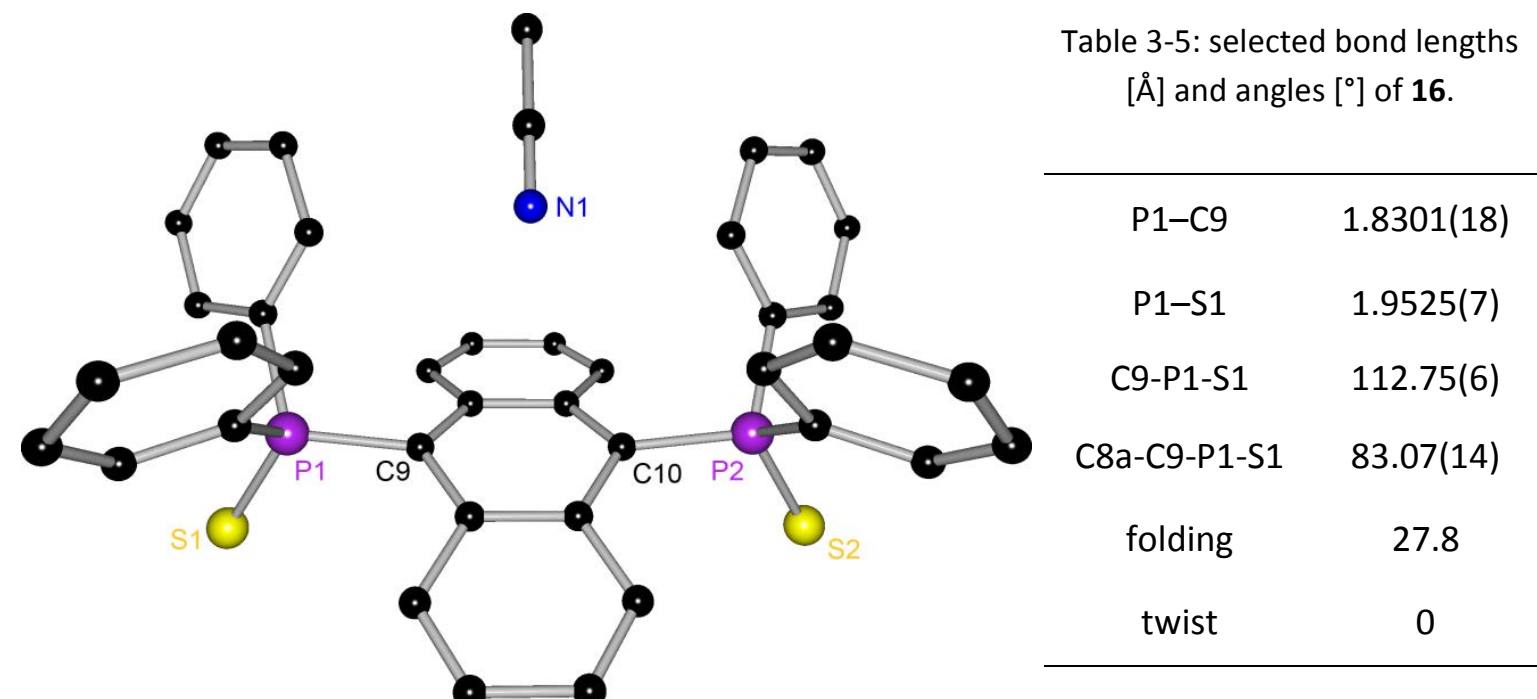

Figure 3-22: crystal structure of SPAnPS@MeCN (16), hydrogen atoms are omitted for clarity.

It is located above the plane of the anthracene moiety at a distance of well over $4 \AA$. 16 crystallizes in the hexagonal space group $P 63 / m$, which is very unusual and has not been previously observed for phosphoryl anthracenes. It is the first compound of this kind which is completely symmetric and has a mirror plane located directly down the middle of the molecule. The acetonitrile molecule is located on this mirror plane. This unique geometry leads to the typical "cis"-folding of the anthracene moiety, but no twist deformation whatsoever. The folding angle measures $27.8^{\circ}$. In this respect SPAnPS@MeCN (16) is the direct opposite of SPAnPS@tol (15), which shows only twist deformation but no folding. The $\mathrm{P}-\mathrm{S}$ and $\mathrm{P}-\mathrm{C}$-bond distances are very similar to those found in SPAnPS@tol (15). As expected, there is no $\pi$ - $\pi$-overlap in the structure of 
SPAnPS@MeCN (16). The only noteworthy intermolecular interactions present are two $\mathrm{C}-\mathrm{H} \cdots \pi$ bonds of the 2,3-hydrogen atoms to the central ring of an adjacent anthracene moiety (Figure 3-23).
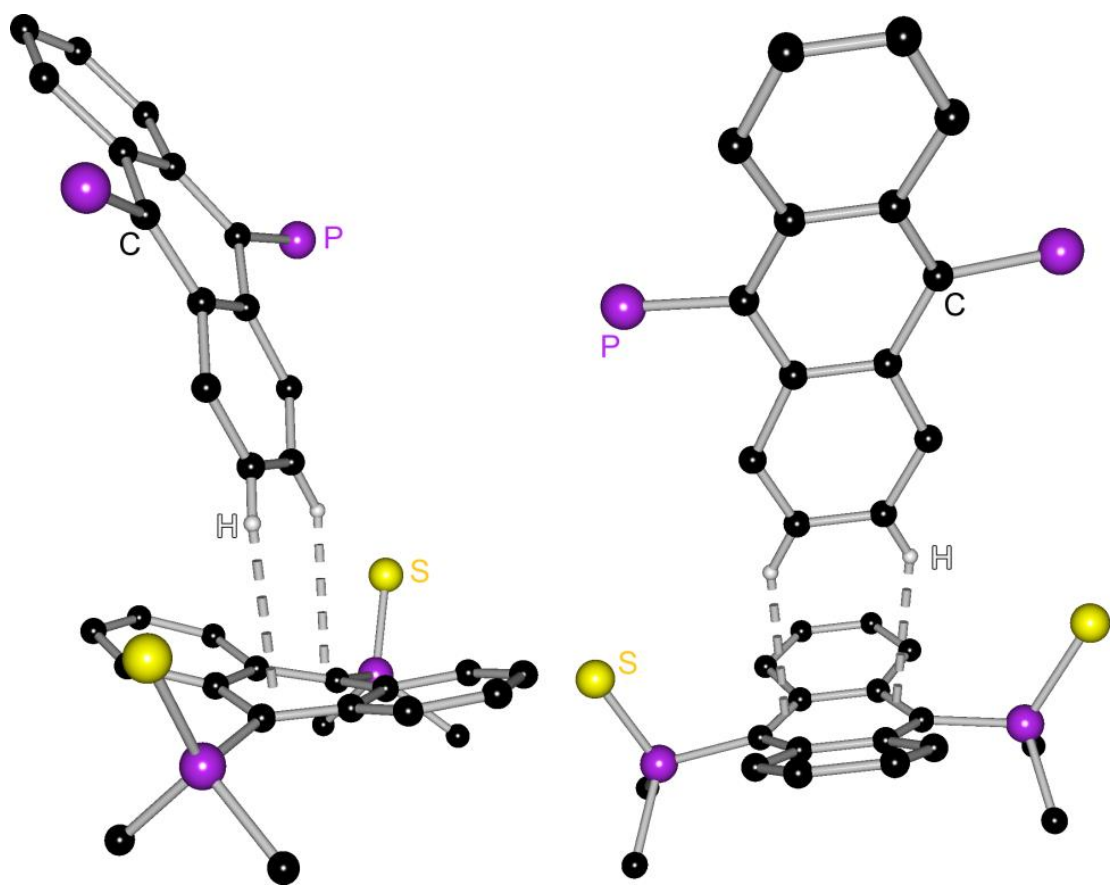

Figure 3-23: C-H“” $\pi$ bonding in the structure of SPAnPS@MeCN (16), phenyl substituents are omitted for clarity.

Due to the symmetry of the molecule both interactions show identical distances and angles of $2.667 \AA / 56.9^{\circ}$. Though this distance is fairly short, the hydrogen atoms are located above the very edge of the central $C_{6}$-perimeter, which makes the $\pi$ density in this region at least questionable and therewith makes this interaction weaker than the distance and angle imply.

Crystallization of SPAnPS from an acetone/hexane mixture again affords a structure with a cisoid conformation of the SPAnPS molecule. SPAnPS@Ace (17) crystallizes in the monoclinic space group $P 2_{1} / c$ and the asymmetric unit contains one molecule as well as disordered fragments of acetone and hexane molecules. The observed bond distances do not differ significantly from those observed in the structure of 16. A fairly strong folding angle of the anthracene moiety of $28.3^{\circ}$ is found, as well as a twist deformation of $3.1^{\circ}$. As in 15 and 16 , no noteworthy $\pi-\pi$ interactions are present in the structure of 17 . A single $\mathrm{C}-\mathrm{H}^{\cdots} \pi \pi$ interaction between a para $\mathrm{C}-\mathrm{H}$ of a phenyl substituent and a peripheral anthracene ring is observed (Figure 3-24). It measures $2.714 \AA$ and encloses a moderate angle of $60.5^{\circ}$ with the $\pi$ system. 


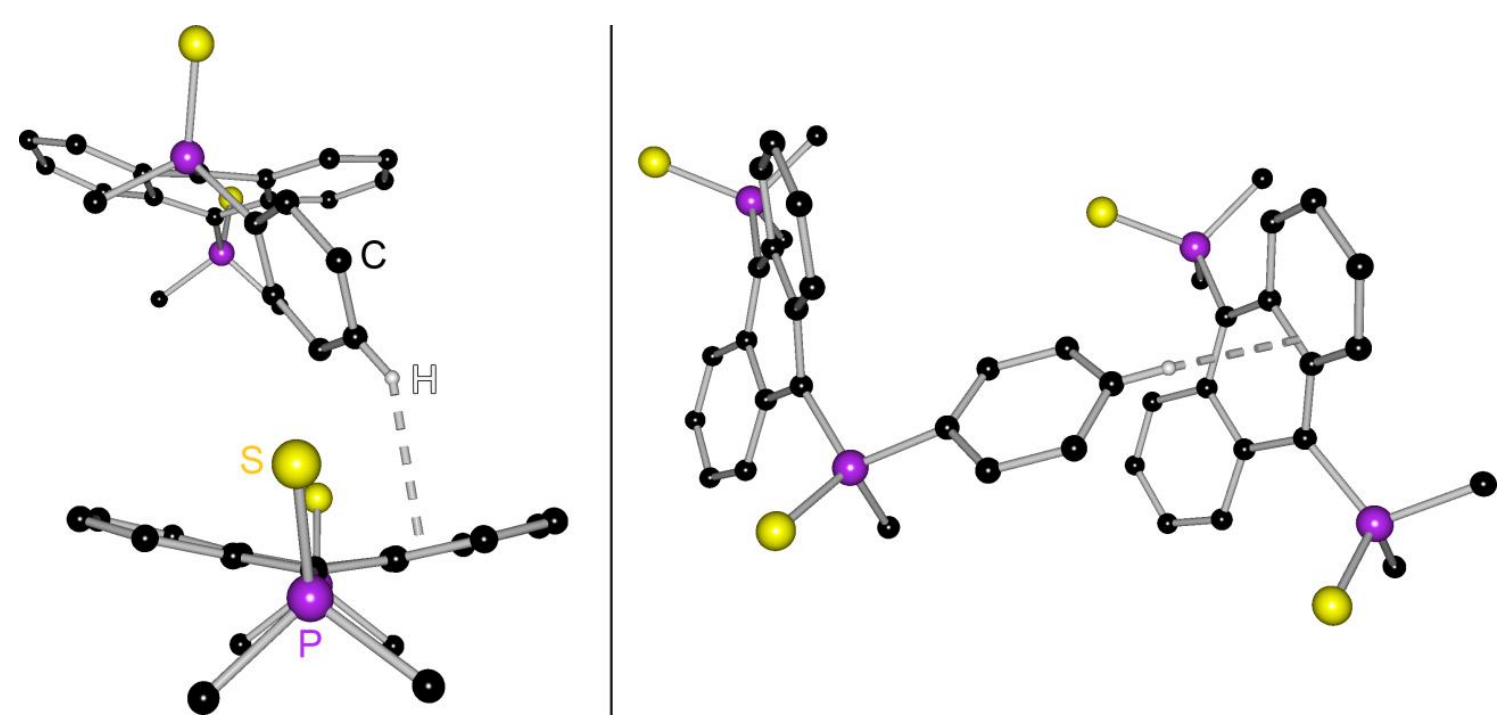

Figure 3-24: $\mathrm{C}-\mathrm{H} \cdots \pi$ bonding in the structure of SPAnPS@Ace (17), phenyl substituents are omitted for clarity.

When SPAnPS is crystallized from DCM/MeOH 3:1, SPAnPS@DCM (18) is obtained. Two molecules of DCM are co-crystallized in this structure, $\mathrm{MeOH}$ is not intercalated. 18 crystallizes in the monoclinic space group $P 2_{1} / c$ and the asymmetric unit contains one molecule of SPAnPS@DCM. The P-S and P-C bond distances are in the expected range, showing only marginal deviations compared the previous structures of SPAnPS. A cisoid conformation of the SPAnPS molecule is found which is nearly perfectly symmetric. SPAnPS@MeCN (16) - which is perfectly symmetric - shows a twist angle of $0^{\circ}$.SPAnPS@DCM (18) which is structurally very closely related exhibits a twist angle of $1.1^{\circ}$ which deviates only minimally. The symmetry of the structure of SPAnPS@DCM is lowered by the intercalated DCM molecules.
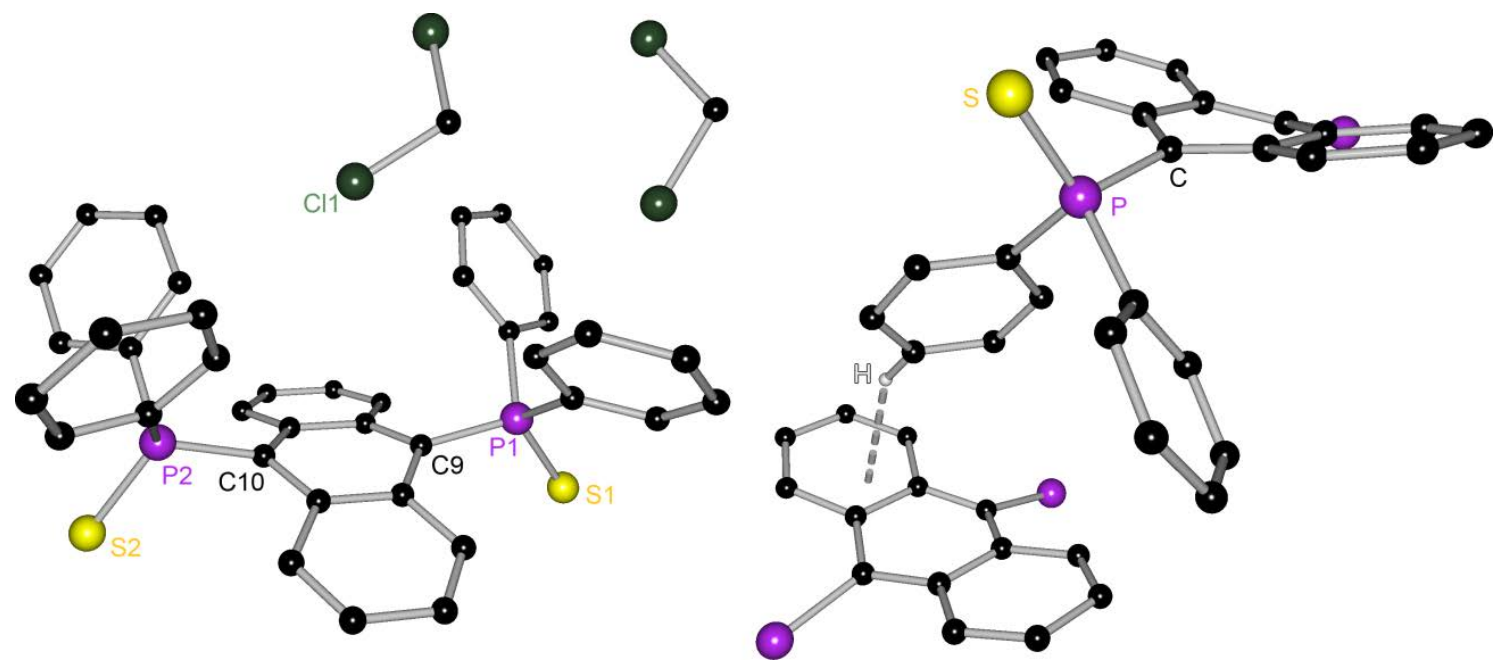

Figure 3-25: left: crystal structure of SPAnPS@DCM (18), hydrogen atoms are omitted for clarity; right: $\mathrm{C}-\mathrm{H}^{\cdots} \pi \mathrm{r}$ bonding in the structure of 18 , phenyl substituents are omitted for clarity. 
Table 3-6: selected bond lengths $[\AA]$ and angles $\left[{ }^{\circ}\right]$ of $\mathbf{1 8 .}$

\begin{tabular}{cc}
\hline P1-C9 & $1.821(3)$ \\
P1-S1 & $1.9503(9)$ \\
C9-P1-S1 & $114.04(9)$ \\
C8a-C9-P1-S1 & $-84.1(2)$ \\
folding & 25.0 \\
twist & 1.1 \\
\hline
\end{tabular}

While the twist angle is very small, a folding angle of $25.0^{\circ}$ is found, which is slightly smaller than that of SPAnPS@MeCN (16). Again, no $\pi-\pi$ interaction is present in the structure of 18 , but a $\mathrm{C}-\mathrm{H}^{\prime \prime} \pi$ bond of the para-C-H of a phenyl substituent to a peripheral ring of the anthracene moiety is found. The distance to the $\pi$-system measures $2.796 \AA$ at an angle of $59.0^{\circ}$. So far, all solvents/solvent mixtures used were nonaromatic. All intercalation structures resulting from crystallization from these solvents showed cisoid

conformation of the SPAnPS molecule with large folding angles and small twist angles.

Crystallization of SPAnPS from EtOAc surprisingly does not lead to an intercalation of solvent molecules (Figure 3-26). When crystallized at $22^{\circ} \mathrm{C}$, the first completely lattice solvent free structure of SPAnPS is obtained, which will be referred to as SPAnPS_pure (19). Crystallization temperatures below $0^{\circ} \mathrm{C}$ lead to a structure which contains strongly disordered EtOAc molecules. However the quality of the acquired Xray diffraction data was too poor for structure refinement, which is why this structure is not included in this thesis. The quantitative formation of solvent-free crystals at $22^{\circ} \mathrm{C}$ crystallization temperature was also confirmed by NMR experiments.

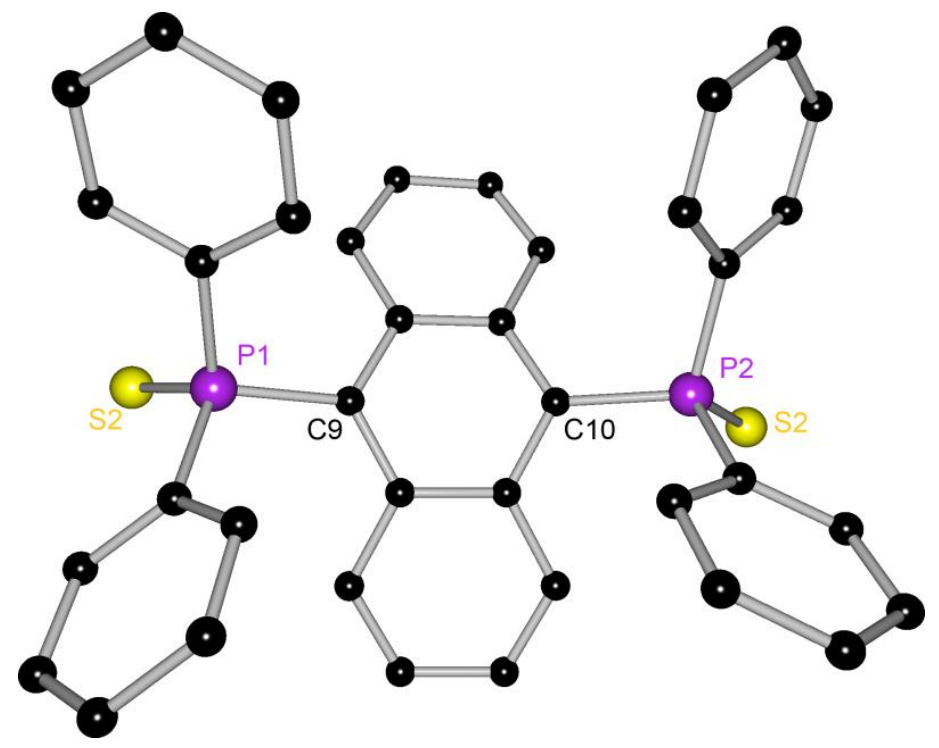

Table 3-7: selected bond lengths $[\AA]$ and angles $\left[^{\circ}\right]$ of 19.

\begin{tabular}{cc}
\hline P1-C9 & $1.824(3)$ \\
P1-S1 & $1.9551(8)$ \\
C9-P1-S1 & $114.55(8)$ \\
C8a-C9-P1-S1 & $85.7(2)$ \\
folding & 32.1 \\
twist & 8.0 \\
\hline
\end{tabular}

Figure 3-26: Crystal structure of SPAnPS_pure (19), hydrogen atoms are omitted for clarity. 


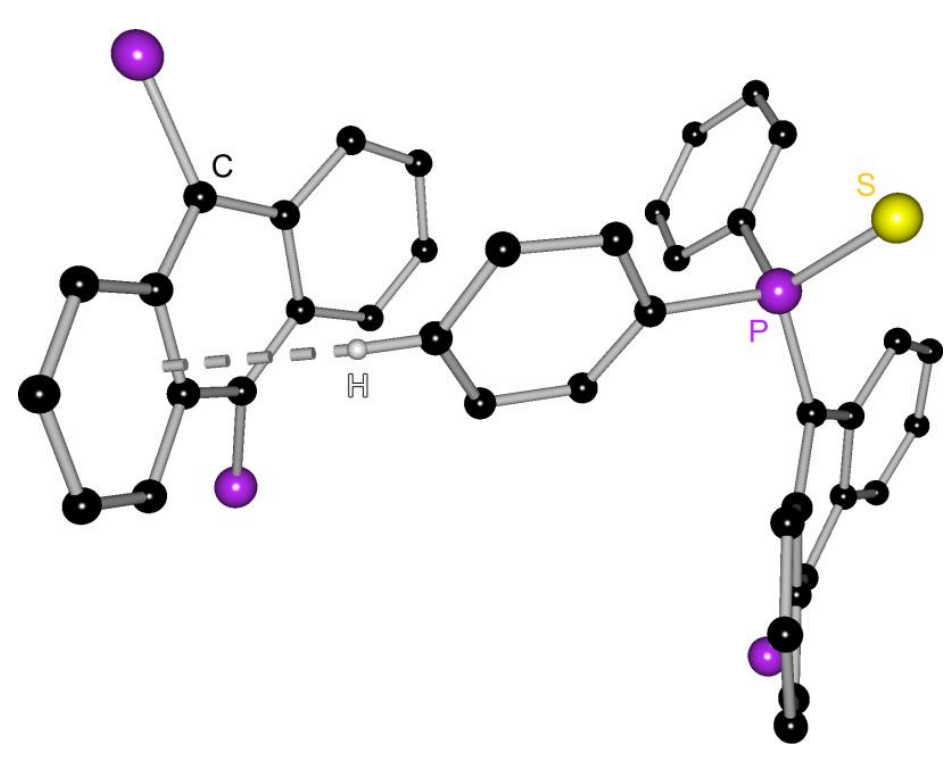

Figure 3-27: C-H'” $\pi$ bonding of SPAnPS_pure (19), phenyl substituents are omitted for clarity.
SPAnPS_pure

crystallizes in the monoclinic space group $P 2_{1}$, and the asymmetric unit contains one SPAnPS molecule. The absence of intercalated solvent leads to the strongest deformation of the anthracene fluorophore encountered so far. A folding angle of $32.1^{\circ}$ is found, and additionally a twist angle of $8.0^{\circ}, \pi-\pi$ overlap is not observed. The $\mathrm{P}-\mathrm{S}$ and $\mathrm{P}-\mathrm{C}$ bond distances are not

affected by the stronger distortion of the molecule.

A single $\mathrm{C}-\mathrm{H}^{\prime \prime} \pi$ bond from the para $\mathrm{C}-\mathrm{H}$ of a phenyl substituent to the adjacent $\pi$ system is found, which is very similar in orientation and geometry to the interaction found in SPAnPS@DCM (18). It measures only $2.613 \AA$, which is quite short, and encloses an angle of $53.0^{\circ}$ with the $\pi$-system.

Finally, SPAnPS was crystallized from toluene $d_{8}$. The obtained compound SPAnPS@tol_d $d_{8}$ (20) was prepared to determine whether the change from hydrogen to deuterium would affect the $\mathrm{C}-\mathrm{H} \cdots \pi$ interactions present in SPAnPS@tol (15). Also a sufficient amount of $\mathbf{2 0}$ was prepared for acquisition of solid state fluorescence spectra. This way comparison of $\mathrm{C}-\mathrm{H}^{\cdots}{ }^{\prime \prime} \pi$ bond distances from the crystal structures, as well as of emission properties was possible. Because an identical mode of intercalation in the host/guest complex was proposed, the contribution of $\mathrm{C}-\mathrm{H} \cdots \pi$ bonding to fluorescence emission by weighing up of small structural alterations induced by differences between $C-H^{\cdots} \pi$ and $C-D \cdots \pi$ bonding was sighted.

Surprisingly, the unit cells of SpAnPS@tol (15) and SPAnPS@tol_d8 (20) differed significantly, the latter showing a unit cell volume of $9682.8 \AA^{3}$, which is more than the fourfold volume observed for SPAnPS@tol (15). A second dataset using a new crystal was collected which confirmed the cell parameters. To eliminate uncertainties, the 
crystals were re-dissolved and crystallized again. Matrix scans of several new crystals were recorded and the cell parameters were identical every time, confirming the results of the first batch of crystals.

While 15 crystallizes in the monoclinic space group $P 2_{1} / n, 20$ crystallizes in the triclinic space group $P \overline{1}$. The symmetry of $\mathbf{2 0}$ is lowered due to the disorder of toluene guest molecules. The asymmetric unit contains four whole SPAnPS molecules and two half SPAnPS molecules. Additionally, seven toluene $d_{8}$ molecules are intercalated, 5 of them in disordered positions. Though all SPAnPS molecules have very similar transoid geometries, they all differ slightly in terms of folding and twist angles which are listed in Table 3-8.

Table 3-8: Folding and twist angles found in SPAnPS@tol and SPAnPS@tol_ds.

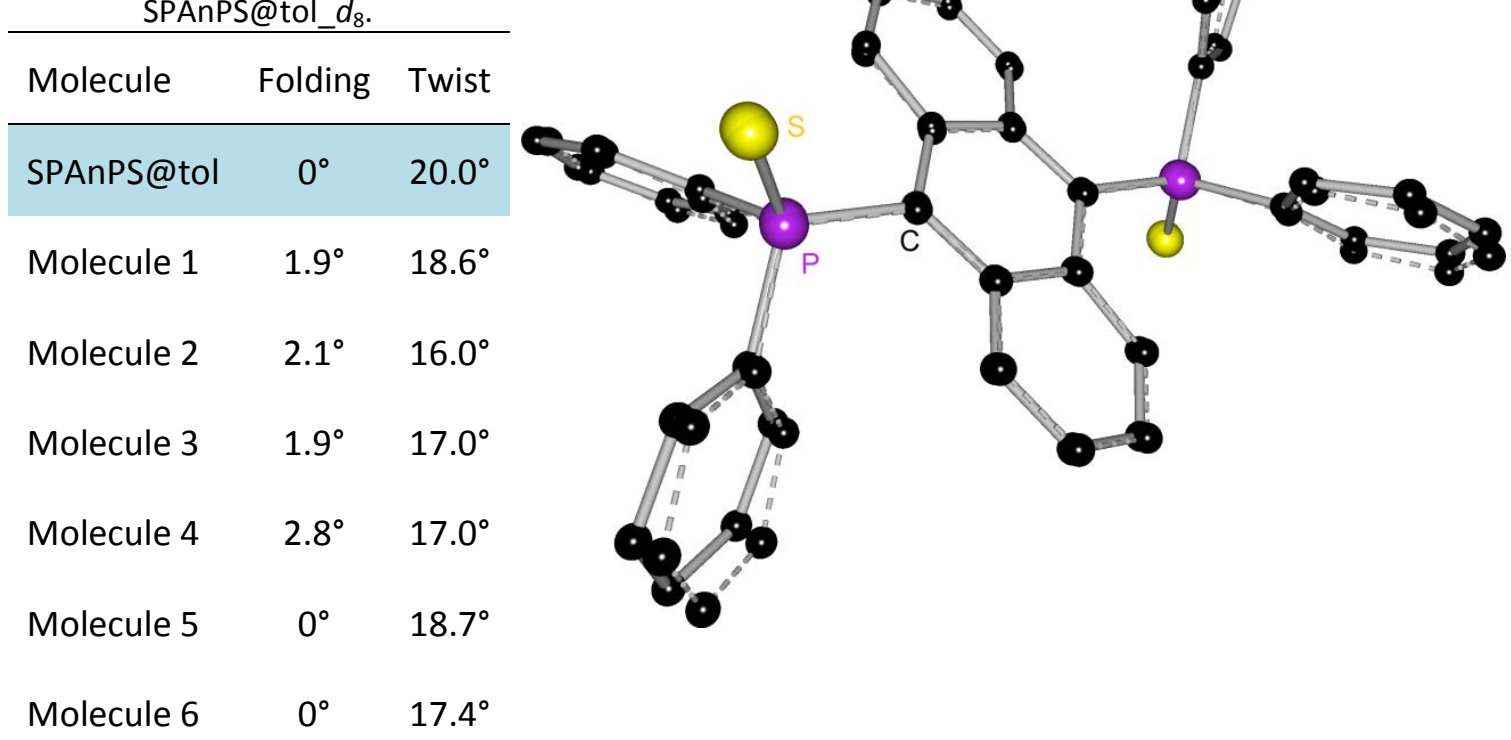

Figure 3-28: Superposition of SPAnPS@tol (15) and SPAnPS@tol_d (20), molecule 4 (dashed).

Moreover, all molecules found in the structure of $\mathbf{2 0}$ also minimally differ from SPAnPS@tol (15) (Figure 3-28). While all molecules of 20 exhibit slightly smaller twist angles than 15 , four of the molecules show weak folding around $2^{\circ}$. Hence, the weaker twisting of the molecules is compensated by slight folding of the fluorophores. Exceptions are the two molecules which are generated by symmetry operations, similar to SPAnPS@tol (15). Due to symmetry reasons their folding angle is also $0^{\circ}$ and the observed twist angle is at approximately $18^{\circ}$, resulting in an overall weaker deformation than found in SPAnPS@tol (15). Several phenyl substituents of 20 show slight disorder. Although the toluene $d_{8}$ molecules are also located above and below 
the anthracene planes, they are with few exceptions strongly disordered and do not adopt fixed positions as the normal toluene molecules do in 15 . Even the nondisordered toluene $d_{8}$ molecules are not positioned in accordance with the normal toluene molecules in 15. Figure 3-29 shows the structures of SPAnPS@tol (15) (left) and the structure of SPAnPS@tol_d $d_{8}$ (20), molecule 3 (right) including the respective $C-H^{\cdots \cdots} \pi / C-D \cdots \pi$ bonded toluene molecules. While in 15 , the toluene molecules are associated by symmetry, they are not in $\mathbf{2 0}$, which is why they are marked with $\mathbf{A}$ and B. $\mathbf{A}$ is not disordered and adopts a fixed position, while $B$ is one of three stationary disordered positions. It is obvious at first sight that the toluene molecules in $\mathbf{1 5}$ assume an almost orthogonal position relative to the anthracene plane, which is often referred to as a T-shaped or "herringbone" arrangement. In contrast, the toluene molecules in 20 are arranged in a more flat angled manner.
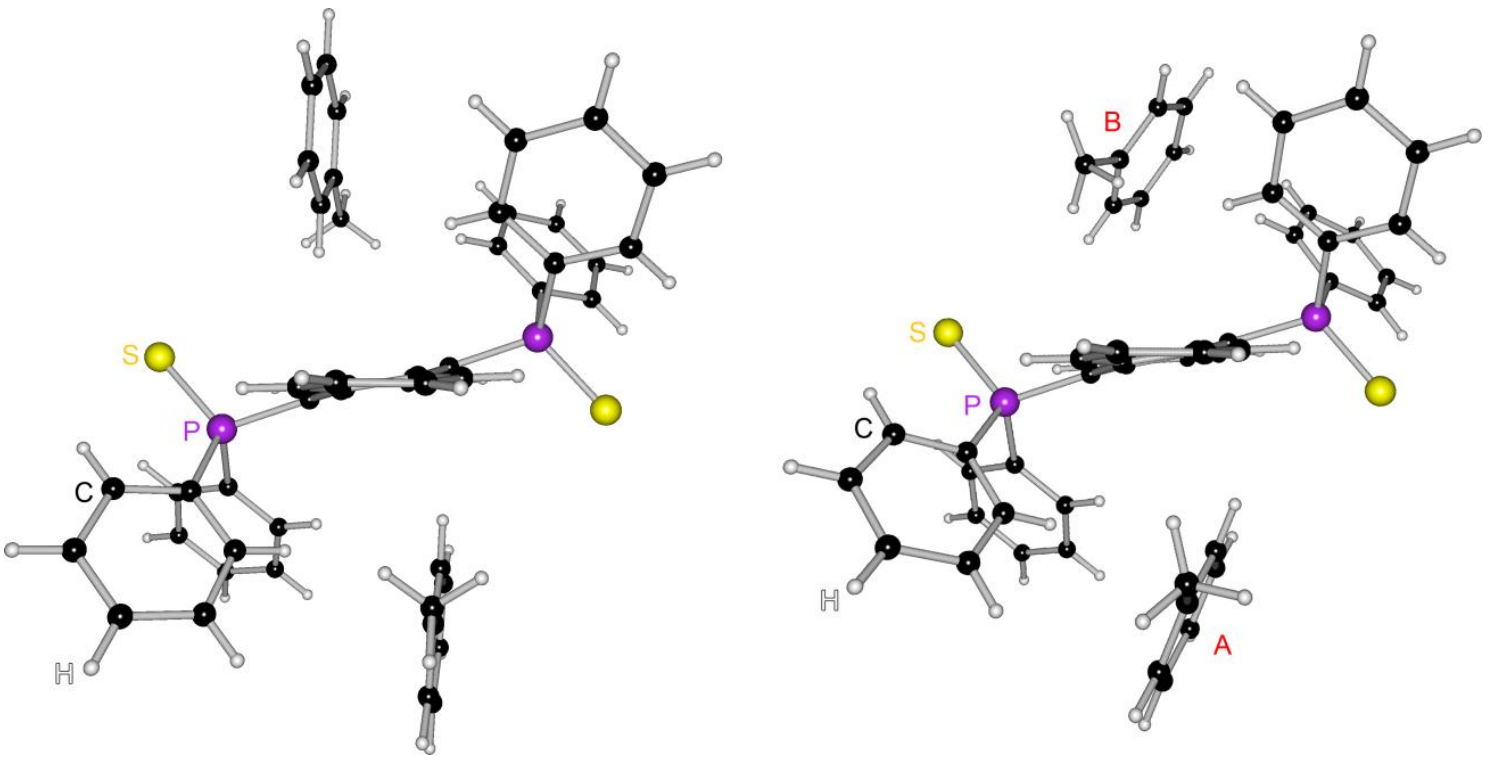

Figure 3-29: comparison between SPAnPS@tol (15) (left) and SPAnPS@tol_d $d_{8}(20)$, molecule 3 (right);

$\mathbf{A}=$ toluene $d_{8}$ molecule in fixed position, $\mathbf{B}=$ disordered toluene $d_{8}$ molecule (only one position depicted).

This also reflected by the lengths and angles of the $C-H \cdots \pi / C-D \cdots \pi$ interactions. The two $\mathrm{sp}^{2} \mathrm{C}-\mathrm{H}^{\cdots} \pi$ bonds found in 15 measure $2.816 \AA$ at $76.8^{\circ}$ to the ring plane. For the disordered toluene $d_{8}$ molecules in $\mathbf{2 0}$, an accurate determination of C-D $\cdots \pi$ distances is difficult, but the angles to the $\pi$-system can be acquired at least roughly. For the disordered toluene molecules these angles range from $35^{\circ}$ to $65^{\circ}$, depending on the examined molecule. For the toluene molecules in fixed positions a more accurate determination is possible. Here the $\mathrm{sp}^{2} \mathrm{C}-\mathrm{D} \cdots \pi$ distances range from $2.850 \AA$ to $3.076 \AA$. 
Moreover the angles enclosed with the $\pi$-system deliver values between $55^{\circ}$ and $65^{\circ}$. Some toluene $d_{8}$ molecules are displaced from the "optimum" position so far that the aromatic hydrogen atoms are not positioned above the $\pi$-system, making C-D $\cdots \pi$ bonding unlikely. In some cases also C-D $\pi \pi$ interactions of the toluene methyl group and the $\pi$-system are found ranging from $\sim 2.75$ to $\sim 3.00 \AA$.

Overall, the C-D" $\pi$ interactions found in the structure of SPAnPS@tol_d $d_{8}$ (20) appear to be weaker than those found in SPAnPS@tol (15), which can be deduced from the distances and angles of these interactions. While normal toluene is only found in fixed positions within the structure of 15 , toluene $d_{8}$ mostly appears in disordered positions, and only few fixed positions. This indicates that the interactions of carbon bound deuterium atoms with aromatic $\pi$-systems seem to be weaker than those of hydrogen atoms. The stronger interaction produces nearly orthogonal arrangements in fixed positions, the weaker interaction produces flat-angled arrangements and is not capable of binding the toluene $d_{8}$ molecules in fixed positions. These differences in bonding should also become manifest in the solid state emission properties of $\mathbf{1 5}$ and 20.

\subsubsection{Solid State Fluorescence}

The solid state fluorescence properties of 15-20 were investigated to fathom whether structural features and intermolecular interactions gathered in 2.3.2 are also reflected by the observed fluorescence emission.

For this purpose, sufficient amounts of single crystals of 15-20 were removed from the mother liquor by filtration and immediately ground and filled into the solid state sample cell for measurement. This way evaporation of lattice solvent was largely suppressed, which is important for consistent results. The evaporation of solvent leads to destruction of the crystalline structure of the sample, which is accompanied by fluorescence quenching. Depending on the volatility of the lattice solvent, this can be a slow or fast process. Although the resulting decomposition is at first only limited to the surface of the microcrystalline particles of the sample, the impact on fluorescence emission is not negligible. To quantify this effect a sample of SPAnPS@tol (15) was filled into the solid state sample cell and repeatedly subjected to identical fluorescence measurements over the course of ten hours. Though the sample cell is a closed device, 
it is not completely air proof and allows the diffusion of evaporated lattice solvent from the sample. Nevertheless this process most likely proceeds even faster in an open vessel.

Figure 3-30 shows the decay of fluorescence intensity over elapsed time. It clearly indicates the quenching of emission upon loss of lattice solvent and therewith of crystalline structure. The speed of crystal decomposition is also dependent on the particle size, because finely ground particles possess a larger surface than larger particles and allow more lattice solvent to evaporate in a defined time interval.

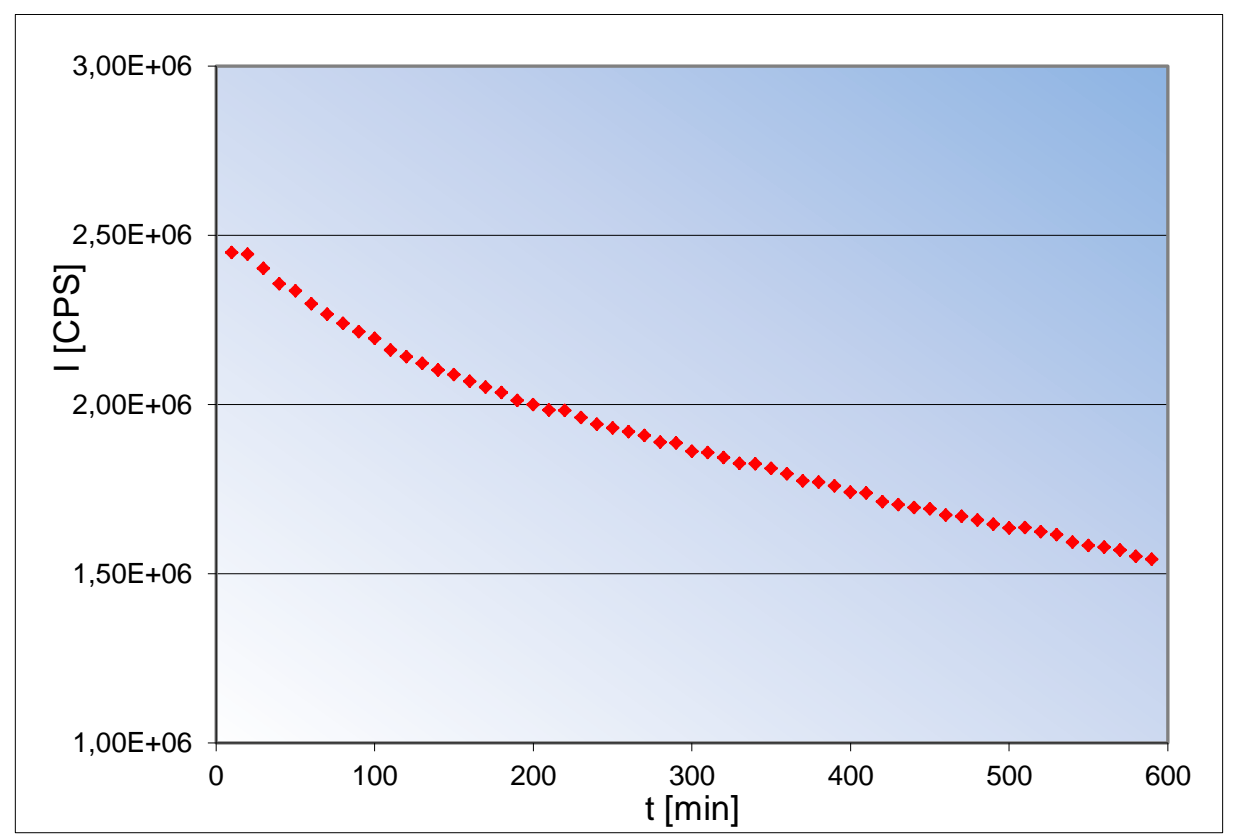

Figure 3-30: Time dependent fluorescence decay of SPAnPS@tol (15) by gradual evaporation of lattice solvent.

If the evaporation process were monitored over an even longer period of time, eventually the emission intensity of solvent free SPAnPS powder would be reached, which was produced by Fei et al. by drying of SPAnPS@tol crystals under heating and reduced pressure. ${ }^{[44 b, 44 c]}$

The observations made by Schwab (which were founded exclusively on optical inspection) that only structures of SPAnPS in a transoid conformation show strong solid state fluorescence were generally confirmed by solid state fluorescence experiments. Because the weakly fluorescent cisoid structures require different measurement conditions than the strongly fluorescent structures in order to obtain suitable data, comparison is only reasonable among compounds of similar 
conformation. The only transoid structures obtained were those of SPAnPS@tol (15) and SPAnPS@tol_d $d_{8}$ (20).As a crystalline analogue to the vacuum dried crystals which were used by Fei et al. for comparison, the lattice solvent free SPAnPS_pure (19) was employed. It will therefore serve as a comparison for molecules in both the transoid and the cisoid conformation.
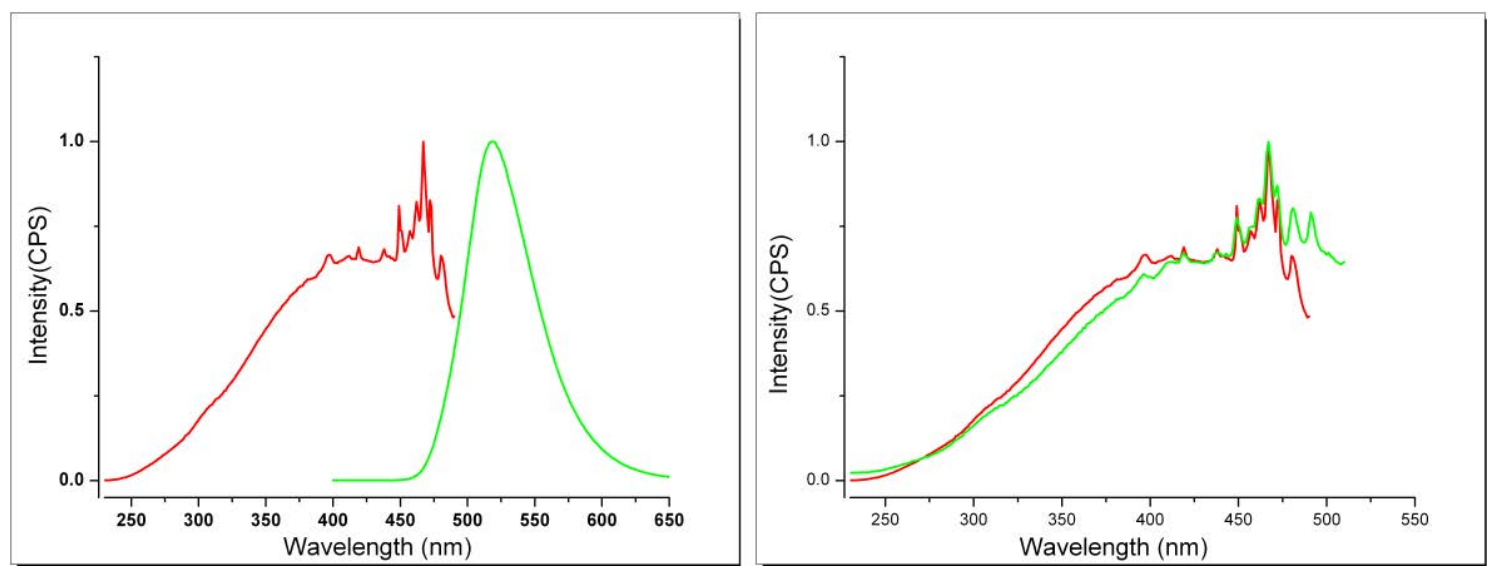

Figure 3-31: Left: normalized excitation (red) and emission (green) spectra of SPAnPS@tol (15); right: normalized excitation spectra of SPAnPS@tol (15) (red) and SPAnPS@tol_d8 (20) (green).

Figure 3-31 (left) shows the normalized solid state excitation and emission spectra of SPAnPS@tol (15).In a broad range from $350 \mathrm{~nm}$ to $480 \mathrm{~nm} 15$ shows strong fluorescence, reaching a sharp maximum at an excitation wavelength of $467 \mathrm{~nm}$. The emission band is not quite as broad but also spans over $50 \mathrm{~nm}$. It reaches its maximum at $519 \mathrm{~nm}$ and does not exhibit the typical anthracene vibrational structure, which has been previously observed for several phosphorylanthracenes in solution (c.f. 3.1). The excitation spectra of SPAnPS@tol (15) and SPAnPS@tol_d $d_{8}(20)$ (Figure 3-31, right) are of nearly identical shape and show identical maxima, but also deviate slightly in some regions, which already indicates that the differences observed in the crystal structures may also be reflected by the fluorescence properties of both compounds. Also the maximum emission wavelengths are affected and are surprisingly not identical (Figure 3-33, left). Though they only differ by $4 \mathrm{~nm}$, the differences induced co-crystallized toluene and toluene $d_{8}$ are undeniable. The lattice solvent free SPAnPS_pure (19) shows even stronger deviation from 15 in its maximum emission wavelength, which exhibits a bathochromic shift of $8 \mathrm{~nm}$. Though $\pi-\pi$ overlap has repeatedly been named as an influential factor on maximum emission wavelengths, ${ }^{[50,}{ }^{53]}$ the large $\pi-\pi$ distances within the structures of 15, 19, and 20 virtually preclude this option. 


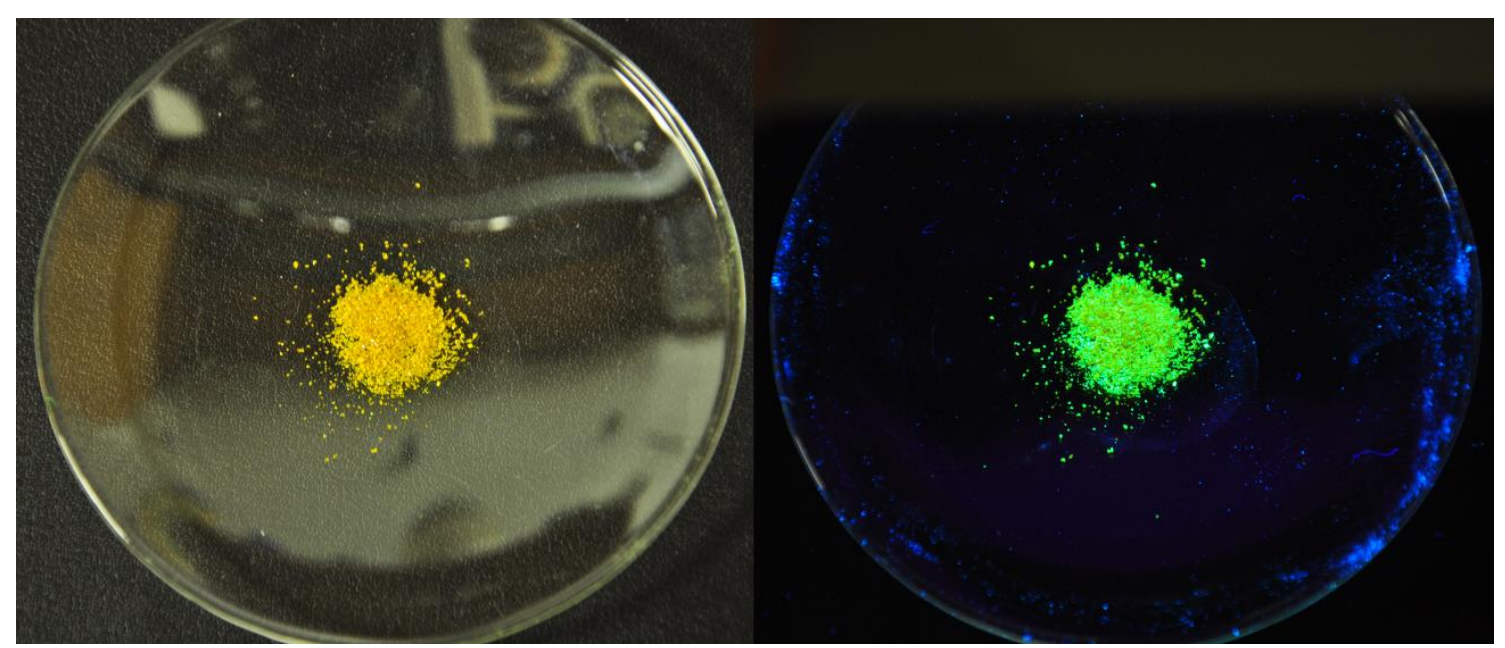

Figure 3-32: Sample of SPAnPS@tol_d8 (20) in daylight (left) and under exposure to UV light, $\lambda_{\mathrm{Ex}}=366 \mathrm{~nm}$ (right).

The differences between these three compounds become even more evident when comparing their emission intensities (Figure 3-33, right). The emission of the lattice

Table 3-9: Compiled maximum emission wavelengths and relative emission intensities of 15, 19, 20.

\begin{tabular}{ccccc}
\hline & $\lambda_{E m}(\max )[\mathrm{nm}]$ & $\mathrm{I}_{\mathrm{rel}}{ }^{\mathrm{a}}$ & $\mathrm{I}_{\text {rel }}^{\mathrm{b}}$ & $\mathrm{I}_{\text {rel }}^{\mathrm{c}}$ \\
20 & 519 & 1 & 1 & 1 \\
19 & 515 & 0.62 & 0.66 & 0.70 \\
19 & 527 & 0.018 & $\mathrm{n} / \mathrm{A}$ & $\mathrm{n} / \mathrm{A}$
\end{tabular}

a) $\lambda_{E x}=380 \mathrm{~nm}$; b) $\lambda_{E x}=449 \mathrm{~nm} ; \lambda_{E x}=467 \mathrm{~nm}$. solvent free 19 is nearly completely quenched compared to SPAnPS@tol (15) and SPAnPS@tol_d $d_{8}$ (20). The intensity ratio of $1: 0.018$ between the emission of 15 and 19 (equates to factor 56) is in the range of the intensity ratio reported by $\mathrm{Fei}$ et al. between SPAnPS@tol and vacuum dried crystals. ${ }^{[44 b, 44 c]}$ But also 15 and 20 differ significantly in terms of emission intensity. Independent of the excitation wavelength, the structure containing toluene $d_{8}$ as lattice solvent only reaches between $60 \%$ and $70 \%$ of the emission intensity produced by the structure containing regular toluene (Table 3-9). 

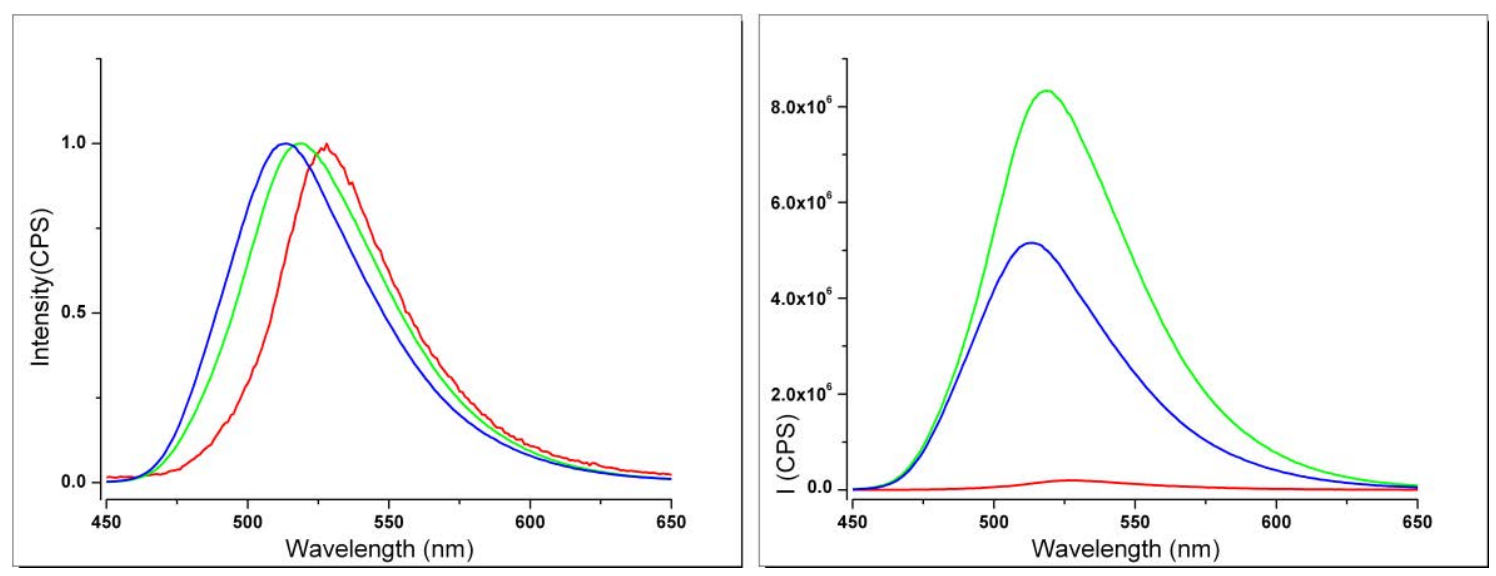

Figure 3-33: Left: normalized solid state emission spectra of SPAnPS_pure (19) (red), SPAnPS@tol (15) (green), and SPAnPS@tol_d8 (20) (blue); right: solid state emission spectra of SPAnPS_pure (19) (red), SPAnPS@tol (15) (green), and SPAnPS@tol_d8 (20) (blue).

Explanations for the observed phenomena can be deduced from the structural properties discussed in 3.2.2. Compared to both compounds with a transoid conformation of the SPAnPS molecule (15 and 20), the cisoid structure of 19 features an enormous folding angle of the anthracene moiety. It measures $32.1^{\circ}$, while the transoid structures exhibit folding angles of $0^{\circ}(15)$ and an averaged angle of $1.15^{\circ}(20)$, respectively. Thus, the folding of the anthracene moiety appears to be obstructive for fluorescence emission, while twist deformation (which is strong for both 15 and 20) does not appear to have a similarly strong quenching effect. On the other hand, the small deviations in fluorophore deformation between SPAnPS@tol (15) and SPAnPS@tol_d $d_{8}$ (20) do not seem to justify the loss of over one third of emission intensity from 15 to 20. Fei et al. have assigned the strong emission of SPAnPS@tol (15) to the formation of an exciplex between the fluorophore and the co-crystallized toluene molecules. The T-shaped arrangement and the formation of it by $\mathrm{C}-\mathrm{H} \cdots \pi$ bonding were shown play a key role in enabling of fluorescence emission. While the toluene molecules in the structure of $\mathbf{1 5}$ are in fixed positions in the T-shaped complex and exhibit no disorder, the toluene $d_{8}$ molecules in the structure of $\mathbf{2 0}$ show strong disorder and a much flatter averaged angle to the fluorophore. In some cases the orientation of the toluene $d_{8}$ molecules even prevents interaction with the fluorophore altogether. Hence, the weaker $\mathrm{C}-\mathrm{H}^{\cdots} \pi$ bonding of the toluene $d_{8}$ molecules to the fluorophore leads to flat angled arrangements and in consequence to weaker fluorescence emission. This clearly demonstrates the importance of $\mathrm{C}-\mathrm{H}^{\cdots \cdots} \pi$ interactions for the formation of strongly emitting molecular arrangements. 

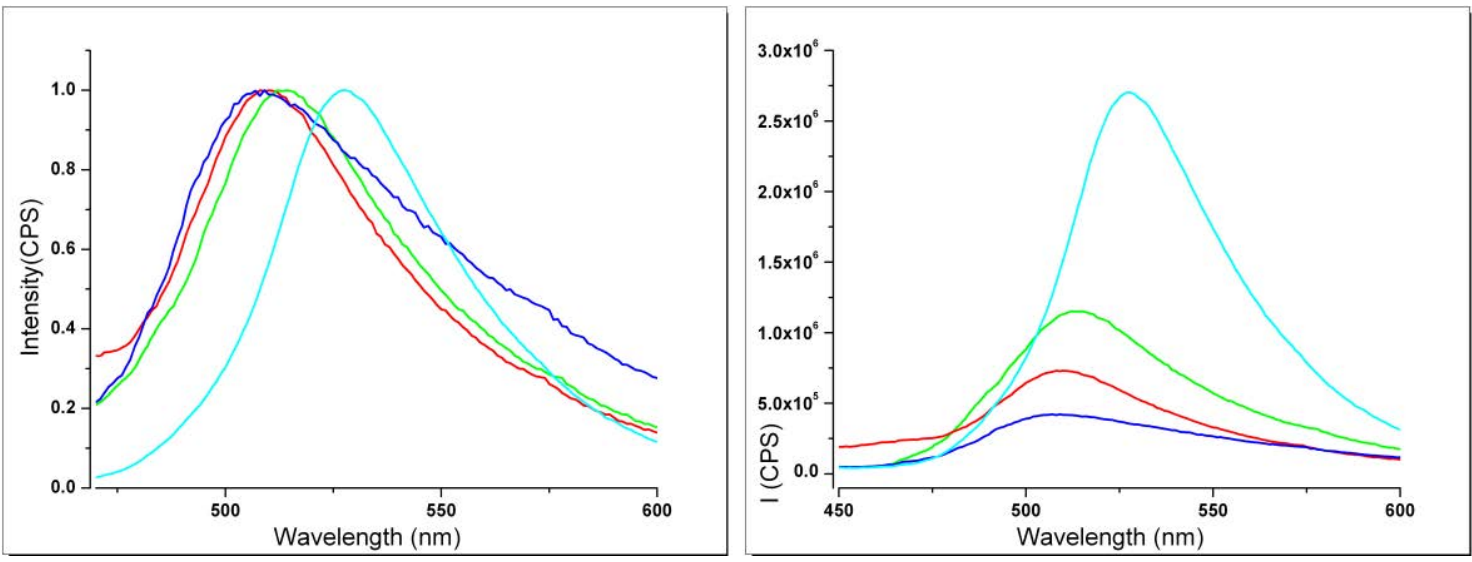

Figure 3-34: Left: normalized solid state emission spectra of SPAnPS@Ace (17) (red), SPAnPS@DCM (18) (green), SPAnPS@MeCN (16) (blue), and SPAnPS_pure (19) (cyan); right: solid state emission spectra of SPAnPS@Ace (17), SPAnPS@DCM (18) (green), SPAnPS@MeCN (16) (blue), and SPAnPS_pure (19) (cyan).

Although by a magnitude weaker fluorescent, the emission properties of the cisoid structures can also be compared.While SPAnPS@MeCN (16), SPAnPS@Ace (17), and SPAnPS@DCM (18) all exhibit very similar maximum emission wavelengths, the emission maximum of the lattice solvent free SPAnPS_pure (19) is again red-shifted by approximately $10 \mathrm{~nm}$ (Figure 3-34, left). As pointed out before, this effect cannot be produced by $\pi-\pi$ overlap which is completely absent in all four structures. Though all four compounds are weakly fluorescent, SPAnPS_pure (19) shows the clearly strongest emission. This is quite surprising because it also features the strongest deformation of the anthracene moiety, which would make particularly weak emission expectable. The remaining three compounds show emission intensities that roughly correlate with their degree of deformation: SPAnPS@DCM (18) is stronger fluorescent than SPAnPS@Ace (17) and SPAnPS@MeCN (16) and exhibits the weakest folding of the fluorophore $\left(25.0^{\circ}\right) .16$ and $\mathbf{1 7}$ have nearly identical folding angles and differ only in terms of twist deformation. The stronger emitting $\mathbf{1 7}$ features a slightly twisted $\left(3.1^{\circ}\right)$ fluorophore, while 16 does not show any twist deformation. Also the in comparison strongly fluorescent SPAnPS_pure (19) exhibits a noteworthy twist angle of $8.0^{\circ}$, while being strongly folded. This suggests that twisting of the fluorophore may in fact promote fluorescence emission in contrast to folding which causes quenching. This also correlates with the strong fluorescence of SPAnPS@tol (15) and SPAnPS@tol_d (20) which both show strong twist deformation and virtually no folding. 
Furthermore, $\mathrm{C}-\mathrm{H}^{\cdots} \pi$ bonding within the structures of 16-19 can be consulted to explain the observed emission intensities. SPAnPS_pure (19) exhibits a strong C-H' $\pi$ interaction, which may also contribute to the observed fluorescence emission. The structures of SPAnPS@Ace (17) and SPAnPS@DCM (18) also contain noteworthy C-H”' $\pi$ interactions, while the weakest fluorescent SPAnPS@MeCN (16) shows the supposed weakest interaction of this kind. Hence, the combination of the effects of fluorophore deformation and $\mathrm{C}-\mathrm{H}^{\cdots} \pi$ bonding are able to give a conclusive explanation for the measured emission intensities.

When recalling the initial problem whether $\mathrm{Fei}$ - who attributed the solid state fluorescence of SPAnPS@tol (15) to exciplex formation via C-H“” $\pi$ bonding - or Schwab - who assigned the occurrence of fluorescence to a transoid orientation of the phosphoryl substituents - was right with his assumptions, the only valid answer is: both were right. Although Schwab did not explicitly address the deformation of the fluorophore as a quenching factor, the transoid conformation of the SPAnPS molecule to which he assigned strong solid state fluorescence simultaneously leads to evenly distributed steric strain and therewith to weak deformation. The possible emission enhancing effect of twist deformation of the fluorophore was also taken into consideration by Schwab. ${ }^{[59 b]}$ On the other hand the effects of weakened of $\mathrm{C}-\mathrm{H}^{\prime \prime} \pi$ bonding on emission properties was impressively demonstrated by comparison of SPAnPS@tol_d (20) and SPAnPS@tol (15).The observed decrease of emission caused by flat-angled orientation and disordered positioning of toluene $d_{8}$ molecules relative to the anthracene plane emphasizes the importance of $\mathrm{C}-\mathrm{H} \cdots \pi$ interactions for solid state fluorescence of SPAnPS.

\subsection{9-(Bis(diethylamino)phosphoryl)-10-methylanthracenes and their Gold(I) complexes.}

The results presented in 3.2 have illustrated the sensitivity of the solid state emission of SPAnPS towards structural alterations resulting from co-crystallized solvent molecules. While in the case of SPAnPS, where the molecule bearing the fluorophore was always identical, the transferability of the derived findings to other compounds had yet to be investigated. Hence, asymmetrically substituted 9-(bis(diethylamino)phosphoryl)-10-methylanthracenes were synthesized with varying 
substituents. By variation of the phosphoryl substituents it was attempted to monitor the effects of altered steric demand on packing motifs, and consequently, on fluorescence properties. Furthermore, the corresponding gold(I) complexes were prepared to investigate the effects of metal coordination on the solid state structures and on solid state- and in-solution fluorescence. Gold(I) was chosen for complex synthesis due to the high affinity of sulfur and gold towards one another. Furthermore, literature reports on alteration of fluorescence properties upon coordination of gold(I) further supported the choice of this cation. ${ }^{[65]}$

\subsubsection{Synthesis}<smiles>Brc1c2ccccc2c(Br)c2ccccc12</smiles>

1.) $n \mathrm{BuLi}$

2.) $\mathrm{Mel}$

2.) - Lil<smiles>Cc1c2ccccc2c(Br)c2ccccc12</smiles>

1.) $n \mathrm{BuLi}$

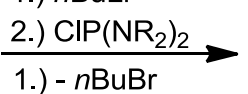

2.) $-\mathrm{LiCl}$

$\mathrm{R}=\mathrm{Me}, \mathrm{Et}$<smiles></smiles><smiles>[R20]NP([R20])(=C[Al]Cl)c1c2ccccc2c(C)c2ccccc12</smiles>
$[\mathrm{Ox}]$

22: $E=S, R=M e$

24: $E=S, R=E t$

26: $E=S e, R=M e$

28: $E=S e, R=E t$

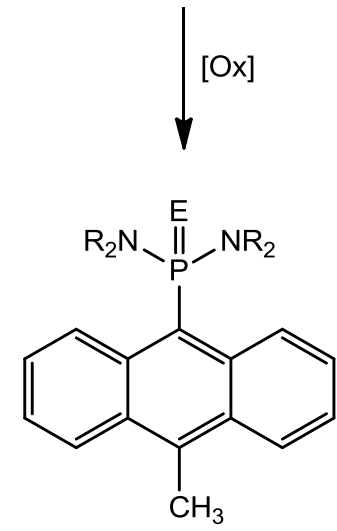

21: $E=S, R=M e$

23: $E=S, R=E t$

25: $E=S e, R=M e$

27: $E=S e, R=E t$

Scheme 3-6: Synthesis of 21-28.

By selective monolithiation of 9,10-dibromoanthracene, asymmetric anthracene derivatives are accessible. ${ }^{[59 a, 60]}$ The phosphorylanthracenes 21, 23, 25, and 27 were synthesized by monolithiation of 9,10-dibromoanthracene and subsequent reaction with methyl iodide. To introduce the phosphane substituent, the lithiation step was repeated and the lithiated species was reacted with the respective chlorophosphane. All chlorophosphanes were prepared according to literature procedures. ${ }^{[68]}$ The subsequent oxidation was carried out with elemental sulfur and selenium, 
respectively. All compounds were crystallized for purification and structurally characterized. In the following, the resulting compounds will be referred to as:

$\begin{array}{ll}\text { MeAnPS}\left(\mathrm{NMe}_{2}\right)_{2}(\mathbf{2 1}) & \text { MeAnPS}\left(\mathrm{NEt}_{2}\right)_{2}(\mathbf{2 3}) \\ \mathrm{MeAnPSe}\left(\mathrm{NMe}_{2}\right)_{2}(\mathbf{2 5}) & \mathrm{MeAnPSe}\left(\mathrm{NEt}_{2}\right)_{2}(\mathbf{2 7})\end{array}$

The gold complexes $\mathbf{2 2}, \mathbf{2 4}, \mathbf{2 6}$, and $\mathbf{2 8}$ were obtained by reaction of the respective phosphorylanthracene with dimethylsulfidechlorogold(I). All metal complexes were also crystallized for purification and structurally characterized. In the following, these compounds will be referred to as:

$$
\begin{array}{ll}
{\left[\mathrm{MeAnP}\left(\mathrm{NMe}_{2}\right)_{2}(\mathrm{~S}) \mathrm{AuCl}\right](\mathbf{2 2})} & {\left[\mathrm{MeAnP}\left(\mathrm{NEt}_{2}\right)_{2}(\mathrm{~S}) \mathrm{AuCl}\right](\mathbf{2 4})} \\
{\left[\mathrm{MeAnP}\left(\mathrm{NMe}_{2}\right)_{2}(\mathrm{Se}) \mathrm{AuCl}\right](\mathbf{2 6})} & {\left[\mathrm{MeAnP}\left(\mathrm{NEt}_{2}\right)_{2}(\mathrm{Se}) \mathrm{AuCl}\right](\mathbf{2 8})}
\end{array}
$$

The methyl substituent in the 10-position is introduced because methyl substituted phosphorylanthracenes have repeatedly shown conspicuous solid state fluorescence phenomena in past research. Moreover, the methyl group is used as a wild-card for various organic moieties. While basically every organic moiety in the 10-position would most likely lead to a different packing motif in the solid state, fluorescence properties in solution are only marginally altered by the steric demand of alkyl substituents in the 10-position, hence a methyl group represents all alkyl substituents quite well for insolution experiments.

\subsubsection{Structural Comparison}

Although the four anthracene derivatives, as well as their gold complexes, are structurally closely related, only $\mathbf{2 3}$ and $\mathbf{2 7}$ are isostructural. In none of the structures lattice solvent molecules are co-crystallized, which ensures good comparability. For all compounds the asymmetric unit contains one molecule, independent of the space group. In consideration of the packing behaviour of substituted anthracenes which have been described in previous publications, a packing motif with a large $\pi-\pi$ overlap and bulky phosphoryl substituents facing in opposite directions appears to be most favourable. ${ }^{[54,59]}$ For explanations on quantification of structural properties such as folding angle, twist deformation or $\mathrm{C}-\mathrm{H}^{\prime}{ }^{\mathrm{m}} \pi$ bonds, please see 3.2.1.

MeAnPS $\left(\mathrm{NMe}_{2}\right)_{2}$ (21) crystallizes in the monoclinic space group $P 2_{1} / n$. The anthracene moiety is not perfectly planar, because it is slightly folded along the 9,10 - 
vector with the dimethylamino and the methyl group both located at the wider side of the anthracene plane. The folding angle adds up to $9.9^{\circ}$, while the twist deformation is weak at $2.0^{\circ}$.

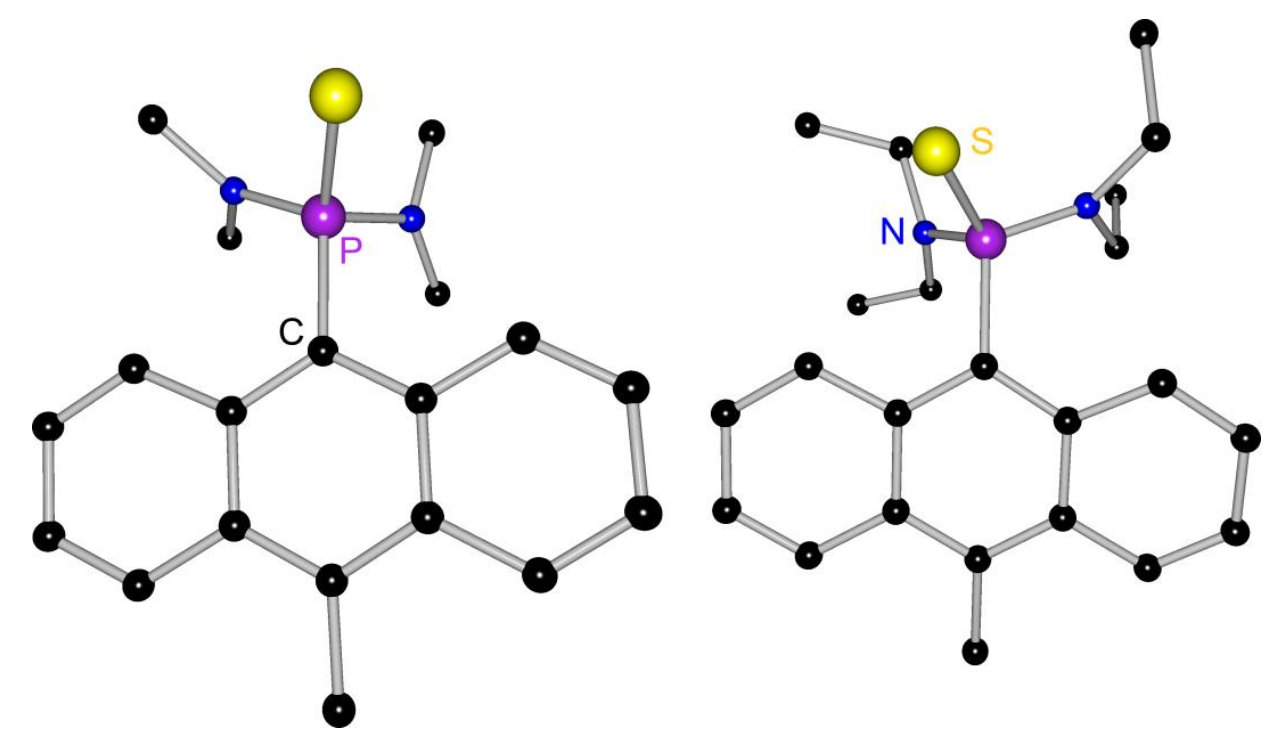

Figure 3-35: Crystal structures of MeAnPS(NMe $)_{2}(\mathbf{2 1} \text {, left) and MeAnPS(NEt })_{2}(\mathbf{2 3}$, right), hydrogen atoms are omitted.

The phosphorus atom in 9-position and the methyl group in 10-position are located almost exactly in the C9-C10-axis. The torsion angle of the C8-C9-P-S-bond to the anthracene plane $70.9^{\circ}$, hence the molecule is not symmetrical, which would require a $90^{\circ}$ angle. Packing plots reveal the intermolecular interactions of $\mathbf{2 1}$ in the solid state. Every two molecules show nearly exact parallel orientation of the anthracene planes with the convex sides facing each other and the phosphoryl substituents pointing to the outside. The observed offset in the face-to-face $\pi$-stacking results in a $\pi$ - $\pi$ overlap of approximately $35 \%$ with a $\pi-\pi$ distance of $3.51 \AA$ (Figure 3-36). Additionally, a methyl $\mathrm{C}-\mathrm{H} \cdots \pi$ interaction between an aminomethyl group and an outer ring of the anthracene $\pi$ system can be found. 


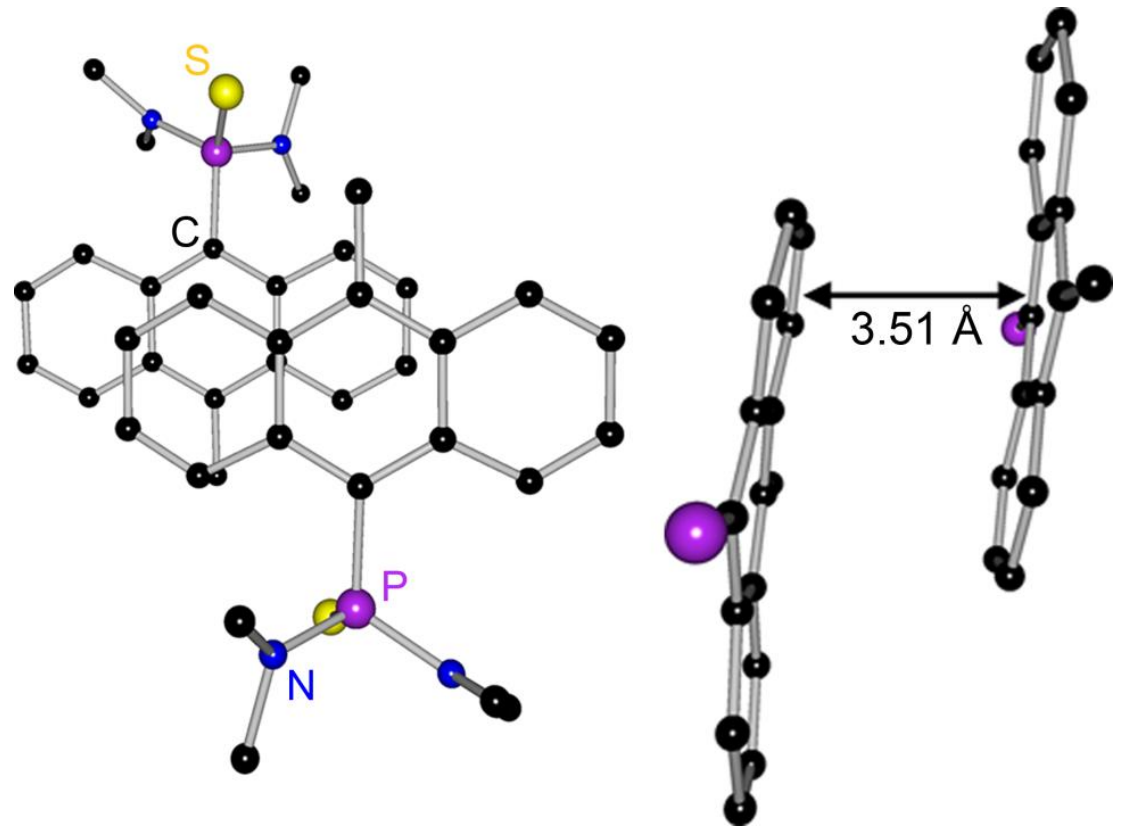

Figure 3-36: $\pi-\pi$ overlap in the structure of MeAnPS(NMe $)_{2}$ (21): top view (left) and side view (right).

In the solid state structure of $21, \mathrm{sp}^{3} \mathrm{C}-\mathrm{H} \cdots \pi$ distances of $2.946 \AA$ and $2.993 \AA$, following from two methyl $\mathrm{C}-\mathrm{H}$-bonds, are observed. The angles to the anthracene ring-plane measure $48.8^{\circ}$ and $25.7^{\circ}$, respectively, which is considerably more acute than the optimum T-shaped arrangement. Therefore these interactions can be considered comparatively weak.

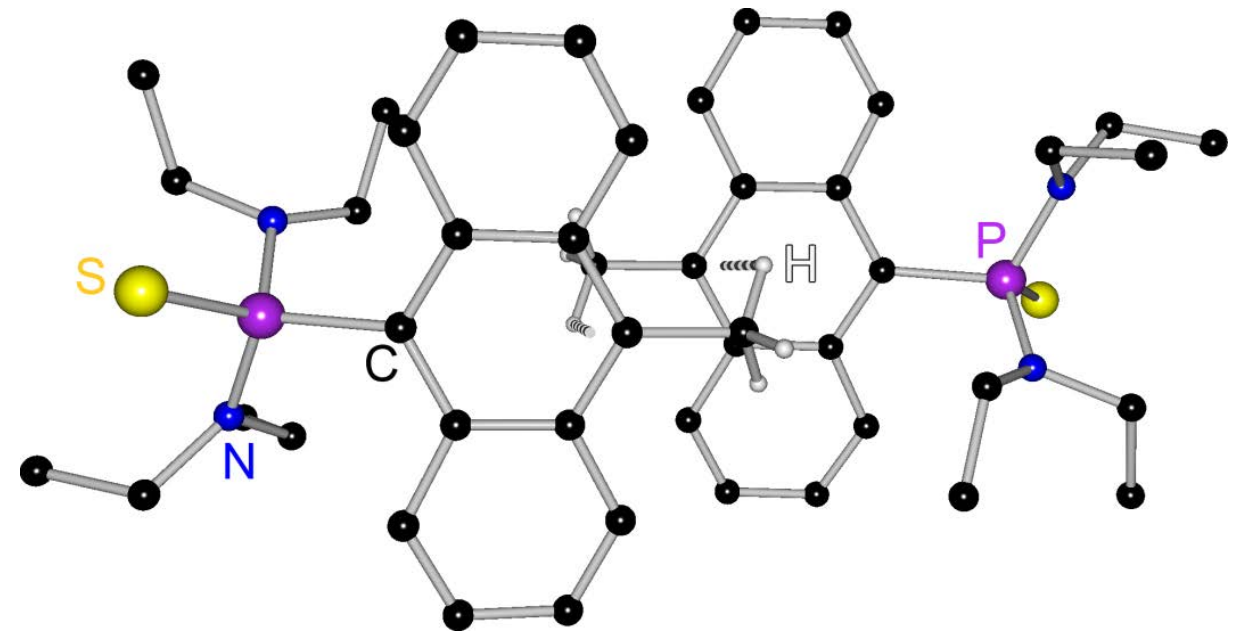

Figure 3-37: Low angled C-H“” $\pi$ interaction in the structure of $\mathbf{2 3}$ without $\pi-\pi$ overlap.

Compared to MeAnPS(NMe $)_{2}$ (21), the steric bulk of the phosphoryl substituent is increased in MeAnPS(NEt $)_{2}$ (23) by replacing the dimethylamino groups with diethylamino groups. This is reflected by a larger folding angle of the anthracene moiety of $13.8^{\circ}$. As in $\mathbf{2 1}$, both amino-substituents are located on the same side of the 
anthacene plane, leading to a stronger folding and also a slight twist angle of $8.8^{\circ}$ of the ring system. Also, the phosphorus atom is forced out of the C9*C10-axis. Furthermore, the intermolecular interactions in the solid state structure of $\mathbf{2 3}$, which crystallizes in the monoclinic space group $P 2_{1} / c$, differ significantly from those found in 21. In 23 , no noteworthy $\pi-\pi$ interactions are found: the shortest distance between two $\pi$-systems measures nearly $7 \AA$, which is negligible. Though a "head-to-tail" arrangement is also found in $\mathbf{2 3}$, the molecules are shifted so far that the $\pi$ systems no longer overlap and the only significant interaction found is an $\mathrm{sp}^{3} \mathrm{C}-\mathrm{H}^{\cdots} \pi$ interaction of the methyl group in 10-position with the central ring of the adjacent anthracene moiety. One $\mathrm{C}-\mathrm{H}$-bond is directed towards the anthracene $\pi$ system showing a distance/angle of $2.899 \AA / 31.2^{\circ}$, which again can be considered fairly weak (Figure 3-37).
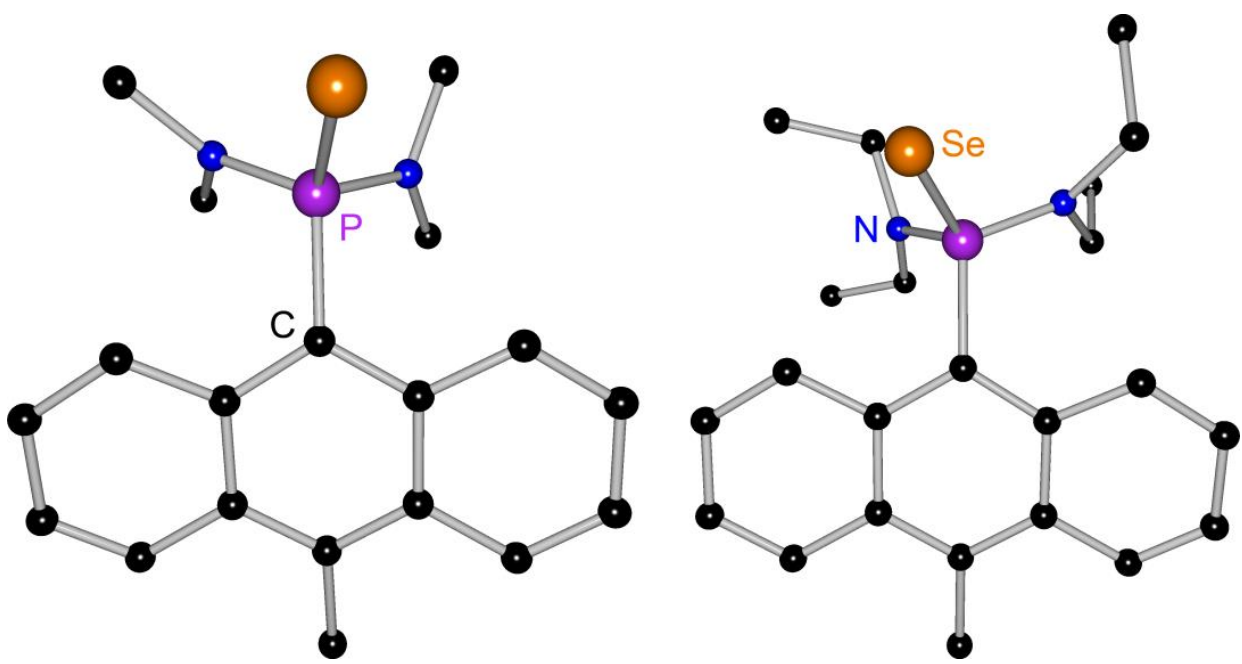

Figure 3-38: Crystal structures of MeAnPSe(NMe $)_{2}$ (25, left) and MeAnPSe(NEt $)_{2}$ (27, right), hydrogen atoms are omitted.

Although MeAnPS $\left(\mathrm{NMe}_{2}\right)_{2}$ (21) and MeAnPSe $\left(\mathrm{NMe}_{2}\right)_{2}$ (25) are identical molecules apart from the P-bound chalcogen, their solid state structures differ significantly. As in 21 and 23, both bulky amino-groups are located on the same side of the anthracene plane, leading to a folding of the anthracene moiety of $16.7^{\circ}$, which is nearly twice as large as the folding angle in $\mathbf{2 1}$. Moreover a distinctly stronger twist deformation of $8.0^{\circ}$ is found. Also, the phosphorus atom is - as observed in MeAnPS(NEt $)_{2}(23)-$ forced out of the C9*C10-axis. The intermolecular interactions found for $\mathbf{2 5}$ combine the packing phenomena already discussed for $\mathbf{2 1}$ and 23. Two different "head-to-tail" type arrangements occur, one of them shows a $\pi-\pi$ overlap of approximately $35 \%$ and 
a distance of $3.60 \AA$ with aromatic hydrogen atoms located above the ring centres of the opposite $\pi$ system. The second interaction found is between a $\mathrm{C}-\mathrm{H}$ bond of the methyl group in 10-position and an outer anthracene ring. The $\mathrm{C}-\mathrm{H} \cdots \pi$ distance here is $2.917 \AA$, the angle to the ring plane measures $41.2^{\circ}$. Additionally, an aromatic $\mathrm{sp}^{2}$ $\mathrm{C}-\mathrm{H} \cdots \pi$ bond in 2-position of the anthracene moiety to an adjacent $\pi$ system is present. It is also fairly accute $\left(40.8^{\circ}\right)$ and emulates a distance of $3.096 \AA$.

As mentioned before, MeAnPS(NEt $)_{2}$ (23) and MeAnPSe(NEt $)_{2}$ (27) are isostructural. While the same exchange of the chalcogen in $\mathbf{2 1}$ and $\mathbf{2 5}$ completely changed the packing motif, the exchange of the sulfur atom to selenium does not influence the packing arrangement of $\mathbf{2 7}$ compared to 23. This shows that the steric demand of the diethylamino groups outnumbers the effect of the heavier chalcogen in 27. Hence, the deviations of cell parameters and intermolecular interactions are marginal. The folding- and twist angle of the anthracene moiety is identical to the angle found in 23. The methyl $\mathrm{C}-\mathrm{H}^{\cdots} \boldsymbol{} \pi$ interaction exhibits minimal deviations from the one found in 23 , measuring $2.978 \AA\left(29.5^{\circ}\right)$.

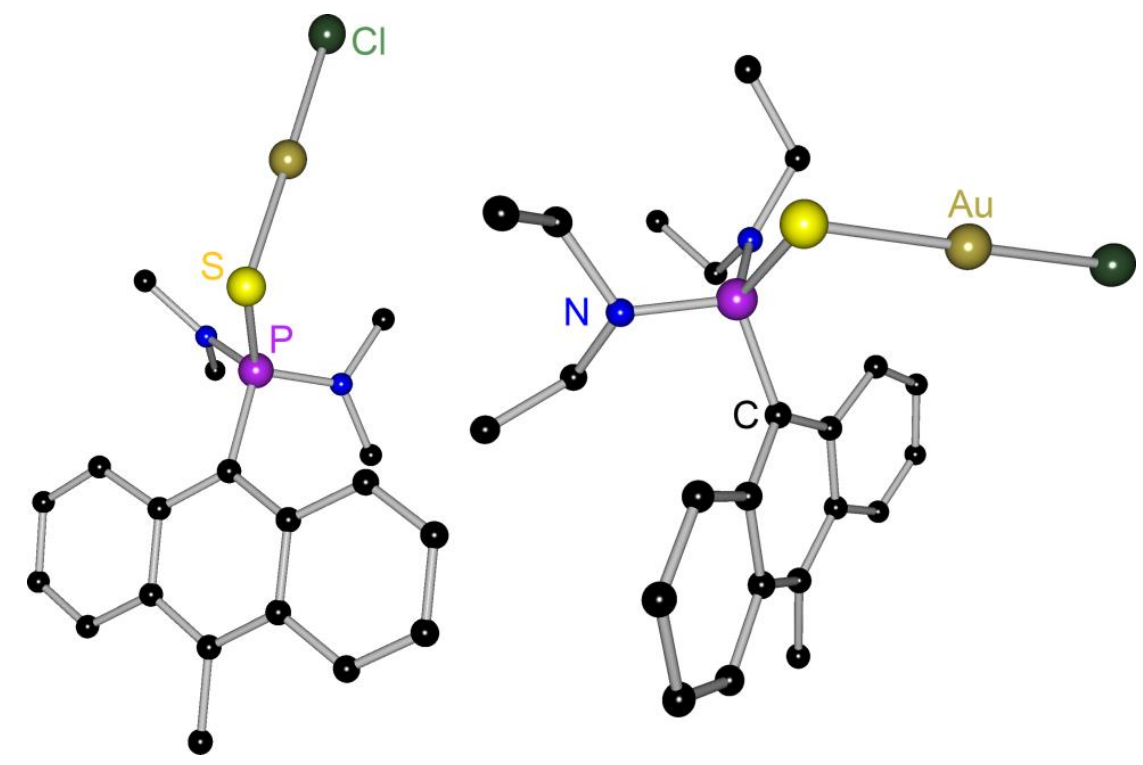

Figure 3-39: Crystal structures of $\left.\left[\mathrm{MeAnP}\left(\mathrm{NMe}_{2}\right)_{2}(\mathrm{~S}) \mathrm{AuCl}\right](\mathbf{2 2} \text {, left) and [MeAnP(NEt })_{2}(\mathrm{~S}) \mathrm{AuCl}\right](\mathbf{2 4}$, right); hydrogen atoms are omitted.

All gold complexes show a linear $\mathrm{E}-\mathrm{Au}-\mathrm{Cl}$ coordination geometry, which is characteristic of gold(I) compounds. ${ }^{[69]}$ However, the orientation of the linear E-Au-Cl fragment differs among the four compounds. Surprisingly no gold-gold interactions were found in any of the complexes. 
Due to the coordination of gold(I) the structure of $\left[\mathrm{MeAnP}\left(\mathrm{NMe}_{2}\right)_{2}(\mathrm{~S}) \mathrm{AuCl}\right]$ (22) clearly is different from the structure of MeAnPS $\left(\mathrm{NMe}_{2}\right)_{2}(\mathbf{2 1})$. In 22, the typical "headto-tail" orientation is also observed, with the anthracene moieties shifted in a similar way as described for MeAnPS(NEt $)_{2}$ (23), leading to virtually no $\pi-\pi$ overlap. Nevertheless aromatic $\mathrm{C}-\mathrm{H}$ bonds are located over the ring centres of the adjacent anthracene moiety in parallel orientation. As in $\mathbf{2 1}$, both amino groups are located at the same side of the anthracene plane. The linear $\mathrm{S}-\mathrm{Au}-\mathrm{Cl}$ fragment is directed away from the anthacene moiety. Although the phosphorus atom is located distinctly further outside of the C9.'C10-axis, the folding angle of the anthracene ring system is smaller than in $\mathbf{2 1}$, enclosing only $6.0^{\circ}$. The twist deformation of the fluorophore is however slightly stronger $\left(4.4^{\circ}\right)$. The "head-to-tail" arrangement produces a $\mathrm{C}-\mathrm{H}^{\prime \cdots} \pi$ bond from the 10-methyl $\mathrm{C}-\mathrm{H}$ bond to a peripheral ring of the anthracene moiety. The distance of $2.776 \AA$ and an angle of $48.2^{\circ}$ to the ring plane suggest that this $\mathrm{C}-\mathrm{H}^{\cdots} \pi$ interaction can be considered stronger than the ones described before. A second $\mathrm{C}-\mathrm{H}^{\cdots} \pi \mathrm{r}$ bond resulting from the interaction of an aromatic $\mathrm{C}-\mathrm{H}$ bond in 2-position with a neighbouring anthracene ring is also found, measuring $2.980 \AA$ and $53.7^{\circ}$.
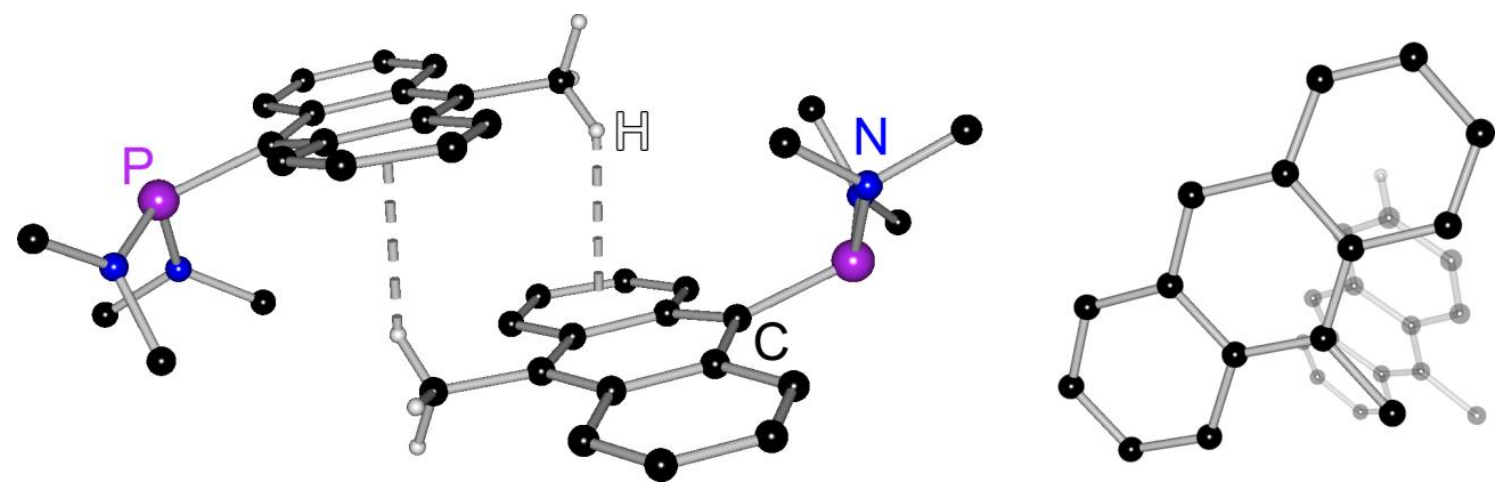

Figure 3-40: Left: $\mathrm{sp}^{3} \mathrm{C}-\mathrm{H}{ }^{\prime \prime} \pi$ bonding in the structure of [MeAnP(NMe $\left.)_{2}(\mathrm{~S}) \mathrm{AuCl}\right]$ (22), S-Au-Cl fragments are omitted for clarity; right: $\mathrm{sp}^{2} \mathrm{C}-\mathrm{H} \cdots \pi$ bonding in the structure of 22, substituents are omitted.

[MeAnP(NEt $)_{2}(\mathrm{~S}) \mathrm{AuCl}$ (24) again shows a different arrangement. Though, like [MeAnP(NMe $)_{2}(\mathrm{~S}) \mathrm{AuCl}$ ] (22), 24 also crystallizes in $P 2_{1} / C$, the orientation of the linear $\mathrm{S}-\mathrm{Au}-\mathrm{Cl}$ fragment is antipodal to the one observed in $\mathbf{2 2}$. While in $\mathbf{2 2}$ this fragment points away from the anthracene moiety, here it is located behind the anthracene ring system on the reverse side of the bulky diethylamino groups. Driven by the steric demand of these groups, this arrangement leads to a notable distortion of the anthracene moiety. A folding angle of $20.7^{\circ}$ is reached, accompanied by a twist angle of $4.4^{\circ}$, and the phosphorus atom is clearly displaced from the C9.. $\mathrm{C} 10$-axis, to which 
the P-C9-bond encloses an angle of $13.5^{\circ}$. The "head-to-tail" arrangement of every two molecules is also found in $\mathbf{2 4}$. They face each other with their concave side, while the $\pi-\pi$ distance measures only $3.21 \AA$, achieving an overlap of approximately $25 \%$. Additionally an aromatic $\mathrm{sp}^{2} \mathrm{C}-\mathrm{H}^{\cdots} \pi \mathrm{r}$ interaction very similar to the one found in $\mathbf{2 2}$ is observed. Again originating from a $\mathrm{C}-\mathrm{H}$ bond in 2-position, it measures $3.055 \AA$ and $42.6^{\circ}$ to the adjacent $\pi$ system.

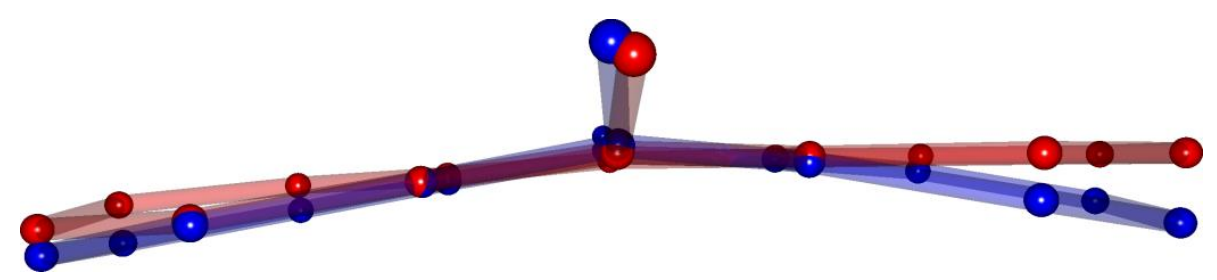

Figure 3-41: differences in fluorophore deformation between [MeAnP(NMe $\left.)_{2}(\mathrm{~S}) \mathrm{AuCl}\right](22)$ (red) and [MeAnP(NEt $\left.)_{2}(\mathrm{~S}) \mathrm{AuCl}\right]$ (24) (blue).

The structure of $\left[\mathrm{MeAnP}\left(\mathrm{NMe}_{2}\right)_{2}(\mathrm{Se}) \mathrm{AuCl}\right]$ (26) is the first structure among the compounds in this chapter that shows a rotated phosphoryl substituent with the amino groups located on opposite sides of the anthracene plane. However the arrangement is not symmetric which would require the P-Se-bond to be located in the anthracene plane. In fact, one N-P-bond has a torsion angle of nearly $90^{\circ}$ with respect to the anthracene plane. The supposedly more relaxed arrangement with both bulky amino substituents located on different sides surprisingly does not lead to less deformation of the ring system. The folding angle adds up to $12.7^{\circ}$, but the deviation of the phosphorus atom from the C9'C10-axis is significantly smaller than the one found in 24. The twist angle of the fluorophore is in the same range as observed for $\mathbf{2 4}$, measuring $5.4^{\circ}$. As in $\mathbf{2 2}$, the linear Se-Au-Cl fragment is directed away from the anthracene moiety. While there is virtually no $\pi-\pi$ overlap, a $\mathrm{C}-\mathrm{H}^{\cdots \cdots} \pi$ interaction of the methyl group in 10-position is present. The short distance of only $2.617 \AA$ and the fairly acute angle of $65.5^{\circ}$ to the $\pi$-system makes this $\mathrm{C}-\mathrm{H}^{\cdots} \pi$ bond the supposed strongest found among 21-28. 

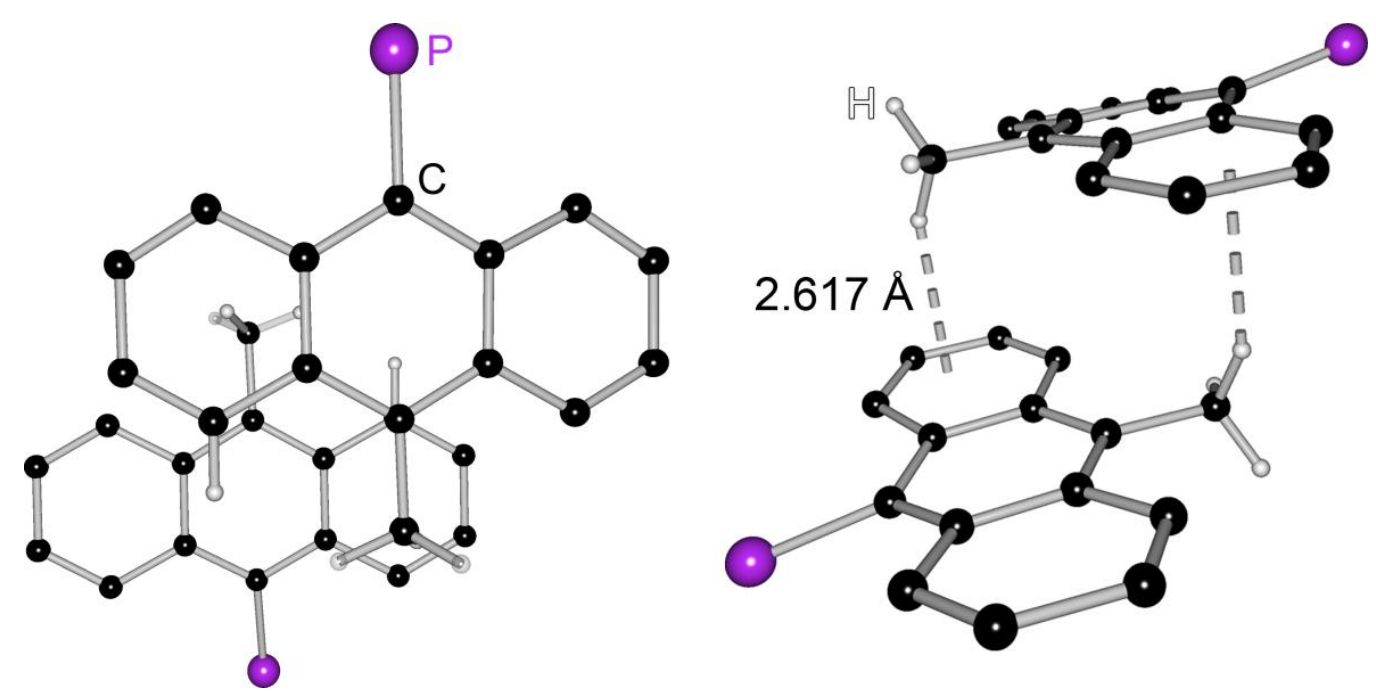

Figure 3-42: $\mathrm{sp}^{3} \mathrm{C}-\mathrm{H}^{\cdots} \pi$ bonding in the structure of $\left[\mathrm{MeAnP}\left(\mathrm{NMe}_{2}\right)_{2}(\mathrm{Se}) \mathrm{AuCl}\right]$ (26): top view (left) and side view (right).

Like in [MeAnP(NMe $\left.)_{2}(\mathrm{Se}) \mathrm{AuCl}\right](\mathbf{2 6})$, the bulky amino groups are also located on opposite sides of the anthracene plane in $\left[\mathrm{MeAnP}\left(\mathrm{NEt}_{2}\right)_{2}(\mathrm{Se}) \mathrm{AuCl}\right]$ (28). Though in the structure of $\left[\mathrm{MeAnP}\left(\mathrm{NEt}_{2}\right)_{2}(\mathrm{~S}) \mathrm{AuCl}\right](\mathbf{2 4})$, the sulfur oxidized equivalent of $\mathbf{2 8}$, this was not the case, the linear Se-Au-Cl fragment is directed away from the anthracene moiety. This results in less deformation of the ring system, reflected by a folding angle of $11.1^{\circ}\left(20.7^{\circ}\right.$ in 24$)$ and a twist angle of $5.1^{\circ}$.

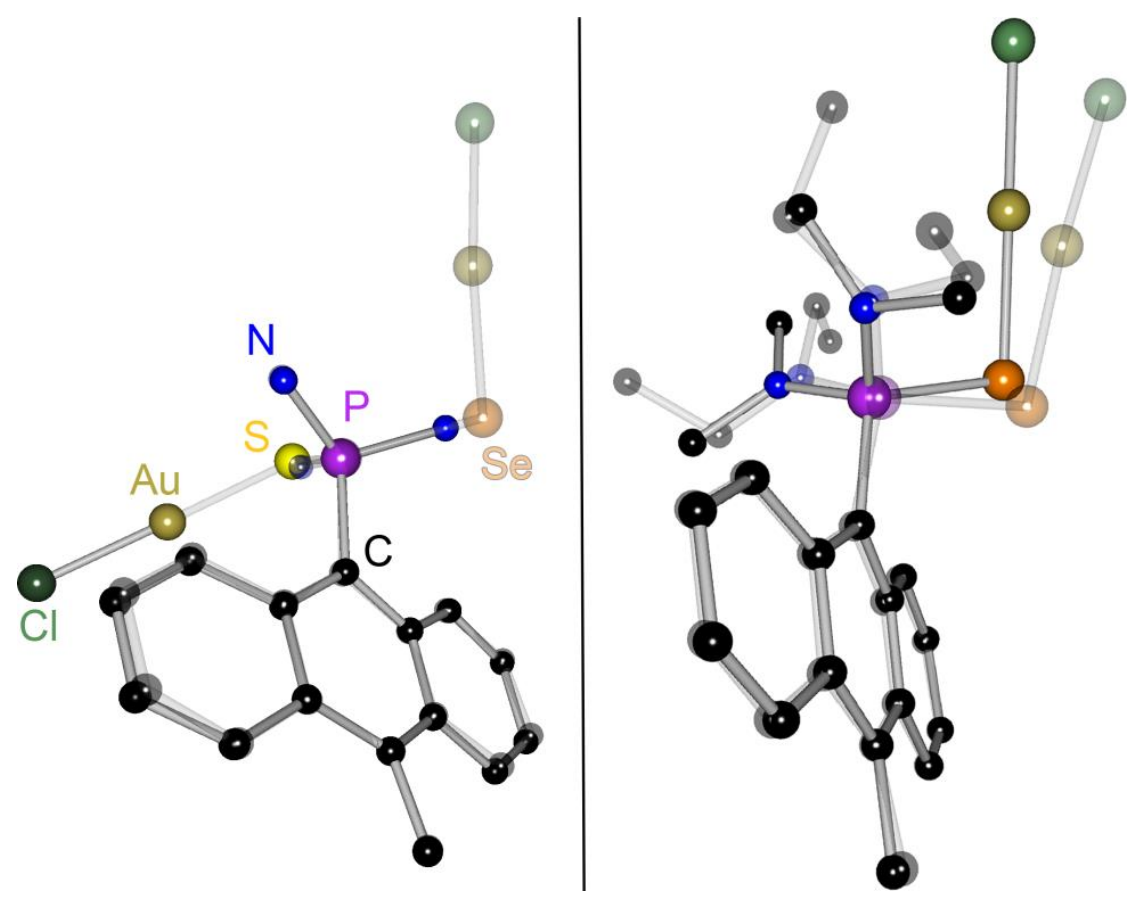

Figure 3-43: Left: Superposition of $\mathbf{2 4}$ and $\mathbf{2 8}$ (transparent), ethyl groups are omitted for clarity; right: superposition of $\mathbf{2 6}$ and $\mathbf{2 8}$ (transparent). 
The $\pi-\pi$ distance in the typical "head-to-tail" arrangement measures $3.53 \AA$, though the overlap of approximately $15 \%$ is not very strong.

Moreover a $\mathrm{C}-\mathrm{H}^{\cdots}{ }^{\prime} \pi$ interaction $\left(2.772 \AA \mathrm{A} / 63.1^{\circ}\right)$ between an aminoethyl group and an outer ring of the anthracene moiety is observed, as well as a similar interaction of the 10 -methyl group with the central anthracene ring $\left(2.935 \AA / 64.8^{\circ}\right)$. One further fairly long $\mathrm{C}-\mathrm{H}^{\cdots \cdots} \pi$ interaction of $3.074 \AA$ is also found, but the combination of this long distance and the acute angle of $35.7^{\circ}$ to the $\pi$ system renders it less important.

As anticipated, the bulky phosphoryl substituents of every two molecules are oriented in opposite directions in all eight structures, leading to a "head-to-tail" packing motif in all cases. The resulting $\pi-\pi$ overlap, however, varies considerably, as well as the distortion of the fluorophores and the number and strength of $\mathrm{C}-\mathrm{H}^{\cdots}{ }^{\prime \prime} \pi$ type interactions. Although MeAnPS(NEt $)_{2}$ (23) and MeAnPSe $\left(\mathrm{NEt}_{2}\right)_{2}$ (27) are isostructural, their corresponding gold complexes [MeAnP(NEt $\left.)_{2}(\mathrm{~S}) \mathrm{AuCl}\right]$ (24) and [MeAnP(NEt $\left.)_{2}(\mathrm{Se}) \mathrm{AuCl}\right]$ (28) are not. All gold complexes feature a linear E-Au-Cl fragment directed away from the anthracene moiety except for $\mathbf{2 4}$, where the fragment is located behind the anthracene plane leading to a significantly stronger distortion than found in the other structures. Despite the close relation among the eight compounds and the similarity of the substituents, a prediction of the resulting packing motif and the corresponding intermolecular interactions is not possible. Nevertheless the information acquired from the detailed evaluation of the solid state structures can be consulted to understand and explain solid state fluorescence properties of the compounds at hand.

\subsubsection{Solid state fluorescence properties}

In the following the solid state fluorescence properties of 21-28 will be presented. Previous research has shown that in general all selenium oxidized phosphoryl anthracenes show distinctly weaker fluorescence emission than their sulfur oxidized counterparts. ${ }^{[44 b, 44 c]}$ This can be assigned to the presence of a heavy atom in the molecule which may generally produce a red-shift of the emission maximum and also favour non-radiative decay pathways over fluorescence emission. ${ }^{[7 a, 7 b, 7 d, 70]}$ Hence 
comparison is only reasonable among the four sulfur oxidized compounds 21-24 and among the four selenium oxidized compounds 25-28.

The solid state excitation spectra of 21-24 are nearly identical in shape. They show a broad range of circa $100 \mathrm{~nm}$ of possible excitation wavelengths, reaching a maximum at $450 \mathrm{~nm}$. Similar behavior has also been observed for SPAnPS (c.f. 3.2). The excitation band is located in the spectral range of visible light, which is particularly attractive for fluorescent materials, leaving the employment of UV light for excitation obsolete. Furthermore, due to the broadness of the band, monochromatic light is not required for excitation, which again is beneficial for fluorescent materials.

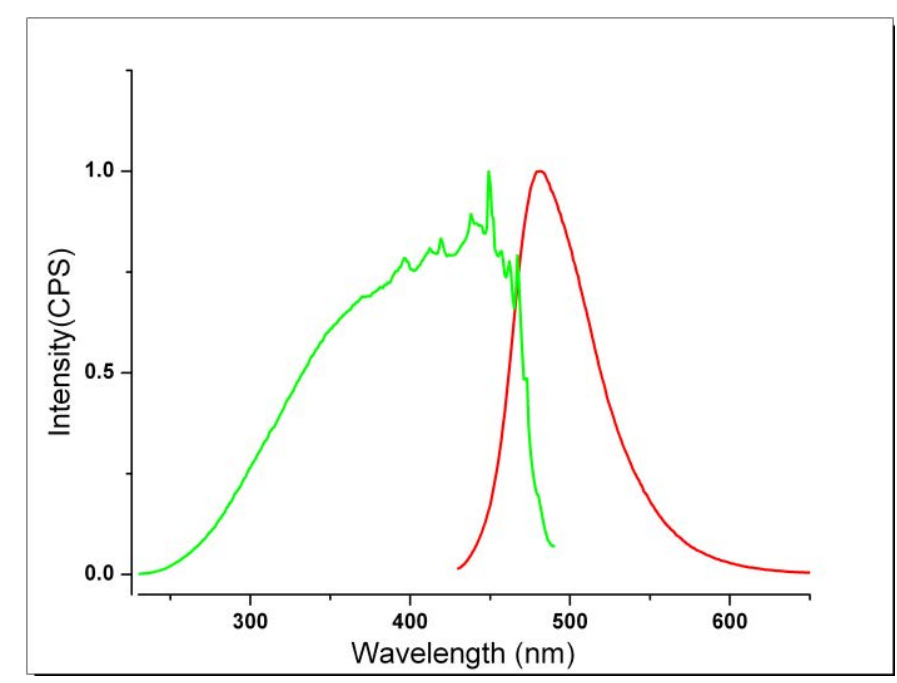

Figure 3-44: Normalized solid state excitation (green) and emission (red) spectra of MeAnPS(NMe $)_{2}$ (21).

The corresponding emission spectra show each one fairly broad maximum, lacking the characteristic vibrational band structure of many anthracene derivatives. ${ }^{\text {[49-50] }}$ Additionally, the emission maximum is red-shifted by circa $70-80 \mathrm{~nm}$ compared to unsubstituted anthracene. This phenomenon was also observed for the strongly emitting SPAnPS structures $\mathbf{1 5}$ and $\mathbf{2 0}$ (c.f. 3.2) The lack of vibrational bands has often been assigned to exciplex formation in solid state for other anthracene derivatives, ${ }^{[52,}$ 55] but does not appear to be present in this case in view of the molecular arrangement. The normalized emission spectra of 21-24 show a notable bathochromic shift of the emission maxima of the gold complexes ( $15 \mathrm{~nm})$ compared to free phosphoryl anthracenes. This is in accordance with theory that predicts a possible redshift of emission in the presence of heavy atoms in the structure. Also the emission maximum of MeAnPS(NEt $)_{2}(\mathbf{2 3})$ is slightly red-shifted compared to MeAnPS $\left(\mathrm{NMe}_{2}\right)_{2}$ 
(21), though there is no heavy atom present in either of the structures. Similar phenomena have repeatedly been explained by $\pi-\pi$ interaction, with increasing overlap and shorter $\pi-\pi$ distance leading to a stronger red-shift of emission. ${ }^{\text {[42c, 53-54] }}$ This does not apply to $\mathbf{2 1}$ and $\mathbf{2 3}$. While $\mathbf{2 1}$ exhibits a $\pi-\pi$ overlap of circa $35 \%$ with a comparatively short $\pi-\pi$ distance of $3.51 \AA$, there is virtually no $\pi-\pi$ interaction in the solid state structure of $\mathbf{2 3}$. Though the bathochromic shift of emission between $\mathbf{2 1}$ and 23 is fairly small, the expected effect of the $\pi-\pi$ overlap is not observed.

Table 3-10: Summarized structural properties of 21, 22, 23, and 24.

\begin{tabular}{|c|c|c|c|c|}
\hline & 21 & 22 & 23 & 24 \\
\hline Space group & $P 2_{1} / n$ & $P 2_{1} / c$ & $P 2_{1} / c$ & $P 2_{1} / c$ \\
\hline Folding angle $\left[{ }^{\circ}\right]$ & 9.9 & 6.0 & 13.8 & 20.7 \\
\hline Twist angle $\left[{ }^{\circ}\right]$ & $2.0^{\circ}$ & 4.4 & 8.8 & 4.4 \\
\hline$\pi-\pi$ overlap (\%) & 35 & - & - & 25 \\
\hline$\pi-\pi$ distance $(\AA ̊)$ & 3.51 & - & - & 3.21 \\
\hline $\begin{array}{c}\mathrm{C}-\mathrm{H}^{\cdots} \pi \text { Interactions } \\
\text { (distance }[\AA \AA] \text { / angle } \\
{\left[^{\circ}\right] \text { ) }}\end{array}$ & $\begin{array}{c}2.946 / 28.8^{\circ} \\
\left(\mathrm{sp}^{3}\right)\end{array}$ & $\begin{array}{c}2.946 / 28.8^{\circ} \\
\left(s p^{3}\right) \\
2.980 / 53.7 \\
\left(\mathrm{sp}^{2}\right)\end{array}$ & $\begin{array}{c}2.899 / 31.2^{\circ} \\
\left(\mathrm{sp}^{3}\right)\end{array}$ & $\begin{array}{c}3.055 / 42.6 \\
\left(\mathrm{sp}^{2}\right)\end{array}$ \\
\hline Offset C-H- $\pi$ & yes & yes & no & yes \\
\hline
\end{tabular}

[MeAnP(NMe $\left.)_{2}(\mathrm{~S}) \mathrm{AuCl}\right]$ (22) and [MeAnP(NEt $)_{2}(\mathrm{~S}) \mathrm{AuCl}$ (24) also differ significantly in terms of $\pi-\pi$ overlap, with $\pi$ systems in $\mathbf{2 4}$ achieving an overlap of $25 \%$ at $3.21 \AA$ and 22 showing virtually none. Again the expected red shift of emission for $\mathbf{2 4}$ is not found, as $\mathbf{2 2}$ and $\mathbf{2 4}$ have nearly identical maximum emission wavelengths.

Emission intensities of aromatic molecules can also be influenced by the degree of $\pi-\pi$ interaction. A stronger interaction has often been named as a quenching factor of solid state fluorescence. ${ }^{[7 \mathrm{~d}, 42 \mathrm{c}, 48 \mathrm{~b}, 50]}$ Furthermore, the deformation of the anthracene moiety caused by steric strain on the fluorophore has been shown to have a quenching effect on fluorescence of anthracene derivatives, which was confirmed by the results obtained in 3.2. ${ }^{[49,52]}$ As mentioned before, heavy atoms in the molecular structure can also exert similar effects on emission properties. 

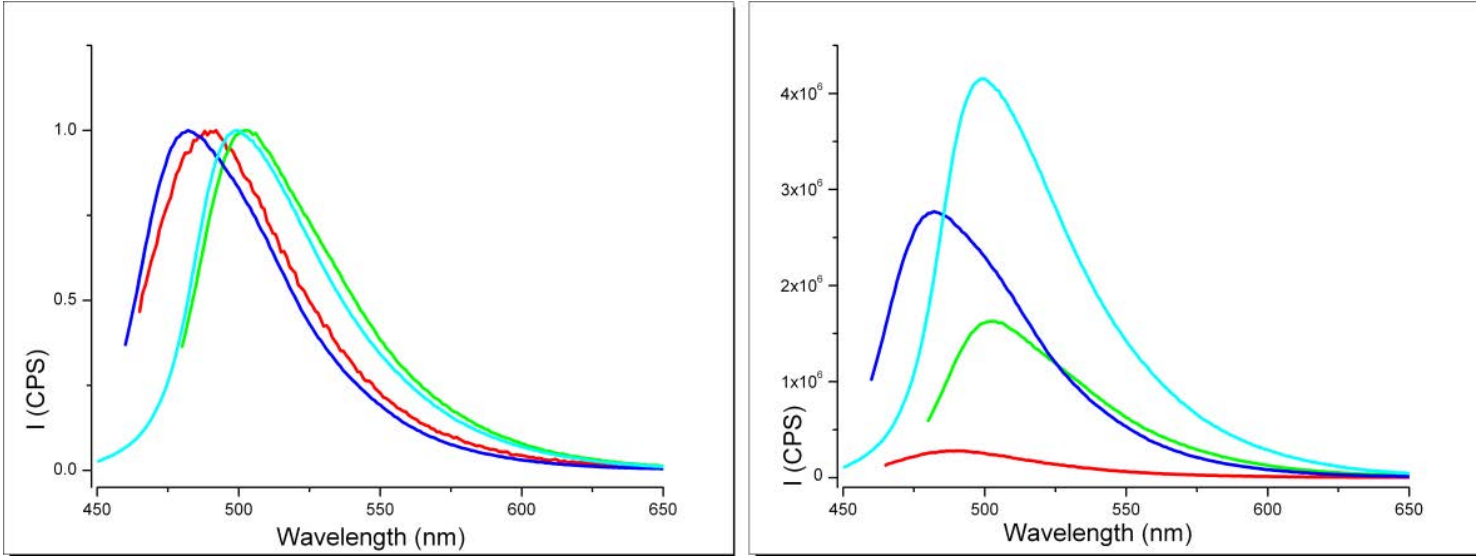

Figure 3-45: Left: Normalized solid state emission spectra of MeAnPS(NMe $)_{2}(\mathbf{2 1}$, blue), $\left[\mathrm{MeAnP}\left(\mathrm{NMe}_{2}\right)_{2}(\mathrm{~S}) \mathrm{AuCl}\right](\mathbf{2 2}, \mathrm{cyan}), \operatorname{MeAnPS}\left(\mathrm{NEt}_{2}\right)_{2}$ (23, red), and [MeAnP(NEt $\left.)_{2}(\mathrm{~S}) \mathrm{AuCl}\right](\mathbf{2 4}$, green); right: solid state emission spectra of $\operatorname{MeAnPS}\left(\mathrm{NMe}_{2}\right)_{2}$ (21, blue), [MeAnP(NMe $\left.)_{2}(\mathrm{~S}) \mathrm{AuCl}\right]$ (22, cyan), $\operatorname{MeAnPS}\left(\mathrm{NEt}_{2}\right)_{2}(\mathbf{2 3}, \mathrm{red})$, and $\left[\mathrm{MeAnP}\left(\mathrm{NEt}_{2}\right)_{2}(\mathrm{~S}) \mathrm{AuCl}\right](\mathbf{2 4}$, green).

When comparing the emission intensities of 21-24 it becomes clear at first sight that the presence of the heavy gold ion in the molecular structures of [MeAnP(NMe $\left.)_{2}(\mathrm{~S}) \mathrm{AuCl}\right]$ (22) and [MeAnP(NEt $\left.)_{2}(\mathrm{~S}) \mathrm{AuCl}\right]$ (24) does not lead to appreciable fluorescence quenching, as both gold complexes show stronger emission than the corresponding free ligands $\mathbf{2 1}$ and 23, respectively. Considering the fluorescence quenching factors named above, excluding the effect of heavy atoms, the strongest emission of $\mathbf{2 2}$ seems plausible, as the solid state structure of $\mathbf{2 2}$ displays no $\pi-\pi$ overlap and the weakest steric deformation of the fluorophore.

Table 3-11: Solid state fluorescence properties of 21-24.

\begin{tabular}{ccccc}
\hline & $\mathbf{2 1}$ & $\mathbf{2 2}$ & $\mathbf{2 3}$ & $\mathbf{2 4}$ \\
\hline$\lambda_{\mathrm{Ex}}(\max )[\mathrm{nm}]$ & 449 & 449 & 449 & 449 \\
$\lambda_{\mathrm{Em}}(\max )[\mathrm{nm}]$ & 482 & 499 & 488 & 502 \\
$\mathrm{I}_{\text {rel }}$ & 0.67 & 1 & 0.07 & 0.39
\end{tabular}

Consequently the similar emission of $\mathrm{MeAnPS}\left(\mathrm{NMe}_{2}\right)_{2}(\mathbf{2 1})$ and 24 is due to their comparable $\pi-\pi$ interactions (slightly larger overlap in $\mathbf{2 1}$, slightly shorter $\pi-\pi$ distance in 24). 24 exhibits a stronger deformation of the fluorophore than $\mathbf{2 1}$. The significantly weaker emission of MeAnPS(NEt $)_{2}$ (23) surprisingly does not fit into this series. The solid state structure of $\mathbf{2 3}$ shows no $\pi-\pi$ overlap (as does the strongest fluorescent [MeAnP(NMe $\left.\left.)_{2}(\mathrm{~S}) \mathrm{AuCl}\right](22)\right)$ and only moderate deformation of the anthracene 
moiety, which suggests that other factors must be taken into account to explain the observed emission properties.

\section{$\underline{\mathrm{C}-\mathrm{H} \cdots} \pi$ hydrogen bonds}

The results derived from the investigation of SPAnPS (c.f. 3.2) have indicated the sensitivity of fluorescence emission of phosphoryl anthracenes towards $\mathrm{C}-\mathrm{H}$ interactions with the aromatic $\pi$ system. Two strong and nearly orthogonal $\mathrm{sp}^{2} \mathrm{CH}^{\cdots \cdots} \pi$ bonds to each fluorophore dictate the presence of fluorescence emission in the case of

SPAnPS@tol (15). ${ }^{[44 b, 44 c]}$ As stated in the description of the crystal structures, C-H ${ }^{\cdots} \pi$ type interactions are also found in 21-28. When these are taken into account in addition to the factors considered above, the emission properties of 21-24 become conclusive. Employing the assumptions made earlier regarding the influence of length, polarity and angle of $\mathrm{CH}^{\cdots} \pi$ bonds, the strength of the interactions found in the packing plots can be classified. MeAnPS(NEt $)_{2}$ (23) displays the weakest and least directed C$H^{\cdots \cdots} \pi$ interaction of all four compounds, as the methyl group is not rotated to the optimum position, producing a low angle of only $31.2^{\circ}$. Furthermore the anthracene moieties of the "head-to-tail" arrangement are shifted to a degree that the methyl $\mathrm{C}-\mathrm{H}$ can barely interact with the $\pi$ system. This also prevents the aromatic $\mathrm{C}-\mathrm{H}$-bonds from adopting a position above the ring centres of the adjacent anthracene moiety like in the structures of $\mathbf{2 1}, \mathbf{2 2}$, and $\mathbf{2 4}$. The strongly fluorescent [ $\mathrm{MeAnP}\left(\mathrm{NMe}_{2}\right)_{2}(\mathrm{~S}) \mathrm{AuCl}$ ] (22) shows one fairly short $\mathrm{sp}^{3} \mathrm{C}-\mathrm{H}^{\cdots} \pi$ bond $\left(2.776 \AA\right.$ ) and one $\mathrm{sp}^{2} \mathrm{C}-\mathrm{H}^{\cdots} \pi \pi$ bond $(2.980 \AA$ ), both enclosing larger angles with the anthracene plane. These can both be considered clearly stronger than the single $\mathrm{C}-\mathrm{H}^{\prime \prime} \pi$ interaction found in 23. $\mathrm{MeAnPS}\left(\mathrm{NMe}_{2}\right)_{2}$ (21) and $\left[\mathrm{MeAnP}\left(\mathrm{NEt}_{2}\right)_{2}(\mathrm{~S}) \mathrm{AuCl}\right]$ (24) exhibit $\mathrm{C}-\mathrm{H}^{\prime \prime} \pi$ bonding of similar strength and additionally, as 22, have aromatic $\mathrm{C}-\mathrm{H}$ bonds located above the ring centres of the parallel $\pi$ system. Merely the distortion of the fluorophore is clearly stronger in $\mathbf{2 4}$ which is reflected by a doubled folding angle. Accordingly the degree of $\mathrm{C}-\mathrm{H}-$ interaction with the $\pi$ system found in $\mathbf{2 1 - 2 4}$ follows the trend of the emission intensities. 


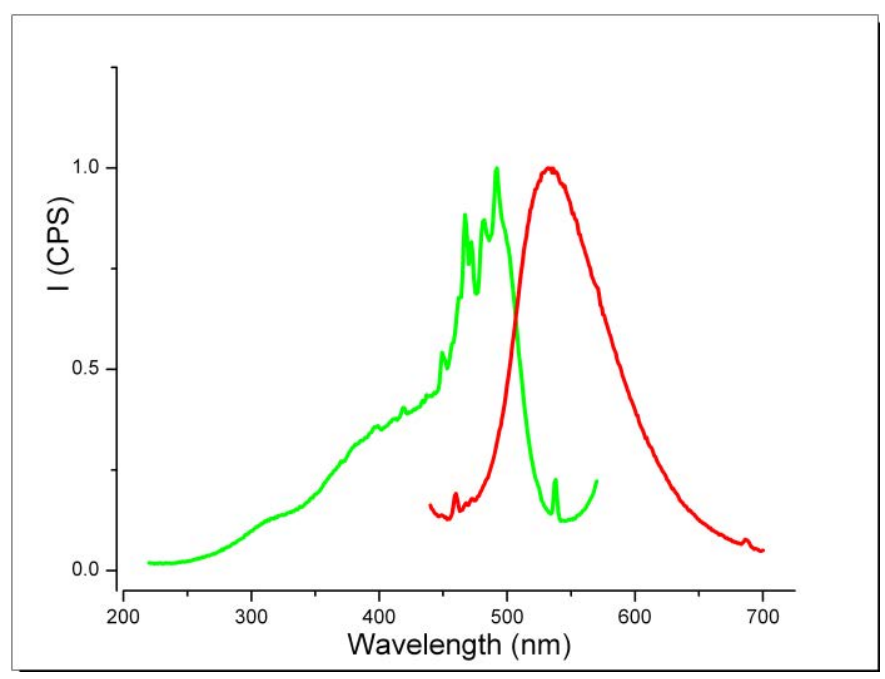

Figure 3-46: Normalized solid state excitation (green) and emission (red) spectra of $\left[\mathrm{MeAnP}\left(\mathrm{NMe}_{2}\right)_{2}(\mathrm{Se}) \mathrm{AuCl}\right](\mathbf{2 6})$.

Though the emission of $\mathbf{2 5 - 2 8}$ is distinctly weaker than of the sulfur oxidized compounds (approximately by the factor of 15 ) the fluorescence phenomena are directly comparable. The excitation spectra of 25-28 again are nearly identical, showing a broad maximum ( $110 \mathrm{~nm})$. The emission band is also fairly broad and lacks a vibrational band structure as previously observed for 21-24. Compared to the sulfur oxidized species, the emission maxima of the selenium derivatives are red-shifted by approximately $40 \mathrm{~nm}$.

Table 3-12: Summarized structural properties of 25, 26, 27, and 28.

\begin{tabular}{|c|c|c|c|c|}
\hline & 25 & 26 & 27 & 28 \\
\hline Space group & $P 2_{1} / c$ & $\mathrm{P} \overline{1}$ & $P 2_{1} / c$ & $P 2_{1} / c$ \\
\hline Folding angle $\left[^{\circ}\right]$ & 16.7 & 12.7 & 13.8 & 11.1 \\
\hline Twist angle $\left[{ }^{\circ}\right]$ & 8.0 & 5.4 & 8.8 & 5.1 \\
\hline$\pi-\pi$ overlap (\%) & 35 & - & - & 15 \\
\hline$\pi-\pi$ distance $(\AA)$ & 3.60 & - & - & 3.53 \\
\hline \multirow{4}{*}{$\begin{array}{c}\mathrm{C}-\mathrm{H}-\pi \text { Interactions } \\
\text { (distance }[\AA]] / \\
\text { angle }\left[^{\circ}\right] \text { ) }\end{array}$} & 2.917 / 41.2 & & & 2.772 / 63.1 \\
\hline & $\left(\mathrm{sp}^{3}\right)$ & $2.617 / 65.5$ & 2.978 / 29.5 & $\left(s p^{3}\right)$ \\
\hline & $3.096 / 40.8$ & $\left(s p^{3}\right)$ & $\left(s p^{3}\right)$ & $2.935 / 64.8$ \\
\hline & $\left(\mathrm{sp}^{2}\right)$ & & & $\left(s p^{3}\right)$ \\
\hline Offset $\mathrm{C}-\mathrm{H}-\pi$ & yes & yes & no & yes \\
\hline
\end{tabular}


Especially MeAnPSe(NMe $)_{2}$ (25) and MeAnPSe(NEt $)_{2}$ (27) show excitation and emission maxima which lie very close together, separated only by $15-20 \mathrm{~nm}$. The normalized emission spectra of 25-28 reveal similar tendencies as deduced for 21-24. The emission maxima of the gold complexes [MeAnP(NMe $)_{2}(\mathrm{Se}) \mathrm{AuCl}$ (26) and 28 show a bathochromic shift of nearly $35 \mathrm{~nm}$ compared to the uncoordinated phosphoryl anthracenes $\mathbf{2 5}$ and 27. Again, no significant shift of emission caused by $\pi-\pi$ overlap is found. 25 and $\mathbf{2 7}$ have nearly identical emission maxima despite the fact that MeAnPSe $\left(\mathrm{NMe}_{2}\right)_{2}$ (25) shows $\pi-\pi$ overlap of $35 \%$ at a distance of $3.60 \AA$ while 27 shows no overlap at all. This also applies to $\left[\mathrm{MeAnP}\left(\mathrm{NMe}_{2}\right)_{2}(\mathrm{Se}) \mathrm{AuCl}\right]$ (26) and [MeAnP(NEt $\left.)_{2}(\mathrm{Se}) \mathrm{AuCl}\right]$ (28) with identical emission maxima but clear differences in $\pi$ $\pi$ overlap. Thus, the observed red-shift of the gold complexes can solely be assigned to the presence of a heavy gold atom in the structures.

The emission intensities of $\mathbf{2 5 - 2 8}$ indicate that the gold atoms in $\mathbf{2 6}$ and $\mathbf{2 8}$ do not lead to significant fluorescence quenching, as both gold complexes show the strongest emissions of the four compounds. The weakest emission is observed for MeAnPSe $\left(\mathrm{NEt}_{2}\right)_{2}$ (27), which is isostructural to 23, which exhibited the weakest emission of all sulfur oxidized compounds. Hence, the structural constitution of both MeAnPS(NEt $)_{2}$ (23) and 27 appears to be particularly obstructive for fluorescence emission. The comparatively strong deformation of the anthracene moiety $\left(13.8^{\circ}\right.$ folding angle) and a single low-angle $\mathrm{C}-\mathrm{H} \cdots \pi$ interaction account for these findings like in 23.
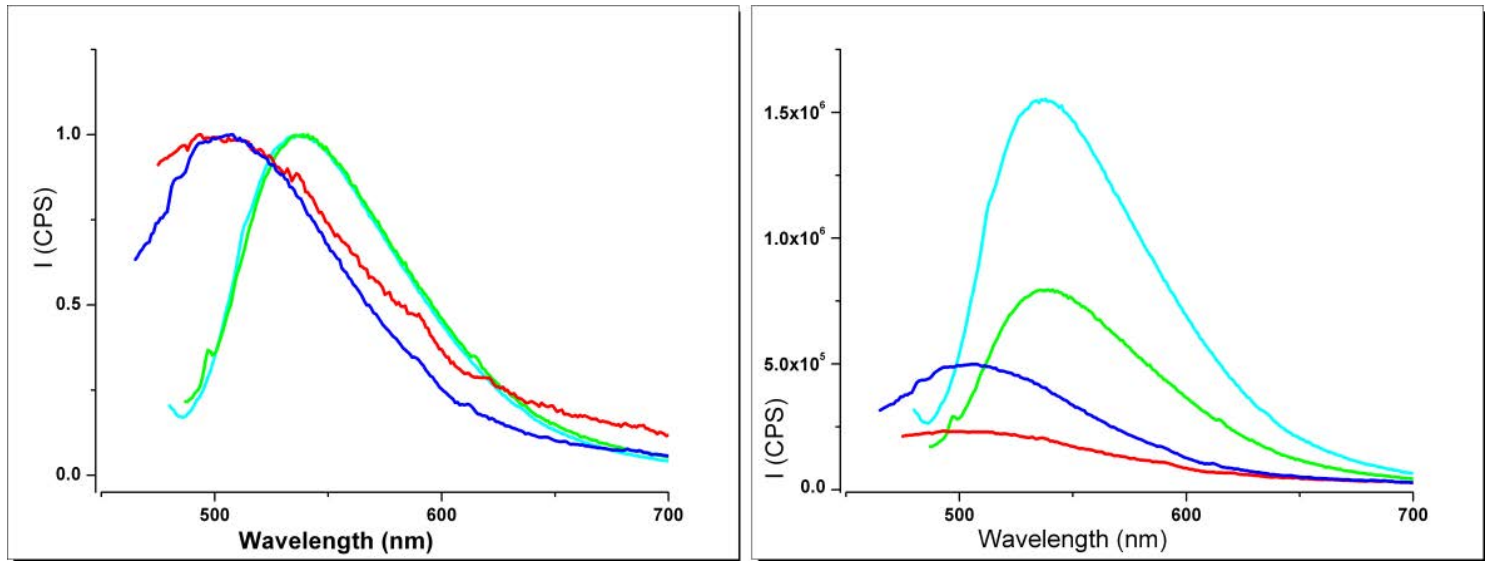

Figure 3-47: Left: normalized solid state emission spectra of MeAnPSe( $\left.\mathrm{NMe}_{2}\right)_{2}$ (25) (blue), [MeAnP(NMe $\left.)_{2}(\mathrm{Se}) \mathrm{AuCl}\right]$ (26) (cyan), MeAnPSe$\left(\mathrm{NEt}_{2}\right)_{2}$ (27) (red), and [MeAnP(NEt $\left.)_{2}(\mathrm{Se}) \mathrm{AuCl}\right]$ (28) (green); right: solid state emission spectra of MeAnPSe(NMe $)_{2}$ (25) (blue), [MeAnP(NMe $\left.)_{2}(\mathrm{Se}) \mathrm{AuCl}\right]$ (26) (cyan), MeAnPSe( $\left.\mathrm{NEt}_{2}\right)_{2}$ (27) (red), and [MeAnP(NEt $\left.)_{2}(\mathrm{Se}) \mathrm{AuCl}\right]$ (28) (green). 
MeAnPSe( $\left(\mathrm{NMe}_{2}\right)_{2}$ (25), which shows the second weakest emission, has the strongest $\pi-\pi$ overlap ( $35 \%$ at $3.60 \AA$ ) and the strongest distortion of the fluorophore $\left(16.7^{\circ}\right.$ folding angle) of all four compounds. These factors would generally lead to an expected emission even weaker than of MeAnPSe(NEt $)_{2}$ (27). But the solid state structure of 25 also contains a sp ${ }^{3} \mathrm{C}-\mathrm{H}^{\cdots} \pi$ and a $\mathrm{sp}^{2} \mathrm{C}-\mathrm{H}^{\cdots} \pi$ interaction, both of medium strength. Taking these into account, the emission of $\mathbf{2 5}$ becomes comprehensible. Slightly stronger emission is observed for $\left[\mathrm{MeAnP}\left(\mathrm{NEt}_{2}\right)_{2}(\mathrm{Se}) \mathrm{AuCl}\right]$ (28), which has a smaller $\pi-\pi$ overlap ( $15 \%$ ) than 25 in combination with a moderate folding angle of $11.1^{\circ}$.

Table 3-13: Solid state fluorescence properties of 25-28.

\begin{tabular}{lllll}
\hline & $\mathbf{2 5}$ & $\mathbf{2 6}$ & $\mathbf{2 7}$ & $\mathbf{2 8}$ \\
\hline$\lambda_{\text {Ex }}(\max )[\mathrm{nm}]$ & 483 & 491 & 479 & 491 \\
$\lambda_{\mathrm{Em}}(\max )[\mathrm{nm}]$ & 505 & 537 & 506 & 538 \\
$\mathrm{I}_{\text {rel }}$ & 0.32 & 1 & 0.15 & 0.51
\end{tabular}

These factors are accompanied by two medium strength $\mathrm{sp}^{3} \mathrm{C}-\mathrm{H} \cdots \pi$ interactions $\left(2.772 \AA / 61.3^{\circ}\right.$ and $\left.2.935 \AA / 64.8^{\circ}\right)$. The strongest emission was recorded for $\left[\mathrm{MeAnP}\left(\mathrm{NMe}_{2}\right)_{2}(\mathrm{Se}) \mathrm{AuCl}\right]$ (26). Here the anthracene moiety exhibits a moderate folding angle of $12.7^{\circ}$ which is close to the angle observed in the structure of $\mathbf{2 8}$. While no $\pi-\pi$ overlap is found, anthracene bound hydrogen atoms are located exactly above the $\pi$ systems of the parallel oriented anthracene moiety at a distance of only $3.41 \AA$. Additionally the shortest $\mathrm{C}-\mathrm{H}^{\cdots} \pi$ bond of all eight compounds is found, measuring only $2.617 \AA$ with a fairly acute angle of $65.5^{\circ}$ to the anthracene plane. As for $\mathbf{2 1 - 2 4}$, the observed emission properties follow the trend of number and strength of $\mathrm{C}-\mathrm{H} \cdots \pi$ interactions present.

The analysis of the solid state fluorescence properties of 21-28 suggests that the influence of $\mathrm{C}-\mathrm{H}^{\prime \prime} \pi$ interactions on emission is an important factor in the process of understanding the basic requirements for solid state fluorescence phenomena. When excluding these from argumentation, by just considering quenching factors as deformation of the fluorophore and $\pi-\pi$ overlap, the observed properties cannot be explained conclusively. Especially the effects of $\pi-\pi$ overlap, which are widely acknowledged in the fluorescence literature, do not occur in the expected manner. Although the observed $\pi-\pi$ distances are fairly short, no bathochromic shift of 
emission was detected for any of the compounds showing $\pi-\pi$ overlap. The proposed quenching from $\pi-\pi$ overlap also does not appear to be the predominant factor in the quenching of emission, as compounds $\mathrm{MeAnPS}\left(\mathrm{NMe}_{2}\right)_{2}$ (21), $\left[\mathrm{MeAnP}\left(\mathrm{NEt}_{2}\right)_{2}(\mathrm{~S}) \mathrm{AuCl}\right]$ (24) and MeAnPSe $\left(\mathrm{NMe}_{2}\right)_{2}$ (25) exhibit considerable emission in spite of $\pi-\pi$ overlap. Similar phenomena have been documented before, e.g. in the work of Dreuw et al. where $\pi$ stacked naphthalene derivatives show stronger emission than the corresponding monomer arrangement. ${ }^{[2 \mathrm{~b}]}$ The deformation of the fluorophore, represented by folding of the anthracene moiety ranging from $6.0^{\circ}$ to $20.7^{\circ}$, seems to have a stronger influence on the emission properties of 21-28. The twist deformation does not appear to have a strong impact on the emission properties. While for the different structures of SPAnPS, even a fluorescence enhancing effect of twist deformation was presumed, the solid state fluorescence of 21-28 cannot confirm this principle. As stated earlier, the concept of solid state fluorescence quenching by distortion of the anthracene moiety was introduced by Mizobe et al. in the course of their work on 2,6-anthracenedisulfonic acid (2,6-ADS). ${ }^{[49,51-52]}$ The distortions found in their compounds are, however, minimal compared to the downright deformed fluorophores of 21-28. All anthracene moieties are almost perfectly planar in the work of Mizobe et al. and IR-spectroscopy was necessary to detect small deviations in steric distortion. Therefore folding angles of over $20^{\circ}$ and additional twisting of the ring system generating ring angles clearly deviating from the ideal $120^{\circ} \mathrm{sp}^{2}$-angle are expected to give nearly complete fluorescence quenching, which again is not observed. On the other hand T-shaped or "herringbone"-like arrangements described in literature are often strongly fluorescent. Though the $\mathrm{C}-\mathrm{H}^{\cdots} \mathrm{\pi} \pi$ interactions resulting from this arrangement are sometimes recognized (as in Mizobe's work) ${ }^{[49]}$, they often are not identified as direct contributors to the fluorescence properties of the given compound. Especially the T-shaped arrangement of anthracene moieties in the solid state often provides several short aromatic $\mathrm{C}-\mathrm{H} \cdots \pi$ interactions per molecule which can be considered strong due to the polarity of the aromatic $\mathrm{C}-\mathrm{H}$ bonds and the steep angle to the $\pi$ system of frequently close to $90^{\circ}$ (Figure 3-48). Especially in the case of 2,6-ADS, large differences between virtually non-fluorescent one-dimensional arrangements and strongly fluorescent two-dimensional "zig-zag" arrangements of the molecules are found. While by the geometrical operations applied for quantification of deformation in this thesis no difference between both forms is determinable, the two dimensional form exhibits strong $\mathrm{C}-\mathrm{H}^{\prime \prime} \pi$ bonding (Figure 3-48), which is absent in the 
one-dimensional structure. The This arrangement comes close to that found in SPAnPS@tol (15) which has shown the possible impact of such interactions and the results from 21-28 also suggest that $\mathrm{C}-\mathrm{H} \cdots \pi$ bonds cause a decisive influence on the solid state fluorescence properties of anthracene derivatives.

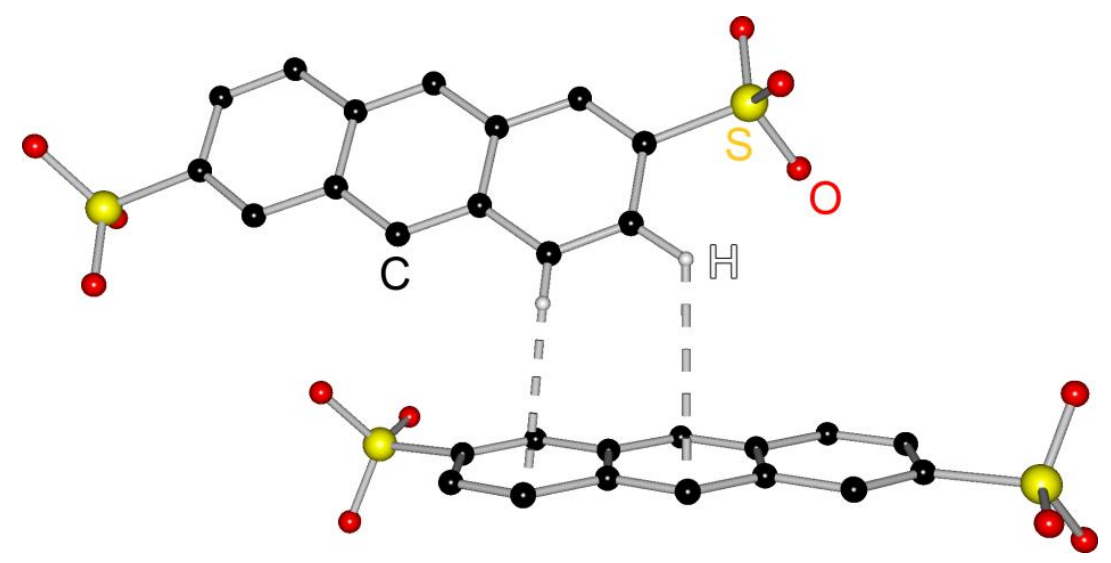

Figure 3-48: $\mathrm{C}-\mathrm{H}^{\cdots} \pi \mathrm{r}$ interactions in a strongly solid state fluorescent compound by Mizobe et al. ${ }^{[49]}$

Summarizing the solid state fluorescence properties of 21-28 from a more remote perspective, it was found, despite the chalkogen or complexed gold atom, that in general every dimethylamino substituted compound showed stronger emission than the diethylamino substituted equivalent ( $21>23 ; 22>24 ; 25>27 ; 26>28)$. Broken down to a simple resumé, bulkier substituents produce a stronger deformation and stronger steric repulsion in the solid state which again leads to larger distances and weaker $\mathrm{C}-\mathrm{H}^{\prime \prime} \pi$ interactions between the fluorophores, which in the case of 21-28 results in suppression of fluorescence emission.

\subsubsection{In-Solution Fluorescence}

The solution fluorescence properties of 21-28 were also investigated. As anticipated, the increased dynamic and rotation of molecules in solution leads to considerably different fluorescence properties than in the solid state. In general, the fluorescence of 21-28 in solution is weak. In 2001 Yip reported on fluorescence quenching of a phosphane substituted anthracene derivative, ascribing the observed suppression of emission to rapid electron transfer from the electron rich diphenylphosphanyl substituent to excited fluorophore. ${ }^{[65]}[x]$ 

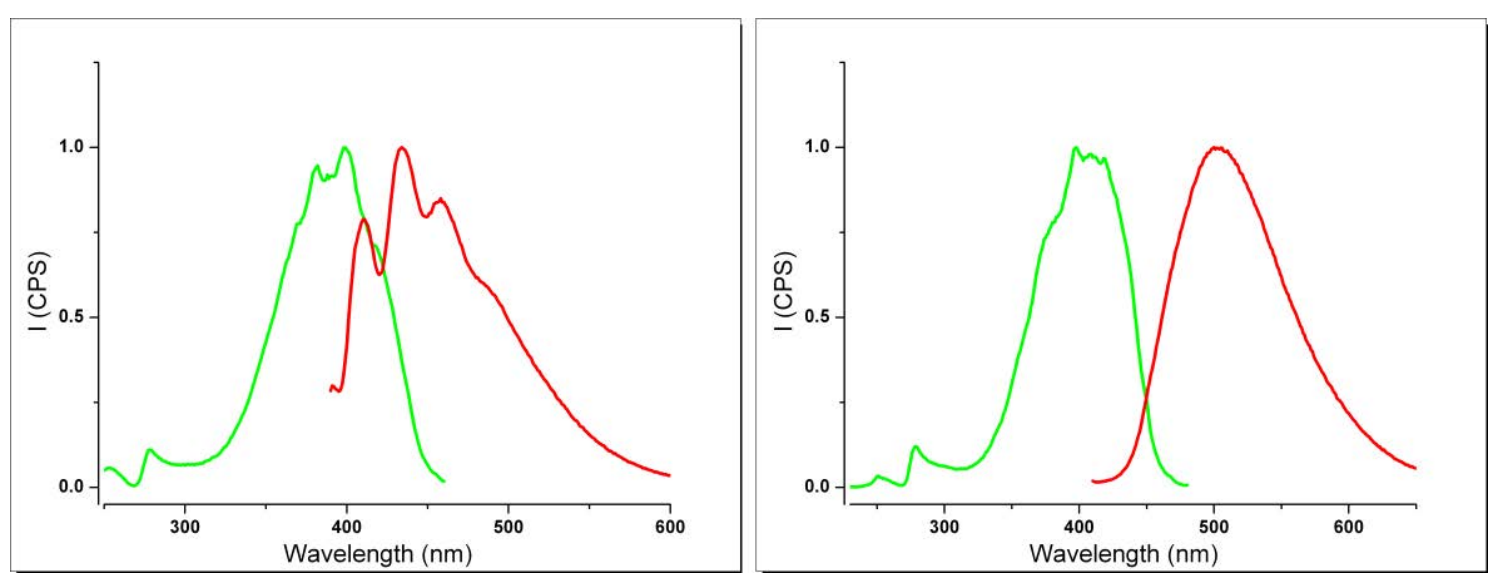

Figure 3-49: Excitation (green) and emission (red) spectra of MeAnPS(NEt $)_{2}$ (23) (left) and [MeAnP(NEt $)_{2}(\mathrm{~S}) \mathrm{AuCl}$ (24) (right), $5 \cdot 10^{-5} \mathrm{M}$ in dichloromethane.

Although the phosphorus atom has neither a lone-pair nor is substituted by phenyl groups in 21-28, electron transfer is nevertheless the cause of the weak fluorescence discussed vide infra.

Alike in solid state, the emission in solution of all selenium oxidized compounds is by about the factor of 10 weaker than of the sulfur oxidized derivatives. Thus only molecules containing the same chalcogens will be compared to one another. For the sulfur oxidized compounds 21-24 a distinct red shift of emission is observed for both gold complexes compared to the free ligands. Figure 3-49 illustrates this bathochromic shift for MeAnPS(NEt $)_{2}$ (23) and the corresponding gold complex $\left[\mathrm{MeAnP}\left(\mathrm{NEt}_{2}\right)_{2}(\mathrm{~S}) \mathrm{AuCl}\right]$ (24). While for the free ligand the excitation and emission maxima are in close proximity $(\sim 45 \mathrm{~nm})$ they separate by nearly $100 \mathrm{~nm}$ upon complexation of gold(I). While the excitation maximum is only slightly shifted, the emission maximum is red shifted by over $50 \mathrm{~nm}$. Also the vibrational structure of the free ligand's emission band is not present in the emission spectrum of the gold complex which shows a single broad emission band. A similar shift is also observed for MeAnPS(NMe $)_{2}$ (21) and its gold complex $\left[\mathrm{MeAnP}\left(\mathrm{NMe}_{2}\right)_{2}(\mathrm{~S}) \mathrm{AuCl}\right](\mathbf{2 2})$, although not as strong as for $\mathbf{2 3}$ and $\mathbf{2 4 .}$ 


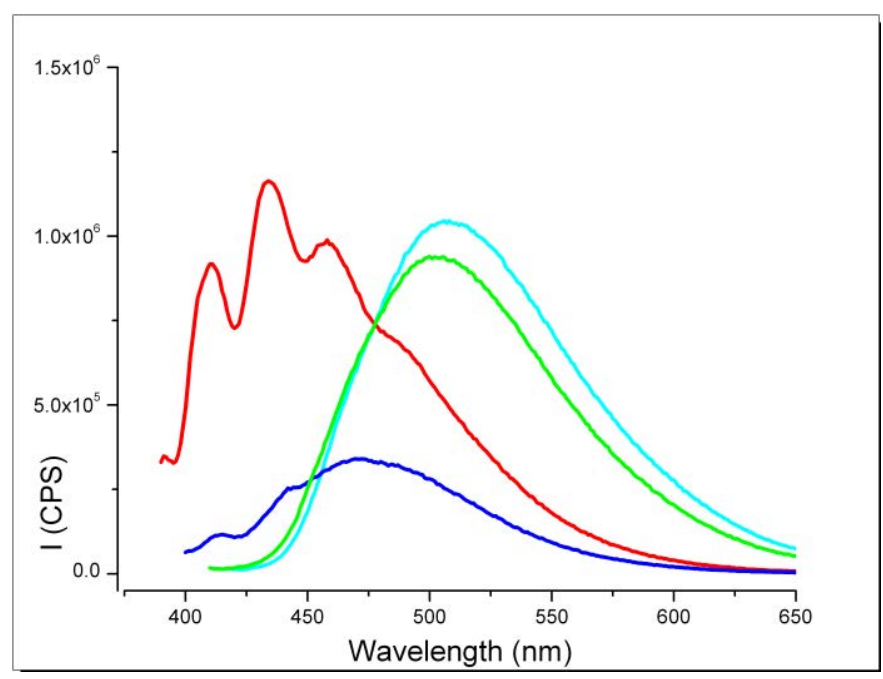

Figure 3-50: Emission spectra of MeAnPS(NMe $)_{2}$ (21) (blue), [MeAnP(NMe $\left.)_{2}(\mathrm{~S}) \mathrm{AuCl}\right]$ (22) (cyan), 23 (red) and $\left[\mathrm{MeAnP}\left(\mathrm{NEt}_{2}\right)_{2}(\mathrm{~S}) \mathrm{AuCl}\right]$ (24) (green), $5 \cdot 10^{-5} \mathrm{M}$ in dichloromethane.

While the emission intensities of 22, 23, and $\mathbf{2 4}$ are nearly identical and observed differences can well be ascribed to small deviations of sample concentrations, MeAnPS(NMe $)_{2}$ (21) shows notably weaker emission. This can be attributed to electron transfer processes. Alkyl amines are known to be efficient quenchers of anthracene fluorescence. ${ }^{[12-13]}$

Table 3-14: Solution fluorescence of 21-24.

This phenomenon has been exploited in

\begin{tabular}{lcccc}
\hline & $\mathbf{2 1}$ & $\mathbf{2 2}$ & $\mathbf{2 3}$ & $\mathbf{2 4}$ \\
\hline$\lambda_{\mathrm{Ex}}(\max )[\mathrm{nm}]$ & 397 & 397 & 400 & 411 \\
$\lambda_{\mathrm{Em}}(\max )[\mathrm{nm}]$ & 469 & 504 & 434 & 508 \\
$\mathrm{I}_{\text {rel }}$ & 0.29 & 0.81 & 1 & 0.90
\end{tabular}
many sensor molecules which carry receptor moieties with amino groups as quenchers, usually linked to the anthracene fluorophore by methylene spacers. ${ }^{[23 c]}$ They have delivered the most effective quenching rates compared to longer spacers in systems of this type. ${ }^{[13,22]}$ This is due to the optimum arrangement achieving maximum orbital overlap facilitating electron transfer to the excited fluorophore. ${ }^{[13]}$ In most cases the methylene spacer carries only one amino group and the required orbital overlap is promoted by rapid rotation around the single bonds of the spacer, resulting in a faster electron transfer than the lifetime of the excited state. In the case of 21-28 two amino groups are present in a geometric arrangement very similar to those of the methylene spacer (Scheme 3-7). 


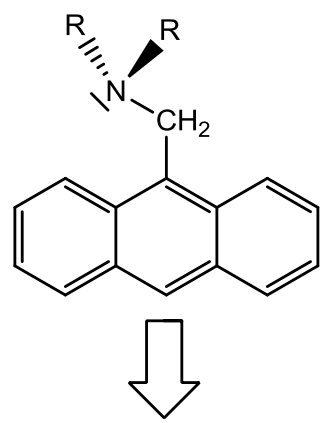

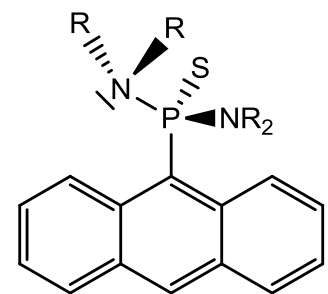<smiles>CC(C)(C)Cc1c2ccccc2cc2ccccc12</smiles><smiles>CC(C)(C)P(N)(=O)c1c2ccccc2cc2ccccc12</smiles>

Scheme 3-7: Geometrical analogies of methylene bridged amine quenchers and amino substituents in 21-28.

Obviously the presence of two quenchers leads to an even higher probability of orbital overlap and electron transfer to the excited fluorophore. Hence, quenching is quite effective and the emission of 21-28 is weak. In contrast to sensor molecules made for metal ion detection, the energy of the amino-lone pairs is not lowered in the gold complex, because only the soft chalcogen donors participate in complexation and not the harder nitrogen donors. Therefore the quenching mechanism is not influenced. The only remaining factor to modify the emission intensity is the rate of rotation about the An-P and P-N bonds. A higher rate of rotation would lead to a higher probability of orbital overlap and consequently to effective quenching. As the steric bulk of the phosphoryl substituent should affect the rate of rotation of the same, this should also become apparent in the effectiveness of quenching.

MeAnPS $\left(\mathrm{NMe}_{2}\right)_{2}(\mathbf{2 1})$, the compound with the smallest substituent, indeed shows the weakest emission. In MeAnPS(NEt $)_{2}$ (23) with a distinctly bulkier substituent and lower rotation rates the quenching is less effective. Both gold complexes $\left[\mathrm{MeAnP}\left(\mathrm{NMe}_{2}\right)_{2}(\mathrm{~S}) \mathrm{AuCl}\right](22)$ and $\left[\mathrm{MeAnP}\left(\mathrm{NEt}_{2}\right)_{2}(\mathrm{~S}) \mathrm{AuCl}\right.$ (24) show similar emissions to 23. In the case of $\mathbf{2 4}$ this is not surprising, as it also carries bulky diethylamino groups. The less effective quenching of $\mathbf{2 2}$ can only be attributed to the complexation of gold(I) which should also notably hinder rotation of the phosphoryl substituent. Proof of a sufficient interaction between ligand and gold ion is given by the strong red shift of emission for both gold complexes. The observed phenomena follow the hypothesis of reduced quenching by steric inhibition of rotation of the phosphoryl substituent. 

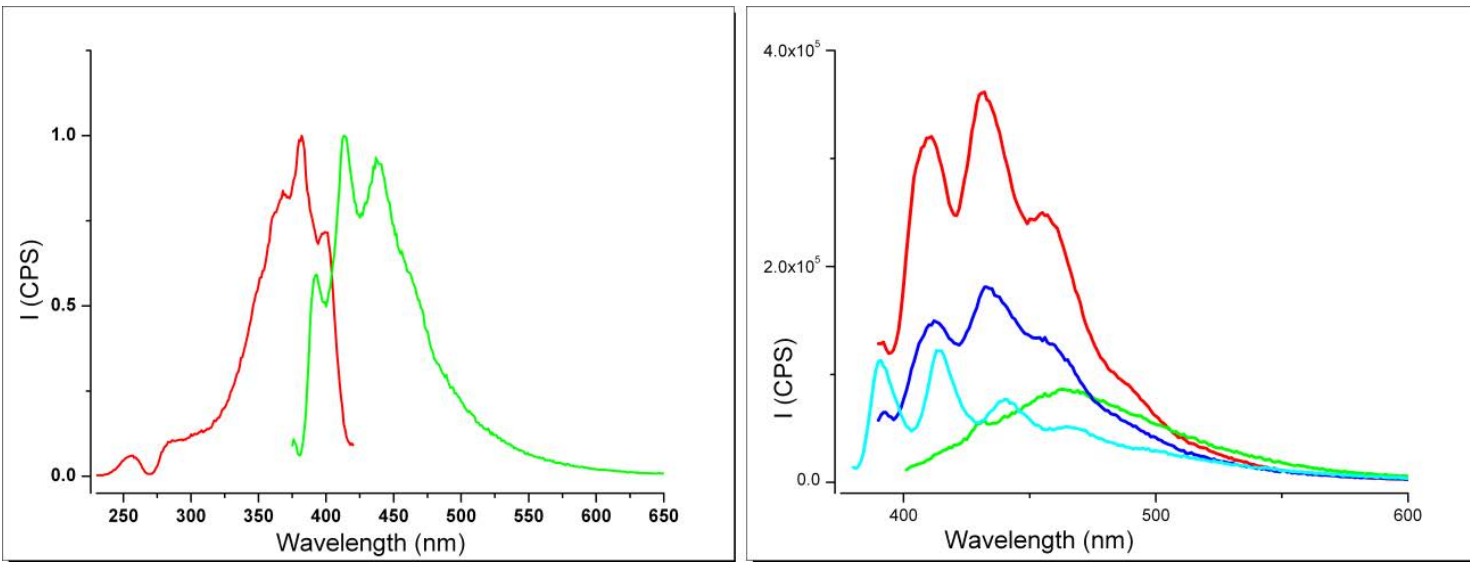

Figure 3-51: Excitation (green) and emission (red) spectra of MeAnPSe(NEt $)_{2}$ (27) (left), and emission spectra of MeAnPSe( $\left(\mathrm{NMe}_{2}\right)_{2}$ (25) (blue), [MeAnP(NMe $)_{2}(\mathrm{Se}) \mathrm{AuCl}$ (26) (cyan), MeAnPSe$\left(\mathrm{NEt}_{2}\right)_{2}$ (27) (red), and [MeAnP(NEt $\left.)_{2}(\mathrm{Se}) \mathrm{AuCl}\right]$ (28) (green) (right), all $5 \cdot 10^{-5} \mathrm{M}$ in dichloromethane.

The selenium oxidized compounds 25-28 show several similarities to $\mathbf{2 1 - 2 4}$ regarding their in-solution fluorescence properties. The most important finding is that the free ligands MeAnPSe $\left(\mathrm{NMe}_{2}\right)_{2}$ (25) and MeAnPSe $\left(\mathrm{NEt}_{2}\right)_{2}$ (27) follow the trend established for their sulfur oxidized counterparts, showing very similar emission proportions as $\mathbf{2 1}$ and $\mathbf{2 3}$. Again the compound which carries the less bulky phosphoryl substituent exhibits more effective fluorescence quenching. In contrast, the gold complexes [MeAnP(NMe $\left.)_{2}(\mathrm{Se}) \mathrm{AuCl}\right]$ (26) and [MeAnP(NEt $\left.)_{2}(\mathrm{Se}) \mathrm{AuCl}\right]$ (28) do not show the characteristic red shift of emission observed for [MeAnP(NMe $\left.)_{2}(S) A u C l\right](22)$ and 24.

Table 3-15: Solution fluorescence of 25-28. This can be explained by the weak Se-Au-

\begin{tabular}{|c|c|c|c|c|c|}
\hline & 25 & 26 & 27 & 82 & raction which most likely leads to a \\
\hline$\lambda_{\mathrm{Ex}}(\max )[\mathrm{nm}]$ & 382 & 384 & 381 & 384 & Additionally \\
\hline$\lambda_{E m}(\max )[n m]$ & 439 & 467 & 432 & 464 & luorescence quenching effect of the \\
\hline$I_{\text {rel }}$ & 0.50 & 0.24 & 1 & 0.14 & selenium atom in the molecular struc \\
\hline
\end{tabular}
of solid state fluorescence appears to be intensified by the presence of a second heavy atom. This leads to very weak emission which lets the results obtained for $\mathbf{2 6}$ and $\mathbf{2 8}$ appear less significant.

In retrospect, it was possible to trace the coordination of gold(I) to MeAnPS $\left(\mathrm{NMe}_{2}\right)_{2}$ (21) and MeAnPS(NEt $)_{2}$ (23) in solution by the means of fluorescence emission. The red shift of the emission maximum by well over $50 \mathrm{~nm}$ enforced by the coordination of 
gold(I) is a significant and well measurable effect. Though overall weakly fluorescent, this behavior upon metal coordination makes this compound class interesting for development of potential sensor systems based on colorimetric detection, rather than typical on/off switching. Furthermore a correlation of emission properties with the steric demand of the phosphoryl substituent was found which lead to a promising hypothesis on rotational rates and their influence on fluorescence quenching.

\subsection{Synthesis of new Phosphoryl Anthracenes and Fluorescence Characterizations}

The previous chapters have outlined the potential of phosphanyl and phosphorylanthracenes in the sectors of fluorescent materials and metal complexation. Variations of phosphorous bound substituents and coordination to cations in solution and in the solid state have been shown to influence the wavelengths and intensities of emitted radiation. Therefore synthetic strategies towards new compounds of this class had to be developed and verified.

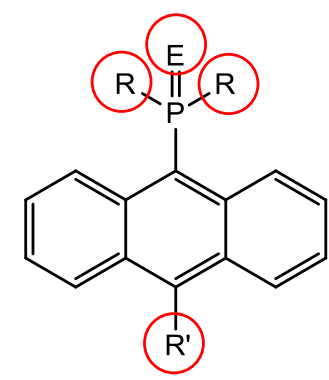

Scheme 3-8: Varied positions of phosphoryl anthracenes.

Furthermore established reaction pathways were utilized to synthesize structurally related compounds for further comparison. Scheme 3-7 shows the positions of phosphoryl anthracenes which were altered by variation of inserted substituents. Not all of the synthesized compounds were subjected to fluorescence experiments. By screening, only those compounds with striking fluorescence properties were closely investigated. The results of these measurements will also be presented in this chapter. For information of the quantification of structural properties as folding angle, twist angle and $\mathrm{C}-\mathrm{H}^{\cdots} \pi \mathrm{r}$ bonding please see 3.2.1. 


\subsubsection{Bis(dialkylamino)phosphanylanthracenes}

In addition to the 9-bis(dialkylamino)phosphoryl-10-methylanthracenes presented in 3.3, related compounds and precursor molecules were synthesized which will be described in the following.

9-Bis(dimethylamino)ohosphanylanthracene (29) and its oxidation products 30-32 were prepared by lithiation of 9-bromoanthracene in diethyl ether at $-15^{\circ} \mathrm{C}$. After subsequent reaction with dimethylaminochlorophosphane, the volatile solvent was evaporated, the crude product was re-dissolved in DCM and the precipitated lithium chloride was removed by filtration. By evaporation of the solvent, $\operatorname{HAnP}\left(\mathrm{NMe}_{2}\right)_{2}$ (29) was obtained as a dark red amorphous solid (Scheme 3-9).

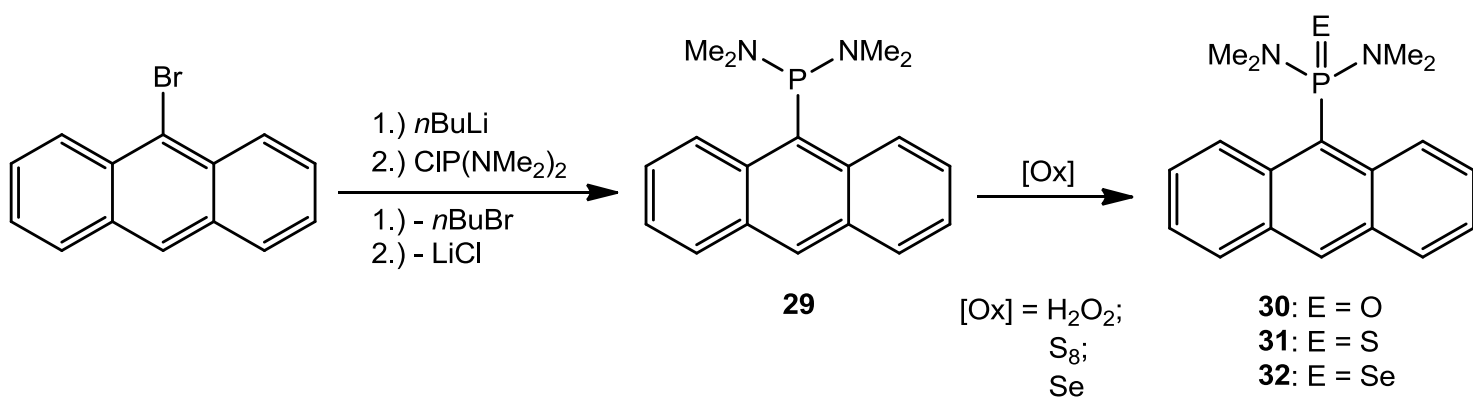

Scheme 3-10: Synthesis of 29-32.

Recrystallization of $\mathrm{HAnP}\left(\mathrm{NMe}_{2}\right)_{2}$ (29) from toluene at $-30^{\circ} \mathrm{C}$ yielded crystals which were suitable for X-ray diffraction experiments. The highly pure crystals of $\mathbf{2 9}$ obtained by recrystallization were used for oxidation reactions. The first oxidation of $\mathbf{2 9}$ was conducted using $\mathrm{H}_{2} \mathrm{O}_{2}$ in a solvent mixture of MeOH/DCM 1:1 at $-15^{\circ} \mathrm{C}$. $\mathrm{HAnPO}\left(\mathrm{NMe}_{2}\right)_{2}$ (30) was obtained by removal of the volatile solvent and recrystallization from $\mathrm{MeOH/DCM}$. Recrystallization also provided crystals of sufficient quality for diffraction experiments. The oxidations using elemental sulfur and selenium were both carried out in toluene at $110^{\circ} \mathrm{C}$. After $6 \mathrm{~h}$ the solutions were filtrated and concentrated to approximately $50 \%$ of the initial volume. Crystallization from toluene gave $\mathrm{HAnPS}\left(\mathrm{NMe}_{2}\right)_{2}(\mathbf{3 1})$ and $\mathrm{HAnPSe}\left(\mathrm{NMe}_{2}\right)_{2}(\mathbf{3 2})$ as yellow crystalline solids.

The crystal structures of 29-32 are depicted in Figure 3-52, crystallographic information on 29-32 is compiled in Table 3-16. 


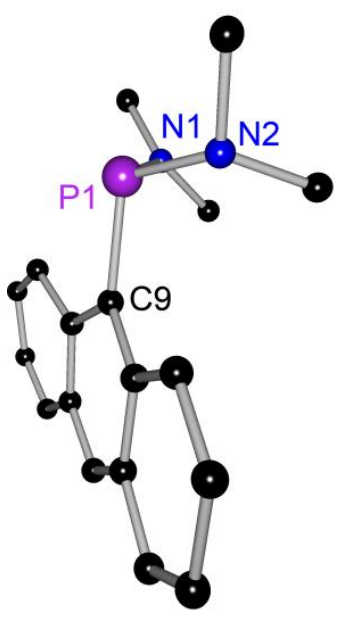

29

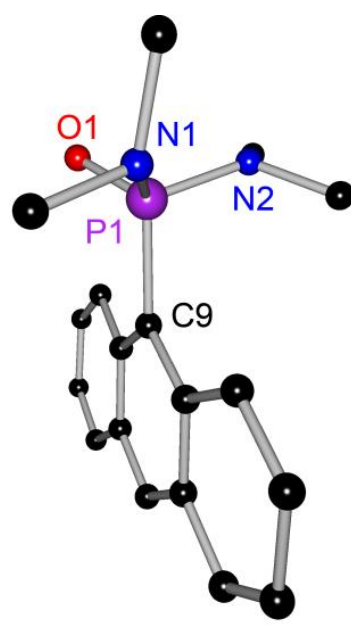

30

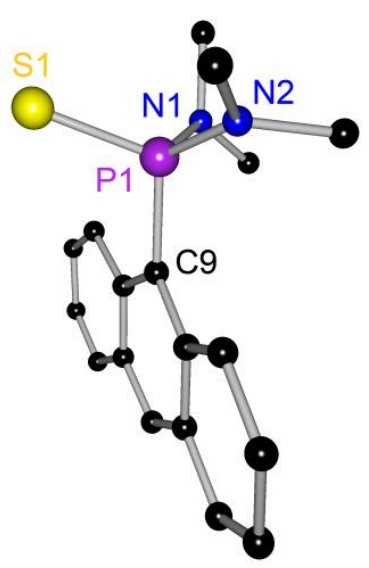

31

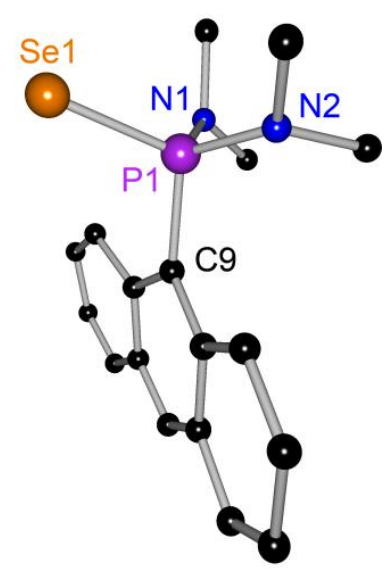

32

Figure 3-52: Crystal structures of 29-32.

Table 3-16: Space groups and selected bond lengths $[\AA]$ and angles $\left[{ }^{\circ}\right]$ of 29-32.

\begin{tabular}{|c|c|c|c|c|c|c|c|}
\hline \multicolumn{2}{|c|}{29} & \multicolumn{2}{|c|}{30} & \multicolumn{2}{|c|}{31} & \multicolumn{2}{|c|}{32} \\
\hline Space gr. & $P 2_{1} / n$ & Space gr. & $\operatorname{Pna}_{1}$ & Space gr. & $P 2_{1} / n$ & Space gr. & $P 2_{1} / C$ \\
\hline \multirow[t]{2}{*}{ P1-C9 } & $1.8614(14)$ & P1-C9 & $1.8468(14)$ & P1-C9 & $1.8363(12)$ & P1-C9 & $1.8295(13)$ \\
\hline & & P1-01 & $1.4805(11)$ & P1-S1 & $1.9587(5)$ & P1-Se1 & $2.1056(4)$ \\
\hline P1-N1 & $1.6866(13)$ & P1-N1 & $1.6607(12)$ & P1-N1 & $1.6572(11)$ & P1-N1 & $1.6651(12)$ \\
\hline Folding & 12.2 & Folding & 3.6 & Folding & 8.9 & Folding & 12.3 \\
\hline Twist & 9.1 & Twist & 4.2 & Twist & 4.0 & Twist & 8.5 \\
\hline
\end{tabular}

Despite the close structural relation among 29-32, none of the compounds are isostructural and they even crystallize in different space groups with exception of $\mathbf{2 9}$ and 31. The $\mathrm{P}-\mathrm{C}$ and $\mathrm{P}-\mathrm{N}$ distances are all in the expected range and differ only marginally among 29-32. Notably, the un-oxidized $\mathrm{HAnP}\left(\mathrm{NMe}_{2}\right)_{2}(29)$ has the longest $\mathrm{P}-\mathrm{C}$ and $\mathrm{P}-\mathrm{N}$ bonds of the four compounds. As expected, the angles around the phosphorous atom indicate that the lone pair in $\mathbf{2 9}$ requires the most space in comparison with the chalcogens in 30-32. In 29, all angles of the phosphorus bound substituents to one another are smaller than the ideal tetrahedral angle. The sulfurand selenium-oxidized $\mathbf{3 1}$ and $\mathbf{3 2}$ also show pronounced deviation from the tetrahedral angle, while the angles surrounding the phosphorus atom in $\mathrm{HAnPO}\left(\mathrm{NMe}_{2}\right)_{2}(30)$ are 
close to the ideal $109.45^{\circ}$. The deformations of the anthracene moieties also differ significantly among the four compounds. Because the deformation is closely linked to the steric strain applied by the phosphanyl substituent, folding and twist deformations can be explained by the orientation of this substituent. $\mathrm{HAnPO}\left(\mathrm{NMe}_{2}\right)_{2}(30)$ exhibits the clearly weakest deformation of the anthracene moiety, in terms of folding and of twist deformation. It also shows the smallest torsion angle of the chalcogenphosphorous bond to the anthracene plane.

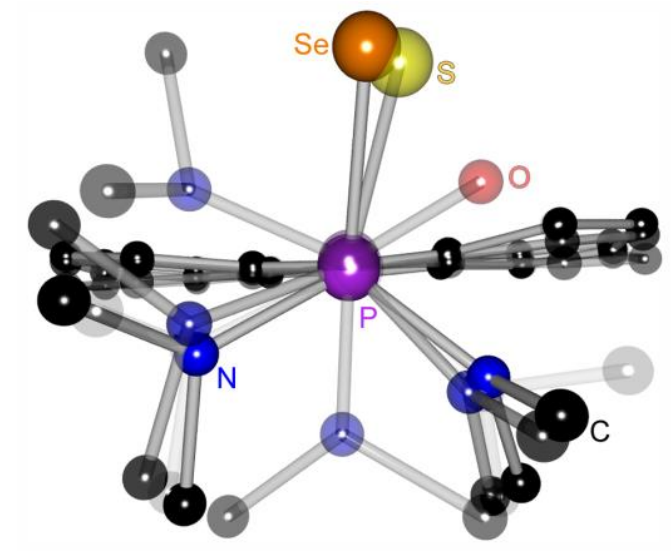

Figure 3-53: superposition of 29-32, transparency decreases from 29 to 32.

Table 3-17: selected torsion angles [ $^{\circ}$ in 29-32.

$\begin{array}{lll}29 & \text { LP-P1-C9-C9a* } & 74.65(11) \\ 30 & \text { O1-P1-C9-C9a } & 28.97(12) \\ 31 & \text { S1-P1-C9-C9a } & 70.38(10) \\ 32 & \text { Se1-P1-C9-C9a } & 79.55(10)\end{array}$

*mean value of the torsion angles N1-P1-C9-C9a and N2-P1-C9-C9a for estimation of the position of the lone pair.
Because the $\mathrm{P}-\mathrm{O}$ bond is located almost in the anthracene plane, the dimethylamino substituents are located on opposite sides of this plane, which leads to a fairly even distribution of steric strain. This explains the weak distortion of the anthracene moiety. All other compounds show distinctly stronger deformation and all feature a nearly orthogonal torsion angle of the chalcogen-phosphorous bond/lone pair to the anthracene plane (Figure 3-53, Table 3-17). This leads to an arrangement in which both amino substituents are located on the same side of the anthracene moiety. Hence, the steric strain is unbalanced and the deformation increases. Taking into account the large steric demand of the lone pair, the torsion angles correlate very well with the observed deformations.

In contrast to the compounds presented in 3.3, which all carry a methyl group in 10position of the anthracene moiety, the majority of 29-32 do not adapt the typical "head-to-tail" arrangement with parallel oriented anthracene planes. Instead, an "edge-to-face" or "herringbone" like arrangement is found in the packing plots of 29, 30, and 32. This can be attributed to the absence of a methyl group in 10-position, which generally prevents an "edge-to-face" arrangement. 

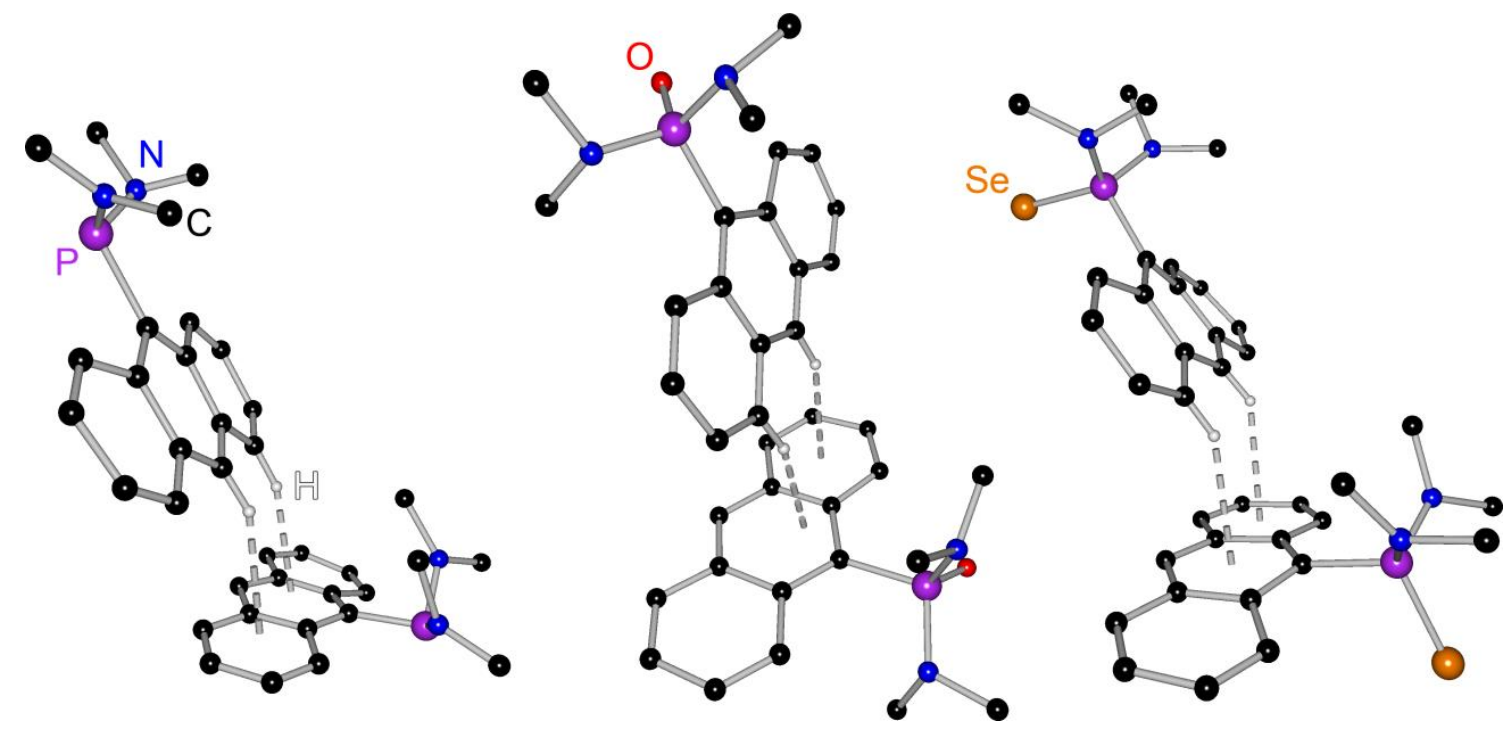

Figure 3-54: C-H“" $\pi$ stabilized "edge-to-face" arrangement in 29 (left), 30 (center) and 32 (right).

The absence of a methyl group in 10-position allows the hydrogen atoms in 4,5- and 10 -position to interact with the aromatic $\pi$-systems of adjacent anthracene moieties, as depicted in Figure 3-54. The resulting $\mathrm{C}-\mathrm{H}^{\cdots} \pi$ bonds stabilize this arrangement and appear to be energetically favoured compared to the optional $\pi$ stacked form which would also be accessible. In all three compounds two $\mathrm{C}-\mathrm{H}^{\prime \prime} \pi$ bonds per molecule are formed, which are listed in Table 3-18.

Table 3-18: $\mathrm{C}-\mathrm{H} \cdots \pi$ Interactions in 29, The fact that these are all $\mathrm{sp}^{2} \mathrm{C}-\mathrm{H} \cdots \pi$ bonds 30, and 32. combined with the very short distances and fairly large angles to the $\pi$ system - underlines the $\mathrm{C}-\mathrm{H}^{\prime} \pi \mathrm{\pi}$ Interactions $[\AA \AA] /\left[^{\circ}\right]$ strength of these interactions. They are in terms of $292.642 / 56.62 .851 / 54.8$ bond distance among the strongest interactions of 30 $2.509 / 61.7 \quad 2.654 / 67.8$ this kind throughout this entire thesis.

$32 \quad 2.644 / 59.4 \quad 2.765 / 53.3$ The packing motif of $\mathrm{HAnPS}\left(\mathrm{NMe}_{2}\right)_{2}$ (31) on the other hand shows the typical "head-to-tail" arrangement with phosphoryl substituents directed in opposite directions. This is quite surprising because $\mathbf{3 1}$ does not show any specific structural or geometrical features which would justify a completely different arrangement than its closely related derivatives. The $\pi-\pi$ distance measures $3.47 \AA$, which is fairly short. The observed $\pi-\pi$ overlap is $\sim 65 \%$ which is also one of the largest overlaps of phosphoryl anthracenes encountered so far (Figure 3-55). 


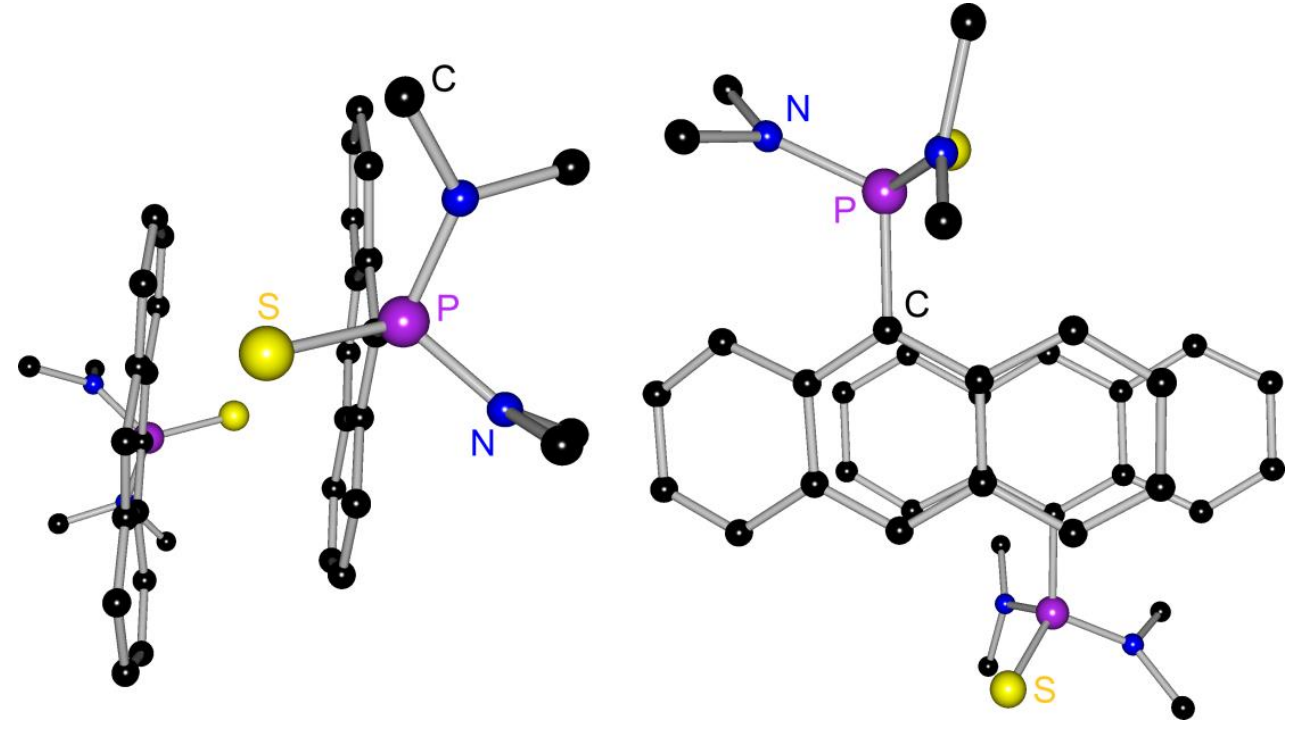

Figure 3-55: $\pi-\pi$ interaction in the structure of $\mathrm{HAnPS}\left(\mathrm{NMe}_{2}\right)_{2}(31)$ : side view (left), top view (right).

The solid state emission properties of $\mathrm{HAnPS}\left(\mathrm{NMe}_{2}\right)_{2}(\mathbf{3 1})$ were also investigated. Both the broad excitation band and the single emission band which shows no vibrational structure (Figure 3-56, left) are very similar to the excitation and emission spectra of the 10-methyl substituted MeAnPS(NMe $)_{2}(\mathbf{2 1})$ and MeAnPS(NEt $)_{2}$ (23) (c.f. 2.2).
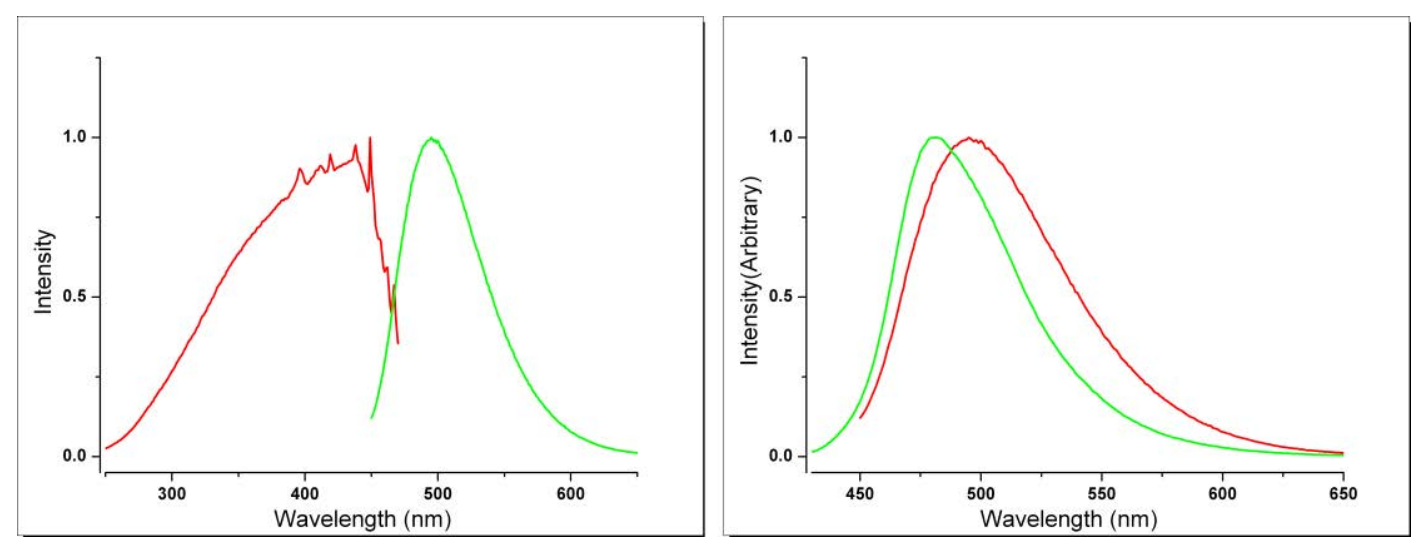

Figure 3-56: left: normalized solid state excitation (red) and emission (green) spectra of $\mathrm{HAnPS}\left(\mathrm{NMe}_{2}\right)_{2}$ (31); right: normalized solid state emission spectra of MeAnPS(NMe $)_{2}(\mathbf{2 1})$ (green) and $\mathrm{HAnP}\left(\mathrm{SNe}_{2}\right)_{2}(\mathbf{3 1})$ (red).

In comparison to its 10-methyl substituted counterpart, $\mathrm{HAnPS}\left(\mathrm{NMe}_{2}\right)_{2}(31)$ shows a nearly identical excitation spectrum, an identical maximum excitation wavelength of $449 \mathrm{~nm}$, but a red-shifted emission maximum. (Figure 3-56, right) The emission maxima differ by $14 \mathrm{~nm}$. Hence, excitation and emission maxima are farther separated for 31. While 21-28 were also structurally closely related, varying maximum emission 
wavelengths could in no case be traced back to $\pi-\pi$ interactions (c.f. 3.3) which have repeatedly been shown to induce red-shifts of emission. ${ }^{[42 c, 50,53-54]}$ Although $\pi-\pi$ overlap ranges from $0 \%$ to $35 \%$ amongst $\mathbf{2 1 - 2 8}$, absolutely no correlation between overlap and maximum emission wavelengths was found. For 31 and 21, however, there is a correlation.

Though the observed bathochromic shift between $\mathbf{2 1}$ and $\mathbf{3 1}$ could be thought to be caused by the absence of the methyl substituent in 10-position in the structure of $\mathbf{3 1}$, this can be regarded as unlikely because a second substituent in 10-position has been shown to rather promote bathochromic shifts of emission in 3.1. Thus, intermolecular interactions appear to be the most probable cause of the red-shift of emission of $\mathbf{3 1}$. And indeed, $\mathbf{2 1}$ and $\mathbf{3 1}$ differ largely in terms of $\pi-\pi$ overlap: $\mathbf{2 1}$ features an overlap of $35 \%$, while 31 shows an overlap of $65 \%$. Because the $\pi-\pi$ distances of 3.51 and $3.49 \AA$ are almost identical, the interaction found in the structure of $\mathbf{3 1}$ can be considered nearly twice as strong as the one in $\mathbf{2 1}$. These two compounds are the first in this thesis to confirm the consequences of $\pi-\pi$ overlap described in literature.

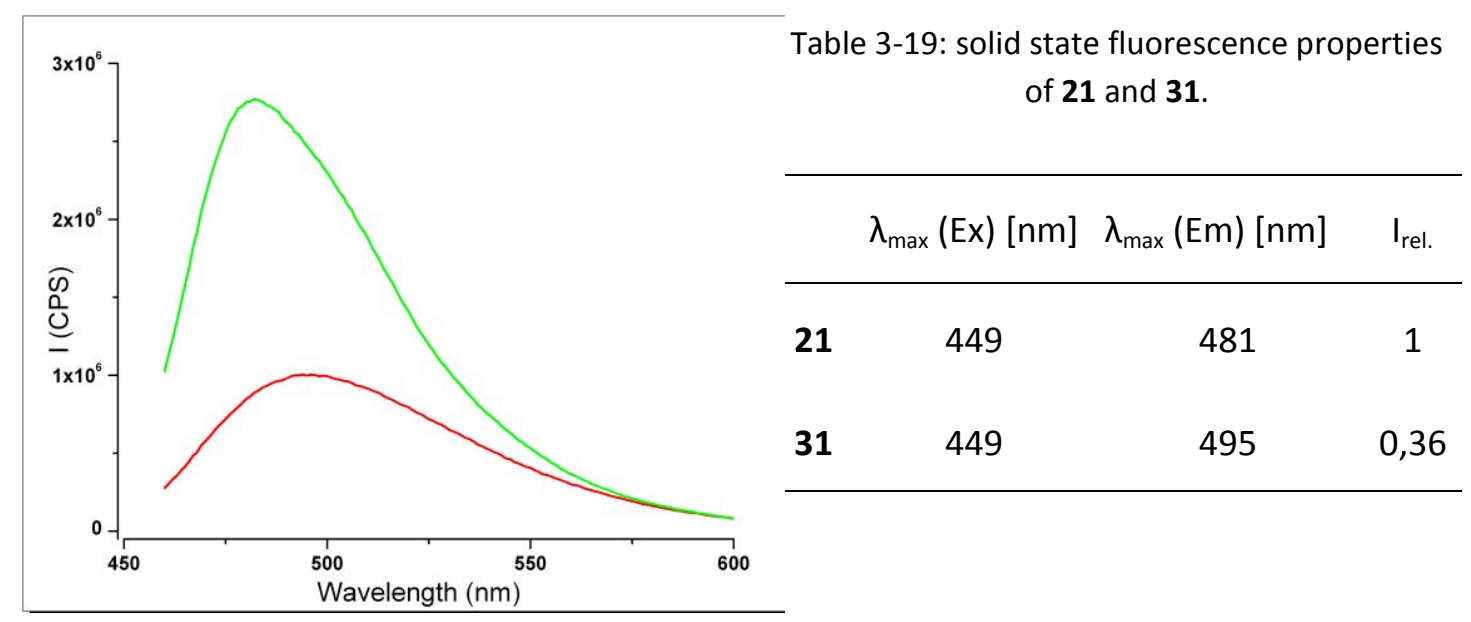

Figure 3-57: maximum solid state emission spectra of $\mathrm{HAnPS}\left(\mathrm{NMe}_{2}\right)_{2}(\mathbf{3 1})(\mathrm{red})$ and $\mathrm{MeAnPS}\left(\mathrm{NMe}_{2}\right)_{2}(\mathbf{2 1})$ (green).

This suggests that this red-shifting effect is by no means negligible for phosphoryl anthracenes, but that it first becomes relevant and its consequences detectable from a certain degree of $\pi-\pi$ overlap onward. For 21-28, which all exhibit an overlap of $35 \%$ or less, the effects of $\pi-\pi$ interaction appear to be comparatively weak and are therefore outnumbered by other effects. In the case of HAnPS(NMe $)_{2}$ (31) they are strong enough to dominate this compound's fluorescence properties. 
This also becomes manifest in the measured emission intensity. Both MeAnPS $\left(\mathrm{NMe}_{2}\right)_{2}(\mathbf{2 1})$ and HAnPS$\left(\mathrm{NMe}_{2}\right)_{2}$ (31) feature similarly strong deformations of the fluorophore (Table 3-20).

Still the observed emission intensity of $\mathbf{2 1}$ is by nearly factor three higher than of $\mathbf{3 1}$ (Figure 3-57). Again this can be ascribed to the stronger $\pi-\pi$ overlap of 31 . This has also

Table 3-20: Fluorophore

deformations of $\mathbf{2 1}$ and $\mathbf{3 1}$.

Folding $\left[^{\circ}\right] \quad$ Twist $\left[^{\circ}\right]$

$21 \quad 9.9$

$31 \quad 8.9$
4.0

2.0 been repeatedly reported in literature and was also not observed for compounds showing $\pi-\pi$ overlap under 35\%. Additionally, 21 features two $\mathrm{C}-\mathrm{H}^{\prime \prime} \pi$ interactions which are completely absent in the structure of $\mathrm{HAnPS}\left(\mathrm{NMe}_{2}\right)_{2}$ (31), which further explains the weak emission of $\mathbf{3 1}$.

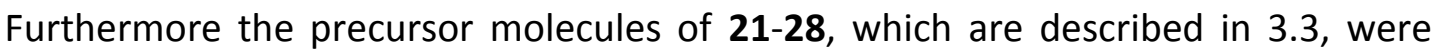
prepared by introduction of a methyl substituent in 10-position prior to the introduction of the phosphane substituent. This was achieved by selective monolithiation of 9,10 -dibromoanthracene at $-15^{\circ} \mathrm{C}$ and subsequent reaction with methyl iodide.<smiles>Brc1c2ccccc2c(Br)c2ccccc12</smiles>

1.) $n \mathrm{BuLi}$

2.) $\mathrm{CH}_{3}$

1.) $-n \mathrm{BuBr}$

2.) - Lil<smiles>Cc1c2ccccc2c(Br)c2ccccc12</smiles>

2.) $-\mathrm{LiCl}$

1.) $n \mathrm{BuLi}$

2.) $\mathrm{CIP}\left(\mathrm{NR}_{2}\right)_{2}$

1.) $-n \mathrm{BuBr}$

2.) $-\mathrm{LiCl}$<smiles>[R16]NP([R16])c1c2ccccc2c(C)c2ccccc12</smiles>

33: $\mathrm{R}=\mathrm{Me}$

34: $\mathrm{R}=\mathrm{Et}$

Scheme 3-11: Synthesis of MeAnP(NMe $)_{2}$ (33) and MeAnP(NEt $)_{2}$ (34).

After aqueous work-up, the obtained 9-bromo-10-methylanthracene was lithiated a second time and then reacted with the respective chlorophosphane. After removal of precipitated lithium chloride and evaportation of the solvent, $\operatorname{MeAnP}\left(\mathrm{NMe}_{2}\right)_{2}(33)$ and $\mathrm{MeAnP}\left(\mathrm{NEt}_{2}\right)_{2}$ (34) were obtained as dark red highly viscous oils (Scheme 3-10). Due to their oily texture, it was not possible to crystallize $\mathbf{3 3}$ and $\mathbf{3 4}$ even at low temperatures. The solid state structures could therefore not be acquired. 
While the oxidations of $\mathbf{3 3}$ and $\mathbf{3 4}$ with elemental sulfur and selenium were successful (as described in 3.3), the oxidations using peroxide were problematic. The reaction conditions used were basically identical to those of previous oxidations with hydrogen peroxide: at $-15^{\circ} \mathrm{C}$ in a solvent mixture of $\mathrm{MeOH}$ and $\mathrm{DCM}$. If the peroxide solution is too concentrated or added to the un-oxidized phosphane too fast, decomposition of the compound is observed.

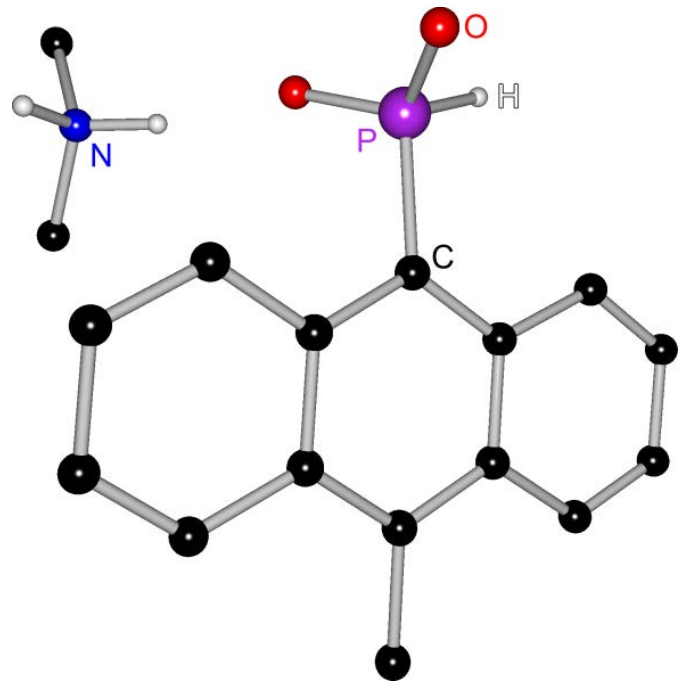

Figure 3-58: Crystal structure of decomposition product 33a.
As depicted in Figure 3-58, the phosphorus bound amino groups were cleaved from the compound and have formed ammonium ions as counter ions to the hypophosphite anion. This underlines the reduced stability of $\mathrm{P}-\mathrm{N}$ bonds compared to $\mathrm{P}-\mathrm{C}$ bonds. Although in this case the instability of the $\mathrm{P}-\mathrm{N}$ bonds has led to decomposition of the compound, this phenomenon can also by synthetically exploited, which will be shown in 3.4.4.

Finally, also symmetrical bis(dialkylamino)phosphanylanthracene derivatives were synthesized. Related symmetrical compounds have previously been utilized for the preparation of cyclic metal complexes, which also makes this compound class chemically relevant. ${ }^{[71]}$

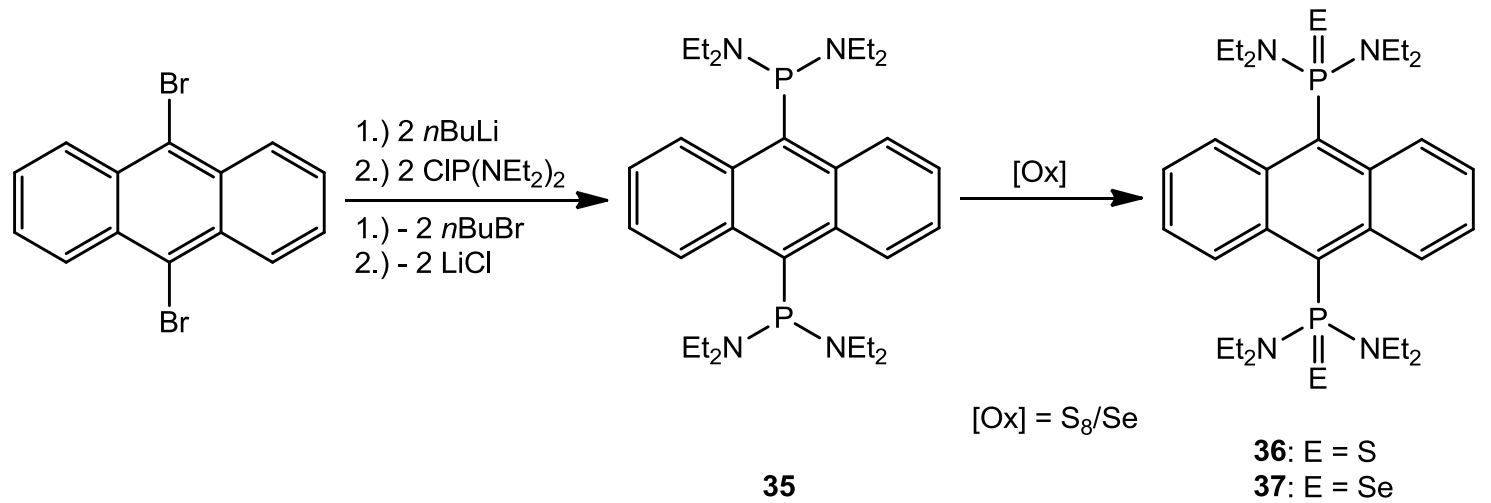


Di-lithiation of 9,10-dibromoanthracene and reaction with two equivalents of chlorophosphane yielded the symmetrical $\left(\mathrm{Et}_{2} \mathrm{~N}\right)_{2} \mathrm{PAnP}\left(\mathrm{NEt}_{2}\right)_{2}(35)$, which can again be converted to its oxidation products by reaction with elemental sulfur or selenium in toluene at $110^{\circ} \mathrm{C}(\mathrm{Scheme} 3-11) \cdot\left(\mathrm{Et}_{2} \mathrm{~N}\right)_{2} \mathrm{SPAnPS}\left(\mathrm{NEt}_{2}\right)_{2}(36)$ and $\left(\mathrm{Et}_{2} \mathrm{~N}\right)_{2} \mathrm{SePAnPSe}\left(\mathrm{NEt}_{2}\right)_{2}$ (37) were obtained by crystallization from toluene. Though 35-37 have been previously synthesized and $\mathbf{3 6}$ and $\mathbf{3 7}$ have also been subjected to X-ray diffraction experiments for structure determination, these compounds were re-synthesized and listed here because they are important precursor molecules of metal complexes described in 3.5.

Moreover the crystallization of $\mathbf{3 5}$ was accomplished for the first time. Generally un-oxidized phosphanyl anthracenes have proven to be far more challenging to crystallize than their oxidized analogues. Especially the presence of numerous aliphatic substituents further hinders the crystallization progress, because many flexible substituents in a single molecule rarely simultaneously assume fixed ordered positions, which is a precondition for obtaining crystalline materials. Hence, most un-oxidized bis(diethylamino)phosphanylanthracenes are highly viscous oils. After a considerably long crystallization time of nearly three years, crystals of $\mathbf{3 5}$ which were suitable for diffraction experiments were obtained. It is the first un-oxidized bis(diethylamino)phosphanylanthracene that has been crystallized so far (Figure 3-59).

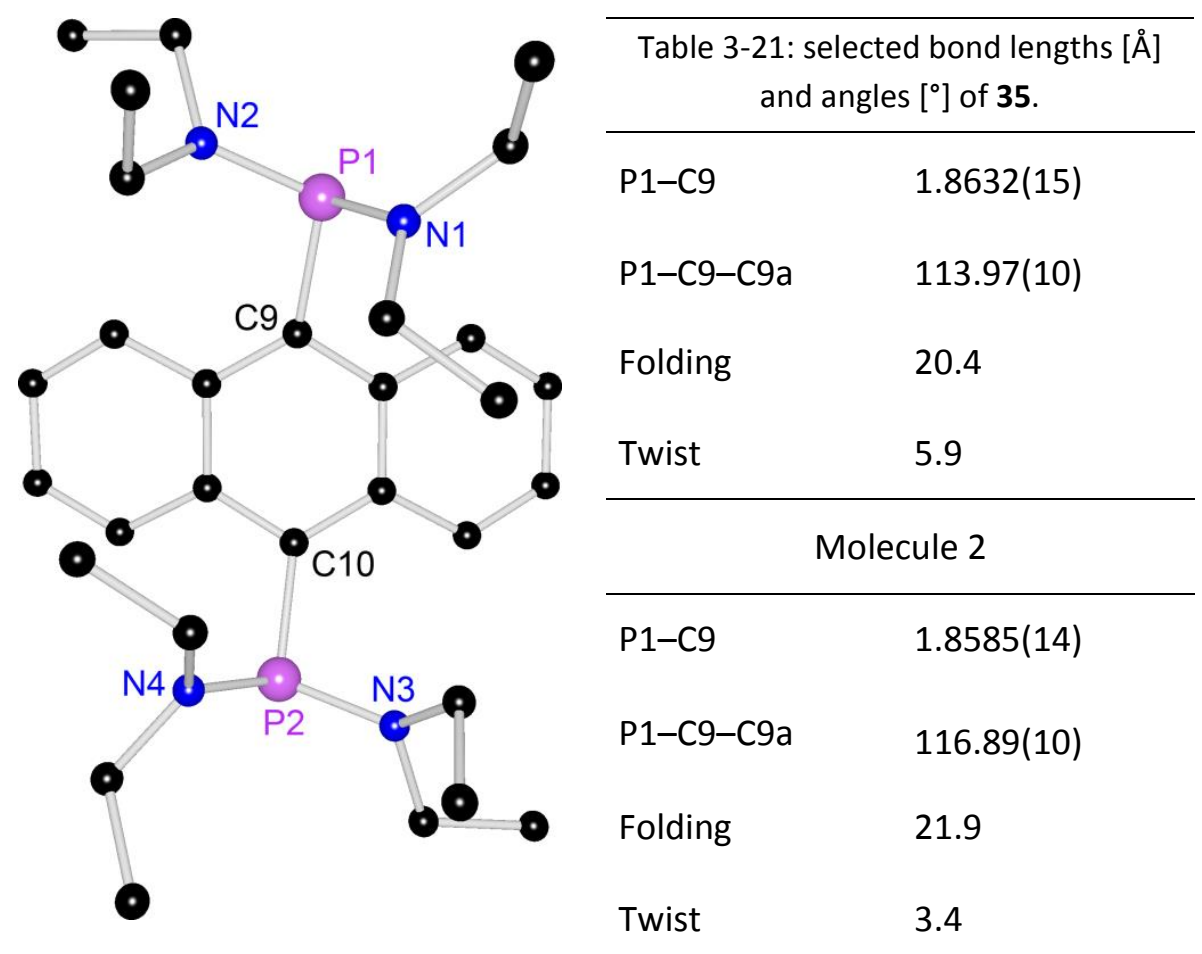

Figure 3-59: Crystal structure of 35, only one of two independent molecules is depicted, hydrogen atoms are omitted. 
As predicted, the numerous very flexible ethyl groups all assume different orientations in the crystal structure, which induces the low symmetry of the structure. 35 crystallizes in the triclinic space group $P \overline{1}$ and two independent molecules of $\mathbf{3 5}$ are present in the asymmetric unit. The two molecules are similar regarding their conformations and deformations of the anthracene moieties, but not identical. Molecule 1 exhibits a folding angle of $20.4^{\circ}$ and a twist angle of $5.9^{\circ}$, while molecule 2 is folded by $21.9^{\circ}$ and twisted by $3.4^{\circ}$. The overall deformation is nevertheless alike and in both cases fairly strong, which is not surprising in view of the steric strain supplied by two bulky substituents. The $\mathrm{P}-\mathrm{C}$ and $\mathrm{P}-\mathrm{N}$ distances also only deviate minimally between molecule 1 and 2 and are in the expected range. The steric demand of the substituents bring about such large intermolecular distances in the packing motif of 35 , that virtually no noteworthy $\pi-\pi$ or $\mathrm{C}-\mathrm{H}^{\cdots} \pi$ interactions are found which is quite rare.

\subsubsection{Synthesis of Phosphanylanthracenes from Triphenyl- phosphite}

The phosphanylanthracenes which have been presented so far were all prepared by reaction of lithiated anthracenes with chlorophosphanes under elimination of lithium chloride. This method is very reliable and provides nearly quantitative conversion which minimalizes side products and purification steps. There is a number of commercially available chlorophosphanes which can be utilized in such reactions, and the majority has found application in one or more compounds. On the other hand the commercially available chlorophosphanes are limited to a small repertoire of aromatic and aliphatic substituents, which again diminishes synthetic options in the preparation of phosphanylanthracenes. Moreover the synthesis of derivatized chlorophosphanes is a difficult process due to the high reactivity, air and moisture sensibility, and toxicity of chlorophosphanes. Also the introduction of more complex substituents leads to high boiling points of the resulting phosphanes, which makes purification tedious. Though e.g. the preparation of bis(dialkylamino)chlorophosphanes was successful (c.f. 3.3), reaction of organometallics with $\mathrm{PCl}_{3}$ - which is the common reaction pathway - is often unselective and leads to virtually inseparable product mixtures.

Because the coordination of metal cations has been shown to alter the emission properties of phosphanyl anthracenes, the preparation of compounds which are 
capable of forming more stable complexes with metal ions with high selectivity is desirable. Hence, the chlorophosphanes of interest should preferably carry functionalized substituents to generate chelating phosphane ligands (Scheme 3-12).

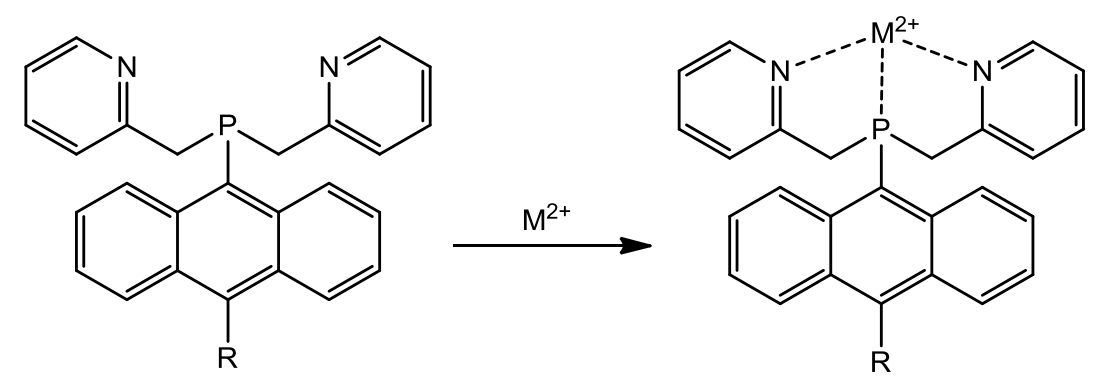

Scheme 3-13: possible structure and coordination mode of a chelating phosphanyl anthracene.

The chlorophosphanes necessary for the preparation of compounds like the one depicted in Scheme 3-12 have not been successfully synthesized, which underlines how challenging the synthesis of such compounds is via established reaction pathways. Consequently, other synthetic routes must be explored to access this type of compounds.

In 2006 Keller et al. published their work on synthetic strategies towards tertiary phosphanes from triphenylphosphite. ${ }^{[72]}$ By conversion with lithiumorganics in one-pot reactions they were able to insert organic substituents to triphenylphosphite. This was among other substrates also actualized with 9-lithioanthracene, introducing the anthracene moiety as a primary substituent (Scheme 3-13).

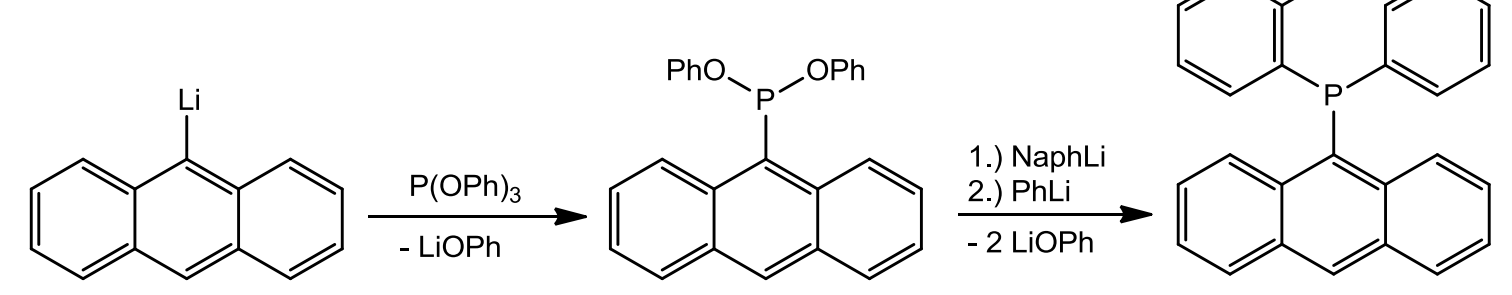
Scheme 3-14: tertiary phosphane synthesis by Keller et al. ${ }^{[72]}$

The introduction of a large substituent in the first reaction step is named as a precondition to assure selectivity in the following reaction steps and avoid multiple substitution with the same substituent. This way Keller and co-workers prepared a number of mainly aromatic, but also aliphatic substituted phosphanyl anthracenes with yields ranging from $46 \%$ to $70 \%$. Highly diluted reaction mixtures as well as low 
temperatures of $-78^{\circ} \mathrm{C}$ or below are also an important factor in achieving high selectivity according to Keller et al.

To verify whether this synthetic strategy is suitable for the introduction of functionalized substituents to prepare chelating phosphanyl anthracenes, $\mathrm{BrAnP}(\mathrm{OPh})_{2}$ (38) was synthesized under conditions identical to those described by Keller (Scheme 3-14).<smiles>Brc1c2ccccc2c(Br)c2ccccc12</smiles>

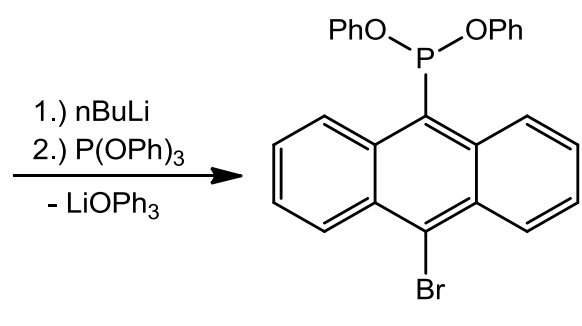

38

Scheme 3-15: Synthesis of $\mathrm{BrAnP}(\mathrm{OPh})_{2}$ (38).

9,10-Dibromoanthracene was mono-lithiated and reacted with one equivalent of triphenylphosphite at $-78^{\circ} \mathrm{C}$ in THF. After completion of the reaction the solvent was evaporated and the crude product was dissolved in diethyl ether and extracted with $\mathrm{H}_{2} \mathrm{O}$ to remove the formed lithium phenolate. $\mathbf{3 8}$ was obtained by crystallization from diethyl ether. Although $\mathbf{3 8}$ was the main product, also noteworthy amounts of disubstituted byproduct were formed.<smiles>Brc1c2ccccc2c(Br)c2ccccc12</smiles>

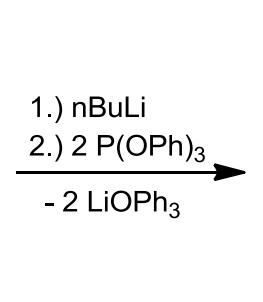<smiles>Brc1c2ccccc2c(P(Oc2ccccc2)c2ccccc2)c2ccccc12</smiles>

39

Scheme 3-16: Formation of di-substituted byproduct ( $\mathrm{BrAn})_{2} \mathrm{OPh}(39)$.

This is problematic because $\mathbf{3 8}$ and $\mathbf{3 9}$ show very similar solubility and were crystallized under identical conditions from the same sample. Separation of product and byproduct is therefore difficult. The crystal structures of $\mathbf{3 8}$ and $\mathbf{3 9}$ are depicted in Figure 3-60, crystallographic information is compiled in Table 3-22. 


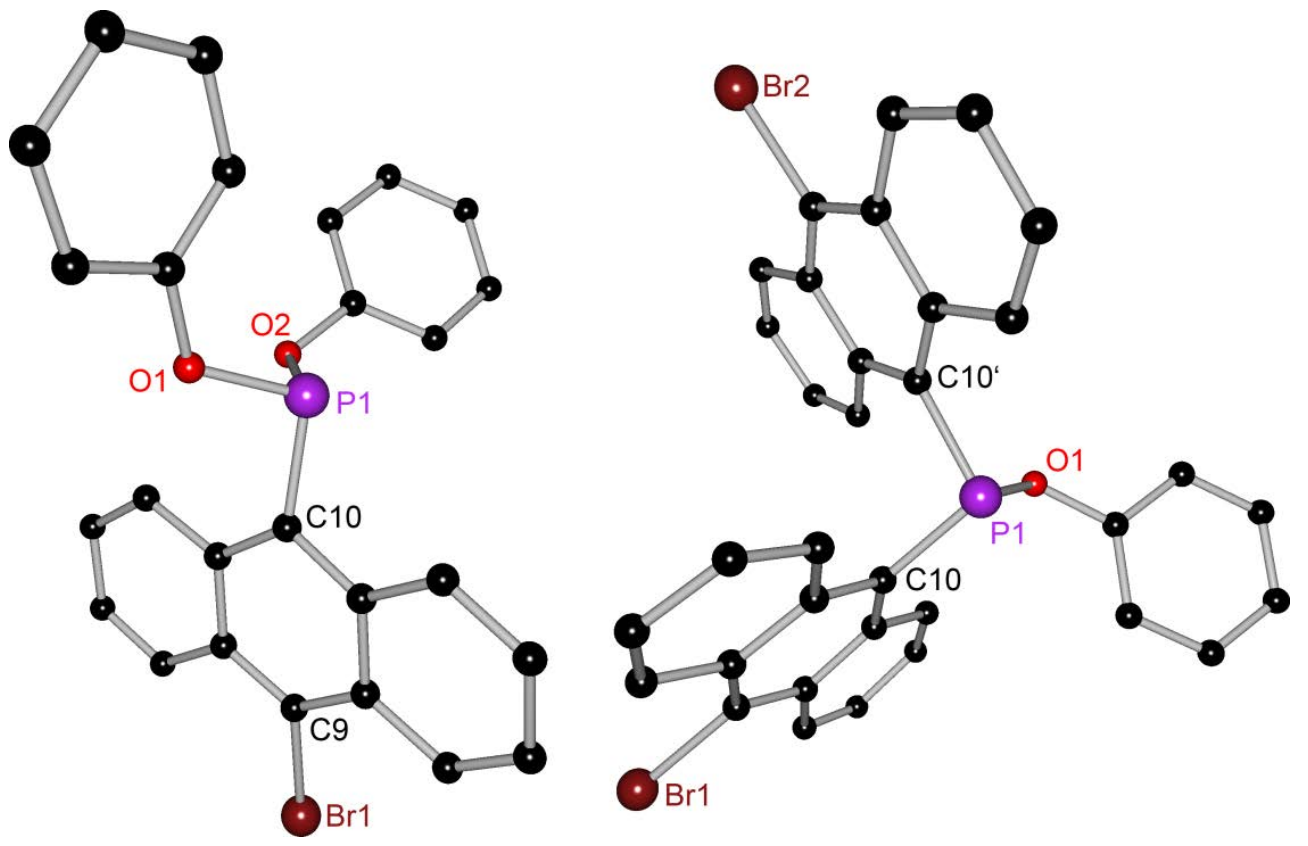

Figure 3-60: left: crystal structure of $\mathrm{BrAnP}(\mathrm{OPh})_{2}(38)$, right: crystal structure of $(\mathrm{BrAn})_{2} \mathrm{POPh}(39)$, hydrogen atoms are omitted for clarity.

\begin{tabular}{|c|c|c|c|}
\hline \multicolumn{4}{|c|}{$\begin{array}{r}\text { Table 3-22: space groups and selected bon } \\
\qquad \mathbf{3 8} \text { and } \mathbf{3 9 .}\end{array}$} \\
\hline \multicolumn{2}{|c|}{$\operatorname{BrAn}(\mathrm{OPh})_{2}(\mathbf{3 8})$} & \multicolumn{2}{|c|}{$(\mathrm{BrAn})_{2} \mathrm{OPh}(39)$} \\
\hline Space gr. & $P 2_{1} / c$ & Space gr. & $P \overline{1}$ \\
\hline P1-C10 & $1.828(2)$ & P1-C10 / P1-C10' & $1.855(3) / 1.865(3)$ \\
\hline $\mathrm{P} 1-\mathrm{O} 1$ & $1.6510(14)$ & $\mathrm{P} 1-\mathrm{O} 1$ & $1.664(2)$ \\
\hline C10-P1-O1 & $98.43(8)$ & C10-P1-O1 & $98.27(11)$ \\
\hline C10-P1-O2 & $99.95(8)$ & $\mathrm{C} 10^{\prime}-\mathrm{P} 1-01$ & $108.04(12)$ \\
\hline O1-P1-O2 & $98.57(8)$ & C10-P1-C10' & 101.92(12) \\
\hline Folding & 7.5 & Folding & $9.0 / 7.8$ \\
\hline Twist & 4.5 & Twist & $4.1 / 8.4$ \\
\hline
\end{tabular}

$\mathrm{BrAnP}(\mathrm{OPh})_{2}$ (38) crystallizes in the monoclinic space group $P 2_{1} / c$ and the asymmetric unit contains one complete molecule of 38 . While the $\mathrm{P}-\mathrm{C}$ and $\mathrm{P}-\mathrm{O}$ bond distances are in the expected range, the angles around the phosphorus atom all clearly deviate from the ideal $109.45^{\circ}$. All angles are smaller than $100^{\circ}$, which demonstrates the strong distortion of the tetrahedral geometry, with the lone pair demanding distinctly more space than the other substituents. The deformation of the anthracene 
moiety is moderate at a folding angle of $7.5^{\circ}$ and a twist angle of $4.5^{\circ}$. This may be attributed to the increased flexibility of the phenyl groups. Compared to phosphanylanthracenes in which phenyl substituents are bound directly to the phosphorus atom, the oxygen atoms in $\mathbf{3 8}$ allow the phenyl rings to turn away from the anthracene moiety, reducing the steric strain which they apply to it. Furthermore there is no noteworthy $\pi-\pi$ overlap statable, but a single $\mathrm{sp}^{2} \mathrm{C}-\mathrm{H}^{\cdots}{ }^{\prime \prime} \pi$ interaction is present, which is quite strong at $2.595 \AA$ and an angle of $76.3^{\circ}$ to the ring plane.

$(\mathrm{BrAn})_{2} \mathrm{POPh}(39)$ on the other hand crystallizes in the triclinic space group $P \overline{1}$. The single molecule contained in the asymmetric unit has two anthracene moieties which differ slightly in terms of deformation (see Table 3-22). Although still moderately distorted, the observed deformation of both anthracene moieties is stronger than of 38, which is caused by the higher steric demand of the second phosphorus bound anthracene moiety. The bond distances are very similar to those found in the structure of 38, but the angles around the phosphorus atom are slightly larger. Although the geometry can still be considered a distorted tetrahedron, the deviations from the ideal tetrahedral angle are smaller than in 38. Due to the fact that two anthracene moieties are contained in every molecule, the probability of $\pi-\pi$ overlap is clearly increased for 39 compared to 38 . Thus, two $\pi-\pi$ interactions are found in the packing plot of $\mathbf{3 9}$, one achieving an overlap of $\sim 35 \%$ at a distance of $3.35 \AA$, the other achieving an overlap of $\sim 20 \%$ at $3.45 \AA$ A. Because both $\operatorname{BrAnP}(\mathrm{OPh})_{2}(38)$ and $(\mathrm{BrAn})_{2} \mathrm{POPh}(39)$ were precursor molecules, their fluorescence properties were not investigated.

Keller et al. have stated in their work that intermediate products in which the phosphane carries an anthracenyl moiety and two phenoxy substituents (as the case in 38), are quite stable towards oxidation with aerial oxygen. Hence, Keller et al. handled their intermediate products without inert gas atmospheres and even extracted their crude products with water in a separating funnel. ${ }^{[72]}$ To determine whether selective oxidation was yet possible, $\mathrm{BrAnP}(\mathrm{OPh})_{2}(38)$ was oxidized in established manner with elemental sulfur in toluene (Scheme 3-16). 
<smiles>Brc1c2ccccc2c(P(Oc2ccccc2)Oc2ccccc2)c2ccccc12</smiles>

Scheme 3-17: Oxidation of $\mathbf{3 8}$ with elemental sulfur.

After a reaction time of $6 \mathrm{~h}$ at $110^{\circ} \mathrm{C}$ the solution was filtrated and concentrated. $\mathrm{BrAnPS}(\mathrm{OPh})_{2}(40)$ was crystallized from toluene and obtained as a yellow crystalline solid. The crystal structure of $\mathbf{4 0}$ was acquired by $\mathrm{X}$-ray diffraction.
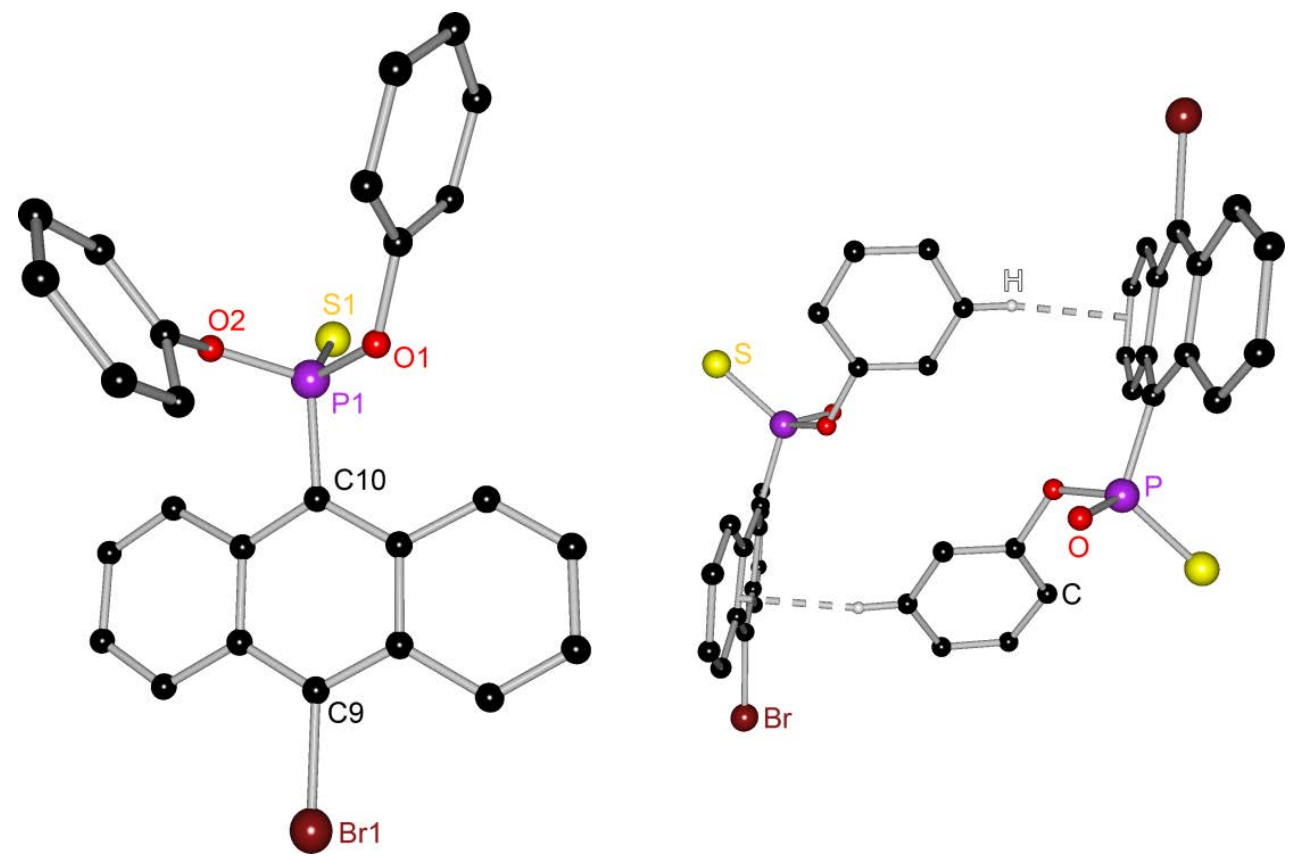

Figure 3-61: left: crystal structure of $\mathrm{BrAnPS}(\mathrm{OPh})_{2}$ (40), hydrogen atoms are omitted for clarity; right: aromatic $\mathrm{C}-\mathrm{H}^{\prime \prime} \pi$ interaction in the structure of 40 , hydrogen atoms and two phenyl groups are omitted for clarity.

BrAnPS $(\mathrm{OPh})_{2}(40)$ also crystallizes in the space group $P \overline{1}$. Compared to the unoxidized 38, the oxidation with elemental sulfur does not lead to drastic structural changes in terms of fluorophore deformation. The values of folding and twist deformation are similar for $\mathbf{3 8}$ and $\mathbf{4 0}$, therefore the steric pressure enforced by the phosphane substituent does not appear to change notably by oxidation. 
Table 3-23: space group and selected bond lengths $[\AA]$ and angles $\left[{ }^{\circ}\right]$ of $\mathbf{4 0 .}$

\begin{tabular}{ll} 
Space gr. & $P \overline{1}$ \\
P1-S1 & $1.9121(8)$ \\
P1-C10 & $1.812(2)$ \\
P1-O1 & $1.6021(17)$ \\
O1-P1-O2 & $103.47(9)$ \\
C10-P1-S1 & $115.84(8)$ \\
C10-P1-O1 & $107.72(10)$ \\
folding & 8.5 \\
twist & 5.4 \\
\hline
\end{tabular}

The geometry around the phosphorus atom is also of distorted tetrahedral character, although the angles are all closer to the ideal tetrahedral angle than in 38, which indicates the smaller spatial demand of the $\mathrm{P}=\mathrm{S}$ bond compared to the lone pair. Like in the structure of 38 , there is no $\pi-\pi$ overlap in 40 , but a very strong $\mathrm{C}-\mathrm{H}^{\cdots} \pi \mathrm{r}$ bond is found between a phenyl C$\mathrm{H}$ and the adjacent anthracene moiety (Figure 3-61, right). It measures only $2.537 \AA$, which is very short, and shows a nearly orthogonal orientation towards the $\pi$-system at $79.4^{\circ}$, making it one of the strongest interactions of this kind observed so far in solid state structures of phosphoryl anthracenes.

Next, functionalized substituents were introduced to the precursor molecule $\mathrm{BrAnP}(\mathrm{OPh})_{2}$ (38). Because the nucleophilic substitution mechanism proposed by Keller et al. only applies to lithiumorganics, the first step was to choose a functionalized residue which can be converted to a lithiumorganic reagent. The geometry of the resulting compound should be suitable for complexation of metal cations and additionally be flexible enough for a variety of metal ions with varying radii and coordination geometries. Furthermore, the lithiation of the functionalized substrate should preferably happen quantitatively to foreclose unnecessary contamination with side products. 2-Picoline unites all of the required criteria. ${ }^{[73]}$ By reaction with $n$-BuLi in THF it can be lithiated quantitatively and bis(picolyl)posphanes have previously been prepared and successfully employed in metal complexation. ${ }^{[74]}$

Two equivalents of 2-picoline were dissolved in THF and reacted with $n$-BuLi at $-15^{\circ} \mathrm{C}$ over the course of $30 \mathrm{~min}$. The resulting dark red solution was added to a diluted THF solution of $\mathrm{BrAnP}(\mathrm{OPh})_{2}$ (38) at $-78^{\circ} \mathrm{C}$ over 30 min (Scheme 3-17: A). After completed addition the reaction mixture was slowly warmed to ambient temperature and stirred overnight. After quenching of the reaction with $1 \mathrm{~mL}$ of degassed $\mathrm{H}_{2} \mathrm{O}$, the solvent was evaporated and the crude product was dissolved in diethyl ether and extracted with degassed $\mathrm{H}_{2} \mathrm{O}$. The organic layers were dried over $\mathrm{MgSO}_{4}$. Evaporation of the solvent yielded a dark red oily product. 


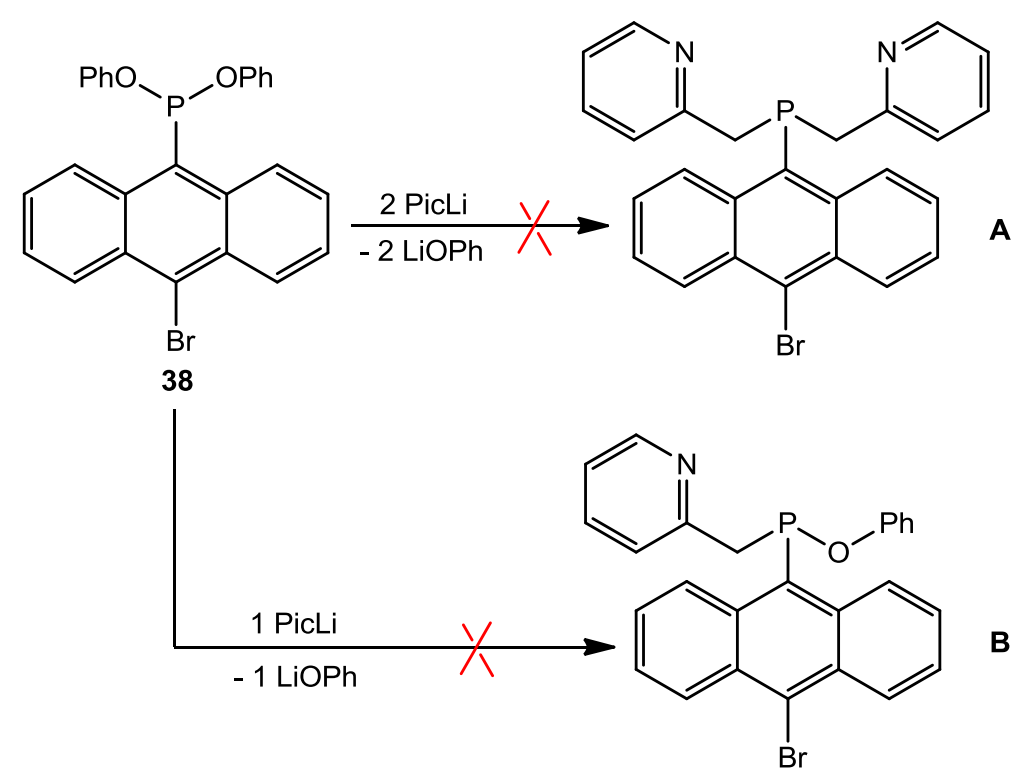

Scheme 3-18: Mono- and di-substitution of $\mathbf{3 8}$ with 2-picolyl lithium.

The ${ }^{31} \mathrm{P}-\mathrm{NMR}$ spectrum of the reaction product showed well over 10 peaks and also the ${ }^{1} \mathrm{H}-\mathrm{NMR}$ spectrum revealed a multitude of overlaying signals, indicating a vast mixture of reaction products. Crystallization of the desired compound from various solvents $\left(\mathrm{Et}_{2} \mathrm{O}\right.$, toluene, THF, DCM) at different temperatures for purification was unsuccessful. Although MS-El spectrometry indicated that the di-substituted derivative was contained in the product mixture, the NMR spectra showed that it was clearly not the main product. Therefore a new approach with step-wise substitution of the phenoxy groups with intermediate isolation and purification of the mono-substituted product was chosen (Scheme 3-17: B). Reaction conditions were chosen analogous to those described above, except only one equivalent of 2-picolyl lithium was added. After completion of the reaction the product was again dissolved in ether and extracted with degassed $\mathrm{H}_{2} \mathrm{O}$. Drying over $\mathrm{MgSO}_{4}$ and evaporation of the solvent afforded a wax-like amorphous solid. As for the attempted di-substitution before, the ${ }^{31} \mathrm{P}-\mathrm{NMR}$ spectrum showed a multitude of signals, although indicating to monosubstituted compound as the main product. The formation of the mono-substituted product was also confirmed by MS-El spectrometry, but again as part of an inseparable product mixture.

To reduce the exposure to aerial oxygen and moisture in the work-up process, and therewith eliminate a possible source of side product formation or decomposition, the synthesis was repeated as a "one-pot" reaction (Scheme 3-18). This option was also 
repeatedly underlined by Keller et al., who were able to synthesize the majority of their compounds via "one-pot" reactions. ${ }^{[72]}$

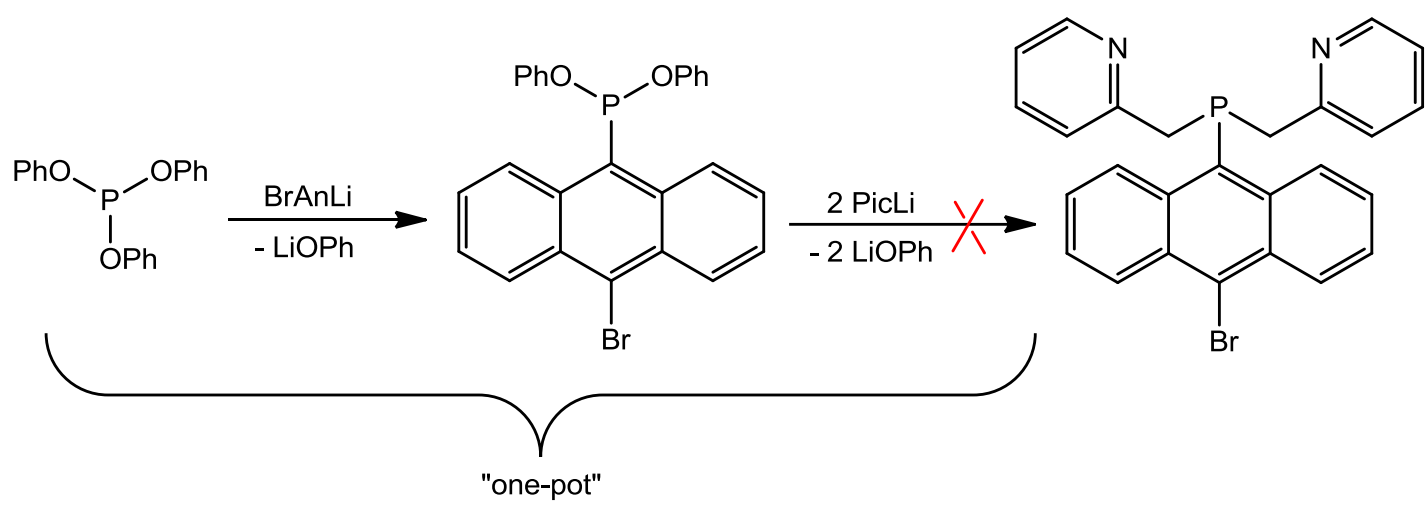

Scheme 3-19: "One-pot” synthesis of a di-functionalized product.

The obtained product from this reaction was very similar in colour and texture to the product from the first reaction of $\mathbf{3 8}$ with two equivalents of 2-picolyl lithium. Again the desired product was only traceable by mass-spectrometry.

The problems encountered can be explained by comparison of the reactions and products published by Keller et al. to the reactions described above. The majority of tertiary phosphanes synthesized via the mechanism presented above (especially those which were prepared at satisfactory yields) are tertiary aryl phosphanes. These are - as for example $\mathrm{PPh}_{3}$ - quite unreactive and stable towards oxidation. Compounds like these can be exposed to oxygen in processes like aqueous extraction. The stability of anthryl phosphonites like $\mathbf{3 8}$ is undeniable, but with increasing number of aliphatic substituents the sensitivity of the phosphane towards oxidation rapidly increases. This is most likely one source of the observed side products. Furthermore, the yields from reactions of aryl lithium compounds with $\mathrm{P}(\mathrm{OPh})_{3}$ which Keller et al. reported are by far higher than from reactions with aliphatic lithiumorganics (13-26\%). ${ }^{[72]}$ Hence the yields from reactions with 2-picolyl lithium can be expected to be quite low as well. The methods of purification described by Keller are also limited to crystallization. This is particularly problematic for non-solid products which cannot be crystallized and therewith not separated from accruing side products. Overall, this synthetic strategy is only conditionally suitable for preparation of functionalized phosphanyl anthracenes. 


\subsubsection{Synthesis via 9-Anthracenyldichlorophosphanes}

Because the synthetic strategy towards functionalized phosphanyl anthracenes employing triphenylphosphite did not yield the desired results, alternative synthetic routes needed to be exploited. Preferably quantitative conversions or effective purification options were of top priority to avoid contamination with side products. An synthetic option was found in 9-anthracenyldichlorophosphanes.

This compound class was first accessed by Schmutzler et al. in 1992 by reaction of 9 lithioanthracene with $\mathrm{PCl}_{3}$, which is a very straightforward approach. ${ }^{[75]}$ Although the isolation of 9-anthracenyldichlorophosphane was successful, the formation of disubstituted byproduct was described as problematic because the two products are particularly difficult to separate in addition to their pronounced air and moisture sensitivity. Hence, this synthetic strategy would contradict the premise of avoiding formation of secondary products. Furthermore Yang et al. prepared 9anthracenyldichlorophosphane by reaction of anthracenyl magnesium bromide with $\mathrm{PCl}_{3}$ but encountered similar problems. ${ }^{[76]}$ A different and far more selective route to aryldichlorophosphanes was introduced by Duff and Shaw in 1972, who were able to synthesize 1-naphtyldichlorophosphane from 1-naphtylbis(diethylamino)phosphane by cleavage of the $\mathrm{P}-\mathrm{N}$ bonds (Scheme 3-19). ${ }^{[77]}$

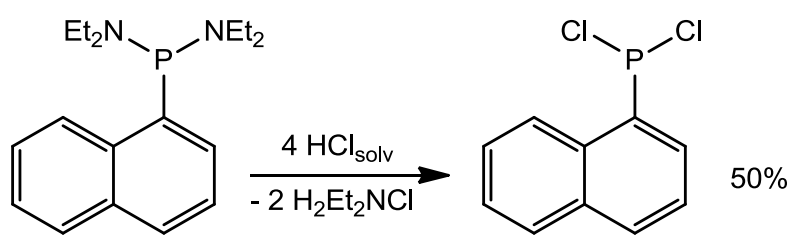

Scheme 3-20: Synthesis of 1-naphtyldichlorophosphane by Duff and Shaw. ${ }^{[77]}$

The reduced stability of aminophosphanes compared to alkylphosphanes has repeatedly become manifest in the course of this thesis and this reaction pathway takes advantage of this phenomenon. By addition of an ethereal solution of $\mathrm{HCl}$ to 1naphtylbis(diethylamino)phosphane, the amino groups were substituted under formation of diethylammonium chloride and the desired dichlorophosphane. The poor yield of $50 \%$ was later improved by Schmidbaur et al. to $83 \%$ using gaseous $\mathrm{HCl}^{[78]}$ This synthetic route was first applied to the anthracene moiety in a similar reaction by the workgroup around Tokitoh, who were able to prepare 9- 
anthracenyldichlorophosphane in high purity. ${ }^{[79]}$ This procedure was adapted and again slightly refined by Schwab. ${ }^{[59 b]}$

Although 9-anthracenyldichlorophosphane has been previously prepared via different reaction pathways, the outstanding synthetic potential in synthesis of functionalized phosphanylanthracenes was not recognized or not yet exploited.<smiles>Brc1c2ccccc2c(Br)c2ccccc12</smiles>

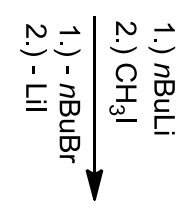<smiles>Cc1c2ccccc2c(Br)c2ccccc12</smiles>

1.) $n \mathrm{BuLi}$ $\frac{\text { 2.) } \mathrm{CIP}\left(\mathrm{NEt}_{2}\right)_{2}}{\text { 1.) }-n \mathrm{BuBr}}$ 2.) $-\mathrm{LiCl}$

1.) $n \mathrm{BuLi}$ 2.) $\mathrm{CIP}(\mathrm{NE}$ 2.) $-\mathrm{LiCl}$<smiles>CCNP(c1c2ccccc2c(C)c2cc(CCN(CC)CC)ccc12)N(CC)CC</smiles>

Scheme 3-21: Synthesis of $\mathrm{BrAnPCl}_{2}$ (41) and $\mathrm{MeAnPCl}_{2}$ (42).<smiles>ClP(Cl)c1c2ccccc2c(Br)c2ccccc12</smiles>

41<smiles>Cc1c2ccccc2c(P(Cl)Cl)c2ccccc12</smiles>

42

Although the syntheses of $\mathbf{4 1}$ and $\mathbf{4 2}$ were mainly carried out according to the procedures described by Tokitoh and Schwab, further optimizations were made to improve yields and purities of the resulting products. The starting materials $-9,10-$ dibromoanthracene and 9-bromo-10-methylanthracene - were both reacted with one equivalent of $n$-BuLi in diethyl ether at $-15^{\circ} \mathrm{C}$ and subsequently reacted with one equivalent of bis(diethylamino)chlorophosphane. The solvent was evaporated from both reaction mixtures and the crude products were dissolved in DCM and filtrated. Evaporation of the solvent afforded 9-bromo-10-bis(diethylamino)phosphanylanthracene and 9-bis(diethylamino)phosphanyl-10-methylanthracene, respectively, as dark red highly viscous oils (Scheme 3-22). Both products were dissolved in hexane without further purification. The solutions were cooled to $-15^{\circ} \mathrm{C}$ and $\mathrm{HCl}$ gas was discharged into the solutions over $5 \mathrm{~min}$ followed by $15 \mathrm{~min}$ of stirring at $-15^{\circ} \mathrm{C}$. This procedure was repeated three times. Afterwards the suspensions of insoluble ammonium salt and 9-anthryldichlorophosphanes were stirred for $2 \mathrm{~h}$ at room 
temperature. Then the solvent was evaporated. The obtained solid mixtures of product and ammonium salt were extracted with toluene using a Soxhlet apparatus under inert gas atmosphere. Evaporation of the solvent finally afforded 9-bromo-10dichlorophosphanylanthracene $\left(\mathrm{BrAnPCl}_{2} \quad(\mathbf{4 1})\right)$ and 9-dichlorophosphanyl-10methylanthracene $\left(\mathrm{MeANPCl}_{2}(\mathbf{4 2})\right)$ as yellow crystalline solids. The utilization of a Soxhlet apparatus clearly improves the yield of the reaction compared to the method used by Schwab who merely rinsed the mixture of ammonium salt and anthrylphosphane three times with toluene. The continuous extraction of the crude product ensures nearly quantitative yields of the anthryldichlorophosphanes. Both $\mathbf{4 1}$ and $\mathbf{4 2}$ were re-crystallized from hexane; the obtained crystals were suitable for single crystal x-ray diffraction experiments.

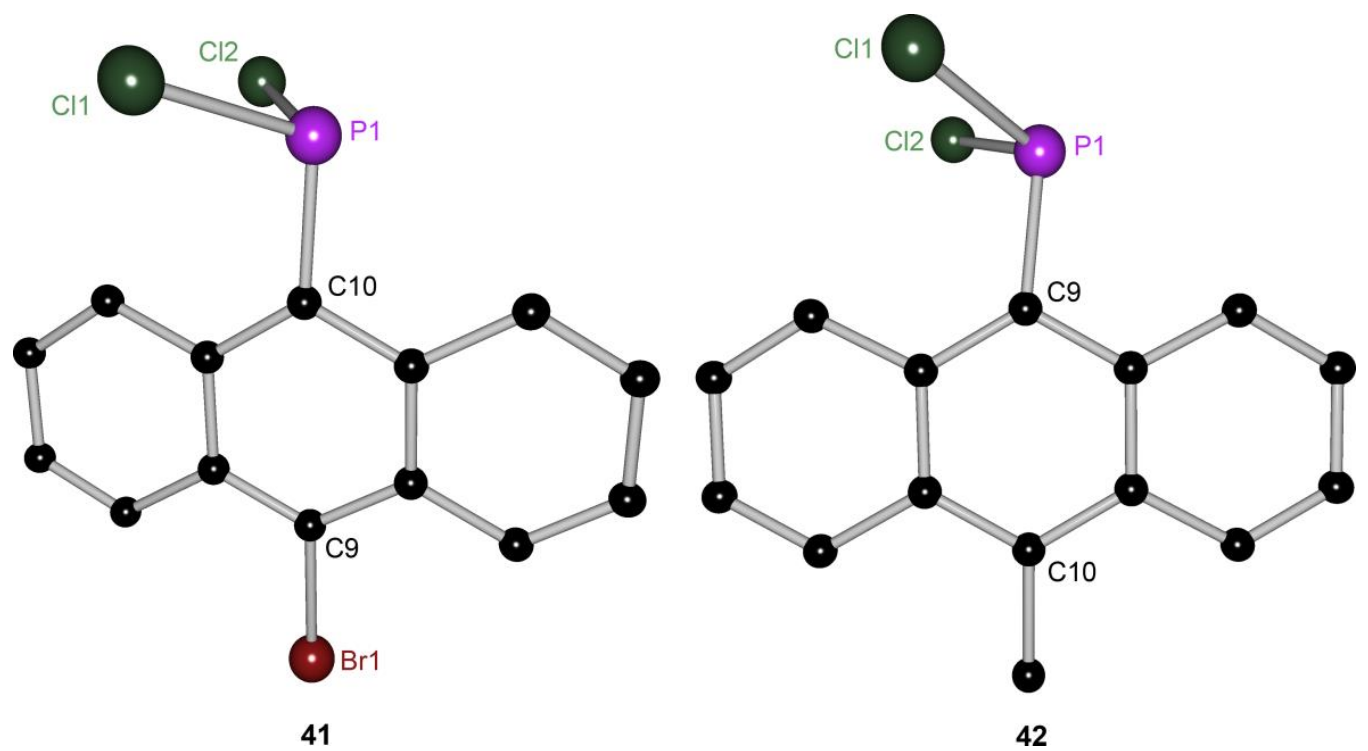

Figure 3-62: crystal structures of $\mathrm{BrAnPCl}_{2}$ (41) (left) and $\mathrm{MeAnPCl}_{2}$ (42) (right).

Despite their fairly simple structures, both 41 and $\mathbf{4 2}$ crystallize in the low symmetry space group $P \overline{1}$. The obviously close structural relation between 41 and $\mathbf{4 2}$ is reflected by the observed bond distances and angles which only differ minimally. Astonishingly the lone pair of the phosphorus atom is not located in the anthracene plane in either of the compounds. The torsion angles of the lone pairs to the anthracene plane are the only angles that differ significantly in the structures of $\mathbf{4 1}$ and $\mathbf{4 2 .}$ 
Table 3-24: selected bond lengths $\left[\AA ̊ \Omega\right.$ and angles $\left[{ }^{\circ}\right]$ of $\mathbf{4 1}$ and $\mathbf{4 2 .}$

\begin{tabular}{ll|ll}
\hline \multicolumn{2}{c|}{$\mathrm{BrAnPCl}_{2}(41)$} & \multicolumn{2}{c}{$\mathrm{MeAnPCl}_{2}(42)$} \\
$\mathrm{P} 1-\mathrm{C} 10$ & $1.830(3)$ & $\mathrm{P} 1-\mathrm{C} 9$ & $1.8161(14)$ \\
$\mathrm{P} 1-\mathrm{Cl} 1$ & $2.0723(10)$ & $\mathrm{P} 1-\mathrm{Cl} 1$ & $2.0760(5)$ \\
$\mathrm{Cl1}-\mathrm{P} 1-\mathrm{Cl} 2$ & $99.95(4)$ & $\mathrm{Cl} 1-\mathrm{P} 1-\mathrm{Cl} 2$ & $100.86(2)$ \\
$\mathrm{C} 10-\mathrm{P} 1-\mathrm{Cl} 1$ & $103.06(9)$ & $\mathrm{C} 9-\mathrm{P} 1-\mathrm{Cl} 1$ & $102.61(5)$ \\
C10a-C10-P1-LP & 12.6 & $\mathrm{C} 9 \mathrm{a}-\mathrm{C} 9-\mathrm{P} 1-\mathrm{LP}$ & 4.4 \\
Folding / Twist & $2.4 / 3.0$ & Folding / Twist & $1.8 / 0.5$ \\
\hline
\end{tabular}

The torsion angle measures $12.6^{\circ}$ in the structure $\mathbf{4 1}$ and only $4.4^{\circ}$ in the structure of 42, which indicates that it is at least nearly located in the anthracene plane for the latter. As previously observed, the torsion angle also affects the deformation of the anthracene moiety. Though distortion is comparatively weak in both molecules, the larger torsion angle of the lone pair in $\mathbf{4 1}$ also induces a stronger deformation of the fluorophore (Table 3-24).
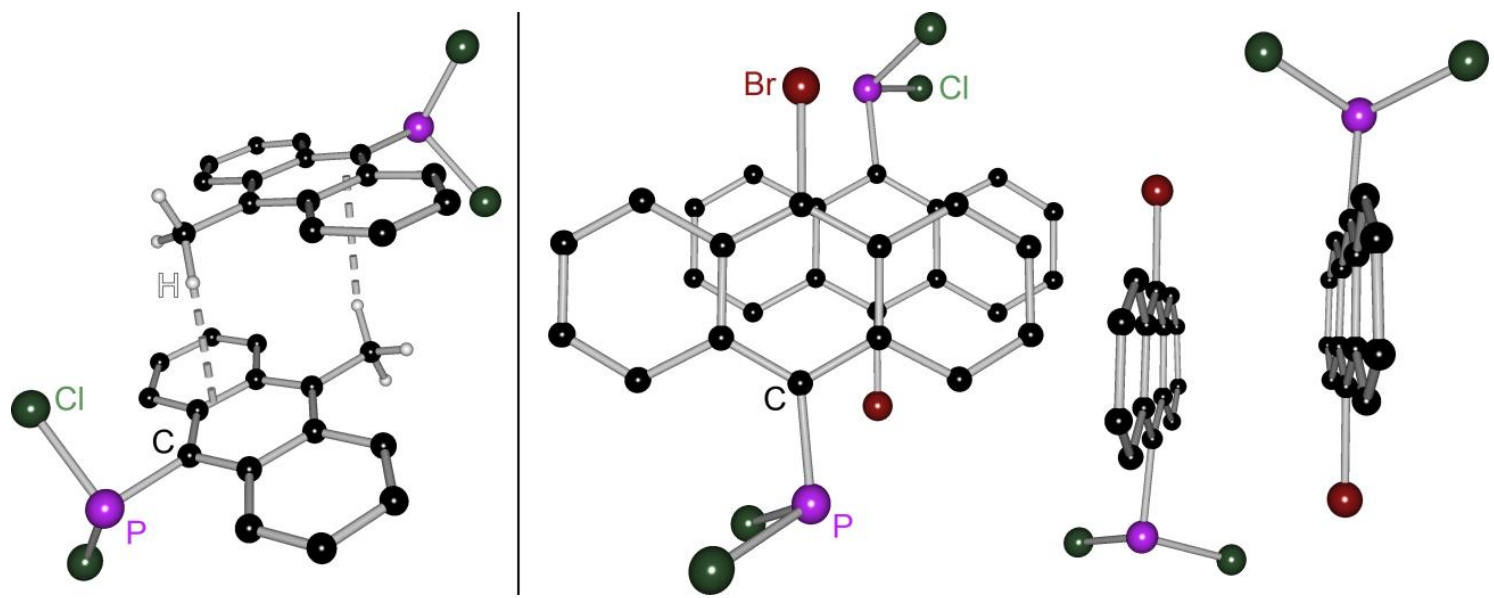

Figure 3-63: Intermolecular interactions in the structures of $\mathbf{4 2}$ (left) and $\mathbf{4 1}$ (right). On the right the top view and side view of the $\pi-\pi$ interaction are depicted. Hydrogen atoms are omitted for clarity.

The intermolecular interactions within the packing motifs also differ remarkably in spite of the similarities between both compounds. The typical "head-to-tail" arrangement is found in both cases but while 41 exhibits strong $\pi-\pi$ overlap of $\sim 65 \%$ at a distance of $3.44 \AA$, there is no $\pi-\pi$ interaction present in the structure of $\mathbf{4 2}$. Vice versa, a $\mathrm{C}-\mathrm{H}^{\cdots} \pi \mathrm{r}$ interaction is found in the structure of $42\left(2.727 \AA / 42.2^{\circ}\right)$, which is absent in the structure of $\mathbf{4 1}$ (Figure 3-63). 
The obtained 9-anthryldichlorophosphanes are highly reactive and offer a multitude of possibilities for substitution of the phosphorus bound chlorine atoms. This discloses a corresponding number of options and synthetic strategies for introduction of functionalized substituents to the phosphorus atom, generating chelating phosphanyl anthracenes. Scheme 3-22 shows the possible reaction types and the corresponding substance classes produced.

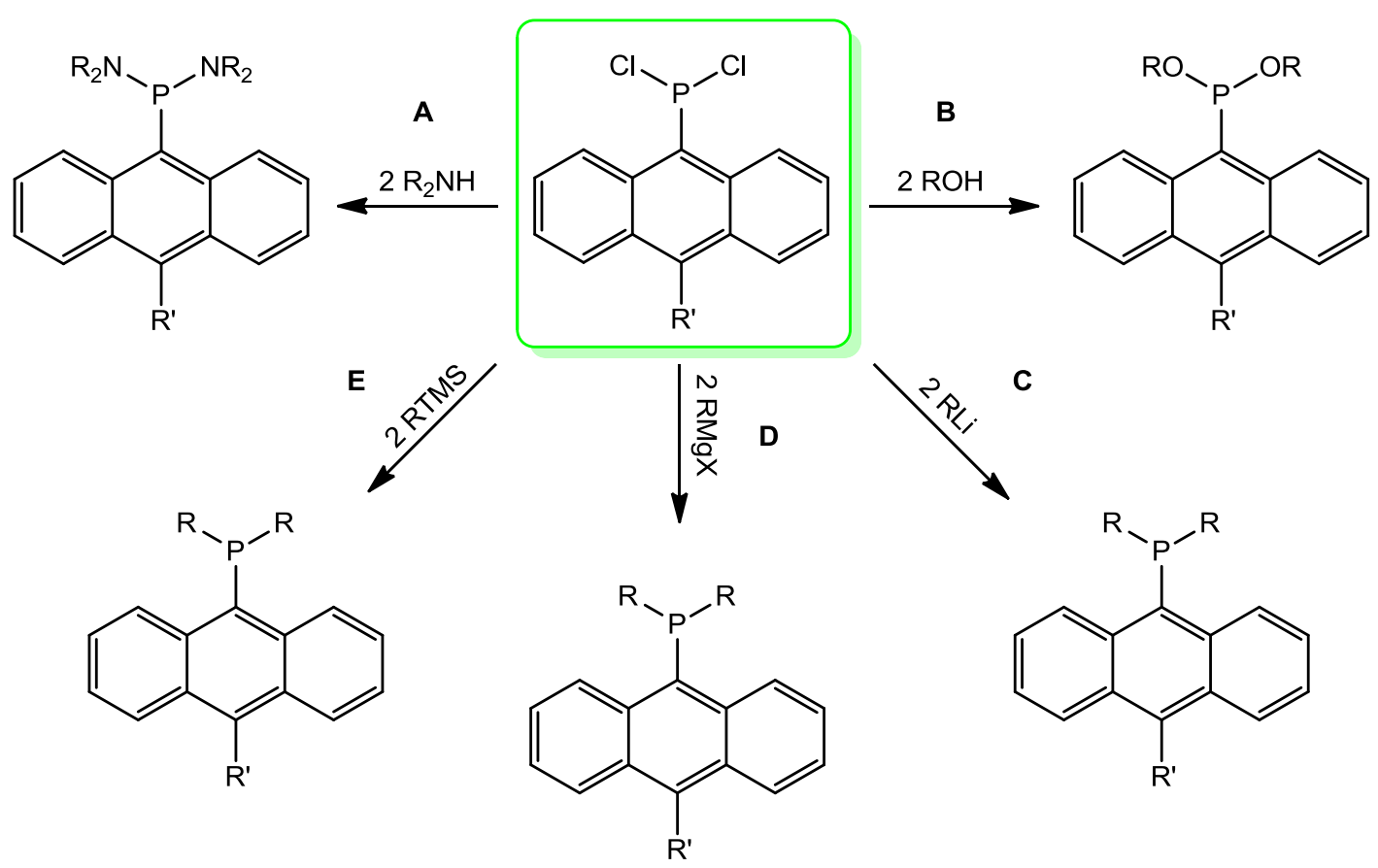

Scheme 3-23: Possible conversions of 9-anthryldiclorophosphanes.

Reaction type $\mathbf{A}$ is the reversal of the cleavage employed in the final step of synthesis of $\mathbf{4 1}$ and $\mathbf{4 2}$. The reaction of secondary amines with chlorophosphanes has been shown to be effective and reliable e.g. in the synthesis of bis(dialkylamino)chlorophosphanes which were utilized for preparation of the compounds described in 3.3. ${ }^{[68]}$ By utilization of functionalized secondary amines, donating side-arms can be introduced, creating chelating ligands. Reaction type $\mathbf{B}$ affords 9-anthrylphosphonites by reaction with alcohols. Analogue to reaction type $\mathbf{A}$, usage of functionalized alcohols leads to chelating phosphanylanthracenes. In both reaction types $\mathbf{A}$ and $\mathbf{B}, \mathrm{HCl}$ is eliminated which needs to be removed from the reaction. To achieve this, a tertiary amine like $\mathrm{NEt}_{3}$ must be added to the reaction mixture which forms an inert ammonium salt with the generated $\mathrm{HCl}$ without reacting 
with the dichlorophosphane. The reaction of lithiumorganics with chlorophosphanes (reaction type $\mathbf{C}$ ) under elimination of lithium chloride is also a well-established procedure. Although in this type of reaction selectivity has often been described as a problem, the sighted di-substitution is unproblematic in this aspect. Even excess amounts of lithiumorganics could be used and unreacted residues could be quenched after completion of the reaction. Via this reaction pathway Schwab was successful in preparing di(2-pyridyl)phosphanylanthracene (Scheme 3-23). ${ }^{[59 b]}$ Although this compound bears functionalized substituents at the phosphorus atom, its structural and geometrical properties prevent metal coordination as a tridentate donor and rather promote metal coordination in Janus-Head type coordination motifs. ${ }^{\left[{ }^{80]}\right.}$<smiles>ClP(Cl)c1c2ccccc2cc2ccccc12</smiles>

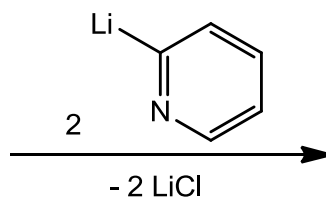<smiles>c1ccc(P(c2ccccn2)c2c3ccccc3cc3ccccc23)nc1</smiles>

Scheme 3-24: preparation of di(2-pyridyl)phosphanylanthracene by Schwab. ${ }^{[59 b]}$

Analogue to lithiumorganics, Grignard reagents can also be reacted with chlorophosphanes under elimination of magnesium halides (reaction type D). Finally, alkyl or aryl trimethylsilanes can be reacted with dichlorophosphanes to introduce functionalized substituents (reaction type E). Braunstein et al. used this method to introduce 2-picolyl substituents to dichlorophenylphosphane ${ }^{[81]}$ and Whitesides and Moore were able to insert benzothiazolyl substituents to $\mathrm{PCl}_{3}$ via this reaction pathway. ${ }^{[82]}$ The trimethylchlorosilane eliminated in the course of the reaction can simply be removed under reduced pressure.

Reaction type A was not used, but several reactions according to path B were conducted. The reactions were carried out following a standard procedure. The respective 9-anthrayldichlorophosphane was dissolved in diethyl ether and cooled to $-15^{\circ} \mathrm{C}$, then 5 equivalents of $\mathrm{dry} \mathrm{NEt}_{3}$ were added. After $5 \mathrm{~min}$ two equivaltents of the respective alcohol dissolved in diethyl ether or THF were added over $30 \mathrm{~min}$ at $-15^{\circ} \mathrm{C}$. After the addition was completed, the solution was warmed to ambient temperature and stirred for $3 \mathrm{~h}$. The resulting suspension was concentrated and the insoluble diethylammonium chloride was removed by filtration. Evaporation of the solvent afforded the phosphonites as yellow oily products. 


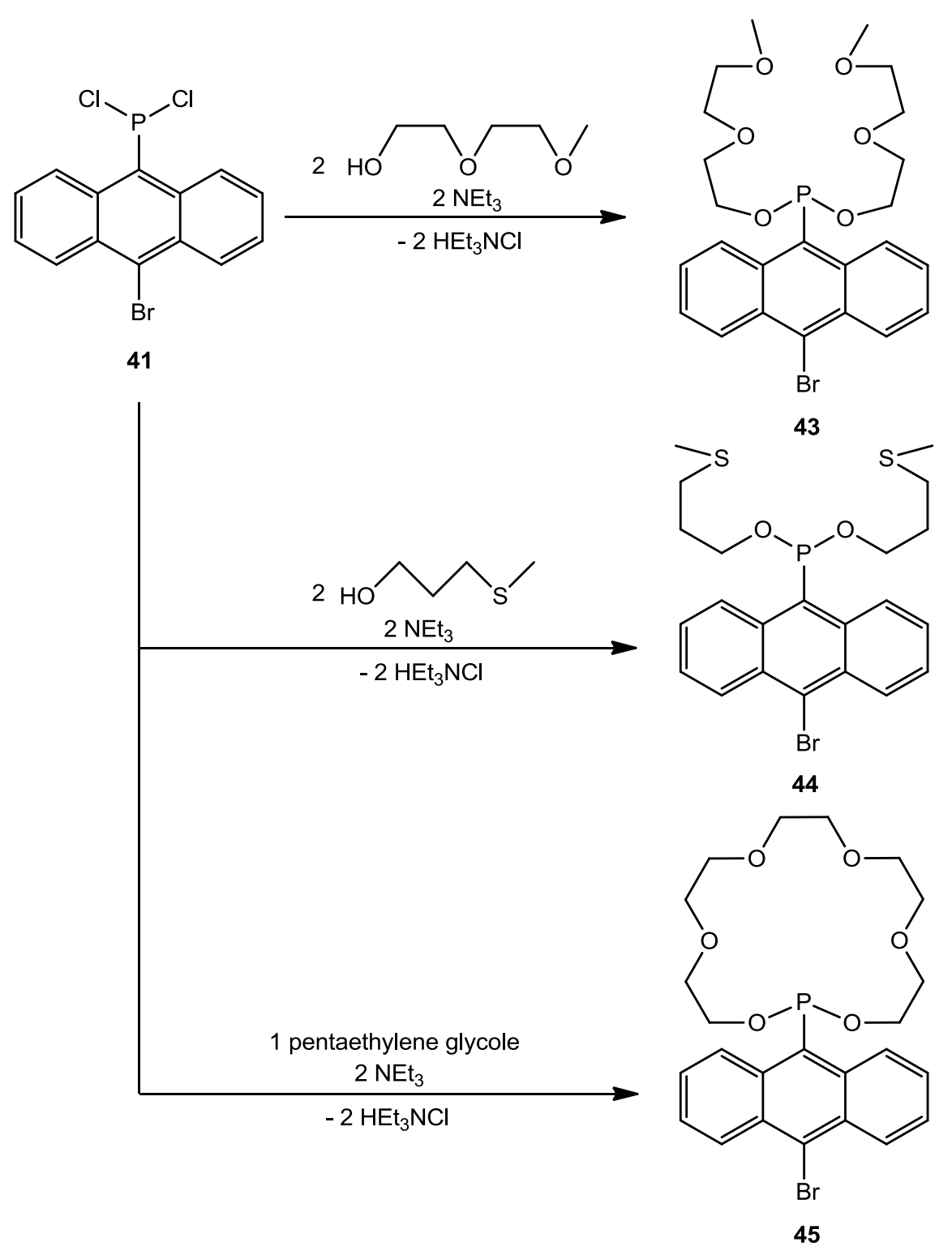

Scheme 3-25: Reactions of $\mathbf{4 1}$ with alcohols.

Though the presented mechanism was verified, some problems occurred in the syntheses of 43-45. Only $\mathbf{4 3}$ was obtained in high purity, while $\mathbf{4 4}$ and $\mathbf{4 5}$ contained byproducts. Although for the synthesis of $\mathbf{4 5}$ a very high dilution of the reactants was applied to promote the intramolecular formation of the cycle and to avoid intermolecular reactions which would generate polymer products, this side reaction could apparently not be fully suppressed. Furthermore $\mathbf{4 4}$ and $\mathbf{4 5}$ contain oxidation products, which most likely do not originate from aerial oxygen, but rather from moisture contained in the alcohols which were reacted with the 9-anthryldichlorophosphane. Because these could not be sufficiently dried prior to the reaction, reaction of the contained water with the highly reactive chlorophosphanes leads to the 
observed contaminations. Also the $\mathrm{HCl}$ which is formed during the reaction can promote the formation of side products, which was reported by Braunstein et al. who encountered similar problems in reactions of alcohols with dichlorophosphanes. Again purification was a major issue due to the oily texture of the products which hindered crystallization of the desired products. In the case of $\mathbf{4 4}$, the particularly unpleasant odour further complicated the handling and purification of the compound. Nevertheless the reaction of dichlorophosphanylanthracenes with alcohols is a potent method for introduction of functionalized substituents. In the future, side reactions could be further supressed by deprotonation of the alcohols prior to reaction with the chlorophosphanes, which has also been successfully actualized. ${ }^{[81]}$<smiles>[R]c1c2ccccc2c(P(Cl)Cl)c2ccccc12</smiles>

$\mathrm{R}=\mathrm{Br}, \mathrm{Me}$

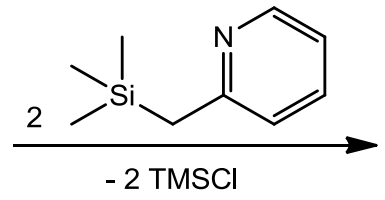<smiles>[R]c1c2ccccc2c(P(Cc2ccccn2)Cc2ccccn2)c2ccccc12</smiles>

46: $\mathrm{R}=\mathrm{Br}$ 47: $\mathrm{R}=\mathrm{Me}$

Scheme 3-26: Substitution of dichlorophosphanylanthracenes with trimethylsilylpicoline.

Furthermore, functionalized substituents were inserted by reaction of the dichlorophosphanes with 2-(trimethylsilyl)picoline under elimination of trimethylchlorosilane according to reaction type E. 2-(Trimethylsilyl)picoline was prepared according to literature procedures ${ }^{[83]}$ and dissolved in a mixture of THF and ether. A solution of the respective phosphane $\mathbf{4 1}$ or $\mathbf{4 2}$ in THF was added at $-78^{\circ} \mathrm{C}$. The reactions were stirred overnight and removal of the solvent and drying in vacuo at $60^{\circ} \mathrm{C}$ yielded crude products of $\mathrm{BrAnP}(\mathrm{Pic})_{2}(46)$ and $\mathrm{MeAnP}(\mathrm{Pic})_{2}(47)$ as red oils (Scheme 3-25). 46 and 47 were obtained as the main products, confirmed by the characteristic shifts in the ${ }^{31} \mathrm{P}$ NMR spectra of both compounds as well as the distinctive coupling pattern of the methylene protons in the ${ }^{1} \mathrm{H}$ NMR spectrum. While Braunstein and co-workers achieved a yield of $68 \%$ and report no side products and no further purification of their bis(2-picolyl)phenylphosphane which was prepared using the identical procedure, the crude products of both $\mathbf{4 6}$ and $\mathbf{4 7}$ contained noteworthy impurities. This may be caused by the stronger steric demand of the anthryl moiety compared to the phenyl moiety, which may have led to incomplete turnover. The 
reactions were repeated identically, but after stirring overnight the reaction mixtures were refluxed for $30 \mathrm{~min}$, which did not affect the ratios of products and side products. Purification was again problematic due to the oily texture of the products which countervails crystallization.

Another possibility of accessing alternative synthetic strategies is reduction of 9anthryldichlorophosphanes. The reduction of dichlorophosphanes with lithiumaluminiumhydride is a widespread synthetic procedure and has even been applied to anthrylphosphanes by Kubiak et al. ${ }^{[76]}$

$\mathrm{MeAnPCl}_{2}$ (42) was suspended in diethyl ether and added to a suspension of two equivalents of lithium aluminium hydride in diethyl ether over the course of $3 \mathrm{~h}$ at $-78^{\circ} \mathrm{C}$. The resulting reaction mixture was slowly warmed to room temperature and then stirred for $2 \mathrm{~h}$. After heating to $35^{\circ} \mathrm{C}$ for $30 \mathrm{~min}$, degassed $\mathrm{NH}_{4} \mathrm{Cl}$ solution was added, the organic phase was removed and dried over $\mathrm{MgSO}_{4}$. Evaporation of the solvent afforded $\mathrm{MeAnPH}_{2}(\mathbf{4 8})$ as a yellow powder.<smiles>Cc1c2ccccc2c(P(Cl)Cl)c2ccccc12</smiles>

Scheme 3-27: Synthesis of $\mathrm{MeANPH}_{2}$ (48).

The quantitative reduction was monitored by NMR spectroscopy. The most prominent feature of the ${ }^{1} \mathrm{H}$ NMR spectrum is the doublet at $\delta=4.36 \mathrm{ppm}$. It is produced by the two phosphorus bound hydrogen atoms and the ${ }^{1} J_{\mathrm{P}-\mathrm{H}}$-coupling generates a very large coupling constant of $203.9 \mathrm{~Hz}$ (Figure 3-64). 


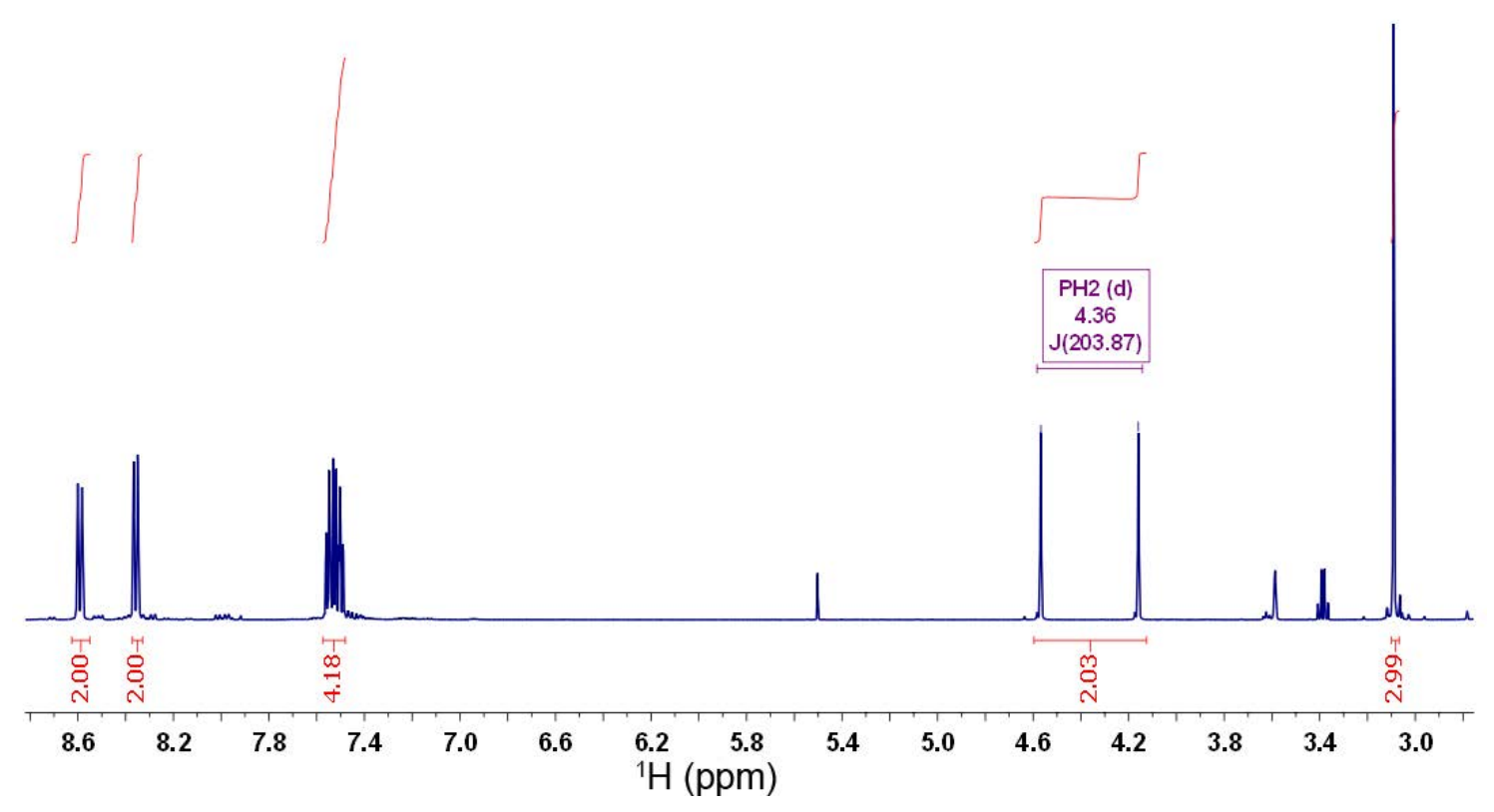

Figure 3-64: ${ }^{1} \mathrm{H}$ NMR spectrum of $\mathrm{MeAnPH}_{2}$ (48).

The ${ }^{31} \mathrm{P}\left\{{ }^{1} \mathrm{H}\right\}$ NMR spectrum shows a single sharp peak at $\delta=-161.0 \mathrm{ppm}$ which underlines the purity of the compound and the quantitative reduction of the dichlorophosphane (Figure 3-65, left). ${ }^{31} \mathrm{P}$ NMR experiments without proton decoupling produce a triplet signal resulting from the coupling of hydrogen atoms with the phosphorus atom, which further proves the presence of two hydrogen atoms at the phosphorus atom (Figure 3-65, right).
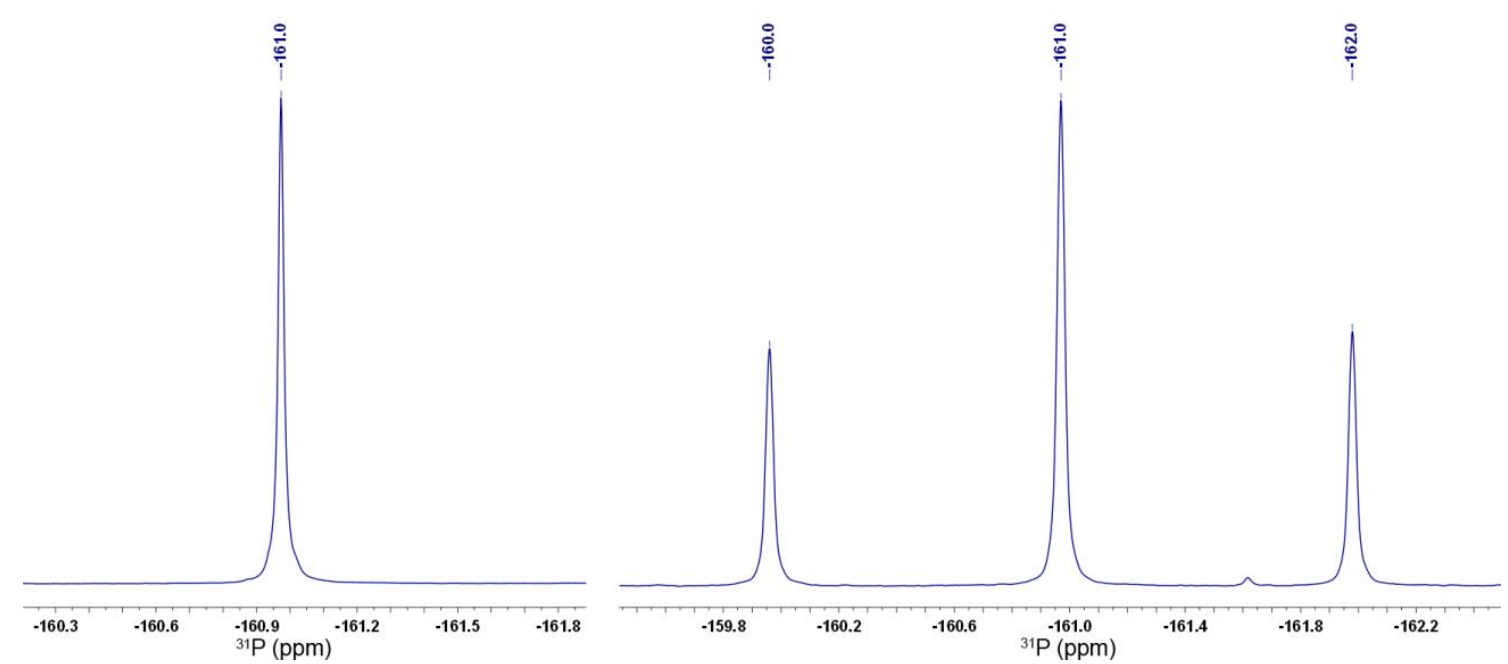

Figure 3-65: left: ${ }^{31} \mathrm{P}\left\{{ }^{1} \mathrm{H}\right\}$ NMR spectrum of 48 ; right: ${ }^{31} \mathrm{P}$ NMR spectrum of $4 \mathbf{8}$. 
Despite crystallization attempts from a variety of solvents, no single crystals of

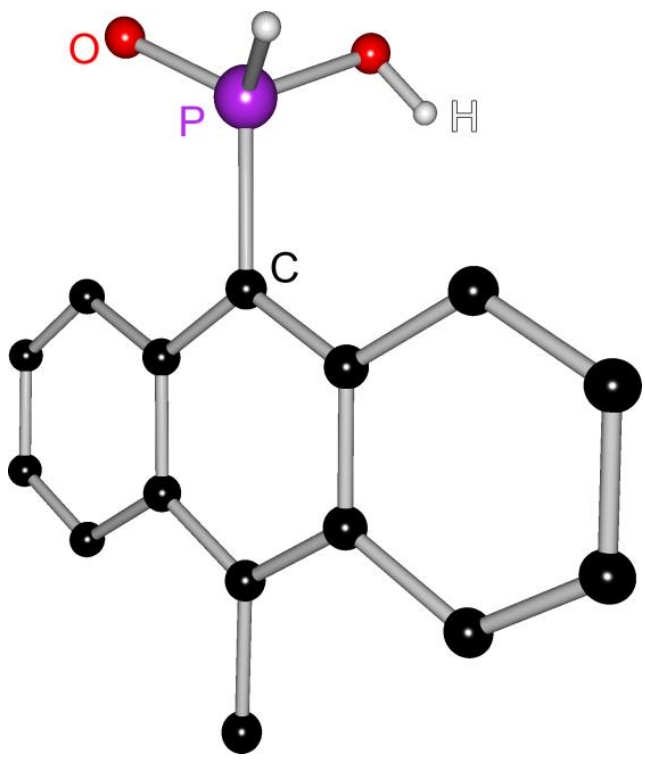

Figure 3-1: Crystal structure of 48 a. $\mathrm{MeAnPH}_{2}$ (48) could be obtained. The compound shows a strong tendency of forming powder precipitates when surpassing the solubility limit. The high sensitivity of $\mathbf{4 8}$ towards oxidation became apparent when the oxidation product 48a was crystallized from a flask which had been briefly opened when taking a sample of precipitated solid material under argon counter flow. Two days after taking this sample, a notable amount of crystalline material had formed, which turned out to be the oxidation product 48a (Figure 3-66).

By reduction of 9-anthryldichlorophosphane the reactivity at the phosphorus atom is inverted, almost in the manner of an umpolung. While the phosphorus atom is highly electrophilic in dichloroposphanes, the reduction converts it to a nucleophile, which can be largely increased in strength by deprotonation. Deprotonated phosphanes have been shown to react with a variety of electrophiles such as alkyl halides or aldehydes affording acceptable yields. In 1999 Kubiak et al. published a reaction of 9anthrylphosphane with 1,2-dichloroethane which yielded 9-anthraylposphirane (Scheme 3-27). ${ }^{[76]}$

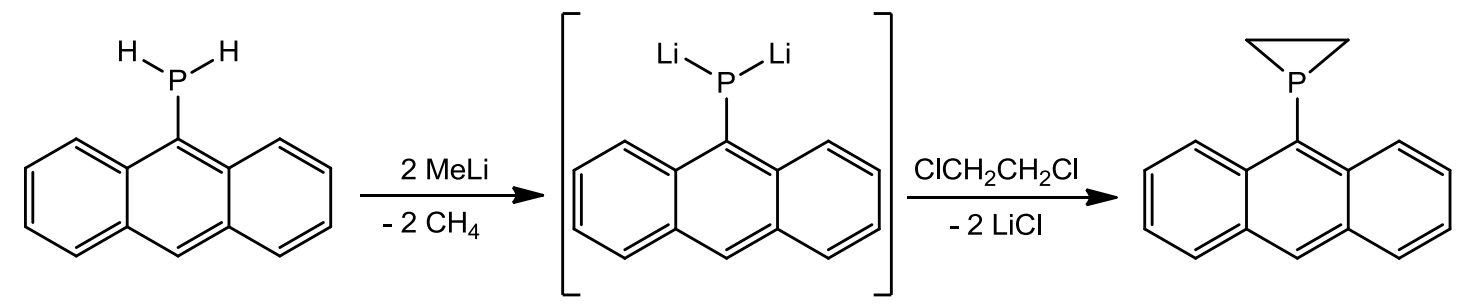
Scheme 3-28: 9-anthrylphosphirane synthesis by Kubiak et al. ${ }^{[76]}$

Kubiak and co-workers reacted their phosphane with two equivalents of methyl lithium, followed by addition of 1,2-dichloroethane. They postulated a di-lithiated intermediate to be formed upon addition of two equivalents of methyl lithium. Although the yield of $77 \%$ of the desired phosphirane suggests that the proposed mechanism is valid, there is no other report of a di-lithiated or double deprotonated 
phosphane documented in literature and the stability of such an arrangement appears more than questionable. This was confirmed by reaction of $\mathrm{MeAnPH}_{2}$ (48) with methyl lithium and bromophenetole as an electrophile. The colour changes of the reaction mixture from yellow over purple to green upon addition of two equivalents of methyl lithium which were reported by Kubiak et al. were also observed. However reaction with two equivalents of bromophenetole yielded a vast mixture of products which contained only traces of the desired di-substituted product (Scheme 3-28).

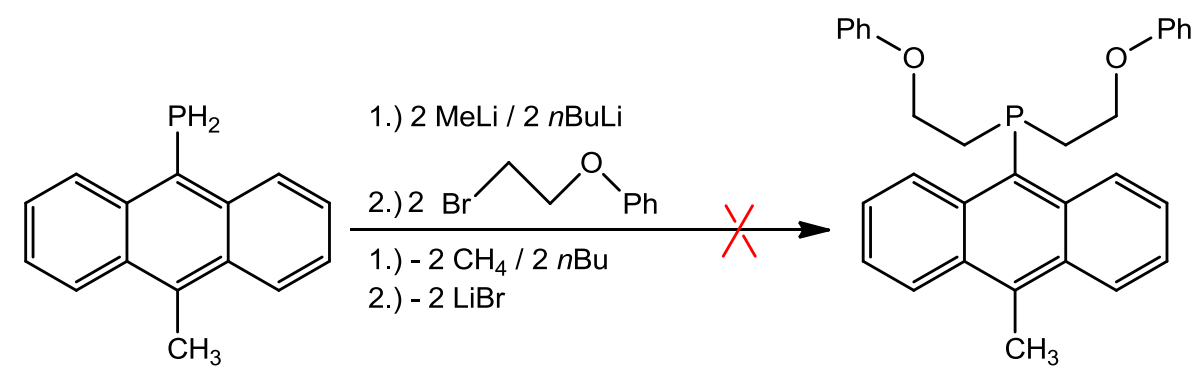

Scheme 3-29: Attempted di-substitution of $\mathbf{4 8}$ with bromophenetole.

The reaction was repeated and reaction temperatures were varied which did not notably affect the composition of the product. Also $n$-BuLi was tried as a deprotonation agent, which did not improve the outcome of the reaction. All products contained coupling products of the electrophile and the deprotonation agent, which indicates that after addition of two equivalents of MeLi or $n$-BuLi to the phosphane there are still noteworthy amounts of deprotonation agent present in the reaction mixture which then react with the electrophile in a nucleophilic substitution. Furthermore, P-P coupled products were found. These results suggest that the dilithiation of the phosphane postulated by Kubiak et al. does not occur quantitatively and that a step-wise deprotonation of the phosphane is more likely. The fact that Kubiak and co-workers still obtained a yield of nearly $80 \%$ of their phosphirane may be assigned to the close proximity of nucleophile and electrophile in the intramolecular formation of the phosphirane.

Because bromophenetole is a fairly poor electrophile, its low reactivity towards the deprotonated phosphane was spotted as a possible reason for the unsatisfying results. Therefore an electrophile with enhanced reactivity was chosen to verify the feasibility of this reaction. The benzylic position of the halogen atom in benzyl bromide is activated and therewith beneficial for substitution reactions. Therefore the reaction was repeated and benzyl bromide was used as the electrophile. Again a product 
mixture containing the coupling product of MeLi and benzyl bromide was obtained. One more attempt was made, but this time the substitution was performed step-wise, which means that two consecutive single-deprotonations of the phosphane were conducted, each followed by reaction with one equivalent of benzyl bromide. The reaction was done in a "one-pot" manner, with no work-up or purifications between the reaction steps (Scheme 3-29).<smiles>Cc1c2ccccc2c(P)c2ccccc12</smiles>

1.) MeLi $\underset{\text { 2.) } \mathrm{BrCH}_{2} \mathrm{Ph}}{-\mathrm{CH}_{4}}$ 2.) $-\mathrm{LiBr}$<smiles>Cc1c2ccccc2c(PCc2ccccc2)c2ccccc12</smiles>

1.) $\mathrm{MeLi}$ $\underset{\text { 2.) }) \mathrm{BrCH}_{2} \mathrm{Ph}}{\longrightarrow}$ 2.) $-\mathrm{LiBr}$<smiles>Cc1c2ccccc2c(P(Cc2ccccc2)Cc2ccccc2)c2ccccc12</smiles>

49

Scheme 3-30: Synthesis of $\mathrm{MeAnP}\left(\mathrm{CH}_{2} \mathrm{Ph}\right)_{2}$ (49).

After completion of the reaction, the solvent was removed under reduced pressure, the crude product was re-dissolved in DCM and the lithium bromide was removed by filtration. Evaporation of the solvent afforded $\operatorname{MeAnP}\left(\mathrm{CH}_{2} \mathrm{Ph}\right)_{2}(49)$ as a yellow solid. Though conversion was also not quantitative, $\mathbf{4 9}$ was clearly the primary product with only minor byproduct contamination. $\mathbf{4 9}$ is a solid, hence it was possible to crystallize the product for purification. Because $\mathbf{4 9}$ contains no donor functions and was rather prepared as a proof of principle, the step-wise reaction was also attempted with bromophenetole. This reaction again afforded an inseparable product mixture. These findings show that a quantitative di-lithiation of the phosphane is not possible and that on the one hand, a step-wise deprotonation and reaction of the phosphane is the preferential method for di-substitution of phosphanes. On the other hand they show that not every electrophile is suitable for achieving high yields of the desired disubstituted product.

The crystals obtained of $\mathrm{MeAnP}\left(\mathrm{CH}_{2} \mathrm{Ph}\right)_{2}$ (49) were subjected to single crystal x-ray diffraction experiments. 


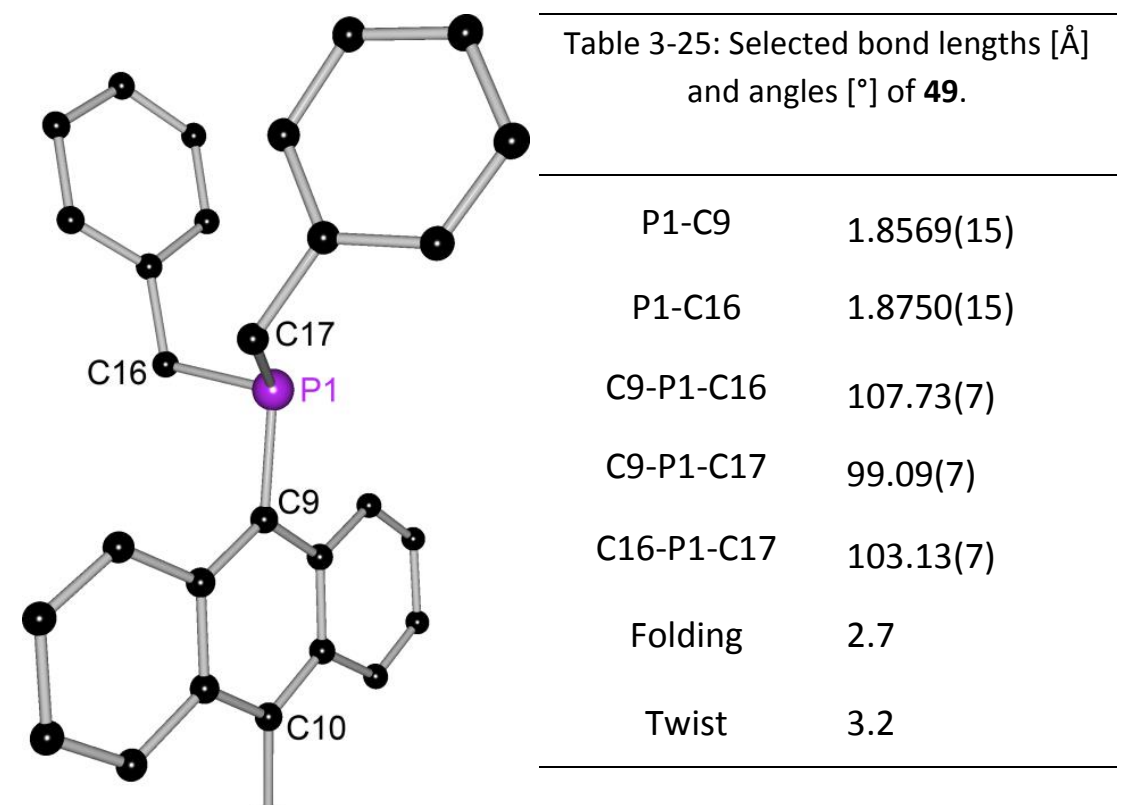

Figure 3-67: Crystal structure of $\mathrm{MeAnP}\left(\mathrm{CH}_{2} \mathrm{Ph}\right)_{2}$ (49).

MeAnP( $\left(\mathrm{CH}_{2} \mathrm{Ph}\right)_{2}$ (49) crystallizes in the monoclinic space group $C_{2} / c$ and the asymmetric unit contains one molecule of 49. As observed for other phosphorus(III) compounds, the angles between the phosphorus bound substituents deviate strongly from the ideal tetrahedral angle which is caused by the large spatial demand of the lone pair. The lone pair is not located in the anthracene plane, it has a torsion angle of $35.6^{\circ}$ relative to the anthracene plane. The methylene groups between the phosphorus atom and the phenyl rings increase the flexibility of the phosphorus bound substituents. The weaker steric strain resulting from this flexibility is reflected by the

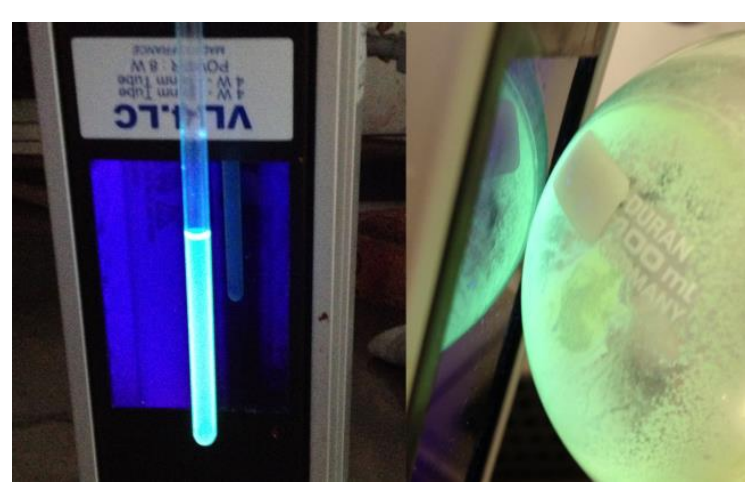

Figure 3-68: Fluorescnce phenomena of $\mathbf{4 9}$ in solution (left) and in the solid state (right); $\lambda_{\mathrm{Ex}}=366 \mathrm{~nm}$. weak deformation of the fluorophore of $2.7^{\circ}$ folding angle and $3.2^{\circ}$ twist angle.

Both in solution and in the solid state 49 exhibits striking fluorescence phenomena (Figure 3-68). In the packing plot of 49 , a $\pi-\pi$ overlap of $\sim 35 \%$ is found at a distance of $3.50 \AA$, which is a common value. Additionally a 
$\mathrm{C}-\mathrm{H}^{\prime \prime} \pi$ interaction between a meta phenyl hydrogen atom and a peripheral ring of the anthracene moiety is observed. It measures $2.691 \AA$ at an angle of $63.5^{\circ}$ to the ring plane (Figure 3-69).
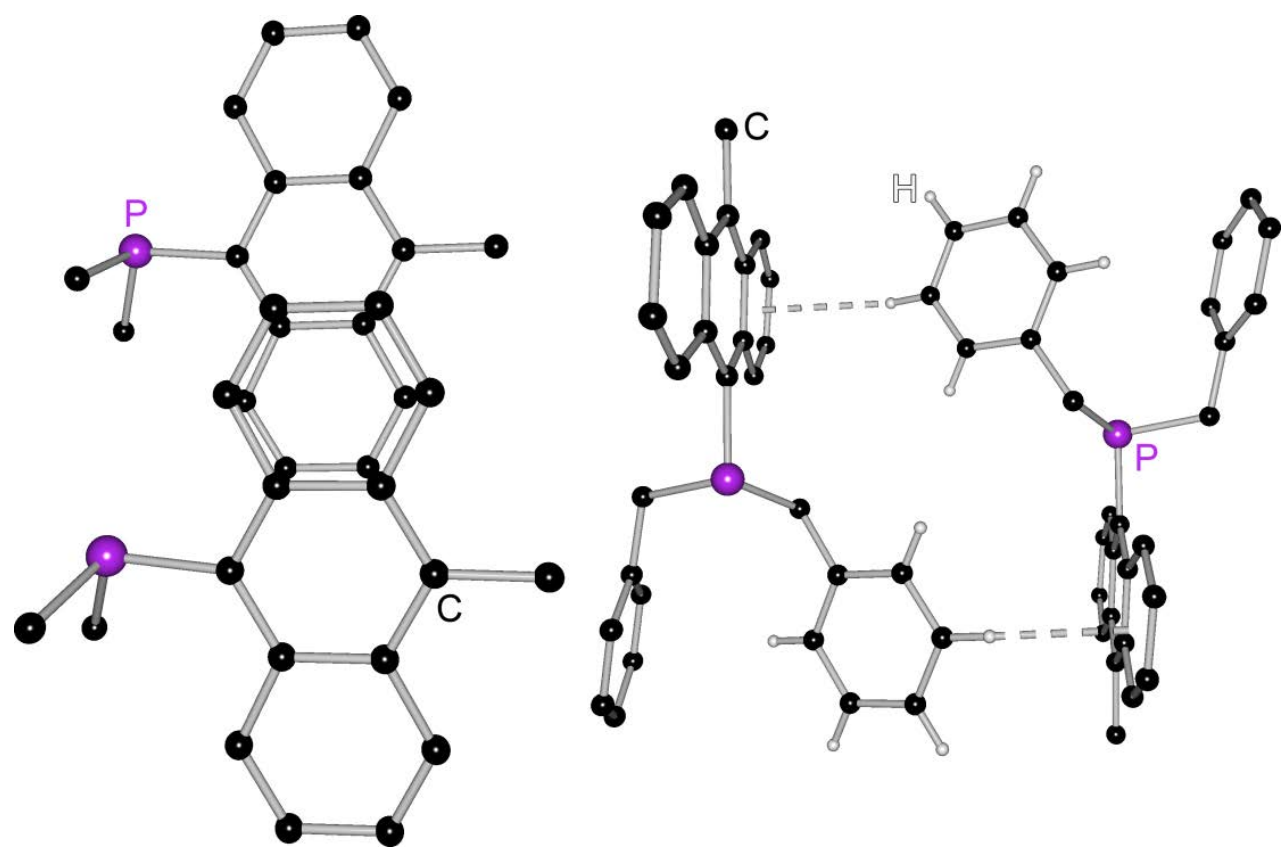

Figure 3-69: Intermolecular interactions in the packing plot of 49. Left: $\pi-\pi$ overlap, phenyl rings and hydrogen atoms are omitted for clarity; right: $\mathrm{C}-\mathrm{H} \cdots{ }^{\prime \prime} \pi$ interaction.

Although 49 could not be obtained in sufficient amounts in high purity for fluorescence experiments, the low deformation of the fluorophore as well as the strong phenyl $\mathrm{C}-\mathrm{H}^{\cdots}{ }^{\prime} \pi$ bonding depicted in Figure 3-69 (right) account for the observed fluorescence phenomena.

\subsection{Metal Complexes of Phosphanyl and Phosphorylanthracenes}

Apart from the gold(I) complexes presented in 3.3, several other metal complexes of previously described phosphanyl and phosphoryl anthracenes were prepared. The following chapter is dedicated to the analysis of these compounds with reference to their coordination behavior and their structural properties in the solid state. Moreover, also changes of fluorescence phenomena arising from metal coordination were described if the synthesized complexes exhibited striking fluorescence behavior and enough crystalline material was obtained for fluorescence experiments. 


\subsubsection{Gold(I) Complexes}

The complexes were synthesized by reaction of the respective phosphoryl anthracenes with $\left[\mathrm{Me}_{2} \mathrm{SAuCl}\right]$ in acetone. Displacement of the $\mathrm{SMe}_{2}$ donor afforded the gold(I) complexes $\left[\mathrm{HAnPPh}_{2}(\mathrm{~S}) \mathrm{AuCl}\right]$ (50), $\left[\mathrm{HAnP}^{i} \mathrm{Pr}_{2}(\mathrm{~S}) \mathrm{AuCl}\right]$ (51), and [MeAnPPh 2 (S)AuCl] (52) (Scheme 3-30). All complexes were crystallized from acetone at $-30^{\circ} \mathrm{C}$. The obtained crystals were suitable for single crystal $\mathrm{X}$-ray structure analysis.

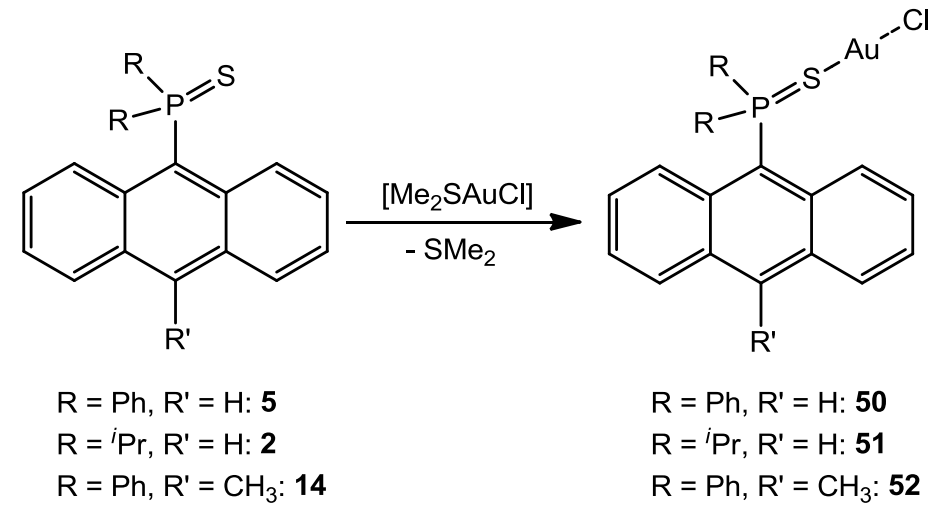

Scheme 3-31: Synthesis of gold(I) complexes 50-52.

[HAnPPh 2 (S)AuCl] (50) crystallizes in the monoclinic space group $P 2_{1} / n$. The typical linear S-Au-Cl fragment ${ }^{[69]}$ is not directed away from the anthracene moiety, but rather located behind it on the opposite side of the phenyl groups relative to the anthracene plane. This arrangement was also found in the complex [MeAnP(NEt $\left.)_{2}(\mathrm{~S}) \mathrm{AuCl}\right](\mathbf{2 4})$.

The S-Au- $\mathrm{Cl}$ fragment encloses an angle of $173.8^{\circ}$, which is nearly perfectly linear. The S-Au bond distance measures $2.257 \AA$ which is slightly shorter than corresponding $\mathrm{S}-\mathrm{Au}$ bonds found in previous gold complexes of sulfur oxidized compounds. The tetrahedral geometry surrounding P1 is slightly distorted. The deformation of the anthracene moiety is moderately strong with folding and twist angles of $14.1^{\circ}$ and $7.7^{\circ}$, respectively, which in fact indicates weaker deformation than observed for the mere ligand $\mathrm{HAnPSPh}_{2}(4)$. 
Intermolecular interactions in the structure of $\mathbf{5 0}$ are limited to a single $\mathrm{C}-\mathrm{H} \cdots \pi$ bond between a meta hydrogen atom of a phenyl substituent and a peripheral ring of the anthracene moiety which measures $2.818 \AA$ at an angle of $53.8^{\circ}$ to the ring plane (Figure 3-70, right).

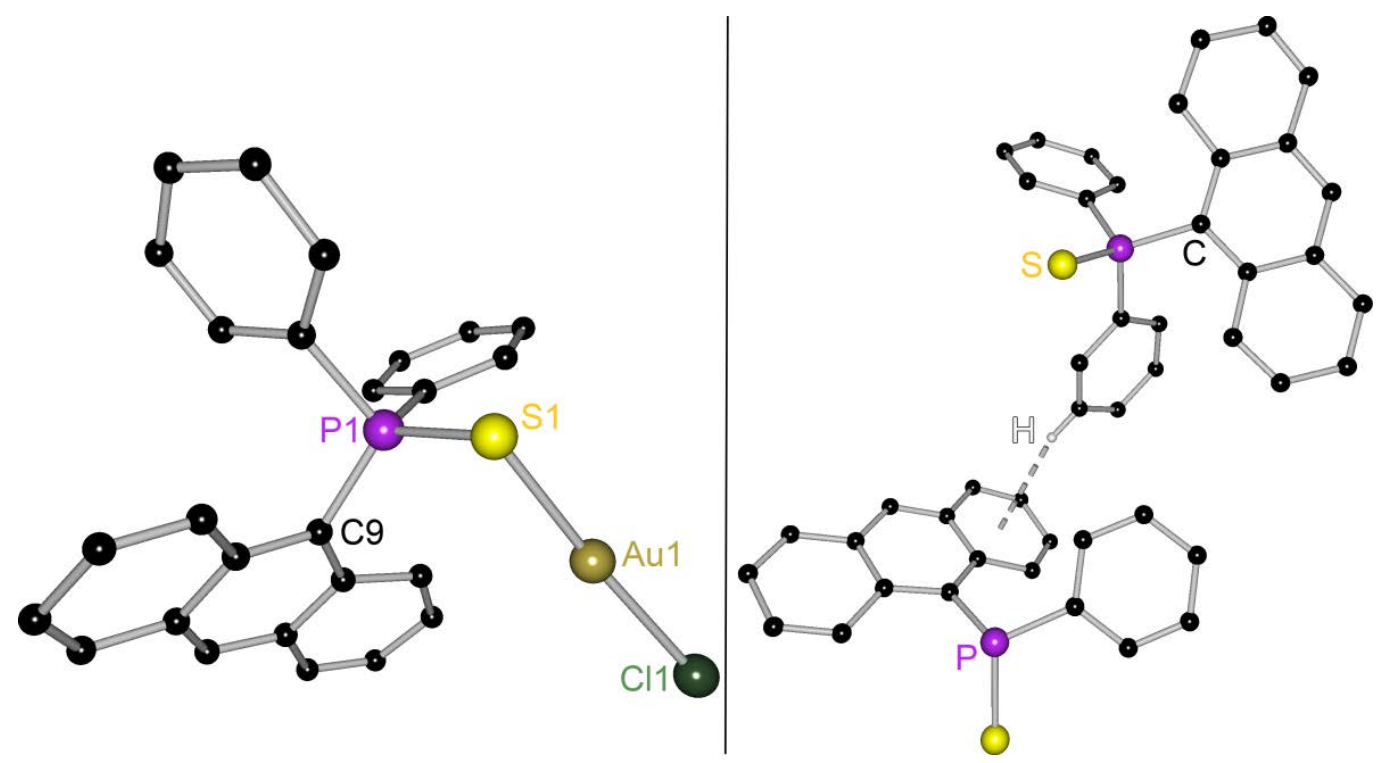

Figure 3-70: left: crystal structure of $\left[\mathrm{HAnPPh}_{2}(\mathrm{~S}) \mathrm{AuCl}\right](\mathbf{5 0})$, hydrogen atoms are omitted for clarity; right: $\mathrm{C}-\mathrm{H}^{\cdots} \pi$ interaction in $\mathbf{5 0}$, one phenyl substituent and $\mathrm{Au}-\mathrm{Cl}$ fragments are omitted for clarity.

Table 3-26: Selected bond lengths $[\AA ̊]$ and angles $\left[^{\circ}\right]$ of $[\mathrm{HAnPPh}(\mathrm{S}) \mathrm{AuCl}](\mathbf{5 0})$.

\begin{tabular}{lc|lc}
\hline P1-S1 & $2.0217(9)$ & S1-Au1-Cl1 & $173.77(3)$ \\
S1-Au1 & $2.2571(7)$ & C9a-C9-P1-S1 & $80.00(2)$ \\
C9-P1-S1 & $116.18(9)$ & Folding & 14.1 \\
P1-S1-Au1 & $105.99(3)$ & Twist & 7.7 \\
\hline
\end{tabular}

The gold complex [ $\left.\mathrm{HAnP}^{i} \mathrm{Pr}_{2}(\mathrm{~S}) \mathrm{AuCl}\right]$ (51) shows a different orientation of the S-Au-Cl fragment from the one observed in $\mathbf{5 0}$. While in $\mathbf{5 0}$, the torsion angle of the $\mathrm{P}=\mathrm{S}$ bond to the anthracene plane measures $80^{\circ}$, it measures only $14.4^{\circ}$ in the structure of 51 . Therewith it is nearly located in the anthracene plane, with both iso-propyl groups located on opposite side of the anthracene plane. This leads to an almost orthogonal orientation of the S-Au-Cl fragment to the anthracene plane. 

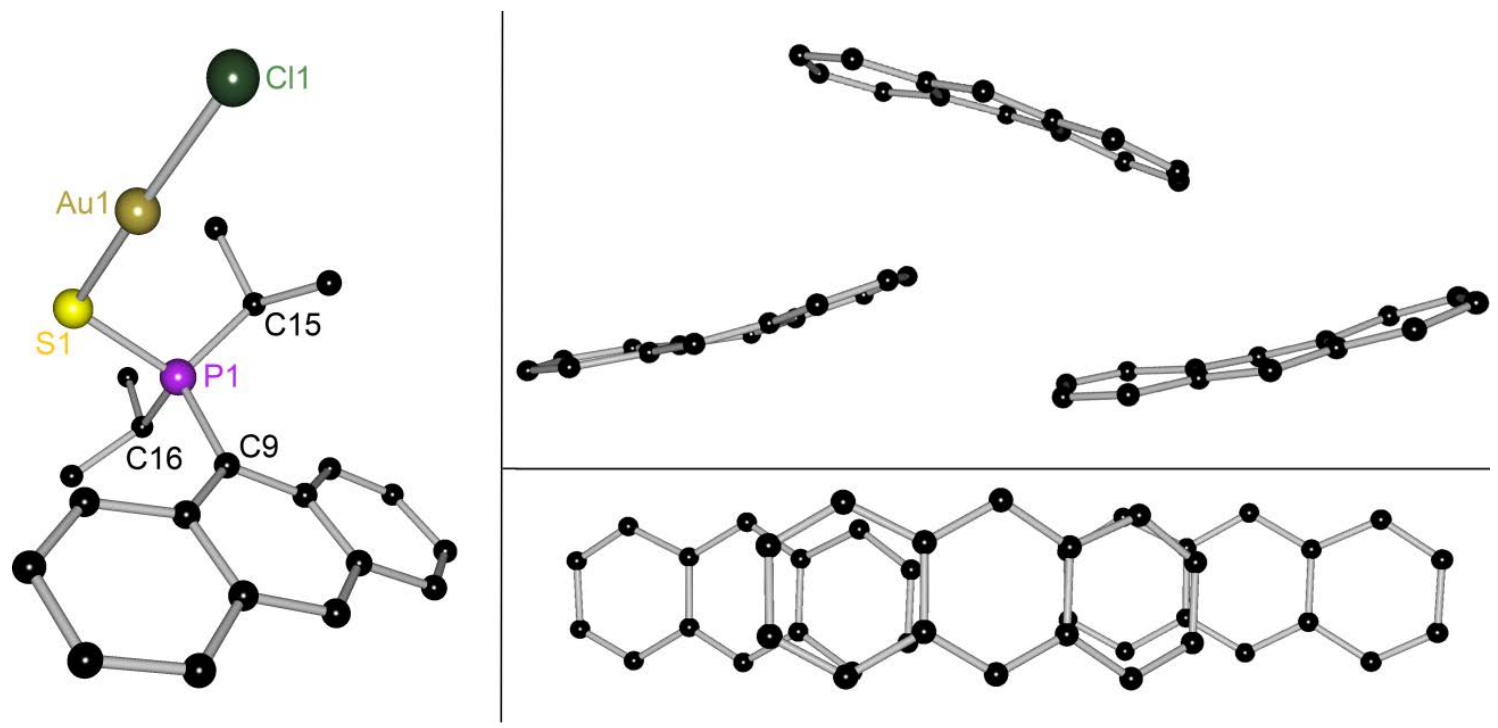

Figure 3-71: Left: crystal structure of $\left[\mathrm{HAnP}^{i} \mathrm{Pr}_{2}(\mathrm{~S}) \mathrm{AuCl}\right]$ (51), hydrogen atoms are omitted for clarity; right: non-parallel $\pi-\pi$ overlap of fluorophores in $\mathbf{5 1}$, side view (top) and top view (bottom).

Moreover, one would predict well distributed steric strain applied by phosphoryl substituent due to its nearly symmetrical alignment, which would result in a small expected deformation of the fluorophore. Though the actual deformation is moderate at folding and twist angles of $12.6^{\circ}$ and $3.7^{\circ}$, it is stronger than expected. The observed distortion is induced by the $\mathrm{Au}-\mathrm{Cl}$ fragment, as the anthracene moiety is folded in the opposite direction of it. The $\mathrm{S}-\mathrm{Au}-\mathrm{Cl}$ fragment is even closer to perfectly linear than in the structure of $\mathbf{5 0}$, enclosing an angle of $176.74^{\circ}$. The observed bond distances are within the expected range and differ only minimally, the tetrahedral geometry of the P-bound substituents is slightly distorted. While $\mathbf{5 0}$ crystallizes in a monoclinic space group, $\mathbf{5 1}$ crystallizes in the orthorhombic space group Fdd2. This leads to a distinctly different packing motif of the molecules. In the structure of [ $\left.\mathrm{HAnP}^{i} \mathrm{Pr}_{2}(\mathrm{~S}) \mathrm{AuCl}\right](\mathbf{5 1})$ the phosphanylanthracene molecules do not adapt the typical "head-to-tail" positioning with parallel orientation of the anthracene moieties. Although $\pi-\pi$ overlap is found, the involved fluorophores are not parallel oriented, but exhibit an angle of $\sim 35^{\circ}$ to one another (Figure 3-71, right). In addition to the non-parallel $\pi-\pi$ interactions, two $\mathrm{sp}^{3}$ type $\mathrm{C}-\mathrm{H} \cdots \pi$ bonds are present in the structure of $\mathbf{5 1}$ between methyl hydrogen atoms and the $\pi$ systems of both a central and a peripheral $C_{6}$-perimeter. They measure $2.880 \AA\left(45.9^{\circ}\right)$ and $2.936 \AA\left(44.0^{\circ}\right)$, respectively (Figure 3-72). That makes them both fairly weak, taking into account the weak polarization of the $\mathrm{sp}^{3} \mathrm{C}-\mathrm{H}$ bonds. 


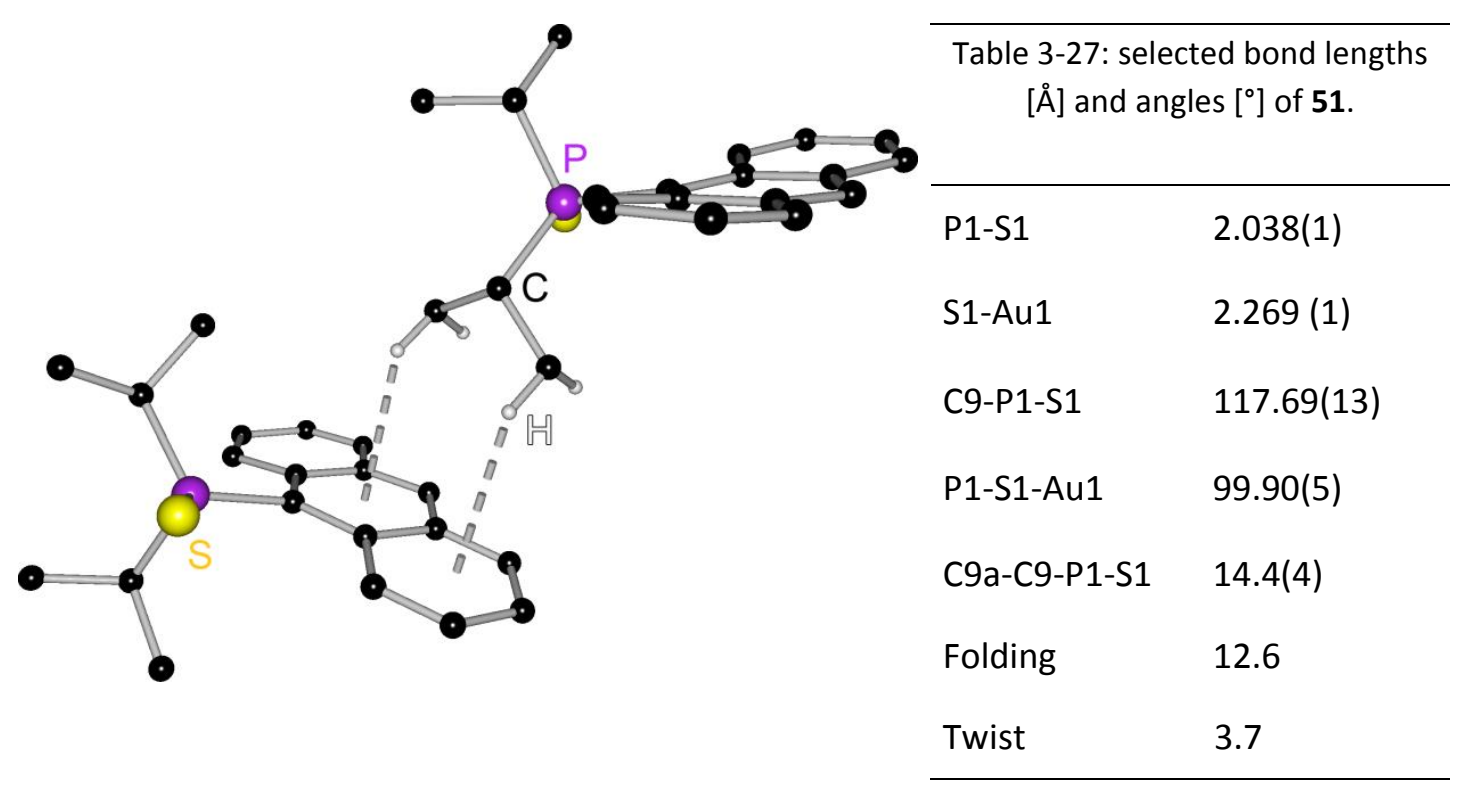

Figure 3-72: $\mathrm{C}-\mathrm{H}^{\cdots}{ }^{\mathrm{m}} \pi$ interactions in $\mathbf{5 1}$. Au-Cl fragments are omitted for clarity.

The structure of [MeAnPPh $2(S) A u C l] ~(52)$ is the first gold complex described so far which bears co-crystallized solvent molecules in its crystal structure. The asymmetric unit contains one molecule of $\mathbf{5 2}$ as well as one molecule of acetone. Aside from the lattice solvent, the local symmetry of the molecule is closely related to that of $\mathbf{5 0 .}$

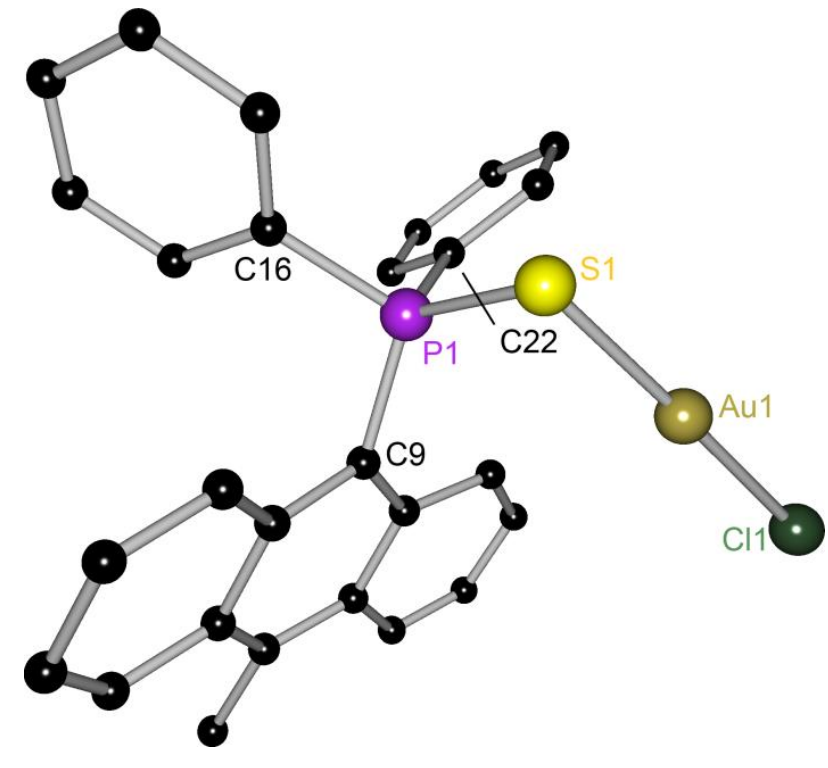

Table 3-28: Selected bond lengths $[\AA]$ and angles $\left[^{\circ}\right]$ of $\mathbf{5 2}$.

$\overline{P 1-S 1 \quad 2.0287(15)}$

S1-Au1 2.2722(12)

C9-P1-S1

$115.16(15)$

P1-S1-Au1 95.93(6)

C9a-C9-P1-S1 79.6(3)

Folding $\quad 10.6$

Twist $\quad 4.8$

Figure 3-73: Crystal structure of [ $\left.\mathrm{MeAnPPh}_{2}(\mathrm{~S}) \mathrm{AuCl}\right]$ (52),

lattice solvent and hydrogen atoms are omitted for clarity. 
This is especially reflected by the similar torsion angles of the $\mathrm{P}=\mathrm{S}$ bonds to the anthracene plane which deviate by only $0.4^{\circ}$. The resultant orientation of the S-Au-Cl fragment is accordingly also nearly identical, as well as the steric strain applied to the anthracene moiety. The induced deformation of the fluorophore shows only minor deviations from that found in the structure of $\mathbf{5 0}$, which is underlined by the superposition of both compounds depicted in Figure 3-74. The predominant difference in structure between the two complexes is the remarkably small P-S-Au angle of only $95.9^{\circ}$ in $\mathbf{5 2}$, which leads to the deviation in the orientations of both S-Au-Cl fragments. The S-Au- $\mathrm{Cl}$ angle of $\mathbf{5 2}$ measures $178.5^{\circ}$, which is the closest to ideal linear geometry encountered so far.
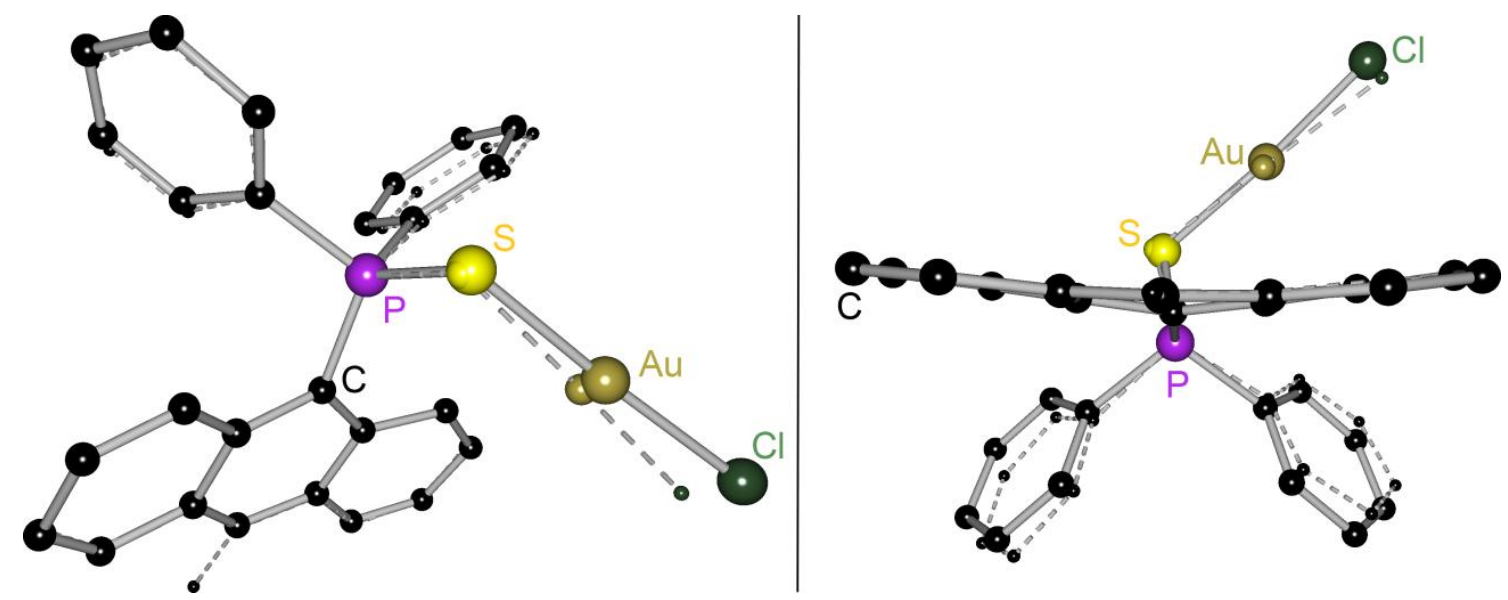

Figure 3-74: Superposition of [ $\left.\mathrm{HAnPPh}_{2}(\mathrm{~S}) \mathrm{AuCl}\right](\mathbf{5 0})$ (regular spheres, solid bonds) and $\left[\mathrm{MeAnPPh}_{2}(\mathrm{~S}) \mathrm{AuCl}\right]$ (52) (small spheres, dashed bonds). Hydrogen atoms are omitted for clarity.

The distorted tetrahedral geometry surrounding the phosphorus atom is nearly identical to the corresponding geometry in $\mathbf{5 0 . ~ I n ~ s p i t e ~ o f ~ t h e ~ s t r o n g ~ s i m i l a r i t i e s ~ i n ~ t h e ~}$ local structures of $\mathbf{5 0}$ and $\mathbf{5 2}$, the methyl group in 10-position of $\mathbf{5 2}$ leads to a completely different packing motif than observed for 50 . It not only crystallizes in the orthorhombic space group $\mathrm{Pbca}$, also the intermolecular interactions differ significantly. While $\mathbf{5 0}$ showed virtually no $\pi-\pi$ overlap, [MeAnPPh $2(S) A u C l] ~(52)$ exhibits an overlap of $\sim 40 \%$ at a distance of $3.61 \AA$ (Figure $3-75$ ). 

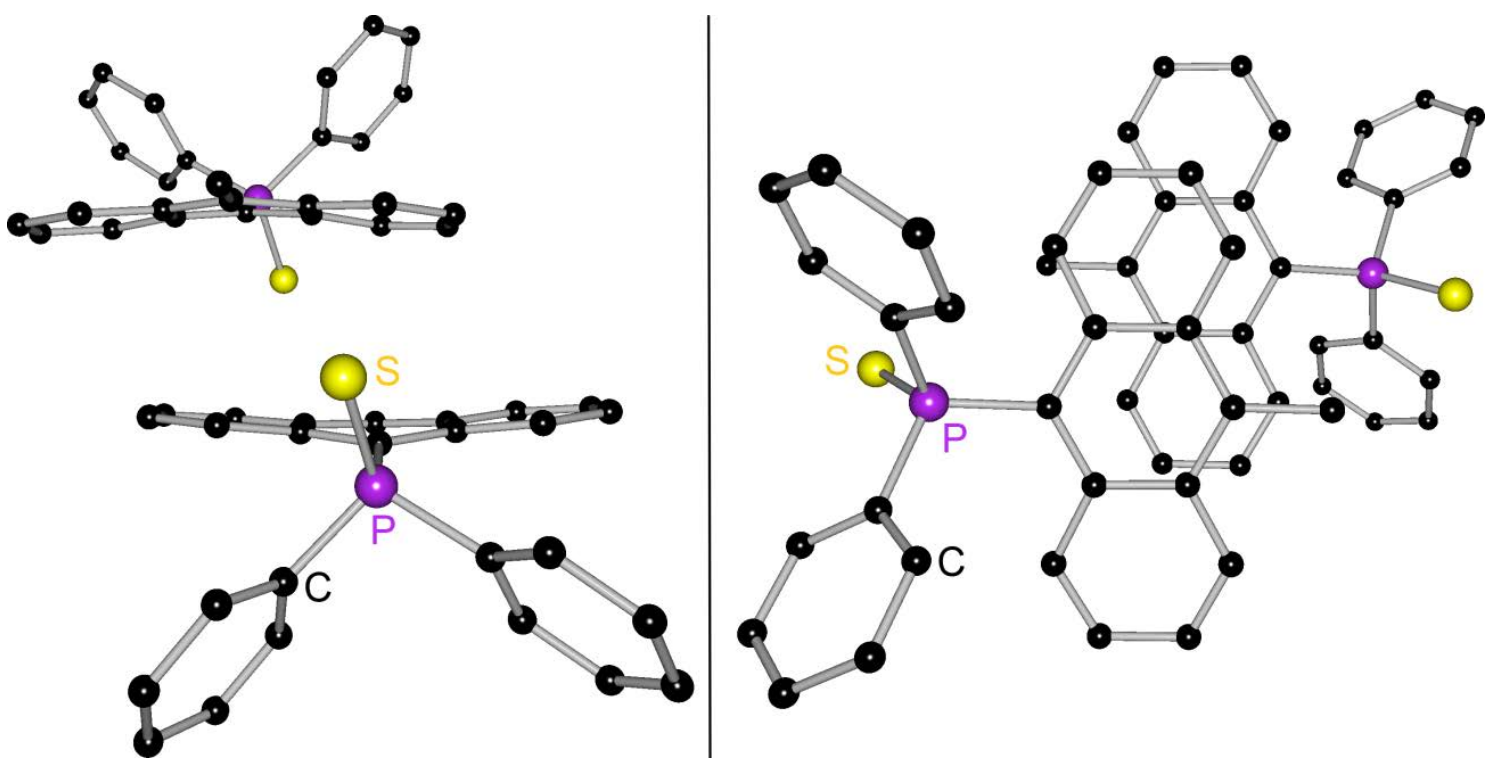

Figure 3-75: $\pi-\pi$ overlap in the structure of 52, side view (left) and top view (right), hydrogen atoms and $\mathrm{Au}-\mathrm{Cl}$ fragments are omitted for clarity.

Reactions of symmetric phosphanylanthracenes with gold(I) and silver(I) salts have repeatedly produced cyclic complexes in the past. ${ }^{[65,71,84]}$ Therefore the feasibility of producing cyclic complexes from oxidized symmetric phosphorylanthracenes was

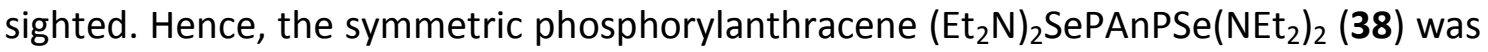
reacted with $\left[\mathrm{Me}_{2} \mathrm{SAuCl}\right]$.<smiles>CCNP(=O)(c1c2ccccc2c([PH](=[Se])(=[Se])NCC)c2ccccc12)N(CC)CC</smiles>

Scheme 3-32: Synthesis of gold (I) complex 53.

Although the molecule bears two selenium donor atoms, only one equivalent of [ $\left.\mathrm{Me}_{2} \mathrm{SAuCl}\right]$ was added to promote the formation of a cyclic complex which also exhibits a ligand/metal ion ratio of $1: 1$. Crystallization from acetone at $-30^{\circ} \mathrm{C}$ surprisingly afforded the symmetric gold complex

[ClAu(Se)(Et $\left.\left.{ }_{2} \mathrm{~N}\right)_{2} \mathrm{PAnP}\left(\mathrm{Et}_{2} \mathrm{~N}\right)_{2}(\mathrm{Se}) \mathrm{AuCl}\right]$ (53), which has a 1:2 ligand/metal ion ratio (Scheme 3-31). Despite the ratio of the utilized substances, the formation of the 
symmetric complex appears to be favored over the formation of a cyclic arrangement. 53 crystallizes in the monoclinic space group $P 2_{1} / c$ and the asymmetric unit contains one molecule. The linear Se-Au-Cl fragments are directed in opposite directions away from the anthracene moiety on the identical side of the anthracene plane in a cisoid conformation. Both show nearly perfectly linear geometry, with Se-Au-Cl angles of $174.8^{\circ}$ and $173.2^{\circ}$, respectively (Table $3-29$ ).

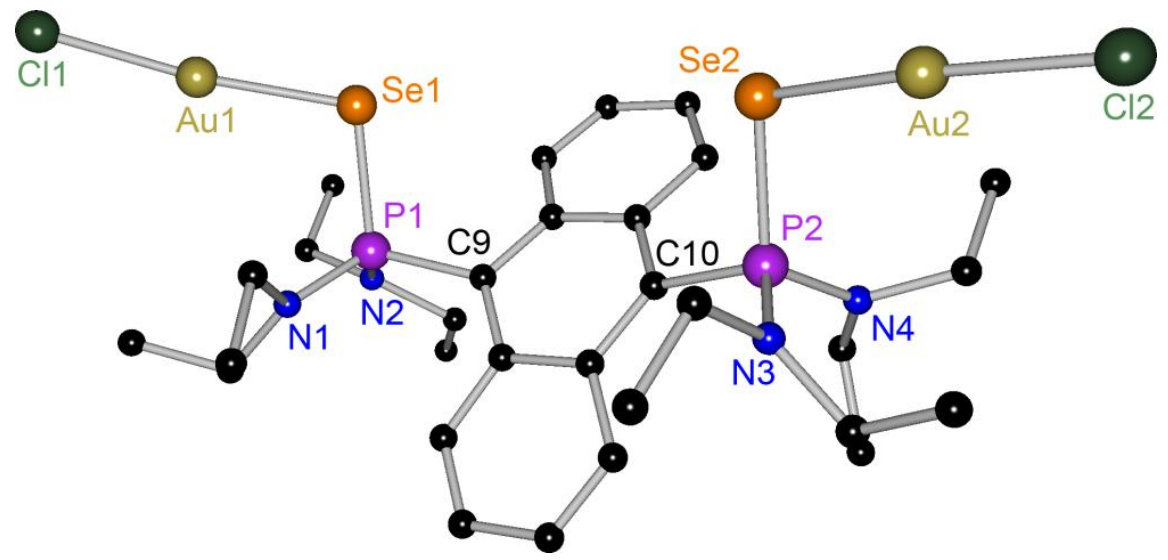

Figure 3-76: Crystal structure of 53, hydrogen atoms are omitted for clarity.

Table 3-29: Selected bond lengths $[\AA]$ and angles $\left[{ }^{\circ}\right]$ of 53.

\begin{tabular}{lc|cc}
\hline Se1-Au1 & $2.3798(7)$ & C9-P1-N1 & $113.2(3)$ \\
Se2-Au2 & $2.346(2)$ & C9a-C9-P1-Se1 & $-131.5(5)$ \\
P1-Se1-Au1 & $98.81(5)$ & C4a-C10-P2-Se2 & $136.3(5)$ \\
P2-Se2-Au2 & $104.70(8)$ & Folding & 19.0 \\
C9-P1-Se1 & $104.4(2)$ & Twist & 1.6 \\
\hline
\end{tabular}

The bonding partners of the phosphorus atoms form distorted tetrahedra, the deviations from the ideal tetrahedral angle are moderate. The Se-Au distances are not identical, but differ by only $0.034 \AA$ and are both in the range of the Se-Au distances observed in $\mathbf{2 6}$ and $\mathbf{2 8}$ (see 3.3). In contrast, P-Se-Au angles are similar but differ by over $5^{\circ}$ which is the strongest deviation found between the two coordination sites of the complex. The presence of two bulky bis(diethylamino)selenophosphoryl substituents in the structure combined with two large coordinated gold ions leads to a strong steric strain on the fluorophore affording a folding angle of $19.0^{\circ}$ and a twist deformation of $1.6^{\circ}$. This is a major difference to the uncoordinated 
$\left(\mathrm{Et}_{2} \mathrm{~N}\right)_{2} \mathrm{SePAnPSe}\left(\mathrm{NEt}_{2}\right)_{2}$ (38) which exhibits an extreme twist deformation of $24.4^{\circ}$. Generally the strong folding and weak twist deformation is in accordance with the deformations observed for other symmetric phosphoryl anthracenes in a cisoid conformation (see 3.2). Due to the strong steric demand of the substituents which prevent the anthracene moieties from close contact, there are no noteworthy intermolecular interactions in terms of $\pi$ interactions present in the structure of $5 \mathbf{5 3}$.

Although the primary goal of synthesizing a cyclic complex was not attained, a different interesting feature of $\mathbf{5 3}$ was discovered. $\mathbf{5 3}$ is the first gold complex of a phosphoryl anthracene to exhibit a gold-gold contact in its solid state structure.

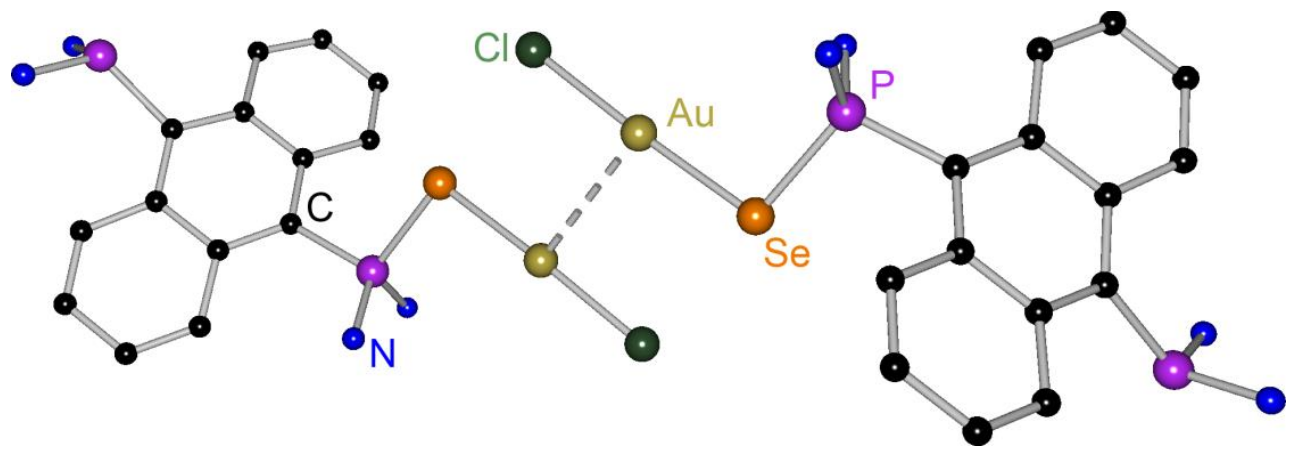

Figure 3-77: Gold-gold contact in the structure of 53. Ethyl groups, hydrogen atoms and terminal $\mathrm{Au}-\mathrm{Cl}$ fragments are omitted for clarity.

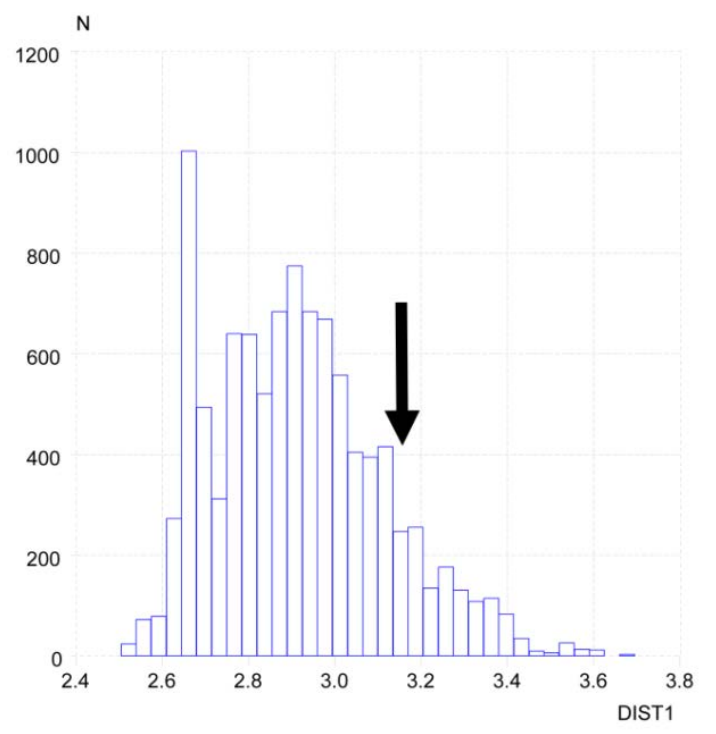

Figure 3-78: CSD statistic distribution of observed AuAu bond distances $[\AA ̊]$.
The Au-Au distance measures $3.153 \AA$, which is slightly longer than the average observed $\mathrm{Au}-\mathrm{Au}$ interaction (Figure 3-78). The $\mathrm{Au}-\mathrm{Au}$ bond is nearly orthogonal to the Se$\mathrm{Au}-\mathrm{Cl}$ fragment with an Se-Au-Au' angle of $100.4^{\circ}$. The formation of the Au-Au bond only occurs between every two molecules in the packing motif, which leads to a dimer-like complex. The terminal Se$\mathrm{Au}-\mathrm{Cl}$ fragments do not show $\mathrm{Au}-\mathrm{Au}-$ interactions, which would generate a linear coordination polymer. The gold-gold interaction also affects Se-Au bonding. The terminal Se-Au bond is slightly 
shorter than the Se-Au bond of the fragment participating in Au-Au bonding. This also applies to the $\mathrm{Au}-\mathrm{Cl}$ bonds.

\subsubsection{Copper(I) Complexes}

So far, only the coordination of phosphorylanthracenes to gold(I) has been described in this thesis. Although several interesting features have been discovered in this class of metal complexes, the linear coordination geometry of the gold(I) complexes is also somewhat limiting. To expand the spectrum of complexed metals in order to investigate the coordination of phosphanylanthracenes to metals in other coordination geometries, the synthesis of copper(I) complexes was peered.

To synthesize a copper(I) complex, the corresponding metal ions must be dissolved in sufficient quantity, which is often a limiting factor. The precursor complex $\left[\mathrm{Cu}\left(\mathrm{PPh}_{3}\right)_{2} \mathrm{BH}_{4}\right]$ shows very good solubility even in weakly or non-donating solvents and the $\mathrm{PPh}_{3}$ donors can be easily replaced which makes it a very potent starting material in complex synthesis. The un-oxidized $\mathrm{MeAnP}\left(\mathrm{NMe}_{2}\right)_{2}$ (33) was reacted with 0.5 equivalents of $\left[\mathrm{Cu}\left(\mathrm{PPh}_{3}\right)_{2} \mathrm{BH}_{4}\right]$ in $\mathrm{DCM}$ at ambient temperature, then the mixture was heated to $35^{\circ} \mathrm{C}$ for $5 \mathrm{~min}$. and then stirred at room temperature for $8 \mathrm{~h}$. Storage of the reaction mixture at $-30^{\circ} \mathrm{C}$ yielded $\left[\left(\mathrm{MeAnP}\left(\mathrm{NMe}_{2}\right)_{2}\right)_{2} \mathrm{CuBH}_{4}\right](\mathbf{5 4})$ as yellow octahedral crystals (Scheme 3-32).

2

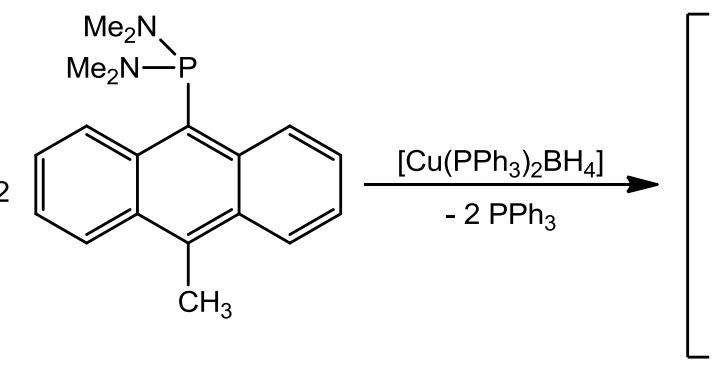

33

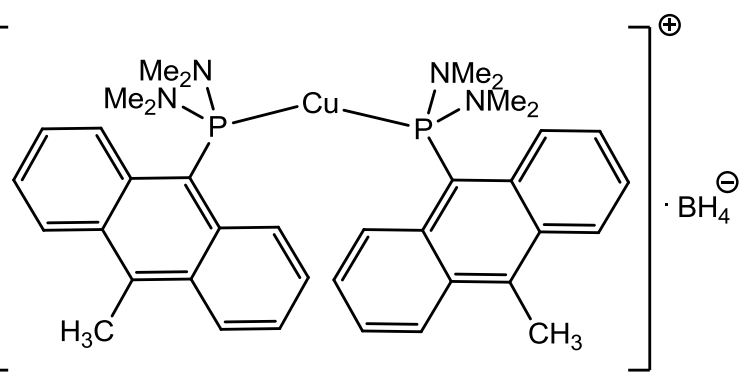

54

Scheme 3-33: Synthesis of [(MeAnP(NMe $\left.\left.)_{2}\right)_{2} \mathrm{CuBH}_{4}\right](\mathbf{5 4})$.

Though an incomplete reaction under exchange of only one $\mathrm{PPh}_{3}$ ligand by the phosphanylanthracene is also thinkable, both $\mathrm{PPh}_{3}$ donors were replaced, yielding a dimer complex. The perfectly octahedral shape of the crystals is visible in Figure 3-80, which shows a crystal of $\mathbf{5 4}$ mounted on a glass fibre for diffraction experiments. 


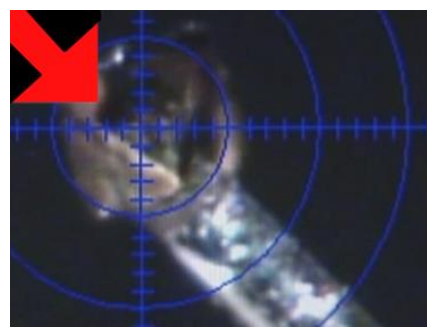

Figure 3-2: Crystal of 54.

Although the precursor molecule $\mathrm{MeAnP}\left(\mathrm{NMe}_{2}\right)_{2}(33)$ is a highly viscous oil which could not be crystallized, the coordination to $\mathrm{Cu}(\mathrm{I})$ affords a solid metal complex. Due to the fixed orientation of the coordinated phosphanyl substituents, an ordered arrangement suitable for crystallization is generated.

The second driving force for crystallization can be derived from the crystal structure of 54. The formation of a dimer complex brings the $\pi$-systems of the donating phosphanyl anthracenes into close proximity to one another (Figure 3-80, left). The

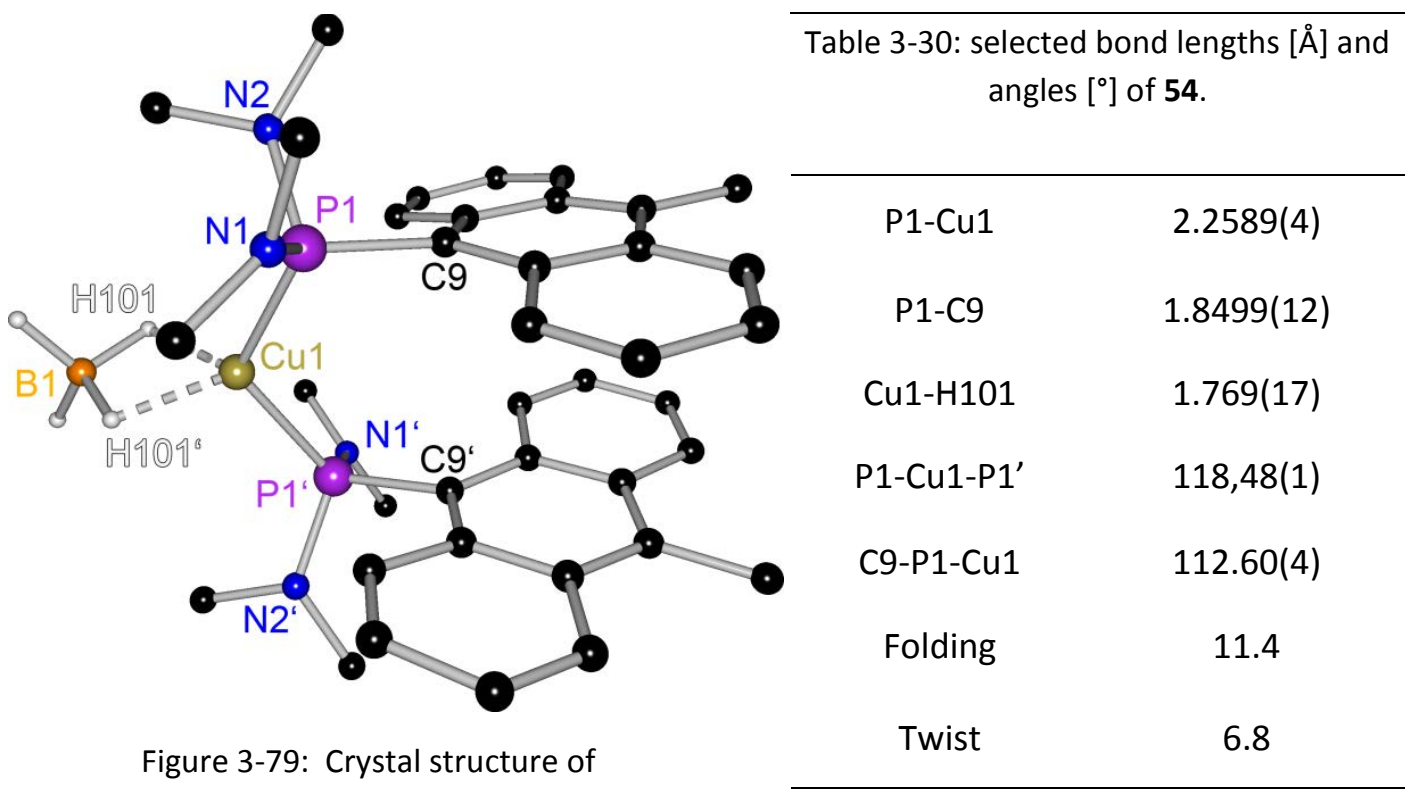

$\left[\left(\mathrm{MeAnP}\left(\mathrm{NMe}_{2}\right)_{2}\right)_{2} \mathrm{CuBH}_{4}\right](\mathbf{5 4})$, hydrogen atoms are omitted for clarity.

resulting $\pi$-stacked arrangement appears to be energetically favored towards crystallization. Although the $\pi$ systems show parallel orientation at a distance of $3.53 \AA$, the anthracene moieties are rotated against each other, rather than assuming a completely ecliptic conformation (Figure $3-80$, right). This way an overlap of only $\sim 40 \%$ is achieved. 


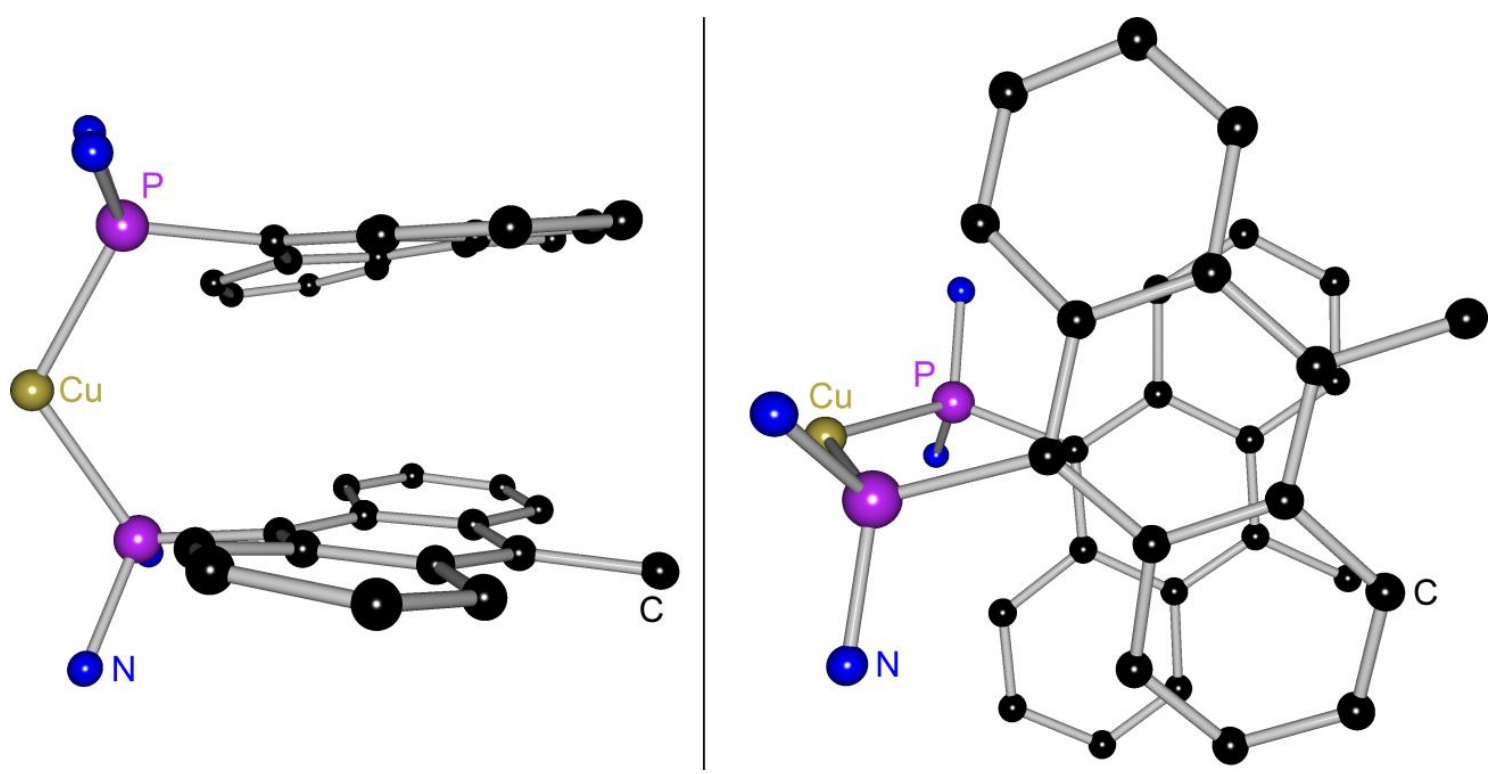

Figure 3-81: $\pi-\pi$ overlap in the crystal structure of 54 , side view (left) and top view (right); amine bound methyl groups, borate ion and hydrogen atoms are omitted for clarity.

The asymmetric unit contains one half of the dimer complex, the other half is produced by symmetry operations. Both the copper atom and the borate anion are located on a mirror plane and are only present to $1 / 2$ in the asymmetric unit. 54 crystallizes in the orthorhombic space group Pbcn. The angles of the P-bound substituents including the coordinated copper(I) ion range from $99.7^{\circ}$ to $113.7^{\circ}$ indicating distorted tetrahedral geometry. The $\mathrm{P}-\mathrm{Cu}$ bond exhibits the clearly highest spatial demand, as all angles to the P-Cu bond are larger than $112^{\circ}$, while the angles between all other substituents are smaller than $110^{\circ}$. The $\mathrm{P}-\mathrm{Cu}$ bond measures $2.259 \AA$, which is very close to the average value of $\mathrm{P}-\mathrm{Cu}$ bonds (Figure 3-81). Moreover, the P1-Cu1-P1' angle is close to $120^{\circ}$ at $118.5^{\circ}$ which is a major deviation from the $109.45^{\circ}$ expected for tetrahedral coordination. This can be attributed to the large steric bulk of the donating phosphanylanthracenes, whose repulsion induces the observed widening of the P1-Cu1-P1' angle. A further factor which may explain the large angle is the absence of further donors to fill the coordination sphere of the copper ion to its preferred coordination number of 4 . Instead of coordination of two additional donors, there is only an interaction between two hydrogen atoms of the borate anion and the copper cation is present. Both $\mathrm{Cu}-\mathrm{H}$ distances are symmetric and measure $1.769 \AA$, which indicates the strength of these interactions. Similar interactions have repeatedly been observed between borate anions and transition metal cations. Besides copper(I), interactions of this kind have also been found 
between hydrogen and various early transition metal ( $\mathrm{Fe}, \mathrm{Cr}, \mathrm{Ni}, \mathrm{Co}, \mathrm{Zn}$ ) and late transition metals ( $\mathrm{Rh}, \mathrm{Ir}, \mathrm{Os}, \mathrm{Nb}, \mathrm{Ru}, \mathrm{Re}$ ). As Figure 3-81 shows, the $\mathrm{Cu}-\mathrm{H}$ distance found in $\mathbf{5 4}$ is distinctly shorter than the average non-hydride $\mathrm{M}-\mathrm{H}$ bond. The H101Cu1- $\mathrm{H} 101^{\prime}$ angle is very small at only $63.9^{\circ}$, which deviates extremely from the tetrahedral angle preferred in the coordination of $\mathrm{Cu}(\mathrm{I})$. This phenomenon probably also contributes to the large P1-Cu1-P1' angle. The fluorophore deformation is moderate and can be ranked between the deformations of the sulfur and selenium oxidation products MeAnPS(NMe $)_{2}(\mathbf{2 1})$ and MeAnPSe$\left(\mathrm{NMe}_{2}\right)_{2}(\mathbf{2 5})$.
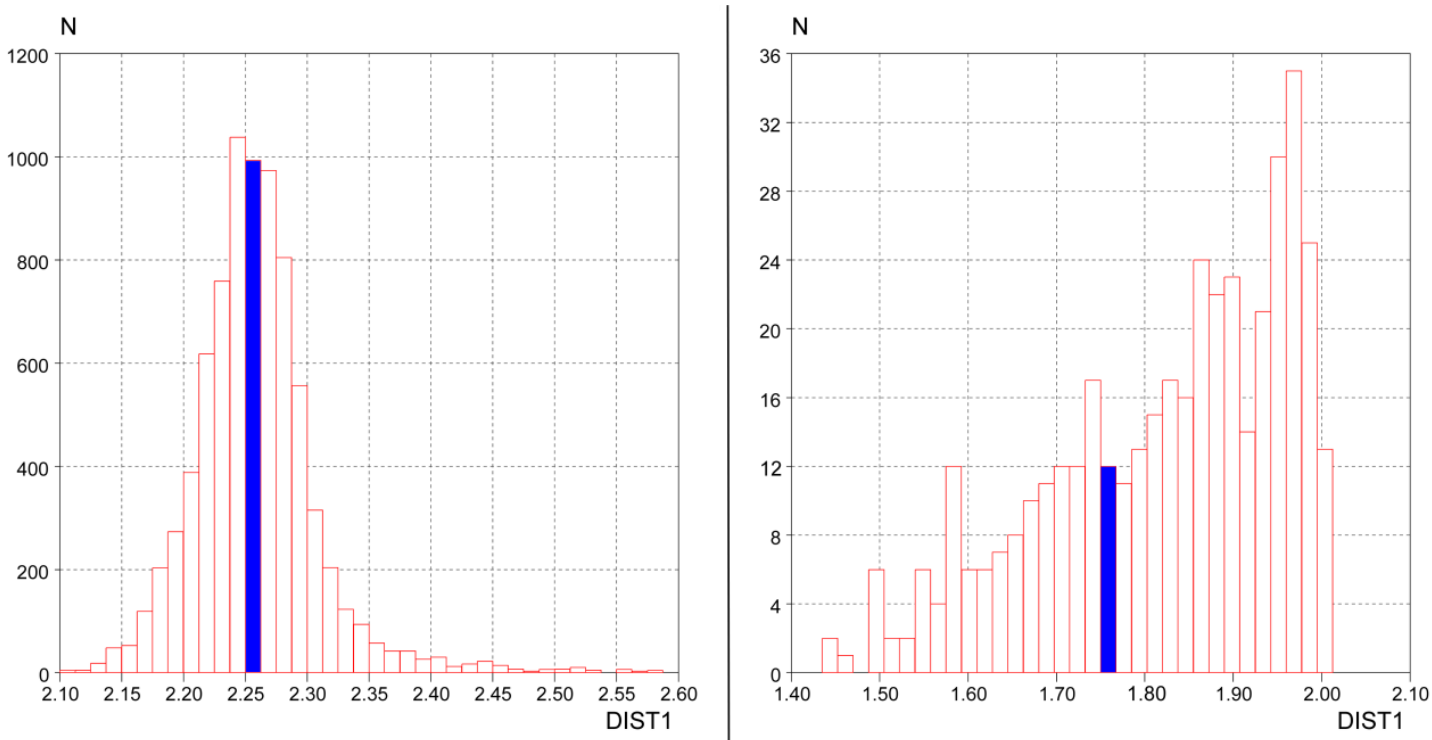

Figure 3-82: left: CSD statistics on P-Cu bond distances, value of $\mathbf{5 4}$ is marked (blue) (total average $=2.259 \AA$ ) , right: CSD statistics on non-hydride transition metal/hydrogen interactions, value of 54 is marked (blue) (total average $=1.822 \AA$ ).

Overall, the change from gold(I) to copper(I) has made a completely different mode and geometry of metal coordination accessible, which afforded a $\pi$ stacked dimer complex. A similar arrangement has been reported by Kubiak et al. who were able to synthesize a platinum(II) complex of their 9 -anthrylphosphirane. ${ }^{[76]}$ The orientation of the fluorophores to one another is comparable to the one observed in $\mathbf{5 4}$, while the $\pi$ $\pi$ distance of $3.40 \AA$ is slightly shorter than the $3.53 \AA$ found in $\mathbf{5 4}$. Additionally, the PPt-P angle of their cis-dichlorobis[1-(9-anthracene)phosphirane]platinum(II) is by approximately $20^{\circ}$ smaller than the P-Cu-P angle of 54 . This can be assigned to the preferred square-planar coordination of platinum(II), which produces angles close to $90^{\circ}$ around the platinum atom. Moreover, the preferred coordination number of four is achieved by coordination of two chloride ions, which also strongly contrasts the 
mere two $\mathrm{Cu}-\mathrm{H}$ interactions found in 54. The smaller P-M-P angle is most likely also the reason for the smaller observed $\pi-\pi$ distance in Kubiak's platinum complex.

\subsubsection{Zinc(II) Complexes}

The results presented in 3.1 have shown that the coordination of $\mathrm{Zn}^{2+}$ can alter the fluorescence properties of phosphanyl and phosphoryl anthracenes in solution. Investigation of the coordination mode in the solid state is therefore of interest. As for the gold(I) complexes presented in 3.3 , the impact of metal coordination on solid state fluorescence is a key phenomenon in understanding the mechanisms of solid state fluorescence which has not been exploited to date.

So far - with one exception - no zinc complexes of phosphanyl or phosphoryl anthracenes are known. The only successfully synthesized zinc complex by Stern from 2009 is merely an oxidation product of the desired compound, ${ }^{[59 c]}$ which further fuels the interest in preparation of this class of compounds.

The solubility of zinc salts in organic solvents is likewise other metal salts a limiting factor in complex synthesis. Though zinc chloride shows good solubility in THF, the utilization of strongly donating solvents leads to complex formation with the stronger donor and often the desired complex is not obtained. The fact that zinc bromide is well soluble in the weakly donating solvent DCM opens up new vistas in the synthesis of zinc complexes of fairly weak monodentate ligands. The reaction of $\mathrm{HAnPPh}_{2}$ (4) with one equivalent of zinc bromide in DCM at ambient temperature over $30 \mathrm{~min}$ and subsequent cooling and storage at $-30^{\circ} \mathrm{C}$ yielded $\left[\mathrm{HAnPPh} \mathrm{ZnBr}_{2}\right]_{2}$ (55) as yellow crystals (Scheme 3-33).

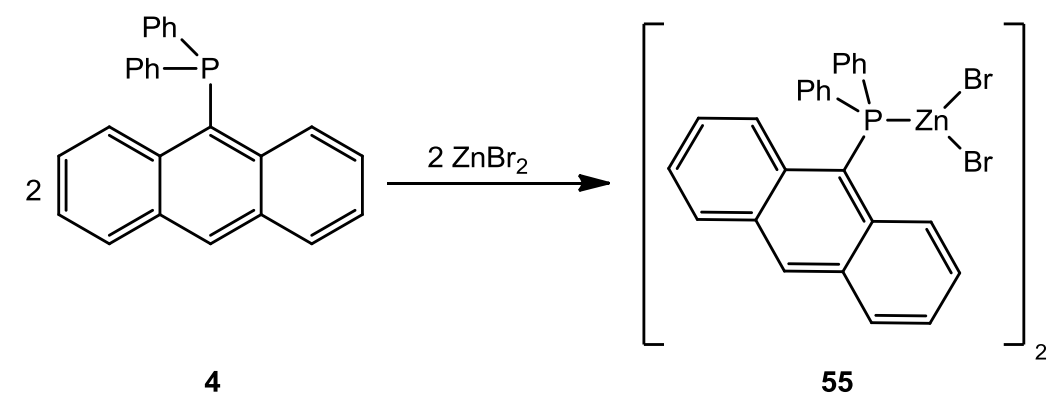

Scheme 3-34: Synthesis of [HAnPPh $\left.\mathrm{ZnBr}_{2}\right]_{2}$ (55). 
A dimer complex is formed which differs significantly from the copper(I) complex 54. Although both $\mathrm{Zn}^{2+}$ and $\mathrm{Cu}^{+}$prefer tetrahedral coordination geometry, $\mathbf{5 4}$ exhibits a different dimer structure with two ligands bound directly to the copper(I) atom. [HAnPPh $\left.\mathrm{ZnBr}_{2}\right]_{2}$ (55), on the other hand, features a four-membered $\mathrm{Zn}_{2} \mathrm{Br}_{2}$ ring with two $\mu^{2}$-bridging bromide ions (Figure 3-82). This fragment connects the two phosphanylanthracene molecules to form the dimer complex. The donor/metal ion ratio is $1: 1$, while it is $2: 1$ in the case of [(MeAnP(NMe $\left.\left.)_{2}\right)_{2} \mathrm{CuBH}_{4}\right](54)$. The cause of this phenomenon can be found in the counter ions of the utilized cations. Because both complexes $\mathbf{5 4}$ and $\mathbf{5 5}$ were crystallized from DCM, no additional donor molecules besides the phosphanylanthracenes and the components of the metal salts were present in the reaction mixtures. In the case of 54, this circumstance led to the formation of a dimer complex with a formal coordination number of 2 , and two agostic interactions between the copper ion and the borate anion. In absence of suitable additional donors, this was the only stable arrangement, because the expelled $\mathrm{PPh}_{3}$ donors are too bulky for coordination in addition to the two phosphanylanthracenes. In the case of $\mathbf{5 5}$, the availability of bromide donors allows the fulfillment of the coordination number of four along with the preferred tetrahedral coordination geometry by formation of the central four-membered $\mathrm{Zn}_{2} \mathrm{Br}_{2}$ ring. This way an orientation of both phosphanylanthracene donors in opposite directions is possible, minimalizing the steric repulsion of the donor molecules. The bromide ions are small enough to assume nearly ideal positions.

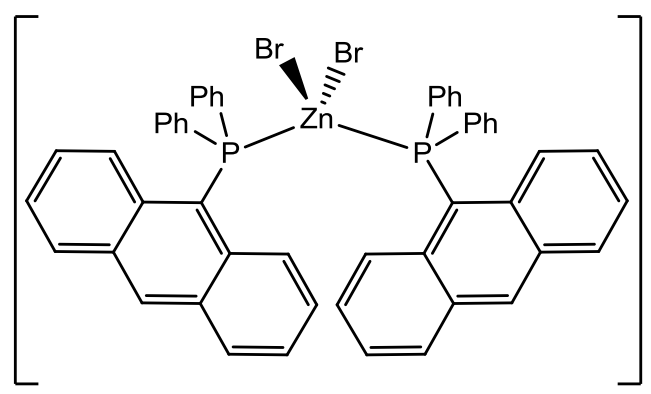

Scheme 3-35: Possible alternative arrangement of $\mathbf{5 5 .}$

Therefore the formation of the dimer $\mathbf{5 5}$ appears to be energetically favored over the imaginable arrangement depicted in Scheme 3-34 with two phosphanylanthracene donors and two bromide ions coordinating the zinc ion, which would be the analogue structure of 54. The phenomenon of of four-membered zinc halide ring formation is best known from its occurance in $\left[\mathrm{Zn}_{2} \mathrm{X}_{6}\right]^{2-}$ complex anions. Coordination of additional 
donors to this aggregate is fairly rare. There are only five reported complexes of phosphorus donors coordinated to a $\mathrm{Zn}_{2} \mathrm{X}_{2}$ cycle, and not a single one is bridged by bromide ions.

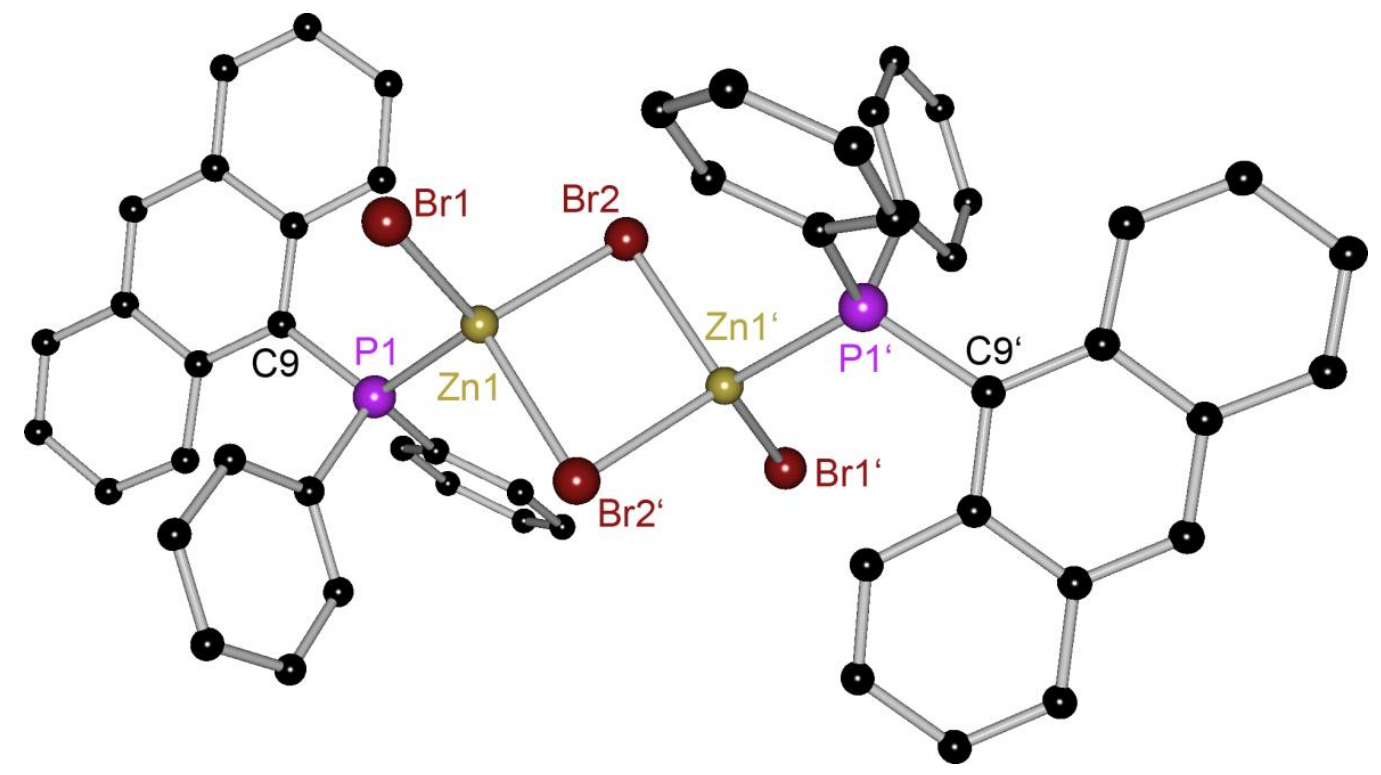

Figure 3-83: Crystal structure of 55, only one of two independent dimers is depicted (molecule 1), lattice solvent molecules and hydrogen atoms are omitted for clarity.

Table 3-31: Selected bond lengths $\left[\AA ̊ \AA\right.$ and angles $\left[{ }^{\circ}\right]$ of 55.

\begin{tabular}{cc|cc}
\multicolumn{2}{c|}{ Molecule 1 } & \multicolumn{2}{c}{ Molecule 2 } \\
P1-Zn1 & $2.3959(6)$ & $\mathrm{P} 2-\mathrm{Zn2}$ & $2.3873(6)$ \\
Zn1-Br1 & $2.3346(3)$ & $\mathrm{Zn2}$-Br3 & $2.3246(3)$ \\
Zn1-Br2 & $2.4448(3)$ & $\mathrm{Zn2}-\mathrm{Br} 4$ & $2.4772(3)$ \\
Zn1-Br2' & $2.4957(3)$ & $\mathrm{Zn2-Br4^{ \prime }}$ & $2.4804(3)$ \\
Br2-Zn1-Br2' & $95.963(11)$ & Br4-Zn2-Br4' & $95.555(11)$ \\
Zn1-Br2-Zn1' & $84.037(11)$ & Zn2-Br4-Zn2' & $84.445(11)$ \\
P1-Zn1-Br1 & $113.702(17)$ & P2-Zn2-Br3 & $115.096(17)$ \\
C9-P1-Zn1 & $118.03(7)$ & C9-P2-Zn2 & $112.97(7)$ \\
Folding & 10.2 & Folding & 5.5 \\
Twist & 7.3 & Twist & 5.6 \\
\hline
\end{tabular}


The asymmetric unit of $\mathbf{5 5}$ contains two half dimer molecules which are symmetryindependent of one another. The corresponding dimer complexes are generated by symmetry operations. Two lattice solvent molecules of DCM are co-crystallized. For the sake of clarity and to visualize the dimer structure, only one of two independent dimers in the structure of $\mathbf{5 5}$ is depicted in Figure 3-82. The $\mathrm{Zn}_{2} \mathrm{Br}_{2}$ rings of both dimers are perfectly planar and the ring angles are nearly identical (Table 3-31). Although the Br2-Zn1-Br2' angle $\left(95.96^{\circ}\right)$ is larger than the Zn1-Br2-Zn1' angle (84.04 $)$, it still deviates strongly from the ideal tetrahedral angle. The angles between all other bonding partners of $\mathrm{Zn} 1$ on the other hand are all larger than $110^{\circ}$, the P1-Zn1-Br2 angle even measures $118.48^{\circ}$. This shows that the formation of the four-membered ring leads to massive distortion of the tetrahedral geometry surrounding Zn1.

As expected, the terminal $\mathrm{Zn} 1-\mathrm{Br} 1$ bond is over $0.1 \AA$ shorter than the bond from $\mathrm{Zn} 1$ to the $\mu^{2}$-bridging $\mathrm{Br} 2$, which can be assigned to the less directed character of the bridging interaction. The P1-Zn bond measures $2.396 \AA$, which is a common value for this type of bond (c.f. Figure 3-89). Except for minor deviations, all these observations apply to both dimers in the structure of 55 .

Noteworthy differences between the two dimers are only found in the geometry of phosphorus bound residues. The tetrahedral geometry surrounding P1 exhibits stronger distortion than the corresponding geometry of bonding partners around P2. This is well described by the C9-P-Zn angles, which measure 118.03 in molecule 1 and 112.97 in molecule 2.
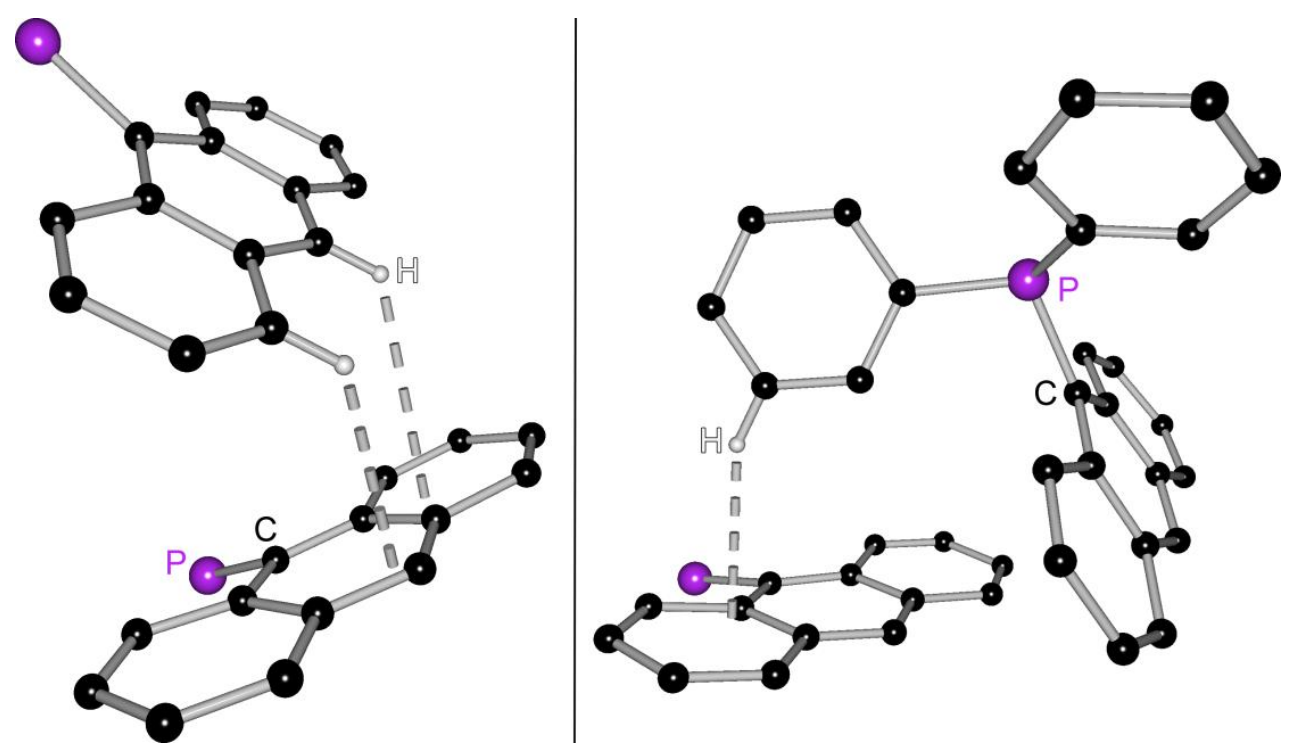

Figure 3-84: $\mathrm{C}-\mathrm{H}^{\cdots} \pi$ interactions in the structure of $\left[\mathrm{HAnPPh}_{2} \mathrm{ZnBr}_{2}\right]_{2}(55), \mathrm{ZnBr}_{2}$ fragments and phenyl groups are omitted for clarity. 
Also the torsion angles of the P-Zn bonds to the anthracene plane differ by nearly $10^{\circ}$. Moreover, the deformations of the anthracene moieties vary between molecule 1 and molecule 2 , the latter showing clearly weaker deformation.

Within the packing motif of $\mathbf{5 5}$ several intermolecular interactions are found. Although there is no appreciable $\pi-\pi$ overlap, several $\mathrm{C}-\mathrm{H} \cdots \pi$ type interactions are present. The hydrogen atoms in 4- and 10-position form $\mathrm{C}-\mathrm{H}^{\cdots} \pi$ bonds with the adjacent $\pi$ system in a "herringbone"-like arrangement (Figure 3-83, left), which has been previously found for three other compounds which were un-substituted in 10position (see 3.4). The Distances and angles of the interactions measure $2.758 \AA / 45.0^{\circ}$ ( $\mathrm{H}-4)$ and $3.271 \AA / 49.8^{\circ}(\mathrm{H}-10)$, respectively. These two medium strength interactions are complemented by a very strong $\mathrm{C}-\mathrm{H}^{\prime \prime} \pi$ bond between a meta-hydrogen atom of a phenyl substituent and a peripheral ring of the neighboring anthracene moiety (Figure $3-83$, right). The short distance of $2.671 \AA$ and the steep angle of $72.4^{\circ}$ to the ring plane underline its strength.

Besides the solid state structures, also the in-solution and solid state fluorescence spectra of $\mathbf{5 5}$ could be acquired from crystalline material. When exposed to UV light, crystals of $\mathbf{5 5}$ exhibit strong green-blue fluorescence (Figure 3-84).

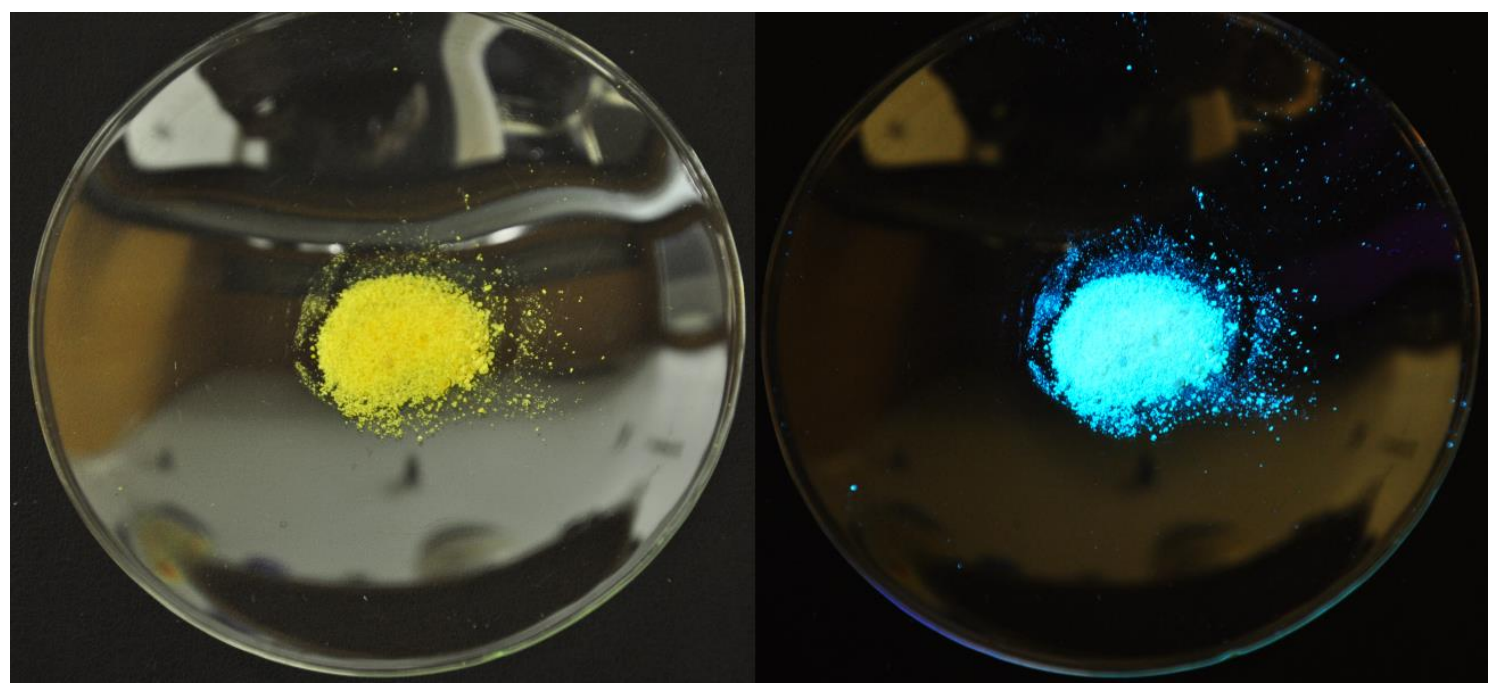

Figure 3-85: Crystalline sample of $\left[\mathrm{HAnPPh}_{2} \mathrm{ZnBr}_{2}\right]_{2}$ (55) in daylight (left) and under exposure to UV light, $\lambda_{\mathrm{Ex}}=366 \mathrm{~nm}$ (right).

The solid state excitation spectrum of $\mathbf{5 5}$ shows a broad band with a width of nearly $150 \mathrm{~nm}$ which reaches a maximum at $438 \mathrm{~nm}$, which is by ca. $15 \mathrm{~nm}$ blue-shifted compared to the emission maxima of the various sulfur oxidized compounds described 
in 3.2 and 3.3 (Figure 3-85, left). The emission spectrum shows no vibrational band structure and shows strong similarities to the emission spectra of previously investigated compounds.
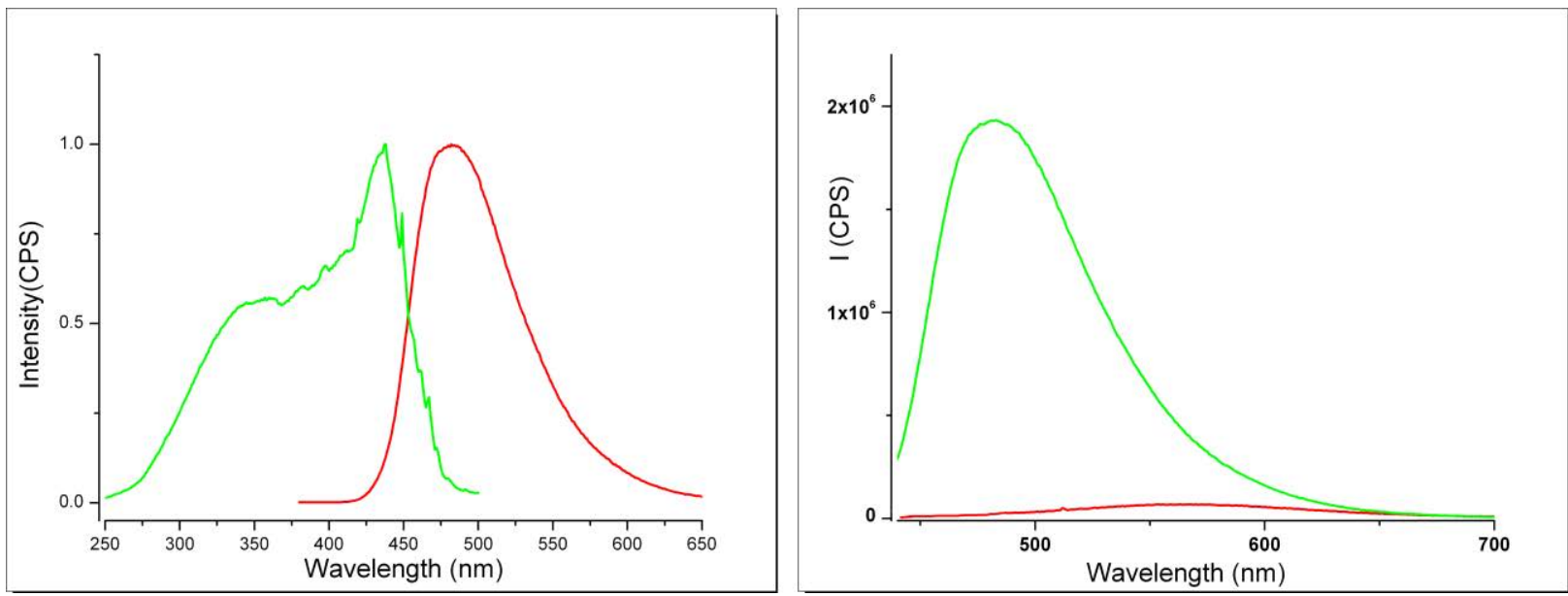

Figure 3-86: Left: normalized solid state excitation (green) and emission (red) spectra of $\left[\mathrm{HAnPPh} \mathrm{ZnBr}_{2}\right]_{2}$ (55); right: emission spectra of $\mathrm{HAnPPh}_{2}(\mathbf{4})$ (red) and [HAnPPh $\left.\mathrm{ZnBr}_{2}\right]_{2}$ (55) (green).

It reaches the emission maximum at a wavelength of $483 \mathrm{~nm}$. Compared to the uncoordinated ligand $\mathrm{HAnPPh}_{2}(4)$, the complex $\left[\mathrm{HAnPPh} \mathrm{ZnBr}_{2}\right]_{2}(55)$ shows a massive increase of emission intensity (Figure 3-85, right). While for the free ligand solid state emission is virtually completely quenched, coordination of $\mathrm{Zn}^{2+}$ leads to a 89-fold enhancement. Although $\mathbf{4}$ and $\mathbf{5 5}$ differ slightly in terms of fluorophore deformation, these minor structural differences are unlikely to induce such extremes in terms of solid state fluorescence.
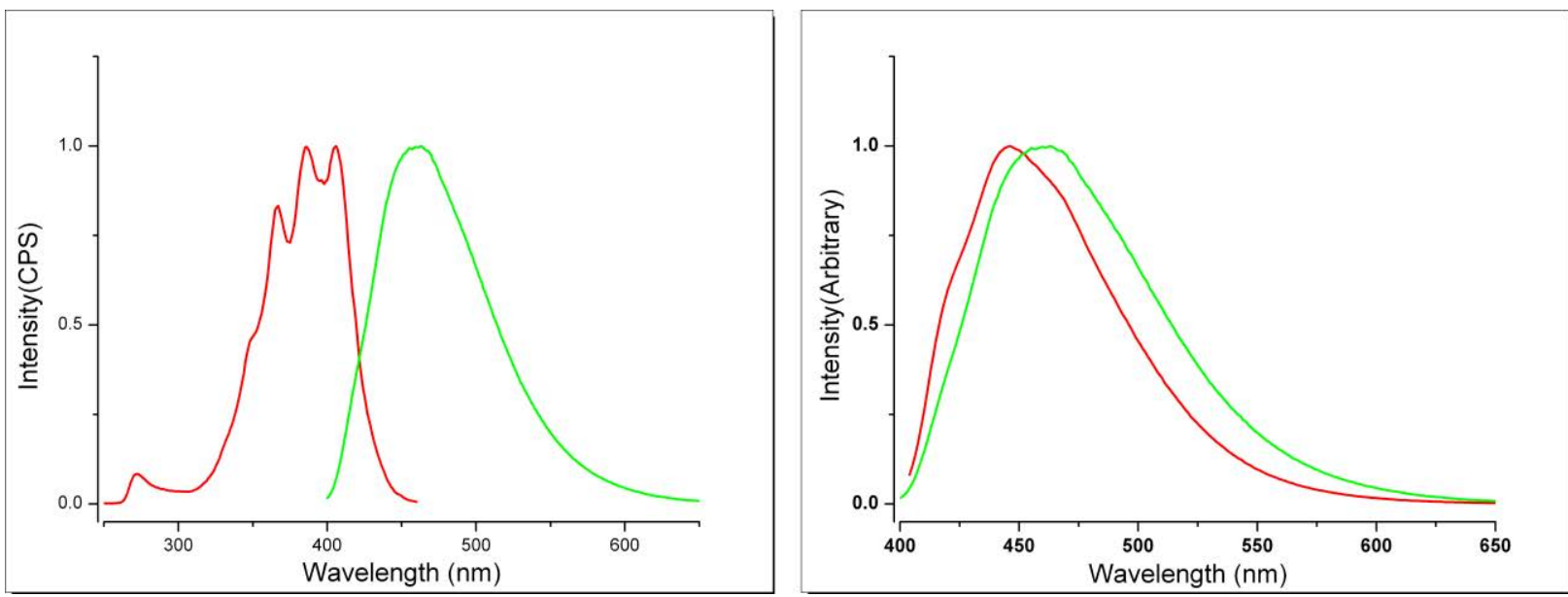

Figure 3-87: Lleft: normalized excitation (red) and emission (green) spectra of [ $\left.\mathrm{HAnPPh}_{2} \mathrm{ZnBr}_{2}\right]_{2}$ (55) $5 \cdot 10^{-5} \mathrm{M}$ in DCM; right: normalized emission spectra of $\mathrm{HAnPPh}_{2}(\mathbf{4})$ (red) and $\left[\mathrm{HAnPPh}_{2} \mathrm{ZnBr}_{2}\right]_{2}(\mathbf{5 5})$ (green). 
The strong emission of $\mathbf{5 5}$ can be ascribed to strong $\mathrm{C}-\mathrm{H}^{\cdots} \pi \mathrm{r}$ bonding in its structure, as especially the interaction between a meta hydrogen atom of a phenyl substituent with the fluorophore very similar to the strongly fluorescent exciplex arrangement in SPAnPS@tol (15) in terms of distance and angle. The extremely weak emission of $\mathrm{HAnPPh}_{2}$ (4) on the other hand cannot be conclusively explained and is probably caused by other factors. While the emission spectrum of the in-solution fluorescence measurement is nearly identical to the solid state emission spectrum, the excitation spectrum shows a narrower band with three distinct maxima (Figure 3-86, left). As observed for the gold(I) complexes in 3.3 and as indicated in 3.1, the coordination of $\mathrm{Zn}^{2+}$ induces is slight red-shift of the emission maximum, which is reflected by the shift of $10 \mathrm{~nm}$ between $\mathrm{HAnPPh}_{2}(4)$ and its zinc complex 55 (Figure 3-86, right).

Additionally, the 10-methyl substituted derivative of $\left[\mathrm{HAnPPh}_{2} \mathrm{ZnBr}_{2}\right]_{2}$ (55) was synthesized. Reaction of the un-oxidized phosphane $\mathrm{MeAnPPh}_{2}$ (13) with $\mathrm{ZnBr}_{2}$ in $\mathrm{DCM}$ afforded the analogue complex of $55,\left[\mathrm{MeAnPPh} \mathrm{ZnBr}_{2}\right]_{2}(56)$. Crystallization at $-30^{\circ} \mathrm{C}$ yielded light yellow crystals which were suitable for X-ray structure determination.

Apart from the stronger disorder of co-crystallized solvent molecules in 56, the structures of $\mathbf{5 5}$ and $\mathbf{5 6}$ are very much alike.

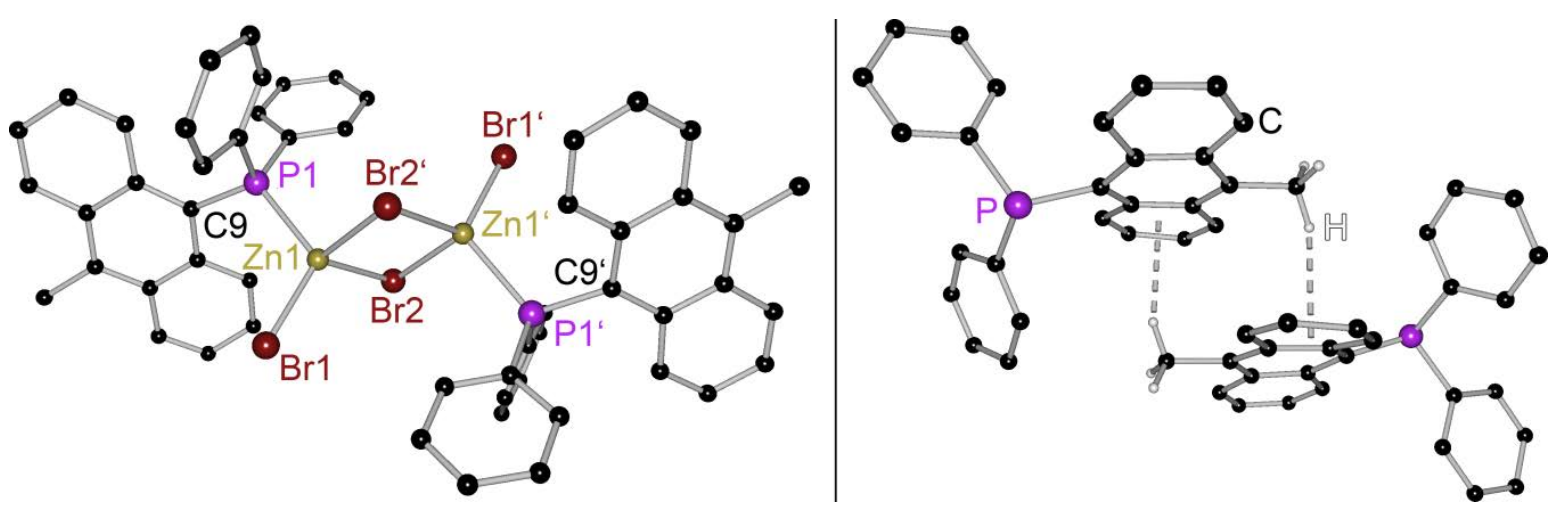

Figure 3-88: Left: crystal structure of [MeAnPPh $\left.\mathrm{ZnBr}_{2}\right]_{2}$ (56), only one of two independent dimers is depicted, lattice solvent molecules and hydrogen atoms are omitted for clarity; right: $\mathrm{C}-\mathrm{H}$ “' $\pi$ bonding in the structure of $\mathbf{5 6 .}$

As observed in the structure of $\mathbf{5 5}$, the asymmetric unit of $\mathbf{5 6}$ contains two half dimer molecules, the second half of each is generated by symmetry operations. The donor molecules are linked by a planar $\mathrm{Zn}_{2} \mathrm{Br}_{2}$ four-membered ring with two $\mu^{2}$ coordinated bromide ions. The formation of these rings can again be attributed to the 
availability of bromide donors, which allow the formation an arrangement of minimum steric repulsion between the bulky phosphanyl anthracene molecules. The ring angles found in both dimer molecules are very close to the corresponding angles in $\mathbf{5 5}$ Table 3-32). The terminal $\mathrm{Zn}-\mathrm{Br}$ bonds are by over $0.1 \AA$ shorter than the bridging $\mathrm{Zn}-\mathrm{Br}$ bonds.

Table 3-32: selected bond lengths $[\AA ̊]$ and angles $\left[{ }^{\circ}\right]$ of 56.

\begin{tabular}{cc|cc}
\hline \multicolumn{2}{c|}{ Molecule 1 } & \multicolumn{2}{c}{ Molecule 2 } \\
P1-Zn1 & $2.4203(19)$ & $\mathrm{P} 2-\mathrm{Zn2}$ & $2.3761(18)$ \\
$\mathrm{Zn1}$-Br1 & $2.3403(10)$ & $\mathrm{Zn2}-\mathrm{Br} 3$ & $2.3272(9)$ \\
Zn1-Br2 & $2.4837(10)$ & $\mathrm{Zn2}-\mathrm{Br} 4$ & $2.4559(10)$ \\
$\mathrm{Br2-Zn1-Br2}$ & $93.36(3)$ & $\mathrm{Br} 4-\mathrm{Zn} 2-\mathrm{Br} 4^{\prime}$ & $95.90(3)$ \\
$\mathrm{P} 1-\mathrm{Zn} 1-\mathrm{Br} 1$ & $113.40(5)$ & $\mathrm{P} 2-\mathrm{Zn} 2-\mathrm{Br} 3$ & $114.47(5)$ \\
Folding & 11.1 & Folding & 7.7 \\
Twist & 7.2 & Twist & 5.9 \\
\hline
\end{tabular}

As indicated by the similar angles, the distortion of the tetrahedral coordination geometry of $\mathrm{Zn} 1$ and $\mathrm{Zn} 2$ is in the same range as observed for $\mathbf{5 5}$. Though it deviates only slightly, the tetrahedral geometry surrounding the phosphorus atoms is slightly less distorted in $\mathbf{5 6}$ than in 55. The torsion angles of the P-Zn bonds to the corresponding anthracene planes measure $39.4^{\circ}$ and $36.8^{\circ}$ in the structure of $\mathbf{5 6}$. These values are closer together and overall larger than observed in $\mathbf{5 5}$. The deformation of the anthracene moieties is moderate at values close to those found for $\mathbf{5 5}$, and the presence of one stronger and one weaker distorted anthracene residue is also in accordance with the findings gathered from the structure of $\mathbf{5 5}$.

Despite the high compliance of the "local" structures of both complexes, the methyl group in 10-position of $\left[\mathrm{MeAnPPh}_{2} \mathrm{ZnBr}_{2}\right]_{2}$ (56) generates a completely different packing motif than found for 55. Instead of a "herringbone" arrangement as in 55, the typical "head-to-tail" type packing is observed, which has been found for a number of other 10-methyl substituted compounds (see 3.3). The resulting intermolecular interactions are consequently also completely unalike. In the structure of $\mathbf{5 6}$ a $\mathrm{sp}^{3}$ $\mathrm{C}-\mathrm{H}^{\cdots} \pi$ bond is found from a methyl $\mathrm{C}-\mathrm{H}$ to a peripheral ring of the opposing 
anthracene moiety with a distance of $2.610 \AA$ at an angle of $60.7^{\circ}$ to the ring plane, which is the shortest $\mathrm{sp}^{3} \mathrm{C}-\mathrm{H}^{\cdots} \pi \pi$ bond observed throughout this thesis (Figure 3-87, right).

Finally, a zinc(II) complex of an oxidized phosphorylanthracene was synthesized. This way the feasibility of zinc coordination via sulfur donors was investigated. Additionally comparison between the coordination of zinc(II) and gold(I) regarding structural properties as well as fluorescence properties was facilitated.

For preparation of a zinc complex of a thiophosphorylanthracene, MeAnPS $\left(\mathrm{NMe}_{2}\right)_{2}$ (21) was reacted with one equivalent of $\mathrm{ZnBr}_{2}$ in $\mathrm{DCM}$ and crystallized at $-30^{\circ} \mathrm{C}$ (Scheme 3-35). [MeAnP(NMe $)_{2}(\mathrm{~S}) \mathrm{ZnBr}_{2}$ ] (57) was obtained as a yellow microcrystalline solid. By recrystallization from DCM single crystals of suitable size for X-ray diffraction experiments were obtained.

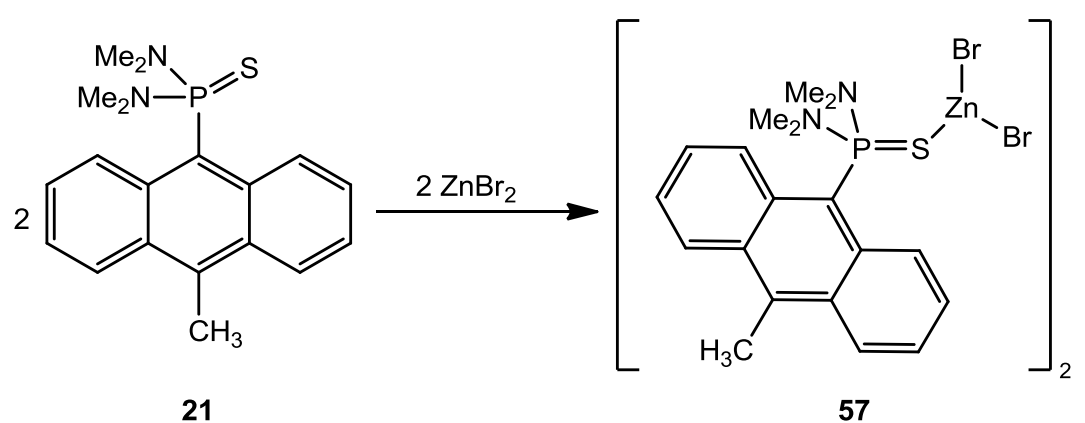

Scheme 3-36: Synthesis of [MeAnP(NMe $\left.)_{2}(\mathrm{~S}) \mathrm{ZnBr}_{2}\right]$ (57).

Analogue to the two previous zinc(II) complexes, a dimer complex with two $\mu^{2}$ bridging bromide ions in a $\mathrm{Zn}_{2} \mathrm{Br}_{2}$ four-membered ring is formed (Figure 3-88). In contrast to the two other zinc complexes which both crystallize in the triclinic space group $P \overline{1}$, [MeAnP(NMe $\left.)_{2}(\mathrm{~S}) \mathrm{ZnBr}_{2}\right]$ (57) crystallizes in the higher symmetric monoclinic space group $P 2_{1} / c$. The asymmetric unit contains one molecule of $\mathbf{5 7}$ which is equivalent to one half dimer.

Again the formation of a planar four-membered $\mathrm{Zn}_{2} \mathrm{Br}_{2}$ ring allows an arrangement of the phosphorylanthracenes in opposite directions, minimizing steric repulsion. The coordination of a double bonded terminal sulfur atom to a $\mathrm{Zn}_{2} \mathrm{X}_{2}$ cycle has not been previously reported. 


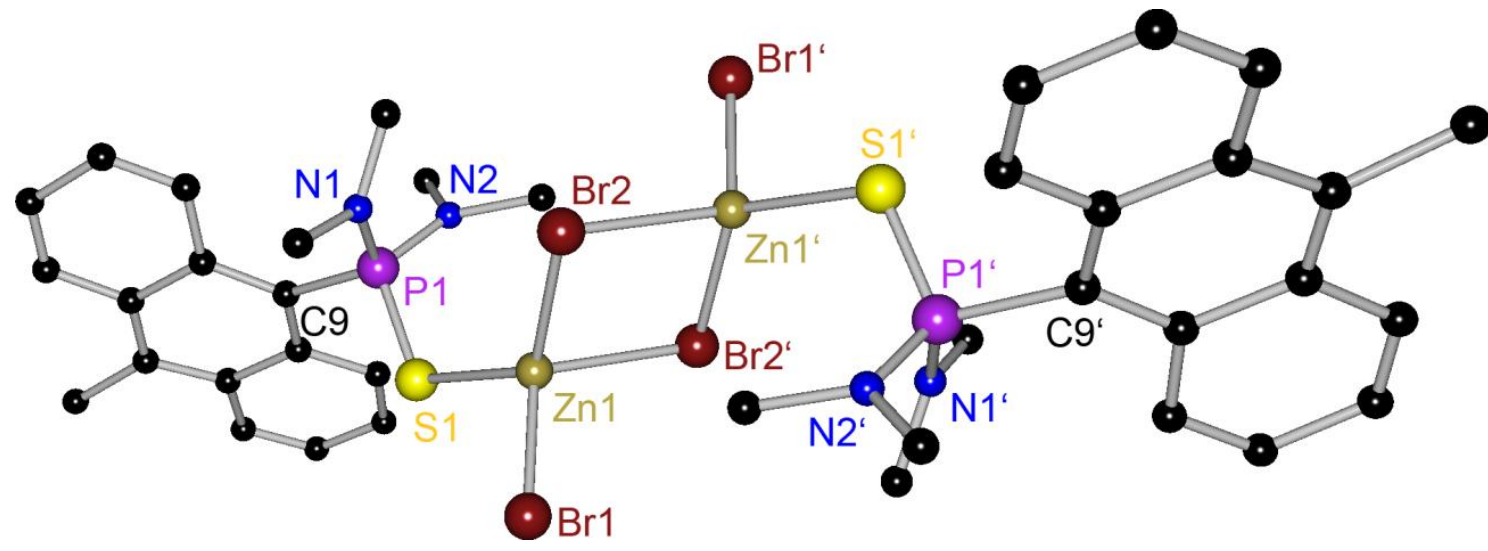

Figure 3-89: Crystal structure of [MeAnP(NMe $\left.)_{2}(\mathrm{~S}) \mathrm{ZnBr}_{2}\right]$ (57), one half of the depicted dimer is generated by symmetry; hydrogen atoms are omitted for clarity.

Table 3-33: selected bond lengths $\left[\AA ̊ \AA\right.$ and angles $\left[{ }^{\circ}\right]$ of $\mathbf{5 7}$.

\begin{tabular}{ll|ll}
\hline S1-Zn1 & $2.3235(11)$ & Zn1-Br2-Zn1' & $87.213(19)$ \\
P1-S1 & $2.0222(13)$ & S1-P1-Zn1 & $113.30(6)$ \\
Zn1-Br1 & $2.3534(5)$ & C9a-C9-P1-S1 & $51.1(3)$ \\
Zn1-Br2 & $2.4702(6)$ & Folding & 11.4 \\
Br2-Zn1-Br2' & $92.786(19)$ & Twist & 6.8 \\
\hline
\end{tabular}

While the $\mathrm{Zn-Br}$ distances deviate only marginally from the values observed in $\mathbf{5 5}$ and $\mathbf{5 6}$ - with a shorter terminal $\mathrm{Zn}-\mathrm{Br}$ distance and a slightly longer bridging $\mathrm{Zn}-\mathrm{Br}$ bond - the S-Zn bond is by $0.1 \AA$ shorter than the P-Zn bonds in $\mathbf{5 5}$ and $\mathbf{5 6}$. This is in accordance with the statistics of P-Zn and S-Zn bonds deposited in the CSD, which display a slightly longer average P-Zn bond distance of $2.400 \AA$ than average S-Zn bond distance $(2.358 \AA)$. The tetrahedral coordination geometry of $\mathrm{Zn} 1$ is even more distorted than in both previous zinc complexes with a Br2-Zn-Br2' angle of only $92.8^{\circ}$. The geometry surrounding P1 is also of distorted tetrahedral character and the observed angles are close to those observed for the mere ligand MeAnPS $\left(\mathrm{NMe}_{2}\right)_{2}(\mathbf{2 1})$. 

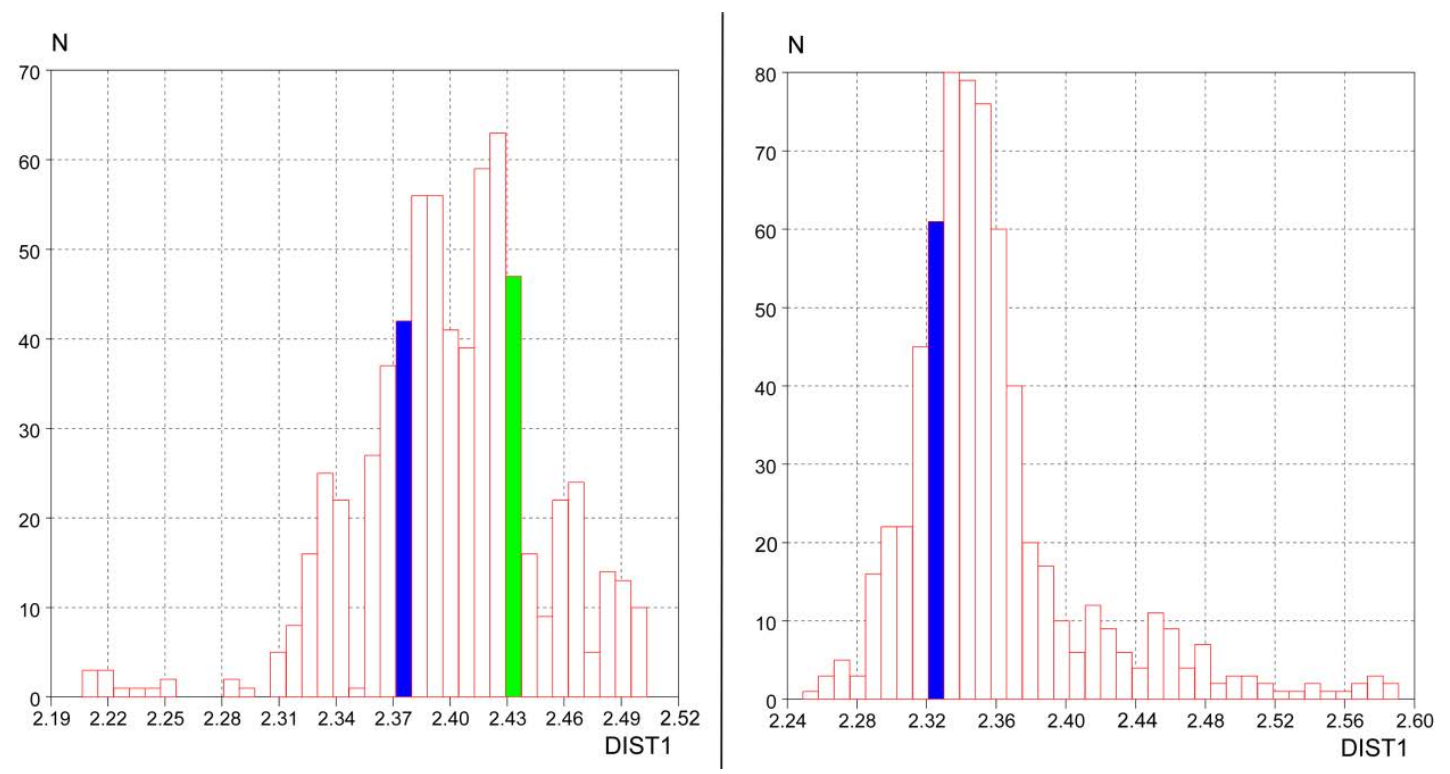

Figure 3-90: Left: CCDC statistics on P-Zn bond distances, minimum (blue) and maximum (green) values for $\mathbf{5 5}$ and $\mathbf{5 6}$ are marked (total average $=2.400 \AA$ ); right: CCDC statistics on S-Zn bond distances, value for 57 (blue) is marked (total average $=2.358 \AA$ ).

The torsion angle of the $\mathrm{P}=\mathrm{S}$ bond to the anthracene plane measures $51.1^{\circ}$ for $\mathbf{5 7}$, which is clearly smaller than for the un-coordinated ligand $\mathbf{2 1}\left(76.5^{\circ}\right)$. The deformation of the anthracene moiety in $\mathbf{5 7}$ is in fact slightly weaker than for $\mathbf{2 1}$, with folding and twist angles of $11.4^{\circ}$ and $6.8^{\circ}$, respectively.
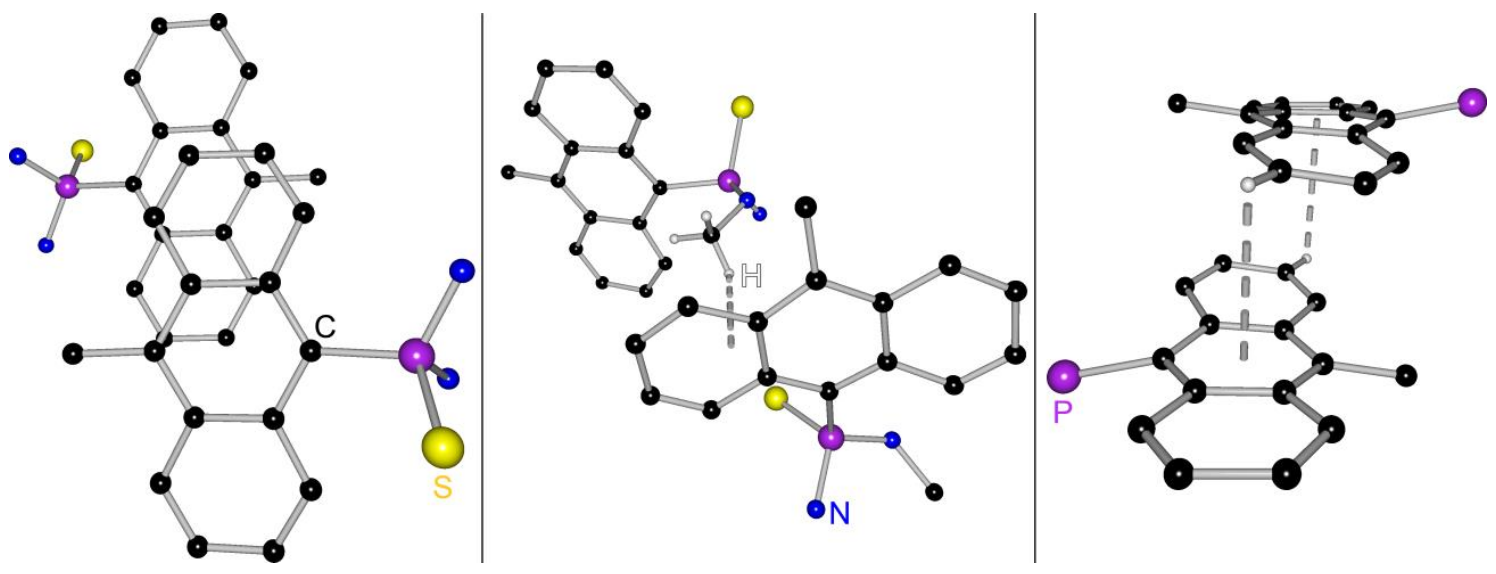

Figure 3-91: Intermolecular interactions in the structure of 57: $\pi-\pi$ overlap (left), $\mathrm{ZnBr}_{2}$ fragments and methyl groups are omitted for clarity; $\mathrm{sp}^{3} \mathrm{C}-\mathrm{H} \cdots \pi$ interaction (center), $\mathrm{ZnBr}_{2}$ fragments and methyl groups are omitted for clarity; $\mathrm{sp}^{2} \mathrm{C}-\mathrm{H}^{\cdots} \pi \mathrm{r}$ interaction (right), $\mathrm{ZnBr}_{2}$ fragments, sulfur atoms and amino substituents are omitted for clarity.

Several intermolecular interactions a present in the packing motif of [MeAnP(NMe $\left.)_{2}(\mathrm{~S}) \mathrm{ZnBr}_{2}\right]$ (57). The "head-to-tail" arrangement of the anthracene moieties which is typical of 10-methyl substituted phosphoryl anthracenes is found, 
producing a $\pi-\pi$ overlap of $\sim 30 \%$ at a distance of $3.54 \AA$ (Figure 3-90, left). Moreover, $\mathrm{C}-\mathrm{H} \cdots \pi$ type interactions are present, namely an $\mathrm{sp}^{3} \mathrm{C}-\mathrm{H} \cdots \pi$ bond from a methyl $\mathrm{C}-\mathrm{H}$ to a peripheral anthracene ring $\left(2.757 \AA / 36.3^{\circ}\right.$ ) (Figure $3-90$, center) and an $\mathrm{sp}^{2} \mathrm{C}-\mathrm{H}^{\cdots} \pi$ interaction (Figure 3-90, right).

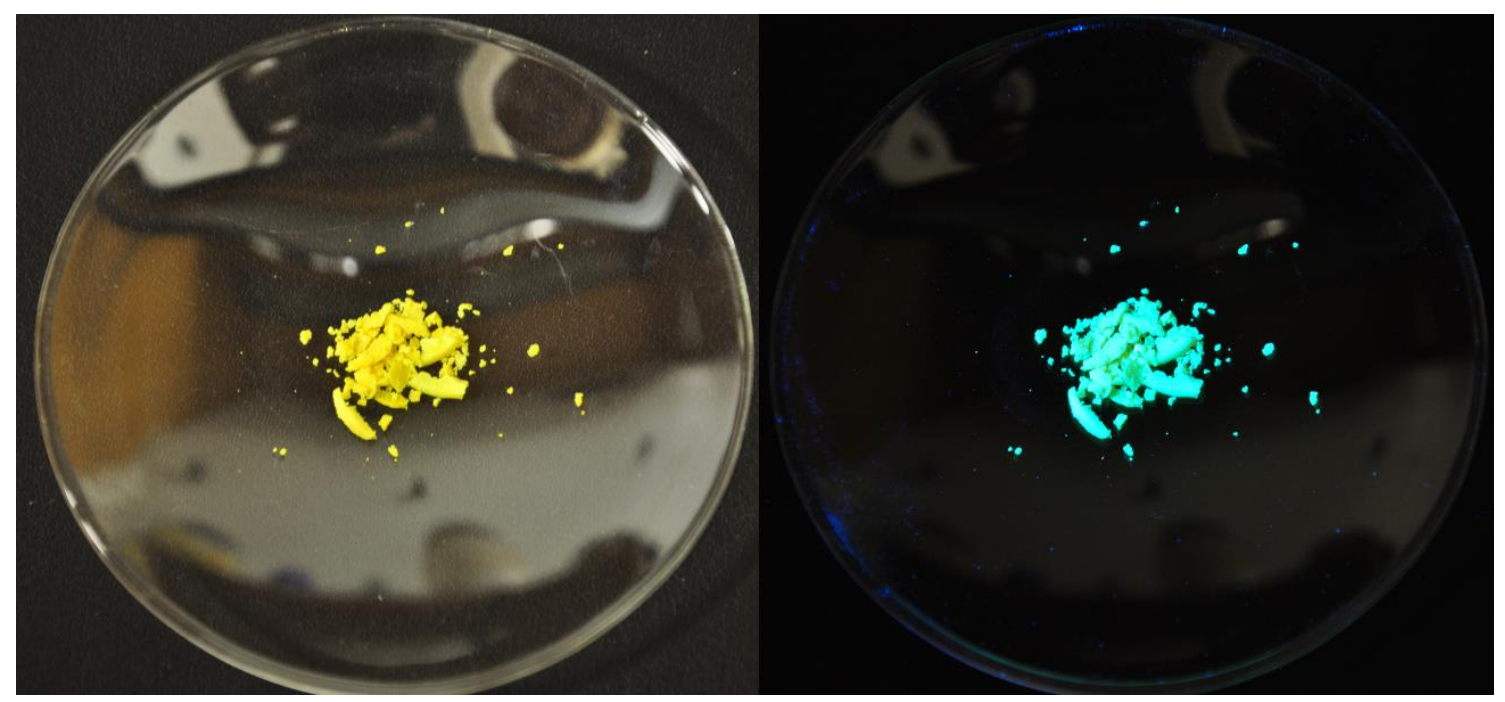

Figure 3-92: [MeAnP(NMe $\left.)_{2}(\mathrm{~S}) \mathrm{ZnBr}_{2}\right](57)$ in day light (left) and under exposure to UV light $(\lambda=366 \mathrm{~nm})$.

[MeAnP(NMe $)_{2}\left(\mathrm{~S}_{\left.\mathrm{ZnBr}_{2}\right]}\right.$ (57) exhibits striking solid state fluorescence over a wide range of excitation wavelengths (Figure 3-91). Excitation wavelengths between $320 \mathrm{~nm}$ and $460 \mathrm{~nm}$ almost constantly generate strong emission intensities, reaching a maximum at $449 \mathrm{~nm}$. The Single emission maximum lacks a band structure, which is in accordance with the observations made for the free ligand $\mathbf{2 1}$ and the corresponding gold(I) complex 22.
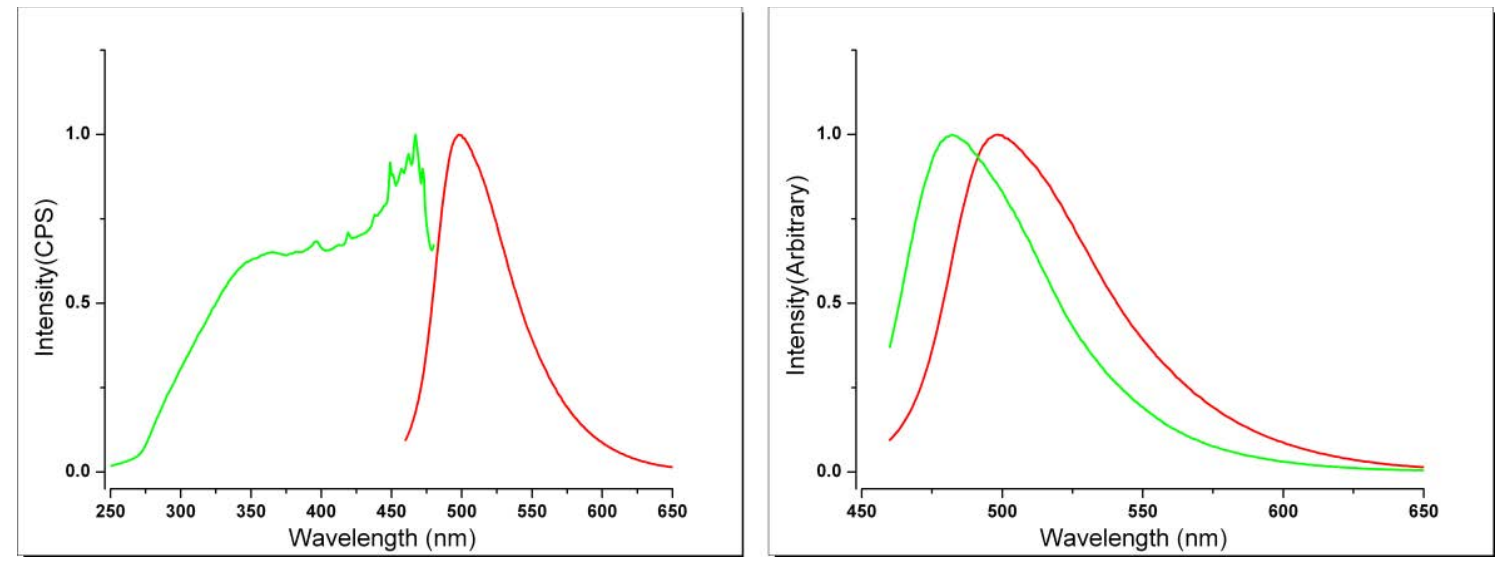

Figure 3-93: left: normalized solid state excitation (green) and emission (red) spectra of 57; right: normalized solid state emission spectra of MeAnPS(NMe $)_{2}$ (21) (green) and 57 (red). 
The coordination of zinc(II) induces a red-shift of the emission maximum by $16 \mathrm{~nm}$ compared to the free ligand (Figure 3-92, right). This is the exact bathochromic shift which was also found for the gold(I) complex 22. Apparently, the coordination of metals affects the emission wavelength, but not the type of metal coordinated. The observed emission intensity of [MeAnP( $\left.\left(\mathrm{NMe}_{2}\right)_{2}(\mathrm{~S}) \mathrm{ZnBr}_{2}\right]$ (57) is doubled compared to the free ligand 21, making it one of the strongest emitting compounds described so far. The intensity is even 50\% stronger than of the gold complex 22 (Figure 3-93).

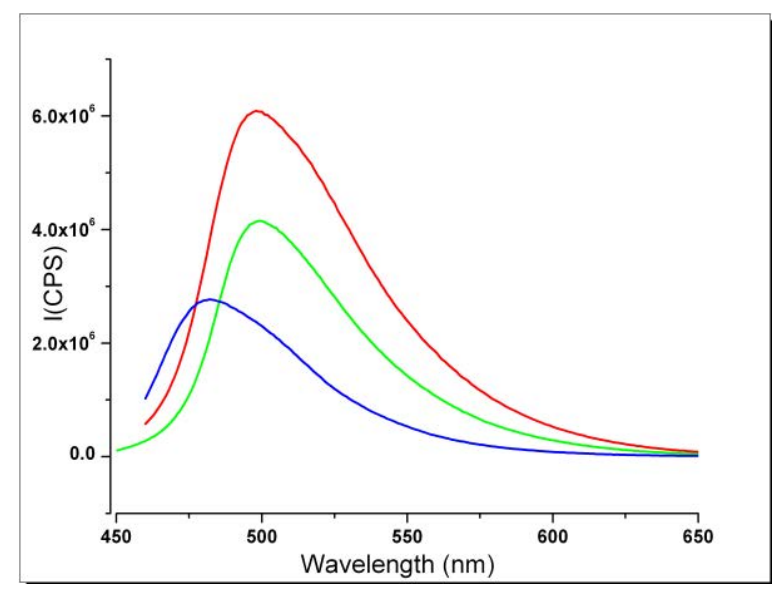

\begin{tabular}{|c|c|c|}
\hline \multicolumn{3}{|c|}{$\begin{array}{l}\text { Table 3-34: Maximum emission } \\
\text { wavelengths and relative emission } \\
\text { intensities of } \mathbf{2 1}, \mathbf{2 2} \text {, and } \mathbf{5 7} \text {. }\end{array}$} \\
\hline & $\lambda_{\max }[\mathrm{nm}]$ & $I_{\text {rel. }}$ \\
\hline 21 & 483 & 0,45 \\
\hline 22 & 499 & 0,67 \\
\hline 57 & 499 & 1,00 \\
\hline
\end{tabular}

Figure 3-94: Solid state emission spectra of MeAnPS( $\left.\mathrm{NMe}_{2}\right)_{2}$ (21) (blue), [MeAnP(NMe $\left.)_{2}(\mathrm{~S}) \mathrm{AuCl}\right]$ (22) (green), and $\left[\mathrm{MeAnP}\left(\mathrm{NMe}_{2}\right)_{2}(\mathrm{~S}) \mathrm{ZnBr}_{2}\right]$ (57) (red).

\subsection{Boranyl Anthracenes}

Having thoroughly investigated and analyzed the syntheses, structures and fluorescence properties of numerous phosphane-substituted anthracene derivatives, a new compound class was opened up. The following chapter is dedicated to synthesis and fluorescence behavior of boranylanthracenes. By comparing their structural features and fluorescence phenomena to those of phosphoryl anthracenes described in previous chapters, similarities and differences between these compound classes will be illustrated.

Borane substituted anthracenes have previously been used as precursor molecules in the syntheses of light emitting devices and chromophores which raised interest in the use of borane substituents for the synthesis of fluorescent compounds. ${ }^{[85]}$ Moreover, boranes have also been used in sensing devices for water by Ooyama et. 
al. ${ }^{[86]}$ Not only the direct influence of borane substituents on emission properties was of note, also possible applications in sensing devices fueled interest in these compounds. Boranes are Lewis acidic compounds which are known to form Lewis acid/base pairs. The Lewis acidity of boranes can be influenced by introduction of electron withdrawing substituents. In 2002, Yamashita et al. synthesized a boranyl anthracene which impressively underlines this phenomenon. Their 9-catecholeboranyl1,8-bis(dimethylamino)-anthracene carries two amino groups in 1,8-position as Lewis bases. As Figure 3-94 shows, intramolecular formation of a Lewis acid/base pair leads to a distinct distortion of the molecule's geometry. ${ }^{[87]}$ The formed N1-B1-bond measures $1.809 \AA$, and leads to deformation of the initially planar geometry around the boron atom towards tetrahedral geometry. Furthermore the formation of the fivemembered ring leads to displacement of the boron atom from the C10 “c9 axis. The C9-B1-bond now encloses an angle of $13.3^{\circ}$ with this axis.

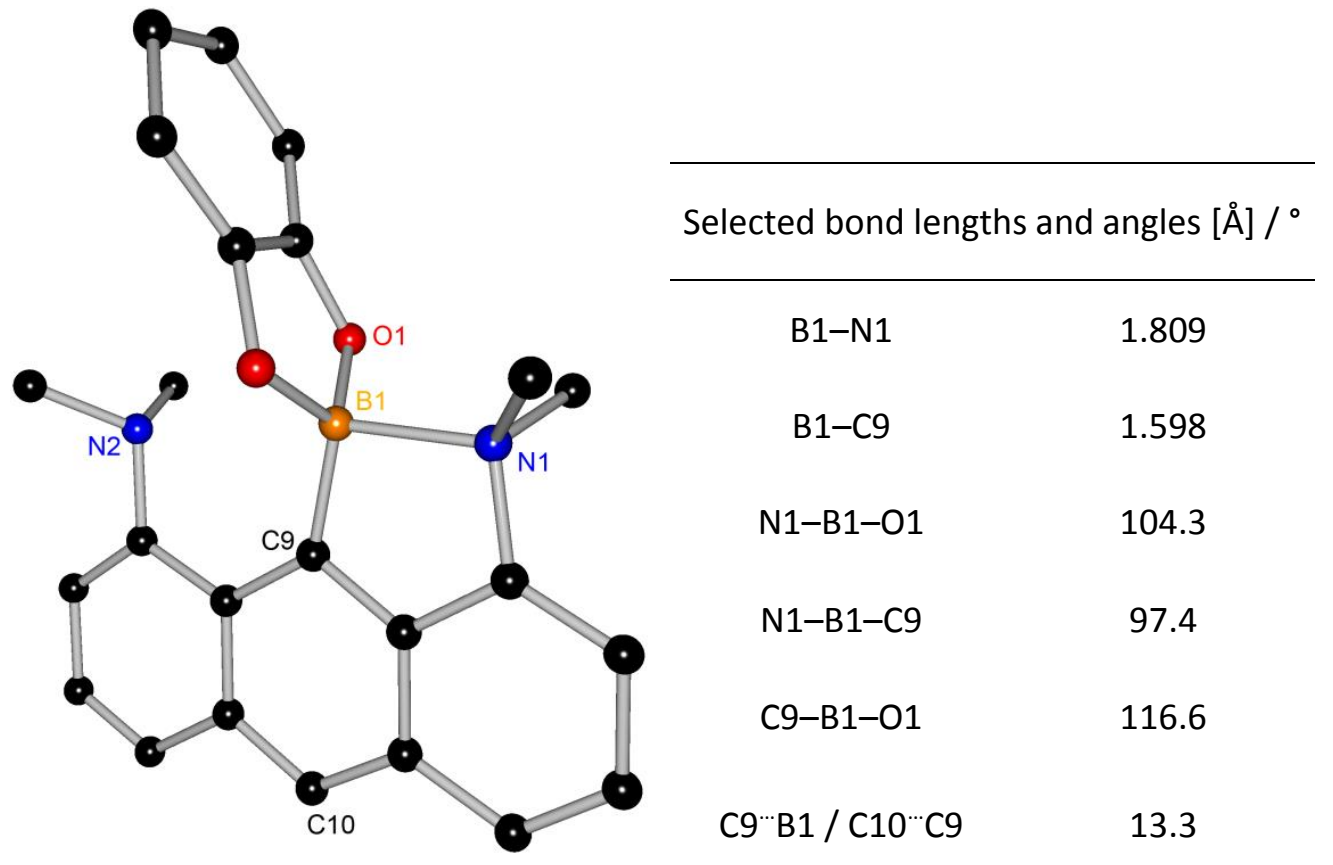

Figure 3-95: Crystal structure of 9-catecholeboranyl-1,8-bis(dimethylamino)anthracene by Yamashita et al. ${ }^{[87]}$ Hydrogen atoms are omitted for clarity.

These deformations indicate the strength of the N-B-interaction and the tendency of adduct formation between Lewis bases and electron deficient boranes. Similar observations were also made for the phosphane analogue. ${ }^{[88]}$ Though Yamashita et al. did not investigate the fluorescence properties of this molecule and rather used the anthracene moiety as a rigid backbone for their compounds, this compound class 
might bear significant potential in the field of chemical sensors. Because the formation of a Lewis acid/base pair generates a formal negative charge at the boron atom (which again is bound directly to the fluorophore), this process is likely to alternate the electronic properties of the fluorophore (Scheme 3-36). Hence, the coordination of a Lewis base to the borane may trigger a shift of the molecule's emission maximum (in solution or solid state) or lead to fluorescence suppression or enhancement. The inverse structural motif of adduct formation between anthracene bound amines and boranes in a PET on/off sensing device has also been reported. ${ }^{[89]}$ While in Yamashita's 9-catecholeboranyl-1,8-bis(dimethylamino)anthracene the Lewis basic donor atoms are in permanent proximity to the boron atom, a hypothetic sensor molecule would carry no donor atoms in 1,8-position, allowing intermolecular bonding of Lewis bases (Scheme 3-36).

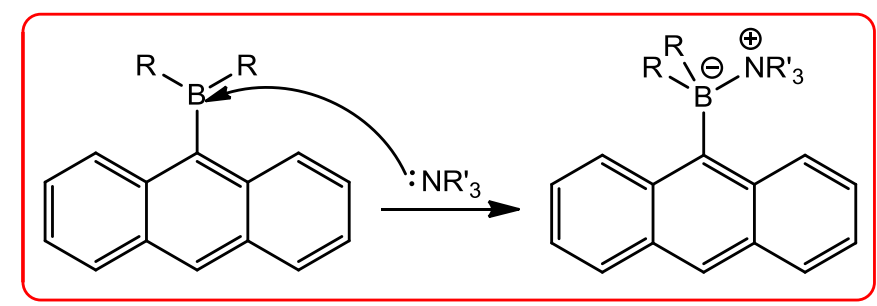

Scheme 3-37: Generation of a negative charge at the boron atom by intermolecular formation of a Lewis acid/base adduct.

Boron bound substituents could be used to control the Lewis acidity of the sensor molecule and therewith the selectivity towards different donors. Further selectivity could be achieved via steric control by variation of the steric demand of the boron bound substituents or by introduction of bulky substituents in the 1,8-positions of the anthracene moiety. The first steps of validating the general concept of such a sensor system will be described in the following pages. 


\subsubsection{Synthesis}<smiles>Clc1c2ccccc2c(Br)c2cc(C(Br)C(Br)Br)ccc12</smiles>

58<smiles>[R]B([R])c1cccc2c(Cl)c3ccccc3c(B([R])[R])c12</smiles>

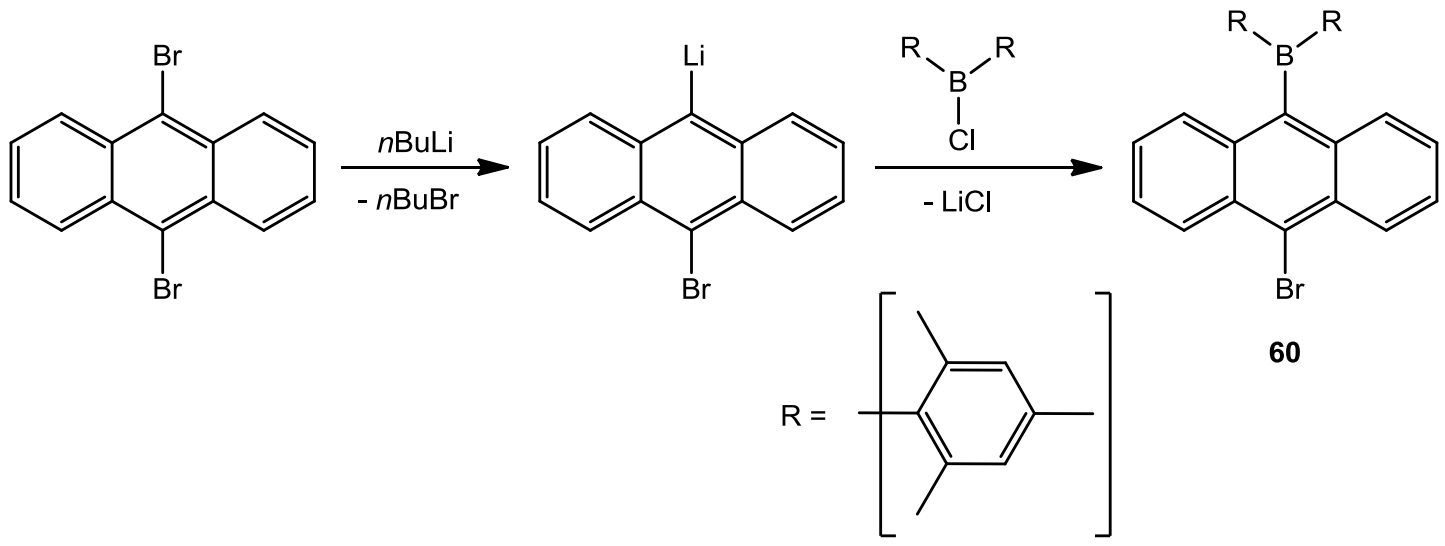

Scheme 3-38: Synthesis of 58, 59 and $\mathbf{6 0 .}$

9-Chloro-10-(catecholeboranyl)anthracene ClAnBCat (58), 9-Chloro-10-(diisopinocampheylboranyl)anthracene $\mathrm{ClAnBIPC}_{2}$ (59), and 9-Bromo-10-(dimesithylboranyl)anthracene BrAnBMes$_{2}(60)$ were synthesized via similar reaction pathways. 9-Bromo-10-chloroanthracene and dibromoanthracene, respectively, were mono lithiated in diethyl ether at $-15 \mathrm{C}$ using $n$-BuLi and subsequently reacted with chloro boranes (dissolved in $5 \mathrm{~mL}$ of diethyl ether) under elimination of lithium chloride (Scheme 3-37). After stirring the reaction mixtures overnight, the solvent was evaporated, the crude products were dissolved in anhydrous DCM and lithium chloride 
was removed by filtration. The solvent was again evaporated and the products were re-crystallized from THF (58), MeCN (59) and THF/toluene (60). This way, single crystals of $\mathbf{5 9}$ and $\mathbf{6 0}$ which were suitable for X-ray diffraction experiments were obtained.

Because - as stated before - the 9- and 10-positions of the anthracene moiety strongly influence one another, all synthesized boranylanthracenes bear an electron withdrawing halogen substituent in 9-position. This way the -1 effect should be transferred to the boranyl substituent in 10-position, leading to stronger electron deficiency at the boron atom. Especially in the case of ClAnBCat (58) this should lead to an even stronger tendency of bonding to Lewis bases than observed for Yamashita's 9catecholeboranyl-1,8-bis(dimethylamino)anthracene.

\subsubsection{In-solution Fluorescence}

Even if only exposed to daylight, solutions of $\operatorname{BrAnBMes}_{2}(60)$ reveal a green-blue fluorescent glow, which underlines the conspicuous fluorescent behavior of this compound class (Figure 3-95).

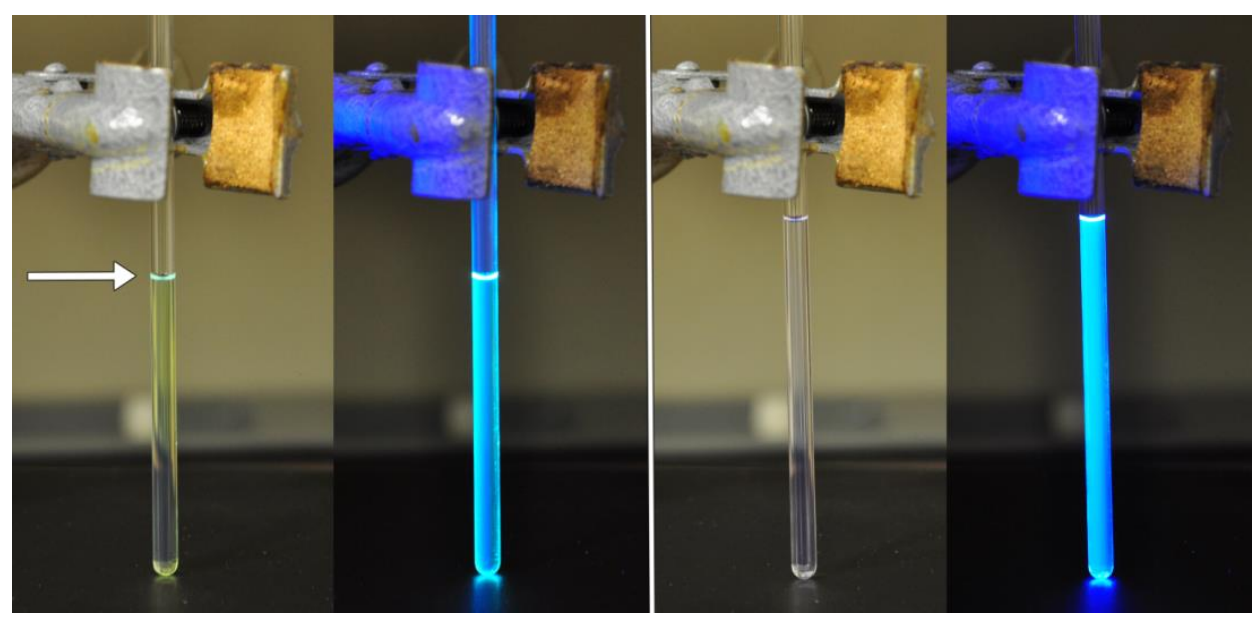

Figure 3-96: Left side: DCM solution of $\operatorname{BrAnBMes}_{2}(60)$, under exposure to daylight (far left), fluorescent glow highlighted with an arrow; under irradiation with UV light, $\lambda=366 \mathrm{~nm}$ (mid. Left). Right side: DCM solution of BrAnBCat (58); under exposure to daylight (mid. right), under irradiation with UV light, $\lambda=366 \mathrm{~nm}$ (far right). 

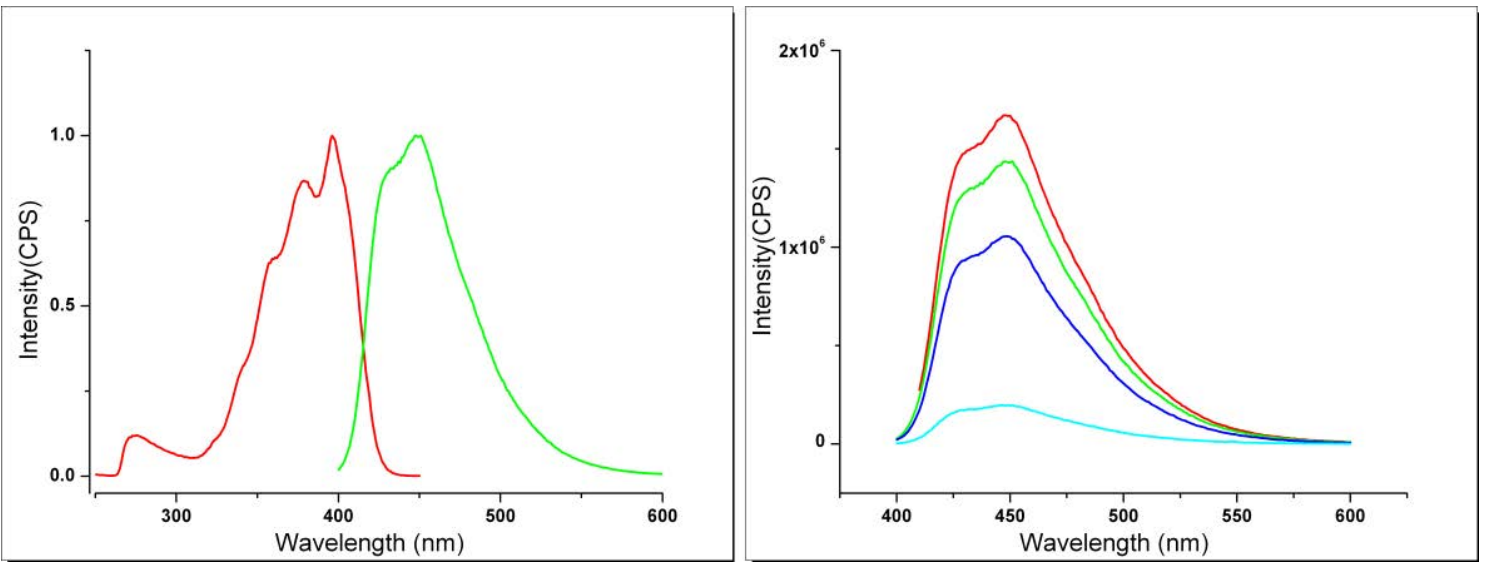

Figure 3-97: Left: normalized excitation (red) and emission (green) spectra of ClAnBCat (58); right: emission spectra of ClAnBCat (58) at $274 \mathrm{~nm}$ (cyan), $359 \mathrm{~nm}$ (blue), $379 \mathrm{~nm}$ (green), and $396 \mathrm{~nm}$ (red).

This first impression is confirmed by fluorescence experiments which certify the strong fluorescence of ClAnBCat (58). Though the emission spectrum shows a small shoulder at $425 \mathrm{~nm}$, a defined band structure is not visible, very much like observed for the majority of alkyl- and arylphosphane substituted compounds inspected before. The excitation spectrum exhibits a single broad band, and the excitation and emission maxima are well separated by $\sim 50 \mathrm{~nm}$. The Emission maximum is also red-shifted by nearly $50 \mathrm{~nm}$ compared to unsubstituted anthracene (Figure 3-96).
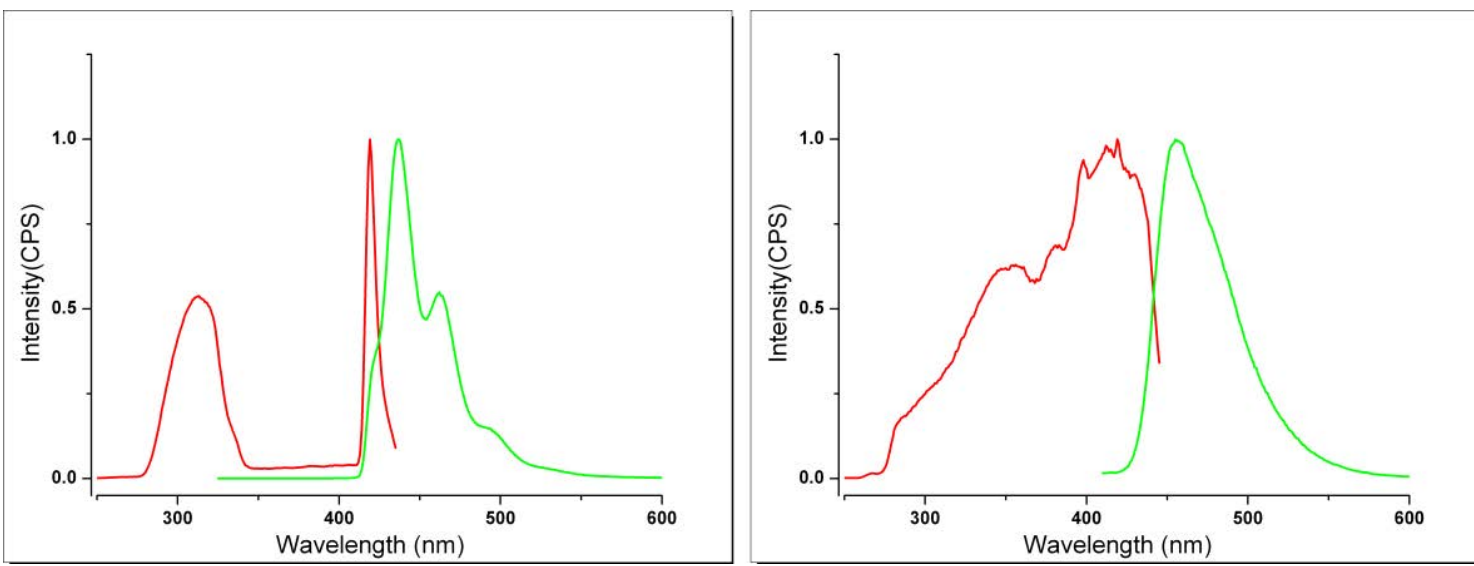

Figure 3-98: left: normalized excitation (red) and emission (green) spectra of $\mathrm{ClAnBCy}_{2}$ (59); right: normalized excitation (red) and emission (green) spectra of BrAnBMes $_{2}$ (60).

When changing from strongly electron withdrawing oxygen substituents at the boron atom as in ClAnBCat (58) to alkyl substituents in $\mathrm{ClAnBIPC}_{2}$ (59), these drastic differences become apparent in the fluorescence spectra (Figure 3-97, left). Accordingly, excitation and emission spectra of $\mathrm{CIAnBIPC}_{2}(59)$ differ significantly from 
those of 58. The excitation spectrum now shows two maxima, which are separated by over $100 \mathrm{~nm}$.

Table 3-35: maximum excitation andemission wavelengths of 58,59 , and 60 .

\begin{tabular}{lcc} 
& $\lambda_{\max }(\mathrm{Ex})[\mathrm{nm}]$ & $\lambda_{\max }(\mathrm{Em})[\mathrm{nm}]$ \\
\hline ClAnBCat (58) & 396 & 449 \\
$\mathrm{ClAnBIPC}_{2}$ (59) & 313,419 & 437 \\
BrAnBMes $_{2}(60)$ & 419 & 455 \\
\hline
\end{tabular}

Also the emission spectrum features a typical anthracene band structure and the emission maximum lies at a shorter wavelength of only $437 \mathrm{~nm}$. Furthermore excitation and emission maximum are separated by merely $18 \mathrm{~nm}$, which is articulately

less than found for ClAnBCat (58). The overall fluorescence intensity is also clearly weaker.

Moving on to BrAnBMes $2(60)$, the boron bound substituents are now aromatic, and hence of clearly more electron withdrawing character than the aliphatic substituents in (59). This alteration is again visible in the fluorescence properties of 60 . Both the excitation and emission spectrum bear resemblance to those of ClAnBCat (58), showing a single broad excitation band and a broad emission band lacking vibrational structures. The emission maximum at $455 \mathrm{~nm}$ is red-shifted even farther than the emission maximum of $\mathbf{5 8}$. The only serious difference lies in the distance between excitation and emission maximum, which adds up to only $36 \mathrm{~nm}$ for $\mathbf{6 0}$.

Derivable tendencies are that boron bound $+\mid$-alkyl substituents lead to emission properties similar to un-substituted anthracene and to a small gap between excitation and emission maxima. This gap increases the stronger the electron withdrawing character of the substituents becomes $(59<60<58)$. In comparison to a corresponding phosphanyl anthracene which also carries a halogen substituent in 9position, the fluorescence phenomena of $\mathrm{ClAnBCat}(58)$ and $\operatorname{BrAnBMes}_{2}(60)$ can be regarded as similar in respect to the resulting emission wavelengths (Figure 3-98). In terms of emission intensity, the boranyl anthracenes produce largely stronger fluorescence than their phosphane substituted counterparts. This finding can explained by the fact that there is a clearly smaller probability of electron transfer from the electron deficient boranyl substituent to the excited fluorophore than from the electron rich phosphanyl substituent. Hence, the observed quenching in solution is stronger for compounds carrying the latter substituent. 


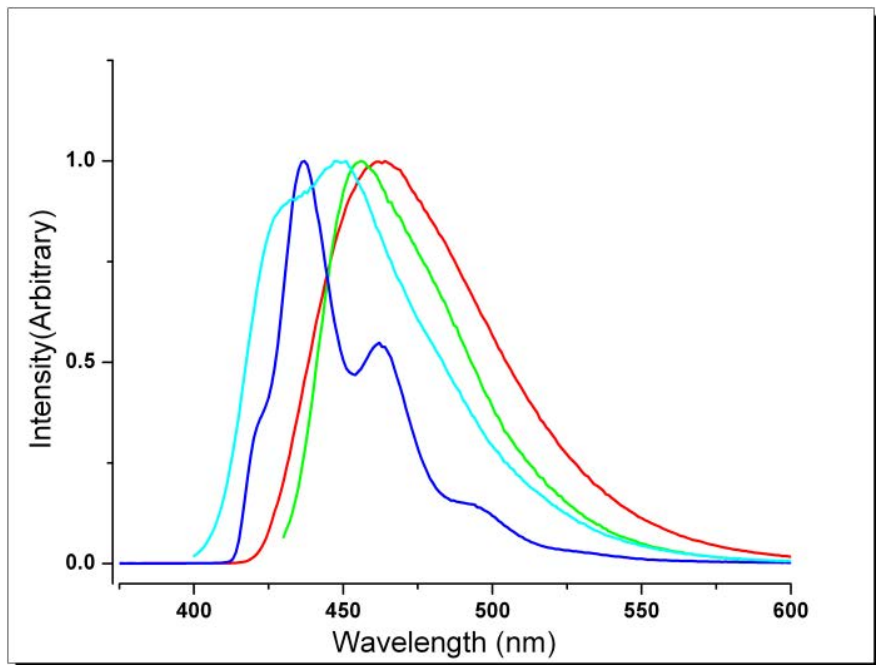

Figure 3-99: Normalized emission spectra of ClAnBCat (58, cyan), $\operatorname{ClAnBIPC}_{2}$ (59, blue), $\operatorname{BrAnBMes}_{2}$ (60, green), and $\mathrm{BrAnPPh}_{2}(\mathbf{9}, \mathrm{red})$.

In order to function as a potential sensor, the observed fluorescence phenomena of 58-60 must be altered to a detectable degree by formation of a Lewis acid/base adduct, resulting in a concrete "yes/no" signal. By estimating the effect of the used substituents, 58-60 can be ordered by Lewis acidity. The aliphatic, aromatic and hetero atomic substituents lead to an order of $\mathrm{ClAnBIPC}_{2}(\mathbf{5 9})<\mathrm{BrAnMes}_{2}(\mathbf{6 0})<\mathrm{ClAnBCat}(\mathbf{5 8})$ of acidity. The steric demand of the substituents also differs significantly, from the very bulky isopinocampheyl substituent, to the fairly bulky mesityl substituent to the planar and undemanding catachole substituent, which leads to a reverse order of the compounds 58-60 in terms of steric shielding of the boron atom: $\mathrm{CIAnBIPC}_{2}$ (59) > BrAnMes $_{2}(60)>$ ClAnBCat $(58)$. These two factors combined lead to a very weak expected tendency of adduct formation for $\mathrm{CIAnBIPC}_{2}$ (59), a slightly stronger tendency for $\mathrm{BrAnMes}_{2}$ (60) and the clearly strongest for ClAnBCat (58).

To monitor the adduct formation in situ via fluorescence spectroscopy, solutions of 58-60 in non-Lewis basic solvents (hexane, heptane, toluene) were prepared. The analyte solutions of Lewis bases were prepared in corresponding solvents. The following Bases were used: MeCN, NEt 3 , TMEDA, $\mathrm{PPh}_{3}$, THF. These donors were chosen due to their variety in steric demand (from linear $\mathrm{MeCN}$ to bulky $\mathrm{PPh}_{3}$ ) and due to the different heteroatoms included. Because amines are in general potential quenchers of anthracene fluorescence, the applied concentrations were kept low. All experiments were carried out under inert gas atmosphere to avoid decomposition of 58-60 by oxidation or hydrolysis. 
Unfortunately, no noteworthy alterations of the emission properties upon addition of Lewis bases could be found for any of the compounds. A possible explanation is that the acid/base interaction is too weak in solution. This may be assigned to too weak Lewis acidity of 58-60. Even the supposed strongest Lewis acid CIAnBCat (58) does not offer optimum electron pare acceptor properties, as the boron bound oxygen atoms bear lone pairs which can interact with the empty p-orbital of the boron atom, reducing its electron deficiency (Scheme 3-38).
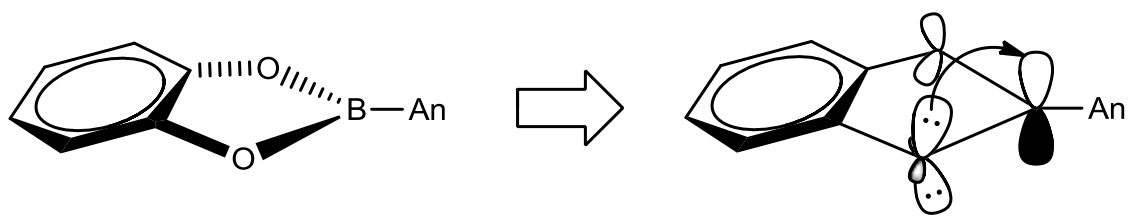

Scheme 3-39: Interaction of an oxygen lone pair with the empty p-orbital of the boron atom.

Additionally, the steric demand of the boron bound substituents in $\mathbf{5 9}$ and $\mathbf{6 0}$ is quite large which may also hinder the adduct formation. Moreover, the low concentrations of the sample and analyte solutions might decelerate the adduct formation to a degree that in situ fluorescence measurements are not suitable for detecting this process. Finally, the interaction between Lewis acid and base may generally be too weak to alter the electronic properties of the fluorophore in solution. Despite these unsatisfying results, the general concept presented above is not disproved. Synthetic alterations of the boranyl anthracenes could solve the problems stated, or at least verify the crucial factors inhibiting the function of such a detecting device. Possible solutions will be addressed in 3.6.5.

\subsubsection{Solid State Structures}

The crystal structures of $\mathrm{ClAnBIPC}_{2}$ (59) and $\mathrm{BrAnBMes}_{2}(\mathbf{6 0})$ were also determined. Despite considerable efforts, no crystals of ClAnBCat (58) of sufficient quality could be obtained. CIAnBIPC 2 (59) crystallizes in the orthorhombic space group $P 2_{1} 2_{1} 2_{1}$, and the asymmetric unit contains one molecule of 59. All B-C-bonds are nearly identical at $1.58 \AA$, which is a common bond distance between carbon an boron. 


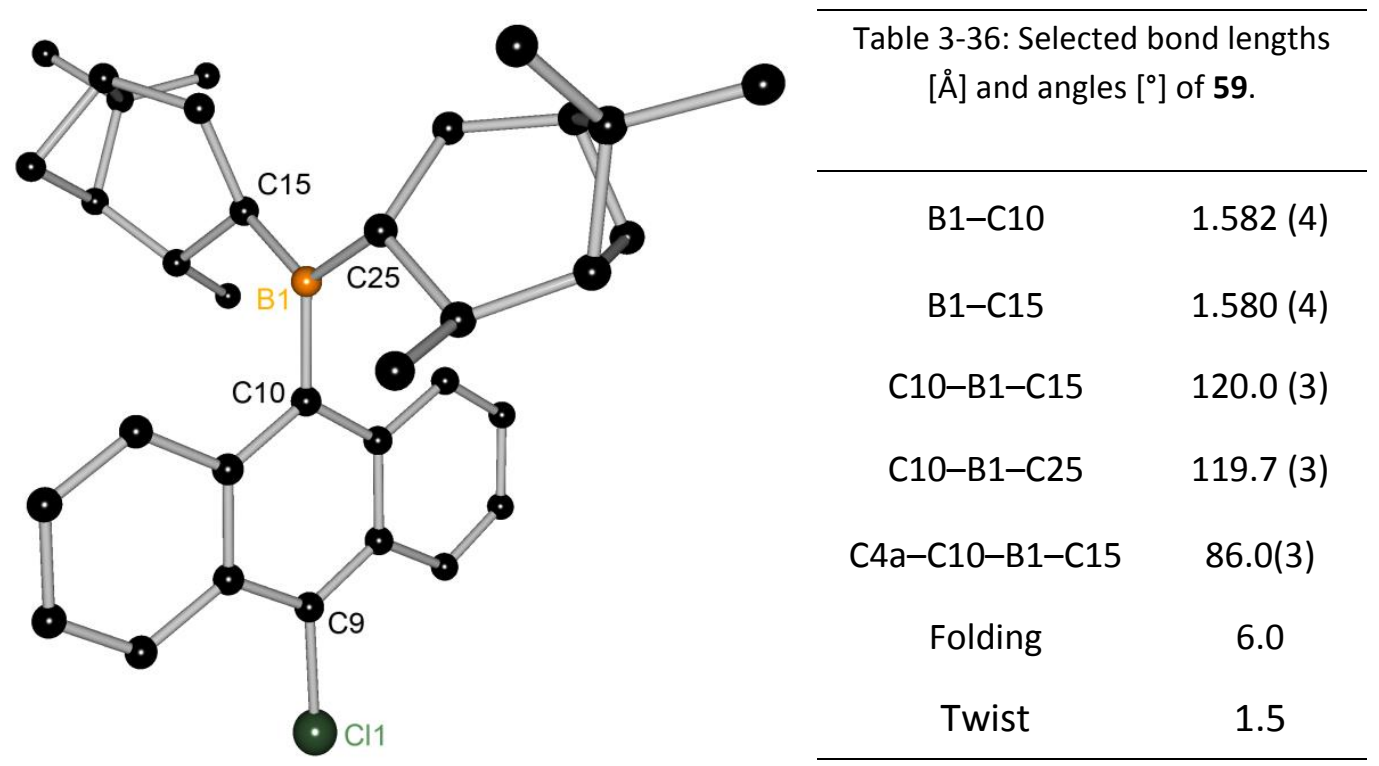

Figure 3-100: Solid state structure of $\mathrm{ClAnBIPC}_{2}$ (59), hydrogen atoms are omitted for clarity.

The angles around the boron atom almost perfectly match the expected $120^{\circ}$ angle. Surprisingly, none of the six methyl groups show rotational disorder. Despite the bulky substituents at the boron atom, the anthracene moiety is weakly distorted, showing folding and twist angles of only $2.6^{\circ}$ and $1.5^{\circ}$. This can be attributed to the nearly symmetrical alignment of the substituents on opposite sides of the anthracene plane (Figure 3-100, left). The torsion angle of the B1-C15-bond to the anthracene plane is nearly right-angled at $86.0^{\circ}$. The boron atom in 10-position and the chlorine atom in 9position are only minimally displaced from the C9*'C10 vector.

The steric demand of the boron bound substituents is so large that it prevents the molecules from coming close to one another in the packing motif of 59. Even the typical "head-to-tail" orientation found for most anthracene derivatives is inhibited. Thus, there is no $\pi$ - $\pi$-overlap, because the fluorophores are so far apart. The only interaction found is an aromatic $\mathrm{C}-\mathrm{H}^{\cdots} \pi$ bond from hydrogen atom in 3-position to a peripheral ring of the adjacent anthracene moiety. It measures $2.742 \AA$ while enclosing an angle of $67.2^{\circ}$ with the ring plane (Figure 3-100, right) which can be considered comparatively strong due to the fairly short distance and the steep angle. 

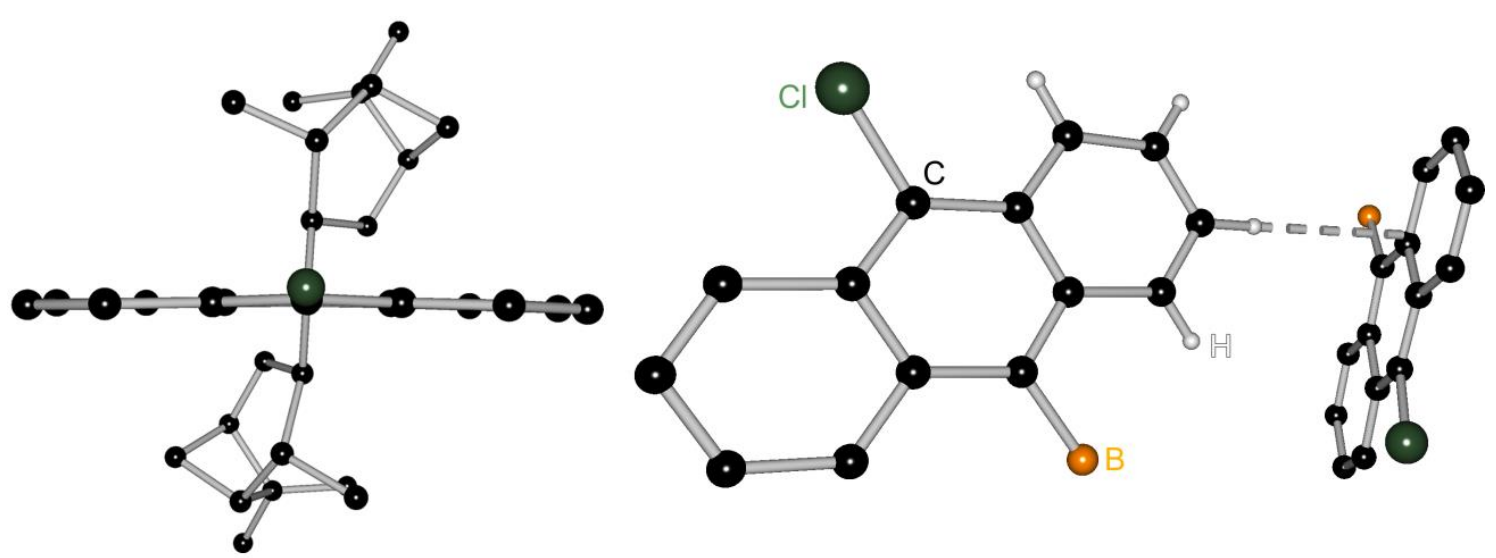

Figure 3-101: Left: nearly orthogonal orientation of the boron bound substituents to the anthracene plane in $\mathbf{5 9}$ (hydrogen atoms are omitted for clarity); right: $\mathrm{C}-\mathrm{H}^{\cdots} \mathrm{\pi}$ interaction between two anthracene moieties in $\mathbf{5 9}$ (boron bound substituents and hydrogen atoms in 5-8-position are omitted for clarity).

BrAnBMes $_{2}$ (60) also crystallizes in the orthorhombic space group $P 2_{1} 2_{1} 2_{1}$. In contrast to 59, here the asymmetric unit contains two independent molecules which differ in their geometries. They will be referred to as molecule 1 and molecule 2 . While the bond distances and angles surrounding the boron atom in molecule 1 and 2 are very similar and in the expected range, the deformation of the fluorophore is clearly stronger in molecule 1 . This is reflected by the folding angle of $10.1^{\circ}$, which is nearly twice as large as in molecule $2\left(5.4^{\circ}\right)$. The measured twist angles of the anthracene moiety are nearly identical for both molecules $\left(4.6^{\circ} / 4.8^{\circ}\right)$. Furthermore the boron atom is located notably outside the C9 “'C10 axis in molecule 1 , assuming a position almost $0.3 \AA$ above the anthracene plane. In contrast to $\mathrm{ClAnBCy}_{2}$ (59), the torsion angles of the boron bound substituents to the anthracene plane are far from orthogonal in both molecule 1 and 2 . They measure $47.9^{\circ}$ and $126.4^{\circ}$, respectively. This leads to unbalanced steric strain applied by the mesityl groups, which again leads to distinctly stronger deformation of the fluorophore than observed for $\mathbf{5 9}$.

Unlike 59, the packing motif of $\mathrm{BrAnBMes}_{2}(\mathbf{6 0})$ produces a multitude of intermolecular interactions. Though the steric demand of the mesityl substituents also prevents a "head-to-tail" orientation of the molecules and therewith hinders $\pi-\pi$ overlap, there are several $\mathrm{C}-\mathrm{H}^{\cdots} \pi \mathrm{r}$ interactions present in the solid state structure of $\mathbf{6 0}$. 


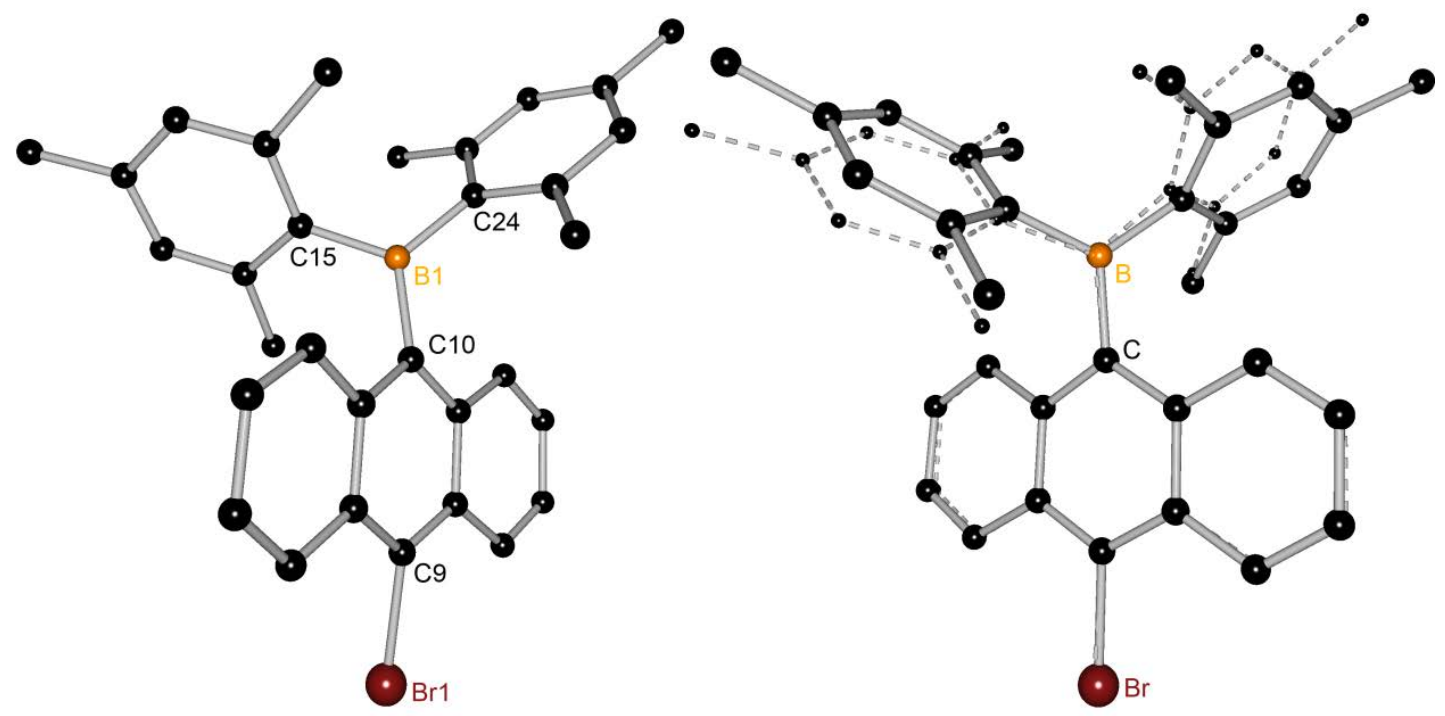

Figure 3-102: Left: solid state structure of $\operatorname{BrAnBMes}_{2}(60)$, molecule 1 (hydrogen atoms are omitted for clarity); right: superposition of molecule 1 and molecule 2 (dashed), hydrogen atoms are omitted for clarity.

Table 3-37: Selected bond lengths $[\AA]$ and angles $\left[{ }^{\circ}\right]$ of molecule 1

\begin{tabular}{ll}
\hline B1-C9 & $1.592(5)$ \\
B1C15 & $1.575(5)$ \\
C9B1C15 & $116.9(3)$ \\
C15B1C24 & $121.0(3)$ \\
Folding & 10.1 \\
Twist & 4.8 \\
\hline
\end{tabular}

Table 3-38: Selected bond lengths $[\AA]$ and angles $\left[{ }^{\circ}\right]$ of molecule 2

\begin{tabular}{ll}
\hline B1-C9 & $1.580(5)$ \\
B1C15 & $1.579(5)$ \\
C9B1C15 & $118.4(3)$ \\
C15B1C24 & $121.0(3)$ \\
Folding & 5.4 \\
Twist & 4.6 \\
\hline
\end{tabular}

The mesityl substituents offer numerous aromatic and aliphatic hydrogen atoms for interactions of this kind. A total of three $\mathrm{C}-\mathrm{H} \cdots \pi$ interactions originating from aromatic anthracene protons are found (Table 3-39). The clearly weakest is an offset face-toface interaction (Figure 3-102, left), which has nearly parallel orientation and has a distance of $3.26 \AA$, which makes it one of the shortest face-to-face interactions found throughout this entire thesis. Two more $\mathrm{sp}^{2}$ type $\mathrm{C}-\mathrm{H}^{\cdots} \pi \pi$ bonds are found between the hydrogen atoms in 1,2-position of molecule 2 to an adjacent $\pi$-system. (Figure 3-102, center) These can be considered fairly strong due to distances/angles of $2.812 \AA / 56.9^{\circ}$ and $2.920 \AA 8 / 52.5^{\circ}$, respectively. 

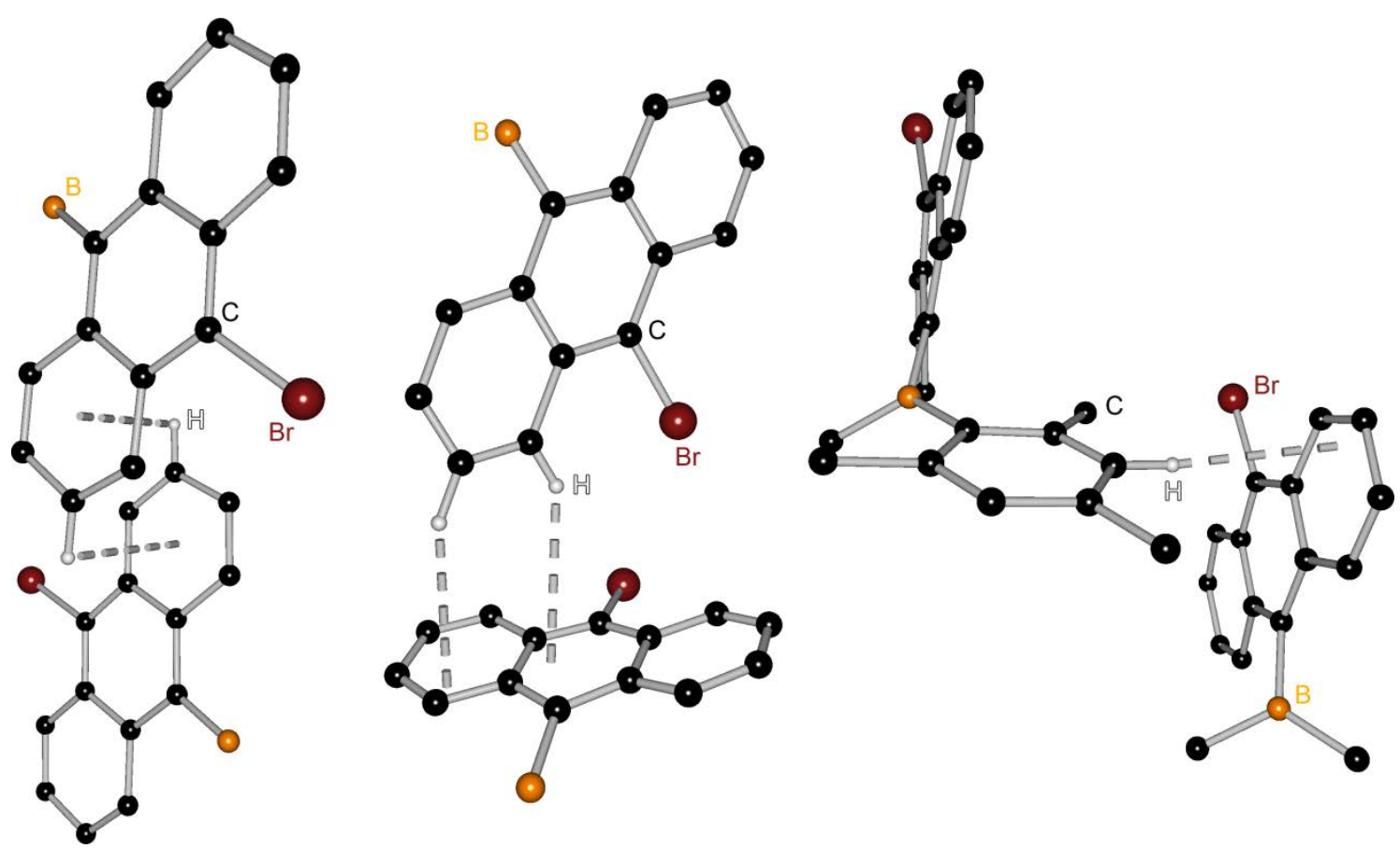

Figure 3-103: Aromatic C-H“' $\pi$ interactions in the structure of BrAnBMes $_{2}$ (60). Left: parallel C-H“' $\pi$ interaction between molecule 1 and 2 (mesityl substituents are omitted for clarity); center: $\mathrm{C}-\mathrm{H}^{\cdots} \pi$ interactions of the hydrogen atoms in 2,3-position (mesityl substituents are omitted for clarity); right: C$H^{\prime \prime} \pi$ interaction of a mesityl substituent (residual mesityl groups are omitted for clarity).

Table 3-39: $\mathrm{C}-\mathrm{H}^{\cdots} \mathrm{\pi} \pi$ interactions in the structure of 60 .

\begin{tabular}{ccc}
\hline Type & Distance $[\AA]$ & Angle $\left[^{\circ}\right]$ \\
\hline $\mathrm{sp}^{3}$ & 2.755 & 81.6 \\
$\mathrm{sp}^{2}$ & 2.889 & 82.7 \\
$\mathrm{sp}^{2}$ & 2.812 & 56.9 \\
$\mathrm{sp}^{2}$ & 2.920 & 52.5 \\
$\mathrm{sp}^{2}$ & 3.260 & 2.1
\end{tabular}

Furthermore, compounds 58-60 were also crystallized from solvent mixtures containing the donors used for the in-solution experiments in 3.6.2 ( $\mathrm{MeCN} \mathrm{NEt}_{3}$, TMEDA, THF, $\left.\mathrm{PPh}_{3}\right)$. This way it was intended to monitor the adduct formation in solid
Additionally, a medium strength $\mathrm{sp}^{3} \mathrm{C}-\mathrm{H} \cdots \pi$ interaction is found which measures $2.755 \AA$ while enclosing a steep angle of $81.6^{\circ}$ with the ring plane. The proposed strongest $\mathrm{C}-\mathrm{H}^{\cdots} \pi$ bond is found between an aromatic proton of a mesityl substituent and a peripheral ring of the anthracene moiety of molecule 1 . Though it is not the shortest interaction at $2.889 \AA$, the angle of $82.7^{\circ}$ is close to the optimum orthogonal orientation (Figure 3-102, right). The pronounced differences in geometry and intermolecular interactions should also influence the solid state fluorescence phenomena of 59 and 60 , as will be shown in 3.6.4. 
state by co-crystallization of the respective donor and potentially alter the solid state fluorescence properties. In practice, it was only possible to isolate crystals of either the mere boranylanthracenes or of decomposition products (Figure 3-103).
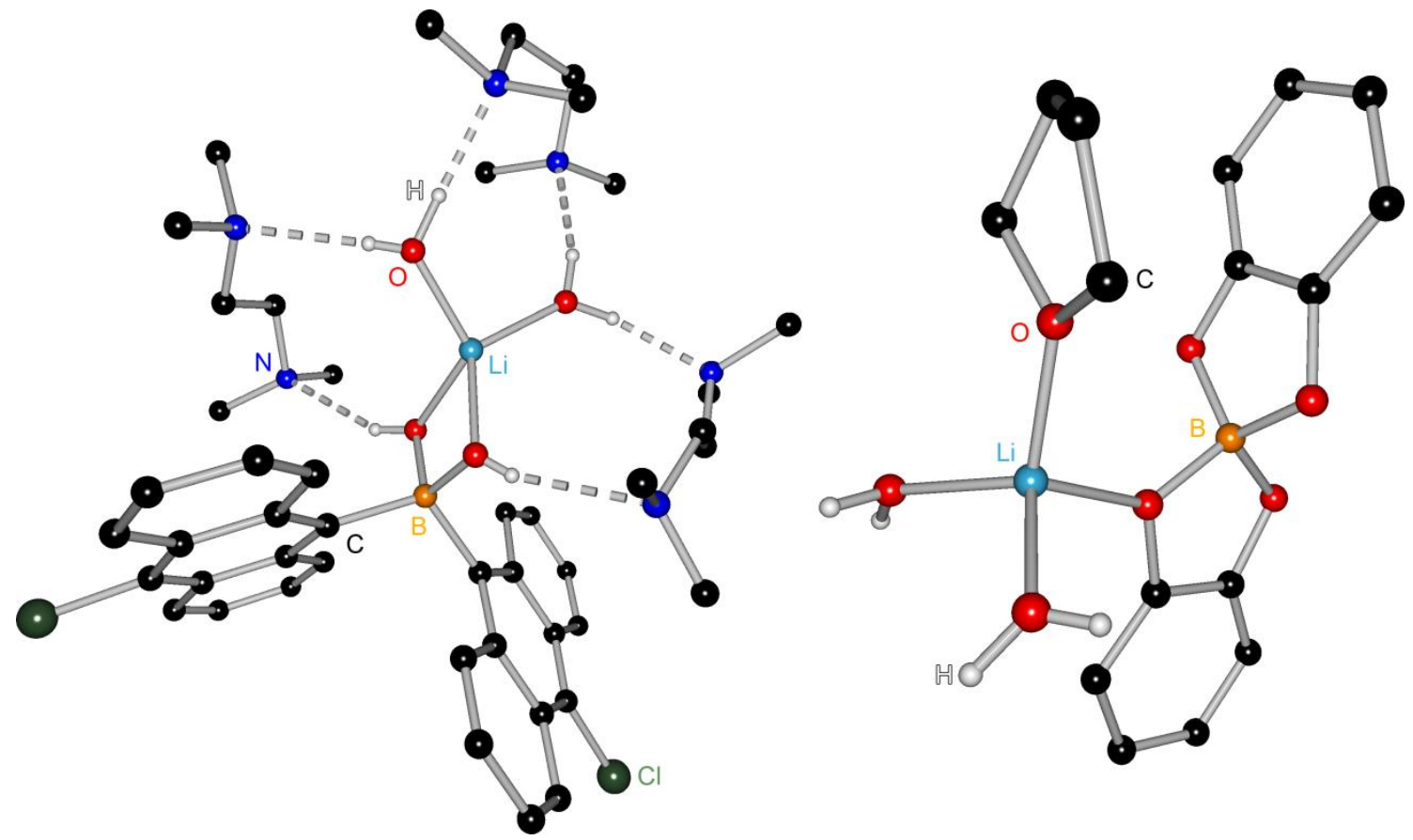

Figure 3-104: Decomposition products of $\mathrm{ClAnBIPC}_{2}$ (59) (left), and of ClAnBCat (58) (right, bottom).

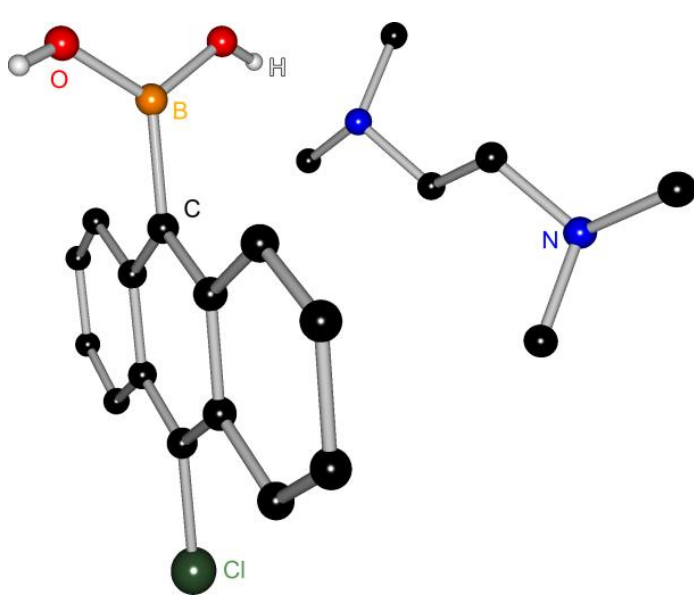

This shows the sensitivity of ClAnBCat (58) and $\mathrm{ClAnBIPC}_{2}$ (59) towards hydrolysis. Even small amounts of moisture contained in the assumed anhydrous donors were sufficient to hydrolyze significant amounts of the boranyl anthracenes. Also traces of lithium chloride originating from the salt elimination during synthesis appear to promote decomposition of $\mathbf{5 8}$ and $\mathbf{5 9}$.

Though lithium chloride was removed by filtration, very small particles are capable of passing through the filter, which explains traces of lithium salt in the products. The only compound that stayed intact independent of the donor bases added was BrAnBMes $_{2}(60)$. This underlines the stability of aromatic substituted anthryl boranes compared to aliphatic or heteroatomic substituted derivatives, which will be taken up in 3.6.5. 


\subsubsection{Solid State Fluorescence}

The air sensitive solid state samples of 58-60 were prepared in an argon glove box and measured immediately after removal from the inert gas atmosphere to avoid oxidation or decomposition.
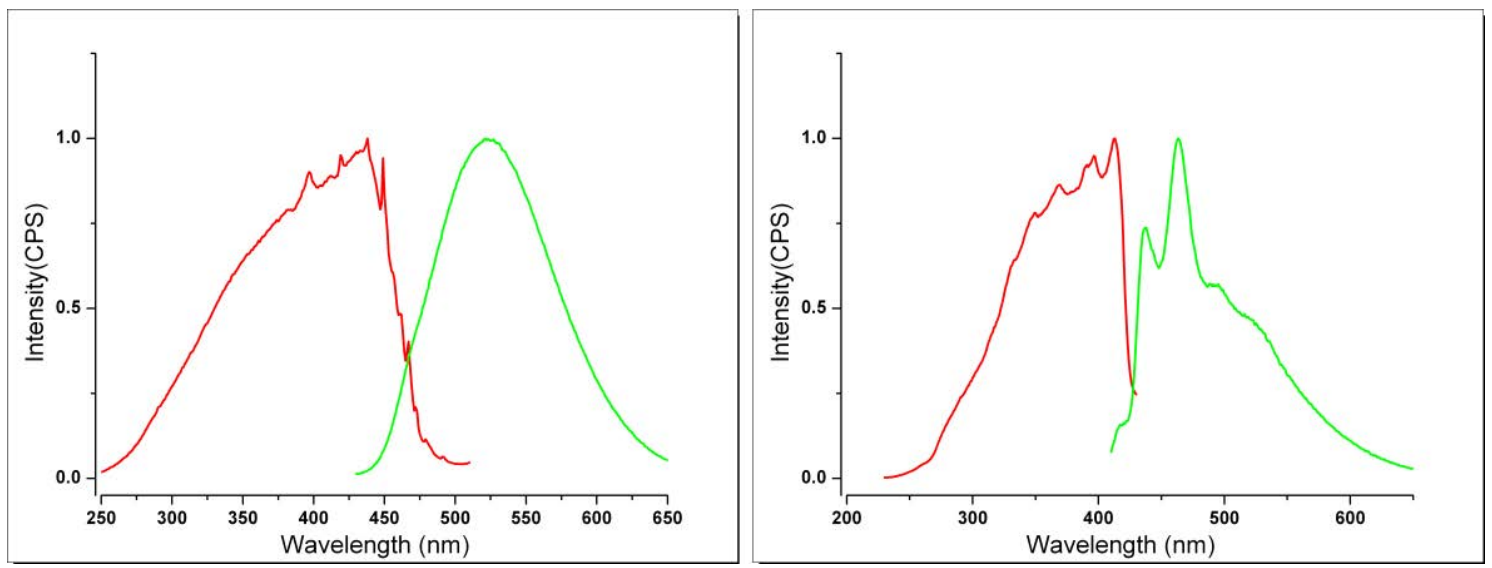

Figure 3-105: Left: normalized solid state excitation (red) and emission (green) spectra of CIAnBCat (58); right: normalized solid state excitation (red) and emission (green) spectra of $\mathrm{ClAnBIPC}_{2}$ (59).

Compared to the in-solution fluorescence, both excitation and emission maxima of 5860 are clearly red-shifted (Figure 3-104). All compounds show a broad excitation band of over $100 \mathrm{~nm}$ width, which has similarly been observed in the solid state fluorescence of phosphanyl and phosphoryl anthracenes (c.f. 3.2/3.3). The difference to in-solution measurements is particularly pronounced for $\mathrm{ClAnBIPC}_{2}$ (59) which exhibits two narrow and well separated excitation maxima in solution. Several other tendencies comply well with the findings of previous in-solution experiments.

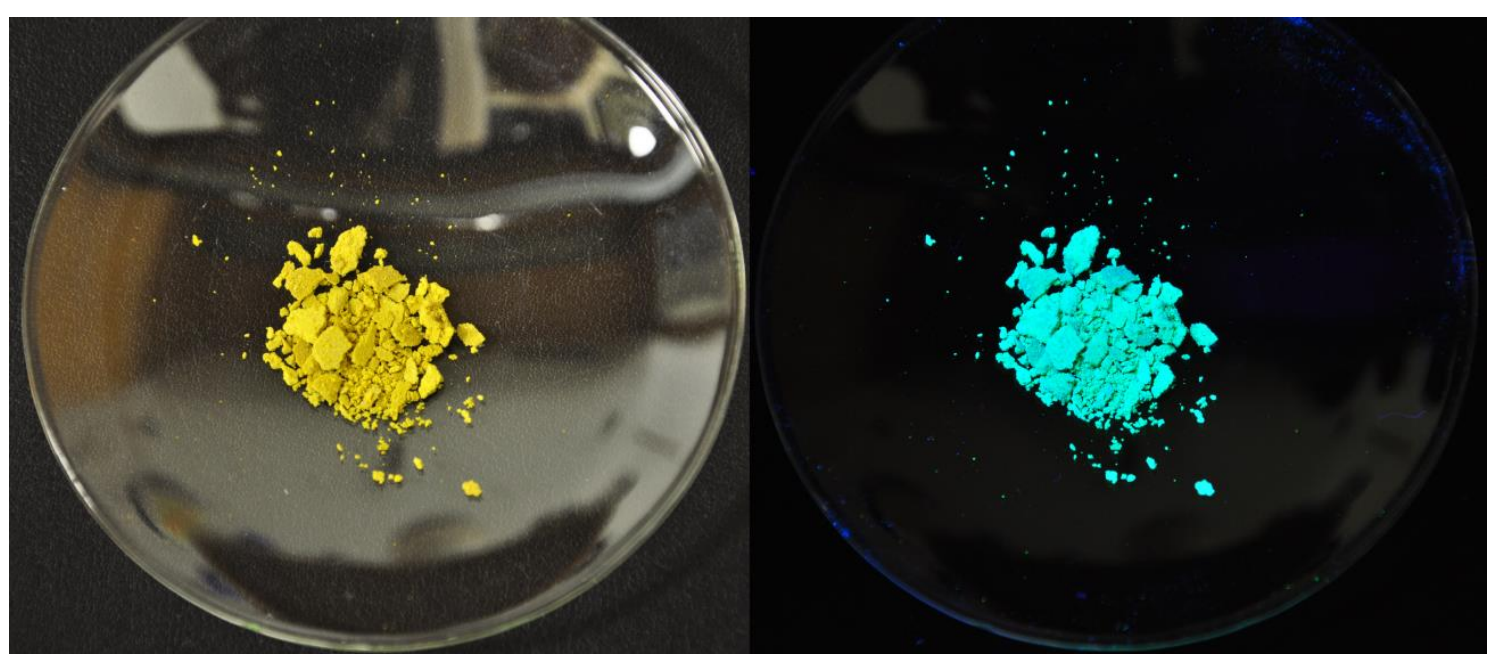

Figure 3-106: Solid sample of $\mathrm{BrAnBMes}_{2}(60)$ in daylight (left) and under exposure to UV light $(\lambda=366 \mathrm{~nm})$ (right). 


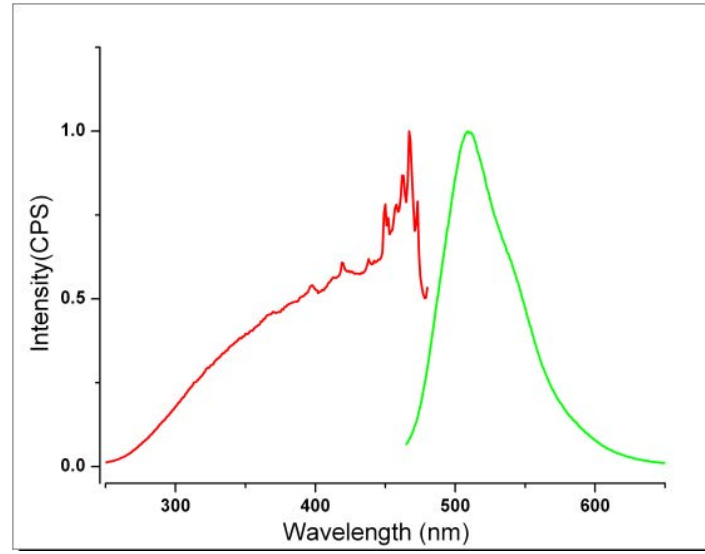

Table 3-40: Maximum excitation an emission wavelengths of 58,59 , and 60 .

\begin{tabular}{ccc} 
& $\lambda_{\max }(\mathrm{Ex})[\mathrm{nm}]$ & $\lambda_{\max }(\mathrm{Em})[\mathrm{nm}]$ \\
\hline ClAnBCat (58) & & 525 \\
$\mathrm{ClAnBIPC}_{2}$ (59) & 413 & 463 \\
BrAnBMes $_{2}(\mathbf{6 0})$ & 467 & 509 \\
\hline
\end{tabular}

Figure 3-107: normalized solid state excitation (red) and emission (green) spectra of $\mathrm{BrAnBMes}_{2}$ (60).

E.g. ClAnBIPC 2 (59) solely shows a band structure in its emission spectrum, as it did in solution. Furthermore CIAnBCat (58) exhibits the largest gap between excitation and emission maximum - in this case of nearly $100 \mathrm{~nm}$ - as observed in solution. In terms of emission, $\mathrm{ClAnBIPC}_{2}$ (59) shows the maximum of shortest wavelength. This also confirms the results acquired in solution. In contrast, ClAnBCat (58) features a distinctly farther red-shifted emission maximum than $\mathrm{BrAnBMes}_{2}$ (60), which was reversed for in-solution experiments. The difference in maximum emission wavelengths between $\mathrm{ClAnBIPC}_{2}$ (59) and BrAnBMes 2 (60) of $45 \mathrm{~nm}$ cannot be explained by differences in $\pi-\pi$ overlap, since neither of the two compounds feature such interactions in their packing motif. Hence, other factors must account for this phenomenon.

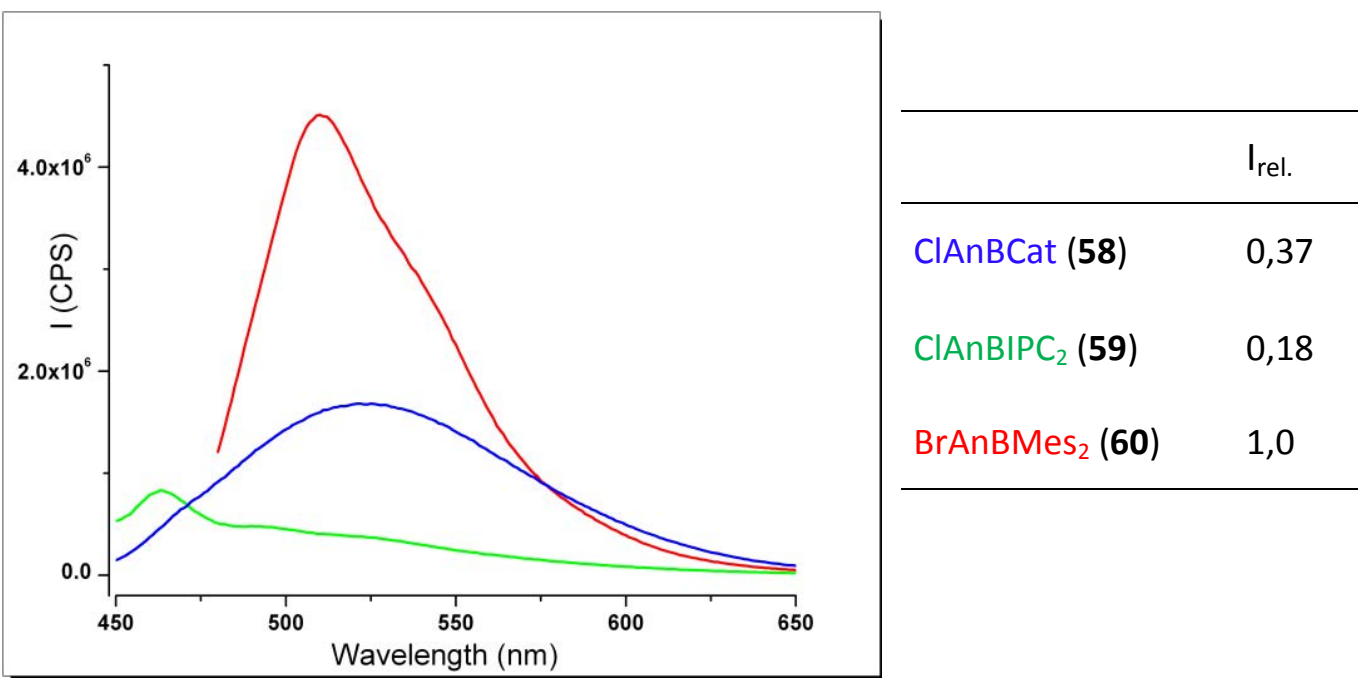

Figure 3-108: Solid state maximum emission spectra of ClAnBCat (58) (blue), $\mathrm{ClAnBIPC}_{2}$ (59) (green), and $\mathrm{BrAnBMes}_{2}$ (60) (red). 
The differences in emission intensity are just as striking. BrAnBMes $2(60)$ shows the clearly strongest emission intensity, followed by 58 and 59 (Figure 3-107). Taking into account the structural properties illustrated in 6.3.4, the observed intensities can be evaluated. Due to the lack of structural information on ClAnCat (58), this compound will be excluded from argumentation. The structural information is summarized in Table 3-41. Because the structure of BrAnBMes$_{2}(60)$ contains two independent molecules the specified folding and twist angles are averaged values of both molecules.

Table 3-41: Summarized structural information on $\mathrm{ClAnBCy}_{2}$ (59) and $\mathrm{BrAnBMes}_{2}$ (60).

\begin{tabular}{|c|c|c|c|c|}
\hline & Folding $\left[^{\circ}\right]$ & Twist $\left[{ }^{\circ}\right]$ & $\mathrm{C}-\mathrm{H}^{\cdots} \pi$ interactions $[\AA \AA] /\left[^{\circ}\right]$ & $I_{\text {rel }}$ \\
\hline $\mathrm{ClAnBIPC}_{2}$ & 2.6 & 1.5 & $1 \times \mathrm{sp}^{2}: 2.742 / 67.2$ & 0.18 \\
\hline $\mathrm{BrAnBMes}_{2}$ & 7.5 & 4.7 & $\begin{array}{l}3 \times \mathrm{sp}^{2}: 2.812 / 56.9 ; 2.920 / 52.5 \\
2.889 / 82.7 \quad 1 \times \mathrm{sp}^{3}: 2.755 / 81.6\end{array}$ & 1.0 \\
\hline
\end{tabular}

The weak emission intensity of $\mathrm{ClAnBIPC}_{2}$ (59) compared to $\mathrm{BrAnBMes}_{2}$ (60) intuitionally suggests a strong deformation of the anthracene moiety in $\mathbf{5 9}$. In fact, the opposite is the case. The weakly fluorescent $\mathbf{5 9}$ shows a by factor three smaller deformation of the fluorophore than the strongly fluorescent ClAnBMes 2 (60). As stated earlier, the often cited effect of $\pi-\pi$ overlap induced quenching is irrelevant here because neither of the packing plots exhibit such interactions. Again $\mathrm{C}-\mathrm{H}^{\cdots} \pi$ interactions are the only possibility of explaining this apparently contradictory result. While CIAnBIPC 2 (59) only forms a single $C-H^{\cdots} \pi$ bond, there are four such interactions present in the structure of BrAnBMes $_{2}(60)$. Especially the aromatic interactions which are oriented almost orthogonal to the $\pi$ system can be considered particularly strong and are similar to those found in SPAnPS@tol (15), which have been shown to generate a strongly fluorescent material by formation of a T-shaped exciplex. Thus, number and strength of the $\mathrm{C}-\mathrm{H}^{\cdots}{ }^{\mathrm{m}} \pi$ interactions on hand outnumber the effect of fluorophore deformation. 


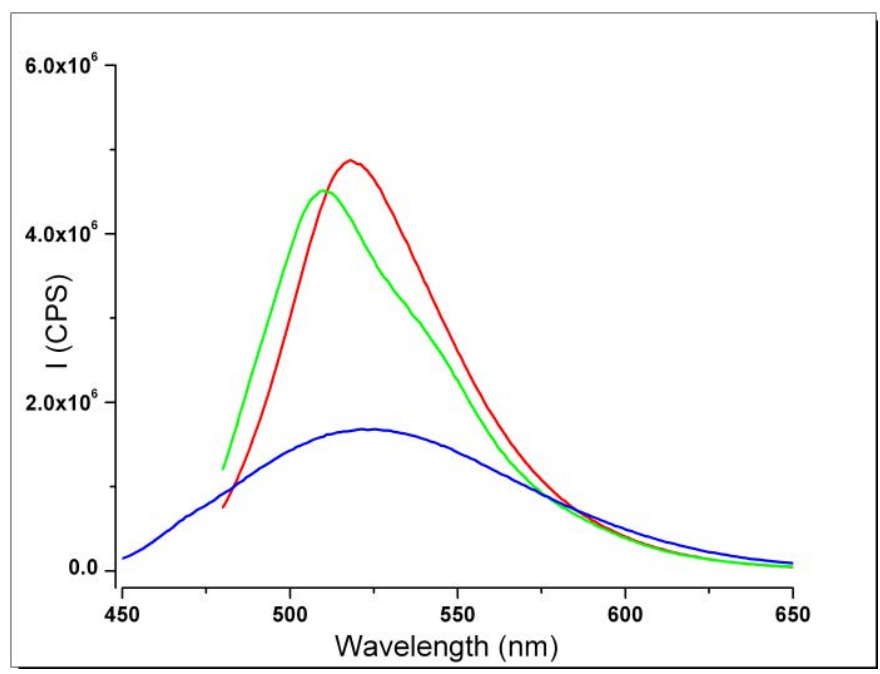

Figure 3-109: Solid state emission spectra of SPAnPS@tol (15, red), BrAnBMes 2 (60, green), and ClAnBCat (58, blue).

The strong fluorescence of these compounds is further confirmed by comparison to SPAnPS@tol (15), which is one of the compounds with the strongest observed solid state fluorescence (Figure 3-108). Especially BrAnBMes 2 (60) comes very close to 15 in terms of emission intensity proving the emission enhancing character of the $\mathrm{C}-\mathrm{H} \cdots \pi$ bonded arrangement described above.

\subsubsection{Further Synthetic Approaches}

In light of the impressive fluorescence properties of boranyl anthracenes, further synthetic work was essential in order to generate more stable and versatile derivatives. Especially the obstructions of formation and detection of Lewis acid/base adducts in solution and in the solid state called for structural alterations, and the problems of regular decomposition upon addition of donor bases had to be addressed. The primary goals were to produce higher stability of the compounds while simultaneously generating enhanced Lewis acidity at the boron atom.

From the crystallization attempts from solutions containing moisture contaminated donor bases, the pronounced stability of the aromatic substituted BrAnBMes 2 (60) compared to the other compounds became apparent. Furthermore electron withdrawing substituents which do not possess lone pairs in direct proximity to the boron atom had to be introduced. This way the interaction of lone pairs with the empty p-orbital of the boron atom is prevented assuring maximum Lewis acidity. The 
substituent which combines aromaticity and electron withdrawing character with no lone pairs in $\alpha$-position is the pentafluoro phenyl substituent. The introduction of this substituent to boranyl anthracenes should supply sufficient Lewis acidity for adduct formation while retaining moderate steric demand and sufficient stability.

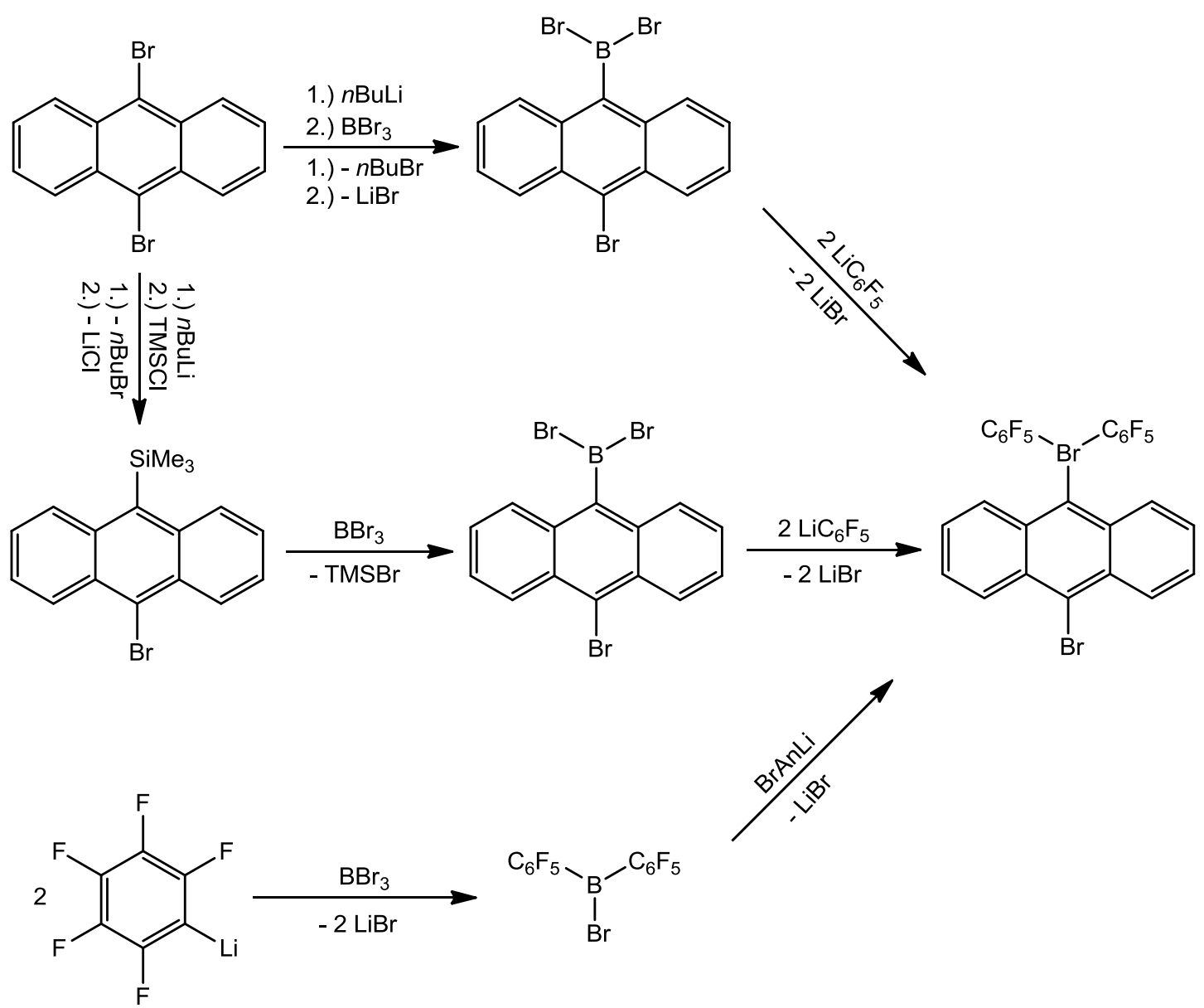

Scheme 3-40: Synthetic approaches towards the preparation of 9-bromo-10-

(bis(pentafluorophenyl)boranylanthracene.

Three different synthetics routes were explored for the synthesis of 9-bromo-10(bis(pentafluorophenyl)boranylanthracene (Scheme 3-39). Unfortunately, in all cases the desired product could only be traced by mass spectrometry and was not obtained as the main product. This can mainly be ascribed to the very high and unselective reactivity of $\mathrm{BBr}_{3}$. For example, the reaction of 9-bromo-10-lithioanthracene with $\mathrm{BBr}_{3}$ in diethyl ether gave 9-bromo-10-ethylanthracene as the primary product. The extreme Lewis acidity of $\mathrm{BBr}_{3}$ had led to adduct formation with ether solvent molecules which were thereby activated. Upon addition of nucleophiles this activation led to ether cleavage and introduction of an ethyl substituent to the anthracene 
moiety. This shows that Milder reaction conditions and more selective reagents must be employed for the synthesis of 9-bromo-10-(bis(pentafluorophenyl)boranylanthracene in future synthetic strategies. A possible alternative could be the utilization of the $\mathrm{C}_{6} \mathrm{~F}_{5}$ stannyl transfer agent $\mathrm{Me}_{2} \mathrm{Sn}\left(\mathrm{C}_{6} \mathrm{~F}_{5}\right)_{2}$ described by Parks et al., which enables highly selective transfer of pentafluorophenyl moieties to $\mathrm{BCl}_{3}$ for the synthesis of bis(pentafluorophenyl)chloroborane. ${ }^{[90]}$ 


\section{Anthracene Derivatives Containing Spacers}

The second part of this thesis is dedicated to anthracene derivatives which bear an alkyl spacer between the fluorophore and the main functional group or substituent (Scheme 4-1). By utilization of spacers, the direct electronic influence of inserted substituents on the fluorophore is minimized, which is why the compounds in the following chapters do not differ significantly in terms of maximum emission wavelengths.

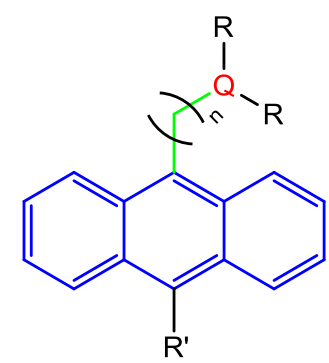

Scheme 4-1: General composition of a sensor molecule according to the "quencher-spacer-fluorophore" principle.

On the other hand the geometry of the molecule is distinctly altered by spacers, generating more rotational degrees of freedom, which again are capable of creating orbital overlap for electron transfer processes, and therewith taking "secondary" influence on the electronic properties of the fluorophore. Molecular arrangements of fluorophores and quencher moieties which are linked by spacers have been successfully applied in countless sensor systems and molecular switches and are a major research target to date, with numerous research groups involved worldwide. ${ }^{[15 c \text {, }}$ 15d, 23a, 23c, 28] There is a large number of known amine based receptor/quencher moieties (some of them highly complex ${ }^{[91]}$ ) and their number is still increasing. Still there is an undeminished demand for new receptors, as there are still numerous metal cations which are not yet sufficiently detectable, as well as a sheer endless variety of other potentially interesting analytes which require corresponding sensor molecules. Furthermore, the findings made for amine quenchers have not been transferred to quencher moieties based on higher homologues which would further broaden the range of applications of fluorescent sensors. Moreover, a combination of emission shifting phosphoryl substituents and the established "receptor-spacer-fluorophore" 
set-up has not yet been attempted, let alone actualized. These challenges will be addressed in the following subchapters.

\subsection{Synthesis of Precursor Molecules}

The first step in synthesis of potential sensor molecules is the acquisition of suitable starting materials. Only a very limited number of receptor/quencher moieties is commercially available, which is why the majority of work is often invested into the synthesis of such compounds. ${ }^{[91 b, 92]}$ In view of the complexity of many lately employed cryptands, this effort is commonly higher than the required synthesis for the unification of receptor and fluorophore. The second sector of precursor synthesis is focused on derivatization of the fluorophore, e.g. by introduction of spacers or conversion of the fluorophore moiety into a reactive intermediates for the introduction of receptor units.

\subsubsection{Synthesis of Receptor Units}

The majority of receptor units is based on amines which are effective quenchers of anthracene fluorescence (c.f. 1.1). Synthesis of chelating amine ligands as receptor units by introduction of functionalized substituents was achieved via two synthetic strategies.

\section{a) Synthesis from HMDS}

In 2008, Azizi et al. published the syntheses of various symmetric bi-functionalized amines from HMDS. ${ }^{[93]}$ Their synthesis of bis(2-picolyl)amine was adopted and reproduced (Scheme 4-2).

2<smiles>O=Cc1ccccn1</smiles>

1.) $\mathrm{LiClO}_{4}, 50^{\circ} \mathrm{C}$

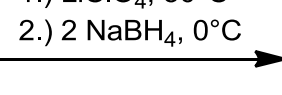<smiles>c1ccc(CNCc2ccccn2)nc1</smiles>

Scheme 4-2: Synthesis of bis(2-picolyl)amine according to Azizi et al.

A suspension of $\mathrm{LiClO}_{4}$ in $\mathrm{HMDS}$ was heated to $50^{\circ} \mathrm{C}$ and 2-pyridylcarbaldehyde was added over 30min. According to Azizi et al, the $\mathrm{LiClO}_{4}$ acts as a Lewis acid and is vital for the success of the reaction. Within minutes of the addition of 2- 
pyridylcarbaldehyde, a solid intermediate product is formed, taking up the entire volume of the reaction mixture as a massive solid clot. This solid is re-dissolved when a slight excess of $\mathrm{NaBH}_{4}$ dissolved in $\mathrm{MeOH}$ is added at $0^{\circ} \mathrm{C}$. Pre-cooling of the $\mathrm{MeOH}$ in advance of dissolving $\mathrm{NaBH}_{4}$ is very important to avoid reaction of the borohydride with the moisture contained in $\mathrm{MeOH}$, which leads to strong gas formation. Evaporation of the solvent, addition of an aqueous $\mathrm{NaHCO}_{3}$ solution and extraction with DCM produces a yellow oily crude product. Purification by column chromatography ( $n$-pentane/ethyl acetate 5:1) affords bis(2-picolyl)amine at a yield of $54 \%$.

This reaction pathway was subsequently applied to other substrates in the general procedure depicted in Scheme 4-3.

2<smiles></smiles>

1.) $\mathrm{LiClO}_{4}, 50^{\circ} \mathrm{C}$

2.) $2 \mathrm{NaBH}_{4}, 0^{\circ} \mathrm{C}$<smiles>[R]CNC[R]</smiles>

61: $R=$<smiles>CC(C)(C)c1cccs1</smiles>

62: $\mathrm{R}=$<smiles>CC12CCC(COC1)C2</smiles>

63: $R=$<smiles>Cc1ccccc1SCc1ccccc1</smiles>

Scheme 4-3: Synthesis of symmetric amines 61, 62, and 63.

Bis(2-methylthienyl)amine (61) was obtained as a yellow oil, while bis(2methoxybenzyl)amine (62) and bis(2-methylthiobenzyl)amine (63) are solids. The yields are acceptable at rates ranging from $45 \%(61)$ to $69 \%(62)$. As Scheme $4-3$ shows, only aromatic aldehydes were reacted, yielding aromatic substituted amines. The attempt of employing aliphatic amines clarifies the limitations of this synthetic route (Scheme 4-4).

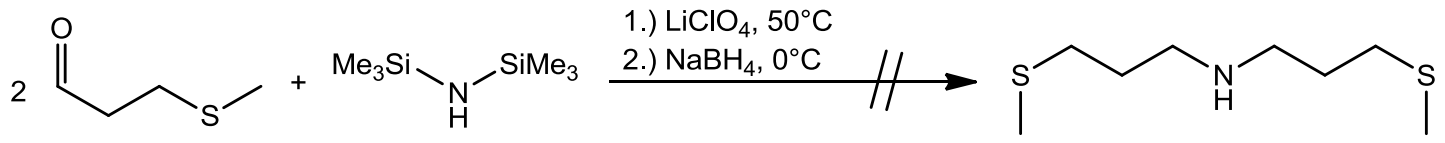

Scheme 4-4: Attempted synthesis of bis(3-methylthiopropyl)amine.

The reaction of 3-methylthiopropionaldehyde with HMDS yields a virtually inseparable product mixture with a polymeric main product (determined by GC/MS). This led to the postulation of a possible mechanism, which surprisingly was not attempted by Azizi et al. ${ }^{[93]}$ (Scheme 4-5). The only explanation for the obtained result 
is the presence of hydrogen atoms in $\alpha$-position to the aldehyde function. This leads to unselective formation of double bonds in the elimination (a) of $\mathrm{Me}_{3} \mathrm{SiOH}$ (in both directions of the carbonyl-carbon atom). Hence, the selective formation of products is dependent on the exclusive formation of the iminium intermediate (b), and thus limited to aromatic substrates or other substrates lacking $\alpha$-hydrogen atoms.

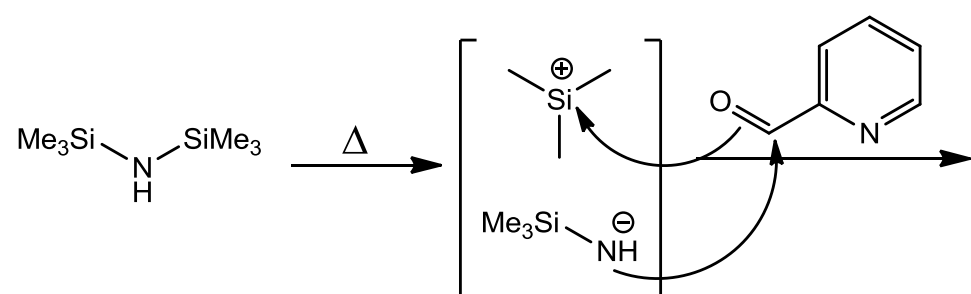<smiles>COCC(C)c1ccccn1</smiles>

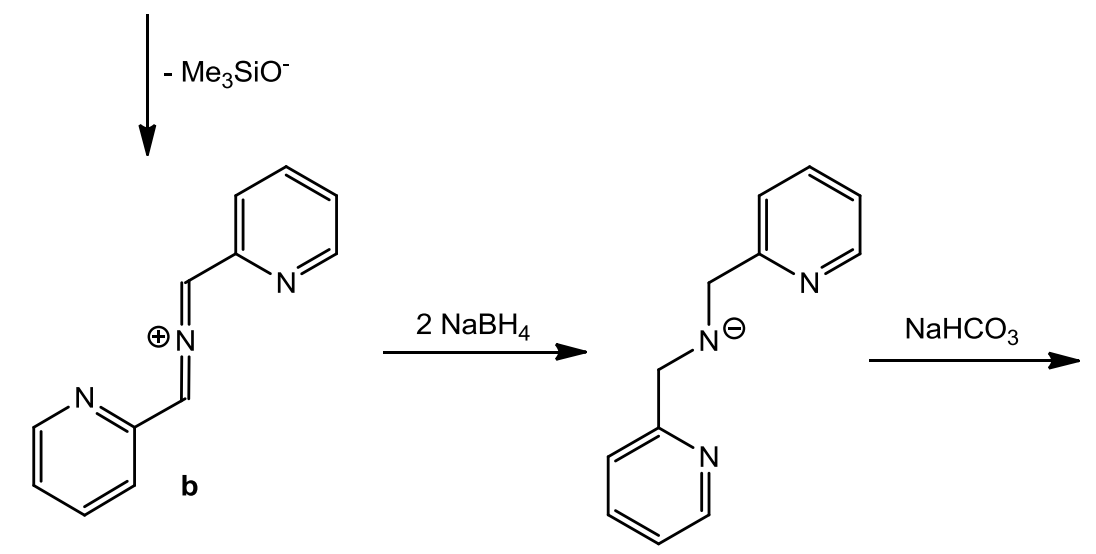

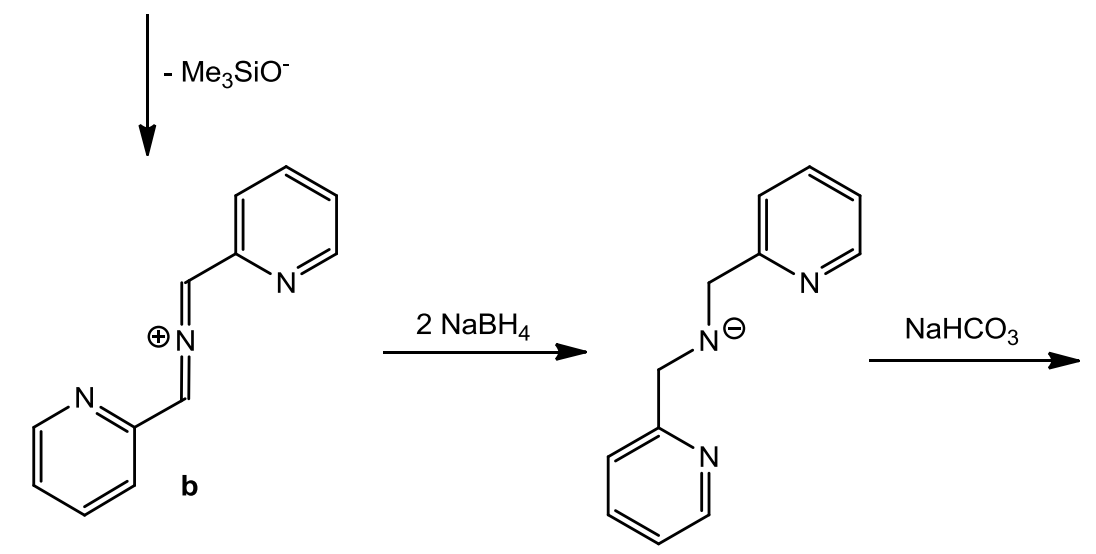

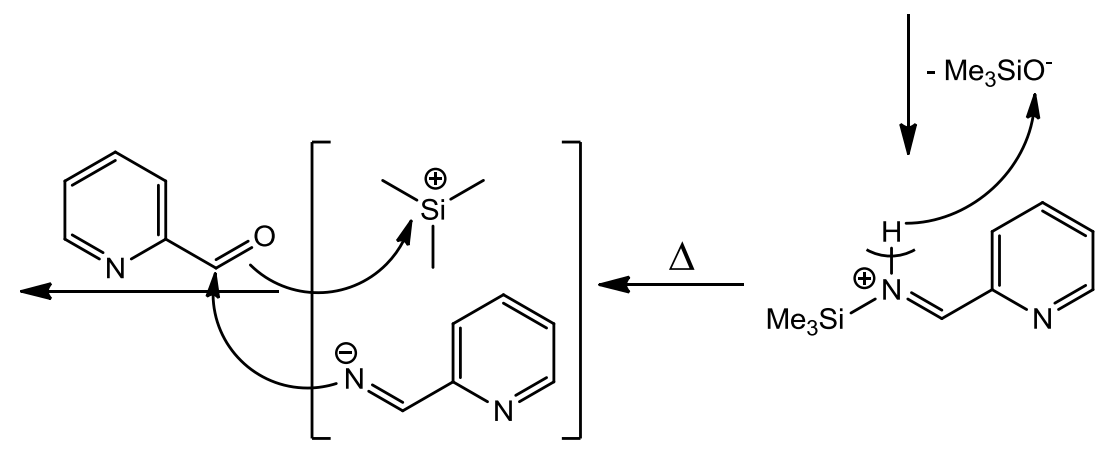<smiles>c1ccc(CNCc2ccccn2)nc1</smiles>

Scheme 4-5: Postulated mechanism of aromatic amine synthesis.

The ionic intermediates are likely to be stabilized by the lithium ions and perchlorate ions in the reaction mixture. It is surprising that although a large excess of HMDS is applied (HMDS is used as a substrate and as a solvent simultaneously), apparently only the di-substituted product is formed. The formation of the iminium ion - which is probably the solid intermediate product formed prior to reduction - is apparently energetically favored. 


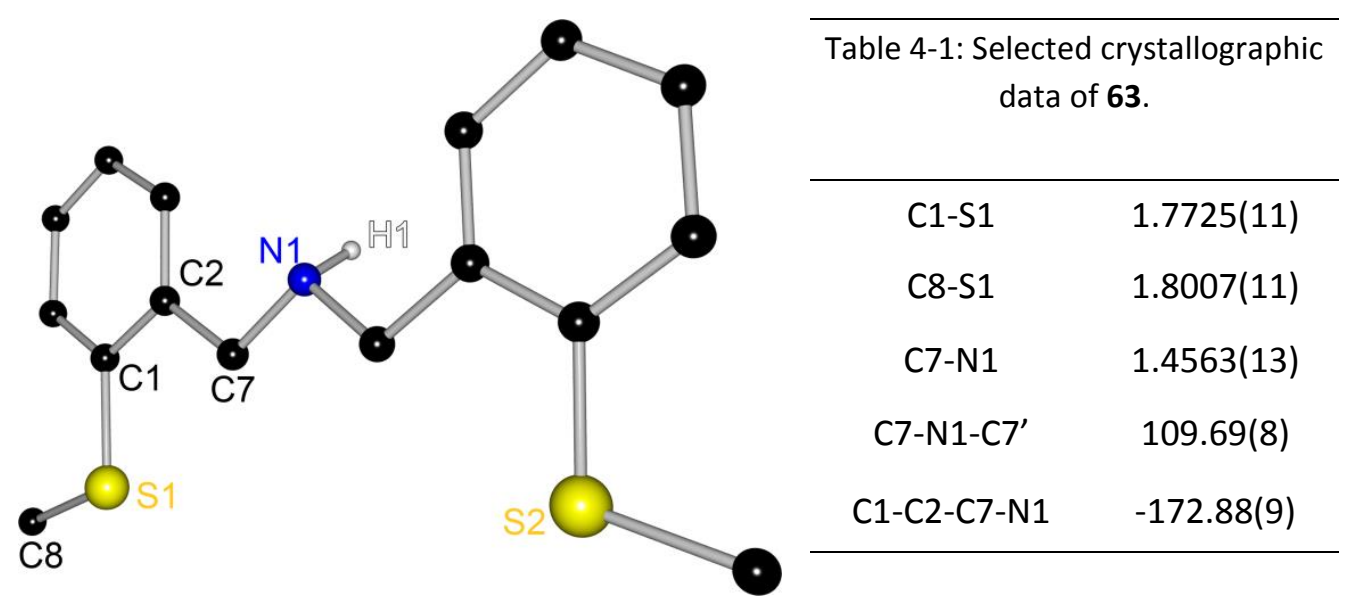

Figure 4-1: Crystal structure of bis(2methylthiobenzyl)amine (63).

Furthermore, the crystal structure of 63 could be determined (Figure 4-1). It was crystallized from DCM and crystallizes in the monoclinic space group $P 2_{1} / n$. Its structure is almost perfectly planar as indicated by the C1-C2-CN1 torsion angle of $172.9^{\circ}$ (Table 4-1). The amine hydrogen position was freely refined.

\section{b) Synthesis from bis(2-chloroethyl)amine hydrochloride}

The second synthetic pathway was used to synthesize di-substituted aliphatic amines. Bis(2-chloroethyl)amine hydrochloride is a widespread starting material in the synthesis of aliphatic amines. ${ }^{[94]}$ The hydrochloride rather than the free bis(2chloroethyl)amine is used because the latter is unstable and undergoes an intramolecular substitution reaction under formation of a three-membered cycle and elimination of $\mathrm{HCl}$. This mechanism is utilized in the closely related compound tris(2chloroethyl)amine, which is better known under its trivial name N-LOST, which has been used as a chemical weapon. ${ }^{\text {[95] }}$

Although some reactions offer direct conversion of the hydrochloride to the desired di-substituted amine in one-pot reactions, ${ }^{[94 b]}$ the alternative introduction of a protecting silyl group is more useful when the bis(2-chloroethyl)amine is to be used in a variety of reactions, as it is stable over longer periods of time. The introduction of the silyl protecting group was carried out by refluxing of bis(2-chloroethyl)amine hydrochloride with one equivalent of trimethylsilyl chloride and catalytic amounts of DMSO in $\mathrm{NEt}_{3}$ over $16 \mathrm{~h}$ (Scheme 4-6). 


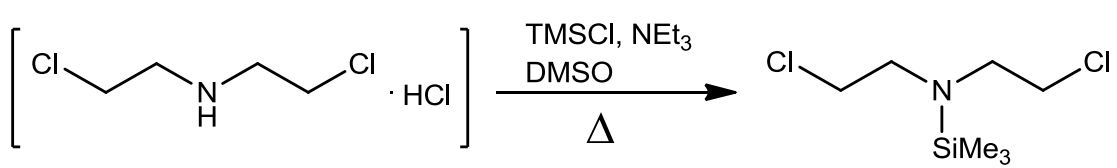

Scheme 4-6: Synthesis of bis(2-chloroethyl)trimethylsilylamine.

After filtration and evaporation of the solvent, the protected amine was obtained as a yellow oil. It was then reacted with a variety of nucleophiles, generating chelating amines (Scheme 4-7).<smiles>CS(C)(C)CCCl</smiles>

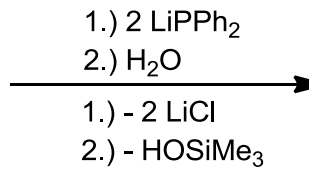

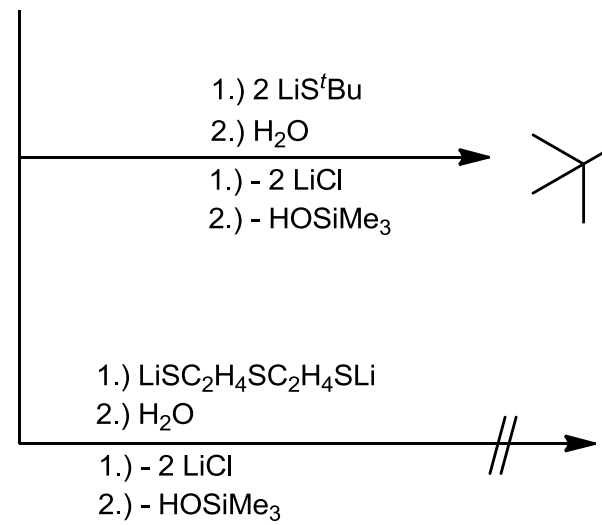<smiles>CC(C)(C)SCCNCCSC(C)(C)C</smiles>
65

Scheme 4-7: Amine synthesis from bis(2-chloroethyl)trimethylsilylamine.

Although $\mathrm{HN}\left(\mathrm{C}_{2} \mathrm{H}_{4} \mathrm{PPh}_{2}\right)_{2}$ (64) has been known since 1968 when it was first synthesized by Sacconi ${ }^{[96]}$ and has been successfully used as a ligand in complex synthesis and catalysis in the past decades, it is described here because minor alterations were made in its synthesis. In contrast to most other synthetic pathways, which have often employed reactions of chlorophosphanes with elemental lithium ${ }^{\text {[97] }}$ or deprotonation agents as $\mathrm{KO}^{t} \mathrm{Bu},{ }^{[94 a]} n$-BuLi was used for deprotonation of the phosphane. This method of phosphane deprotonation has e.g. been described by De Jongh et al. ${ }^{[98]}$ This way the yield was improved and the formation of oxidation products during the work-up process was suppressed. The siliyl group was removed by refluxing in a hexane $/ \mathrm{H}_{2} \mathrm{O}$ mixture. P-N-P type ligands like 64 have been used in synthesis of a multitude of transition metal complexes due to their high flexibility and their soft P-donor side arms. ${ }^{[94 a, 99]}$ The formation of stable complexes with a large number of metal cations makes 64 particularly interesting as a receptor unit in sensor 
molecule synthesis. To date no P-N-P type ligands have been utilized as quencher moieties in the context of chemical sensing.

Furthermore an S-N-S type receptor unit was synthesized (Scheme 4-7). Alike the P$\mathrm{N}-\mathrm{P}$ ligands, S-N-S ligands have repeatedly been shown to form stable complexes with a variety of soft transition metals. ${ }^{[100]}$ Also the closely related but less steric demanding $\mathrm{HN}\left(\mathrm{C}_{2} \mathrm{H}_{4} \mathrm{SEt}\right)_{2}$ has been successfully used as a receptor for detection of $\mathrm{Ag}^{+}$ions, which underlines the aptitude of S-N-S receptors in chemical sensing. ${ }^{[101]}$ The synthesis of $\mathrm{HN}\left(\mathrm{C}_{2} \mathrm{H}_{4} \mathrm{~S}^{t} \mathrm{Bu}\right)_{2}(65)$ has been reported by McGuinness et al. but again the original synthesis was altered. ${ }^{[99 a]}$ While McGuiness and co-workers did not comment on the synthesis of 65 , and similar amines as $\mathrm{HN}\left(\mathrm{C}_{2} \mathrm{H}_{4} \mathrm{SEt}\right)_{2}$ have mostly been prepared by deprotonation of the thiol using $\mathrm{NaOH}^{[94 \mathrm{~b}]}$ a different method was used in the synthesis of $\mathrm{HN}\left(\mathrm{C}_{2} \mathrm{H}_{4} \mathrm{~S}^{t} \mathrm{Bu}\right)_{2}$ (65). The mercaptan was dissolved in THF and deprotonated with $n$-BuLi at $-15^{\circ} \mathrm{C}$. The resulting solution was added to a THF solution of bis(2-chloroethyl)trimethylsilylamine and stirred for $24 \mathrm{~h}$ at ambient temperature. The silyl protecting group was removed analogously to 64 . While 65 is stable as a solid over several weeks, it is instable when stored in solution over longer periods of time. Crystallization of 65 from DCM afforded the dimerization product 65a (Figure 4-2). Reaction of two molecules of $\mathbf{6 5}$ under elimination of two $\mathrm{HS}^{t} \mathrm{Bu}$ moieties leads to the structure depicted in Figure 4-2.
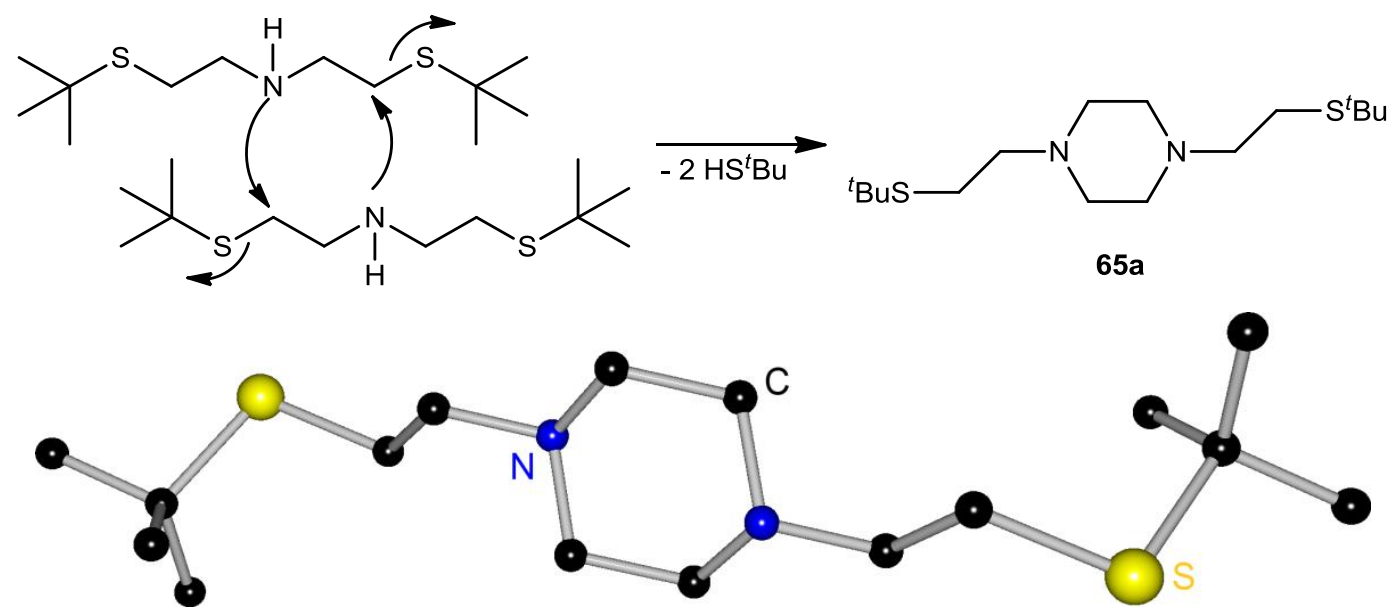

Figure 4-2: Top: dimerization of 65 , bottom: crystal structure of $65 a$.

Although its synthesis was not initially planned, 65a is an interesting ligand which should have outstanding potential in metal complexation due to its high flexibility. 
The synthesis of the cyclic monoazatrithia-12-crown-4 ether, on the other hand, was not successful. Although the synthesis of the compound is known ${ }^{[102]}$ and azathiacrown ethers have also found application in chemical sensing, ${ }^{[103]}$ the compound was not successfully reproduced. Deprotonation of the dithiol with $n$-BuLi led to a poorly soluble polymer-like product. Hence, for the synthesis of cyclic compounds other deprotonation agents appear to be favorable.

\subsubsection{Introduction of Spacers}

As illustrated in 1.1, spacers are absolutely vital parts of sensor molecules for effective fluorescence quenching. Their length and flexibility and the corresponding orbital overlap between quencher and fluorophore are crucial factors which influence the rate of electron transfer and therewith of quenching. ${ }^{[13,22]}$ Effective quenching is a precondition of large on/off emission ratios which are desirable in chemical sensing.

\section{a) Deprotonation of 9-methylanthracene}

Almost all synthetic approaches to sensor molecule preparation described in literature follow the established pathway of introducing alkyl spacers to the fluorophore via conventional organic synthesis, and then installing good leaving groups for introduction of the receptor/quencher moiety. The latter process is almost exclusively limited to $S_{N}$-chemistry. The possibility of reversing the reactivity of the involved reactants using organometallics has not yet been established.

In 2011 Tatić et al. reported on the deprotonation of toluene under mild conditions using trimethylsilylmethyllithium (TMSMeLi). ${ }^{[04]}$ This opens up the possibility of deprotonating the related compound 9-methylanthracene and therewith converting the fluorophore moiety to a nucleophile. Reactions with electrophile quencher moieties would be possible and the former methyl group of the starting material would then automatically become a methylene spacer in the reaction product (Scheme 4-8).

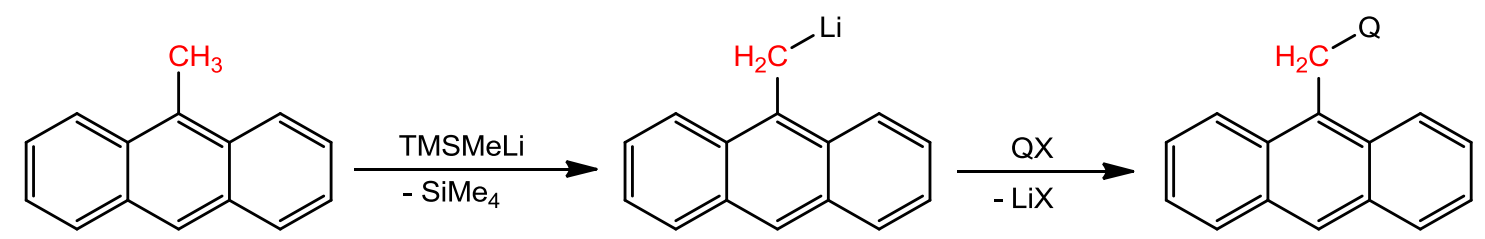

Scheme 4-8: Sensor molecule synthesis via inverted reactivities $(Q=$ quencher). 
To access these types of reactions, the transferability of the deprotonation process from toluene to 9-methylanthracene had to be verified. Hence, isolation and characterization of the lithiated species was sighted. For this purpose, 9methylanthracene was suspended in diethyl ether and 1 equivalent of TMEDA was added. The mixture was cooled to $-15^{\circ} \mathrm{C}$ and reacted with one equivalent of TMSMeLi over 30 minutes (Scheme 4-9). Upon addition of TMSMeLi the color of the solution immediately changed from light yellow to dark green. After completion of the reaction the solution's color was so dark green that it appeared nearly black.

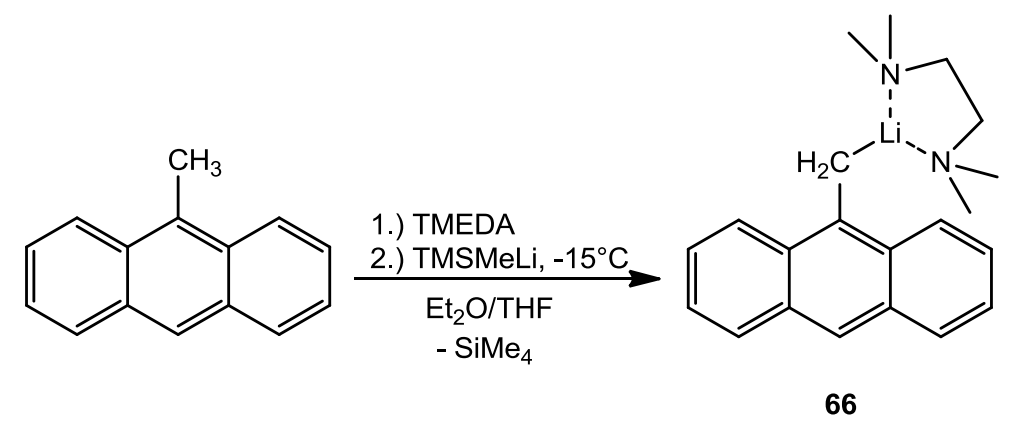

Scheme 4-9: Synthesis of [AnCH $\left.{ }_{2} \mathrm{Li} \cdot T M E D A\right]$ (66).

Within minutes of completion of the reaction a dark crystalline precipitate was formed, leaving the mother liquor almost colorless. Due to the high speed of crystallization, the quality of the obtained crystals was poor. This made the acquisition of good X-ray data problematic. The dark color and very high reactivity of the compound further complicated the selection of a suitable crystal, as the decomposition commenced quickly and the non-transparent crystals could hardly be optically judged in terms of quality. Thus, only poor quality data was obtained at a poor resolution which made description of the disordered structure impossible, despite multiple experiments with several different crystals. Nevertheless, the obtained results indicated a separated ion pair in the solid state (Figure 4-3). 

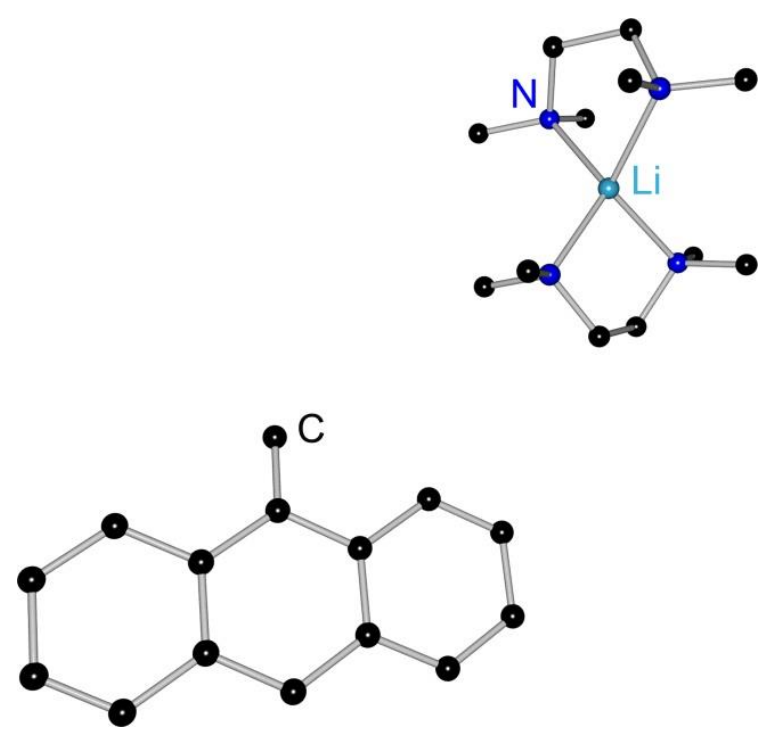

Figure 4-3: Atom coordinates of 66 acquired from first diffraction experiments. Disordered fragments are omitted for clarity.

The asymmetric unit contains one lithium ion and two half methylanthracene molecules, which indicates that each methylanthracene carries one negative charge. The poor resolution of the acquired data disabled the determination of the position of the charge because hydrogen positions cannot be found and freely refined at low resolutions. Furthermore the methylanthracene molecules show static disorder with a $\sim 50: 50$ occupation of two positions with the methyl groups facing in opposite directions.

To avoid the rapid crystallization induced by the poor solubility of 66 in diethyl ether, the reaction was repeated and the precipitate was separated from the solution by filtration. Despite the inert gas atmosphere, the dark solid decomposed to a light yellow product (which turned out to be 9-methylanthracene) within minutes, before it could be re-dissolved in a different solvent. This again underlines the high reactivity and instability of the lithiated species. A third attempt using a cooled filtration device $\left(-78^{\circ} \mathrm{C}\right)$ was successful. The lithiated compound was preserved long enough to be redissolved in pre-cooled THF. 66 showed very good solubility in THF which however again complicated crystallization. Only after several months, single crystals were obtained from the THF solution. At this point the color of the solution had changed from dark green to dark purple. The crystal color was identically black as before.

The new batch of crystals was again used for X-ray diffraction experiments. The slower crystallization process proved to be beneficial for crystal quality and a resolution of $0.78 \AA$ was reached. The unit cell showed strong deviations from the unit cell found in previous diffraction experiments. 


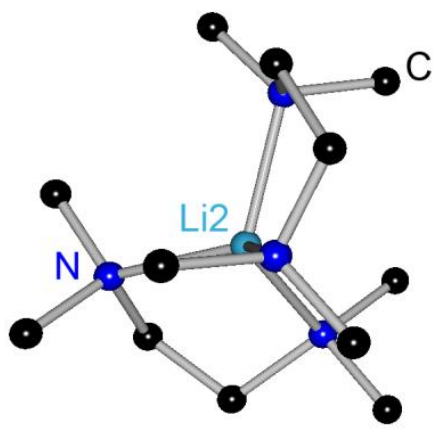

Figure 4-4: Crystal structure of [AnCH $\left.\mathrm{ALi}_{2} \mathrm{TMEDA}\right]$ (66), disordered fragments are omitted for clarity, anthracene moieties are labeled 1-3.

As the cell parameters had already indicated, the obtained structure differed significantly from the structure derived from previous measurements (Figure 4-4). Although the lithium cations and methylanthracenide anions are also separated ion pairs, the asymmetric unit contains two lithium ions and three methylanthracene molecules. This implies that only two of the three methylanthracenes can be deprotonated to generate an overall neutral charge. This is most likely due to slowly proceeding decomposition of the lithiated species over the long crystallization time. This may also explain the color change from dark green to dark purple. Because crystallization was not observed for a fairly long time, possibly only the presence of methyl anthracene (from decomposition) promoted the crystallization process, forming an arrangement which is favorable for crystallization. Despite the clearly slower crystallization, the structure of $\mathbf{6 6}$ exhibits strong disorder. Although the resolution is sufficient to resolve disorder phenomena, the main objective of locating the position of the charge is still considerably hindered.

While both lithium positions are fixed, all four surrounding TMEDA donor molecules assume two different positions each. This would not affect the possibility of locating the position of the charge, but unfortunately the anthracene molecules are also disordered. Methylanthracene molecule 1 is located between both lithium cations. It exhibits a nearly 50:50 "head-to-tail" disorder of two positions with the methyl groups directed in opposite directions (which was also observed in the first diffraction experiments). Additionally, one of the two positions also shows rotational disorder of 
the 9-methyl group. Molecule $\mathbf{2}$ is also disordered in two positions in "head-to-tail" manner, and also one of the two positions exhibits a rotational disorder of the methyl group, which makes molecule 1 and $\mathbf{2}$ very much alike. Molecule $\mathbf{3}$ on the other hand does not assume two positions, but is completely fixed with exception of the methyl group which exhibits rotational disorder. Although all disorder can be described sufficiently, the low occupations (especially of the methyl proton positions) make reliable statements on the positions of the charges difficult.

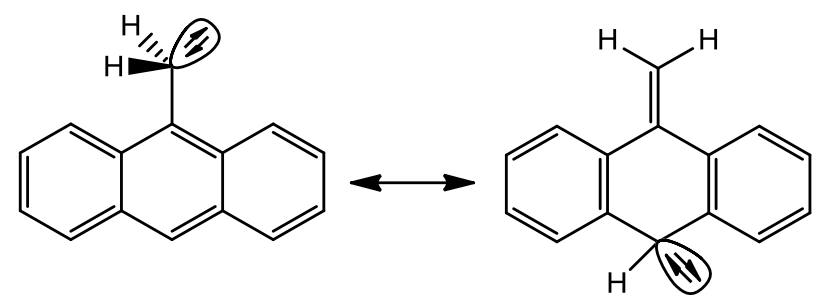

Scheme 4-10: Possible delocalization of the negative charge.

Furthermore, the disorder also makes the $\mathrm{C}-\mathrm{CH}_{2}$ distances - which could also indicate deprotonation of the methyl group - less accurate, which also disables this diagnostic option (Scheme 4-10: delocalization of the charge would induce shortening of the $\mathrm{C} 9-\mathrm{CH}_{2}$ bond). Although deprotonated and non-deprotonated methylanthracene molecules could also be disordered amongst one another, the structural information gathered lets this appear unlikely. The deprotonated methylanthracenes are probably exclusively located in the positions of molecule $\mathbf{1}$ and $\mathbf{2}$, as these both show the "headto-tail" orientation at a 50:50 ratio which was also found in the first structure (Figure 4-3) which did not contain un-deprotonated methylanthracene. The fixed position of molecule $\mathbf{3}$ in turn suggests that only un-deprotonated methylanthracene is located there. Moreover the deprotonation almost certainly takes place at the methyl group because all aromatic hydrogen positions are fully occupied.

Although the information acquired from the crystal structures cannot fully answer all questions concerning the position of the negative charge, it does show that the deprotonation using TMSMeLi in fact works and that the formed structure differs articulately from the structure of benzyl lithium described by Tatic et al. In the case of benzyl lithium, contact ion pairs are on hand which form a cyclic tetramer or a monomer depending on the donor base added. ${ }^{[104]}$ Also the $\mathrm{CH}_{2}$ groups exhibit perfectly planar geometry (tetramer) or nearly planar geometry (monomer), indicating 
a change of hybridization from $\mathrm{sp}^{3}$ to $\mathrm{sp}^{2}$. This was not observed for any of the $\mathrm{CH}_{2}^{-}$ groups in 66 .

To eliminate remaining uncertainties regarding the position of the charge, 66 was subjected to NMR experiments. It was discovered that the lithiation could also be carried out in THF instead of diethyl ether, so the reaction was conducted in situ in an NMR tube with THF- $d_{8}$ as a solvent. ${ }^{1} \mathrm{H},{ }^{13} \mathrm{C}, \mathrm{H}-\mathrm{H}$ COSY, ${ }^{13} \mathrm{C} \mathrm{HSQC}$ and ${ }^{13} \mathrm{C} \mathrm{HMBC}$ experiments were conducted and the structure could be fully described.

At first sight, it is striking that the entire ${ }^{1} \mathrm{H}$ NMR spectrum of 66 appears to be shifted up-field (Figure 4-5, left top \& bottom) compared to 9-methylanthracene. The peaks are also clearly farther apart in the spectrum of 66.

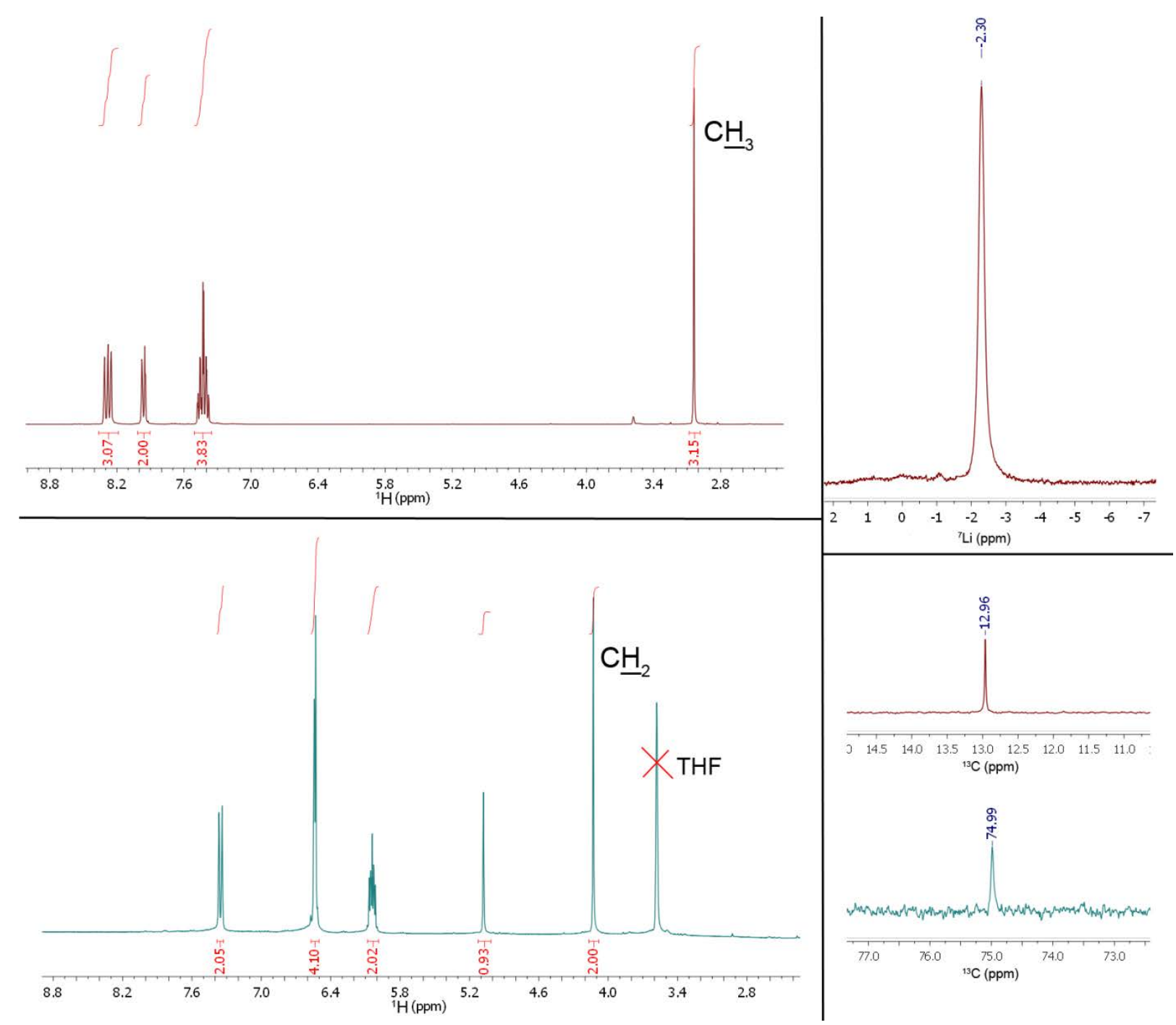

Figure 4-5: Left: ${ }^{1} \mathrm{H}$ NMR spectra of 9-methylanthracene (top) and 66 (bottom); top right: ${ }^{7}$ Li NMR spectrum of 66 ; bottom right: ${ }^{13} \mathrm{C}$ NMR shifts of C15 in 9-methylanthracene (red) and 66 (cyan). 
The general up-field shift indicates stronger shielding of the protons in $\mathbf{6 6}$ compared to 9-methylanthracene, which is imposed by higher electron density. While the chemical shifts of 9-methylanthracene are in the expected range of aromatic protons, the shifts found for $\mathbf{6 6}$ are far lower than expected.

The ${ }^{7}$ Li NMR spectrum shows a single peak at $-2.3 \mathrm{ppm}$ which proves that there is only one single species of lithium ions present in 66 (Figure 4-5, top right). This is in accordance with the results of the diffraction experiment (both lithium atoms coordinated by two TMEDA molecules). Li-C coupling is not observed which further supports the assumption that the separated ion pair which was found in the solid state is also present in solution.

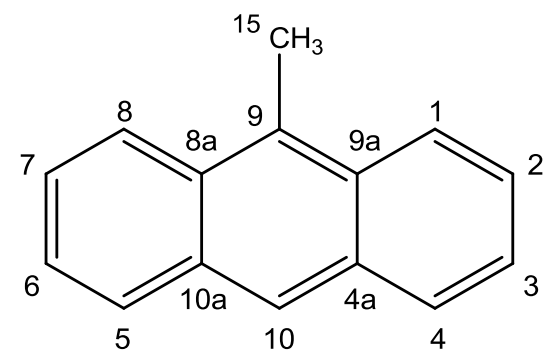

Scheme 4-11: Applied numbering of the anthracene moiety.

The integrals of the ${ }^{1} \mathrm{H}$ NMR spectrum also confirm the observation derived from the crystal structure that the deprotonation exclusively occurred at the methyl group (Figure 4-5, left). The ${ }^{13} \mathrm{C}$ NMR chemical shifts of C15 also indicate the deprotonation of the methyl group; the shift from $13.0 \mathrm{ppm}$ (9-methylanthracene) to $75.0 \mathrm{ppm}(66)$ is very large (Figure 4-5, bottom right). In comparison, the chemical shift of the $\mathrm{CH}_{2}$ carbon atom in benzyl lithium is only 31.7 ppm (tetramer) and 36.1 ppm (monomer).

The signal assignment between starting material and lithiated species depicted in Figure 4-6 confirms that all signals are shifted up-field by deprotonation of the methyl group, except for the signal of the methylene protons themselves. Especially the shift of the H10-singlett from $8.33 \mathrm{ppm}$ to $5.06 \mathrm{ppm}$ is drastic. The down-field shift of the methylene proton signal (formerly methyl protons) from $3.10 \mathrm{ppm}$ to $4.12 \mathrm{ppm}$ is quite surprising, because obverse observations were made for toluene/benzyl lithium, where a slight up-field shift of the methylene proton signal compared to the methyl proton signal of toluene was found. ${ }^{[104]}$ The up-field shift of the aromatic proton signals compared to toluene is however observed for benzyl lithium, although much weaker than in 66. 


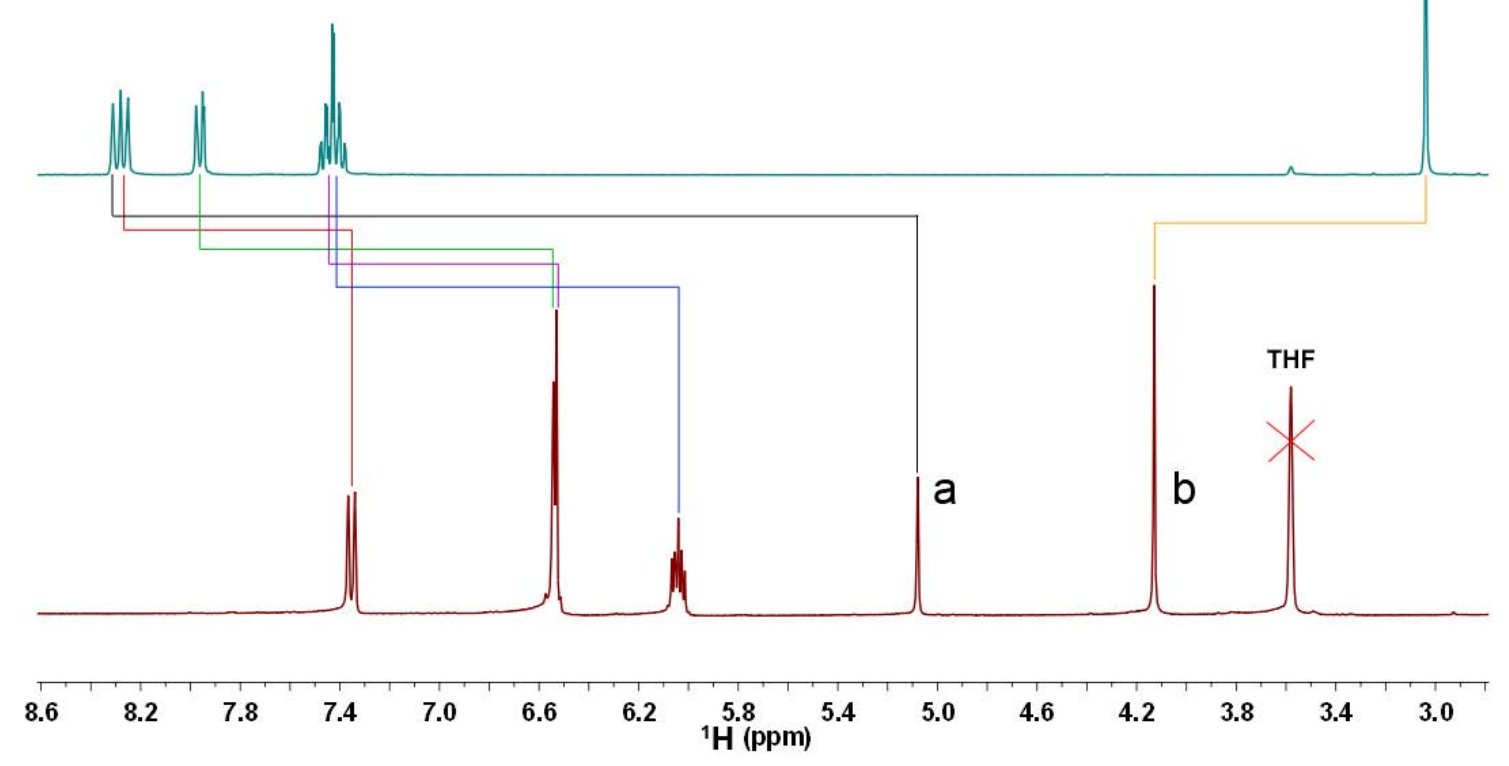

Figure 4-6: ${ }^{1} \mathrm{H}$ NMR spectra and signal assignment of 9-methylanthracene (top) and [AnCH $\left.{ }_{2} \mathrm{Li} \cdot \mathrm{TMEDA}\right]$ (66) (bottom); $\mathbf{a}=\mathrm{H} 10, \mathbf{b}=\mathrm{C}_{2}$ (formerly $\mathrm{C}_{3}$ ).

Though the deprotonation of the methyl group is verified, the localization of the negative charge is still questionable. Delocalization of the charge in the aromatic ring system as well as localization at any position in the aromatic system would require at least partial $\pi$-bonding between $\mathrm{C} 15$ and C9. This would alter the benzylic character of the methylene protons to (at least by trend) that of vinylic protons (Scheme 4-12). The chemical shift of vinyl protons is clearly further down-field, between 4.6 and 5.0 ppm for terminal $\mathrm{C}=\mathrm{C}_{2}$ protons, and between 5.2 and 5.7 for $\mathrm{C}=\mathrm{C} \underline{\mathrm{H} R}$ protons. ${ }^{[105]}$ In the case of benzyl lithium described by Tatic et al., this is by no means observed. The upfield shift of the methylene proton signals indicates that there is virtually no $\pi$-bonding between $\mathrm{C} 7$ and $\mathrm{C} 1$, despite the planar geometry of the methylene group. In the case of 66 the down-field shift of the methylene proton signals suggests at least partial $\pi$ bonding between $\mathrm{C} 9$ and $\mathrm{C} 15$. This is further supported by the strong up-field shifts of the aromatic signals. Especially the drastic up-field shift of the H10-signal implies a decrease of aromatic character in the central anthracene cycle. 

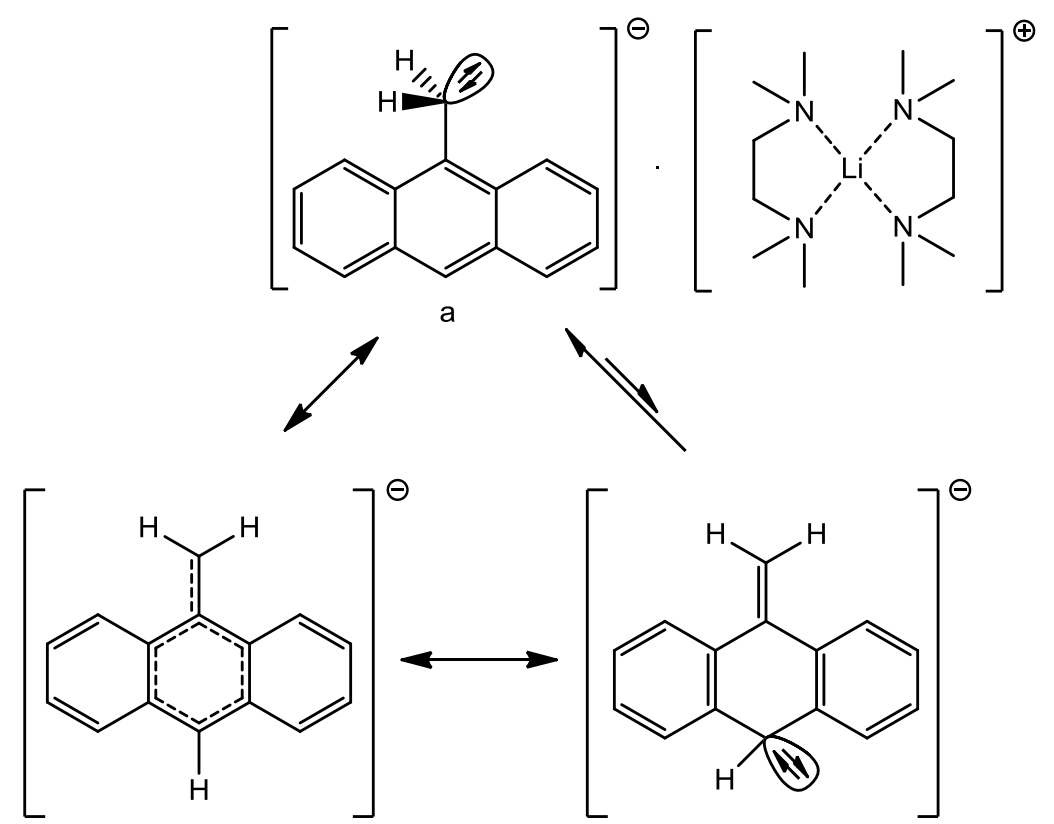

b

Scheme 4-12: Possible localizations of the negative charge in 66.

Taking into account the findings derived from the crystal structure and the observed chemical shifts, a localization of the charge at C15 can be assumed (Scheme 4-12, a), with a minor portion of the charge coupling into the aromatic system. The down-field shift of the methylene protons is clearly too weak to postulate full $\pi$ bonding between C9 and C15. Nevertheless a small fraction of the charge is transferred to the aromatic system, inducing an up-field shift of the corresponding proton signals.

\section{b) Asymmetric introduction of spacers via established synthetic strategies}

Most di-substituted sensor molecules known in literature - independent of whether they are symmetric with two identical receptor units or asymmetric with two different receptor units - contain two identical spacers. ${ }^{[15 d, 23 a, 28]}$ Starting materials are often 9,10-bis(chloromethyl)anthracene or 9,10-bis(bromomethyl)anthracene, which both lead to sensor molecules containing two methylene spacers. Depending on the quencher moieties, different spacer lengths can be beneficial for effective quenching, ${ }^{[23 a]}$ or certain analytes may require increased flexibility of the spacer due to steric demand. For cases like these, the availability of a precursor molecule with two different spacers is desirable. 


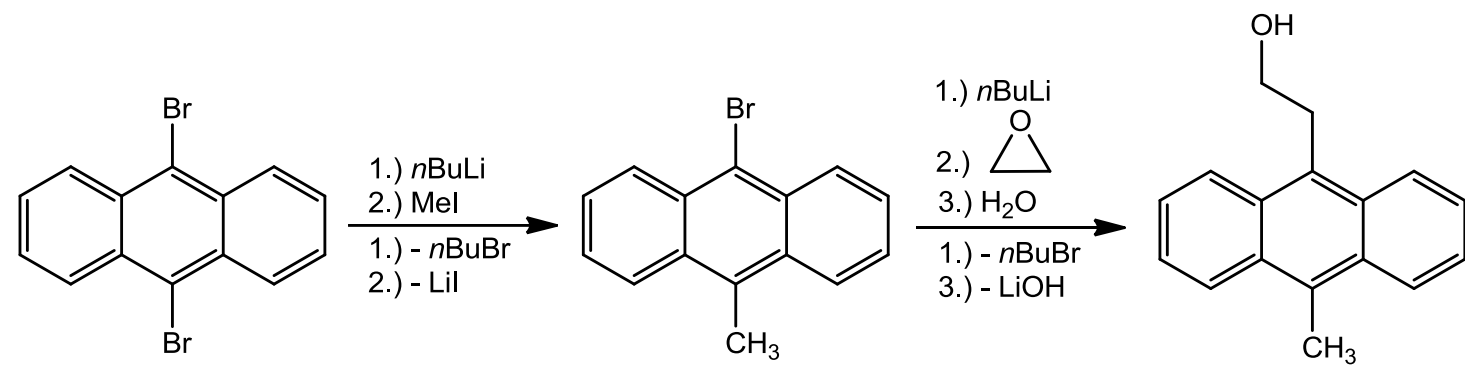

67

Scheme 4-13: Introduction of two different spacers to the anthracene fluorophore.

Synthesis is possible starting from the easily accessible 9,10-dibromoanthracene, which was converted to 9-bromo-10methylanthracene by selective mono-lithiation ${ }^{[59 a,}$ 60] and quenching with methyl iodide (Scheme 4-13). After aqueous work-up for removal of the lithium salt, a second lithiation was conducted in diethyl ether at $-15^{\circ} \mathrm{C}$. The solution of the lithiated intermediate was stirred at $0^{\circ} \mathrm{C}$ for $10 \mathrm{~min}$ and then again cooled to $-15^{\circ} \mathrm{C}$. Subsequently, gaseous oxirane was discharged into the solution with an inlet tube for $25 \mathrm{~min}$. Hydrolysis of the resulting lithium alkoxide, drying over $\mathrm{MgSO}_{4}$ and removal of the solvent afforded 9-(2-hydroxyethyl)-10-methylanthracene (67) as a yellow amorphous solid which was further purified by column chromatography (pentane/EtOAc 4:1). Although the preparation of 67 has previously been reported in literature, ${ }^{[106]}$ a completely different synthetic route was used, which is why its synthesis is described here.

In order to introduce quencher moieties (e.g. amines) to the molecule, the nucleophilic hydroxyl group must be converted to a suitable leaving group. This was achieved by reaction with $\left[\mathrm{BrPPh}_{3}\right] \mathrm{Br}$, - which was prepared by reaction of triphenylphosphane with elemental bromine - in an Appel type reaction (Scheme 4-14). ${ }^{[107]}$

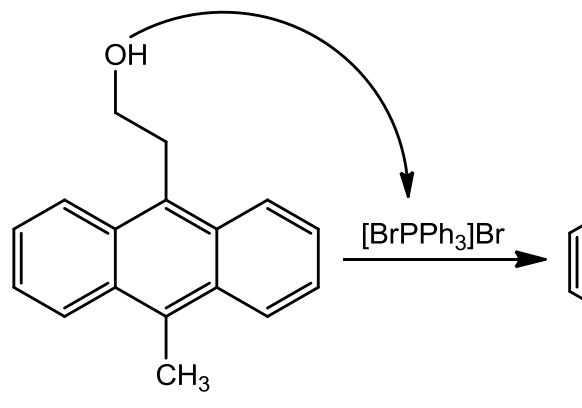

67<smiles></smiles>

68

Scheme 4-14: Synthesis of 9-(2-bromoethyl)-10-methylanthracene (68). 
To a solution of freshly prepared [ $\left.\mathrm{BrPPh}_{3}\right] \mathrm{Br}$ in $\mathrm{MeCN}$ a solution of 67 in $\mathrm{MeCN}$ was added over $30 \mathrm{~min}$ at ambient temperature and stirred for $15 \mathrm{~h}$. Then the solvent was removed and the crude product was dissolved in EtOAc and filtered to remove the insoluble $\mathrm{OPPh}_{3}$. Recrystallization from EtOAc afforded 68 as a yellow crystalline solid. The obtained crystals were also suitable for X-ray diffraction experiments.

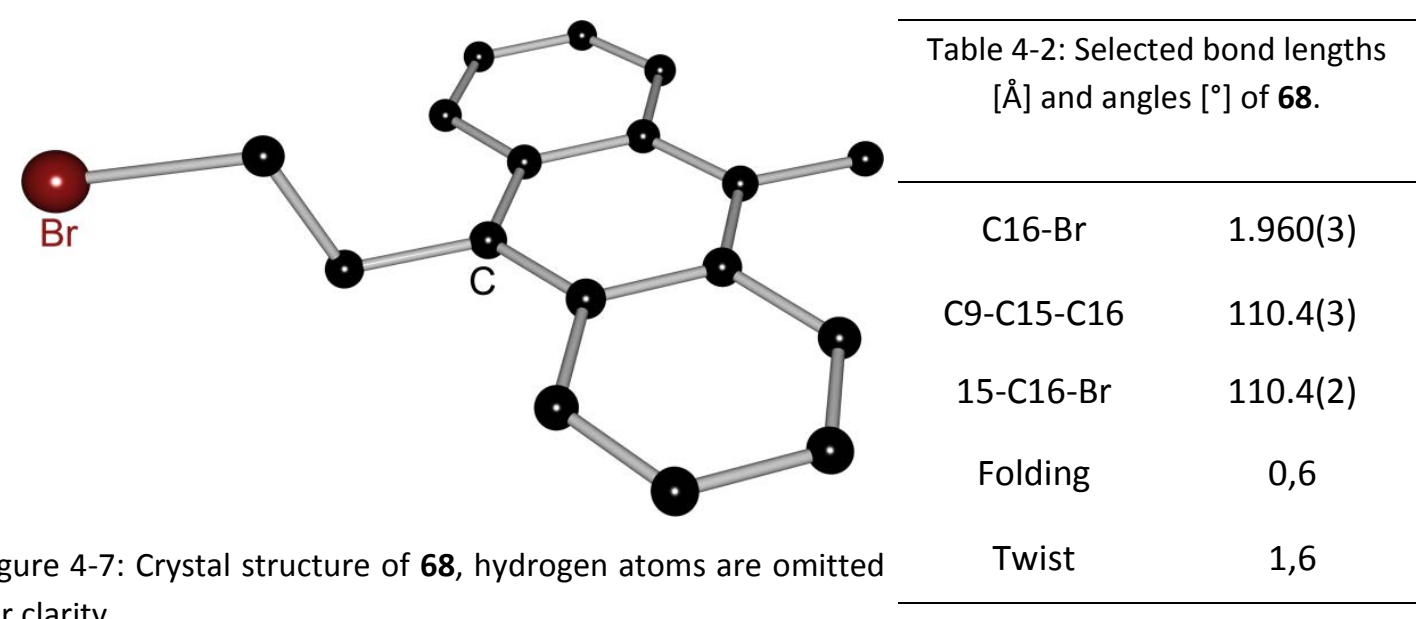
for clarity.

68 crystallizes in the monoclinic space group C2/c. All bond lengths and angles are in the expected range. The anthracene moiety shows very weak deformation due to the low steric demand of the substituents in 9,10-position (Figure 4-7).

68 is an outstanding starting material for asymmetric synthesis of sensor molecules. As shown in Scheme 4-15, 68 can be reacted with a nucleophilic receptor/quencher unit $\left(Q^{1}\right)$, followed by a second bromination at the 10-methyl group.

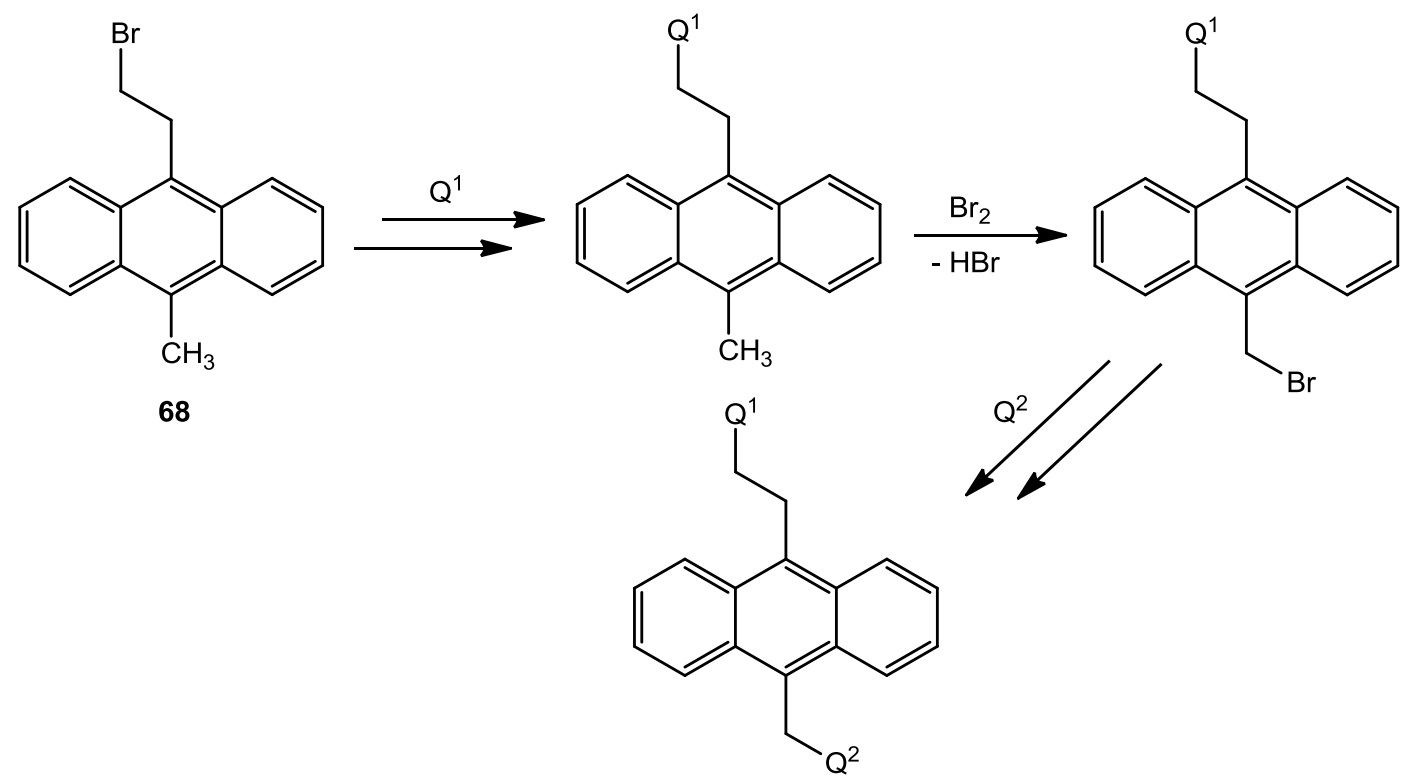

Scheme 4-15: Synthesis of an asymmetric sensor molecule. 
Stern has documented the feasibility of 10-methyl bromination of anthracene derivatives via an addition/elimination mechanism which would also apply to the compound depicted in Scheme 4-15. ${ }^{[59 c]}$ The final step would be a second $S_{N}$ reaction for introduction of a second receptor/quencher moiety $Q^{2}$. Depending on the reactivity of the nucleophiles used and the corresponding reaction conditions required, the primary alcohol 67 can also be converted to a tosilate which represents an even better leaving group than bromide in $\mathrm{S}_{N}$ chemistry. ${ }^{[59 c]}$

\subsection{Amine Based Sensor Molecules}

The next step in generating potential sensor molecules was the unification of the synthesized receptor units with fluorophore/spacer moieties forming quencherspacer-fluorophore arrangements (c.f. 4.0). Besides the synthesized amines described in 4.1.1, also commercially available amines were used. Because the incorporation of such "simple" receptor units in sensing devices has been thoroughly investigated and described in literature, ${ }^{[23 a]}$ the compounds bearing these receptor units were mostly utilized for the investigation of secondary substituent effects (in 10-position) and to gain a general understanding of the occurring fluorescence mechanisms. Although a number of new quenching mechanisms has been discovered in the past years ${ }^{[31]}$ some of them occurring in combination with the established mechanisms - the standard approach towards amine quenchers and anthracene fluorophores is still the PET effect, which will be predominantly addressed in the following subchapters.

\subsubsection{Synthesis of 9-Bromo-10-TMEDA-anthracene (69)}

$N, N, N^{\prime}$-trimethylethylenediamine is a commercially available reagent which can be utilized as a quencher/receptor unit in sensor molecules. Its close relation to the common chelating ligand TMEDA suggests analogous coordination behavior towards a large variety of cations. It has been utilized by Huston et al. in the symmetric sensor molecule 9,10-bis(TMEDA)anthracene for detection of $\mathrm{Zn}^{2+}$ ions. ${ }^{[24]}$ Although Huston and co-workers reported very strong fluorescence enhancement, the selectivity of the sensor is presumably very low due to the affinity of the TMEDA receptor towards multiple metal ions. As most amine based PET sensor molecules, it also shows very 
strong fluorescence enhancement upon protonation at low $\mathrm{pH}$ values. The asymmetric compound with a bromo substituent in 9-position which allows further derivitization has not been synthesized previously. The simple and reliable PET system supplied by the TMEDA receptor is well suitable for the investigation of secondary substituents at the fluorophore on the PET mechanism.

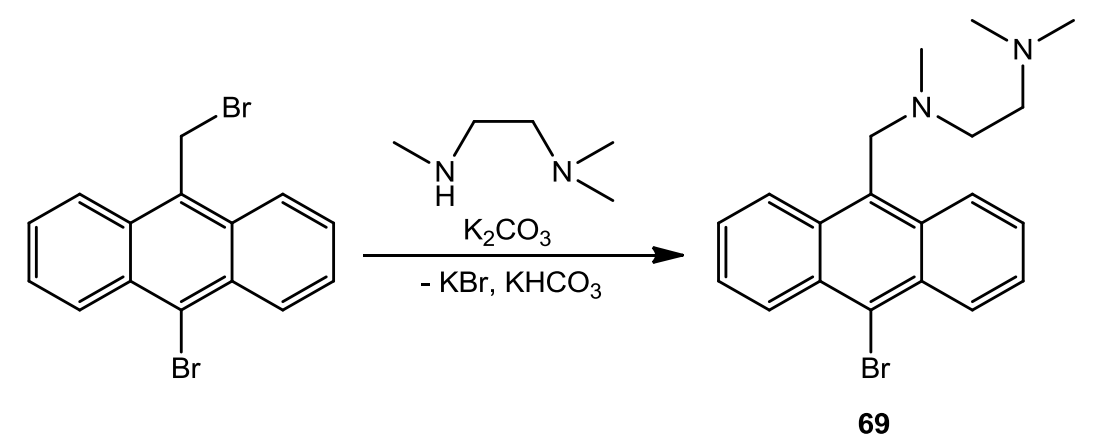

Scheme 4-16: Synthesis of 9-bromo-10-TMEDA-anthracene (69).

9-Bromo-10-bromomethylanthracene was prepared by bromination of 9-bromo-10methylanthracene according to Stern. ${ }^{[59 c]}$ It was reacted with 1.0 equivalents of $N, N, N^{\prime}$ trimethylethylenediamine and 4.0 equivalents of $\mathrm{K}_{2} \mathrm{CO}_{3}$ in $\mathrm{MeCN}$ at $82^{\circ} \mathrm{C}$ over $4 \mathrm{~h}$ (Scheme 4-16). After completion of the reaction the solvent was removed and the crude product was dissolved in DCM and extracted with water. Drying of the organic phase over $\mathrm{MgSO}_{4}$ and evaporation of the solvent afforded 69 as an orange semi-solid (yield: $73 \%$ ).

Next, further alterations in 9-position of the fluorophore by substitution of the bromine atom were attempted. Similar reactions have been used to link sensor molecules to solid state materials or surfaces or e.g. cyano substituents have been introduced to sensor molecules to improve the electron acceptor properties. Generally the 10-position is very sensitive towards the alteration of HOMO and LUMO energies of the fluorophore, as e.g the strong emission shift upon introduction of phosphoryl substituents and boranyl substituents to the anthracene moiety has shown (c.f. 3.13.6).

Because most anthracene derivatives are virtually insoluble in water, the applications of corresponding sensor molecules are often limited to organic solvents. Regarding the poor solubility of most cations in organic media, an enhancement of the sensor's solubility in aqueous environments is desirable, e.g. for in vivo detection of metal ions. For this purpose the introduction of a carboxyl group was attempted by 
lithiation of 69 and subsequent reaction with $\mathrm{CO}_{2}$ by addition of the lithiated intermediate to solid $\mathrm{CO}_{2}$ (Scheme 4-17, Reaction A). This reaction type has been successfully applied to several anthracene derivatives. ${ }^{[59 c, 108]}$ Unfortunately, only 9-TMEDA-anthracene was recovered due to hydrolysis of the lithiated intermediate. In the light of the small amount of only $1.6 \mathrm{mmol}$ of 69 used for the reaction, the moisture on the surface of the dry ice was presumably sufficient for complete hydrolysis of the lithiated species.

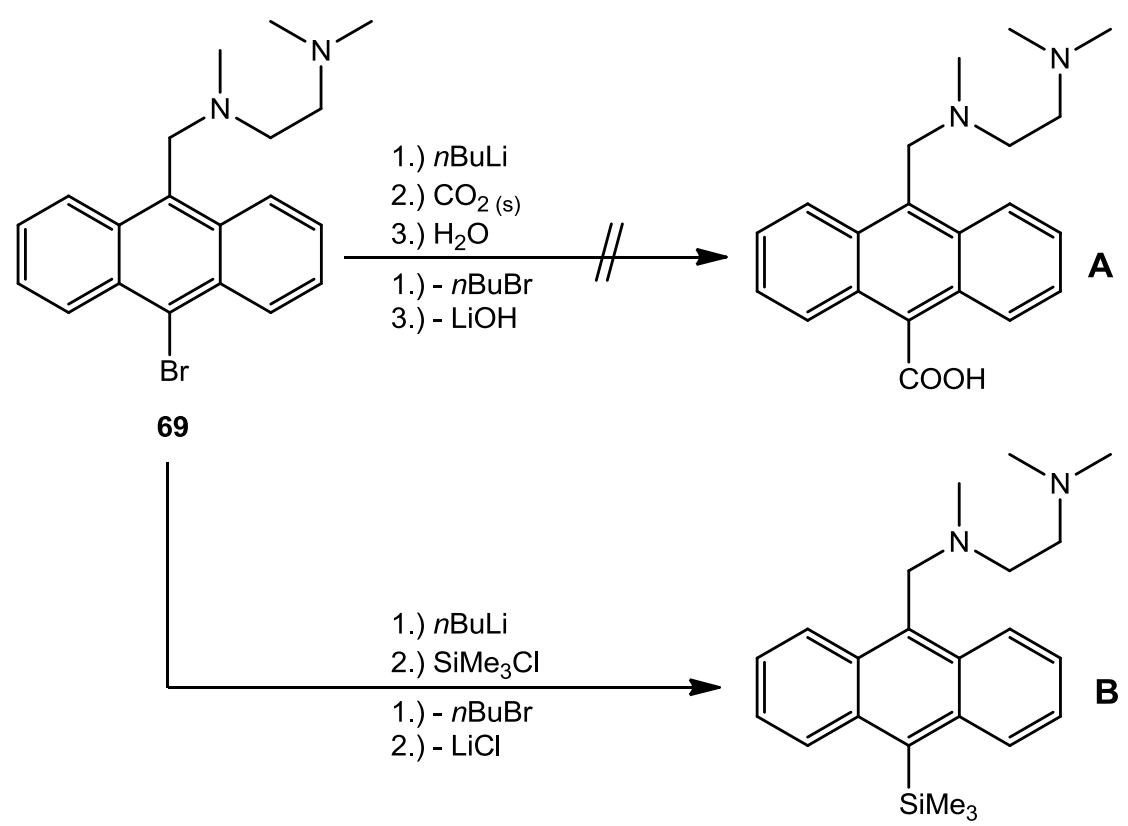

Scheme 4-17: Further reactions of 69.

The reaction was repeated with gaseous $\mathrm{CO}_{2}$ which was discharged into a solution of lithiated 69, but again the contained moisture lead to hydrolysis and only 9-TMEDAanthracene was obtained. Utilization of fresh dry ice or up-scaling of the reaction are possible approaches towards solving this problem.

While influencing general properties like solubility is one option of derivatization, alteration of the electronic properties is another. In 2009 Maeda et al. were able to prepare 9-trimethylsilylanthracene as well as its germyl- and stannyl- analogues and investigate their fluorescence properties. ${ }^{[109]}$ It was found that the TMS substituent produces a pronounced elongation of fluorescence lifetime as well as an increase of emission intensity and a red-shift of the emission maximum compared to unsubstituted anthracene. In combination with a quencher moiety, the longer fluorescence lifetime induced by a silyl substituent may produce more effective 
quenching, because the probability of electron transfer to the fluorophore in the excited state would also increase in proportion to the adaptable time interval. This would produce larger on/off emission ratios.

As described by Maeda et al., the lithiated intermediate was reacted with $\mathrm{TMSCl}$ at $-15^{\circ} \mathrm{C}$ for introduction of the silyl group (Scheme $4-17$, reaction B). After completion of the reaction the solvent was evaporated and the crude product was re-dissolved in DCM and filtrated to remove the contained lithium chloride. The obtained dark yellow oily product contained a mixture of the desired product and unreacted 69 . The attempted separation of both products by column chromatography was not successful, because the exposure to oxygen led to cleavage of the silyl group from the anthracene moiety.

\subsubsection{Synthesis and Fluorescence Properties of 9-Bromo-10-bis(2- methoxyethyl)aminomethylanthracene (70)}

The second commercially acquired amine receptor used was bis(2methoxyethyl)amine. Its close structural relation to diglyme and PMDTA should provide good conditions for formation of chelate complexes. Although bis(2methoxyethyl)amine has been used as a receptor unit in chemical sensing, it not been used for cation detection as a chelating ligand, but in the attempted detection of boranes. After synthesizing a PET on/off sensor system, Arimori et al. intended to inhibit the electron transfer process by lowering the redox potential of the receptor unit through formation of adducts between the quencher amine and free boranes. ${ }^{[110]}$ Although they did observe an increase of emission, revision of their results revealed that the observed phenomena did not follow from B-N bonding, but rather from protonation of the amine. The moisture contained in the utilized solvents formed adducts with the Lewis acidic boranes which again were acidic enough to protonate the amine quencher.

A very similar compound based on bis(2-hydroxyethyl)amine was published in 2000 by Wang et al. who also postulated an adduct formation between their sensor molecule and Lewis acidic boranes, which led to emission enhancement. ${ }^{[89]}$ In light of the conclusions drawn by Arimori and due to the $\mathrm{MeOH}$ (which is most probably not 
water free) used as a solvent by Wang et al., the results reported by latter group seem at least questionable.

An analogous compound having a bromine atom in 10-position for further derivatization has not yet been reported. 9-Bromo-10-bromomethylanthracene was reacted with one equivalent of bis(2-methoxyethyl)amine and four equivalents of $\mathrm{K}_{2} \mathrm{CO}_{3}$ in $\mathrm{MeCN}$ under reflux for $4 \mathrm{~h}$ (Scheme 4-18). The solvent was evaporated, water was added and the product extracted with DCM. The organic layer was dried over $\mathrm{MgSO}_{4}$ and the solvent was removed, yielding 9-bromo-10-(bis(2-methoxyethyl)aminomethylanthracene (70) as a dark yellow oil (70\%).<smiles>COCCNCCOC(=O)C(C(=O)OC)(C(C)(C)C)C(C)(C)C</smiles>

Scheme 4-18: Synthesis of 9-bromo-10-(bis(2-methoxyethyl)aminomethylanthracene (70).

Although Arimori et al. had indirectly verified the PET mechanism for the bis(2methoxyethyl)amine quencher by protonation, ${ }^{[110]}$ a protonation was also conducted for the 9-bromo derivative under more accurate conditions. This way remaining insecurities regarding the suitability of the quencher could be eliminated.
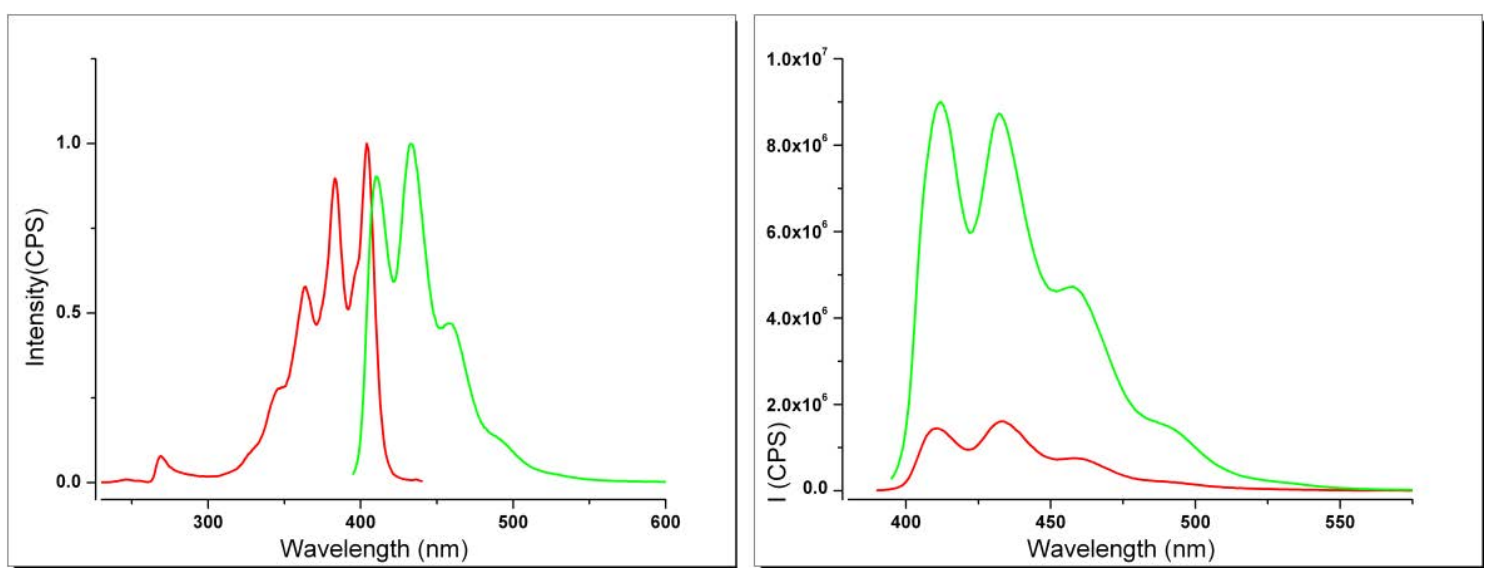

Figure 4-8: Left: normalized excitation (red) and emission (green) spectra of $70,5 \cdot 10^{-5} \mathrm{M}$ in DCM; right: emission spectra of 70 at $\mathrm{pH}=\sim 7$ (red) and $\mathrm{pH}=\sim 3$ (green), $5 \cdot 10^{-5} \mathrm{M}$ in $\mathrm{MeOH}$. 
The excitation and emission spectra of $\mathbf{7 0}$ (Figure 4-8, left) are nearly identical in shape and position of excitation and emission maxima to those of 9-bromo-10methylanthracene (BrAnMe). This confirms that the spacer minimizes the direct influence of the quencher substituent on the electronic properties of the fluorophore. As commonly observed, the excitation and emission spectra act like two symmetrically mirrored images. By addition of $\mathrm{HCl}$ to a methanol solution of 70 , the effects of protonation can be monitored. Lowering of the $\mathrm{pH}$ from nearly neutral to $\mathrm{ca}$. $\mathrm{pH}=3$, an increase of emission by a factor of 6.5 is achieved. This certifies a functioning PET mechanism for $\mathbf{7 0}$, which is suppressed by protonation-induced lowering of the redox potential of the amine quencher.

After confirming the validity of the PET mechanism, also the inhibition of PET by metal coordination had to be confirmed, because the bis(2-methoxyethyl)amine receptor has not yet been investigated in the context of cation detection. In 2002 Yang et al. published a fluorescence study of an anthracene based sensor with a 25,27-bis(1-propyloxy)calix[4]azacrown-5 receptor. ${ }^{[111]}$ Though more complex in structure, this receptor contains the identical O-N-O fragment as bis(2-methoxyethyl)amine. The fact that the calix[4]azacrown receptor showed pronounced sensitivity towards $\mathrm{Zn}^{2+}$ ions suggested to also investigate the sensitivity of $\mathbf{7 0}$ towards this cation.
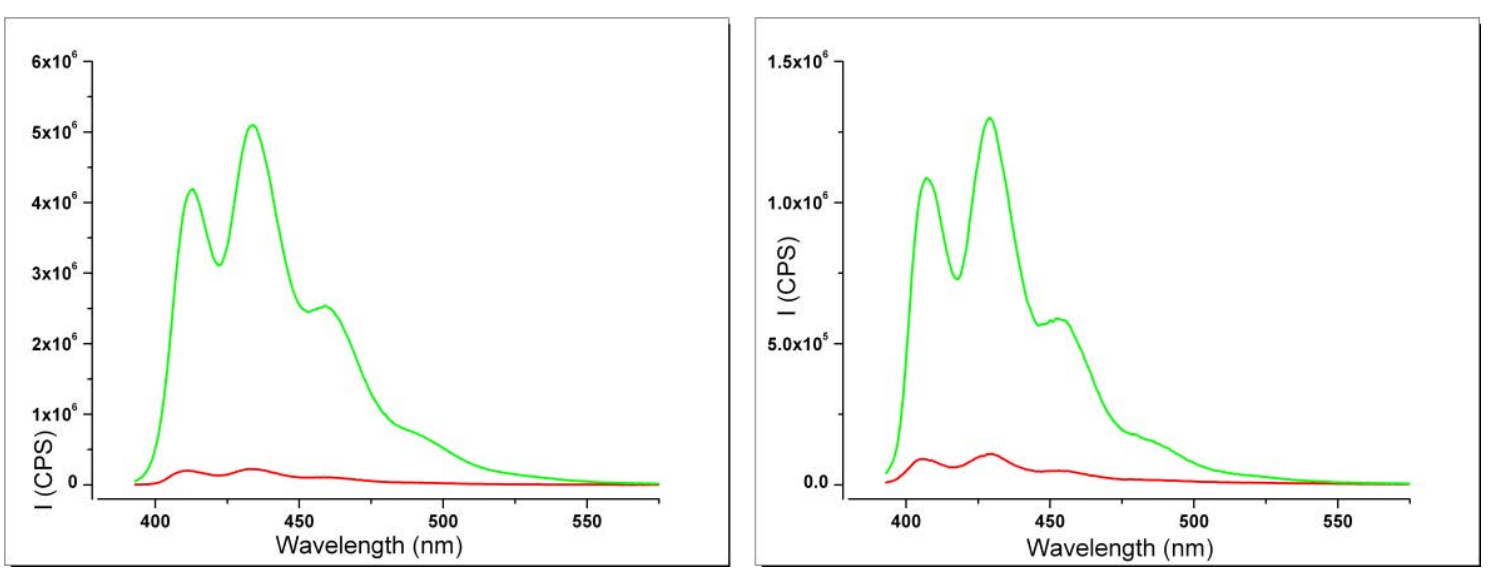

Figure 4-9: Left: emission spectra of $70\left(5 \cdot 10^{-5} \mathrm{M}\right.$ in DCM) before addition of $\mathrm{ZnBr}_{2}$ (red) and after addition of $\mathrm{ZnBr}_{2}$ (green); right: emission spectra of $70\left(5 \cdot 10^{-5} \mathrm{M}\right.$ in $\mathrm{MeOH}$ ) before addition of $\mathrm{ZnCl}_{2}$ (red) and after addition of $\mathrm{ZnCl}_{2}$ (green).

As Figure 4-9 shows, the addition of $\mathrm{Zn}^{2+}$ leads to a strong increase of emission intensity. Both bromide and chloride counter ions were used in $\mathrm{DCM}$ and $\mathrm{MeOH}$ solutions to eliminate possible secondary effects originating from the solvent or counter ions. Although the overall emission was stronger in DCM at identical 
concentration, the relative amplification of emission was similar in both cases. This confirms that (though with supposed low selectivity) the complexation of $\mathrm{Zn}^{2+}$ inhibits the electron transfer process of $\mathbf{7 0 .}$

\subsubsection{Introduction of a Bis(2-pydridyl)amine Receptor}

The third and last commercially acquired amine used as a potential quencher was bis(2-pyridyl)amine. Although Stern has reported on synthesis and structure of 9bromo-10-(bis(2-pyridyl)aminomethyl)anthracene, ${ }^{[59 c]}$ the florescence properties of this compound were not previously investigated. Therefore the synthesis was reproduced, but via a different synthetic strategy. Stern had deprotonated bis(2pyridyl)amine with $n$-BuLi and then reacted the resulting amide with 9-bromo-10bromomethylanthracene. Due to the poor yield of this reaction the reaction conditions, which were previously successfully applied in the syntheses of 69 and 70 , were also applied to the reaction of bis(2-pyridyl)amine with 9-bromo-10bromomethylanthracene. Thus, both precursor compounds were reacted with four equivalents of $\mathrm{K}_{2} \mathrm{CO}_{3}$ in $\mathrm{MeCN}$ at $82^{\circ} \mathrm{C}$ (Scheme 4-19).<smiles>BrCc1c2ccccc2c(Br)c2ccccc12</smiles><smiles>C=C(C)C(=O)ONc1ccccn1</smiles><smiles>CCCN(CC)Cc1c2ccccc2c(Br)c2ccccc12</smiles><smiles>Cl[n+]1ccccc1N=c1ccccn1Cc1c2ccccc2c(Br)c2ccccc12</smiles>

Scheme 4-19: Reaction of bis(2-pyridyl)amine with 9-bromo-10-bromomethylanthracene. 
After completion of the reaction the solvent was evaporated, DCM was added and the organic layer was extracted with water and saturated $\mathrm{NaCl}$ solution. Drying over $\mathrm{MgSO}_{4}$ and removal of the solvent gave a yellow solid. Surprisingly, not the expected 9bromo-10-(bis(2-pyridyl)aminomethyl)anthracene was obtained, but the pyridinium salt 71 (Scheme 4-19, bottom). 71 was obtained in high purity at a yield of 85\% (in relation to $\mathrm{BrAnCH}_{2} \mathrm{Br}$ ) which indicates that it is clearly the main product under the reaction conditions described above. Possibly the steric shielding of the central amine nitrogen atom makes the nucleophilic attack of both pyridyl nitrogen atoms kinetically favorable.

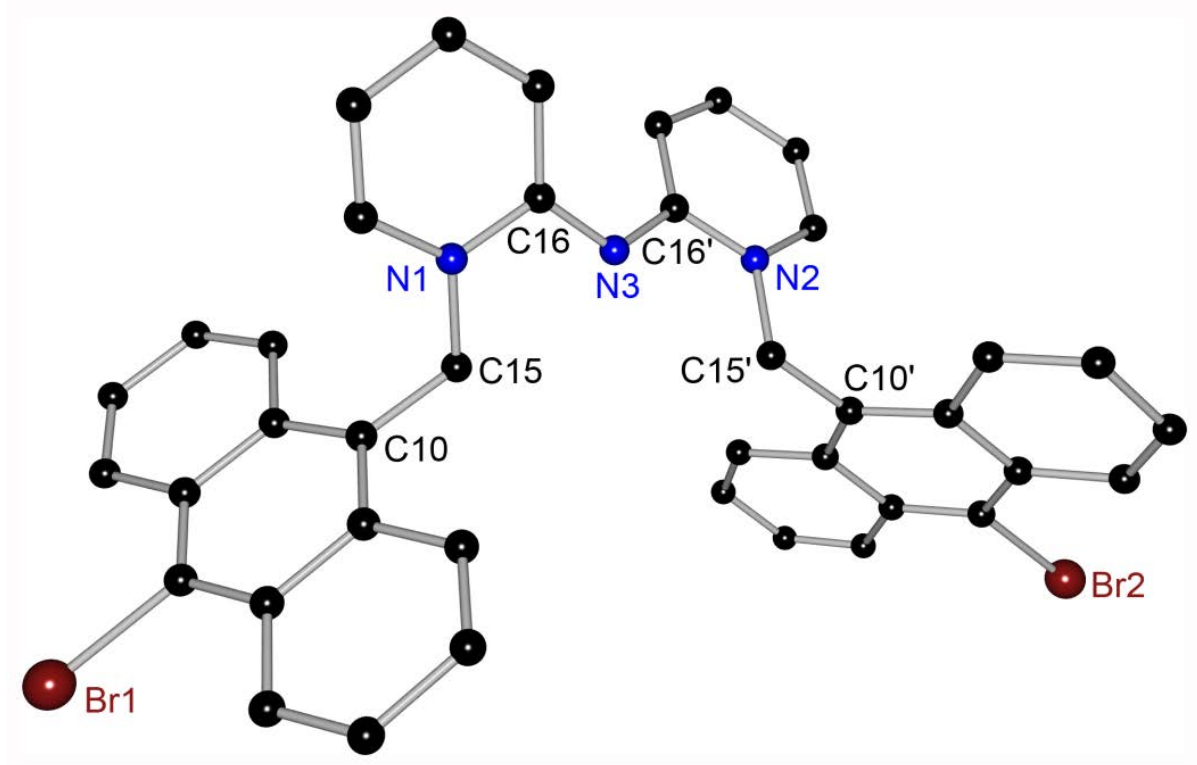

Figure 4-10: Crystal structure of 71, chloride counter ion, lattice solvent molecules and hydrogen atoms are omitted for clarity.

Table 4-3: Selected bond lengths $[\AA ̊]$ and angles $\left[{ }^{\circ}\right]$ of 71.

Crystallization of $\mathbf{7 1}$ from $\mathrm{MeOH}$ afforded crystals

\begin{tabular}{|c|c|c|c|c|}
\hline C15-N1 & $1.494(4)$ & C16-N3-C16' & $123.4(2)$ & which were also suitable for \\
\hline $\mathrm{C} 15^{\prime}-\mathrm{N} 2$ & $1.497(3)$ & C10-C15-N1 & $113.5(2)$ & X-ray \\
\hline N1-C16 & $1.373(4)$ & $\mathrm{C} 10^{\prime}-\mathrm{C} 15^{\prime}-\mathrm{N} 2$ & $114.5(2)$ & $\begin{array}{l}\text { determination.71 crystallizes } \\
\text { in the triclinic space group } P \overline{1}\end{array}$ \\
\hline $\mathrm{N} 2-\mathrm{C} 16^{\prime}$ & $1.385(4)$ & C10-C15-N1-C16 & $172.3(3)$ & and the asymmetric un \\
\hline C16-N3 & $1.332(4)$ & N1-C16-N3-C16' & 161.7(3) & contains the \\
\hline $\mathrm{C} 16^{\prime}-\mathrm{N} 3$ & $1.330(4)$ & N2-C16'N3-C16 & $153.4(3)$ & depicted in Figure 4-10 \\
\hline
\end{tabular}

and one $\mathrm{MeOH}$ and one water molecule. The nitrogen atom N3 does not carry a 
hydrogen atom. The bond distances and angles indicate that the positive charge is delocalized throughout both pyridyl rings as well as the bridging nitrogen atom N3. The C16-N3-C16' angle of nearly $120^{\circ}$ indicates $\mathrm{sp}^{2}$ hybridization of N3. Also the C16-N3 bond and the C16'-N3 bond are equally long which also confirms the delocalization of the charge. Otherwise N3 would carry one double bond and one single bond, which would notably differ in length.

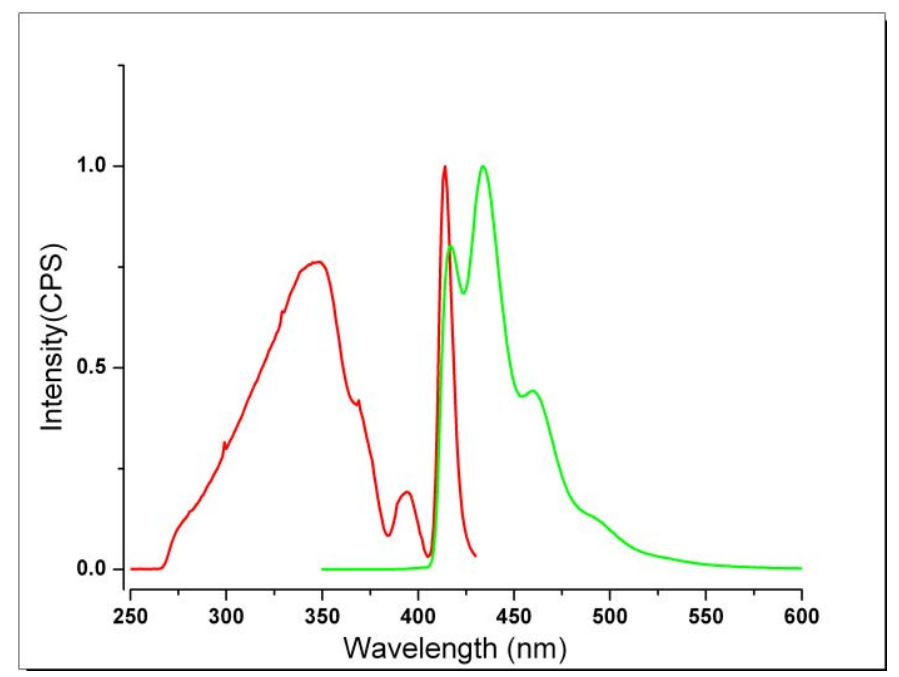

Figure 4-11: Normalized excitation (red) and emission (green) spectra of $71\left(5 \cdot 10^{-5} \mathrm{M}\right.$ in DCM).

The fluorescence properties of $\mathbf{7 1}$ were also investigated.The unusual structure of 71 also induces remarkable alterations of the fluorescence properties compared to previously described amine based compounds. While the shape of the emission spectrum shows the typical band structure and only differs minimally in the position of its emission maximum, the excitation spectrum differs largely from those of other amines (Figure 4-11). The regularly observed shape which resembles a mirrored image of the emission spectrum with three maxima is not found. Instead a broad excitation band at $340 \mathrm{~nm}$ is apparent, which is clearly blue-shifted compared to other amines. Furthermore a second very sharp band at $418 \mathrm{~nm}$ is present which was also not previously observed.

Due to the positive charge of the compound, electron transfer processes are not observed, which makes $\mathbf{7 1}$ strongly fluorescent. Accordingly, the observed emission intensity is not altered by the addition of metal cations or lowering of the $\mathrm{pH}$ value of the solution. 


\subsubsection{Synthesis and Fluorescence Properties of 9,10-Bis[bis(2- thienylmethyl)aminomethyl]anthracene (72).}

The first of the synthesized amine quenchers (c.f. 4.1.1) which was reacted with a fluorophore moiety was bis(2-thienylmethyl)amine (61).

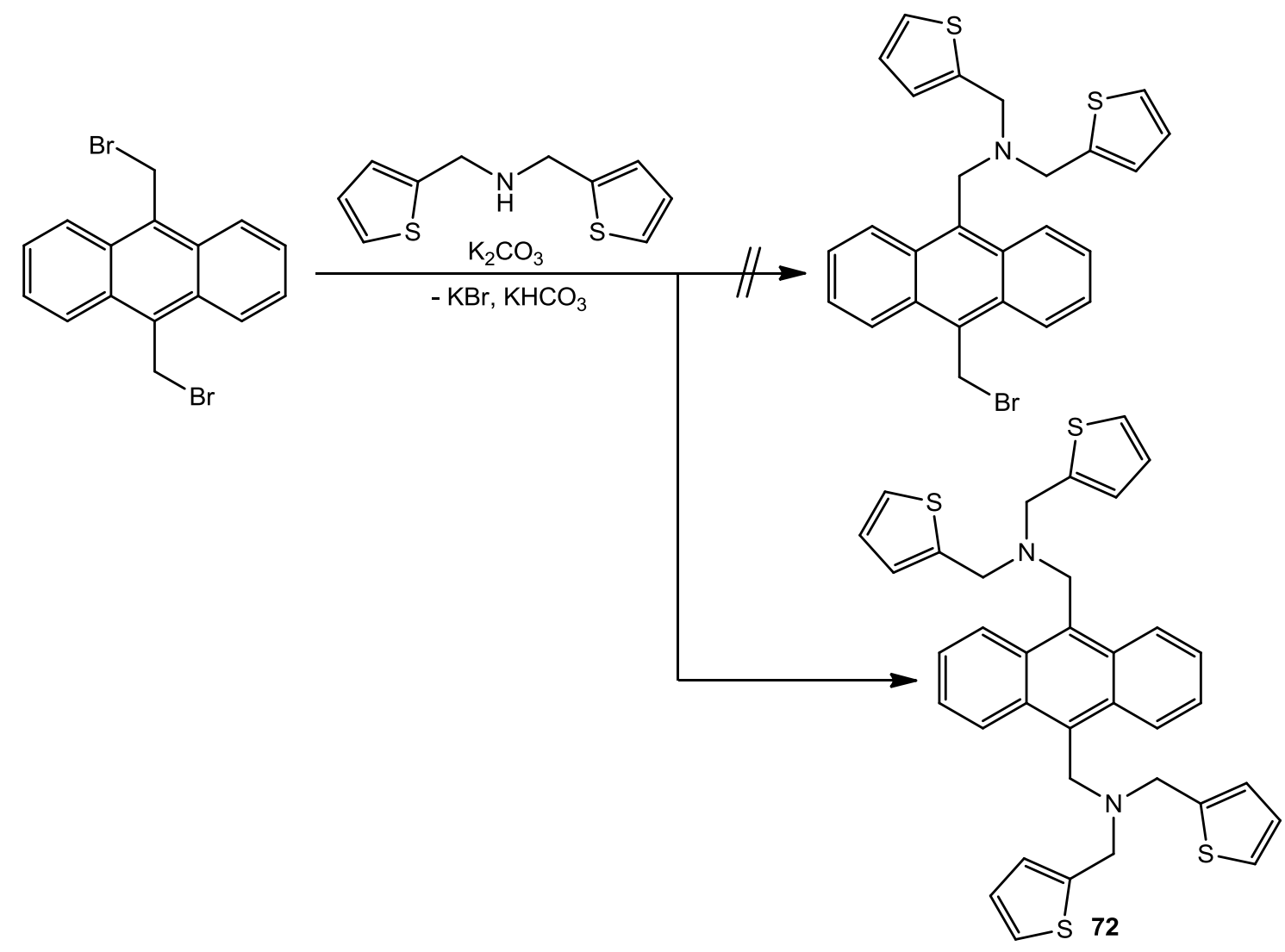

Scheme 4-20: Synthesis of $\mathbf{7 2 .}$

By reacting bis(2-thienylmethyl)amine (61) with one equivalent of the symmetric starting material 9,10-bis(bromomethyl)anthracene, it was attempted to synthesize the mono-substituted product (Scheme 4-20, top right). The recovered product however revealed that this substitution reaction cannot be controlled via stoichiometry, as exclusively the di-substituted compound $\mathbf{7 2}$ was formed. The product was purified by column chromatography and separated from unreacted 9,10bis(bromomethyl)anthracene. The yellow solid was recrystallized from DCM and thus obtained in high purity. Furthermore the solid state structure was determined (Figure 4-12). 


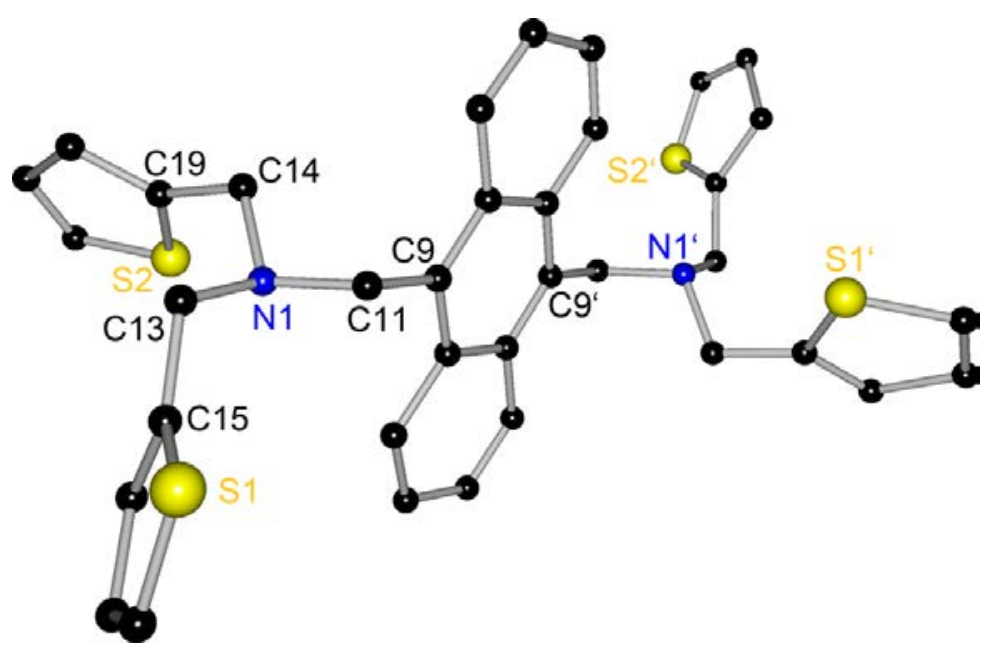

Figure 4-12: Crystal structure of $\mathbf{7 2}$, hydrogen atoms and disordered fragments are omitted for clarity.

The asymmetric unit contains one half molecule of $\mathbf{7 2}$, the other half is generated by an inversion center at the midpoint of the central $C_{6}$-perimeter. $\mathbf{7 2}$ crystallizes in the monoclinic space group $P 2_{1} / c$ and the symmetry of the molecule leads to a folding angle of the anthracene moiety of $0^{\circ}$. Though a twist angle of $5.0^{\circ}$ is observed, the overall deformation of the fluorophore is weak, which is ensured by the flexibility of the spacers which allow favorable orientations of the bulky amine substituents. As expected, the $\mathrm{C}-\mathrm{S}$ bond distances within the thienyl rings are distinctly longer than the C-C bonds (1.74 $\AA$ vs. $1.40 \AA$ ). But also the C-C bond distances vary strongly within the thienyl moieties, assuming values between $1.34 \AA$ and $1.41 \AA$. The geometry surrounding the nitrogen atoms is very close to ideal tetrahedral geometry.

Both symmetry independent thienyl rings exhibit disorder. One ring shows rotational disorder about the C14-C19 axis, generating two positions with an occupation of 66:34. The second ring also disordered in a 67:33 ratio, but in identical orientation without rotation.

The fluorescence properties of $\mathbf{7 2}$ were also investigated. The standard procedure of recording the compound's excitation and emission spectra, then verifying the PET mechanism by protonation of the amine, and then screening for sensitivity towards different metal cations was also applied here.

The electron transfer between quencher moiety and excited fluorophore was verified by protonation (Figure 4-13). The strong increase of emission intensity can be explained by the presence of two quencher moieties in the structure of $\mathbf{7 2}$. This leads 
to particularly effective fluorescence quenching in the "off" mode of the sensor molecule and therewith to very large on/off emission ratios (Figure 4-13).

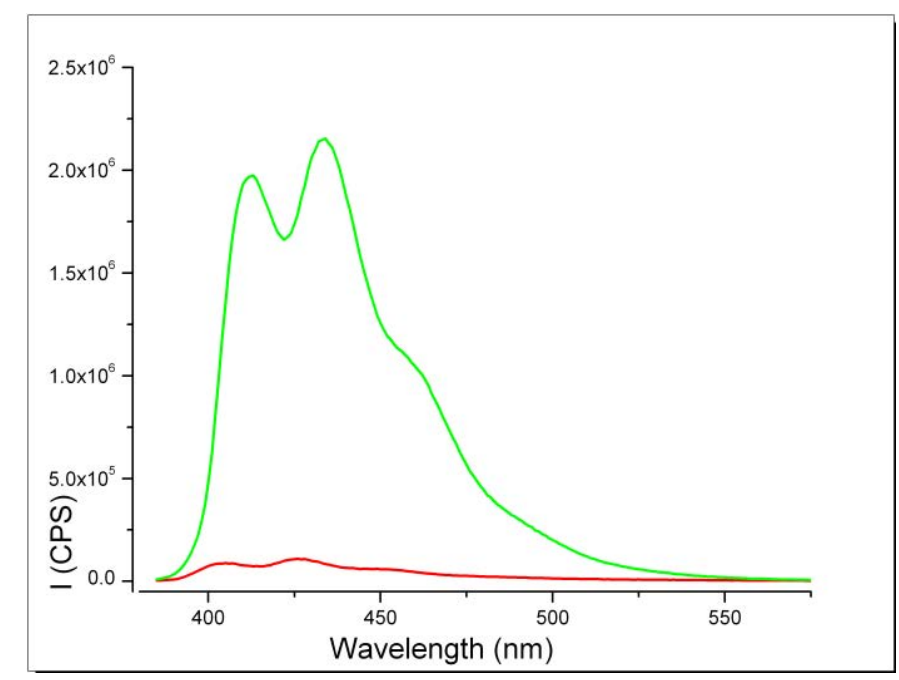

Figure 4-13: Emission spectra of $\mathbf{7 2}\left(5 \cdot 10^{-5} \mathrm{~m}\right.$ in $\left.\mathrm{MeOH}\right)$ before (red) and after protonation (green).

The screening for sensitivity of $\mathbf{7 2}$ towards cations was in contrast less fruitful. Strong coordination of cations and the corresponding increase of emission intensity were not found at high rates or noteworthy selectivity. A lately published electron density study on S-heteroaromatic compounds by Hey et al. reveals that the population of the sulfur lone pairs in S-heteroaromatic cycles is particularly low, which makes possible interactions with cations improbable or negligibly weak. ${ }^{[112]}$ This was also confirmed by the empirical results obtained by Granitzka, who found no favored orientation of thienyl donors towards electron pair accepting cations in his crystallized metal complexes of dithienylphosphanes. ${ }^{[113]}$ Therewith the receptor units of $\mathbf{7 2}$ can be virtually considered as monodentate ligands which makes the occurrence of chelation enhanced fluorescence (CHEF) unlikely.

\subsubsection{Synthesis and Fluorescence Properties of 9-[bis(2-tert- butylthioethyl)aminomethyl]anthracene (73)}

The next amine which was linked to the anthracene fluorophore was bis(2-(tertbutylthio)ethyl)amine (65). Again the well-proven reaction conditions of refluxing both starting materials with four equivalents of $\mathrm{K}_{2} \mathrm{CO}_{3}$ in $\mathrm{MeCN}$ were chosen (Scheme 4-21). 


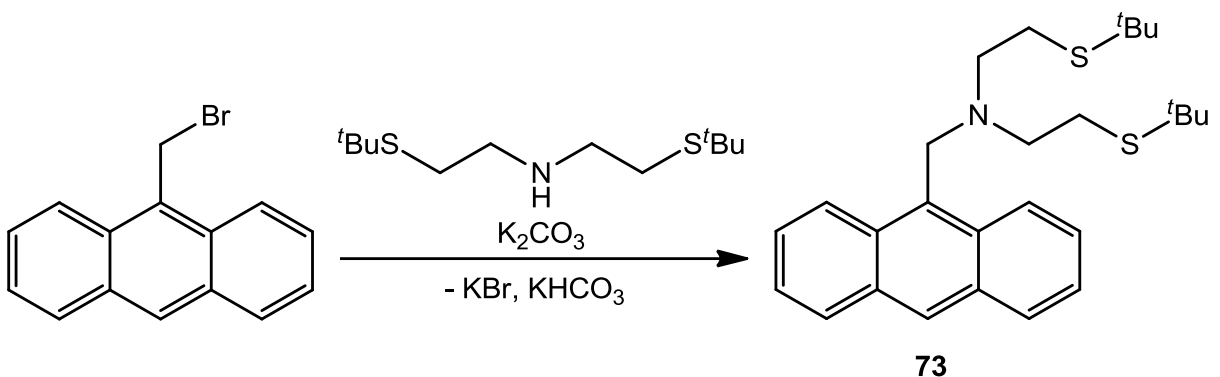

Scheme 4-21: Synthesis of 9-[bis(2-tert-butylthioethyl)aminomethyl]anthracene (73).

Because the receptor unit $\mathbf{6 5}$ had not been previously tested in chemical sensing, the possibility of further derivitization of $\mathbf{7 3}$ was set aside and 9(bromomethyl)anthracene was used as starting material, which lacks a second bromo substituent. The focus was thus clearly set on the properties of the receptor. After aqueous work-up and column chromatography, 73 was obtained as a particularly malodorous oily semi-solid. Crystallization of $\mathbf{7 3}$ from various solvents and at different temperatures was not successful. It was also attempted to crystallize the hydrochloride of $\mathbf{7 3}$, but the addition of $\mathrm{HCl}$ only afforded crystallization of decomposition products.

In 1999 Ishikawa et al. reported on synthesis of the closely related compound 6-(9'anthrylmethyl)-3,9-dithia-6-azaundecane which differs from $\mathbf{7 3}$ only in the terminal ethyl groups (instead of tert-butyl) of the functionalized amine side-arms. ${ }^{[101]}$ Along with several similar compounds, Ishikawa and co-workers investigated the sensitivity of this molecule's emission properties towards coordination of $\mathrm{Ag}^{+}$.
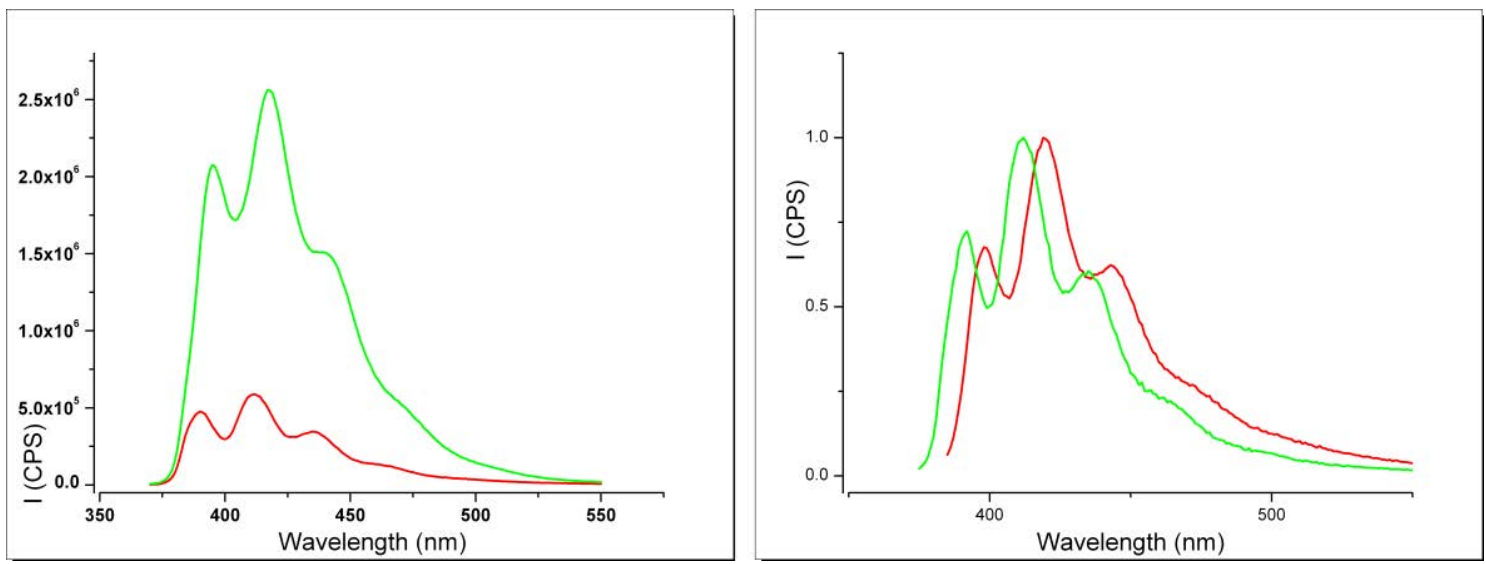

Figure 4-14: Left: emission spectra of $73\left(5 \cdot 10^{-5} \mathrm{M}\right.$ in $\left.\mathrm{MeOH}\right)$ at $\mathrm{pH} \approx 7$ (red) and $\mathrm{pH} \approx 2$ (green); right: normalized emission spectra of 73 in $\mathrm{MeOH}$ (green) and DCM (red). 
Although they were able to verify the PET mechanism of their compound by protonation and were also able to detect the coordination of $\mathrm{Ag}^{+}$, the on/off ratios as well as the selectivity were fairly low. This may be improved by the stronger steric demand of the terminal tert-butyl substituents in 73. The observed increase of emission upon protonation of $\mathbf{7 3}$ proves the presence of an electron transfer process to the excited fluorophore in the free ligand (Figure 4-14, left). This in accordance with the results of Ishikawa, who made identical observations for the structurally related 6(9'-anthrylmethyl)-3,9-dithia-6-azaundecane. Furthermore it was found that $\mathbf{7 3}$ shows a shift of the emission maximum by $5 \mathrm{~nm}$ when dissolved ion DCM instead of methanol, which was not observed to this degree for other amines (Figure 4-14, right).

The screening for sensitivity of $\mathbf{7 3}$ towards complexation of metal cations was conducted by addition of various metal salt solutions to samples of $\mathbf{7 3}$. The soft sulfur donors were thought to make coordination of soft transition metal ions particularly favorable, which is why the focus was set mainly on this group of metals. The sensitivity of Ishikawa's structurally closely related 6-(9'-anthrylmethyl)-3,9-dithia-6azaundecane towards $\mathrm{Ag}^{+}$ions could not be confirmed for 73 . This may be attributed to the unconventional approach chosen by Ishikawa and co-workers for detection of $\mathrm{Ag}^{+}$. Instead of lowering the redox potential of the amine quencher by coordination of $\mathrm{Ag}^{+}$and therewith inhibiting the PET, they used the protonated (strongly fluorescent) species of their compound. Upon addition of $\mathrm{Ag}^{+}$, the decrease of emission observed by Ishikawa and co-workers was ascribed to displacement of protons from the amine coordination site by strong binding of $\mathrm{Ag}^{+}$. The resulting lower rate of protonation was presumed to produce the observed weaker emission.

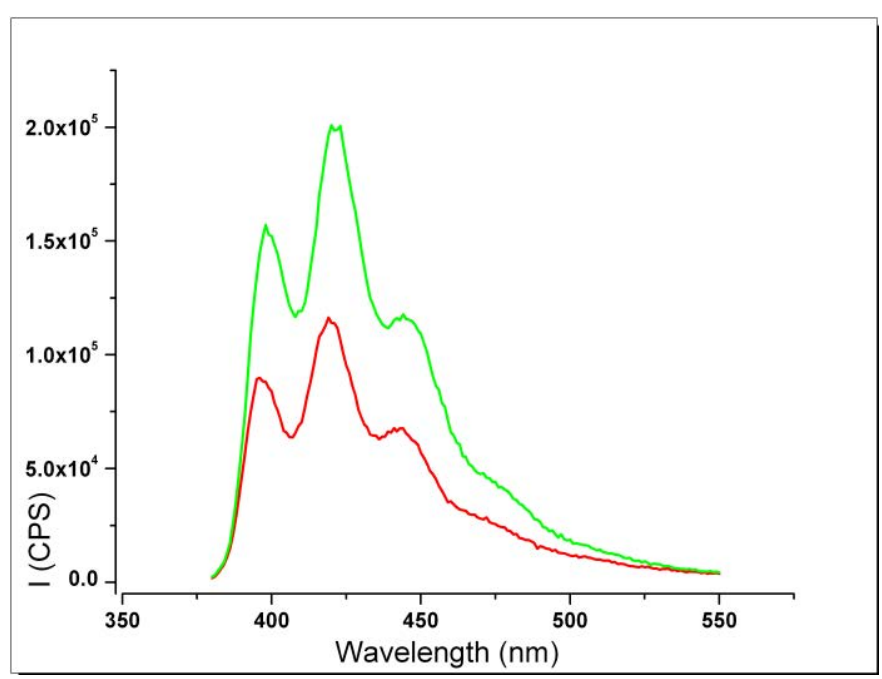

Figure 4-15: Emission spectra of $73\left(5 \cdot 10^{-5} \mathrm{M}\right.$ in DCM) before (red) and after addition of $\mathrm{Au}^{+}$ions (green). 
This method is not particularly established, and the "regular" approach of fluorescence increase by chelation enhanced fluorescence (CHEF) of $\mathbf{7 3}$ with $\mathrm{Ag}^{+}$was not successful.

However the addition of $\mathrm{Au}^{+}$to $\mathbf{7 3}$ induces an emission increase by a factor of 1.8 , which is noteworthy (Figure 4-15). Possibly further screening with an even larger variety of cations can reveal further sensitivities of $\mathbf{7 3}$ towards different transition metals or even lanthanides.

\subsubsection{Synthesis of $\mathrm{AnCH}_{2} \mathrm{~N}\left(\mathrm{C}_{2} \mathrm{H}_{4} \mathrm{PPh}_{2}\right)_{2}$ (74)}

Furthermore, the P-N-P type receptor 64 was reacted with the anthracene fluorophore. Compared to the previously used amines, this receptor was sensitive towards oxidation with aerial oxygen which required inert conditions for synthesis. Of course a resulting sensor molecule would also instable towards aerial oxygen and therefore need to be handled in an inert atmosphere at all times. On the other hand the benefits of this ligand in transition metal coordination may well outnumber the disadvantages of complicated handling. Despite the large number of amine based sensor compounds described in literature, P-N-P type receptors have not been previously utilized with the exception of one compound described by $X u$ et al. which exhibits a cyclic structure in presence o group 11 metal cations, which in turn shows sensitivity towards intercalation of benezenediacetonitrile. ${ }^{[114]}$ In view of the large variety of metal complexes synthesized from P-N-P ligands, this receptor bears pronounced potential for the formation of stable complexes between sensor and analyte. Also the effect of electron rich phosphane donors within receptor units on electron transfer processes has not been previously documented.

For introduction of the P-N-P receptor to the fluorophore 9(bromomethyl)anthracene was reacted with one equivalent of 64 in anhydrous degassed $\mathrm{MeCN}$ at $82^{\circ} \mathrm{C}$ for $4 \mathrm{~h}$ in presence of four equivalents of $\mathrm{K}_{2} \mathrm{CO}_{3}$ (Scheme 4-22, reaction $\mathbf{A})$. 


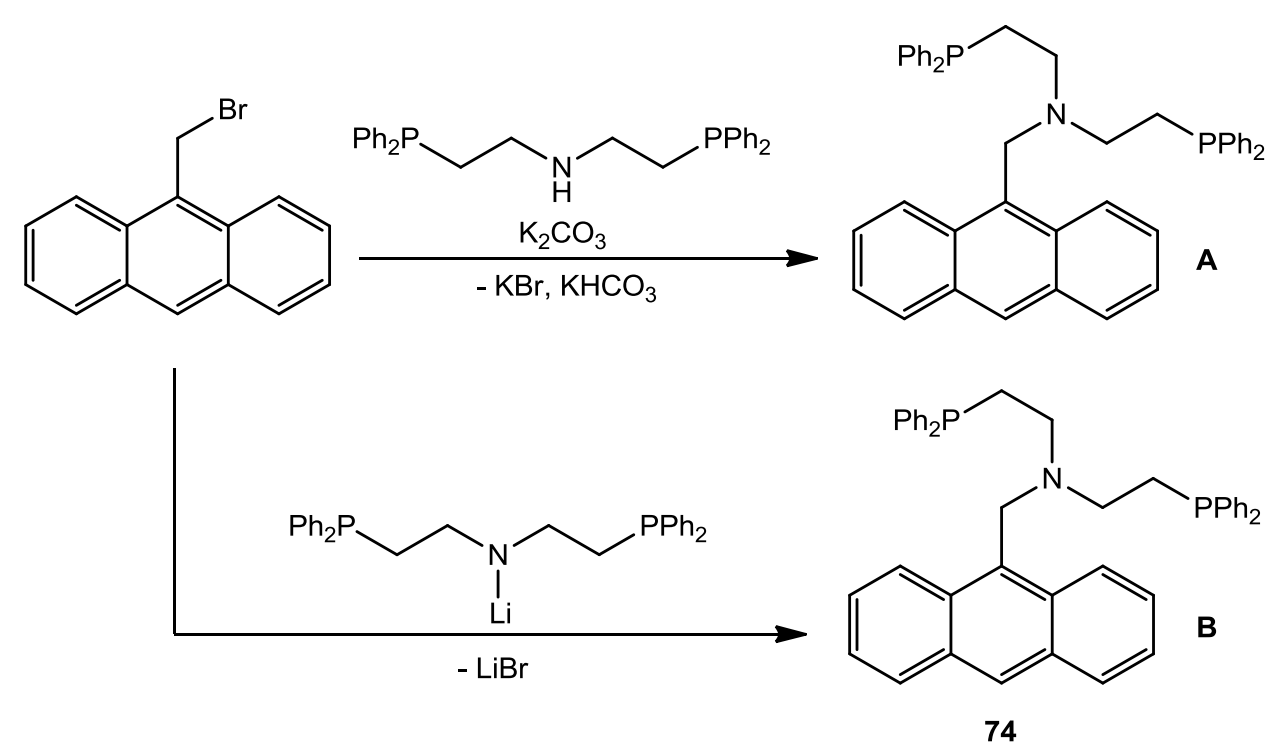

Scheme 4-22: Synthesis of $\mathrm{HAnCH}_{2} \mathrm{~N}\left(\mathrm{C}_{2} \mathrm{H}_{4} \mathrm{PPh}_{2}\right)_{2}$ (74).

After completion of the reaction the solvent was removed and the crude product was extracted with degassed DCM and degassed water. The organic layer was dried over $\mathrm{MgSO}_{4}$, filtrated and the volatile solvent was evaporated. Although inert conditions were preserved at all times during the reaction and work-up process, the ${ }^{31} \mathrm{P}$ NMR spectrum indicated the presence of several byproducts in the crude product. Because the P-N-P receptor $\mathbf{6 4}$ had been shown to be stable towards water (cleavage of the protecting group in its synthesis was achieved by refluxing in degassed water), formation of oxidation products in the aqueous extraction of $\mathbf{7 4}$ appeared unlikely. Furthermore the oxidation of one or both phosphorus atoms would not account for the observed number of byproducts. The most probable explanation is that the phosphorus donors also underwent $S_{N}$ reactions with 9-(bromomethyl)anthracene in addition to the nitrogen atom. This way numerous different products, including polymeric products are thinkable. Although $\mathbf{7 4}$ was determined to be the main product by NMR experiments, separation of the byproducts was problematic. The oily texture of the crude product hindered purification by recrystallization, and its sensitivity towards oxidation disabled purification via column chromatography.

Therefore a second reaction was conducted under milder conditions. To prevent the phosphane donors from reacting with 9-(bromomethyl)anthracene the reaction temperature was held below $20^{\circ} \mathrm{C}$. To enhance the reactivity of the amine it was deprotonated with $n$-BuLi at $-15^{\circ} \mathrm{C}$ in THF and added drop wise to a cooled solution of 9-(bromomethyl)anthracene in THF (Scheme 4-22, reaction B). The resulting mixture 
was stirred for $20 \mathrm{~h}$ at ambient temperature. After completion of the reaction the solvent was evaporated and the crude product was dissolved in degassed DCM and filtrated. This way contact with water was completely avoided. Removal of the solvent afforded an amorphous yellow solid. While the ${ }^{31} \mathrm{P}$ NMR spectrum of the crude product showed only a single signal, the ${ }^{1} \mathrm{H}$ NMR spectrum indicated incomplete turnover and noteworthy contaminations from starting materials. Though again $\mathbf{7 4}$ was the main product, the problems of purification were similar to those encountered in the first synthesis. Unfortunately $\mathbf{7 4}$ could not be obtained in sufficient purity for fluorescence experiments.

\subsubsection{Syntheses and Fluorescence Properties of $\mathrm{AnCH}_{2} \mathrm{~N}\left(\mathrm{CH}_{2} \mathrm{PhOMe}\right)_{2}(75)$ and $\mathrm{AnCH}_{2} \mathrm{~N}\left(\mathrm{CH}_{2} \mathrm{PhSMe}\right)_{2}(76)$}

Finally, the two aromatic amines bis(2-methoxybenzyl)amine (62) and bis(2methylthiobenzyl)amine (63) were inserted to the anthracene fluorophore. Because neither of the amines had been previously synthesized, also the resulting sensor molecules were consequently novel.

62 and 63 were reacted with 9-(bromomethyl)anthracene under identical conditions. The respective amine was dissolved in $\mathrm{MeCN}$ and one equivalent of 9(bromomethyl)anthracene and four equivalents of $\mathrm{K}_{2} \mathrm{CO}_{3}$ were added. The reactions were heated to $82^{\circ} \mathrm{C}$ for $8 \mathrm{~h}$ and then cooled to ambient temperature. The solvent was removed and both crude products were dissolved in DCM and extracted with water and saturated $\mathrm{NaCl}$ solution. The organic layers were dried over $\mathrm{MgSO}_{4}$ and filtrated. After removal of the solvent the crude products were purified by column chromatography (75: petrol ether/ethyl acetate 10:1), (76: petrol ether/ethyl acetate 15:1). $\mathrm{HAnCH}_{2} \mathrm{~N}\left(\mathrm{CH}_{2} \mathrm{PhOMe}\right)_{2}$ (75) and $\mathrm{HAnCH}_{2} \mathrm{~N}\left(\mathrm{CH}_{2} \mathrm{PhSMe}\right)_{2}$ (76) were obtained as light yellow solids. Recrystallization from DCM (75) and chloroform (76) afforded crystals which were suitable for X-ray structure determination. 


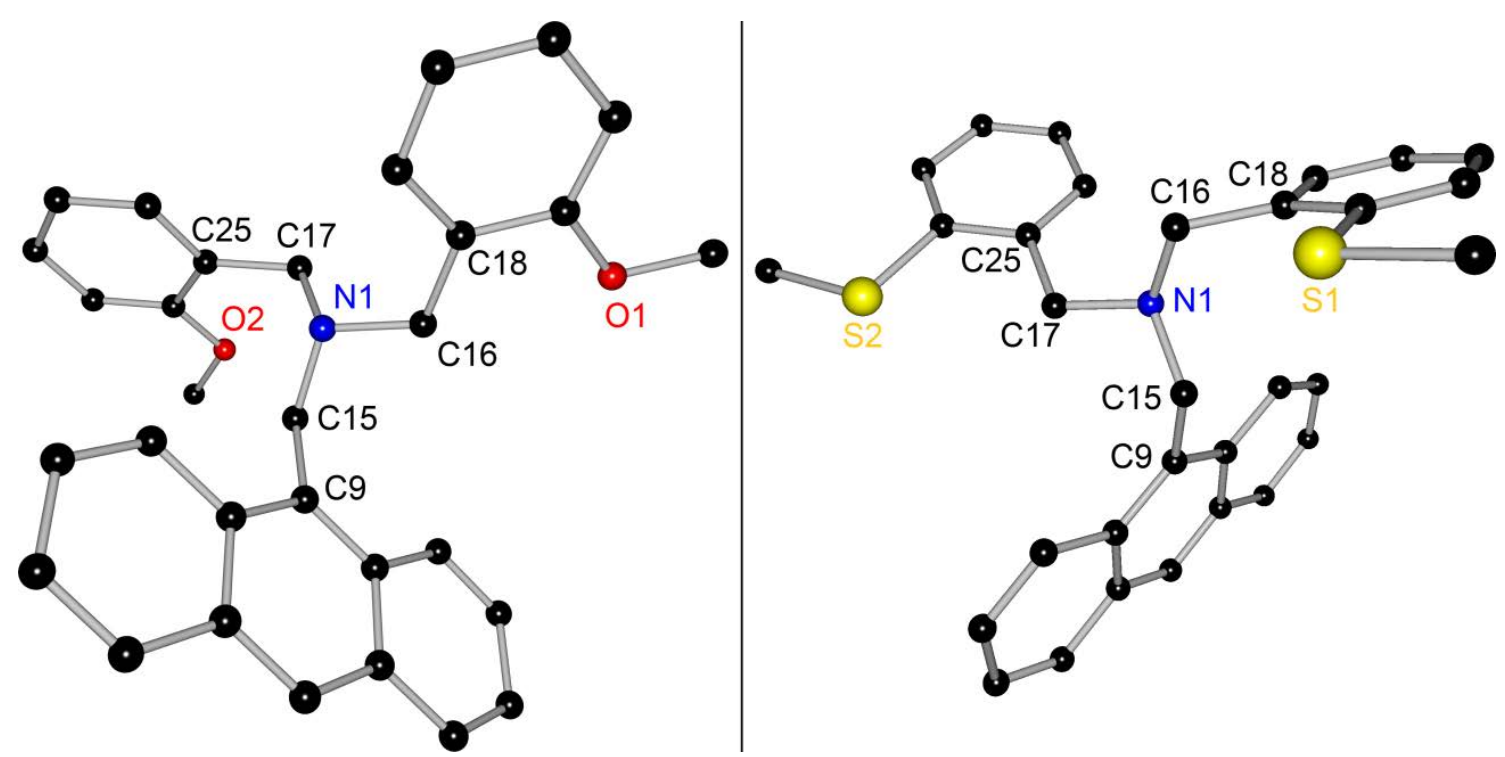

Figure 4-16: Crystal structures of $\mathrm{HAnCH}_{2} \mathrm{~N}\left(\mathrm{CH}_{2} \mathrm{PhOMe}\right)_{2}\left(75\right.$, left) and $\mathrm{HAnCH}_{2} \mathrm{~N}\left(\mathrm{CH}_{2} \mathrm{PhSMe}\right)_{2}$ (76, right), hydrogen atoms and lattice solvent (76) are omitted.

Table 4-4: Selected bond lengths $[\AA ̊]$ and angles $\left[{ }^{\circ}\right]$ of 75 and 76.

\begin{tabular}{cc|cc}
\hline \multicolumn{2}{c|}{$\mathrm{HAnCH}_{2} \mathrm{~N}_{\left(\mathrm{CH}_{2} \mathrm{PhOMe}\right)_{2}(75)}$} & \multicolumn{2}{c}{$\mathrm{HAnCH}_{2} \mathrm{~N}\left(\mathrm{CH}_{2} \mathrm{PhSMe}\right)_{2}(76)$} \\
$\mathrm{C} 15-\mathrm{N} 1$ & $1.4719(11)$ & $\mathrm{C} 15-\mathrm{N} 1$ & $1.473(3)$ \\
$\mathrm{C} 9-\mathrm{C} 15-\mathrm{N} 1$ & $114.89(7)$ & $\mathrm{C} 9-\mathrm{C} 15-\mathrm{N} 1$ & $114.7(2)$ \\
$\mathrm{C} 15-\mathrm{N} 1-\mathrm{C} 16$ & $110.99(7)$ & $\mathrm{C} 15-\mathrm{N} 1-\mathrm{C} 16$ & $110.9(2)$ \\
C16-N1-17 & $109.21(7)$ & $\mathrm{C} 16-\mathrm{N} 1-17$ & $110.73(19)$ \\
C9a-C9-C15-N1 & $76.89(10)$ & C9a-C9-C15-N1 & $71.2(3)$ \\
Folding & 2.6 & Folding & 3.9 \\
Twist & 2.9 & Twist & 2.7 \\
\hline
\end{tabular}

Apart from the C-O and C-S bond lengths, $\mathbf{7 5}$ and $\mathbf{7 6}$ do not differ strongly in their geometrical features, as indicated by bond distances and angles compiled in Table 4-4. The geometry surrounding N1 is nearly perfectly tetrahedral for both compounds. Even the deformation of the anthracene moiety is nearly equally weak in both structures due to the flexibility provided by the spacer. While both asymmetric units contain one molecule of $\mathbf{7 5}$ and $\mathbf{7 6}$, respectively, a chloroform molecule is cocrystallized in the structure of 76. Additionally one benzyl moiety is slightly disordered in 76, which is not observed in the structure of 75. Moreover, $\mathbf{7 5}$ crystallizes in the 
monoclinic space group $P 2_{1} / n$ while 76 crystallizes in $P \overline{1}$. Also the phenyl rings in the structure of $\mathbf{7 5}$ are nearly parallel oriented, while they are notably tilted against one another in 76. On the other hand the distance between the oxygen/sulfur donors is quite similar at $7.0 \AA$ (75) and $7.5 \AA$ (76).

In order to investigate the fluorescence properties of both compounds, solutions in DCM and MeCN were prepared. MeCN is a particularly versatile solvent because it is mixable with water and several organic solvents. The prepared MeCN solutions were used for the obligatory protonation experiments to validate the suitability of the newly synthesized receptor units in terms of redox potential by inhibiting the PET mechanism.
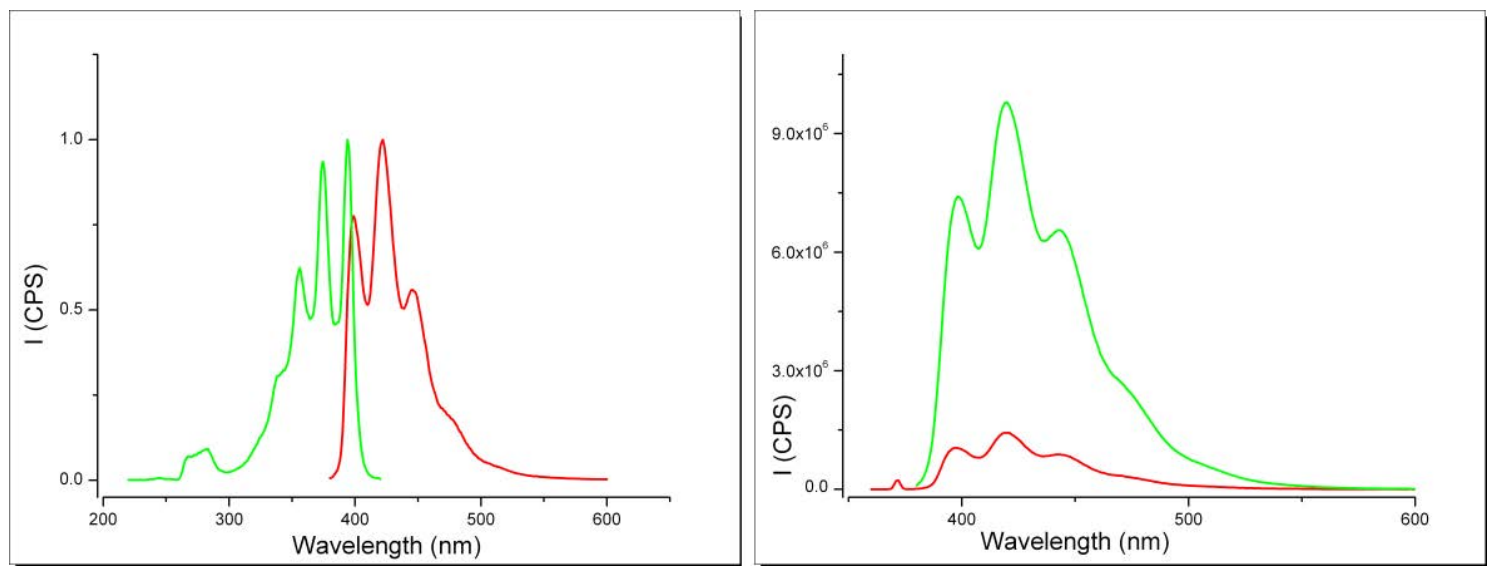

Figure 4-17: Left: normalized excitation (green) and emission (red) spectra of $\mathrm{HAnCH}_{2} \mathrm{~N}\left(\mathrm{CH}_{2} \mathrm{PhOMe}\right)_{2}$ (75), $5 \cdot 10^{-5} \mathrm{M}$ in DCM; right: emission spectra of 75 at $\mathrm{pH}=7$ (red) and $\mathrm{pH}=3$ (green), both $5 \cdot 10^{-5} \mathrm{M}$ in MeCN.

The excitation- and emission spectra of $\mathrm{HAnCH}_{2} \mathrm{~N}\left(\mathrm{CH}_{2} \mathrm{PhOMe}\right)_{2}$ (75) exhibit the expected shape and also the position of excitation and emission maxima comply well with the properties found for other amines (Figure 4-17, left). Upon protonation a strong increase of emission is visible which verifies the PET mechanism and therewith the suitability of the newly synthesized amine as a quencher moiety (Figure 4-17, right).

As Figure 4-18 (left) shows, the excitation- and emission spectra of $\mathrm{HAnCH}_{2} \mathrm{~N}\left(\mathrm{CH}_{2} \mathrm{PhSMe}\right)_{2}(76)$ are nearly identical to those of 75 , which is not particularly surprising as both compounds only differ in their terminal donor functions. The increase of emission intensity upon protonation is even stronger for $\mathbf{7 6}$ than observed 
for $\mathbf{7 5}$, which can partly be explained by the slightly lower $\mathrm{pH}$ applied for $\mathbf{7 6}$ (Figure 4-18, right).
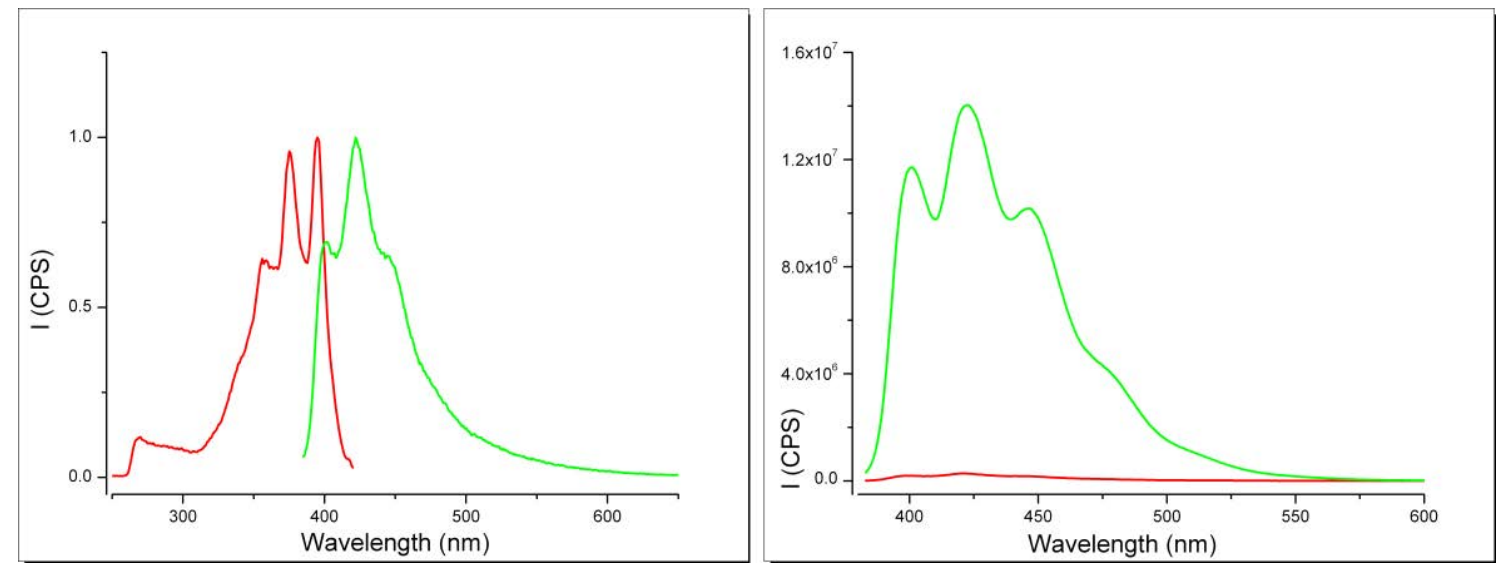

Figure 4-18: Left: normalized excitation (red) and emission (green) spectra of $\mathrm{HAnCH}_{2} \mathrm{~N}\left(\mathrm{CH}_{2} \mathrm{PhSMe}\right)_{2}$ (76), $5 \cdot 10^{-5} \mathrm{M}$ in DCM; right: emission spectra of 76 at $\mathrm{pH}=7$ (red) and $\mathrm{pH}=2$ (green), both $5 \cdot 10^{-5} \mathrm{M}$ in $\mathrm{MeCN}$.

Furthermore, the DCM solutions of $\mathbf{7 5}$ and $\mathbf{7 6}$ were used to evaluate the behavior of both compounds upon $\mathrm{Zn}^{2+}$ addition in a non-donating solvent. This way coordination of the sensor molecules to the cations was ensured by disabling "competition" for the coordination of cations between donating solvent molecules and the low concentrated sensor molecules.
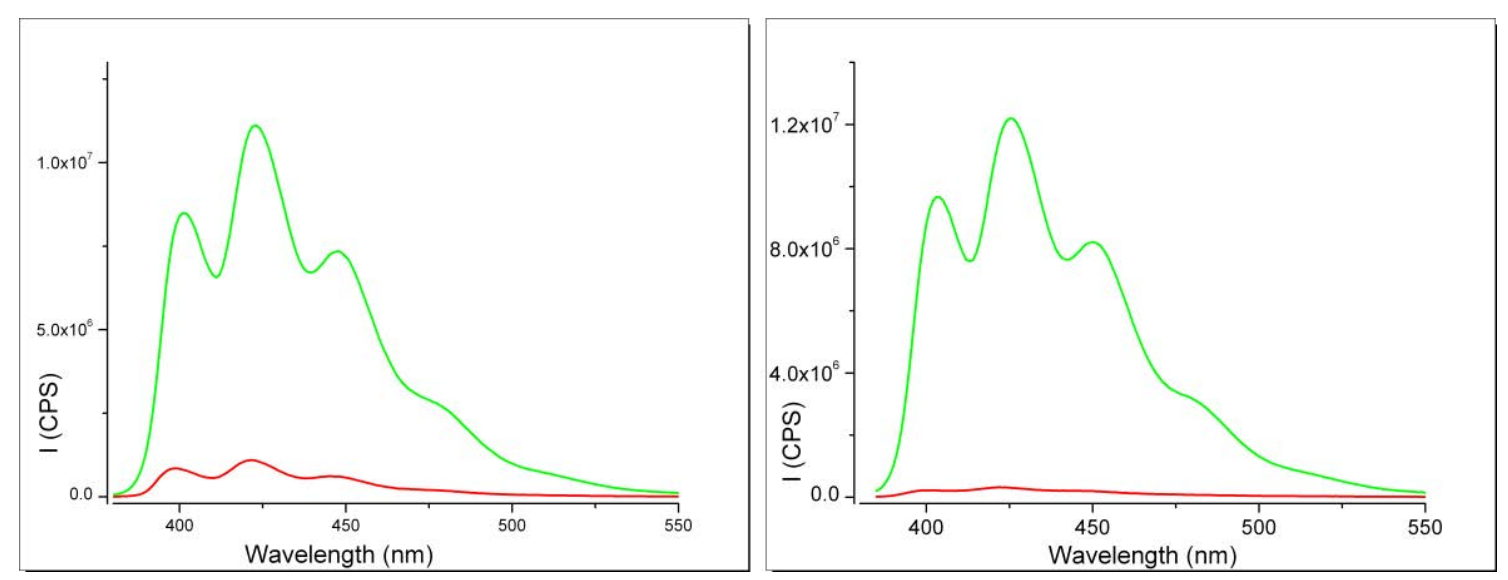

Figure 4-19: Left: emission spectra of $75\left(5 \cdot 10^{-5} \mathrm{M}\right.$ in DCM) before (red) and after (green) addition of $\mathrm{ZnBr}_{2}$; right: emission spectra of $76\left(5 \cdot 10^{-5} \mathrm{M}\right.$ in DCM) before (red) and after (green) addition of $\mathrm{ZnBr}_{2}$.

The obtained results are nearly identical to those of the previous protonation experiments (Figure 4-19). Both compounds exhibit a strong increase of fluorescence emission upon addition of $\mathrm{ZnBr}_{2}$. Though both compounds reach nearly identical intensities, the on/off ratio is larger for $\mathbf{7 6}$, because it shows even weaker fluorescence 
than 75 before addition of the $\mathrm{Zn}^{2+}$ solution (red lines). The strong increase of emission confirmed the inhibition of electron transfer by coordination of both sensor molecules to $\mathrm{Zn}^{2+}$. The next step was to investigate the coordination of metal ions in donating media.

Having verified the occurrence of electron transfer from the receptor to the excited fluorophore, $\mathbf{7 5}$ and $\mathbf{7 6}$ were subjected to extensive screening experiments to monitor their sensitivity towards various main group- and transition metal cations. The miscibility of MeCN with water enabled the utilization of aqueous metal salt solutions which made a large variety of cations accessible. The screening was conducted by recording background emission spectra of the $5 \cdot 10^{-5} \mathrm{M}$ solutions of 75 and $\mathbf{7 6}$, then adding an excess amount of the respective aqueous metal salt solution, and then recording a second emission spectrum under identical experimental conditions.

$\mathrm{HAnCH}_{2} \mathrm{~N}\left(\mathrm{CH}_{2} \mathrm{PhOMe}\right)_{2}$ (75) was the first compound which was subjected to screening experiments. The compound appeared to show sensitivity towards a number of cations, as the additions of most aqueous solutions to the MeCN solution of $\mathbf{7 5}$ triggered noteworthy increases of emission intensity. However the acquired results did not appear to be consistent, as e.g. identical cations with different counter ions produced deviating intensities. During the process of finding the cause for the observed deviations, different solvent mixtures of $\mathrm{MeCN}$ and water were tested to preclude solubility differences as the origin of the inconsistencies. It soon became evident that even before the addition of metal cations, there were strong variations of the emission intensities of the background spectra. Therefore a systematic investigation of the dependency of emission intensity on water concentration within the sample solution was conducted.

A fluorescence sample was regularly prepared by filling $0.3 \mathrm{~mL}$ of the previously prepared $5 \cdot 10^{-4} \mathrm{M}$ sensor compound solution (in $\mathrm{MeCN}$ ) into the fluorescence cuvette (which holds $3 \mathrm{~mL}$ of sample solution) and then adding $2.7 \mathrm{~mL}$ of solvent to achieve the desired concentration of $5 \cdot 10^{-5} \mathrm{M}$. This method produces smaller errors than the preparation of a $5 \cdot 10^{-5} \mathrm{M}$ solution to start with, which would require weighing and handling of much smaller substance masses. For the water concentration dependent series of measurements the infilling of $0.3 \mathrm{~mL}$ of $5 \cdot 10^{-4} \mathrm{M}$ sensor compound solution in MeCN was retained. The remaining $2.7 \mathrm{~mL}$ of additional solvent was varied in its 
composition from water and $\mathrm{MeCN}$ in $0.1 \mathrm{~mL}$ steps. The resulting $\mathrm{V} / \mathrm{V} \%$ ratios are compiled in Table 4-5.

Table 4-5: Solvent mixtures prepared for the water concentration dependent experiments.

\begin{tabular}{lcccccccccc}
\hline Exp. No. & 1 & 2 & 3 & 4 & 5 & 6 & 7 & 8 & 9 & 10 \\
$\mathrm{~V}\left(\mathrm{H}_{2} \mathrm{O}\right)(\mathrm{mL})$ & 0 & 0.1 & 0.2 & 0.3 & 0.4 & 0.5 & 0.6 & 0.7 & 0.8 & 0.9 \\
$\mathrm{~V}(\mathrm{MeCN})(\mathrm{mL})$ & 2.7 & 2.6 & 2.5 & 2.4 & 2.3 & 2.2 & 2.1 & 2.0 & 1.9 & 1.8 \\
$\mathrm{~V} / \mathrm{V} \%\left(\mathrm{H}_{2} \mathrm{O}\right)$ & 0 & 3.33 & 6.67 & 13.33 & 16.67 & 20.00 & 23.330 & 26.67 & 30.00 & 33.33 \\
\hline
\end{tabular}

This series was continued until the $0.3 \mathrm{~mL}$ of sensor compound solution were mixed with $2.7 \mathrm{~mL}$ of pure water which equates to $90 \%$ water in the sample solution.

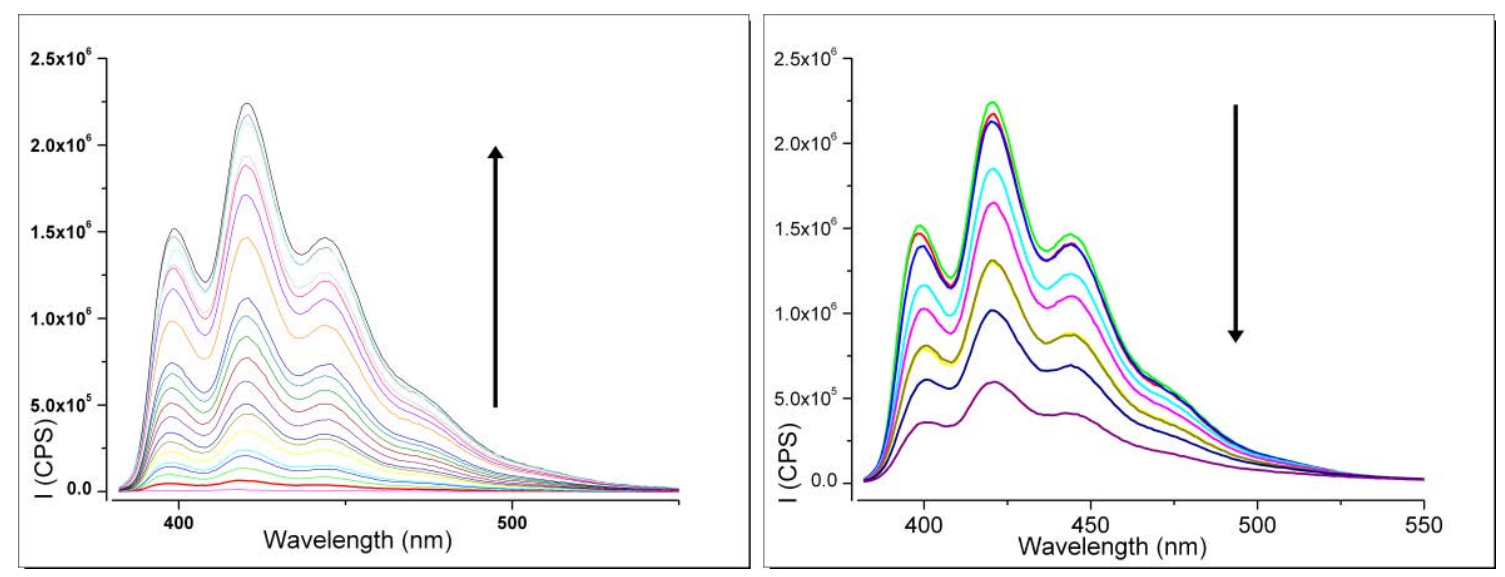

Figure 4-20: Water concentration dependent emission of $\mathrm{HAnCH}_{2} \mathrm{~N}\left(\mathrm{CH}_{2} \mathrm{PhOMe}\right)_{2}$ (75); left: increase of emission intensity from $0 \%$ water to $66.7 \%$ water $\left(2.0 \mathrm{~mL} \mathrm{H}{ }_{2} \mathrm{O} / 1.0 \mathrm{~mL} \mathrm{MeCN}\right)$ in the direction of the arrow. Right: decrease emission intensity from $66.7 \%$ water $\left(2.0 \mathrm{~mL} \mathrm{H} \mathrm{H}_{2} \mathrm{O} / 1.0 \mathrm{~mL} \mathrm{MeCN}\right.$ ) to $90.0 \%$ water (2.7 $\mathrm{mL} \mathrm{H} \mathrm{H}_{2} \mathrm{O} / 0.3 \mathrm{~mL} \mathrm{MeCN}$ ) in the direction of the arrow.

The resulting water concentration dependent data are depicted in Figure 4-20 and show a stunning increase of emission intensity of $\mathbf{7 5}$ with rising water concentration within the sample solution (Figure 4-20, left). This trend continues to a maximum which is reached at $66,7 \%$ water in the sample, which is equivalent to $2.0 \mathrm{~mL}$. At this point an emission enhancement by factor 180 compared to the solution in pure MeCN is achieved, which is an astonishing value. At water concentrations beyond $66.7 \%$ or $2.0 \mathrm{~mL}$ the measured intensity declines fairly quickly, in fact even faster than it had previously increased (Figure 4-20, right). The plot of the maximum emission intensity against the water concentration at a fixed wavelength of $425 \mathrm{~nm}$ is depicted in Figure 
4-21. It reveals a gradual non-linear increase of emission to a maximum at $66.7 \%$ water concentration, followed by a steep linear decrease of emission towards $90 \%$ water.

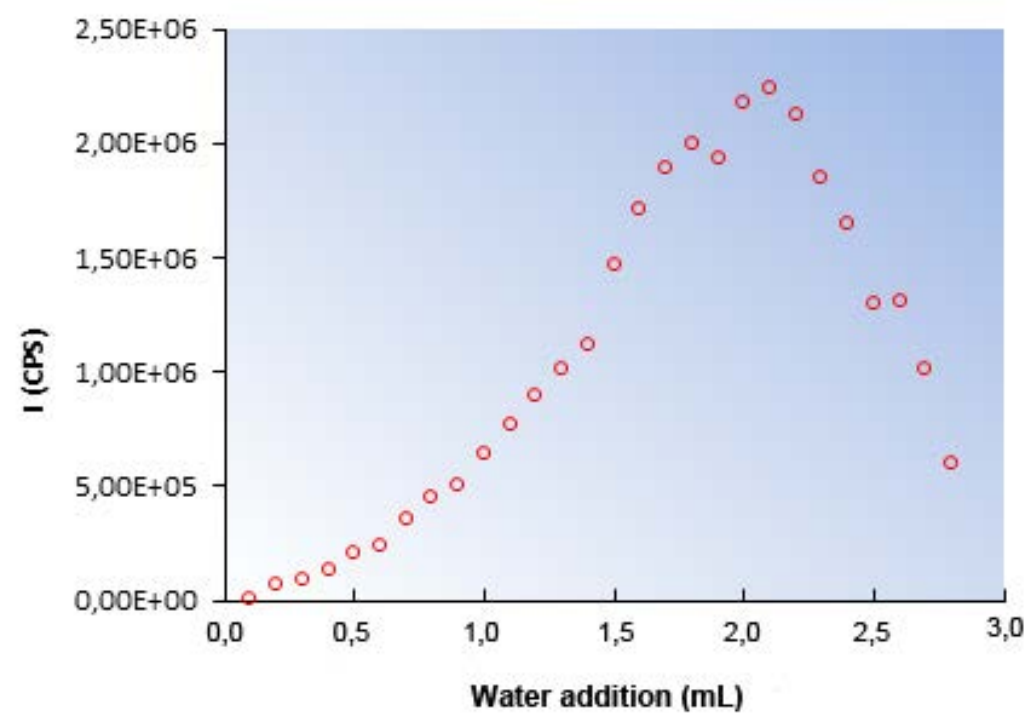

Figure 4-21: Plot of 75's emission intensity vs. water addition $\left(\lambda_{\mathrm{Em}}=425 \mathrm{~nm}\right)$.

To find an explanation for this remarkable phenomenon, the identical series of measurements was repeated with $\mathrm{HAnCH}_{2} \mathrm{~N}\left(\mathrm{CH}_{2} \mathrm{PhSMe}\right)_{2}$ (76). Again the samples were prepared on the basis of a $5 \cdot 10^{-4} \mathrm{M}$ solution of 76 in $\mathrm{MeCN}$.
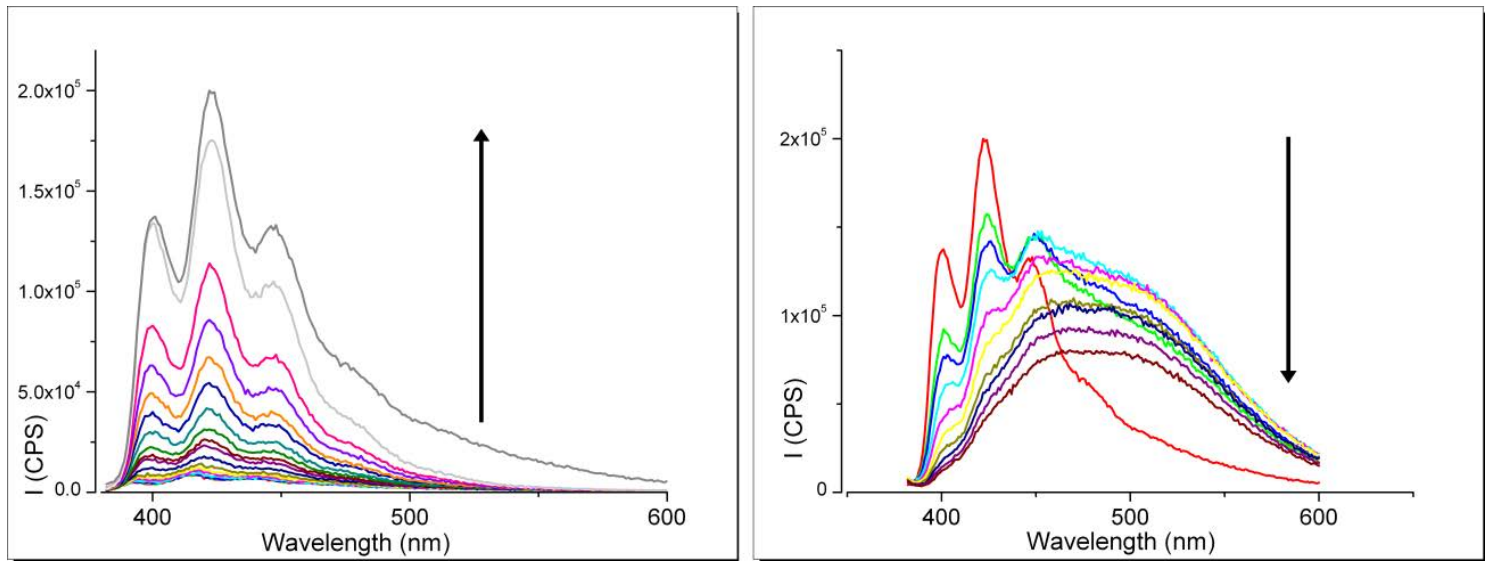

Figure 4-22: Water concentration dependent emission of $\mathrm{HAnCH}_{2} \mathrm{~N}\left(\mathrm{CH}_{2} \mathrm{PhSMe}\right)_{2}$ (76); left: increase of emission intensity from $0 \%$ water to $60.0 \%$ water $(1.8 \mathrm{~mL} \mathrm{H} 2 \mathrm{O} / 1.2 \mathrm{~mL} \mathrm{MeCN})$ in the direction of the arrow. Right: decrease emission intensity from $60.0 \%$ water $\left(1.8 \mathrm{~mL} \mathrm{H} \mathrm{H}_{2} \mathrm{O} / 1.2 \mathrm{~mL} \mathrm{MeCN}\right)$ to $90.0 \%$ water (2.7 $\mathrm{mL} \mathrm{H} \mathrm{H}_{2} \mathrm{O} / 0.3 \mathrm{~mL} \mathrm{MeCN}$ ) in the direction of the arrow.

As Figure 4-22 (left) shows, 76 also exhibits an increase of emission with rising water concentration. The overall emission enhancement is however much weaker than 
observed for $\mathbf{7 5}$ and the overall emission intensity is also much weaker. While at the $\mathrm{MeCN} /$ water ratio of maximum intensity an emission increase by the factor of 180 was observed for 75, only a factor of 19 is reached for 76. Also the maximum emission intensity is reached at a lower percentage of water (66.7\% for $75,60.0 \%$ for 76$)$. At water concentrations beyond $60 \%$ the behavior of $\mathbf{7 6}$ differs even stronger from that observed for 75 (Figure 4-22, right). Although the emission intensity also decreases after surpassing the maximum at $60 \%$ water, the decrease is less steep than for $\mathbf{7 5}$. Moreover the shape of the emission spectrum gradually changes from the typical pronounced band structure to a broad red-shifted maximum which lacks a vibrational structure.

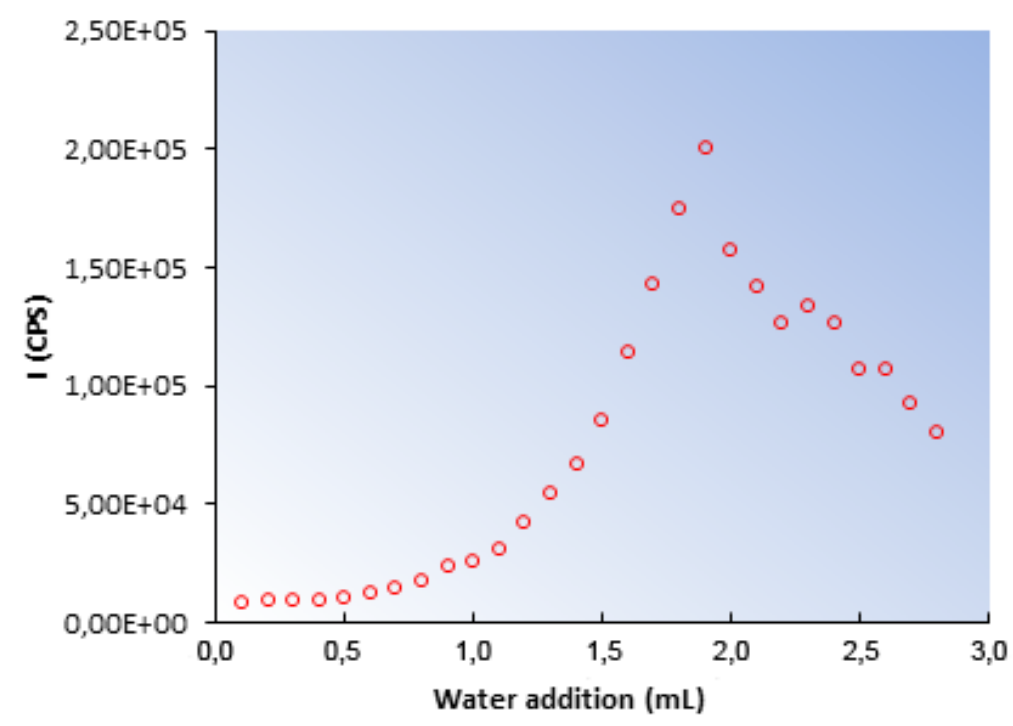

Figure 4-23: Plot of 76's emission intensity vs. water addition $\left(\lambda_{\mathrm{Em}}=425 \mathrm{~nm}\right)$.

The slightly scattered data points in the decreasing part of the plot of emission intensity vs. addition of water can be traced to the overall weak emission which enables small errors to induce strong deviations (Figure 4-23). Furthermore the altered shape of the emission spectrum affects this plot because all data points were acquired at a fixed emission wavelength of $425 \mathrm{~nm}$ which does not account for the unexpected red-shift of the emission maximum of $\mathbf{7 6}$ beyond water concentrations of $60 \%$.

Obviously 75 and 76 differ strongly in their behavior upon addition of water although both compounds only differ structurally in their terminal donor functions. The results acquired to this point indicated that the addition of water leads to a stronger inhibition of the emission suppressing electron transfer for $\mathbf{7 5}$ than it does for 
76. To determine to which degree the PET was still active at the water concentration which leads to maximum emission intensity, samples of $\mathbf{7 5}$ and $\mathbf{7 6}$ were prepared at the maximal emitting water ratio (66.7\% water for 75 and $60.0 \%$ water for 76$)$. After recording background spectra of both samples, $1 \mathrm{M}$ hydrochloric acid was added, generating a low $\mathrm{pH}$ value of 1 , which ensures virtually quantitative protonation of the amines of both sensor molecules. Hence, at this $\mathrm{pH}$ value all remaining electron transfer is suppressed and maximum intensity is emitted.
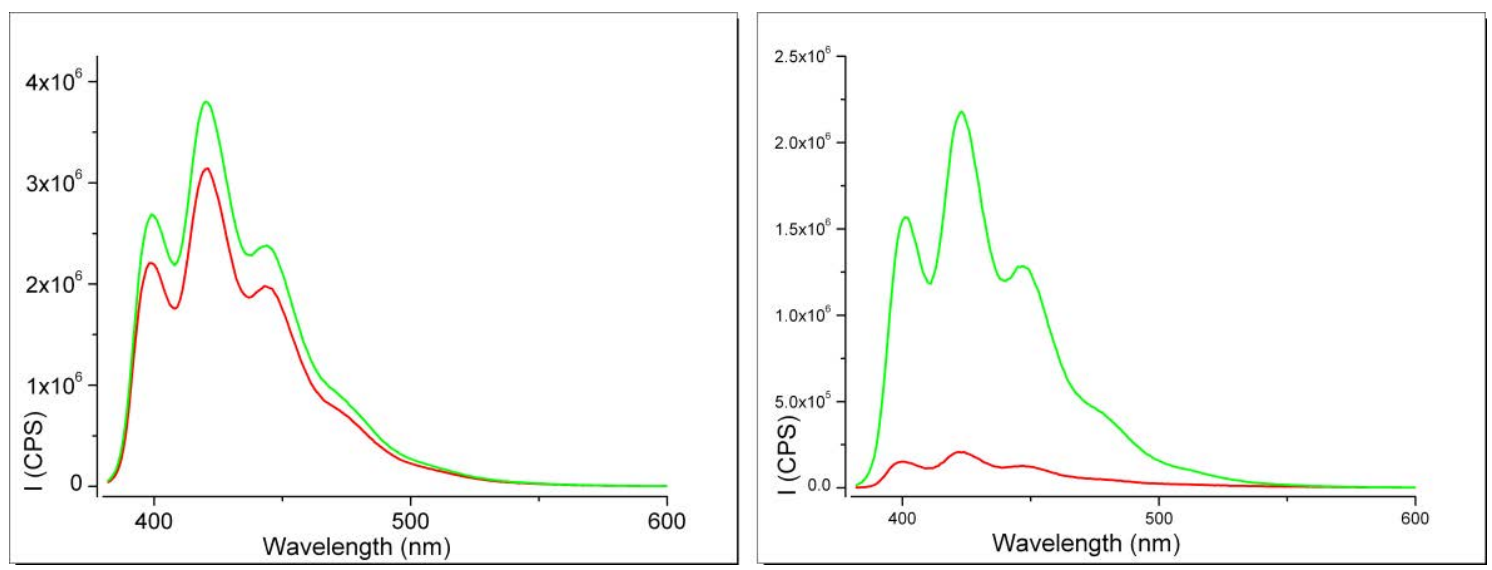

Figure 4-24: Left: emission spectra of $75\left(5 \cdot 10^{-5} \mathrm{M}\right.$ in $\left.\mathrm{H}_{2} \mathrm{O} / \mathrm{MeCN} 66.7 / 33.3 \%\right)$ before (red) and after protonation (green); right: emission spectra of $76\left(5 \cdot 10^{-5} \mathrm{M}\right.$ in $\left.\mathrm{H}_{2} \mathrm{O} / \mathrm{MeCN} 60.0 / 40.0 \%\right)$ before (red) and after protonation (green).

In the case of 75 , the additional protonation only induces an increase of emission by the factor of 1.2 (Figure 4-24, left). This small enhancement confirms the nearly complete suppression of electron transfer for 75 at a water concentration of $66.7 \%$. In contrast, the additional protonation of $\mathbf{7 6}$ triggers a strong increase of emission intensity by the factor of 19 . This shows that even at the water concentration of maximum emission (60\%) the electron transfer process is still active and only marginally suppressed for 76 .

These differences in suppression of the PET must arise from the terminal methoxy (75) and methylthio (76) substituents. A higher degree of protonation of the amine of 75 in an aqueous environment would explain the observed suppression of the PET, but this would require distinctly higher basicity of $\mathbf{7 5}$ compared to $\mathbf{7 6}$ to justify this assumption. Because the chemical environment of the amine nitrogen atoms is identical in $\mathbf{7 5}$ and $\mathbf{7 6}$ and the terminal functional groups are too far away from the amine nitrogen atom to take influence on its basicity, this approach appears unlikely. 
There are several examples in literature in which sensor compounds show high sensitivity towards the polarity of solvents. ${ }^{[62 a]}$ The observed effect is however mostly contrary to the enhancement found for 75. Because the electron transfer process briefly generates a radical ion pair, this intermediate is stabilized by strongly polar solvents or even by hydrogen bonding. ${ }^{[115]}$ This in turn favors the electron transfer process and leads to weaker emission in strongly polar solvents or upon hydrogen bonding. Therefore this approach is also not constructive. In literature there are few other reports on comparable sensitivity towards water in solvent mixtures. The majority of those found are - in the bottom line - based on protonation of an amine quencher. One example is a zwitterionic compound by Ooyama et al. having a terminal carboxyl group which undergoes an intramolecular protonation of an amine quencher in aqueous media. ${ }^{[116]}$ A second example is a boronic acid ester which is located in close proximity to an amine quencher, which was also reported by Ooyama et al. By adduct formation between water molecules and the electron deficient borane, the acidity of the water protons is increased, again leading to a protonation of the amine, which switches on the emission of the anthracene fluorophore. ${ }^{[86]}$ Both of these mechanisms cannot be applied to $\mathrm{HAnCH}_{2} \mathrm{~N}\left(\mathrm{CH}_{2} \mathrm{PhOMe}\right)_{2}$ (75), as the structural preconditions are not given.

A phenomenon lately reported by Shellaiah et al. described very similar behavior as observed for 75. $^{[34]}$ Their anthracene derivative also exhibited fluorescence enhancement upon addition of water, reaching a maximum at a water concentration of $60 \%$, which is close to the value determined for 75 . After surpassing this maximum, Shellaiah's compound also exhibited a strong decrease of emission, which is a further similarity to 75. On the other hand their compound's emission underwent a noteworthy red-shift in addition to the emission enhancement. Shellaiah et al. ascribed this phenomenon to aggregation induced emission (AIE, c.f. 1.1), which was assumed to originate from restriction of intramolecular rotation within their compound (Scheme 4-23).<smiles>Oc1ccccc1/C=N/c1ccc2cc3ccccc3cc2c1</smiles>

Scheme 4-23: Water sensitive anthracene derivative by Shellaiah et al. ${ }^{[34]}$ 
Although they also considered hydrogen bonding as a possible inhibitor of electron transfer, the AIE approach was maintained due to the observed emission shifts. The decrease of emission at high water concentrations was ascribed to unspecified solvent effects. The cause of the rotation restricting aggregation was also not explicitly stated.

Though this approach could also be applied to 75, there is no tangible evidence for an aggregation process upon increase of water concentration of the sample solutions. As the concentration of $\mathbf{7 5}$ and $\mathbf{7 6}$ was identical in all samples, there is no reason to assume an aggregation process as stated by Shellaiah et al. Furthermore there is no concrete argument for postulation of an aggregate formation in the case of $\mathbf{7 5}$ which would not also apply to $\mathbf{7 6}$ which did not exhibit comparable emission enhancement in aqueous environments. Therefore the assumption of AIE is not reasonable for explaining the fluorescence phenomena of 75 . The only conclusive explanation must be founded on a participation of the terminal oxygen- and sulfur donors of $\mathbf{7 5}$ and $\mathbf{7 6 .}$ Although both oxygen and sulfur donors form hydrogen bonds, the formed bonds differ significantly in strength and stability. Oxygen donors form strong hydrogen bonds while sulfur donors form weak hydrogen bonds. The proximity of the oxygen donors to the amine nitrogen atom is well suitable for the formation of stable hydrogen bonded formations (Scheme 4-24).

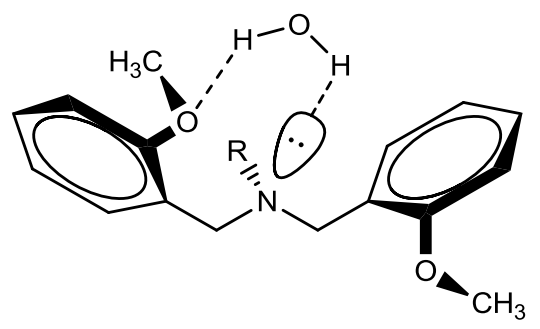

Scheme 4-24: Possible hydrogen bonded arrangement of 75.

Arrangements like these are likely to be stable enough for suppression of the PET. On the other hand the weaker hydrogen bonds between the sulfur donors and water molecules are too weak to generate arrangements which are sufficient in stability for suppression of the PET.

The stability of such arrangements may explain the observed emission increase, but does not account for the decrease of emission at high water concentrations. This effect is probably caused by the solubility of $\mathbf{7 5}$ and $\mathbf{7 6}$ in aqueous media. Complete dissolution of the fluorescent compounds is a precondition of fluorescence emission 
under the given experimental conditions. Because aromatic compounds like anthracene are generally poorly soluble or even insoluble in water, water concentrations above $60 \%$ may lead to an exceeding of the solubility limit of $\mathbf{7 5}$ and 76. With a decreasing percentage of dissolved molecules within the sample, the observed emission intensity decreases in proportion. The broadening and shifting of the emission band of $\mathbf{7 6}$ at high water concentrations can be interpreted as the (at least partial) formation of a finely suspended precipitate of un-dissolved 76, which further supports the assumption of insufficient solubility. This broadening and redshifting of the emission band may also occur is the case of $\mathbf{7 5}$, but is probably overlain by the overall much stronger emission of $\mathbf{7 5}$, and therefore not visible.

A second possible explanation for the strong emission enhancement of $\mathbf{7 5}$ is also founded on hydrogen bonding. Because the rate of electron transfer in flexible systems like that of $\mathbf{7 5}$ is dependent on the ability of the quencher moiety to rotate freely, strong interaction with the solvent (by hydrogen bonding) may produce a similar effect as strongly increased viscosity of the solvent. The exposure of sensor molecules to viscous environments can also produce noteworthy fluorescence enhancements by hindering rotation of the molecule and therewith inhibiting the electron transfer. ${ }^{[62 a, 63-64,117]}$ The stronger interactions of the methoxy substituents of 75 with the polar and hydrogen bonding aqueous environment compared to the methylthio groups of 76 could therefore also be the cause of the observed phenomenon.

For a final experiment regarding the effects of water addition on $\mathbf{7 5}$ and 76, again solutions of both compounds at their maximal emitting water concentrations were prepared. To these solutions a basic salt was added to raise the $\mathrm{pH}$ value of the solutions strongly. Although hydroxide ions are suitable for this purpose, standard reagents like $\mathrm{NaOH}$ or $\mathrm{KOH}$ are not, because possible coordination of the receptor units to the alkali cations could also lead to at least partial inhibition of the PET, which would in turn falsify the measured emission intensities. Therefore a cation had to be found which cannot interact with the receptor units. Tetrabutylammonium ions are extremely bulky and can by no means be coordinated by the by the amine receptor. Therefore tetrabutylammonium hydroxide (TBAH) was added to the previously prepared solutions of $\mathbf{7 5}$ and $\mathbf{7 6 .}$ 

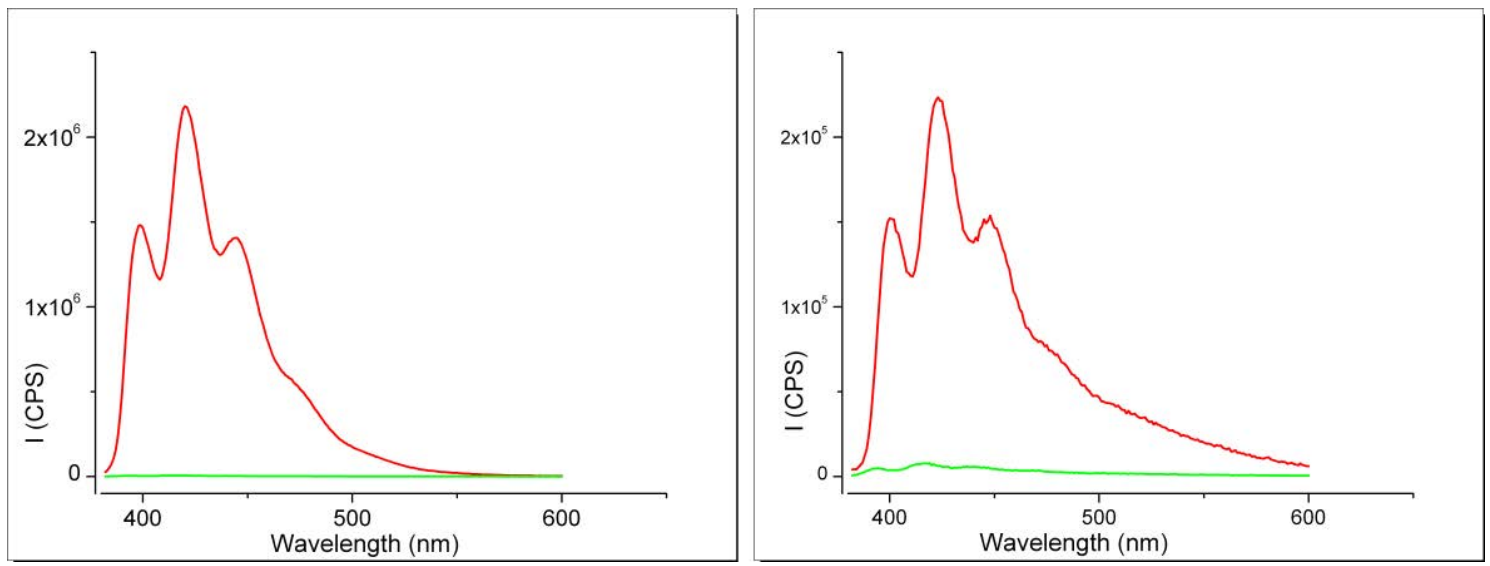

Figure 4-25: Left:emission spectra of $75\left(5 \cdot 10^{-5} \mathrm{M}\right.$ in $\left.\mathrm{H}_{2} \mathrm{O} / \mathrm{MeCN} 66.7 / 33.3 \%\right)$ before (red) and after

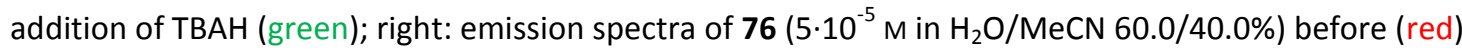
and after addition of TBAH (green).

In both cases the initially observed emission was virtually completely quenched upon addition of TBAH (Figure 4-25). This shows that the suppression of electron transfer which occurs at a neutral $\mathrm{pH}$ is overturned at high $\mathrm{pH}$ values, which causes the again functioning PET mechanism to quench emission. The presence of a stronger base than the amine in the sample solution obviously leads to the decomposition of previous PET suppressing arrangement. This again raises a question regarding the quality of the interaction between the amine nitrogen atom and the water molecules, because despite the presence of hydroxide ions, there are still sufficient amounts of water molecules present for the supposed hydrogen bonding. This also revokes the theory of hindered rotation of the quencher by hydrogen bonding, because the presence of hydroxide ions should not affect the interactions between solvent molecules and receptor.

Taking into account all acquired findings, a compromise between hydrogen bonding and protonation of the amine is the most conclusive explanation for the observed increase of emission in presence of water for 75. Because the amine receptor is of course basic, an equilibrium between the protonated form and the un-protonated form is on hand. This equilibrium is most likely shifted far towards the un-protonated form at a neutral $\mathrm{pH}$. By hydrogen bonding of water molecules to the methoxy groups not only a close proximity between the water protons and the amine nitrogen atom is produced, also the hydrogen bonding may influence the acidity of the water protons slightly. This would again increase the probability of protonation of the amine nitrogen atom or even (locally) shift the equilibrium between protonated und un-protonated 
species. Because the PET is a sensitive process, even small alterations can produce strong effects like the one observed for 75. In presence of hydroxide ions this equilibrium is virtually destroyed and only the un-protonated form is found.

Although a definitive statement is difficult to make, the hypothesis presented above best represents all aspects of the gathered experimental data. Independent of the cause of the observed fluorescence increase, $\mathrm{HAnCH}_{2} \mathrm{~N}\left(\mathrm{CH}_{2} \mathrm{PhOMe}\right)_{2}$ (75) fulfills all preconditions of a highly potent and versatile water sensor. The observed emission increase and sensitivity outperform those of most other compounds described in literature. By comparison to a calibration curve, water concentrations of solvents could be quickly determined. Finally, the first measured emission spectrum of $\mathbf{7 5}$ in pure MeCN (Figure 4-17/Figure 4-18), which showed distinctly higher emission intensity than that of $\mathbf{7 6}$ at equal concentration, can now be explained. Due to the high sensitivity of $\mathbf{7 5}$ towards water, the moisture contained in MeCN was apparently sufficient to notably increase the emission of 75 compared to $\mathbf{7 6}$, producing the observed difference.

\subsection{Phosphane Derivatives Containing Spacers}

One more very interesting and important aspect of this thesis was exploring the transferability of the PET mechanism and the quencher-spacer-fluorophore concept to other quencher moieties than the established amines. The results of the previous chapter as well as numerous reports in literature have shown that detection of soft transition metal ions is particularly challenging via amine receptor units because the interaction between the comparatively hard nitrogen donor and soft metal cations is weak. Not always is it possible to construct a coordination geometry of the sensor molecule which "forces" the amine nitrogen atom into a bonding situation with the metal ion which is sufficiently strong to notably inhibit the PET process. For cases like these, a quencher moiety based on a softer heteroatom would be eligible. Therefore phosphorus - being a higher homologue of nitrogen - was selected to generate a model system for investigation of the PET process. 


\subsubsection{Structures and PET Mechanism}

Experiments throughout this thesis (c.f. 3.1) as well as few reports in literature have indicated electron transfer processes from phosphane moieties to anthracene fluorophores. ${ }^{[65]}$ These findings were mostly derived from observed fluorescence enhancement upon metal coordination. Yet, none of these compounds contained a spacer between the phosphane substituent and fluorophore moiety which is - in contrast - an important element of amine based sensor molecules for achievement of the required orbital overlap for electron transfer. The only sensor molecule containing a spacer and a phosphane quencher was reported by Onoda et al. in 2003. ${ }^{[118]}$ As Scheme 4-25 shows, their compound contained a heteroaromatic fluorophore which<smiles>O=[N+]([O-])c1ccc(NCCP(c2ccccc2)c2ccccc2)c2nonc12</smiles>

Scheme 4-25: Phosphane based PET sensor by Onada et al. most likely differs significantly in terms of HOMO and LUMO energies from the anthracene fluorophore. Transferability to a different fluorophore is therefore not self-evident. Hence, a utilization of phosphanes as quenchers of anthracene fluorescence in quencherspacer-fluorophore systems has not been realized. Onoda and co-workers were able to detect organic peroxides by emission enhancement. By oxidation of the phosphorus atom, the lone pair is no longer available as an electron donator in the PET mechanism and quenching is inhibited, leading to an increase of emission.

In order to validate an electron transfer between phosphane quenchers and anthracene fluorophores, a corresponding system of both moieties linked by an alkyl spacer had to be synthesized.

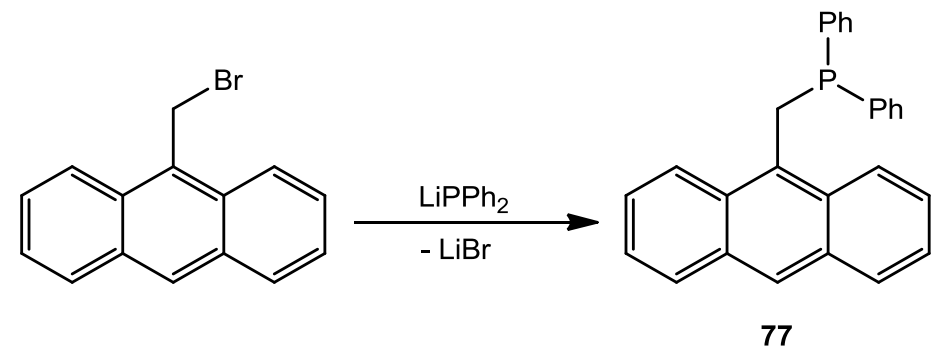

Scheme 4-26: Synthesis of 77. 
Diphenylphosphane was dissolved in diethyl ether and cooled to $-15^{\circ} \mathrm{C}$. One equivalent of $n$-BuLi was added and the solution was stirred for $15 \mathrm{~min}$. The lithiated phosphane was slowly added to a solution of 9-(bromomethyl)anthracene in diethyl ether at $-15^{\circ} \mathrm{C}$. After the addition was completed the reaction mixture was warmed to ambient temperature and stirred for $15 \mathrm{~h}$ (Scheme 4-26). After completion of the reaction a light yellow precipitate had formed. It was separated from the solution by filtration and dried in vacuo. It was then re-dissolved in toluene and again filtrated for removal of lithium bromide. Evaporation of the solvent and recrystallization from diethyl ether afforded crystals of $\mathrm{AnCH}_{2} \mathrm{PPh}_{2}$ (77) which were suitable for X-ray structure determination.

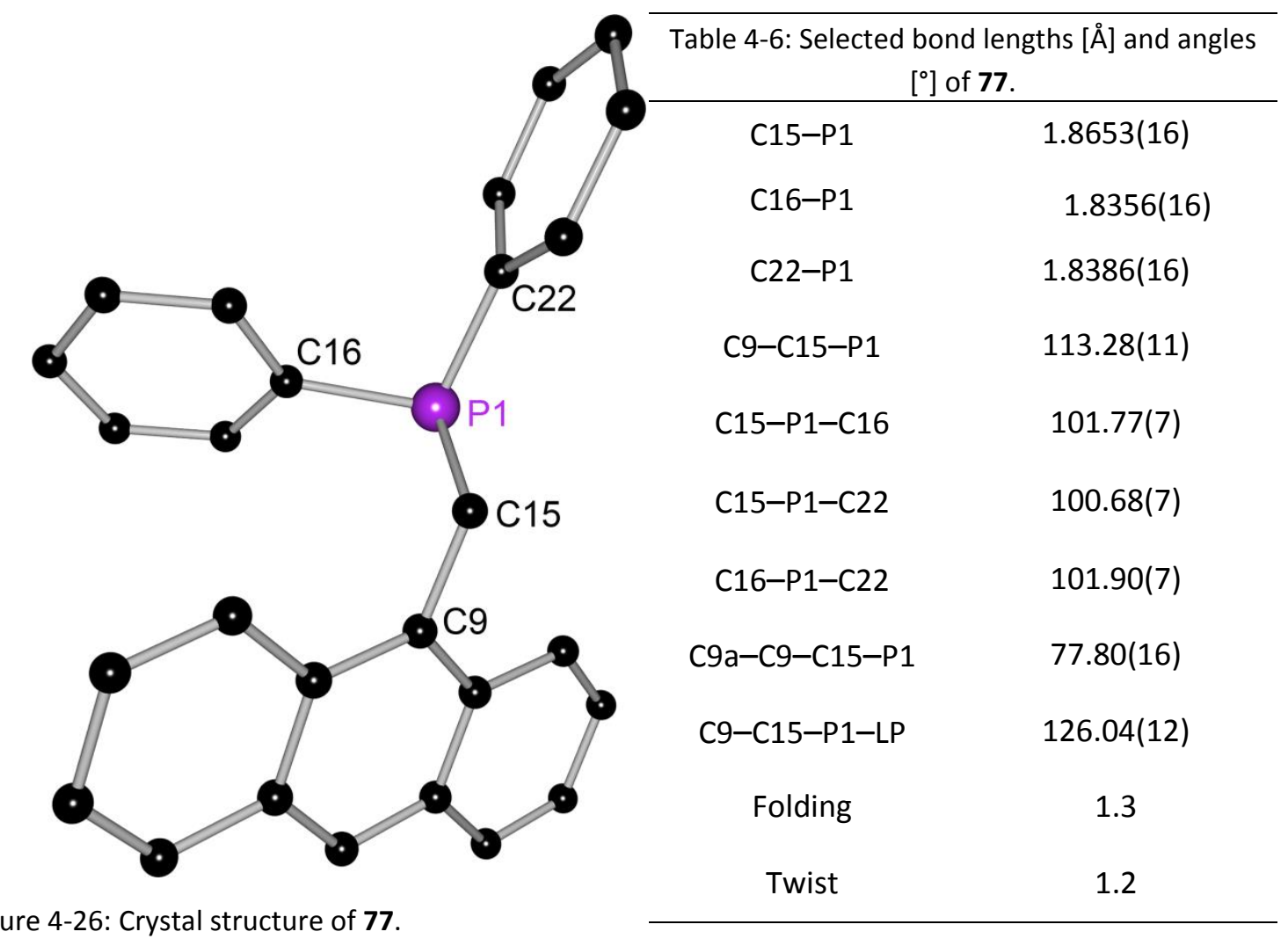

Figure 4-26: Crystal structure of 77.

Despite the low complexity of the molecule, $\mathbf{7 7}$ crystallizes in the low symmetry space group $P \overline{1}$. The asymmetric unit contains one molecule of 77 . Compared to the corresponding compound without a spacer between phosphane substituent and fluorophore $\left(\mathrm{HAnPPh}_{2}(4)\right)$, the deformation of the anthracene moiety is clearly weaker in 77. This can be ascribed to the enhanced flexibility provided by the spacer, which minimizes the steric strain applied by the phosphanyl substituent. All angles of P- 
bound substituents to one another are close to $100^{\circ}$ which demonstrates the spatial demand of the lone pair. The $\pi-\pi$ overlap of ca. $15 \%$ is small at a distance of $3.45 \AA$.

For further comparison, the sulfur oxidation product of $\mathbf{7 7}$ was also synthesized to represent an electron richer counterpart of the regular oxidation product $\mathrm{HAnCH}_{2} \mathrm{POPh}_{2}$ (78). For this purpose $\mathrm{HAnCH}_{2} \mathrm{PPh}_{2}$ (77) was dissolved in toluene and one equivalent of elemental sulfur was added. After heating to $110^{\circ} \mathrm{C}$ for $6 \mathrm{~h}$ and subsequent filtration, $\mathrm{HAnCH}_{2} \mathrm{PSPh}_{2}$ (79) was crystallized from toluene and obtained as a yellow crystalline solid (Scheme 4-27).<smiles>c1ccc(P(Cc2c3ccccc3cc3ccccc23)c2ccccc2)cc1</smiles>

77<smiles>S=P(Cc1c2ccccc2cc2ccccc12)(c1ccccc1)c1ccccc1</smiles>

79

Scheme 4-27: Synthesis of $\mathrm{HAnCH}_{2} \mathrm{PSPh}_{2}$ (79).

Likewise 77, 79 crystallizes in the triclinic space group $P \overline{1}$, but the asymmetric unit contains two independent molecules of 79 (Figure 4-27). Although both molecules are very similar in geometry and orientation of the phosphoryl substituent relative to the anthracene moiety, they do differ in terms of deformation.

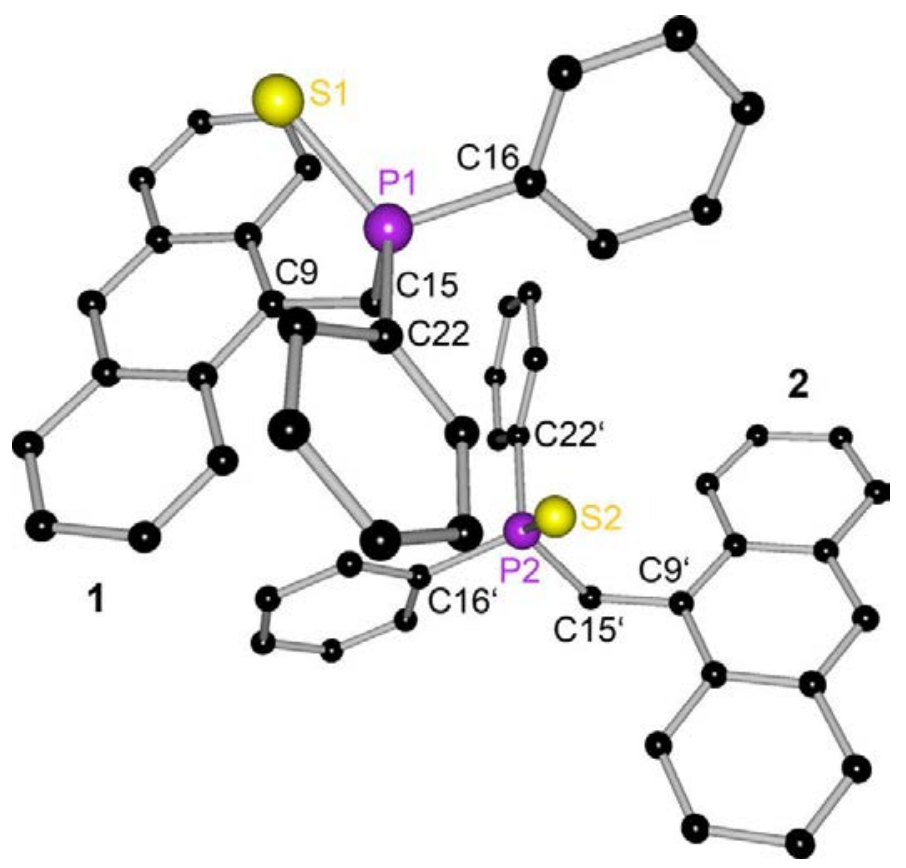

Figure 4-27: Crystal structure of $\mathrm{HAnCH}_{2} \mathrm{PSPh}_{2}$ (79), 1 = molecule 1, 2 = molecule 2; hydrogen atoms are omitted for clarity. 
While molecule 1 exhibits weak distortion of the fluorophore (only slightly stronger than the un-oxidized 77), molecule 2 shows more than twice as strong folding and twist deformation. This is quite surprising in view of the identical structure and similar conformation of both molecules and is likely to be induced by packing.

Table 4-7: Selected bond lengths $[\AA]$ and angles $\left[{ }^{\circ}\right]$ of 79.

\begin{tabular}{cc|cc}
\hline \multicolumn{2}{c|}{ Molecule 1 } & \multicolumn{2}{c}{ Molecule 2 } \\
\hline C9-C15-P1 & $115.12(9)$ & C9'-C15'-P2 & $113.57(9)$ \\
C15-P1-S1 & $114.97(5)$ & C15'-P2-S2 & $114.81(5)$ \\
C16-P1-C22 & $104.73(6)$ & C16'-P1-C22' & $103.92(6)$ \\
C9-C15-P1-S1 & $-28.44(12)$ & C9'-C15'-P2-S2 & $-30.13(11)$ \\
Folding & 3.3 & Folding & 6.0 \\
Twist & 2.9 & Twist & 7.3 \\
\hline
\end{tabular}

Both molecules have a similar distorted tetrahedral geometry surrounding the phosphorus atom. Furthermore, there are two $\mathrm{sp}^{2}$ type $\mathrm{C}-\mathrm{H} \cdots \pi$ interactions present in the packing motif of 79. They measure $2.937 \AA$ and $2.991 \AA$ at angles of $48.7^{\circ}$ and $58.7^{\circ}$, respectively. $\pi-\pi$ overlap is virtually not observed.

Moreover, $\mathrm{AnCH}_{2} \mathrm{PPh}_{2}$ (77) was reacted with [ $\mathrm{Me}_{2} \mathrm{SAuCl}$ to synthesize the corresponding gold(I) complex (Scheme 4-28). Both reactants were dissolved in anhydrous DCM and stirred for $3 \mathrm{~h}$. Cyrstallization at $-30^{\circ} \mathrm{C}$ afforded $\left[\mathrm{HAnCH} \mathrm{PPh}_{2} \mathrm{AuCl}\right](\mathbf{8 0})$ as light yellow crystals.

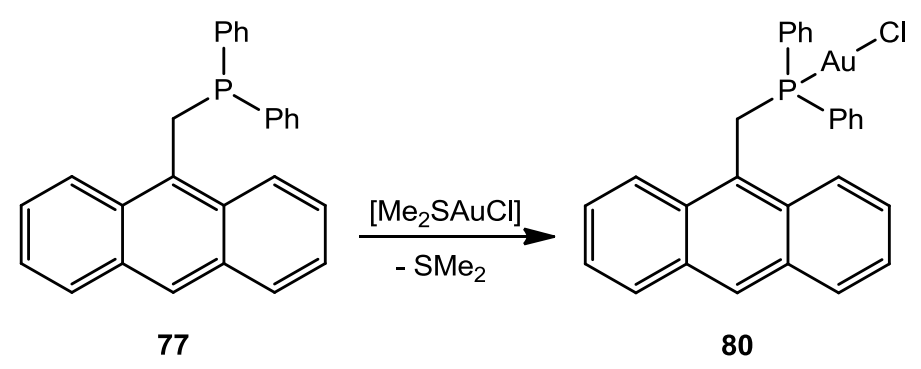

Scheme 4-28: Synthesis of $\left[\mathrm{HAnCH}_{2} \mathrm{PPh}_{2} \mathrm{AuCl}\right](80)$. 


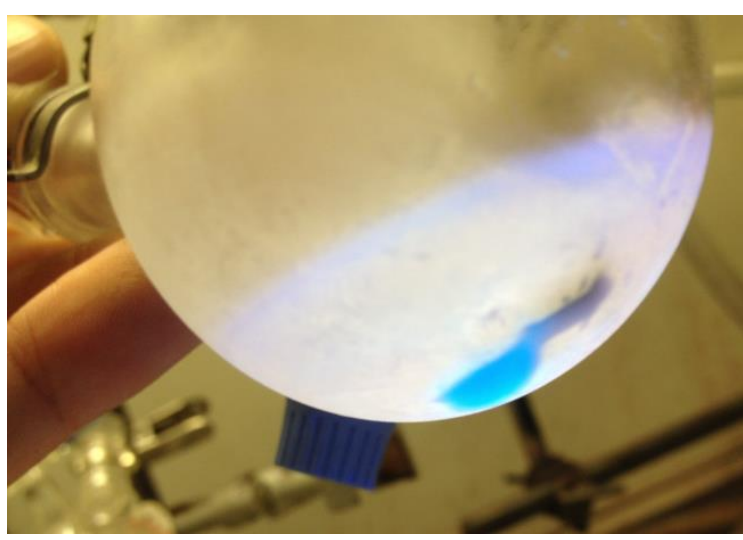

Figure 4-1: [HAnCH $\left.\mathrm{PPh}_{2} \mathrm{AuCl}\right](\mathbf{8 0})$ under exposure to UV light $\left(\lambda_{\mathrm{Ex}}=366 \mathrm{~nm}\right)$.
The crystals exhibit strong deep blue solid state fluorescence upon irradiation with UV light (366 nm) (Figure 4-28). Unfortunately, the obtained amount of crystalline material was not sufficient for acquisition of solid state fluorescence spectra. Analogous to $\mathbf{7 7}$ and 79, [ $\left.\mathrm{HAnCH}_{2} \mathrm{PPh}_{2} \mathrm{AuCl}\right](80)$ crystallizes in the space group $P \overline{1}$. The single molecule contained in the asymmetric unit shows very weak deformation of the fluorophore (in the range of its precursor molecule 77).

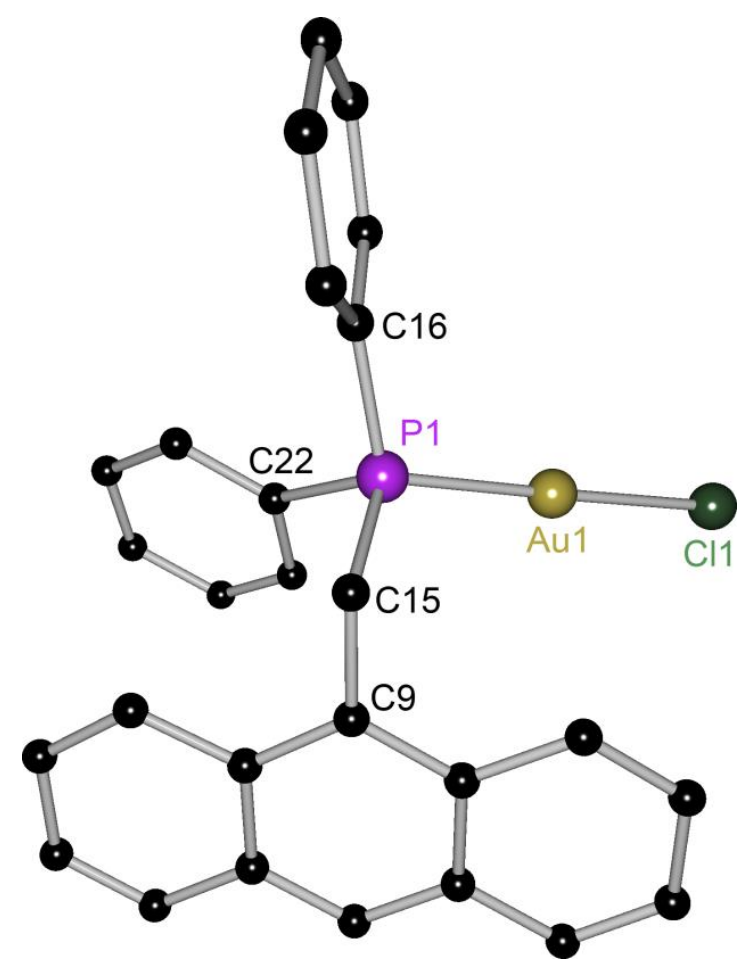

Table 4-8: Selected bond lengths $[\AA]$ and angles $\left[{ }^{\circ}\right]$ of 80 .

\begin{tabular}{cc} 
P1-Au1 & $2.2325(5)$ \\
Au1-Cl1 & $2.2970(5)$ \\
C9-C15-P1 & $112.34(15)$ \\
C15-P1-Au1 & $112.89(7)$ \\
C16-P1-C22 & $105.91(10)$ \\
C16-P1-Au1 & $114.10(7)$ \\
P1-Au1-Cl1 & $177.962(19)$ \\
C9a-C9-C15-P1 & $85.0(2)$ \\
C9-C15-P1-Au1 & $-65.17(16)$ \\
Folding & 2.8 \\
Twist & 1.6 \\
\hline
\end{tabular}

Figure 4-29: Crystal structure of $\mathbf{8 0}$, hydrogen atoms are omitted for clarity.

The tetrahedral geometry surrounding P1 is also less distorted than in $\mathbf{7 7}$ or in the oxidation product 79 (Table 4-8). The P-Au-Cl fragment in nearly linear, enclosing an angle of $177.96^{\circ}$. The shortest Au-Au distance measures $4.62 \AA$, which is too long to postulate Au-Au bonding, which occurs up to an upper limit of ca. $4.0 \AA$ (c.f. Figure 3-78). 


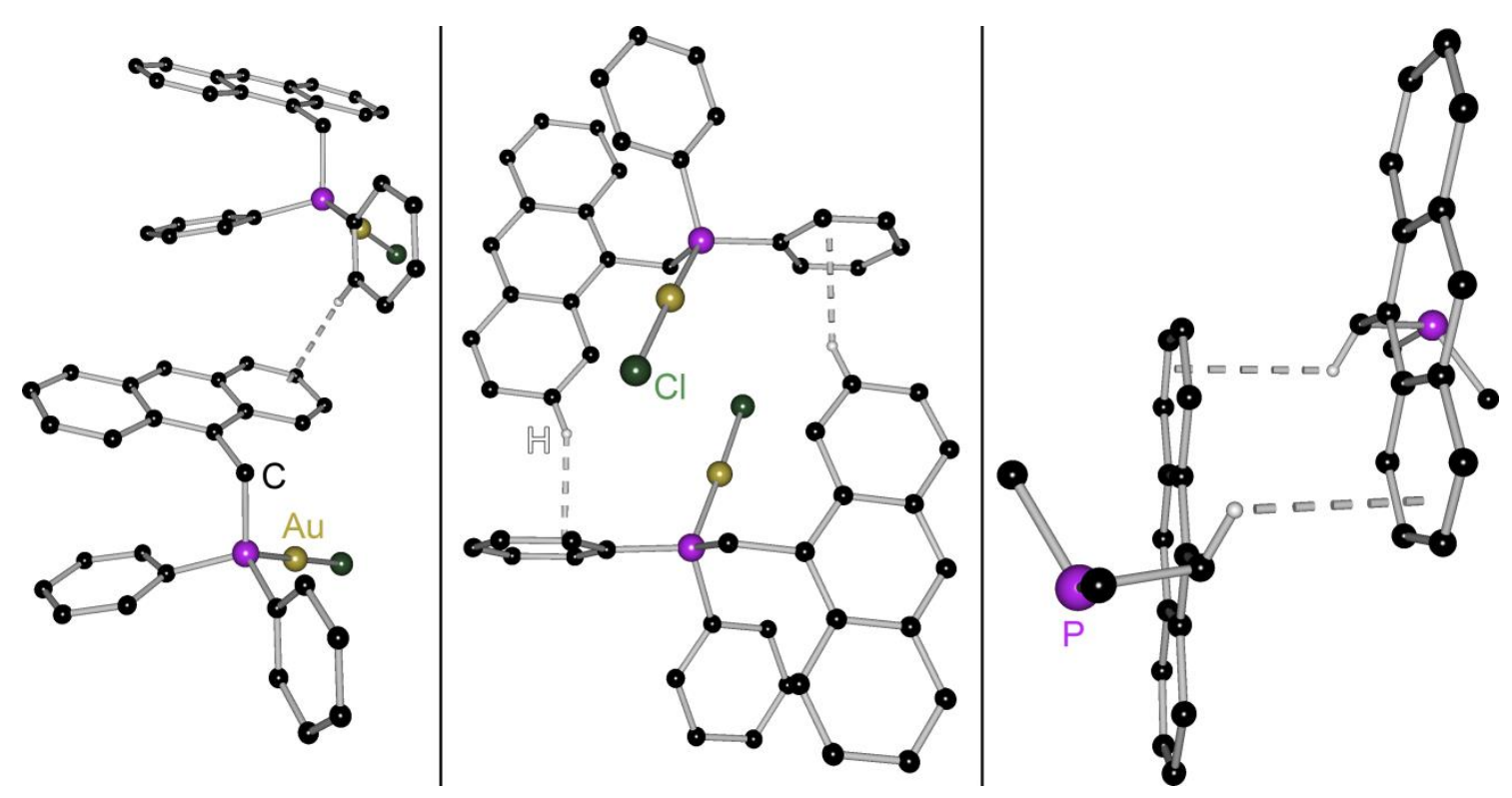

Figure 4-30: $\mathrm{sp}^{2} \mathrm{C}-\mathrm{H}^{\cdots} \pi$ bonding in the structure of 80, hydrogen atoms (left, center) and one phenyl moiety (center) are omitted for clarity; right: $\mathrm{sp}^{3} \mathrm{C}-\mathrm{H}^{\cdots} \pi$ bonding in the structure of $\mathbf{8 0}$, hydrogen atoms, Au-Cl fragments and phenyl moieties are omitted for clarity.

Several intermolecular interactions are present in the structure of $\mathbf{8 0}$. Although no $\pi-\pi$ overlap is found between anthracene moieties, there is a small intramolecular overlap between a phenyl ring and the anthracene $\pi$-system (Figure 4-31). The $\pi-\pi$ distance measures $3.70 \AA$. This interaction is accompanied by a number of $\mathrm{C}-\mathrm{H}^{\cdots} \pi$ bonds. The strongest of these is a $\mathrm{sp}^{2} \mathrm{C}-\mathrm{H}^{\cdots} \pi \mathrm{interaction}$ of an ortho-phenyl hydrogen atom with a peripheral anthracene ring (Figure 4-30, left). It measures $2.716 \AA$ and encloses an angle of $63.5^{\circ}$ with the ring plane. Furthermore, $\mathrm{C}-\mathrm{H} \cdots \pi$ bonding between a hydrogen atom in 2-position and a phenyl ring occurs (Figure 4-30, center), as well as $\mathrm{sp}^{3} \mathrm{C}-\mathrm{H} \cdots \pi$ bonding between the hydrogen atom of the methylene spacer and a peripheral anthracene ring (Figure 4-30, right). The latter interaction measures $2.878 \AA$ at an angle of $30.6^{\circ}$. In view of the findings gathered in previous chapters, the presence of several 


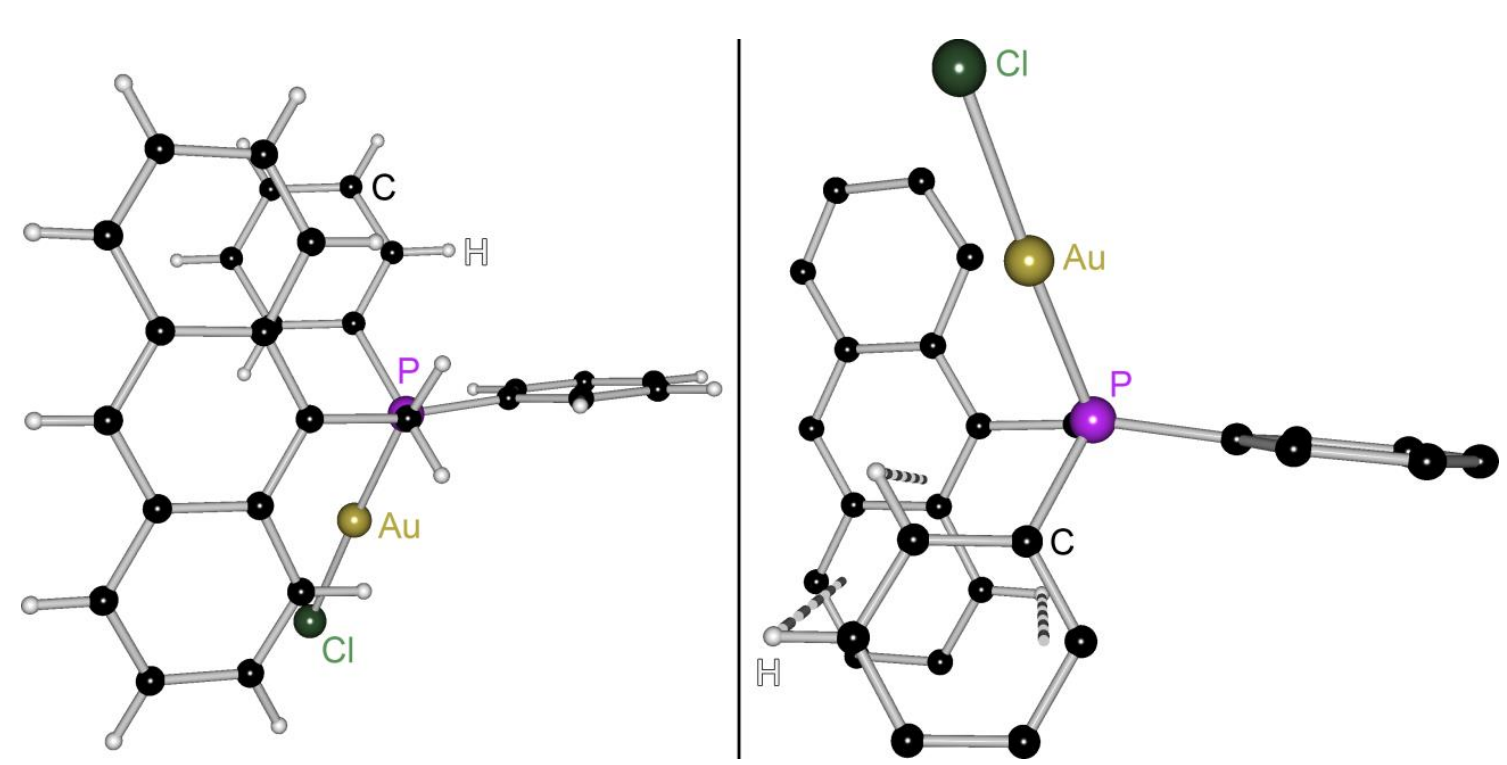

Figure 4-31: Intramolecular $\pi-\pi$ overlap in $\mathbf{8 0 .}$

$\mathrm{C}-\mathrm{H} \cdots \pi$ bonds in combination with the weak deformation of the fluorophore accounts for the observed strong (but not quantified) solid state emission of $\mathbf{8 0}$.

To verify the electron transfer from the phosphane moiety to the excited fluorophore, a dilute solution of $\mathbf{7 7}$ in degassed MeCN was prepared. To inhibit the supposed electron transfer, the lone pair had to be energetically lowered. Because phosphanes cannot be protonated like amines, a different reaction type suitable for phoshanes had to be utilized. Because Onoda et al. had produced this effect by oxidation with organoperoxides, a similar oxidation using hydrogen peroxide was chosen (Scheme 4-29).<smiles>c1ccc(P(Cc2c3ccccc3cc3ccccc23)c2ccccc2)cc1</smiles>

77<smiles>O=P(O)(O)c1cccc2c(CP(=O)(c3ccccc3)c3ccccc3)c3ccccc3cc12</smiles>

78

Scheme 4-29: Oxidation of $\mathbf{7 7}$ with hydrogen peroxide.

In order to visualize the effects of oxidation right away, the oxidation was carried out in situ and monitored by fluorescence spectroscopy. For this purpose a solution of 77 was filled into a Schlenk-cuvette via syringe which was equipped with a magnetic 
stirring bar. After recording a background spectrum of the un-oxidized 77, a slight excess of dilute aqueous $\mathrm{H}_{2} \mathrm{O}_{2}$ solution was added through a septum. From this point onward, an emission spectrum was recorded every $10 \mathrm{~s}$ for the following $20 \mathrm{~min}$ (Figure 4-32).
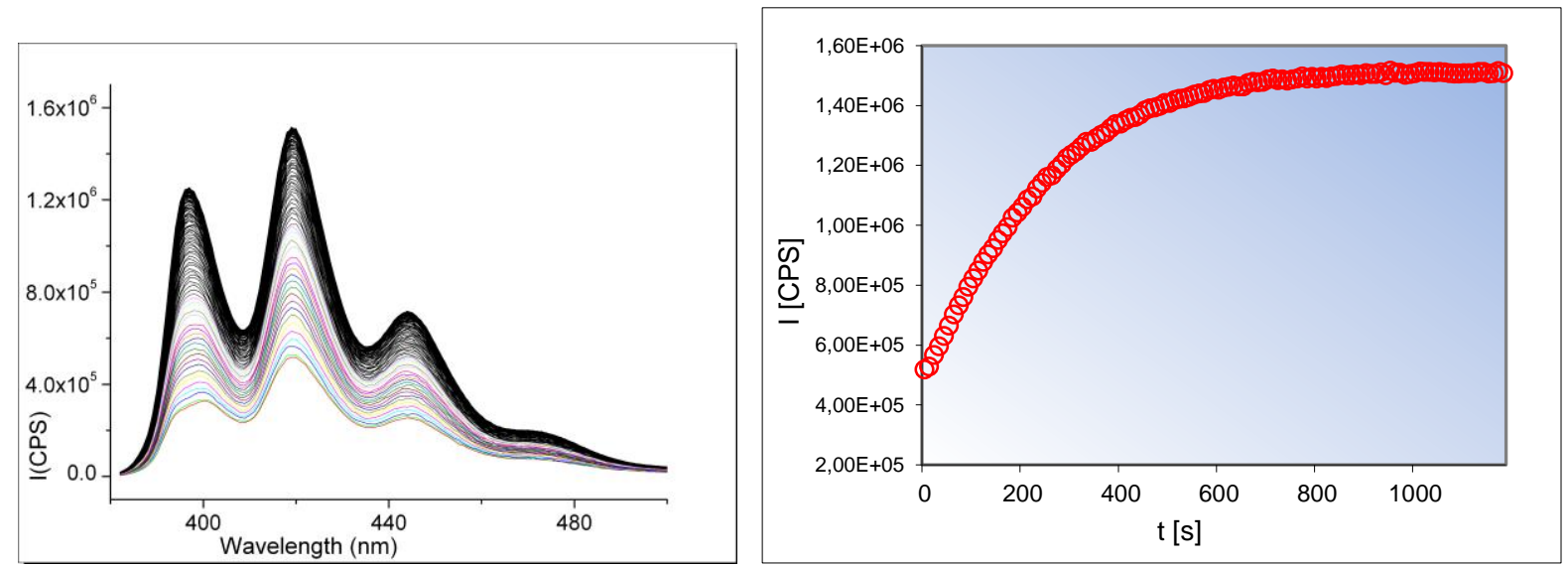

Figure 4-32: Time dependent emission of 77 upon reaction with $\mathrm{H}_{2} \mathrm{O}_{2}$; left: stacked, right: single point plot at $\lambda_{\mathrm{Em}}=432 \mathrm{~nm}$.

A clear increase of emission intensity is visible in Figure 4-32, which proves the inhibition of an electron transfer process by oxidation of the phosphanyl substituent. Within the first four minutes, a linear increase of emission intensity is observed, which then slowly converges to a constant maximum value in the course of the oxidation process. The ca. threefold increase of emission intensity is very close to the emission enhancement ratio reported by Onada et al. for their compound. ${ }^{[18]}$ This clearly qualifies 77 as a sensing device for peroxides. To ensure that the observed emission enhancement is not limited to hydrogen peroxide as an analyte, the identical experiment was repeated with $m C P B A$ as the oxidation agent (Figure 4-33).

Due to the higher reactivity of $m$ CPBA compared to $\mathrm{H}_{2} \mathrm{O}_{2}$, the oxidation was completed within the first $10 \mathrm{~s}$ cycle of the fluorescence measurement. The rapid quantitative oxidation of $\mathbf{7 7}$ lets the emission increase to the maximum value instantly, which then stays constant for the remainder of the experiment. Hence, sensitivity of $\mathbf{7 7}$ is also given towards organic peroxides. 


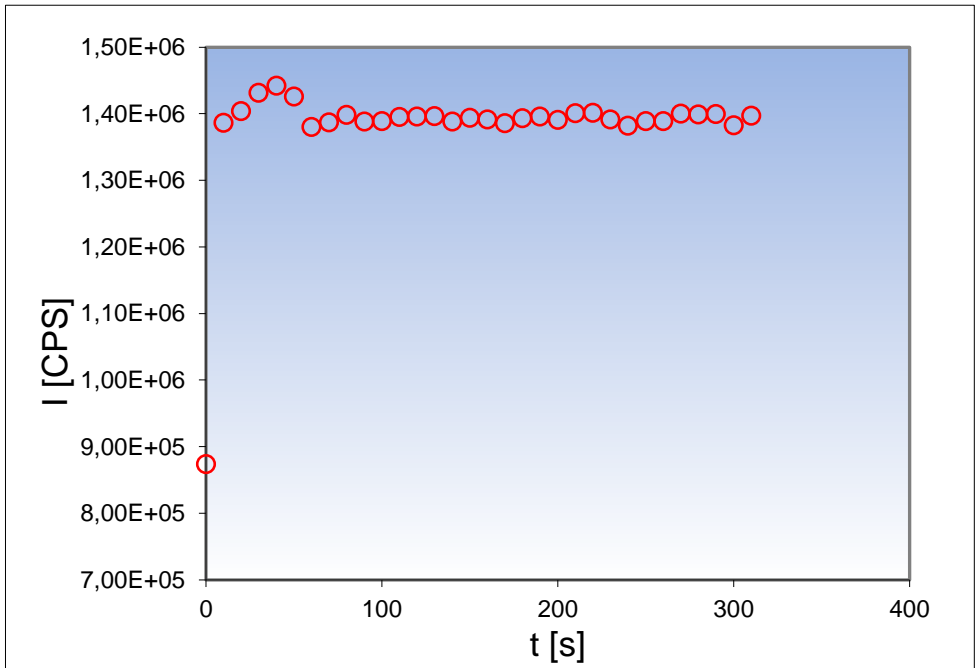

Figure 4-2: Time dependent single point emission of 77 upon reaction with $m C P B A ; \lambda_{E m}=432 \mathrm{~nm}$.
In order to demonstrate the influence of the spacer, the analogue compound of 77 without a spacer $\mathrm{HAnPPh}_{2}$ (4) - was subjected to the identical oxidation experiments as 77. The experiences derived from amine quenchers would predict a weak rate of electron transfer from the quencher to the fluorophore due to unsuitable geometry for the required orbital overlap. This again would lead to a weak expected increase of emission upon oxidation. On the other hand, reports of direct electron transfer from phosphane substituents to anthracene fluorophores (without spacers) suggest contrary results. ${ }^{[65,71 b, 84 a]}$

In identical manner as described for 77, a dilute solution of $\mathrm{HAnPPh}_{2}$ (4) in $\mathrm{MeCN}$ was prepared and filled into the Schlenk-cuvette. Prior to addition of the oxidation agent $\mathrm{H}_{2} \mathrm{O}_{2}$, a background spectrum was recorded, then emission spectra were recorded every $10 \mathrm{~s}$.

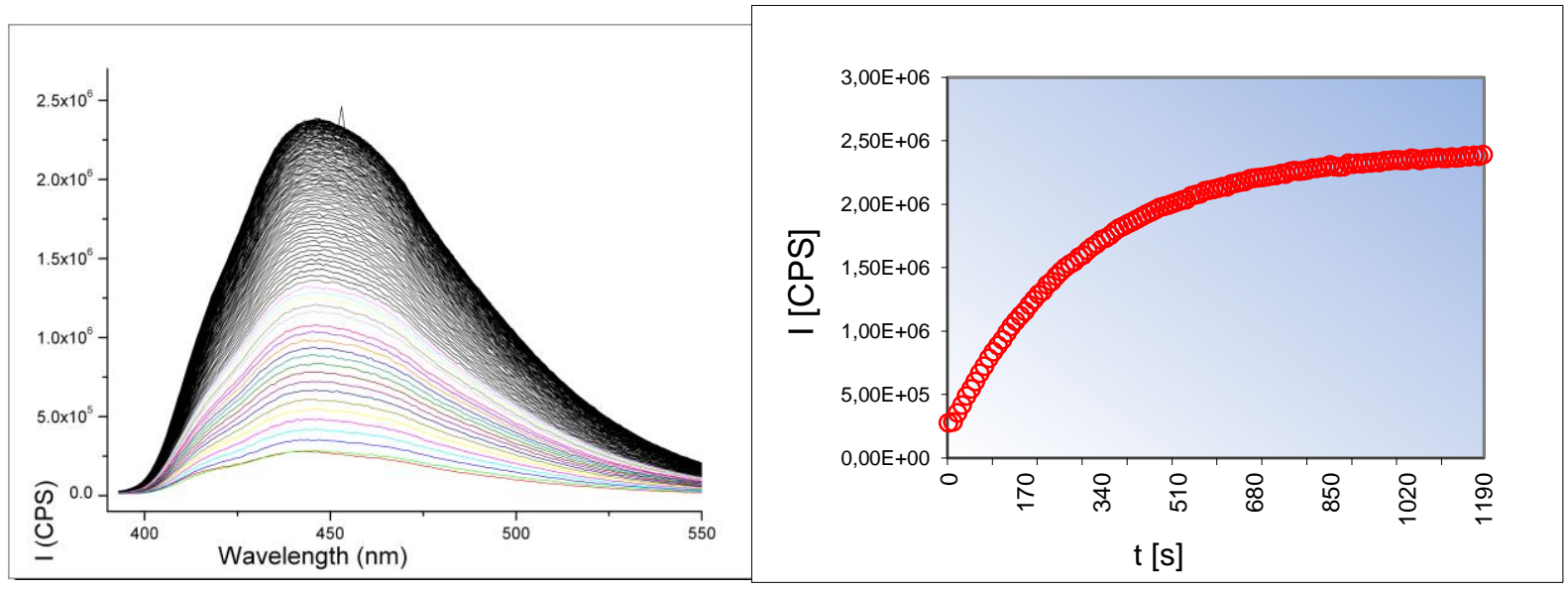

Figure 4-35: Time dependent emission of $\mathrm{HAnPPh}_{2}$ (4) upon reaction with $\mathrm{H}_{2} \mathrm{O}_{2}$; left: stacked, right: single point plot at $\lambda_{\mathrm{Em}}=432 \mathrm{~nm}$. 
As Figure 4-35 shows, 4 also exhibits a strong increase of emission intensity upon oxidation. The run of the curve is very similar to that of $\mathbf{7 7}$, with a linear increase of emission which then slowly converges to a constant maximum. The slightly faster increase of emission in the case of $\mathbf{7 7}$ underlines its higher sensitivity towards oxidation. This is in accordance with the well-established stability of aromatic tertiary phosphanes compared to aliphatic phosphanes towards oxidation. On the other hand the shape of each single emission spectrum demonstrates the influence of the phosphanyl substituent on the HOMO/LUMO energies of the compound, inducing a strong bathochromic shift compared to 77. The typical anthracene band structure of the emission spectrum is missing. Also $\mathbf{4}$ and its oxidation product differ in the wavelength of their emission maxima by $5 \mathrm{~nm}$, which does not apply to 77 and $\mathbf{7 8}$. This effect is also prevented by the spacer in 77 . The oxidation using mCPBA leads to the identical rapid and quantitative formation of the oxidation product as observed for $\mathbf{7 7}$ (Figure 4-36).

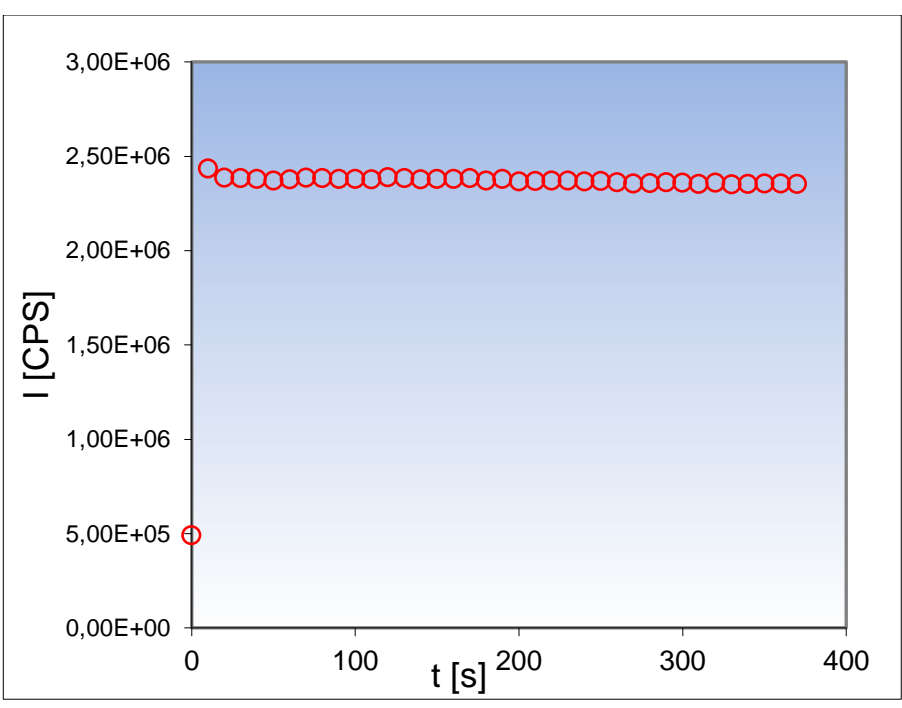

Figure 4-36: Time dependent emission of $\mathbf{4}$ upon reaction with $m$ CPBA
The even stronger (nearly eightfold) increase of emission by oxidation of $\mathrm{HAnPPh}_{2}$ (4) confirms the reports of Yip et al. who had postulated an electron transfer to the excited fluorophore in their compound 9,10-bis(diphenylphosphanyl)anthracene.

Possible explanations for the validity of this process are that phosphanes are generally electron-richer than amines. The supposed obstructive geometry for electron transfer induced by the absence of a spacer may well be compensated by larger and more diffuse orbitals compared to amines which generate the required overlap. Nevertheless, the benefits of spacer - as for example ensuring high flexibility of the receptor unit, inhibiting interactions of the analyte with the $\pi$ system by spatial separation or suppressing the alteration of HOMO/LUMO energies - 
would clearly outnumber the slightly larger on/off ratio in a potential sensor molecule based on a phosphane quencher.

\subsubsection{Further Synthetic approaches}

In addition to the syntheses described in 4.3.1, different other synthetic pathways were explored to generate anthracene derivatives containing spacers and phosphane

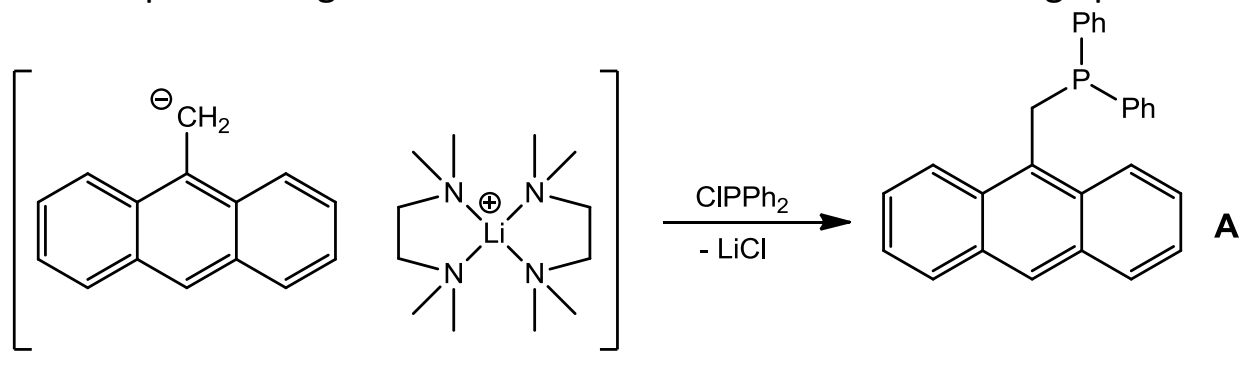<smiles>CCN(CC)Cc1ccc(Cl)cc1CP(CC)N(CC)CCc1ccc2cc3ccccc3c(CP(Cl)Cl)c2c1</smiles>
Scheme 4-30: Reactions of [ $\mathrm{AnCH}_{2}$ Li-TMEDA] (66) with chlorophosphanes.

quenchers. Because chlorophosphanes are strong electrophiles, they are likely to react with the nucleophile [AnCH $\mathrm{AL}_{2} \mathrm{Li}$ TMEDA] (66). Although $\mathrm{HAnCH}_{2} \mathrm{PPh}_{2}$ (77) had been successfully prepared (c.f. 4.3.1), this second approach was taken as a first possible application of 66. [ $\mathrm{AnCH}_{2} \mathrm{Li}$.TMEDA] (66) was prepared by addition of one equivalent of TMSMeLi to a solution of 9-methylanthracene and 2.0 equivalents of TMEDA in diethyl ether at $-15^{\circ} \mathrm{C}$. This solution was added to a solution of diphenylchlorophosphane in diethyl ether at $-78^{\circ} \mathrm{C}$ (Scheme 4-30, reaction A). The identical reaction was carried out with bis(diethylamino)chlorophosphane as an electrophile (Scheme 4-30, reaction B). After completion of both reactions, the solvents were removed and the crude products were dissolved in DCM and filtrated for removal of lithium chloride. As depicted in Scheme 4-30 (reaction B), the bis(diethylamino)phosphane was directly dissolved in hexane and reacted with gaseous $\mathrm{HCl}$ for cleavage of the $\mathrm{P}-\mathrm{N}$ bonds.

Although both reactions were successful and the desired compounds were obtained as the main products, both conversions were not quantitative and separation of byproducts for purification was not successful. This shows that optimum reaction 
conditions for the utilization of $\left[\mathrm{AnCH}_{2} \mathrm{Li}\right.$-TMEDA] (66) must be found to achieve satisfying results in terms of yields and conversion rates.

Because the product of reaction B in Scheme 4-30 bears significant potential for the synthesis of chelating phosphane receptor units in presence of a spacer moiety, synthesis of this compound according to the route described in 4.3.1 was pursued. For this purpose bis(diethylamino)chlorophosphane had to be reduced for subsequent deprotonation. This reduction reaction was described by King and Sundaram in 1984 (Scheme 4-31, reaction A). ${ }^{[68]}$<smiles>CCCCCCCCCNP(CC)NCC</smiles>

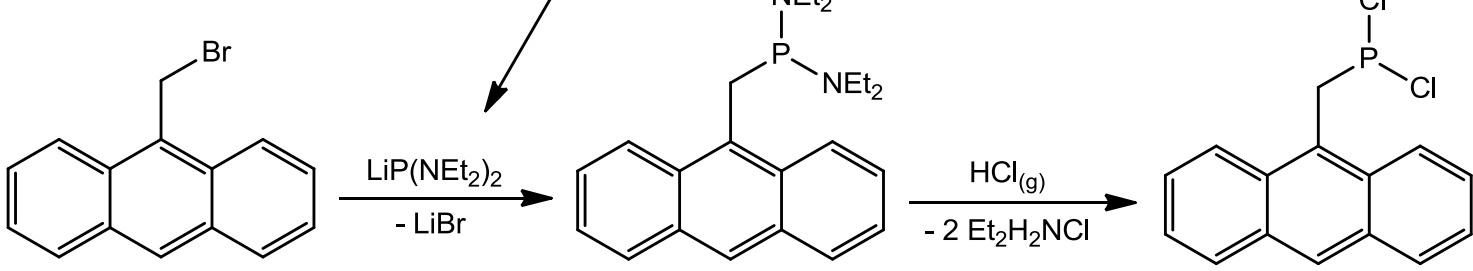

Scheme 4-31: Reduction of $\mathrm{CIP}\left(\mathrm{NEt}_{2}\right)_{2}$ according to King and Sundaram $(\mathbf{A})^{[68]}$ and reaction of the lithiated phosphane with an electrophile.

Bis(diethylamino)chlorophosphane was reacted with a slight excess of lithium aluminumhydride in diethyl ether at $-78^{\circ} \mathrm{C}$ for $3 \mathrm{~h}$. After completion of the reaction the solution was filtrated and the solvent was removed under reduced pressure. The crude product was extracted with pentane, filtrated again and the pentane was evaporated. The crude product was purified by distillation according to the procedure described by King and Sundaram. The obtained clear light yellow liquid crystallized upon cooling to $3^{\circ} \mathrm{C}$ overnight. X-ray structure determination revealed that not the desired phosphane had been prepared, but bis(diethylamino)alane (81) (Scheme 4-32).

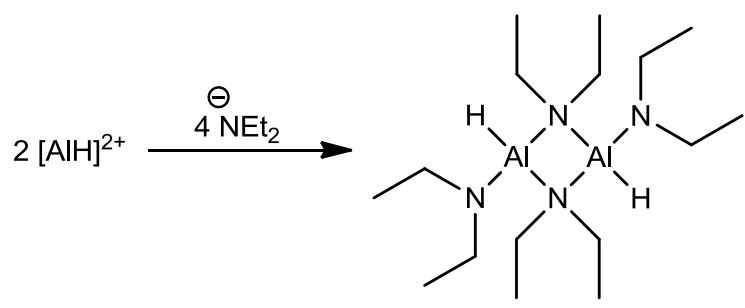

Scheme 4-32: Formation of bis(diethylamino)alane. 
This alane has been synthesized by Kovar and Ashby in 1971 along with a number of other bis(dialkylamino)alanes. ${ }^{[119]}$ While they synthesized the alane derivatives from elemental aluminum, hydrogen and secondary amines, here the formation of the unexpected product clearly originated from amide ions which were generated by decomposition of the employed phosphane. By reaction with aluminum hydride residues which were still dissolved after filtration of the reaction mixture, the alane was formed. Though previously characterized, the crystal structure of bis(diethylamino)alane (81) had not been previously determined.

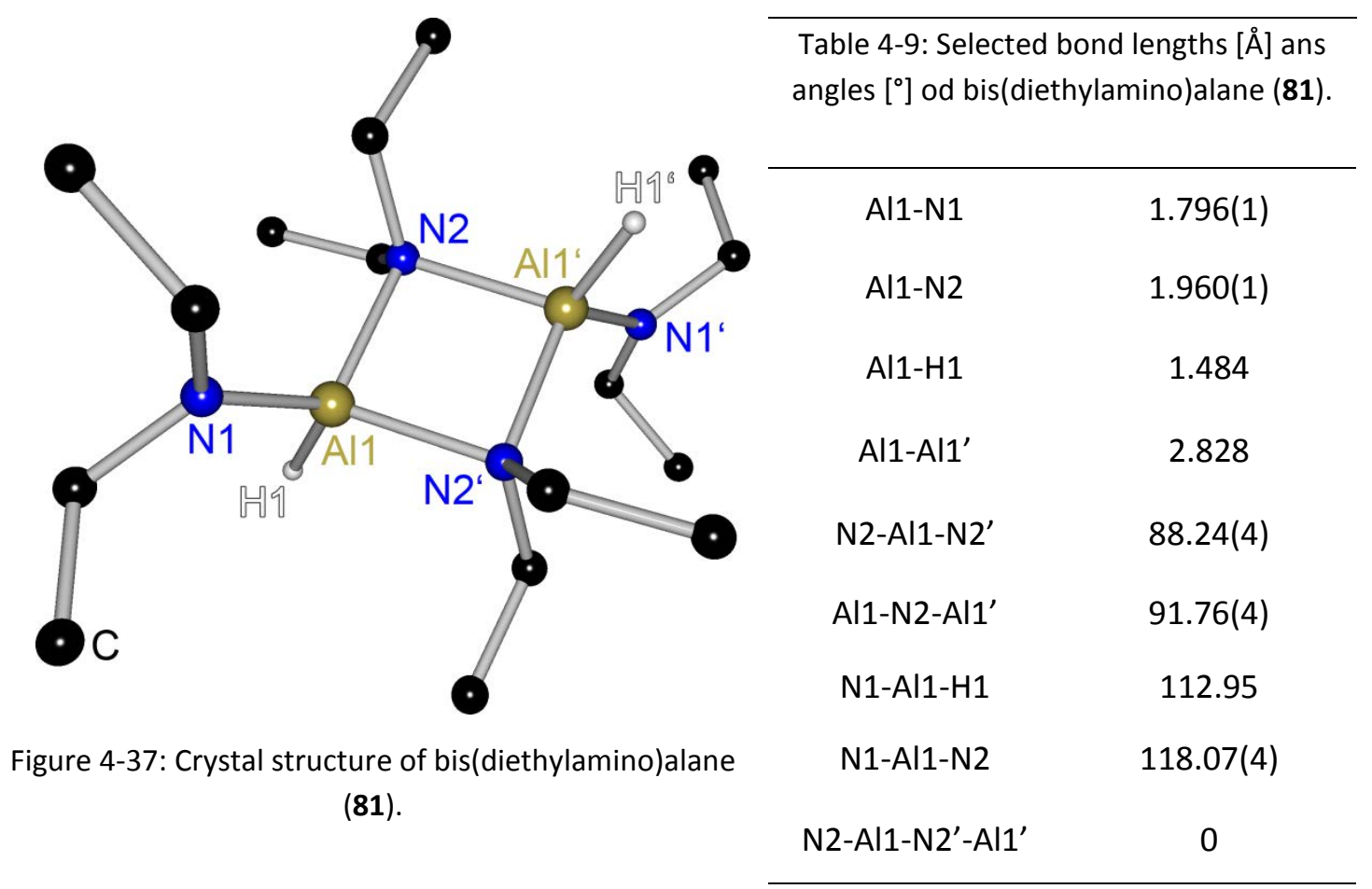

81 crystallizes in the monoclinic space group $P 2_{1} / c$ and the asymmetric unit contains one half of the molecule depicted in Figure 4-37. The central $\mathrm{Al}_{2} \mathrm{~N}_{2}$ fourmembered ring is perfectly planar and all angles are close to $90^{\circ}$. The terminal Al-N bonds are by nearly $0.2 \AA$ shorter than the bridging Al-N bonds within the ring. The geometry at Al1 is of strongly distorted tetrahedral character. The formation of the four-membered ring produces a small N2-Al1-N2' angle of only $88.24^{\circ}$, hence all other angles are distinctly larger than the optimum tetrahedral angle. The short bonds within the $\mathrm{Al}_{2} \mathrm{~N}_{2}$ cycle lead to an Al-Al distance of only $2.828 \AA$. A very similar structure which differs only in the terminal amide donors (diiso-propyl instead of diethyl) has been published by Chang et al. and exhibits nearly identical geometrical parameters as $\mathbf{8 1}$, as does the bis(dimethylamino) analogue. ${ }^{[120]}$ 
Though the formation of $\mathbf{8 1}$ certifies the decomposition of the phosphane, the point of decomposition was not explicitly determinable. The heating of the crude product during the distillation process may well have been the crucial point of decomposition of the desired phosphane. The yield obtained by King and Sundaram was also very poor, and they describe bis(diethylamino)phosphane as highly reactive which may indicate that they also had decomposition issues. Therefore the reaction was repeated under identical conditions, but the distillation was left out to avoid heating of the crude product. Instead, the crude product was dissolved in diethyl ether and one equivalent of $n$-BuLi was added at $-78^{\circ} \mathrm{C}$ for in situ deprotonation of the phosphane. The lithiated phosphane was thought to be separable from impurities by crystallization using this strategy. To further benefit crystallization, the chelating donor base tetramethylpropylenediamine (TMPDA) was added to the reaction mixture and the flask was stored at $-40^{\circ} \mathrm{C}$. Within several days, colorless crystals formed which were suitable for X-ray structure determination.

The obtained product did not turn out to be the deprotonated phosphane, but the lithium alane 82 depicted in Figure 4-38. It is obviously a reaction product of bis(diethylamino)alane (81) and $n$-BuLi as well as the donor base TMPDA. The presence of $\mathbf{8 1}$ in the reaction mixture despite the fact that the reaction was never warmed above $0^{\circ} \mathrm{C}$ proves that the decomposition of the phosphane already occurs during the reduction reaction and not during the distillation. The obtained lithium alane is very reactive and decomposes quickly even in perfluorinated oil at temperatures around $-80^{\circ} \mathrm{C}$. Similar compounds have been reported by Mulvey ${ }^{[121]}$ and Power. ${ }^{[122]}$ Although their compounds contain the identical four-membered Li-N-Al-N cycle as $\mathbf{8 2}$, they all feature bulky bridging amide ligands or bulky alky moieties at the aluminum atom which largely contribute to the kinetic stability of the these compounds. Neither the amide ligands, nor the butyl moieties at the aluminum atom of $\mathbf{8 2}$ exhibit steric properties which would induce kinetic stabilization. Thus, 82 can be assumed to be the most reactive and instable of the reported lithium alanes, and therewith considered the most challenging crystal to mount for the acquisition of $X$-ray data. 


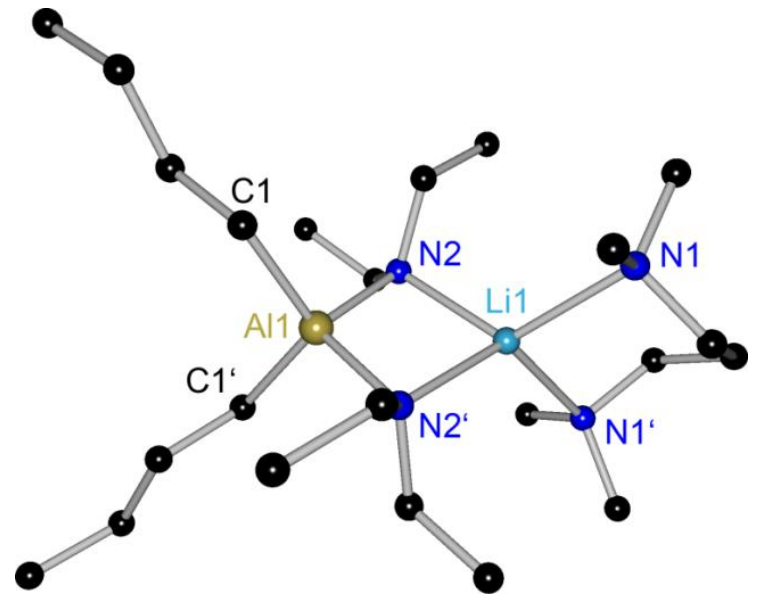

Figure 4-38: Crystal structure of $\mathbf{8 2}$, hydrogen atoms are omitted for clarity.

\begin{tabular}{cc}
\hline $\begin{array}{c}\text { Table 4-10: selected bond lengths }[\AA \AA \\
\text { and angles }\left[{ }^{\circ}\right]\end{array}$ \\
\hline Al1-C1 82.
\end{tabular}

$\mathbf{8 2}$ crystallizes in the orthorhombic space group Fdd2. Analogously to $\mathbf{8 1}$, one half of the molecule depicted in Figure 4-38 is contained in the asymmetric unit. Although the Li-N-Al-N cycle is also perfectly planar, the presence of two different metal ions makes it asymmetric. While the N2-Li1-N2' angle measures $87.1^{\circ}$, the N2-Al1-N2' angle is clearly larger at $102.65^{\circ}$. The Al-N2-Li fragment even encloses an angle of only $85.14^{\circ}$. The Al1-C1 bond to the terminal butyl moiety is slightly longer than the bridging Al1-N2 bond. This is in accordance with the Al-C and Al-N bond distances in other lithium alanes, as well as with the magnitude of the ring angles in these compounds. The Li-Al distance measures $2.760 \AA$ which is slightly longer than the average value derived from the other lithium alanes.

Although the initial goal of synthesizing and deprotonating bis(diethylamino)phosphane for the synthesis of phosphane quenchers was not achieved via this reaction pathway, the crystal structure of bis(diethylamino)alane (81) was determined and the interesting and highly reactive lithium alane $\mathbf{8 2}$ was discovered.

\subsection{Unification of Phosphanes and Sensor Compounds}

The final aspect addressed in this thesis is the challenge of combining the on/off switching of fluorescence provided by the PET mechanism with the wavelength altering effects of phosphoryl substituents. The synthesis of a compound which unites both major aspects described in this work was of great interest. The possibility of 
retaining the on/off switching of emission while being able to tune the emission wavelength and also the shape of the emission profile was particularly appealing. This way excitation and detection of emission could be shifted to convenient wavelengths and the broadening of excitation and emission bands could lead to faster and less complicated detection, requiring less accurate and therewith less expensive monochromatization devices. The main question in this matter was, whether the PET mechanism would still function in spite of the lowering of the HOMO/LUMO gap of the fluorophore which produces the typical bathochromic shift of phosphanylanthracenes. Alterations of these energies may make electron transfer processes less favorable, reducing the quenching rate and therewith the on/off ratio of the sensor. Secondly, the question whether electron transfer would vice versa affect the desired shifting of emission wavelengths arose. To actualize the synthesis of such an asymmetric compound, two different synthetic strategies were pursued.

\subsubsection{Starting from the Phosphane}

The first approach chosen was based on the primary introduction of the phosphoryl substituent to the anthracene fluorophore. 9-(Diphenylthiophosphoryl)-10methylanthracene (14) was synthesized from 9-bromo-10-methylanthracene by monolithiation and reaction with diphenylchlorophosphane, followed by an oxidation with elemental sulfur (c.f. 3.1.1).<smiles>Cc1c2ccccc2c(P(=S)(c2ccccc2)c2ccccc2)c2ccccc12</smiles>

14<smiles>C[C@]1(Br)c2ccccc2[C@@](Br)(P(=S)(c2ccccc2)P(c2ccccc2)c2ccccc2)c2ccccc21</smiles>

$\mathrm{H}_{3} \mathrm{C} \mathrm{Br}$

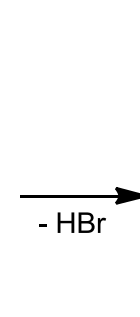

$\mathrm{Br}$<smiles>C1=CCCCC1</smiles><smiles>C=C1c2ccccc2C(Br)(P(=S)(c2ccccc2)c2ccccc2)c2ccccc21</smiles><smiles>S=P(c1ccccc1)(c1ccccc1)c1c2ccccc2c(CBr)c2ccccc12</smiles><smiles>[B][Te]</smiles><smiles>S=P(c1ccccc1)(c1ccccc1)[C@]1(Br)c2ccccc2[C@@](Br)(CBr)c2ccccc21</smiles> 
<smiles>[R10]Cc1c2ccccc2c(P(=S)(c2ccccc2)c2ccccc2)c2ccccc12</smiles>

Scheme 4-34: Introduction of an amine quencher to the brominated compound.

In the next step, the 10-methyl group was brominated with elemental bromine in chloroform according to the addition/elimination mechanism formulated by Stern (Scheme 4-33). ${ }^{[59 c]}$ This compound could then be reacted with a receptor/quencher unit in a final $S_{N}$ type reaction (Scheme 4-34). Although Stern had only verified this mechanism for 9-bromo-10-methylanthacene, which bears a clearly smaller and less complex halogen substituent in 9-position, the identical reaction was attempted with the more complex 14, bearing the emission shifting phosphoryl substituent. 1.1 equivalents of bromine were added to a chloroform solution of 14 over $1 \mathrm{~h}$ at $-15^{\circ} \mathrm{C}$. After the addition was completed, the reaction was slowly warmed to ambient temperature and stirred overnight. Accruing $\mathrm{HBr}$ was vented through a bubble counter. After ca. $14 \mathrm{~h}$ a yellow precipitate had formed which was isolated by filtration and dried in vacuo. The crude product was then crystallized from acetone for purification.

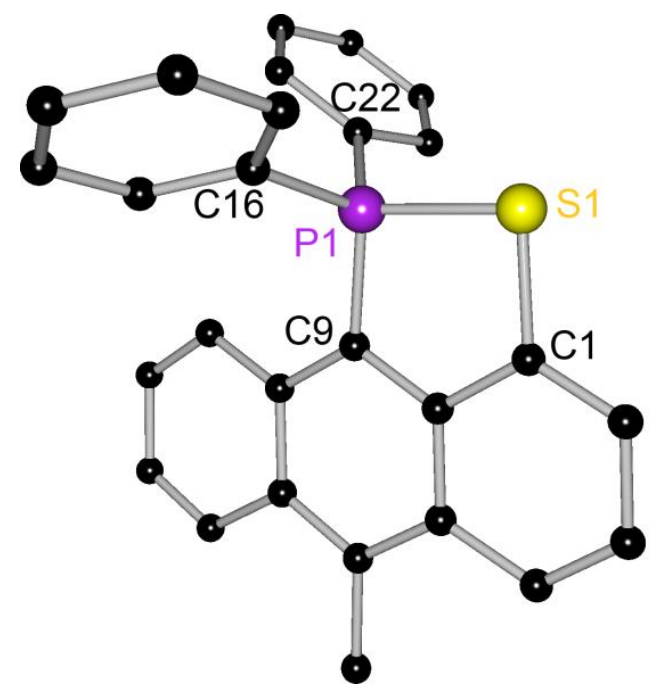

Figure 4-39: Crystal structure of $\mathbf{8 3}$, lattice solvent molecules and hydrogen atoms are omitted for clarity.
Table 4-11: Selected bond lengths $[\AA]$ and angles $\left[{ }^{\circ}\right.$ ] of $\mathbf{8 3}$.

$\begin{array}{ccc}- & \text { P1-S1 } & 2.071(1) \\ \mathrm{Br} 1 & \text { C9-P1 } & 1.805(3) \\ \text { C1-S1 } & 1.781(3) \\ \text { C9-P1-S1 } & 98.13(10) \\ \text { P1-S1-C1 } & 93.03(11) \\ \text { C16-P1-C22 } & 111.80(14) \\ \text { C9a-C9-P1-S1 } & 10.0(2)\end{array}$

Folding

Twist 
Subjection of the obtained crystals to X-ray structure determination revealed, that not the 10-(bromomethyl) derivative had been synthesized, but the P,S-heterocyclic 83 (Figure 4-39).This reaction product - which was obtained at a yield of $85 \%$ - indicates that the addition reaction of $\mathrm{Br}_{2}$ with the anthracene moiety must have been successful, but the elimination step did not proceed in desired manner.The formation of the S1-C1 bond is probably actualized by an intramolecular $\mathrm{S}_{N}{ }^{2}$ reaction between the sulfur atom and C1 (Scheme 4-35). This generates a positive charge which is located at $\mathrm{P} 1$, the bromide counter ion produces an overall neutral charge.

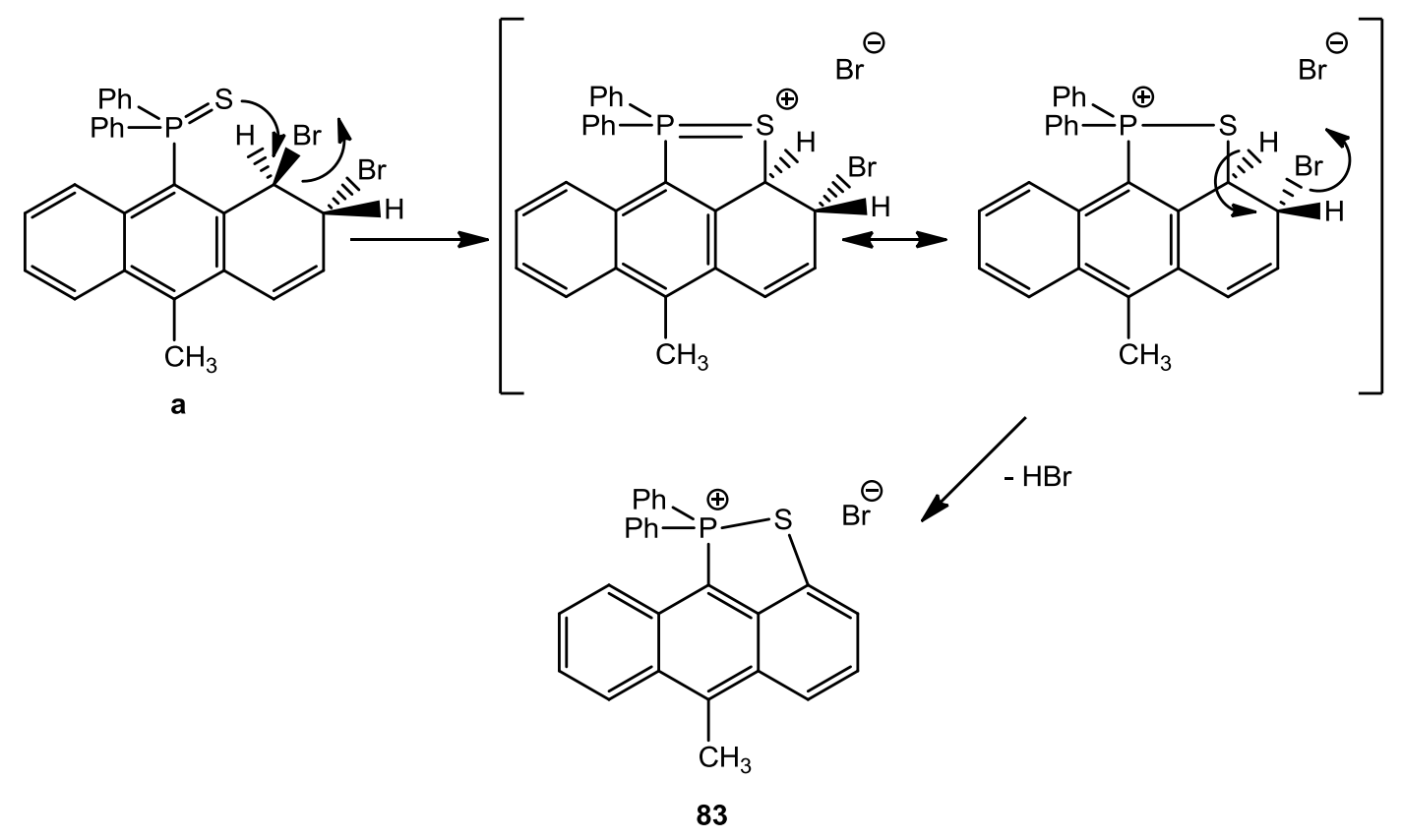

Scheme 4-35: Presumed mechanism of the cyclization reaction.

The precondition of this reaction is the addition of $\mathrm{Br}_{2}$ to the $\mathrm{C} 1=\mathrm{C} 2$ double bond in the first reaction step (a). This has been reported by Cakmak et al. ${ }^{[123]}$ and Hökelek et al., ${ }^{[124]}$ although similar additions usually occur in the 9,10-positions, while retaining the aromaticity of both peripheral rings.

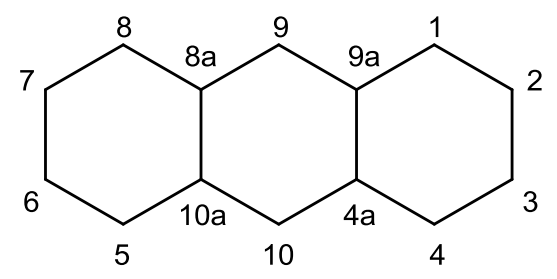

Scheme 4-36: Numbering of the anthracene moiety. 
Possibly the presence of a phosphoryl substituent in 9-position makes this type of addition reaction less favorable, or rather the 9,10 positions less reactive. Even if the addition to the $\mathrm{C} 1=\mathrm{C} 2 \pi$ bond only takes place at minimal rates, with the equilibrium shifted far towards the staring materials, the subsequent intramolecular cyclization reaction removes the addition product (a) from the equilibrium. This again forces the reaction to constantly reproduce the addition product, gradually achieving nearly quantitative turnover. Evidence for the elimination of $\mathrm{HBr}$ in the final reaction step is also given by the crystal structure. A water molecule (contained in acetone) is cocrystallized in the structure of $\mathbf{8 3}$ and an $\mathrm{HBr}$ molecule is associated to this water molecule, forming a hydronium ion which interacts with the associated bromide ion by hydrogen bonding. The fact that the $\mathrm{HBr}$ was not removed in the drying process of the crude product under reduced pressure suggests that the final elimination does not take place before the crystallization of $\mathbf{8 3}$ from acetone, possibly even depending on the presence of moisture.

The formation of this arrangement produces a five-membered cycle with a unique geometry and fairly high tension. It is one of only two five-membered $\mathrm{P}, \mathrm{S}$-heterocycles with neighboring phosphorus and sulfur atoms incorporated in an aromatic backbone. The naphthalene based structure reported by Mizuta et al. does not carry a charge in its structure in contrast to $83 .{ }^{[125]}$ Their compound was synthesized via elimination of a palladium catalyst. The few other P,S-heterocycles which do carry a charge are all aliphatic. ${ }^{[126]}$ The P-S bond in the structure of 83 measures $2.071 \AA$, which is by $0.12 \AA$ longer than the average terminal $\mathrm{P}=\mathrm{S}$ bond. The P-S bond in the structure of Mizuta's naphthalene derivative on the other hand is longer and measures $2.148 \AA$, which can be assigned to the weaker electrostatic attraction between $P$ and $S$ in absence of a positive charge at the phosphorus atom. The P-C and P-S bond lengths are nearly identical to the ones found in $\mathbf{8 3}$. The five-membered cycle is not perfectly planar. While the sulfur atom is located almost exactly in the anthracene plane (as indicated by the C4a-C9a-C1-S1 torsion angle of only $2.1^{\circ}$ ), P1 is slightly displaced from the anthracene plane, producing a C8-C8a-C9-P1 angle of 9.7 $7^{\circ}$ The P1-S1-C1 fragment is nearly right angled at $93.03^{\circ}$, and also the C9-P1-S1 angle is fairly small at only $98.13^{\circ}$. These angles reflect the high tension within this ring, which is mainly induced by the rigid aromatic backbone of the compound. The C1-C9a-C9 angle measures $119.4^{\circ}$, which is very close to the ideal $\mathrm{sp}^{2}$ angle. The corresponding angle in the opposite (un- 
substituted) anthracene ring measures $123.8^{\circ}$, which shows that the formation of the cycle does influence the geometry of the aromatic backbone, but not drastically.

The presence of a positive charge leads to an anti-aromatic character of the fivemembered cycle. Two electrons are supplied by one lone pair of the sulfur atom and two additional electrons are contributed by $\pi$ bonding within the anthracene backbone. Hence, the Hückel's rule of $(4 n+2) \pi$ electrons cannot be fulfilled. ${ }^{[127]}$

The cyclization also prevents rotation of the phosphoryl substituent. The resulting arrangement with the P-S bond located in the angle bisector of both phenyl substituents leads to a very even distribution of the steric strain of the substituent. In turn, this results in a distinctly weaker deformation of the fluorophore than observed for the starting material $\mathrm{MeAnPSPh}_{2}(\mathbf{1 4})$ (folding angle $=16.8^{\circ}$ ). Here both phenyl

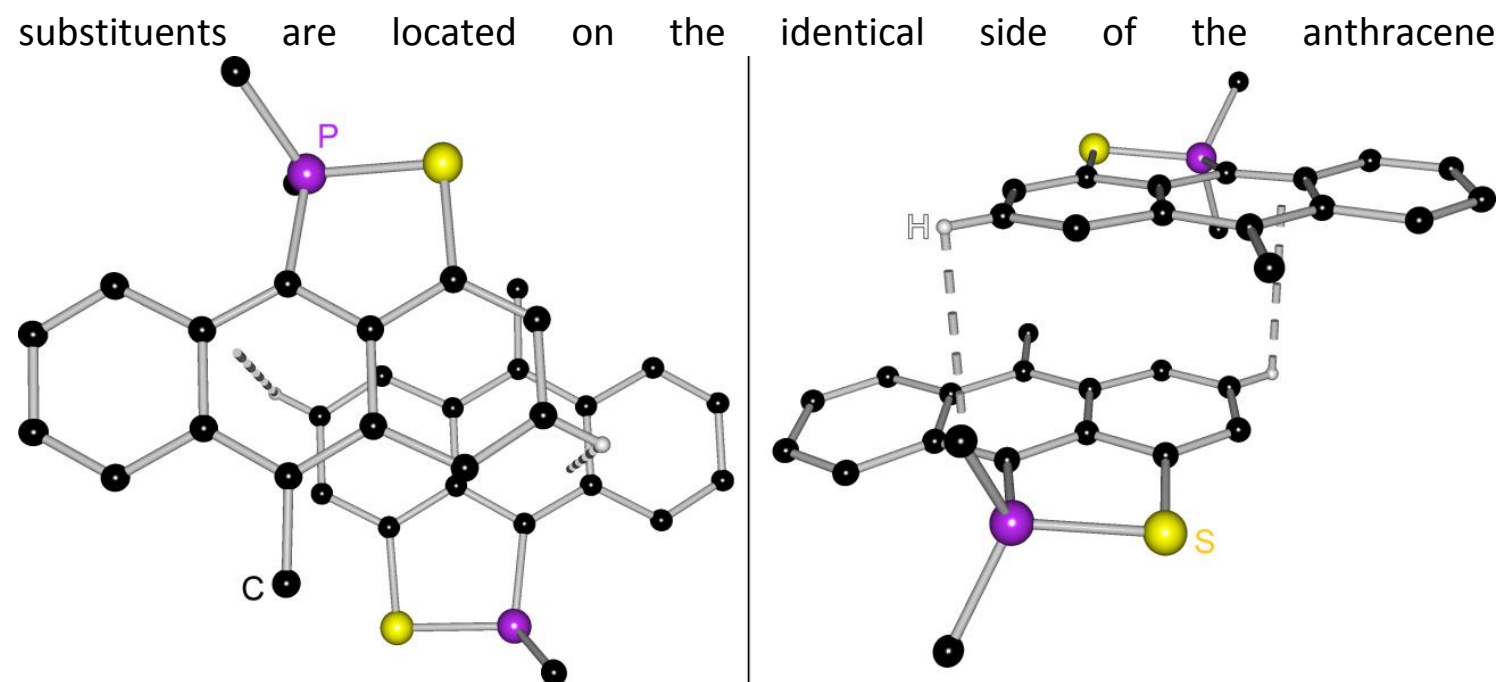

Figure 4-40: Intermolecular interaction in the structure of $\mathbf{8 3}$, bromide ions, phenyl moieties and hydrogen atoms are omitted for clarity.

plane, producing an unbalanced steric strain. The molecules of $\mathbf{8 3}$ assume a "head-totail" arrangement with both phosphoryl substituents directed in opposite directions in the solid state. This arrangement generates a $\pi-\pi$ overlap of $\sim 25 \%$ at a distance of $3.45 \AA$ (Figure 4-40). By the observed offset face-to-face $\pi$ stacking, the aromatic hydrogen atoms are located above the $\pi$ system of the adjacent anthracene moiety.

A sufficient amount of highly pure crystalline material was obtained to conduct solid state fluorescence experiments. 

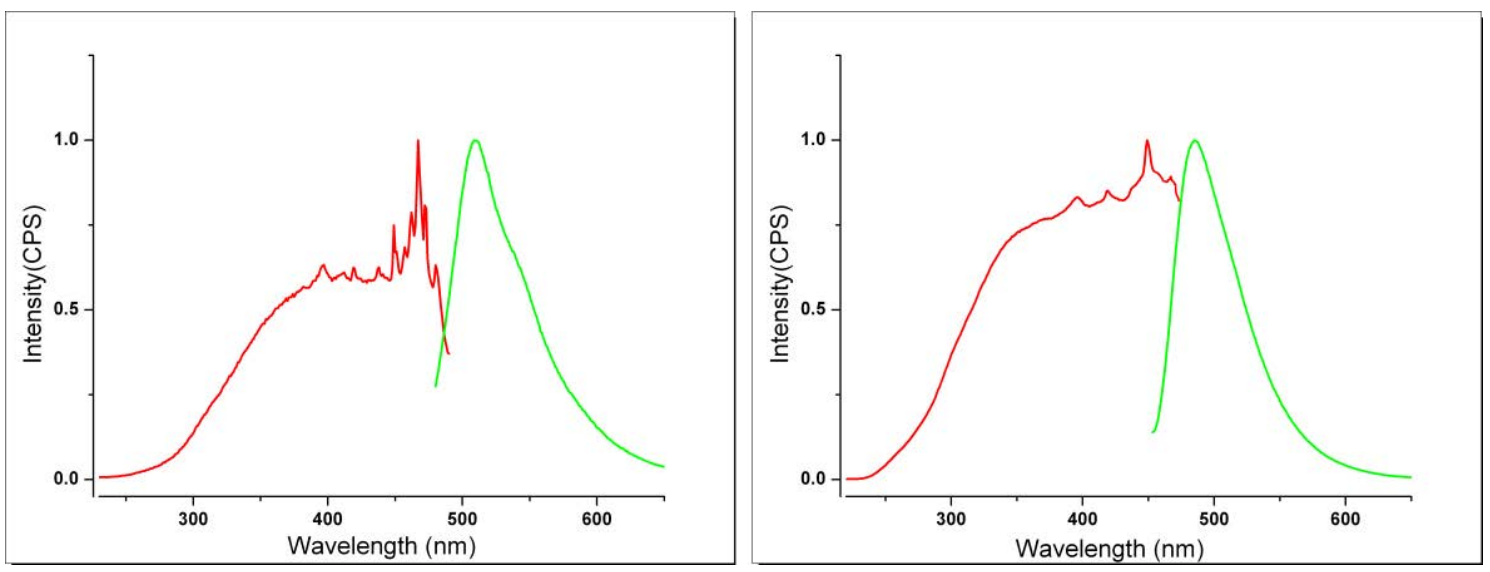

Figure 4-41: Left: normalized solid excitation (red) and emission (green) spectra of 83; right: normalized solid state excitation (red) and emission (green) spectra of $\mathrm{MeAnPSPh}_{2}$ (14).

At first sight, the excitation and emission spectra of $\mathbf{8 3}$ show strong resemblance of those of the non-cyclic precursor compound $\mathrm{MeAnPSPh}_{2}$ (14) (Figure 4-41). The spectral range of possible excitation wavelengths is quite similar, but the excitation maximum is sharper and more accentuated in the case of $\mathbf{8 3}$. Both emission spectra show a similarly broad maximum which lacks a vibrational band structure.
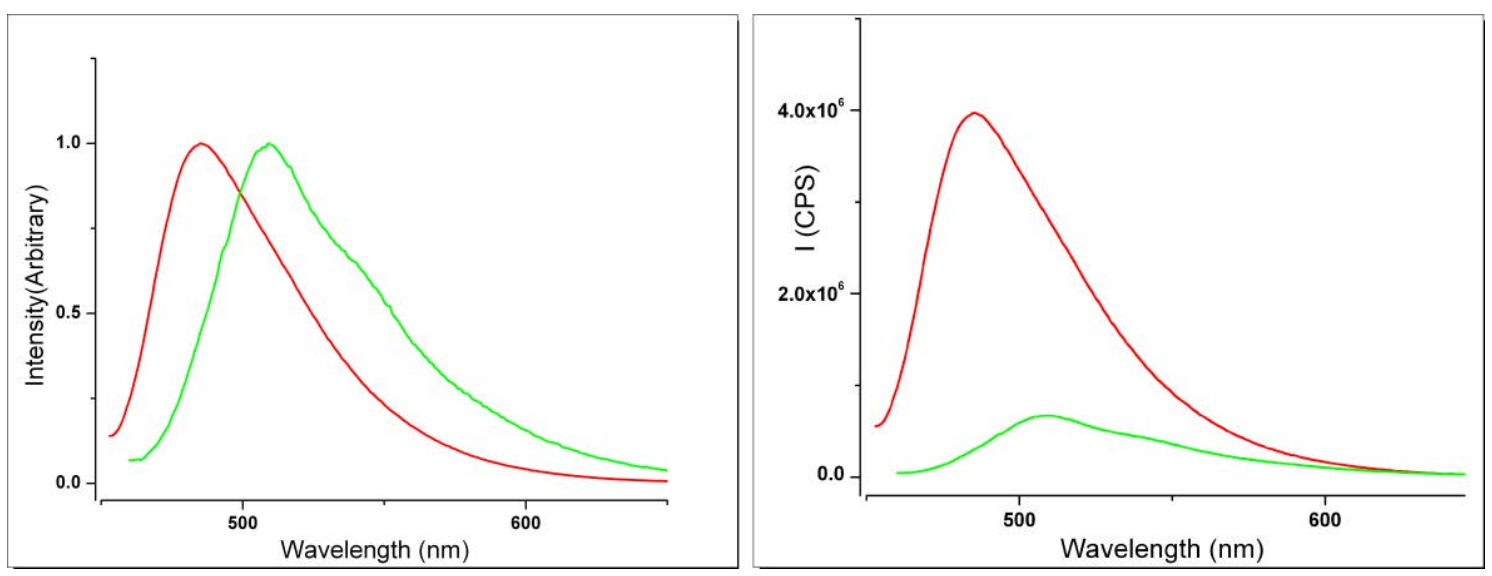

Figure 4-42: Left: normalized solid state emission spectra of $\mathrm{MeAnPSPh}_{2}$ (14) (red) and 83 (green); right: solid state emission spectra of $\mathrm{MeAnPSPh}_{2}$ (14) (red) and 83 (green), $\lambda_{\mathrm{Ex}}=449 \mathrm{~nm}$ in both cases.

When taking a closer look at the emission bands of $\mathbf{8 3}$ and 14, distinct differences become apparent. The emission maximum of $\mathbf{8 3}$ is red-shifted by $25 \mathrm{~nm}$ compared to 14 (Figure 4-42, left), which indicates a smaller HOMO-LUMO gap. This consequently also produces a stronger separation of excitation and emission maximum, as both compounds exhibit identical excitation maxima at $449 \mathrm{~nm}$. The most prominent difference however lies in the observed emission intensities. While $\mathbf{1 4}$ is fairly strongly 
fluorescent, 83 shows only weak emission (Figure 4-42, right), resulting in approximately fivefold emission intensity of $\mathbf{1 4}$ compared to $\mathbf{8 3 .}$

Taking into account the similar intermolecular interactions (both show moderate $\pi-\pi$ overlap) within the packing motifs of both compounds, as well as the clearly stronger deformation of the fluorophore in $\mathbf{1 4}$, this result is unexpected. The structural properties of both compounds would in fact suggest exactly opposite behavior. Hence, the weak solid state emission of $\mathbf{8 3}$ must be assigned to the anti-aromatic cycle present in its structure. While inducing balanced steric strain and therewith weak deformation on the one hand, the cycle also generates a positive charge on the other hand. This charge at the phosphorus atom strongly increases the electron withdrawing effect of the substituent on the $\pi$ system of the fluorophore. This apparently not only leads to a lowering of the HOMO/LUMO gap but also hinders fluorescence emission of 83.

\subsubsection{Synthesis from the Amine}

The second possible synthetic approach is based on introduction of the receptor unit in the first step of synthesis followed by lithiation and introduction of the phosphane in the second step (Scheme 4-37).

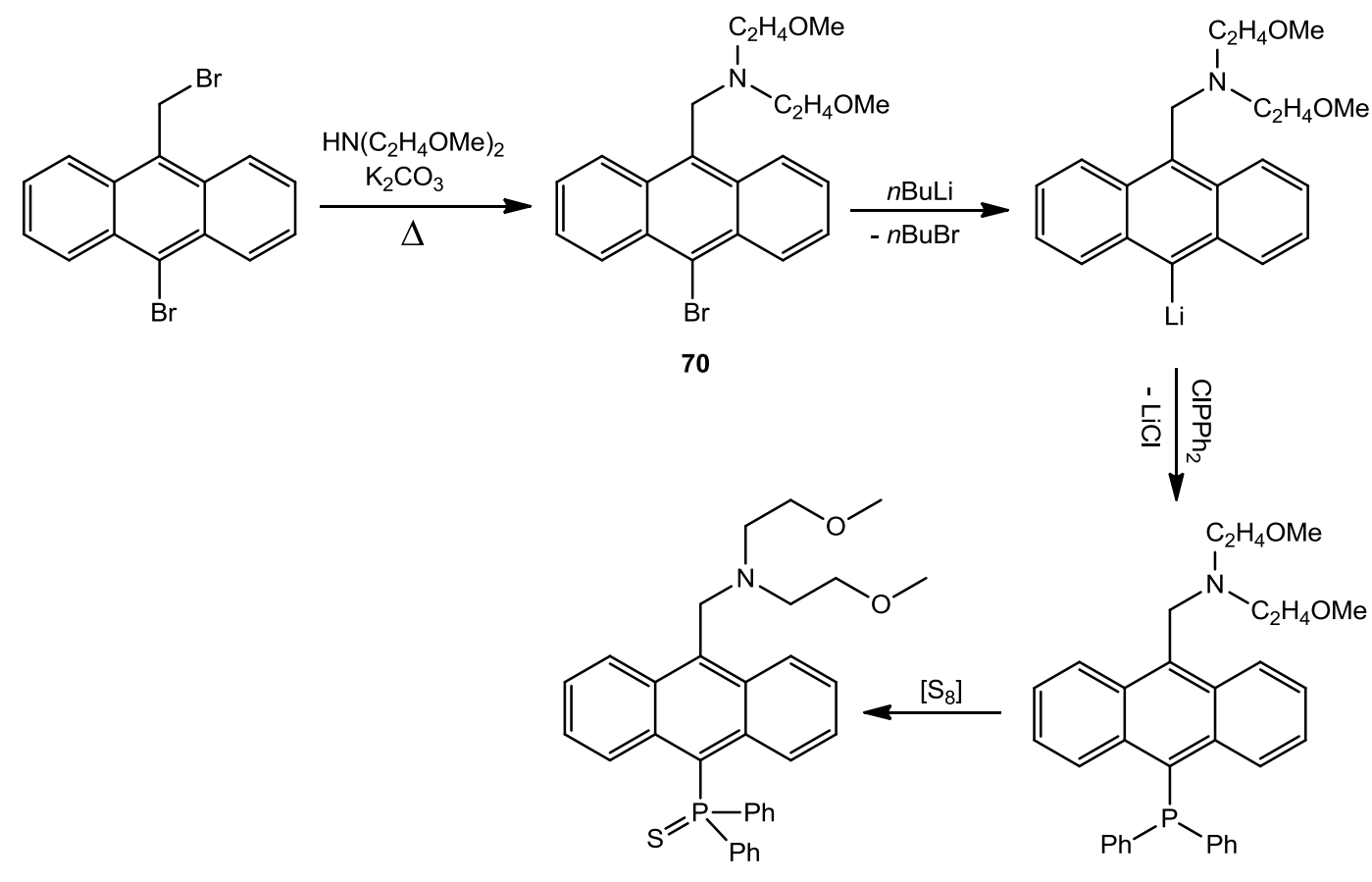

84

Scheme 4-37: Synthesis of $\mathrm{Ph}_{2} \mathrm{SPAnCH}_{2} \mathrm{~N}\left(\mathrm{C}_{2} \mathrm{H}_{4} \mathrm{OMe}\right)_{2}$ (84). 
$\mathrm{BrAnCH}_{2} \mathrm{~N}\left(\mathrm{C}_{2} \mathrm{H}_{4} \mathrm{OMe}\right)_{2}(70)$ was dissolved in diethyl ether and cooled to $-15^{\circ} \mathrm{C}$. Then one equivalent of $n$-BuLi was added over the course of $30 \mathrm{~min}$. After stirring at $0^{\circ} \mathrm{C}$ for $5 \mathrm{~min}$., the lithiated intermediate was again cooled to $-15^{\circ} \mathrm{C}$ and reacted with one equivalent of diphenylchlorophosphane. After $30 \mathrm{~min}$ the cooling bath was removed and the reaction was stirred overnight at ambient temperature. The solvent was evaporated, the crude product was re-dissolved in DCM and filtrated for removal of lithium chloride. Then the solvent was again evaporated and the crude product was dried in vacuo. $\mathrm{A}^{1} \mathrm{H}$ NMR spectrum indicated that the crude product contained noteworthy contaminations from byproducts. Due to the sensitivity of the phosphanyl substituent towards oxidation, purification was postponed and instead the phosphane was selectively converted to a stable phosphorus(V) derivative by direct oxidation with elemental sulfur. For this purpose, the crude product was dissolved in toluene and reacted with 1.1 equivalents (relative to the amount of previously used phosphane) of elemental sulfur. The reaction was heated to $110^{\circ} \mathrm{C}$ for $6 \mathrm{~h}$. After cooling to room temperature, the solution was filtrated over celite and the solvent was removed under reduced pressure. Purification by column chromatography (pentane/ethyl acetate 3:1) afforded $\mathrm{Ph}_{2} \mathrm{SPAnCH}_{2} \mathrm{~N}\left(\mathrm{C}_{2} \mathrm{H}_{4} \mathrm{OMe}\right)_{2}$ (84) as a yellow oil. Crystallization of 84 was not successful due to its oily texture; crystallization attempts from aqueous solvent mixtures to which hydrochloric acid was added (with the intention of crystallizing the hydrochloride of $\mathbf{8 4}$ ) led to gradual decomposition of the compound.

Due to the high purity of the obtained product the in-solution fluorescence properties of 84 could be investigated. Solutions of 84 in DCM and $\mathrm{MeOH}$ were prepared and subjected to fluorescence experiments.
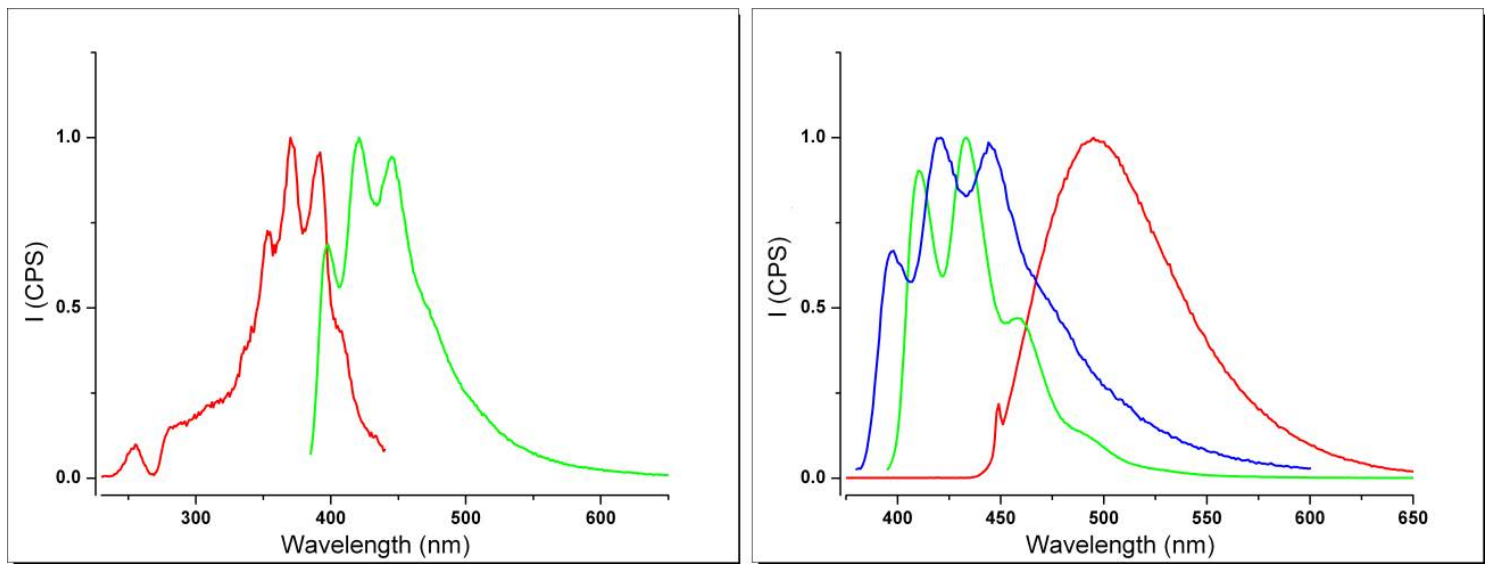

Figure 4-43: Left: normalized excitation (red) and emission (green) spectra of $84\left(5 \cdot 10^{-5} \mathrm{M}\right.$ in DCM); right: normalized emission spectra of $\mathrm{MeAnPSPh}_{2}(\mathbf{1 4}, \mathrm{red}), \mathrm{BrAnCH}_{2} \mathrm{~N}\left(\mathrm{C}_{2} \mathrm{GH}_{4} \mathrm{OMe}\right)_{2}$ (70, green), and $\mathrm{Ph}_{2} \mathrm{SPAnCH}{ }_{2} \mathrm{~N}\left(\mathrm{C}_{2} \mathrm{H}_{4} \mathrm{OMe}\right)_{2}\left(84\right.$, blue), all $5 \cdot 10^{-5} \mathrm{M}$ in DCM. 
Despite the presence of the phosphoryl substituent in 9-position of $\mathrm{Ph}_{2} \mathrm{SPAnCH}_{2} \mathrm{~N}\left(\mathrm{C}_{2} \mathrm{H}_{4} \mathrm{OMe}\right)_{2}(84)$, both the excitation and emission spectra of 84 show strong similarities to those of $\mathrm{BrAnCH}_{2} \mathrm{~N}\left(\mathrm{C}_{2} \mathrm{H}_{4} \mathrm{OMe}\right)_{2}(70)$ and other regular anthracene derivatives containing spacers (c.f. 4.2). The excitation spectrum shows a rather narrow range of activity and both the excitation- and the emission spectrum exhibit a vibrational band structure (Figure 4-43, left). Also the position of the emission maximum is not red-shifted in the expected manner by the phosphoryl substituent. Comparison with the emission spectrum of $\mathrm{MeAnPSPh}_{2}$ (14) clearly underlines this fact (Figure 4-43, right), as the emission maxima of 14 and 84 differ by over $60 \mathrm{~nm}$. Also the differences in shape are striking because $\mathbf{1 4}$ exhibits no band structure of the emission spectrum whatsoever, while it is definitive in the spectrum of $\mathbf{8 4}$. For the time being, this implies that the intended emission shift by introduction of a phosphoryl substituent while preserving the PET mechanism was not successful.
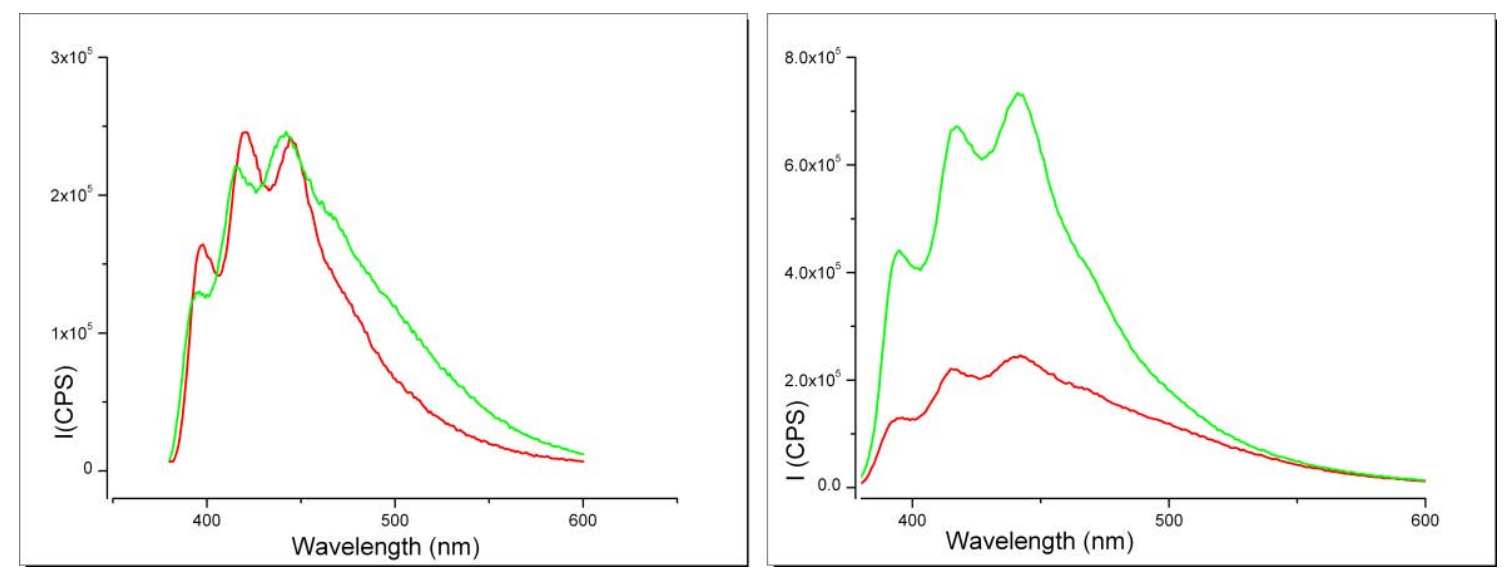

Figure 4-44: Left: emission spectra of $84,5 \cdot 10^{-5} \mathrm{M}$ in DCM (red) and $5 \cdot 10^{-5} \mathrm{M}$ in $\mathrm{MeOH}$ (green); right: emission spectra of $84\left(5 \cdot 10^{-5} \mathrm{M}\right.$ in $\left.\mathrm{MeOH}\right)$ at $\mathrm{pH}=7$ (red) and $\mathrm{pH}=2$ (green).

Nevertheless it was attempted to verify the functioning of the electron transfer mechanism in presence of the phosphoryl substituent. For this purpose a $\mathrm{MeOH}$ solution of $\mathbf{8 4}$ was used for a protonation experiment to ensure good mixing of the employed hydrochloric acid with the solvent. As depicted in Figure 4-44 (left), a change of solvent from DCM to $\mathrm{MeOH}$ also did not influence the emission properties of $\mathbf{8 4}$. Upon addition of aqueous $\mathrm{HCl}$, (to a $\mathrm{pH}$ value of ca. 2), an increase of emission intensity was observed (Figure 4-44, right). Although an increase by a factor of three is clearly detectable, it is weaker than the corresponding increase observed for the precursor compound $\mathrm{BrAnCH}_{2} \mathrm{~N}\left(\mathrm{C}_{2} \mathrm{H}_{4} \mathrm{OMe}\right)_{2}$ (70) and other amines. The overall fluorescence of $\mathrm{Ph}_{2} \mathrm{SPAnCH}_{2} \mathrm{~N}\left(\mathrm{C}_{2} \mathrm{H}_{4} \mathrm{OMe}\right)_{2}$ (84) is also weaker than of 70, which 
suggests that the phosphoryl substituent may promote decay via non radiative pathways. The smaller on/off ratio achieved by protonation indicated that either the electron transfer of the un-protonated compound is less effective than e.g. in $\mathbf{7 0}$, or that a second process contributes to quenching which is not affected by the protonation.

To recruit a second level of comparison between 84 and 70, the titration with $\mathrm{ZnBr}_{2}$ solution was conducted, which had led to a similarly strong increase of emission as protonation for $\mathbf{7 0}$. The obtained results were more than astonishing.
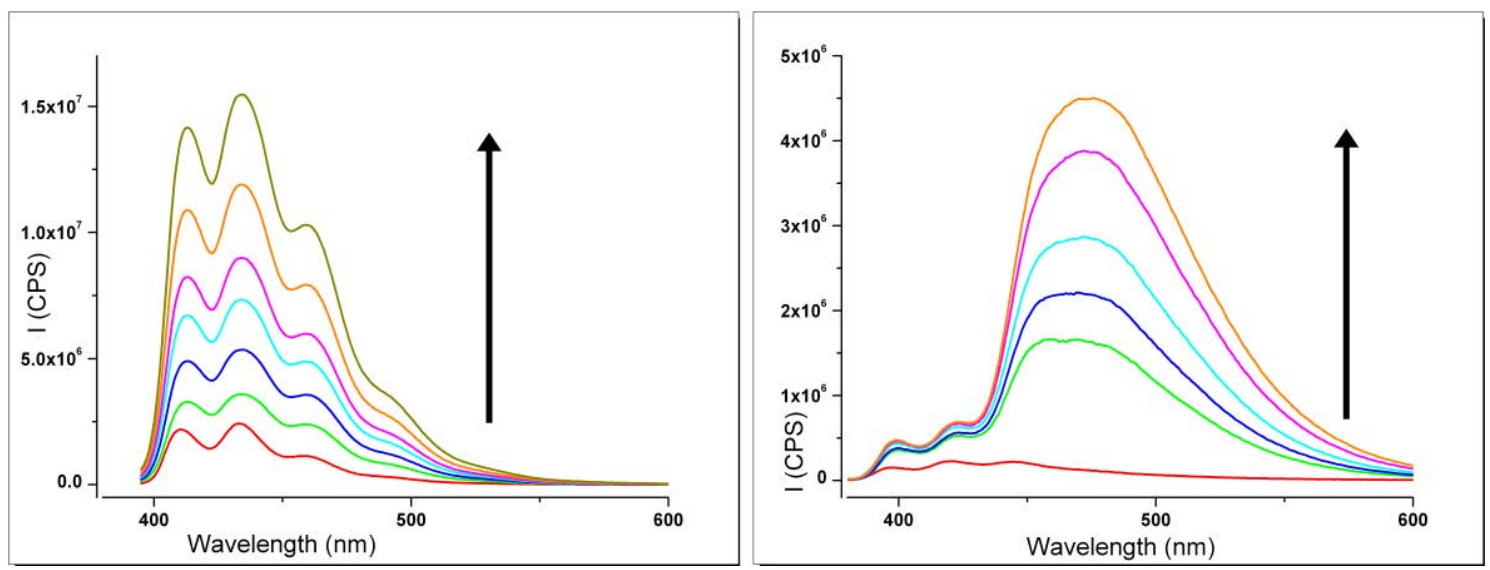

Figure 4-45: Left: titration of $\mathrm{BrAnCH}_{2} \mathrm{~N}\left(\mathrm{C}_{2} \mathrm{H}_{4} \mathrm{OMe}\right)_{2}(70)\left(5 \cdot 10^{-5} \mathrm{M}\right.$ in DCM) with $\mathrm{ZnBr}_{2}$, with $\mathrm{c}\left(\mathrm{Zn}^{2+}\right)=0$

(red), and increasing $\mathrm{Zn}^{2+}$ concentration in the direction of the arrow. Right: titration of $\mathrm{Ph}_{2} \mathrm{SPAnCH}_{2} \mathrm{~N}\left(\mathrm{C}_{2} \mathrm{H}_{4} \mathrm{OMe}\right)_{2}(84)\left(5 \cdot 10^{-5} \mathrm{M}\right.$ in DCM) with $\mathrm{ZnBr}_{2}$, with $\mathrm{c}\left(\mathrm{Zn}^{2+}\right)=0$ (red), and increasing $\mathrm{Zn}^{2+}$ concentration in the direction of the arrow.

While for $\mathbf{7 0}$, the addition of $\mathrm{Zn}^{2+}$ had merely triggered an increase of emission intensity (Figure 4-45, left), a completely different secondary effect is observed for $\mathbf{8 4}$. Though there is also a clearly visible increase of emission in the spectral range of the free ligand (in the range of the band structure), a completely new emission band is generated in a distinctly red-shifted region of the spectrum (Figure 4-45, right). This second maximum is broad and exhibits no band structure and bears strong resemblance of the emission spectrum of $\mathrm{MeAnPSPh}_{2}$ (14). It exhibits a red-shift of $55 \mathrm{~nm}$ compared to the initial emission maximum. Because in this spectral region emission was nearly completely absent for the free ligand $\mathbf{8 4}$, an on/off ratio of over 39:1 is achieved at an emission wavelength of $475 \mathrm{~nm}$. These results show that the $\mathrm{Zn}^{2+}$ ions must not only interact with receptor moiety of $\mathbf{8 4}$, but that a second interaction must be present which becomes apparent in the formation of a second emission band. Both shape and bathochromic shift of the newly generated emission 
band are characteristic of exciplex emission. ${ }^{[7 d]}$ Apperently, by addition of $\mathrm{Zn}^{2+}$ ions, the formation of a strongly emitting exciplex was promoted, which was not produced by protonation. Results like this are exceedingly rare in the context of metal ion detection.

The initially supposed ineffectiveness of the phosphoryl substituent has been impressively disproved by coordination of $\mathrm{Zn}^{2+}$ ions. The influence of the phosphoryl substituent on the shape and wavelength of the emission spectrum upon addition of zinc ions is undeniable, taking into account the numerous emission spectra of phosphorylanthracenes discussed in the course of the thesis. The challenges of discovering the exact coordination mode of $\mathbf{8 4}$ to $\mathrm{Zn}^{2+}$ and therewith the composition of the exciplex could be met by crystallization of the complex and then drawing conclusions from the solid state structure. Furthermore, also mass spectrometry experiments could give insight on the size and composition of the aggregates in solution.

\section{SUMMARY AND OUTLOOK}

In retrospect, a wide range of compounds for diverse applications has been prepared and investigated in the course of this thesis. Thematically these compounds were all linked by the interest in the structure-materials properties relationship. Can fluorescence changes be routed in structurals changes and vice versa? Will different anthracene substitution, various host/guest coordination and metal coordination alter and emphazise or diminish fluorescence?

For the first time, structural properties like fluorophore deformation and $\mathrm{C}-\mathrm{H} \cdots \pi$ bonding of phosphorylanthracenes were quantified and correlated to the corresponding solid state fluorescence properties of the respective compounds. The solid state fluorescence reducing effect of folding the anthracene moiety along the central $C_{6}$-perimeter could be identified unambiguously, while putting some views on the effect of $\pi-\pi$ interaction, which was for long accepted to be true, into perspective. Additionally, the effects of metal coordination on the solid state structures and fluorescence properties of phosphorylanthracenes were monitored. By thorough 
comparison of all these compounds and their sometimes marginal structural changes, their severe influence on the fluorescence properties was pin-pointed.

Furthermore, protocols in the syntheses of chelating phosphorylanthracenes were improved considerably by preparation of versatile precursors, and different synthetic pathways which employ these precursors were highlighted. In addition, the route to the target class of boranylanthracenes was opened. They exhibited remarkable fluorescence properties and possess high potential in future syntheses of fluorescent sensing devices.

Throughout the investigation of solid state fluorescence properties, virtually all strongly fluorescent compounds were shown to exhibit strong $\mathrm{C}-\mathrm{H}^{\cdots} \pi$ bonding within their solid state structures. Especially the formation of T-shaped molecular arrangements was a common feature of all strongly emitting derivatives. In many cases the observed emission intensities could only be explained by including $\mathrm{C}-\mathrm{H}$ "' $\pi$ bonding into the rational argumentation. Therefore the acquired results should contribute to a higher acknowledgement of the emission enhancing effect of weak C$H^{\prime \prime} \pi$ interactions in future evaluations of solid state fluorescence.

Substantial advances were made in the field of in-solution fluorescence. The notable shifting effect of metal coordination on the emission wavelengths of phosphorylanthracenes was shown, which could be applied e.g. in colorimetric sensing devices. Syntheses of amine based sensor molecules according to the receptor-spacerfluorophore principle enabled investigation of the PET mechanism. Among these compounds the strong sensitivity of $\mathrm{HAnCH}_{2} \mathrm{~N}\left(\mathrm{CH}_{2} \mathrm{PhOMe}\right)_{2}$ (75) towards water was discovered which fulfils all prerequisites of a potent water sensor. The transfer of the PET mechanism to phosphanes in a quencher-spacer-fluorophore setup was also actualized and verified by oxidation, which generated noteworthy emission enhancement. Phosphane based sensors could open new perspectives in fluorescent detection of particularly soft cations which exceed the limitations of amine based sensors.

Moreover, the achieved deprotonation of 9-methylanthracene under mild conditions provides a new synthetic route to sensor molecules according to the receptor-spacer-fluorophore principle. 
The successful unification of the PET sensor concept with emission shifting effects by introduction of phosphoryl substituents to the fluorophore has yielded a compound which not only produced large on/off ratios in cation detection, but also exhibited remarkable exciplex emission upon addition of zinc(II) cations. The observed red-shift of emission in addition to the general strong enhancement of emission could be a breakthrough in chemical sensing. This new compound class could revolutionize detection of bound targets by a combining the benefits of both colorimetric and intensity dependent sensing.

In the future, besides further screening for sensitivities of the synthesized sensor molecules towards cationic analytes, two leading concepts appear particularly worth to follow. Firstly, the utilization of phosphane quenchers for the syntheses of chelating receptor units bears great potential. The utilization of the verified PET mechanism in a receptor-spacer-fluorophore setup could enable the chemical recognition of previously undetectable soft metal cations. The application of phosphanes in P-N-P receptor units is also worth mentioning in this context. Secondly, the combination of anthracene bound phosphoryl substituents and the classic PET sensor concept needs to be further investigated. The combination of established quencher/receptor moieties which show highly reliable sensitivity and selectivity towards a certain cation could be combined with different phosphoryl substituents. The emission shifting effect of these substituents could be systematically monitored this way and possibly subsequently applied in chemical sensing devices.

Another future option is to crystallize complexes of sensor molecules coordinating metal cations. Although this had also been attempted in the course of this work, no adaptable complexes were obtained yet. Because to date only metal halides were used in the attempted crystallization of sensor/metal complexes, the utilization of bulkier or larger counter ions like perchlorate or oxalate could be constructive in this context, as these anions have been shown to be beneficial in crystallization. Acquisition of the crystal structures would make tangible information on the strength of receptor/analyte interactions accessible through bond distances and geometrical parameters. These could then again be aligned with the observed emission intensities of sensor and analyte. The combination of both methods could provide key information for tailoring receptor units for selected cations with regard to coordination geometries which provide optimum interaction between analyte and quencher. 
Furthermore the synthesis of highly Lewis acidic boranylanthracenes should be embarked. Verification of the sensor concept of emission shifting by Lewis acid/base adduct formation would open up a wide field of applications of these strongly fluorescent compounds. Not only in-solution detection of neutrally charged molecules would become accessible, also the crystallization of adducts would enable investigation of sensor/analyte interactions in the solid state. Additionally, numerous intercalation structures with different Lewis bases, which may reveal more details on the interconnection of the structural properties and solid state fluorescence would be feasible.

Finally, because the intercalation of solvent molecules in the various structures of SPAnPS has provided impressive insight in and modification of fluorescence phenomena, the synthesis of further intercalation structures should also be followed up. Especially the material science aspect of intercalation structures blends well with the crystallographic approach towards fluorescent compounds taken in this thesis. A possible strategy could be a continuation and expansion of the work of Mizobe et al. on 2,6- and 1,8-athracenedisulfonic acid. ${ }^{[49-53]}$ By alteration of the substituted anthracene positions as well as introduction of phoshite or phosphate substituents instead of sulfonates, and by utilization of varying amines, novel ionic network structures could be synthesized for the intercalation of heterogeneous guest molecules.

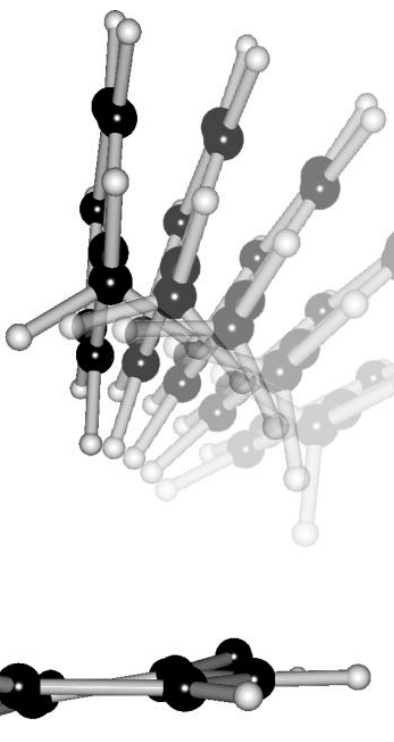

Figure 5-1: Variation of the $\mathrm{C}-\mathrm{H}^{\cdots} \pi$ bonding angle between toluene and the anthracene moiety (side view). 
The effects of interaction between host complexes and guest molecules could also be evaluated using theoretical methods. Especially comparison of SPAnPS@tol (15) and its toluene $d_{8}$ analogue 20 on the basis of theoretical calculations could supplement the experimental data presented in this thesis. The effects of fluorophore deformation and $\mathrm{C}-\mathrm{H}^{*} \pi$ bonding on solid state fluorescence could be simulated by calculations, e.g. by comparison of fluorophore conformations of gradually increasing folding angle or by variation of the $\mathrm{C}-\mathrm{H} \cdots \pi$ bonding angle between intercalated solvent molecules and the $\pi$ system of the fluorophore (Figure 5-1). 


\section{EXPERIMENTAL SECTION}

\subsection{General Procedures}

All manipulations were carried out under dry argon inert gas atmosphere by using modified Schlenk techniques ${ }^{[128]}$ or in an argon glove box. Solvents were freshly distilled from sodium, potassium or sodium potassium alloy prior to use. The employed reactants were commercially available or synthesized according to literature procedures: bis(dimethylamino)chlorophosphane (CIP(NMe $\left.)_{2}\right)$, bis(diethylamino)chlorophosphanes $\left(\mathrm{CIP}\left(\mathrm{NEt}_{2}\right)_{2}\right){ }^{[68]}$ 9-bromo-10-(bromomethyl)anthracene ( $\left.\mathrm{BrAnCH}_{2} \mathrm{Br}\right)$, 9-(bromomethyl)anthracene $\quad\left(\mathrm{HAnCH}_{2} \mathrm{Br}\right), \quad$ 9,10-bis(bromomethyl)anthracene $\left(\mathrm{BrCH}_{2} \mathrm{AnCH}{ }_{2} \mathrm{Br}\right){ }^{[59 \mathrm{c}]}$

\subsection{Spectroscopic and Analytic Methods}

NMR spectra were recorded on a Bruker Avance $500 \mathrm{MHz}$ or $300 \mathrm{MHz}$ spectrometer. Chemical shifts $\delta$ are given in ppm and were calibrated either to solvent signal $\left(\mathrm{C}_{6} \mathrm{D}_{6}: 7.15\left({ }^{1} \mathrm{H}\right), 128.0\left({ }^{13} \mathrm{C}\right)\right.$, THF- $d_{8}: 1.73\left({ }^{1} \mathrm{H}\right), 25.3\left({ }^{13} \mathrm{C}\right)$, DMSO-d $\mathrm{d}_{6}: 2.50\left({ }^{1} \mathrm{H}\right)$, $\left.39.52\left({ }^{13} \mathrm{C}\right), \mathrm{CD}_{2} \mathrm{Cl}_{2}: 5.32\left({ }^{1} \mathrm{H}\right), 53.84\left({ }^{13} \mathrm{C}\right)\right)$ or to the unified $\Xi$-scale $\Xi\left({ }^{15} \mathrm{~N}\right.$, nitromethane $)=0.10136767, \Xi\left({ }^{29} \mathrm{Si}, \mathrm{TMS}\right)=0.19867187, \Xi\left({ }^{31} \mathrm{P}\right.$, phosphoric acid $)=$ $\left.\left.0.40480742, \Xi\left({ }^{119} \mathrm{Sn}, \mathrm{Me}_{4} \mathrm{Sn}\right)=0.37290632\right), \Xi\left({ }^{77} \mathrm{Se}, \mathrm{Me}_{2} \mathrm{Se}\right)=0.19071513\right), \Xi\left({ }^{7} \mathrm{Li}\right.$, $\mathrm{LiCl})=0.38863797) .{ }^{[129]}$ The obtained chemical shifts were assigned according to Scheme 6-1.

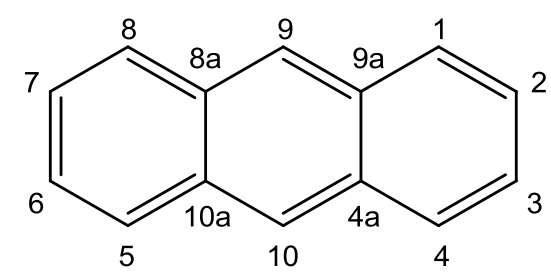

Scheme 6-1. Labeling scheme of NMR signals. 


\subsection{Elemental Analyses}

Elemental analyses were carried out by the Analytische Labor des Instituts für Anorganische Chemie der Georg-August-Universität Göttingen with an Elementar Vario EL3. Some of the determined values of the air and moisture sensitive compounds deviate more than $1.0 \%$ from the calculated ones. This can be explained by the instability of the compounds when handling outside of a Schlenk flask or glove box, the loss of solvent molecules during the drying of the sample in vacuum or the inclusion of argon from canning the samples in an argon glove box.

\subsection{Synthesis and Characterization}

\subsubsection{Synthesis of $\mathrm{HAnP}^{i} \mathrm{Pr}_{2}(1)$}

9-Bromoanthracene $(7,9 \mathrm{~g}, 30,8 \mathrm{mmol})$ was dissolved in diethyl ether $(80 \mathrm{~mL})$ and reacted with $n$-BuLi $(11,2 \mathrm{~mL}(2,75 \mathrm{M}), 30,8 \mathrm{mmol})$ at $-15^{\circ} \mathrm{C}$ over the course of $30 \mathrm{~min}$. The solution was warmed to $10^{\circ} \mathrm{C}$ and stirred for $10 \mathrm{~min}$, then cooled to $-15^{\circ} \mathrm{C}$ and chlorodiisopropylphosphane $(4.70 \mathrm{~g}, 30,8 \mathrm{mmol})$ was added over $30 \mathrm{~min}$. The resulting mixture was stirred for $2 \mathrm{~h}$, then the solvent was evaporated and the crude product was dissolved in DCM $(40 \mathrm{~mL})$. Lithium chloride was removed by filtration. Evaporation of the solvent afforded $\mathrm{HAnP}^{i} \mathrm{Pr}_{2}(\mathbf{1})$ as a yellow solid.

Yield: $6.1 \mathrm{~g}(20.6 \mathrm{mmol}, 67 \%)$

Empirical formula: $\mathrm{C}_{20} \mathrm{H}_{23} \mathrm{P}$

Molar mass $=294.37 \mathrm{~g} / \mathrm{mol}$

${ }^{1} \mathrm{H}-\mathrm{NMR}$

$\left(\mathrm{CDCl}_{3}, 300 \mathrm{MHz}\right)$ :

$\delta[\mathrm{ppm}]=9.20\left(\mathrm{~s}, 2 \mathrm{H}, \mathrm{H}_{1,8}\right), 8.50\left(\mathrm{~s}, 1 \mathrm{H}, \mathrm{H}_{10}\right), 8.00(\mathrm{~d}$, $\left.{ }^{3} J_{H H}=8.6 \mathrm{~Hz}, 2 \mathrm{H}, \mathrm{H}_{4,5}\right), 7.53\left(\mathrm{~m}, 4 \mathrm{H}, \mathrm{H}_{2,7}, \mathrm{H}_{3,6}\right), 2.91(\mathrm{~m}, 2 \mathrm{H}, 2 \mathrm{x}$

$\left.\mathrm{CH}\left(\mathrm{CH}_{3}\right)_{2}\right), 1.50\left(\mathrm{~d},{ }^{3} \mathrm{~J}_{\mathrm{HH}}=6.5 \mathrm{~Hz}, 3 \mathrm{H},{ }^{i} \mathrm{Pr}-\mathrm{CH}_{3}\right), 1.41(\mathrm{~d}$, $\left.{ }^{3} J_{\mathrm{HH}}=6.5 \mathrm{~Hz}, 3 \mathrm{H},{ }^{i} \mathrm{Pr}-\mathrm{CH}_{3}\right), 0.69\left(\mathrm{~m}, 6 \mathrm{H}, 2 \mathrm{x}^{i} \mathrm{Pr}-\mathrm{H}_{3}\right)$.

${ }^{31} \mathrm{P}\left\{{ }^{1} \mathrm{H}\right\}-\mathrm{NMR}$

$\left(\mathrm{CDCl}_{3}, 121 \mathrm{MHz}\right): \quad \delta[\mathrm{ppm}]=0.10(\mathrm{~s})$.

\section{EI-MS}

$m / z(\%):$

294 (53) [M] ${ }^{+}, 252$ (14) $\left[\mathrm{M}-{ }^{i} \mathrm{Pr}\right]^{+}, 209$ (100) $\left[\mathrm{M}^{-}{ }^{-} \mathrm{Pr}_{2}\right]^{+}, 178(22)$

$\left[\mathrm{M}-\mathrm{P}^{\mathrm{i}} \mathrm{Pr}_{2}\right]^{+}$. 


\subsubsection{Synthesis of $\mathrm{HAnPS}^{i} \mathrm{Pr}_{2}$ (2)}

HAnP'Pr ${ }^{\prime}$ (1) (1,0 g, 3,7 mmol) was dissolved in toluene $(20 \mathrm{~mL})$ and sulfur $(0,13 \mathrm{~g}, 4,1 \mathrm{mmol})$ was added. The mixture was stirred and heated to $110^{\circ} \mathrm{C}$ for $6 \mathrm{~h}$. The reaction was cooled to ambient temperature, filtrated and the solvent was evaporated. $\mathrm{HAnPS}^{i} \mathrm{Pr}_{2}$ was obtained by crystallization from toluene as a yellow solid.

Yield: $0.71 \mathrm{~g}(2.2 \mathrm{mmol}, 64 \%)$

Empirical formula: $\mathrm{C}_{20} \mathrm{H}_{23} \mathrm{PS}$

Mlar mass: $326.13 \mathrm{~g} / \mathrm{mol}$

${ }^{1}$ H-NMR

$\left(\mathrm{CDCl}_{3}, 200 \mathrm{MHz}\right)$ :

$\delta=0.83\left(\mathrm{~d},{ }^{3} J_{\mathrm{HH}}=7.00 \mathrm{~Hz}, 3 \mathrm{H}, \mathrm{CH}_{3}\right), 0.92\left(\mathrm{~d},{ }^{3} J_{\mathrm{HH}}=6.80 \mathrm{~Hz}, 3 \mathrm{H}\right.$,

$\left.\mathrm{CH}_{3}\right), 1.40\left(\mathrm{~d},{ }^{3} \mathrm{~J}_{\mathrm{HH}}=6.68 \mathrm{~Hz}, 3 \mathrm{H}, \mathrm{CH}_{3}\right), 1.50\left(\mathrm{~d},{ }^{3} \mathrm{~J}_{\mathrm{HH}}=6.80 \mathrm{~Hz}\right.$,

$\left.3 \mathrm{H}, \mathrm{CH}_{3}\right), 3.27\left(\mathrm{~m}, 2 \mathrm{H}, 2 \times \mathrm{CH}\left(\mathrm{CH}_{3}\right)_{2}\right), 7.50\left(\mathrm{~m}, 4 \mathrm{H}, \mathrm{H}_{2,7}, \mathrm{H}_{3,6}\right)$,

$7.98\left(\mathrm{~m}, 2 \mathrm{H}, \mathrm{H}_{4,5}\right), 8.55\left(\mathrm{~s}, 1 \mathrm{H}, \mathrm{H}_{10}\right), 9.38\left(\mathrm{~d},{ }^{3} \mathrm{~J}_{\mathrm{HH}}=8.38 \mathrm{~Hz}, \mathrm{H}_{1,8}\right)$.

${ }^{31} \mathrm{P}\left\{{ }^{1} \mathrm{H}\right\}-\mathrm{NMR}$

$\left(\mathrm{CDCl}_{3}, 81 \mathrm{MHz}\right): \quad \delta[\mathrm{ppm}]=71.21(\mathrm{~s})$.

EI-MS

$\mathrm{m} / \mathrm{z}(\%)$

$326(100)[\mathrm{M}]^{+}, 284(29)\left[\mathrm{M}-{ }^{i} \mathrm{Pr}\right]^{+}, 241(85)\left[\mathrm{M}^{-}{ }^{i} \mathrm{Pr}_{2}\right]^{+}, 209$ (17)

$\left[\mathrm{Ms}^{i} \mathrm{Pr}_{2}\right]^{+}, 178(68)\left[\mathrm{M}-\mathrm{PS}^{i} \mathrm{Pr}_{2}\right]^{+}$.

\subsubsection{Synthesis of HAnPSe ${ }^{i} \operatorname{Pr}_{2}$ (3)}

HAnP'Pr ${ }_{2}$ (1) $(1,0 \mathrm{~g}, 3,7 \mathrm{mmol})$ was dissolved in toluene $(20 \mathrm{~mL})$ and selenium $(0,41 \mathrm{~g}$, $5,3 \mathrm{mmol}$ ) was added. The mixture was stirred and heated to $110^{\circ} \mathrm{C}$ for $6 \mathrm{~h}$. The reaction was cooled to ambient temperature, filtrated and the solvent was evaporated. $\mathrm{HAnPSe}^{i} \mathrm{Pr}_{2}$ was obtained by crystallization from toluene as a yellow solid.

Yield: $0.91 \mathrm{~g}(2.4 \mathrm{mmol}, 72 \%)$

Empirical formula: $\mathrm{C}_{20} \mathrm{H}_{23} \mathrm{PSe}$

Molar mass: $374.07 \mathrm{~g} / \mathrm{mol}$

${ }^{1}$ H-NMR

$\left(\mathrm{CDCl}_{3}, 200 \mathrm{MHz}\right): \quad \delta=0.83\left(\mathrm{~d}, 3^{3} \mathrm{JHH}_{\mathrm{HH}}=7.00 \mathrm{~Hz}, 3 \mathrm{H}, \mathrm{CH}_{3}\right), 0.93\left(\mathrm{~d},{ }^{3}{ }_{\mathrm{HH}}=6.80 \mathrm{~Hz}, 3 \mathrm{H}\right.$, $\left.\mathrm{CH}_{3}\right), 1.40\left(\mathrm{~d}, 3^{3} \mathrm{HH}_{\mathrm{H}}=6.68 \mathrm{~Hz}, 3 \mathrm{H}, \mathrm{CH}_{3}\right), 1.49\left(\mathrm{~d},{ }^{3} \mathrm{~J}_{\mathrm{HH}}=6.80 \mathrm{~Hz}, 3 \mathrm{H}\right.$, $\left.\mathrm{CH}_{3}\right), 3.34\left(\mathrm{~m}, 2 \mathrm{H}, 2 \times \mathrm{CH}\left(\mathrm{CH}_{3}\right)_{2}\right), 7.50\left(\mathrm{~m}, 4 \mathrm{H}, \mathrm{H}_{2,7}, \mathrm{H}_{3,6}\right), 7.98(\mathrm{~m}$, $\left.2 \mathrm{H}, \mathrm{H}_{4,5}\right), 8.56\left(\mathrm{~s}, 1 \mathrm{H}, \mathrm{H}_{10}\right), 9.40\left(\mathrm{~d},{ }^{3} \mathrm{JHH}_{\mathrm{HH}}=8.38 \mathrm{~Hz}, \mathrm{H}_{1,8}\right)$. 


\section{${ }^{31} \mathrm{P}\left\{{ }^{1} \mathrm{H}\right\}-\mathrm{NMR}$}

$\left(\mathrm{CDCl}_{3}, 81 \mathrm{MHz}\right): \quad \delta[\mathrm{ppm}]=64.60\left(\mathrm{t},{ }^{1} J_{\mathrm{SeP}}=874 \mathrm{~Hz}\right)$.

\section{EI-MS}

$\mathrm{m} / \mathrm{z}(\%)$ :

$374(48)[\mathrm{M}]^{+}, 331(16)\left[\mathrm{M}-{ }^{i} \mathrm{Pr}\right]^{+}, 294(31)\left[\mathrm{M}^{i}{ }^{-} \mathrm{Pr}_{2}\right]^{+}, 209(74)[\mathrm{M}-$

$\left.\mathrm{Se}^{i} \mathrm{Pr}_{2}\right]^{+}, 178(100)\left[\mathrm{M}-\mathrm{SeP}^{i} \mathrm{Pr}_{2}\right]^{+}$.

\subsubsection{Synthesis of $\mathrm{HAnPPh}_{2}(4)$}

9-Bromoanthracene $(8,0 \mathrm{~g}, 31,2 \mathrm{mmol})$ was dissolved in diethyl ether $(80 \mathrm{~mL})$ and reacted with $n$-BuLi $(11,4 \mathrm{~mL}(2,75 \mathrm{M}), 31,2 \mathrm{mmol})$ at $-15^{\circ} \mathrm{C}$ over the course of $30 \mathrm{~min}$. The solution was warmed to $10^{\circ} \mathrm{C}$ and stirred for $10 \mathrm{~min}$, then cooled to $-15^{\circ} \mathrm{C}$ and diphenylchlorophosphane $(6.88 \mathrm{~g}, 31,2 \mathrm{mmol})$ was added over $30 \mathrm{~min}$. The resulting mixture was stirred for $2 \mathrm{~h}$, then the solvent was evaporated and the crude product was dissolved in DCM $(40 \mathrm{~mL})$. Lithium chloride was removed by filtration. Evaporation of the solvent and recrystallization from DCM afforded $\mathrm{HAnPPh}_{2}(\mathbf{4})$ as a yellow solid.

Yield: $8.03 \mathrm{~g}(22.1 \mathrm{mmol}, 71 \%)$

Empirical formula: $\mathrm{C}_{26} \mathrm{H}_{19} \mathrm{P}$

Molar mass $=362,41 \mathrm{~g} / \mathrm{mol}$

\section{${ }^{1} \mathrm{H}-\mathrm{NMR}$}

$\left(\mathrm{CDCl}_{3}, 500 \mathrm{MHz}\right)$ :

$\delta[\mathrm{ppm}]=8.79\left(\mathrm{dd},{ }^{3} \mathrm{~J}_{\mathrm{HH}}=8.0 \mathrm{~Hz},{ }^{4} J_{\mathrm{PH}}=4.5 \mathrm{~Hz}, 2 \mathrm{H}, \mathrm{H}_{1,8}\right), 8.64(\mathrm{~s}$, $\left.1 \mathrm{H}, \mathrm{H}_{10}\right), 8.04\left(\mathrm{dd},{ }^{3} J_{\mathrm{HH}}=8.0 \mathrm{~Hz},{ }^{4} J_{\mathrm{HH}}=1.1 \mathrm{~Hz}, 2 \mathrm{H}, \mathrm{H}_{4,5}\right), 7.46-$ $7.40\left(\mathrm{~m}, 6 \mathrm{H}, o-\mathrm{Ph} / \mathrm{H}_{3,6}\right), 7.33\left(\mathrm{ddd},{ }^{3} J_{\mathrm{HH}}=9.0 \mathrm{~Hz},{ }^{3} J_{\mathrm{HH}}=6.6 \mathrm{~Hz}\right.$, $\left.{ }^{4} \mathrm{~J}_{\mathrm{HH}}=1.1 \mathrm{~Hz}, 2 \mathrm{H}, \mathrm{H}_{2,7}\right), 7.28-7.20(\mathrm{~m}, 4 \mathrm{H}, m-\mathrm{Ph} / p-\mathrm{Ph})$.

\section{${ }^{13} \mathrm{C}\left\{{ }^{1} \mathrm{H}\right\}-\mathrm{NMR}$}

$\left(\mathrm{CDCl}_{3}, 126 \mathrm{MHz}\right)$ :

$$
\begin{aligned}
& \delta[p p m]=136.75\left(\mathrm{~d},{ }^{2} J_{\mathrm{CP}}=12.3 \mathrm{~Hz}, 2 \mathrm{C}, \mathrm{C}_{4 \mathrm{a}, 10 \mathrm{a}}\right), 136.64(\mathrm{~d}, \\
& \left.{ }^{3} \mathrm{~J}_{\mathrm{CP}}=13.0 \mathrm{~Hz}, 2 \mathrm{C}, \mathrm{C}_{9 \mathrm{a}, 8 \mathrm{a}}\right), 131.91\left(\mathrm{~s}, 1 \mathrm{C}, \mathrm{C}_{10}\right), 131.82(\mathrm{~d}, \\
& \left.{ }^{1} \mathrm{~J}_{\mathrm{CP}}=4.7 \mathrm{~Hz}, 2 \mathrm{C}, i-\mathrm{Ph}\right), 131.51\left(\mathrm{~d},{ }^{2} \mathrm{~J}_{\mathrm{CP}}=18.2 \mathrm{~Hz}, 4 \mathrm{C}, 0-\mathrm{Ph}\right), \\
& 129.25\left(\mathrm{~s}, 2 \mathrm{C}, \mathrm{C}_{4,5}\right), 128.39\left(\mathrm{~d},{ }^{3} \mathrm{~J}_{\mathrm{CP}}=24.5 \mathrm{~Hz}, 2 \mathrm{C}, \mathrm{C}_{1,8}\right), 128.37(\mathrm{~d}, \\
& \left.{ }^{3} \mathrm{~J}_{\mathrm{CP}}=18.2 \mathrm{~Hz}, 4 \mathrm{C}, m-\mathrm{Ph}\right), 127.51(\mathrm{~s}, 2 \mathrm{C}, p-\mathrm{Ph}), 125.94(\mathrm{~s}, 2 \mathrm{C}, \\
& \left.\mathrm{C}_{2,7}\right), 125.06\left(\mathrm{~s}, 2 \mathrm{C}, \mathrm{C}_{3,6}\right) .
\end{aligned}
$$

\section{${ }^{31} \mathrm{P}\left\{{ }^{1} \mathrm{H}\right\}-\mathrm{NMR}$}

$\left(\mathrm{CDCl}_{3}, 121 \mathrm{MHz}\right)$ :

$\delta[\mathrm{ppm}]=-24.17(\mathrm{~s})$ 


\section{EI-MS}

$m / z(\%):$ $362(100)[\mathrm{M}]^{+}, 285(3)[\mathrm{M}-\mathrm{Ph}]^{+}, 207$ (9) [M-Ph2] $]^{+}, 176(5)[\mathrm{M}-$ $\left.\mathrm{PPh}_{2}\right]^{+}$.

\subsubsection{Synthesis of $\mathrm{HAnPSPh}_{2}(5)$}

$\mathrm{HAnPPh}_{2}(4)(1,0 \mathrm{~g}, 2,76 \mathrm{mmol})$ was dissolved in toluene (20 mL) and sulfur $(0,11 \mathrm{~g}, 3,43 \mathrm{mmol})$ was added. The mixture was stirred and heated to $110^{\circ} \mathrm{C}$ for $6 \mathrm{~h}$. The reaction was cooled to ambient temperature, filtrated and the solvent was evaporated. $\mathrm{HAnPSPh}_{2}(\mathbf{5})$ was obtained by crystallization from toluene as a yellow solid.

Yield: $0,94 \mathrm{~g}(2, .37 \mathrm{mmol}, 86 \%)$

Empirical formula: $\mathrm{C}_{26} \mathrm{H}_{19} \mathrm{PS}$

Molar mass: $394,47 \mathrm{~g} / \mathrm{mol}$

\section{${ }^{1} \mathrm{H}-\mathrm{NMR}$}

$\left(\mathrm{CDCl}_{3}, 500 \mathrm{MHz}\right)$ :

$\delta[\mathrm{ppm}]=8.61\left(\mathrm{~s}, 1 \mathrm{H}, \mathrm{H}_{10}\right), 8.00\left(\mathrm{dd},{ }^{3} J_{\mathrm{HH}}=9.0 \mathrm{~Hz},{ }^{4} J_{\mathrm{HH}}=1.0 \mathrm{~Hz}\right.$, $\left.2 \mathrm{H}, \mathrm{H}_{1,8}\right), 7.97\left(\mathrm{dd},{ }^{3} J_{\mathrm{HH}}=8.5 \mathrm{~Hz},{ }^{4} \mathrm{~J}_{\mathrm{HH}}=1.5 \mathrm{~Hz}, 2 \mathrm{H}, \mathrm{H}_{4,5}\right), 7.82-$

$7.77(\mathrm{~m}, 4 \mathrm{H}, \mathrm{o}-\mathrm{Ph}), 7.35-7.30\left(\mathrm{~m}, 8 \mathrm{H}, \mathrm{H}_{3,6} / p-\mathrm{Ph}\right), 7.29-7.24$ $(\mathrm{m}, 4 \mathrm{H}, m-\mathrm{Ph}), 7.04$ (ddd, ${ }^{3} J_{\mathrm{HH}}=9.0 \mathrm{~Hz},{ }^{3} J_{\mathrm{HH}}=6.5 \mathrm{~Hz}$, $\left.{ }^{4} J_{\mathrm{HH}}=1.5 \mathrm{~Hz}, 2 \mathrm{H}, \mathrm{H}_{2,7}\right)$.

\section{${ }^{13} \mathrm{C}\left\{{ }^{1} \mathrm{H}\right\}-\mathrm{NMR}$}

$\left(\mathrm{CDCl}_{3}, 126 \mathrm{MHz}\right)$ :

$\delta[p p m]=137.01\left(\mathrm{~d},{ }^{2} \mathrm{~J}_{\mathrm{CP}}=82.5 \mathrm{~Hz}, 2 \mathrm{C}, \mathrm{C}_{8 \mathrm{a}, 9 \mathrm{a}}\right), 133.65(\mathrm{~s}, 1 \mathrm{C}$, $\left.\mathrm{C}_{10}\right), 133.19\left(\mathrm{~d},{ }^{2} J_{\mathrm{CP}}=7.9 \mathrm{~Hz}, 2 \mathrm{C}, \mathrm{C}_{4 \mathrm{a}, 10 \mathrm{a}}\right), 131.46(\mathrm{~d}$, $\left.{ }^{1} J_{\mathrm{CP}}=11.2 \mathrm{~Hz}, 2 \mathrm{C}, i-\mathrm{Ph}\right), 130.81\left(\mathrm{~d},{ }^{2} \mathrm{~J}_{\mathrm{CP}}=10.6 \mathrm{~Hz}, 4 \mathrm{C}, o-\mathrm{Ph}\right)$, $130.55(\mathrm{~s}, 2 \mathrm{C}, p-\mathrm{Ph}), 129.07\left(2 \mathrm{C}, \mathrm{C}_{4,5}\right) 128.42\left(\mathrm{~d},{ }^{3} \mathrm{~J}_{\mathrm{CP}}=12.6 \mathrm{~Hz}\right.$, $4 C, m-P h), 127.28\left(d,{ }^{3} J_{C P}=10.1 \mathrm{~Hz}, 2 C, C_{1,8}\right) 125.65\left(s, 2 C, C_{2,7}\right)$, $125.04\left(s, 2 C, C_{3,6}\right)$.

\section{${ }^{31} \mathrm{P}\left\{{ }^{1} \mathrm{H}\right\}-\mathrm{NMR}$}

$\left(\mathrm{CDCl}_{3}, 81 \mathrm{MHz}\right)$ :

$\delta[\mathrm{ppm}]=35.44(\mathrm{~s})$

\section{EI-MS}

$m / z(\%)$ : $394(100)[\mathrm{M}]^{+}, 362(7)[\mathrm{M}-\mathrm{S}]^{+}, 285$ (33) [M-SPh], 209 (19) [M$\left.\mathrm{SPh}_{2}\right]^{+}$.

\section{Elemental analysis}

in \% (calc.):

C: $75,57(79,17) ; \mathrm{H}: 4,72(4,85)$ 


\subsubsection{Synthesis of $\mathrm{HAnPSePh}_{2}(6)$}

$\mathrm{HAnPPh}_{2}$ (4) $(1,0 \mathrm{~g}, 2,76 \mathrm{mmol})$ was dissolved in toluene $(20 \mathrm{~mL})$ and selenium $(0,28 \mathrm{~g}$, $3,55 \mathrm{mmol}$ ) was added. The mixture was stirred and heated to $110^{\circ} \mathrm{C}$ for $6 \mathrm{~h}$. The reaction was cooled to ambient temperature, filtrated and the solvent was evaporated. $\mathrm{HAnPSPh}_{2}(\mathbf{5})$ was obtained by crystallization from toluene as a yellow solid.

Yield: $0.83 \mathrm{~g}(2.1 \mathrm{mmol}, 76 \%)$

Empirical formula: $\mathrm{C}_{26} \mathrm{H}_{19} \mathrm{PSe}$

Molar mass: $441.37 \mathrm{~g} / \mathrm{mol}$

${ }^{1} \mathrm{H}-\mathrm{NMR}$

$\left(\mathrm{CDCl}_{3}, 500 \mathrm{MHz}\right): \quad \delta[\mathrm{ppm}]=8.62\left(\mathrm{~s}, 1 \mathrm{H}, \mathrm{H}_{10}\right), 8.00\left(\mathrm{~d},{ }^{3} \mathrm{~J}_{\mathrm{HH}}=9.0 \mathrm{~Hz}, 2 \mathrm{H}, \mathrm{H}_{1,8}\right), 7.98$ $\left(\mathrm{dd},{ }^{3} J_{\mathrm{HH}}=8.0 \mathrm{~Hz},{ }^{4} J_{\mathrm{HH}}=1.5 \mathrm{~Hz}, 2 \mathrm{H}, \mathrm{H}_{4,5}\right), 7.87-7.81(\mathrm{~m}, 4 \mathrm{H}, \mathrm{o}-$ $\mathrm{Ph}), 7.34-7.29$ (m, 4H, $\left.\mathrm{H}_{3,6} / p-\mathrm{Ph}\right), 7.28-7.23$ (m, 4H, $\left.m-\mathrm{Ph}\right)$, 7.05 (ddd, ${ }^{3} J_{H H}=9.0 \mathrm{~Hz},{ }^{3} J_{H H}=6.5 \mathrm{~Hz},{ }^{4} J_{\mathrm{HH}}=1.5 \mathrm{~Hz}, 2 \mathrm{H}, \mathrm{H}_{2,7}$ ).

\section{${ }^{13} \mathrm{C}\left\{{ }^{1} \mathrm{H}\right\}-\mathrm{NMR}$}

$\left(\mathrm{CDCl}_{3}, 126 \mathrm{MHz}\right)$ :

$$
\begin{aligned}
& \delta[p p m]=135.29\left(\mathrm{~d},{ }^{2} J_{\mathrm{CP}}=74.2 \mathrm{~Hz}, 2 \mathrm{C}, \mathrm{C}_{8 \mathrm{a}, 9 \mathrm{a}}\right), 133.44(\mathrm{~s}, 1 \mathrm{C}, \\
& \left.\mathrm{C}_{10}\right), 132.94\left(\mathrm{~d},{ }^{3} \mathrm{~J}_{\mathrm{CP}}=7.7 \mathrm{~Hz}, 2 \mathrm{C}, \mathrm{C}_{4 \mathrm{a}, 10 \mathrm{a}}\right), 131.57(\mathrm{~d}, \\
& \left.{ }^{1} \mathrm{~J}_{\mathrm{CP}}=11.2 \mathrm{~Hz}, 2 \mathrm{C}, i-\mathrm{Ph}\right), 131.39\left(\mathrm{~d},{ }^{2} J_{\mathrm{CP}}=10.7 \mathrm{~Hz}, 4 \mathrm{C}, o-\mathrm{Ph}\right), \\
& 130.61(\mathrm{~s}, 2 \mathrm{C}, p-\mathrm{Ph}), 128.95\left(\mathrm{~s}, 2 \mathrm{C}, \mathrm{C}_{4,5}\right), 128.41(\mathrm{~d}, \\
& \left.{ }^{3} \mathrm{JP}_{\mathrm{CP}}=12.6 \mathrm{~Hz}, 4 \mathrm{C}, m-\mathrm{Ph}\right), 127.25\left(\mathrm{~d},{ }^{3} \mathrm{~J}_{\mathrm{CP}}=10.3 \mathrm{~Hz}, 2 \mathrm{C}, \mathrm{C}_{1,8}\right), \\
& 125.47\left(\mathrm{~s}, 2 \mathrm{C}, \mathrm{C}_{2,7}\right), 125.13\left(\mathrm{~s}, 2 \mathrm{C}, \mathrm{C}_{3,6}\right) .
\end{aligned}
$$

${ }^{31} \mathrm{P}\left\{{ }^{1} \mathrm{H}\right\}-\mathrm{NMR}$

$\left(\mathrm{CDCl}_{3}, 81 \mathrm{MHz}\right): \quad \delta[\mathrm{ppm}]=26.13(\mathrm{~s})$

\section{EI-MS}

$\mathrm{m} / \mathrm{z}(\%)$ :

$394(100)[\mathrm{M}]^{+}, 362(7)[\mathrm{M}-\mathrm{S}]^{+}, 285$ (33) [M-SPh] , 209 (19) [M$\left.\mathrm{SPh}_{2}\right]^{+}$.

${ }^{77}$ Se-NMR

$\left(\mathrm{CDCl}_{3}, 95 \mathrm{MHz}\right): \quad \delta[\mathrm{ppm}]=-279.71\left(\mathrm{~d},{ }^{1} J_{\mathrm{SeP}}=729.49 \mathrm{~Hz}\right)$

\section{EI-MS}

$\mathrm{m} / \mathrm{z}(\%): \quad 442(61)[\mathrm{M}]^{+}, 362(78)[\mathrm{M}-\mathrm{Se}]^{+}, 285(100)[\mathrm{M}-\mathrm{SePh}]^{+}, 207(16)\left[\mathrm{M}-\mathrm{SePh}_{2}\right]^{+}$

\section{Elemental analysis}

in \% (calc.):

C: $70,93 \%(70,75 \%) ; H: 4,68 \%(4,34 \%)$ 


\subsubsection{Synthesis of $\mathrm{BrAnP}^{i} \mathrm{Pr}_{2}(7)$}

9,10-Dibromoanthracene $(10,0 \mathrm{~g}, 29,8 \mathrm{mmol})$ was dissolved in diethyl ether $(100 \mathrm{~mL})$ and reacted with $n$-BuLi $(10,8 \mathrm{~mL}(2,75 \mathrm{M}), 29,8 \mathrm{mmol})$ at $-15^{\circ} \mathrm{C}$ over the course of $30 \mathrm{~min}$. The solution was warmed to $10^{\circ} \mathrm{C}$ and stirred for $10 \mathrm{~min}$, then cooled to $-15^{\circ} \mathrm{C}$ and chlorodiisopropylphosphane $(6,60 \mathrm{~g}, 30,0 \mathrm{mmol})$ was added over $30 \mathrm{~min}$. The resulting mixture was stirred for $2 \mathrm{~h}$, then the solvent was evaporated and the crude product was dissolved in $\mathrm{DCM}(50 \mathrm{~mL})$. Lithium chloride was removed by filtration. Concentration of the solution and crystallization from DCM afforded $\mathrm{BrAnP}^{i} \mathrm{Pr}_{2}$ (7) as a yellow solid.

Yield: $7.45 \mathrm{~g}(19.9 \mathrm{mmol}, 67 \%)$

Empirical formula: $\mathrm{C}_{20} \mathrm{H}_{22} \mathrm{PBr}$

Molar mass $=373.26 \mathrm{~g} / \mathrm{mol}$

${ }^{1} \mathrm{H}-\mathrm{NMR}$

$\left(\mathrm{CDCl}_{3}, 300 \mathrm{MHz}\right)$ :

$\delta[\mathrm{ppm}]=9.50-8.60\left(\mathrm{br} \mathrm{s}, 2 \mathrm{H}, \mathrm{H}_{4,5}\right) 8.68\left(\mathrm{~d},{ }^{3} \mathrm{~J}=8.2 \mathrm{~Hz}, 2 \mathrm{H}\right.$,

$\left.\mathrm{H}_{1,8}\right), 7.60\left(\mathrm{ddd},{ }^{3} J=8.3 \mathrm{~Hz},{ }^{3} \mathrm{~J}=6.2 \mathrm{~Hz},{ }^{4} J=1.3 \mathrm{~Hz}, 2 \mathrm{H}, \mathrm{H}_{2,7}\right) 7.56$

(ddd, $\left.{ }^{3} \mathrm{~J}=8.4 \mathrm{~Hz},{ }^{3} \mathrm{~J}=6.1 \mathrm{~Hz},{ }^{4} \mathrm{~J}=1.4 \mathrm{~Hz}, 2 \mathrm{H}, \mathrm{H}_{3,6}\right) 2.92$ (dsept,

$\left.{ }^{3} J=6.9 \mathrm{~Hz}, 2 \mathrm{H}, \mathrm{C} \underline{\mathrm{H}}\right) 1.44\left(\mathrm{dd},{ }^{3} J_{\mathrm{HP}}=17.8 \mathrm{~Hz},{ }^{3} \mathrm{~J}=6.8 \mathrm{~Hz}, 6 \mathrm{H}, \mathrm{CH}_{3}\right)$

$0.69\left(\mathrm{dd},{ }^{3} \mathrm{HP}_{\mathrm{HP}}=13.9 \mathrm{~Hz},{ }^{3} \mathrm{~J}=7.0 \mathrm{~Hz}, 6 \mathrm{H}, \mathrm{C}_{3}\right)$.

${ }^{13} \mathrm{C}\left\{{ }^{1} \mathrm{H}\right\}-N M R$

$\left(\mathrm{CDCl}_{3}, 126 \mathrm{MHz}\right)$

$\delta[\mathrm{ppm}]=130.39\left(\mathrm{~d},{ }^{3} \mathrm{~J}_{\mathrm{CP}}=3.5 \mathrm{~Hz}, 2 \mathrm{C}, \mathrm{C}_{8 \mathrm{a}, 9 \mathrm{a}}\right), 128.92\left(\mathrm{~s}, 2 \mathrm{C}, \mathrm{C}_{4,5}\right)$,

126.78 (s, 2C, C3,6), 126.20 (s, 2C, C C,7), 126.11 (s, 2C, C C 1,8 ),

$125.24\left(d,{ }^{1} J_{C P}=73.6 \mathrm{~Hz}, 1 \mathrm{C}, \mathrm{C}_{10}\right), 26.29\left(\mathrm{~d},{ }^{1} J_{\mathrm{CP}}=13.0 \mathrm{~Hz}, 2 \mathrm{C}\right.$,

$\underline{\mathrm{CH}}), 23.12\left(\mathrm{~d},{ }^{2} \mathrm{~J}_{\mathrm{CP}}=29.7 \mathrm{~Hz}, 2 \mathrm{C}, \underline{\mathrm{C}} \mathrm{H}_{3, \mathrm{Pr}}\right), 21.15\left(\mathrm{~d},{ }^{2} \mathrm{~J}_{\mathrm{CP}}=13.4 \mathrm{~Hz}\right.$,

$\left.2 \mathrm{C}, \underline{\mathrm{C}} \mathrm{H}_{3, \mathrm{Pr}}\right)$.

${ }^{31} \mathrm{P}\left\{{ }^{1} \mathrm{H}\right\}-\mathrm{NMR}$

$\left(\mathrm{CDCl}_{3}, 121 \mathrm{MHz}\right): \quad \delta[\mathrm{ppm}]=1.16(\mathrm{~s})$.

\section{EI-MS}

$m / z(\%):$

$372(58)[\mathrm{M}]^{+}, 330(10)\left[\mathrm{M}-{ }^{i} \mathrm{Pr}\right]^{+}, 287(100)[\mathrm{M}-2 \mathrm{iPr}]^{+}, 207(57)$

$[\mathrm{M}-\mathrm{Br}-2 \mathrm{iPr}]^{+}$. 


\subsubsection{Synthesis of $\mathrm{BrAnPS}^{i} \mathrm{Pr}_{2}(8)$}

$\mathrm{BrAnP}^{\prime} \mathrm{Pr}_{2}$ (7) (1 g, $\left.2.68 \mathrm{mmol}\right)$ was dissolved in toluene $(20 \mathrm{~mL})$ and sulfur $(0.10 \mathrm{~g}, 3.22 \mathrm{mmol})$ was added. The mixture was stirred and heated to $110^{\circ} \mathrm{C}$ for $6 \mathrm{~h}$. The reaction was cooled to ambient temperature, filtrated and the solvent was evaporated. $\operatorname{BrAnPS}^{\prime} \mathrm{Pr}_{2}(\mathbf{8})$ was obtained by crystallization from toluene as a yellow solid.

Yield: $0.81 \mathrm{~g}(2.01 \mathrm{mmol}, 75 \%)$

Empirical formula: $\mathrm{C}_{20} \mathrm{H}_{22} \mathrm{PSBr}$

Molar mass: $405.33 \mathrm{~g} / \mathrm{mol}$.

${ }^{1} \mathrm{H}-\mathrm{NMR}$

$\left(\mathrm{CDCl}_{3}, 300 \mathrm{MHz}\right): \quad \delta[\mathrm{ppm}]=9.27\left(\mathrm{dd},{ }^{3} J=6.5 \mathrm{~Hz},{ }^{4} J=3.5 \mathrm{~Hz}, 2 \mathrm{H}, \mathrm{H}_{4,5}\right)$ 8.39-8.33

$\left(\mathrm{m}, 2 \mathrm{H}, \mathrm{H}_{1,8}\right), 7.55\left(\mathrm{dd},{ }^{3} \mathrm{~J}=6.3 \mathrm{~Hz},{ }^{4} \mathrm{~J}=3.1 \mathrm{~Hz}, 2 \mathrm{H}, \mathrm{H}_{2,7}\right), 7.51$ (dd

${ }^{3} J=6.6 \mathrm{~Hz},{ }^{4} J=3.4 \mathrm{~Hz}, 2 \mathrm{H}, \mathrm{H}_{3,6}$ ), 3.28 (dsept, ${ }^{2} \mathrm{JP}_{\mathrm{CP}}=13.7 \mathrm{~Hz}$,

$\left.{ }^{3} J=6.8 \mathrm{~Hz}, 2 \mathrm{H}, \mathrm{CH}\right), 3.17\left(\mathrm{~s}, 3 \mathrm{H}, \mathrm{CH}_{3}\right), 1.49\left(\mathrm{dd},{ }^{3} J_{\mathrm{HP}}=17.7 \mathrm{~Hz}\right.$,

$\left.{ }^{3} J=6.7 \mathrm{~Hz}, 6 \mathrm{H}, \mathrm{CH}_{3}\right) 0.89\left(\mathrm{dd},{ }^{3} J_{\mathrm{HP}}=18.4 \mathrm{~Hz},{ }^{3} \mathrm{~J}=7.0 \mathrm{~Hz}, 6 \mathrm{H}\right.$,

$\left.\mathrm{CH}_{3}\right)$.

\section{${ }^{13} \mathrm{C}\left\{{ }^{1} \mathrm{H}\right\}-\mathrm{NMR}$}

$\left(\mathrm{CDCl}_{3}, 75 \mathrm{MHz}\right)$ :

$$
\begin{aligned}
& \delta[p p m]=134.16\left(\mathrm{~d},{ }^{2} J_{\mathrm{CP}}=6.5 \mathrm{~Hz}, 2 \mathrm{C}, \mathrm{C}_{4 \mathrm{a}, 10 \mathrm{a}}\right), 130.30(\mathrm{~d}, \\
& \left.{ }^{2} \mathrm{~J}_{\mathrm{CP}}=9.9 \mathrm{~Hz}, 1 \mathrm{C}, \mathrm{C}_{9}\right), 128.60\left(\mathrm{~s}, 2 \mathrm{C}, \mathrm{C}_{1,8}\right), 127.0\left(\mathrm{~d},{ }^{3} J_{\mathrm{CP}}=5.8 \mathrm{~Hz},\right. \\
& \left.2 \mathrm{C}, \mathrm{C}_{4,5}\right), 124.99\left(\mathrm{~d},{ }^{5} \mathrm{~J}_{\mathrm{CP}}=0.5 \mathrm{~Hz}, 2 \mathrm{C}, \mathrm{C}_{2,7}\right), 124.96(\mathrm{~d}, \\
& \left.{ }^{1} \mathrm{~J}_{\mathrm{CP}}=31.0 \mathrm{~Hz}, 1 \mathrm{C}, \mathrm{C}_{10}\right), 32.28\left(\mathrm{~d},{ }^{1} J_{\mathrm{CP}}=48.1 \mathrm{~Hz}, 2 \mathrm{C}, \underline{\mathrm{CH}}\right), 18.34 \\
& \left(\mathrm{~d},{ }^{2} J_{\mathrm{CP}}=1.3 \mathrm{~Hz}, 2 \mathrm{C}, \underline{\mathrm{C}}_{3, \mathrm{Pr}}\right), 17.95\left(\mathrm{~d},{ }^{2} J_{\mathrm{CP}}=1.5 \mathrm{~Hz}, 2 \mathrm{C}, \underline{\mathrm{C}} \mathrm{H}_{3, \mathrm{Pr}}\right), \\
& 15.45\left(\mathrm{~s}, 1 \mathrm{C}, \underline{\mathrm{C}}_{3}\right) .
\end{aligned}
$$

\section{${ }^{31} \mathrm{P}\left\{{ }^{1} \mathrm{H}\right\}-\mathrm{NMR}$}

$\left(\mathrm{CDCl}_{3}, 81 \mathrm{MHz}\right): \quad \delta[\mathrm{ppm}]=70.86(\mathrm{~s})$.

\section{EI-MS}

$m / z(\%):$

$340(100)[\mathrm{M}]^{+}, 308(4)[\mathrm{M}-\mathrm{S}]^{+}, 297(32)[\mathrm{M}-\mathrm{iPr}]^{+}, 255(90)[\mathrm{M}-$

$2 \mathrm{iPr}]^{+}, 239(38)\left[\mathrm{C}_{14} \mathrm{H}_{8} \mathrm{PS}\right]^{+}, 223(28)\left[\mathrm{C}_{14} \mathrm{H}_{8} \mathrm{P}\right]^{+}, 192(42)\left[\mathrm{C}_{14} \mathrm{H}_{8}\right]^{+}$.

\subsubsection{Synthesis of $\mathrm{BrAnPPh}_{2}(9)$}

9,10-Dibromoanthracene $(5,0 \mathrm{~g}, 14,88 \mathrm{mmol})$ was dissolved in diethyl ether $(50 \mathrm{~mL})$ and reacted with $n$-BuLi $(5,4 \mathrm{~mL}(2,75 \mathrm{M}), 14,9 \mathrm{mmol})$ at $-15^{\circ} \mathrm{C}$ over the course of $30 \mathrm{~min}$. The solution was warmed to $10^{\circ} \mathrm{C}$ and stirred for $10 \mathrm{~min}$, then cooled to $-15^{\circ} \mathrm{C}$ and diphenylchlorophosphane $(3,29 \mathrm{~g}, 14,9 \mathrm{mmol})$ was added over $30 \mathrm{~min}$. The resulting mixture was stirred for $2 \mathrm{~h}$, then the solvent was evaporated and the crude product was dissolved in 
DCM $(60 \mathrm{~mL})$. Lithium chloride was removed by filtration. Evaporation of the solvent and recrystallization from DCM afforded $\mathrm{BrAnPPh}_{2}(9)$ as a yellow solid.

Yield: 4,66 g (10.6 mmol, $71 \%)$

Empirical formula: $\mathrm{C}_{26} \mathrm{H}_{18} \mathrm{PBr}$

Molar mass $=441,30 \mathrm{~g} / \mathrm{mol}$

${ }^{1} \mathrm{H}-\mathrm{NMR}$

$\left(\mathrm{CDCl}_{3}, 300 \mathrm{MHz}\right)$ :

$\delta[\mathrm{ppm}]=8.85\left(\mathrm{dd},{ }^{3} J_{\mathrm{HH}}=8.9 \mathrm{~Hz},{ }^{4} \mathrm{~J}_{\mathrm{HP}}=5.3 \mathrm{~Hz}, 2 \mathrm{H}, \mathrm{H}_{4,5}\right) 8.69(\mathrm{~d}$, $\left.{ }^{3} J_{\mathrm{HH}}=8.9 \mathrm{~Hz}, 2 \mathrm{H}, \mathrm{H}_{1,8}\right), 7.58\left(\mathrm{ddd},{ }^{3} \mathrm{~J}=8.9 \mathrm{~Hz},{ }^{3} \mathrm{~J}=6.5 \mathrm{~Hz}\right.$, $\left.{ }^{4} J=1.1 \mathrm{~Hz}, 2 \mathrm{H}, \mathrm{H}_{2,7}\right) 7.44-7.38\left(\mathrm{~m}, 4 \mathrm{H}, \mathrm{H}_{\text {ortho,ph }}\right), 7.37-7.33(\mathrm{~m}$, $\left.2 \mathrm{H}, \mathrm{H}_{3,6}\right) 7.30-7.24\left(\mathrm{~m}, 6 \mathrm{H}, \mathrm{H}_{\text {meta/para, ph }}\right)$.

${ }^{13} \mathrm{C}\left\{{ }^{1} \mathrm{H}\right\}-\mathrm{NMR}$

$\left(\mathrm{CDCl}_{3}, 75 \mathrm{MHz}\right)$ :

$\delta[p p m]=136.7\left(\mathrm{~d},{ }^{1} J_{\mathrm{CP}}=70.2 \mathrm{~Hz}, 2 \mathrm{C}, \mathrm{C}_{\mathrm{ipso}, \mathrm{Ph}}\right), 131.4(\mathrm{~d}$, $\left.{ }^{2} J_{\mathrm{CP}}=18.5 \mathrm{~Hz}, 4 \mathrm{C}, \mathrm{C}_{\text {ortho, } \mathrm{ph}}\right), 130.9$ (d, ${ }^{3} \mathrm{~J}_{\mathrm{CP}}=4.4 \mathrm{~Hz}, 2 \mathrm{C}, \mathrm{C}_{8 \mathrm{a}, \mathrm{ga}}$ ), $129.7\left(\mathrm{~d},{ }^{1} J_{C P}=21.8 \mathrm{~Hz}, 1 \mathrm{C}, \mathrm{C}_{10}\right), 129.2\left(\mathrm{~d},{ }^{3} \mathrm{~J}_{\mathrm{CP}}=1.8 \mathrm{~Hz}, 2 \mathrm{C}, \mathrm{C}_{4,5}\right)$, $128.9\left(\mathrm{~d},{ }^{1} \mathrm{~J}_{\mathrm{CP}}=24.5 \mathrm{~Hz}, 1 \mathrm{C}, \mathrm{C}_{10}\right), 128.8\left(\mathrm{~s}, 2 \mathrm{C}, \mathrm{C}_{1,8}\right), 128.5$ (d, $\left.{ }^{3} J_{C P}=5.5 \mathrm{~Hz}, 4 \mathrm{C}, \mathrm{C}_{\text {meta,ph}}\right), 127.7$ (s, 2C, $\left.\mathrm{C}_{\text {para,ph }}\right), 127.0$ (d, $\left.{ }^{5} J_{C P}=1.6 \mathrm{~Hz}, 2 \mathrm{C}, \mathrm{C}_{2,7}\right), 126.1\left(\mathrm{~d},{ }^{4} \mathrm{JP}_{\mathrm{CP}}=1.6 \mathrm{~Hz}, 2 \mathrm{C}, \mathrm{C}_{3,6}\right)$.

${ }^{31} \mathrm{P}\left\{{ }^{1} \mathrm{H}\right\}-\mathrm{NMR}$

$\left(\mathrm{CDCl}_{3}, 121 \mathrm{MHz}\right)$ :

$\delta[\mathrm{ppm}]=-23.2(\mathrm{~s})$

EI-MS

$m / z(\%):$ $442(100)[\mathrm{M}]^{+}, 361(30)[\mathrm{M}-\mathrm{Br}]^{+}, 283(35)\left[\mathrm{C}_{20} \mathrm{H}_{13} \mathrm{P}\right]^{+}, 207(10)$ $\left[\mathrm{C}_{14} \mathrm{H}_{8} \mathrm{P}\right]^{+}, 176(24)\left[\mathrm{C}_{14} \mathrm{H}_{8}\right]^{+}$.

Elemental analysis

in \% (calc.): $\quad$ C: 69.74 (70.76), H: 4.02 (4.11).

\subsubsection{Synthesis of $\mathrm{BrAnPSPh}_{2}(10)$}

$\mathrm{BrAnPPh}_{2}$ (9) $(1,0 \mathrm{~g}, 2.27 \mathrm{mmol})$ was dissolved in toluene $(20 \mathrm{~mL})$ and sulfur $(0,087 \mathrm{~g}$, $2.72 \mathrm{mmol}$ ) was added. The mixture was stirred and heated to $110^{\circ} \mathrm{C}$ for $6 \mathrm{~h}$. The reaction was cooled to ambient temperature, filtrated and the solvent was evaporated. $\mathrm{BrAnPSPh}_{2}(\mathbf{1 0})$ was obtained by crystallization from toluene as a yellow solid.

Yield: $0.88 \mathrm{~g}$ (1.86 mmol, 82\%)

Empirical formula: $\mathrm{C}_{26} \mathrm{H}_{18} \mathrm{PSBr}$ 
Molar mass: $473.36 \mathrm{~g} / \mathrm{mol}$

${ }^{1} \mathrm{H}-\mathrm{NMR}$

$\left(\mathrm{CDCl}_{3}, 500 \mathrm{MHz}\right)$ :

$\delta[\mathrm{ppm}]=8.61\left(\mathrm{~d},{ }^{3} \mathrm{~J}_{\mathrm{HH}}=9.0 \mathrm{~Hz}, 2 \mathrm{H}, \mathrm{H}_{1,8}\right), 8.10\left(\mathrm{dd},{ }^{3} \mathrm{~J}_{\mathrm{HH}}=8.5 \mathrm{~Hz}\right.$,

$\left.{ }^{4} \mathrm{~J}_{\mathrm{HH}}=1.0 \mathrm{~Hz}, 2 \mathrm{H}, \mathrm{H}_{4,5}\right), 7.78-7.72(\mathrm{~m}, 4 \mathrm{H}, o-\mathrm{Ph}), 7.45$ (ddd, ${ }^{3} \mathrm{~J}_{\mathrm{HH}}$

$\left.=9.0 \mathrm{~Hz},{ }^{3} \mathrm{~J}_{\mathrm{HH}}=6.5 \mathrm{~Hz},{ }^{4} \mathrm{~J}_{\mathrm{HH}}=1.0 \mathrm{~Hz}, 2 \mathrm{H}, \mathrm{H}_{2,7}\right), 7.36-7.32(\mathrm{~m}$,

$2 \mathrm{H}, p-\mathrm{Ph}), 7.30-7.25(\mathrm{~m}, 4 \mathrm{H}, m-\mathrm{Ph}), 7.07\left(\mathrm{ddd},{ }^{3} \mathrm{~J}_{\mathrm{HH}}=8.5 \mathrm{~Hz}\right.$,

$\left.{ }^{3} \mathrm{~J}_{\mathrm{HH}}=6.5 \mathrm{~Hz},{ }^{4} \mathrm{~J}_{\mathrm{HH}}=1.0 \mathrm{~Hz} 2 \mathrm{H}, \mathrm{H}_{3,6}\right)$

${ }^{13} \mathrm{C}\left\{{ }^{1} \mathrm{H}\right\}-\mathrm{NMR}$

$\left(\mathrm{CDCl}_{3}, 126 \mathrm{MHz}\right)$ :

$\delta[\mathrm{ppm}]=136.95\left(\mathrm{~d},{ }^{2} \mathrm{~J}_{\mathrm{CP}}=83.3 \mathrm{~Hz}, 2 \mathrm{C}, \mathrm{C}_{8 \mathrm{a}, 9 \mathrm{a}}\right), 133.54(\mathrm{~d}$,

$\left.{ }^{3} \mathrm{~J}_{\mathrm{CP}}=8.1 \mathrm{~Hz}, 2 \mathrm{C}, \mathrm{C}_{4 \mathrm{a}, 10 \mathrm{a}}\right), 130.73\left(\mathrm{~d},{ }^{2} \mathrm{~J}_{\mathrm{CP}}=10.4 \mathrm{~Hz}, 4 \mathrm{C}, m-\mathrm{Ph}\right)$, $130.64(\mathrm{~s}, 2 \mathrm{H}, p-\mathrm{Ph}), 130.60\left(\mathrm{~d},{ }^{1} \mathrm{~J}_{\mathrm{CP}}=11.2 \mathrm{~Hz}, 2 \mathrm{C}, i-\mathrm{Ph}\right), 128.46$

$\left(d,{ }^{3} J_{C P}=12.7 \mathrm{~Hz}, 4 \mathrm{C}, m-\mathrm{Ph}\right), 128.71\left(\mathrm{~s}, 2 \mathrm{C}, \mathrm{C}_{4,5}\right), 127.62\left(\mathrm{~d},{ }^{3} \mathrm{~J}_{\mathrm{CP}}=\right.$ $\left.10.3 \mathrm{~Hz}, 2 \mathrm{C}, \mathrm{C}_{1,8}\right), 126.94\left(\mathrm{~s}, 2 \mathrm{C}, \mathrm{C}_{3,6}\right), 125.66\left(\mathrm{~s}, 2 \mathrm{C}, \mathrm{C}_{2,7}\right), 123.79$ (d, ${ }^{1} \mathrm{~J}_{\mathrm{CP}}=88.8 \mathrm{~Hz}, 1 \mathrm{C}, \mathrm{C}_{9}$ )

${ }^{31} \mathrm{P}\left\{{ }^{1} \mathrm{H}\right\}-\mathrm{NMR}$

$\left(\mathrm{CDCl}_{3}, 81 \mathrm{MHz}\right): \quad \delta[\mathrm{ppm}]=34.0(\mathrm{~s})$

\section{EI-MS}

$\mathrm{m} / \mathrm{z}(\%)$ :

$473(100)[\mathrm{M}]^{+}, 440(4)[\mathrm{M}-\mathrm{S}]^{+}, 394(15)[\mathrm{M}-\mathrm{Br}]^{+}, 185$ (74) [M-

$\left.\mathrm{C}_{14} \mathrm{H}_{8} \mathrm{BrS}\right]^{+}$

Elemental analysis

in \% (calc.):

C: $65.97 \%(64.54 \%), H: 3.83 \%(4.08 \%)$

\subsubsection{Synthesis of $M e A n P^{i} \operatorname{Pr}_{2}$ (11)}

9-Bromo-10-methylanthracene $(5,0 \mathrm{~g}, 18,44 \mathrm{mmol})$ was dissolved in diethyl ether $(60 \mathrm{~mL})$ and reacted with $n$-BuLi $(6,75 \mathrm{~mL}(2,75 \mathrm{M}), 18,5 \mathrm{mmol})$ at $-15^{\circ} \mathrm{C}$ over the course of $30 \mathrm{~min}$. The solution was warmed to $10^{\circ} \mathrm{C}$ and stirred for $10 \mathrm{~min}$, then cooled to $-15^{\circ} \mathrm{C}$ and chlorodiisopropylphosphane $(2,81 \mathrm{~g}, 18,44 \mathrm{mmol})$ was added over $30 \mathrm{~min}$. The resulting mixture was stirred overnight, then the solvent was evaporated and the crude product was dissolved in DCM $(50 \mathrm{~mL})$. Lithium chloride was removed by filtration. Concentration of the solution and crystallization from DCM afforded MeAnP'Pr $\operatorname{Pr}_{2}(\mathbf{1 1})$ as a yellow solid.

Yield: $4.13 \mathrm{~g}(14.0 \mathrm{mmol}, 76 \%)$

Empirical formula: $\mathrm{C}_{21} \mathrm{H}_{25} \mathrm{P}$

Molar mass $=308.4 \mathrm{~g} / \mathrm{mol}$ 


\section{${ }^{1} \mathrm{H}-\mathrm{NMR}$}

$\left(\mathrm{CDCl}_{3}, 300 \mathrm{MHz}\right)$ :

\section{${ }^{13} \mathrm{C}\left\{{ }^{1} \mathrm{H}\right\}-\mathrm{NMR}$}

$\left(\mathrm{CDCl}_{3}, 126 \mathrm{MHz}\right)$ :

${ }^{31}\left\{{ }^{1} \mathrm{H}\right\}-N M R$

$\left(\mathrm{CDCl}_{3}, 121 \mathrm{MHz}\right)$ :

$\delta[\mathrm{ppm}]=0.02(\mathrm{~s})$

\section{EI-MS}

$m / z(\%):$

$308(42)[\mathrm{M}]^{+}, 293(6)\left[\mathrm{M}-\mathrm{CH}_{3}\right]^{+}, 266(10)\left[\mathrm{M}^{i}{ }^{-} \mathrm{Pr}_{2}\right]^{+}, 223$ (100) $[\mathrm{M}-$ $\left.2^{i} \mathrm{Pr}_{2}\right]^{+}, 192(24)\left[\mathrm{C}_{15} \mathrm{H}_{11}\right]^{+}$.

\section{Elemental analysis}

in \% (calc.):

C: 78.45 (81.79), H: 8.23 (8.17).

\subsubsection{Synthesis of MeAnPS'Pr 2 (12)}

MeAnP'Pr ${ }_{2}$ (11) $(0,99 \mathrm{~g}, 3,21 \mathrm{mmol})$ was dissolved in toluene $(20 \mathrm{~mL})$ and sulfur $(0,11 \mathrm{~g}$, $3,53 \mathrm{mmol}$ ) was added. The mixture was stirred and heated to $110^{\circ} \mathrm{C}$ for $6 \mathrm{~h}$. The reaction was cooled to ambient temperature, filtrated and the solvent was evaporated. $M e A n \operatorname{PS}^{i} \operatorname{Pr}_{2}$ (12) was obtained by crystallization from toluene as a yellow solid.

Yield: $0.74 \mathrm{~g}(2.18 \mathrm{mmol}, 75 \%)$

Empirical formula: $\mathrm{C}_{21} \mathrm{H}_{25} \mathrm{PS}$

Mlar mass: $340.46 \mathrm{~g} / \mathrm{mol}$ 


\section{${ }^{1} \mathrm{H}-\mathrm{NMR}$}

$\left(\mathrm{CDCl}_{3}, 300 \mathrm{MHz}\right): \quad \delta[\mathrm{ppm}]=9.27\left(\mathrm{dd},{ }^{3} J=6.5 \mathrm{~Hz},{ }^{4} J=3.5 \mathrm{~Hz}, 2 \mathrm{H}, \mathrm{H}_{4,5}\right)$ 8.39-8.33

$\left(\mathrm{m}, 2 \mathrm{H}, \mathrm{H}_{1,8}\right), 7.55\left(\mathrm{dd},{ }^{3} \mathrm{~J}=6.3 \mathrm{~Hz},{ }^{4} J=3.1 \mathrm{~Hz}, 2 \mathrm{H}, \mathrm{H}_{2,7}\right), 7.51$ (dd

$\left.{ }^{3} J=6.6 \mathrm{~Hz},{ }^{4} J=3.4 \mathrm{~Hz}, 2 \mathrm{H}, \mathrm{H}_{3,6}\right), 3.28$ (dsept, ${ }^{2} \mathrm{JP}_{\mathrm{CP}}=13.7 \mathrm{~Hz}$,

$\left.{ }^{3} J=6.8 \mathrm{~Hz}, 2 \mathrm{H}, \mathrm{CH}\right), 3.17\left(\mathrm{~s}, 3 \mathrm{H}, \mathrm{CH}_{3}\right), 1.49\left(\mathrm{dd},{ }^{3} J_{\mathrm{HP}}=17.7 \mathrm{~Hz}\right.$,

$\left.{ }^{3} \mathrm{~J}=6.7 \mathrm{~Hz}, 6 \mathrm{H}, \mathrm{CH}_{3}\right) 0.89\left(\mathrm{dd},{ }^{3} J_{\mathrm{HP}}=18.4 \mathrm{~Hz},{ }^{3} \mathrm{~J}=7.0 \mathrm{~Hz}, 6 \mathrm{H}\right.$,

$\left.\mathrm{C}_{3}\right)$.

\section{${ }^{13} \mathrm{C}\left\{{ }^{1} \mathrm{H}\right\}-\mathrm{NMR}$}

$\left(\mathrm{CDCl}_{3}, 75 \mathrm{MHz}\right)$ :

$$
\begin{aligned}
& \delta[p p m]=134.16\left(\mathrm{~d},{ }^{2} J_{\mathrm{CP}}=6.5 \mathrm{~Hz}, 2 \mathrm{C}, \mathrm{C}_{4 \mathrm{a}, 10 \mathrm{a}}\right), 130.30(\mathrm{~d}, \\
& \left.{ }^{2} \mathrm{~J}_{\mathrm{CP}}=9.9 \mathrm{~Hz}, 1 \mathrm{C}, \mathrm{C}_{9}\right), 128.60\left(\mathrm{~s}, 2 \mathrm{C}, \mathrm{C}_{1,8}\right), 127.0\left(\mathrm{~d},{ }^{3} \mathrm{~J}_{\mathrm{CP}}=5.8 \mathrm{~Hz},\right. \\
& \left.2 \mathrm{C}, \mathrm{C}_{4,5}\right), 124.99\left(\mathrm{~d},{ }^{5} \mathrm{~J}_{\mathrm{CP}}=0.5 \mathrm{~Hz}, 2 \mathrm{C}, \mathrm{C}_{2,7}\right), 124.96(\mathrm{~d}, \\
& \left.{ }^{1} \mathrm{~J}_{\mathrm{CP}}=31.0 \mathrm{~Hz}, 1 \mathrm{C}, \mathrm{C}_{10}\right), 32.28\left(\mathrm{~d},{ }^{1} J_{\mathrm{CP}}=48.1 \mathrm{~Hz}, 2 \mathrm{C}, \underline{\mathrm{C}}\right), 18.34 \\
& \left(\mathrm{~d},{ }^{2} J_{\mathrm{CP}}=1.3 \mathrm{~Hz}, 2 \mathrm{C}, \underline{\mathrm{C}}_{3, \mathrm{Pr}}\right), 17.95\left(\mathrm{~d},{ }^{2} \mathrm{~J}_{\mathrm{CP}}=1.5 \mathrm{~Hz}, 2 \mathrm{C}, \underline{\mathrm{C}}_{3, \mathrm{Pr}}\right), \\
& 15.45\left(\mathrm{~s}, 1 \mathrm{C}, \underline{\mathrm{C}}_{3}\right) .
\end{aligned}
$$

\section{${ }^{31} \mathrm{P}\left\{{ }^{1} \mathrm{H}\right\}-\mathrm{NMR}$}

$\left(\mathrm{CDCl}_{3}, 81 \mathrm{MHz}\right)$ :

$\delta[\mathrm{ppm}]=70.86(\mathrm{~s})$

\section{EI-MS}

$m / z(\%):$

$340(100)[\mathrm{M}]^{+}, 308(4)[\mathrm{M}-\mathrm{S}]^{+}, 297(32)[\mathrm{M}-\mathrm{iPr}]^{+}, 255$ (90) [M-

$2 \mathrm{iPr}]^{+}, 239(38)\left[\mathrm{C}_{14} \mathrm{H}_{8} \mathrm{PS}\right]^{+}, 223(28)\left[\mathrm{C}_{14} \mathrm{H}_{8} \mathrm{P}\right]^{+}, 192(42)\left[\mathrm{C}_{14} \mathrm{H}_{8}\right]^{+}$.

\section{Elemental analysis}

in \% (calc.):

C: 67.92 (74.08), H: 7.66 (7.40), S: 8.78 (9.42).

\subsubsection{Synthesis of $\mathrm{MeAnPPh}_{2}(13)$}

9-Bromo-10-methylanthracene $(5,0 \mathrm{~g}, 14,88 \mathrm{mmol})$ was dissolved in diethyl ether $(60 \mathrm{~mL})$ and reacted with $n$-BuLi $(6,71 \mathrm{~mL}(2,75 \mathrm{M}), 18.44 \mathrm{mmol})$ at $-15^{\circ} \mathrm{C}$ over the course of $30 \mathrm{~min}$. The solution was warmed to $10^{\circ} \mathrm{C}$ and stirred for $10 \mathrm{~min}$, then cooled to $-15^{\circ} \mathrm{C}$ and diphenylchlorophosphane $(4,07 \mathrm{~g}, 14,9 \mathrm{mmol})$ was added over $30 \mathrm{~min}$. The resulting mixture was stirred for $2 \mathrm{~h}$, then the solvent was evaporated and the crude product was dissolved in DCM $(60 \mathrm{~mL})$. Lithium chloride was removed by filtration. Evaporation of the solvent and recrystallization from DCM afforded $\mathrm{MerAnPPh}_{2}(\mathbf{1 3})$ as a yellow solid.

Yield: 4,01 g (10.7 mmol, $72 \%)$

Empirical formula: $\mathrm{C}_{27} \mathrm{H}_{21} \mathrm{P}$

Molar mass $=376.43 \mathrm{~g} / \mathrm{mol}$ 


\section{${ }^{1} \mathrm{H}-\mathrm{NMR}$}

$\left(\mathrm{CDCl}_{3}, 300 \mathrm{MHz}\right)$ :

$\delta[\mathrm{ppm}]=8.86\left(\mathrm{dd},{ }^{3} J_{\mathrm{HH}}=8.9 \mathrm{~Hz},{ }^{4} \mathrm{~J}_{\mathrm{HP}}=5.3 \mathrm{~Hz}, 2 \mathrm{H}, \mathrm{H}_{4,5}\right) 8.69(\mathrm{~d}$,

$\left.{ }^{3} J_{\mathrm{HH}}=8.9 \mathrm{~Hz}, 2 \mathrm{H}, \mathrm{H}_{1,8}\right), 7.50\left(\mathrm{ddd},{ }^{3} \mathrm{~J}=8.9 \mathrm{~Hz},{ }^{3} \mathrm{~J}=6.5 \mathrm{~Hz}\right.$,

$\left.{ }^{4} J=1.2 \mathrm{~Hz}, 2 \mathrm{H}, \mathrm{H}_{2,7}\right) 7.48-7.41\left(\mathrm{~m}, 4 \mathrm{H}, \mathrm{H}_{\text {ortho,ph }}\right), 7.34$ (ddd,

$\left.{ }^{3} J=8.9 \mathrm{~Hz},{ }^{3} J=6.5 \mathrm{~Hz},{ }^{4} J=1.2 \mathrm{~Hz}, 2 \mathrm{H}, \mathrm{H}_{3,6}\right) 7.30-7.24(\mathrm{~m}, 6 \mathrm{H}$,

$\mathrm{H}_{\text {meta/para, ph) }} 3.24\left(\mathrm{~s}, 3 \mathrm{H}, \mathrm{CH}_{3}\right)$.

\section{${ }^{13} \mathrm{C}\left\{{ }^{1} \mathrm{H}\right\}-\mathrm{NMR}$}

$\left(\mathrm{CDCl}_{3}, 75 \mathrm{MHz}\right)$ :

$\delta[p p m]=136.5\left(\mathrm{~d},{ }^{1} J_{\mathrm{CP}}=23.3 \mathrm{~Hz}, 2 \mathrm{C}, \mathrm{C}_{\mathrm{ipso}, \mathrm{Ph}}\right), 135.7$ (d,

$\left.{ }^{3} J_{C P}=2.0 \mathrm{~Hz}, 2 \mathrm{C}, \mathrm{C}_{8 \mathrm{a}, 9 \mathrm{a}}\right) 131.5\left(\mathrm{~d},{ }^{2} \mathrm{~J}_{\mathrm{CP}}=18.1 \mathrm{~Hz}, 4 \mathrm{C}, \mathrm{C}_{\text {ortho, ph }}\right)$,

$130.5\left(d,{ }^{2} J_{C P}=4.6 \mathrm{~Hz}, 2 \mathrm{C}, \mathrm{C}_{4 a}, 10 \mathrm{a}\right), 129.3\left(\mathrm{~d},{ }^{1} J_{\mathrm{CP}}=25.4 \mathrm{~Hz}, 1 \mathrm{C}\right.$,

$\left.\mathrm{C}_{10}\right), 129.2\left(\mathrm{~d},{ }^{3} \mathrm{JP}_{\mathrm{CP}}=1.8 \mathrm{~Hz}, 2 \mathrm{C}, \mathrm{C}_{4,5}\right), 128.9\left(\mathrm{~d},{ }^{1} \mathrm{~J}_{\mathrm{CP}}=24.5 \mathrm{~Hz}, 1 \mathrm{C}\right.$,

$\left.\mathrm{C}_{10}\right), 128.8\left(\mathrm{~s}, 2 \mathrm{C}, \mathrm{C}_{1,8}\right), 128.4\left(\mathrm{~d},{ }^{3} \mathrm{~J}_{\mathrm{CP}}=5.5 \mathrm{~Hz}, 6 \mathrm{C}, \mathrm{C}_{\text {meta,para,ph}}\right.$ ),

$127.5\left(\mathrm{~s}, 2 \mathrm{C}, \mathrm{C}_{4,5}\right), 125.4\left(\mathrm{~d},{ }^{4} \mathrm{~J}_{\mathrm{CP}}=1.8 \mathrm{~Hz}, 2 \mathrm{C}, \mathrm{C}_{1,8}\right), 125.3(\mathrm{~s}, 2 \mathrm{C}$,

$\left.C_{2,7}\right) 125.1\left(d,{ }^{4} J_{C P}=1.6 \mathrm{~Hz}, 2 \mathrm{C}, \mathrm{C}_{3,6}\right)$.

${ }^{31} \mathrm{P}\left\{{ }^{1} \mathrm{H}\right\}-N M R$

$\left(\mathrm{CDCl}_{3}, 121 \mathrm{MHz}\right): \quad \delta[\mathrm{ppm}]=-24.1(\mathrm{~s})$.

\section{EI-MS}

$m / z(\%):$

$376(100)[\mathrm{M}]^{+}, 297(15)[\mathrm{M}-\mathrm{Ph}]^{+}, 283$ (15) [M-Ph-CH $]^{+}, 191(8)$ $\left[\mathrm{M}-\mathrm{PPh}_{2}\right]^{+}$.

\section{Elemental analysis}

in \% (calc.): $\quad$ C: 83.24 (86.15), H: 5.97 (5.62).

\subsubsection{Synthesis of MeAnPSPh 2 (14)}

$\mathrm{MeAnPPh}_{2}$ (13) $(1,2 \mathrm{~g}, 3,20 \mathrm{mmol})$ was dissolved in toluene $(20 \mathrm{~mL})$ and sulfur $(0,12 \mathrm{~g}$, $3,82 \mathrm{mmol}$ ) was added. The mixture was stirred and heated to $110^{\circ} \mathrm{C}$ for $6 \mathrm{~h}$. The reaction was cooled to ambient temperature, filtrated and the solvent was evaporated. $\mathrm{MeAnPSPh}_{2}$ (14) was obtained by crystallization from toluene as a yellow solid.

Yield: 0,96 g (2,36 mmol, $80 \%)$

Empirical formula: $\mathrm{C}_{27} \mathrm{H}_{21} \mathrm{PS}$

Molar mass: $408.49 \mathrm{~g} / \mathrm{mol}$ 


\section{${ }^{1} \mathrm{H}-\mathrm{NMR}$}

$\left(\mathrm{CDCl}_{3}, 300 \mathrm{MHz}\right)$ :

$$
\begin{aligned}
& \delta[\mathrm{ppm}]=8.36\left(\mathrm{~d},{ }^{3} J_{\mathrm{HH}}=8.9 \mathrm{~Hz}, 2 \mathrm{H}, \mathrm{H}_{1,8}\right) 8.10\left(\mathrm{~d},{ }^{3} \mathrm{JHH}_{\mathrm{HH}}=9.05 \mathrm{~Hz},\right. \\
& \left.2 \mathrm{H}, \mathrm{H}_{4,5}\right), 7.77\left(\mathrm{ddd},{ }^{3} \mathrm{JPP}_{\mathrm{HP}}=13.3 \mathrm{~Hz},{ }^{3} J=6.4 \mathrm{~Hz},{ }^{4} \mathrm{~J}=1.6 \mathrm{~Hz}, 4 \mathrm{H},\right. \\
& \left.\mathrm{H}_{\text {ortho,ph }}\right) 7.40\left(\mathrm{ddd},{ }^{3} J=8.9 \mathrm{~Hz},{ }^{3} J=6.6 \mathrm{~Hz},{ }^{4} J=1.0 \mathrm{~Hz}, 2 \mathrm{H}, \mathrm{H}_{2,7}\right), \\
& 7.35-7.24\left(\mathrm{~m}, 6 \mathrm{H}, \mathrm{H}_{\text {meta/para, Ph }}\right), 7.06\left(\mathrm{ddd},{ }^{3} J=8.9 \mathrm{~Hz},\right. \\
& \left.{ }^{3} J=6.6 \mathrm{~Hz},{ }^{4} J=1.0 \mathrm{~Hz}, 2 \mathrm{H}, \mathrm{H}_{3,6}\right) 3.23\left(\mathrm{~s}, 3 \mathrm{H}, \mathrm{C}_{3}\right) .
\end{aligned}
$$

\section{${ }^{13} \mathrm{C}\left\{{ }^{1} \mathrm{H}\right\}-\mathrm{NMR}$}

$\left(\mathrm{CDCl}_{3}, 75 \mathrm{MHz}\right)$ :

$$
\begin{aligned}
& \delta[\mathrm{ppm}]=137.0\left(\mathrm{~d},{ }^{2} \mathrm{~J}_{\mathrm{CP}}=82.5 \mathrm{~Hz}, 2 \mathrm{C}, \mathrm{C}_{8 \mathrm{a}, 9 \mathrm{a}}\right), 133.7\left(\mathrm{~s}, 1 \mathrm{C}, \mathrm{C}_{10}\right) \\
& 133.2\left(\mathrm{~d},{ }^{2}{ }_{\mathrm{CP}}=7.9 \mathrm{~Hz}, 2 \mathrm{C}, \mathrm{C}_{4 \mathrm{a}, 10 \mathrm{a}}\right), 131.5\left(\mathrm{~d},{ }^{1} \mathrm{~J}_{\mathrm{CP}}=11.6 \mathrm{~Hz}, 2 \mathrm{C},\right. \\
& \left.\mathrm{C}_{\mathrm{ipso}, \mathrm{Ph}}\right), 130.8\left(\mathrm{~d},{ }^{2} \mathrm{~J}_{\mathrm{CP}}=10.5 \mathrm{~Hz}, 4 \mathrm{C}, \mathrm{C}_{\text {orho,ph }}\right), 130.6(\mathrm{~s}, 2 \mathrm{C}, \\
& \left.\mathrm{C}_{\mathrm{para}, \mathrm{Ph}}\right), 129.1\left(\mathrm{~s}, 1 \mathrm{C}, \mathrm{C}_{4,5}\right), 128.5\left(\mathrm{~d},{ }^{3} \mathrm{~J}_{\mathrm{CP}}=12.6 \mathrm{~Hz}, 4 \mathrm{C}, \mathrm{C}_{\text {meta, Ph }}\right), \\
& 127.3\left(\mathrm{~d},{ }^{3} \mathrm{~J}_{\mathrm{CP}}=10.1 \mathrm{~Hz}, 2 \mathrm{C}, \mathrm{C}_{1,8}\right), 125.7\left(\mathrm{~s}, 2 \mathrm{C}, \mathrm{C}_{2,7}\right), 125.2(\mathrm{~s}, 2 \mathrm{C} \text {, } \\
& \left.\mathrm{C}_{3,6}\right), 15.9\left(\mathrm{~s}, 1 \mathrm{C}, \underline{\mathrm{C}}_{3}\right) .
\end{aligned}
$$

${ }^{31} \mathrm{P}\left\{{ }^{1} \mathrm{H}\right\}-\mathrm{NMR}$

$\left(\mathrm{CDCl}_{3}, 81 \mathrm{MHz}\right)$ :

$\delta[\mathrm{ppm}]=34.43(\mathrm{~s})$.

\section{EI-MS}

$\mathrm{m} / \mathrm{z}(\%)$ :

$408(100)[\mathrm{M}]^{+}, 376(46)[\mathrm{M}-\mathrm{S}]^{+}, 299(46)\left[\mathrm{C}_{21} \mathrm{H}_{16} \mathrm{P}\right]^{+}, 283(10)$

$\left[\mathrm{C}_{15} \mathrm{H}_{11} \mathrm{PS}\right]^{+}, 223(52)\left[\mathrm{C}_{15} \mathrm{H}_{11} \mathrm{P}\right]^{+}$.

Elemental analysis

in \% (calc.):

C: 78.72 (79.39), H: 5.37 (5.18), S: 7.75 (7.85).

\subsubsection{Synthesies of SPAnPS host/guest complexes (16-20)}

For the synthesis of the intercalation structures 16-20, 9,10-Bis(diphenylthiophosphoryl)anthracene $(1.0 \mathrm{~g}, 1.64 \mathrm{mmol})$ was dissolved in the respective solvent/solvent mixture listed in table 6-1 at boiling temperature of the solvent. Then the solutions were filtrated and stored at the crystallization temperatures listed in table 6-1. 


\begin{tabular}{ccc}
\hline \multicolumn{3}{c}{ Table 6-1: Crystallization conditions and compound } \\
nomenclature.
\end{tabular}

For solid state fluorescence experiments the crystals were separated from the mother liquor by filtration, immediately ground to microcrystalline powder and filled into the cavity of the sample cell.

\subsubsection{Synthesis of MeAnPS(NMe $)_{2}(21)$}

MeAnP(NMe $)_{2}(33)(1.45 \mathrm{~g}, 4.68 \mathrm{mmol})$ was dissolved in toluene $(20 \mathrm{~mL})$ and sulfur $(0.16 \mathrm{~g}$, $4.98 \mathrm{mmol}$ ) was added. The mixture was stirred and heated to $110^{\circ} \mathrm{C}$ for $6 \mathrm{~h}$. The reaction was cooled to ambient temperature, filtrated and the solvent was evaporated. MeAnPS $\left(\mathrm{NMe}_{2}\right)_{2}$ (21) was obtained by crystallization from toluene as a yellow solid.

Yield: $1.28 \mathrm{~g}$ ( $3.74 \mathrm{mmol}, 80 \%)$

Empirical formula: $\mathrm{C}_{19} \mathrm{H}_{23} \mathrm{~N}_{2} \mathrm{PS}$

Molar mass: $342.13 \mathrm{~g} / \mathrm{mol}$

\section{${ }^{1} \mathrm{H}-\mathrm{NMR}$}

$\left(\mathrm{CDCl}_{3}, 300 \mathrm{MHz}\right)$ :

$\delta[p p m]=8.85-8.91\left(m, 2 \mathrm{H}, \mathrm{H}_{1,8}\right), 8.26-8.32\left(\mathrm{~m}, 2 \mathrm{H}, \mathrm{H}_{4,5}\right), 7.43-$

$7.52\left(\mathrm{~m}, 4 \mathrm{H}, \mathrm{H}_{2,3,6,7}\right), 3.12\left(\mathrm{~d},{ }^{6} J_{\mathrm{HP}}=1.6 \mathrm{~Hz}, 3 \mathrm{H}, \mathrm{AnC}_{3}\right), 2.70(\mathrm{~d}$,

$\left.{ }^{3} J_{\mathrm{HP}}=10.7 \mathrm{~Hz}, 12 \mathrm{H}, \mathrm{NC}_{3}\right)$.

\section{${ }^{13} \mathrm{C}\left\{{ }^{1} \mathrm{H}\right\}-\mathrm{NMR}$}

$\left(\mathrm{CDCl}_{3}, 76 \mathrm{MHz}\right)$ :

$\delta[\mathrm{ppm}]=136.74\left(\mathrm{~d},{ }^{4} J_{\mathrm{CP}}=4.3 \mathrm{~Hz}, 1 \mathrm{C}, \mathrm{C}_{10}\right), 133.20(\mathrm{~d}$,

$\left.{ }^{3} J_{C P}=8.9 \mathrm{~Hz}, 2 \mathrm{C}, \mathrm{C}_{4 a, 10 a}\right), 130.19\left(\mathrm{~d}^{2} \mathrm{~J}_{\mathrm{CP}}=12.4 \mathrm{~Hz}, 2 \mathrm{C}, \mathrm{C}_{8 \mathrm{a}, 9 \mathrm{a}}\right)$,

$126.63\left(d,{ }^{3} \mathrm{JP}_{\mathrm{CP}}=7.7 \mathrm{~Hz}, 2 \mathrm{C}, \mathrm{C}_{1,8}\right), 125.84\left(\mathrm{~s}, 4 \mathrm{C}, \mathrm{C}_{2,7,3,6}\right), 124.88$

$\left(d,{ }^{4} J_{C P}=7.4 \mathrm{~Hz} 2 \mathrm{C}, \mathrm{C}_{4,5}\right), 122.52\left(\mathrm{~d},{ }^{1} J_{\mathrm{CP}}=129.5 \mathrm{~Hz}, 1 \mathrm{C}, \mathrm{C}_{9}\right)$,

$37.63\left(d,{ }^{2} J_{C P}=4.2 \mathrm{~Hz}, 4 \mathrm{C}, \mathrm{N} \underline{C H}_{3}\right), 15.26\left(d,{ }^{5} J_{C P}=1.0 \mathrm{~Hz}, 1 \mathrm{C}\right.$,

$\left.\mathrm{AnC} \mathrm{CH}_{3}\right)$. 


\section{${ }^{31} \mathrm{P}\left\{{ }^{1} \mathrm{H}\right\}-\mathrm{NMR}$}

$\left(\mathrm{CDCl}_{3}, 122 \mathrm{MHz}\right): \quad \delta[\mathrm{ppm}]=73.72(\mathrm{~s})$.

\section{EI-MS}

$m / z(\%):$

$342(10)[\mathrm{M}]^{+}, 253(20)\left[\mathrm{M}-2 \times \mathrm{NMe}_{2}\right]^{+}, 189(6)\left[\mathrm{M}-\mathrm{PS}\left(\mathrm{NMe}_{2}\right)_{2}\right]^{+}$, $119(100)[\mathrm{M}-\mathrm{AnMe}, \mathrm{S}]^{+}$.

Elemental analysis

in \% (calc.):

C: 66.71 (66.64), H: 7.10 (6.77), N: 8.10 (8.18), S: 9.50 (9.36).

\subsubsection{Synthesis of MeAnPS( $\left(\mathrm{NEt}_{2}\right)_{2}(23)$}

MeAnP(NEt $)_{2}$ (34) $(1.35 \mathrm{~g}, 3.68 \mathrm{mmol})$ was dissolved in toluene $(30 \mathrm{~mL})$ and sulfur $(0.12 \mathrm{~g}$, $3.68 \mathrm{mmol}$ ) was added. The mixture was stirred and heated to $110^{\circ} \mathrm{C}$ for $6 \mathrm{~h}$. The reaction was cooled to ambient temperature, filtrated and the solvent was evaporated. MeAnPS $\left(\mathrm{NEt}_{2}\right)_{2}(\mathbf{2 3})$ was obtained by crystallization from toluene as a yellow solid.

Yield: $1.33 \mathrm{~g}$ (3.34 mmol, $91 \%)$.

Empirical formula: $\mathrm{C}_{23} \mathrm{H}_{31} \mathrm{~N}_{2} \mathrm{PS}$

Molar mass: $398.54 \mathrm{~g} / \mathrm{mol}$.

\section{${ }^{1}$ H-NMR}

$\left(\mathrm{CDCl}_{3}, 300 \mathrm{MHz}\right)$ :

$\delta[p p m]=8.99-9.06\left(m, 2 \mathrm{H}, \mathrm{H}_{1,8}\right), 8.23-8.30\left(\mathrm{~m}, 2 \mathrm{H}, \mathrm{H}_{4,5}\right)$, 7.39-

$7.48\left(m, 4 \mathrm{H}, \mathrm{H}_{2,3,6,7}\right), 3.01-3.30\left(\mathrm{~m}, 8 \mathrm{H}, \mathrm{NC}_{2} \mathrm{CH}_{3}\right), 3.11$ (d,

$\left.{ }^{6} J_{\mathrm{HP}}=1.7 \mathrm{~Hz}, 3 \mathrm{H}, \mathrm{AnCH}_{3}\right), 1.13\left(\mathrm{t},{ }^{3} \mathrm{JHH}_{\mathrm{HH}}=7.1 \mathrm{~Hz}, 12 \mathrm{H}, \mathrm{NCH}_{2} \mathrm{CH}_{3}\right)$.

${ }^{13} \mathrm{C}\left\{{ }^{1} \mathrm{H}\right\}-\mathrm{NMR}$

$\left(\mathrm{CDCl}_{3}, 75 \mathrm{MHz}\right)$

$\delta[p p m]=136.28\left(\mathrm{~d},{ }^{4} J_{\mathrm{CP}}=4.3 \mathrm{~Hz}, 1 \mathrm{C}, \mathrm{C}_{10}\right), 133.04(\mathrm{~d}$,

$\left.{ }^{3} J_{C P}=8.6 \mathrm{~Hz}, 2 \mathrm{C}, \mathrm{C}_{4 a, 10 \mathrm{a}}\right), 130.20\left(\mathrm{~d},{ }^{2} \mathrm{~J}_{\mathrm{CP}}=12.2 \mathrm{~Hz}, 2 \mathrm{C}, \mathrm{C}_{8 \mathrm{a}, 9 \mathrm{a}}\right)$,

$127.52\left(\mathrm{~d},{ }^{3} \mathrm{~J}_{\mathrm{CP}}=7.9 \mathrm{~Hz}, 2 \mathrm{C}, \mathrm{C}_{1,8}\right), 124.98\left(\mathrm{~s}, 2 \mathrm{C}, \mathrm{C}_{4,5}\right), 124.74$ (d,

$\left.{ }^{4,5} J_{C P}=9.5 \mathrm{~Hz}, 4 \mathrm{C}, \mathrm{C}_{2,7,3,6}\right), 123.33\left(\mathrm{~d},{ }^{1} J_{\mathrm{CP}}=127.8 \mathrm{~Hz}, 1 \mathrm{C}, \mathrm{C}_{9}\right)$,

$41.63\left(\mathrm{~d},{ }^{2} \mathrm{~J}_{\mathrm{CP}}=4.3 \mathrm{~Hz}, 4 \mathrm{C}, \mathrm{NCH}_{2} \mathrm{CH}_{3}\right), 15.23\left(\mathrm{~s}, 1 \mathrm{C}, \mathrm{AnCH}_{3}\right)$,

$14.50\left(d,{ }^{3} J_{C P}=3.0 \mathrm{~Hz}, 4 \mathrm{C}, \mathrm{NCH}_{2} \mathrm{CH}_{3}\right)$.

${ }^{31} \mathrm{P}\left\{{ }^{1} \mathrm{H}\right\}-\mathrm{NMR}$

$\left(\mathrm{CDCl}_{3}, 122 \mathrm{MHz}\right)$ :

$\delta[\mathrm{ppm}]=71.4(\mathrm{~s})$ 


\section{EI-MS}

$m / z(\%):$

398 (5) [M] $]^{+}, 326$ (3) [M-NEt $]^{+}, 294$ (5) [M-S, NEt $]^{+}, 253$ (14)

$\left[\mathrm{M}-2 \times \mathrm{NEt}_{2}\right]^{+}, 239$ (6) [M-2 $\times \mathrm{NEt}_{2}, \mathrm{Me}^{+}, 223$ (7) [M-S,

$\left.2 \times \mathrm{NEt}_{2}\right]^{+}, 192(5)\left[\mathrm{M}-\mathrm{PS}\left(\mathrm{NEt}_{2}\right)_{2}\right]^{+}, 175$ (100) [M-PS(NEt $\left.\left.{ }_{2}\right)_{2}, \mathrm{Me}\right]^{+}$, 104 (39) [M-S, AnMe, NEt $]_{2}^{+}$.

\section{Elemental analysis}

in \% (calc.):

C: 68.99 (69.31), H: 8.29 (7.84), N: 6.86 (7.03), S: 8.11 (8.05).

\subsubsection{Synthesis of MeAnPSe $\left(\mathrm{NMe}_{2}\right)_{2}(25)$}

MeAnP(NMe $)_{2}(33)(1.48 \mathrm{~g}, 4.77 \mathrm{mmol})$ was dissolved in toluene $(30 \mathrm{~mL})$ and selenium $(0.40 \mathrm{~g}$, $5.01 \mathrm{mmol}$ ) was added. The mixture was stirred and heated to $110^{\circ} \mathrm{C}$ for $6 \mathrm{~h}$. The reaction was cooled to ambient temperature, filtrated and the solvent was evaporated. MeAnPSe $\left(\mathrm{NMe}_{2}\right)_{2}$ (25) was obtained by crystallization from toluene as a yellow solid.

Yield: $1.64 \mathrm{~g}(4.20 \mathrm{mmol}, 88 \%)$

Empirical formula: $\mathrm{C}_{19} \mathrm{H}_{23} \mathrm{~N}_{2} \mathrm{PSe}$

Molar mass: $390.08 \mathrm{~g} / \mathrm{mol}$.

\section{${ }^{1}$ H-NMR}

$\left(\mathrm{CDCl}_{3}, 300 \mathrm{MHz}\right)$ :

$\delta[p p m]=8.85-8.89\left(m, 2 \mathrm{H}, \mathrm{H}_{1,8}\right), 8.27-8.32\left(\mathrm{~m}, 2 \mathrm{H}, \mathrm{H}_{4,5}\right), 7.42-$ $7.53\left(\mathrm{~m}, 4 \mathrm{H}, \mathrm{H}_{2,3,6,7}\right), 3.10\left(\mathrm{~d}, 3 \mathrm{H}, \mathrm{AnCH}_{3}\right), 2.72\left(\mathrm{~d},{ }^{3} \mathrm{JP}_{\mathrm{HP}}=11 \mathrm{~Hz}\right.$, $\left.12 \mathrm{H}, \mathrm{NC}_{3}\right)$.

\section{${ }^{13} \mathrm{C}\left\{{ }^{1} \mathrm{H}\right\}-\mathrm{NMR}$}

$\left(\mathrm{CDCl}_{3}, 75 \mathrm{MHz}\right)$ :

$\delta[p p m]=136.64\left(\mathrm{~d},{ }^{4} J_{\mathrm{CP}}=4.4 \mathrm{~Hz}, 1 \mathrm{C}, \mathrm{C}_{10}\right), 132.81(\mathrm{~d}$, $\left.{ }^{3} J_{C P}=8.8 \mathrm{~Hz}, 2 \mathrm{C}, \mathrm{C}_{4 a, 10 a}\right), 130.30\left(\mathrm{~d},{ }^{2} \mathrm{~J}_{\mathrm{CP}}=12.2 \mathrm{~Hz}, 2 \mathrm{C}, \mathrm{C}_{8 \mathrm{a}, 9 \mathrm{a}}\right.$ ), $\left.126.44\left(d,{ }^{3}\right)_{C P}=8.0 \mathrm{~Hz}, 2 \mathrm{C}, \mathrm{C}_{1,8}\right), 125.65\left(\mathrm{~s}, 4 \mathrm{C}, \mathrm{C}_{2,7}\right), 124.95$ (s, $\left.2 \mathrm{C}, \mathrm{C}_{3,6}\right), 124.76\left(\mathrm{~s}, 2 \mathrm{C}, \mathrm{C}_{4,5}\right), 121.84\left(\mathrm{~d},{ }^{1} \mathrm{~J}_{\mathrm{CP}}=117.5 \mathrm{~Hz}, 1 \mathrm{C}, \mathrm{C}_{9}\right)$, $38.23\left(\mathrm{~d},{ }^{2} \mathrm{~J}_{\mathrm{CP}}=3.9 \mathrm{~Hz}, 4 \mathrm{C}, \mathrm{N} \mathrm{CH}_{3}\right), 15.32\left(\mathrm{~d},{ }^{5} J_{\mathrm{CP}}=1.2 \mathrm{~Hz}, 1 \mathrm{C}\right.$, $\left.\mathrm{AnCH}_{3}\right)$.

\section{${ }^{31} \mathrm{P}\left\{{ }^{1} \mathrm{H}\right\}-\mathrm{NMR}$}

$\left(\mathrm{CDCl}_{3}, 81 \mathrm{MHz}\right)$ :

$\delta[p p m]=69.84(\mathrm{~s})$.

${ }^{77}$ Se-NMR

$\delta[\mathrm{ppm}]=-150.42\left(\mathrm{~d},{ }^{1} \mathrm{~J}_{\mathrm{SeP}}=760.6 \mathrm{~Hz}\right)$.

$\left(\mathrm{CDCl}_{3}, 57.2 \mathrm{MHz}\right)$ 


\section{EI-MS}

$\mathrm{m} / \mathrm{z}(\%)$

390 (8) [M] $]^{+}, 310$ (4) [M-Se] $]^{+}, 301$ (11) [M-2 x NMe $]^{+}, 266$ (16)

$\left[\mathrm{M}-\mathrm{Se}, \mathrm{NMe}_{2}\right]^{+}, 223$ (9) [M-Se,2 x NMe $]^{+}, 189$ (6) [M-

PSe $\left.\left(\mathrm{NMe}_{2}\right)_{2}\right]^{+}, 119(100)[\mathrm{M}-\mathrm{AnMe}, \mathrm{Se}]^{+}$.

Elemental analysis

in \% (calc.):

C: 58.29 (58.61), H: 6.18 (5.95), N: 7.07 (7.20).

\subsubsection{Synthesis of MeAnPSe(NEt $)_{2}(27)$}

$\operatorname{MeAnP}\left(\mathrm{NEt}_{2}\right)_{2}$ (34) (1.12 g, $\left.3.06 \mathrm{mmol}\right)$ was dissolved in toluene $(20 \mathrm{~mL})$ and selenium $0.24 \mathrm{~g}$, $3.06 \mathrm{mmol}$ ) was added. The mixture was stirred and heated to $110^{\circ} \mathrm{C}$ for $6 \mathrm{~h}$. The reaction was cooled to ambient temperature, filtrated and the solvent was evaporated. $\operatorname{MeAnPSe}\left(\mathrm{NEt}_{2}\right)_{2}$ (27) was obtained by crystallization from toluene as a yellow solid.

Yield: $1.16 \mathrm{~g}(2.60 \mathrm{mmol}, 85 \%)$

Empirical formula: $\mathrm{C}_{23} \mathrm{H}_{31} \mathrm{~N}_{2}$ PSe.

Molar mass: $445.44 \mathrm{~g} / \mathrm{mol}$.

${ }^{1}$ H-NMR

$\left(\mathrm{CDCl}_{3}, 300 \mathrm{MHz}\right): \quad \quad \delta[\mathrm{ppm}]=8.96-9.04\left(\mathrm{~m}, 2 \mathrm{H}, \mathrm{H}_{1,8}\right), 8.23-8.30\left(\mathrm{~m}, 2 \mathrm{H}, \mathrm{H}_{4,5}\right), 7.41-$

7.47 (m, $\left.4 \mathrm{H}, \mathrm{H}_{2,3,6,7}\right), 3.03-3.34$ (m, $\left.8 \mathrm{H}, \mathrm{NC}_{2} \mathrm{CH}_{3}\right), 3.09$ (d,

$\left.{ }^{6} J_{\mathrm{HP}}=1.7 \mathrm{~Hz}, 3 \mathrm{H}, \mathrm{AnC}_{3}\right), 1.14\left(\mathrm{t},{ }^{3} \mathrm{~J}_{\mathrm{HH}}=7.08 \mathrm{~Hz}, 12 \mathrm{H}\right.$,

$\left.\mathrm{NCH}_{2} \underline{\mathrm{H}}_{3}\right)$.

${ }^{13} \mathrm{C}\left\{{ }^{1} \mathrm{H}\right\}-\mathrm{NMR}$

$\left(\mathrm{CDCl}_{3}, 75 \mathrm{MHz}\right)$ :

$\delta[\mathrm{ppm}]=136.12\left(\mathrm{~d},{ }^{4} J_{\mathrm{CP}}=4.4 \mathrm{~Hz}, 1 \mathrm{C}, \mathrm{C}_{10}\right), 132.66(\mathrm{~d}$,

$\left.{ }^{3} J_{C P}=8.4 \mathrm{~Hz}, 2 \mathrm{C}, \mathrm{C}_{4 \mathrm{a}, 10 \mathrm{a}}\right), 130.35\left(\mathrm{~d},{ }^{2} \mathrm{~J}_{\mathrm{CP}}=12.0 \mathrm{~Hz}, 2 \mathrm{C}, \mathrm{C}_{8 \mathrm{a}, 9 \mathrm{a}}\right)$,

$127.38\left(d,{ }^{3} J_{C P}=8.1 \mathrm{~Hz}, 2 \mathrm{C}, \mathrm{C}_{1,8}\right), 124.90\left(\mathrm{~d},{ }^{4,5} \mathrm{~J}_{\mathrm{CP}}=9.5 \mathrm{~Hz}, 4 \mathrm{C}\right.$,

$\left.\mathrm{C}_{2,7,3,6}\right), 124.53\left(\mathrm{~s}, 2 \mathrm{C}, \mathrm{C}_{4,5}\right), 122.67\left(\mathrm{~d},{ }^{1} \mathrm{~J}_{\mathrm{CP}}=116.0 \mathrm{~Hz}, 1 \mathrm{C}, \mathrm{C}_{9}\right)$,

$42.17\left(\mathrm{~d},{ }^{2} J_{\mathrm{CP}}=4.1 \mathrm{~Hz}, 4 \mathrm{C}, \mathrm{NCH}_{2} \mathrm{CH}_{3}\right), 15.30$ (s, $\left.1 \mathrm{C}, \mathrm{AnCH}_{3}\right)$,

$14.39\left(\mathrm{~d},{ }^{3} J_{\mathrm{CP}}=3.0 \mathrm{~Hz}, 4 \mathrm{C}, \mathrm{NCH}_{2} \mathrm{CH}_{3}\right)$.

${ }^{31} \mathrm{P}\left\{{ }^{1} \mathrm{H}\right\}-\mathrm{NMR}$

$\left(\mathrm{CDCl}_{3}, 81 \mathrm{MHz}\right)$ :

$\delta[\mathrm{ppm}]=65.76(\mathrm{~s})$.

${ }^{77}$ Se-NMR

$\delta[\mathrm{ppm}]=-128.79\left(\mathrm{~d},{ }^{1} J_{\mathrm{SeP}}=750.6 \mathrm{~Hz}\right)$.

$\left(\mathrm{CDCl}_{3}, 57.2 \mathrm{MHz}\right)$ 


\section{EI-MS}

$\mathrm{m} / \mathrm{z}(\%)$ :

446 (4) [M] ${ }^{+}, 366$ (6) [M-Se] $]^{+}, 294(22)\left[\mathrm{M}-\mathrm{Se}, \mathrm{NEt}_{2}\right]^{+}, 223(25)$

$\left[\mathrm{M}-\mathrm{Se}, 2 \times \mathrm{NEt}_{2}\right]^{+}, 175$ (100) [M-PSe $\left(\mathrm{NEt}_{2}\right)_{2}, \mathrm{Me}^{+}, 104$ (13) [M-

$\left.\mathrm{Se}, \mathrm{AnMe}, \mathrm{NEt}_{2}\right]^{+}$.

Elemental analysis
in \% (calc.):
C: 62.08 (62.02), H: 7.50 (7.01), N: 6.19 (6.29).

\subsubsection{Synthesis of gold(I) complexes [MeAnP(NMe $\left.)_{2}(\mathrm{~S}) \mathrm{AuCl}\right](22)$, [MeAnP(NEt $\left.\left.)_{2}\right)_{2}(\mathrm{~S}) \mathrm{AuCl}\right]$ (24), [MeAnP(NMe $\left.)_{2}(\mathrm{Se}) \mathrm{AuCl}\right]$ (26), and [MeAnP(NEt $\left.)_{2}(\mathrm{Se}) \mathrm{AuCl}\right](28)$}

$1.00 \mathrm{mmol}$ of the respective phosphorylanthracene $\mathbf{2 1}, \mathbf{2 3}, \mathbf{2 5}$, and 27 as well as $0.29 \mathrm{~g}$ $(1.00 \mathrm{mmol})$ of $\left[\mathrm{Me}_{2} \mathrm{SAuCl}\right]$ were dissolved in acetone $(5-9 \mathrm{~mL})$ and heated to $56^{\circ} \mathrm{C}$ for $2 \mathrm{~min}$. Then the solutions were filtrated and stored at $-30^{\circ} \mathrm{C}$ for crystallization.

Empirical formula: $\mathrm{C}_{19} \mathrm{H}_{23} \mathrm{~N}_{2}$ PSAuCl (22), $\mathrm{C}_{23} \mathrm{H}_{31} \mathrm{~N}_{2}$ PSAuCl (24) $\mathrm{C}_{19} \mathrm{H}_{23} \mathrm{~N}_{2} \mathrm{PSeAuCl}$ (26), $\mathrm{C}_{23} \mathrm{H}_{31} \mathrm{~N}_{2} \mathrm{PSAuCl}(\mathbf{2 8})$

Molar mass: $574.86 \mathrm{~g} / \mathrm{mol}$ (22), $630.96 \mathrm{~g} / \mathrm{mol}$ (24), $621.76 \mathrm{~g} / \mathrm{mol}$ (26), $677.86 \mathrm{~g} / \mathrm{mol}$ (28). NMR data of all gold(I) complexes was identical to the respective phosphorylanthracene $\mathbf{2 1}$,

23, 25 and 27.

ESI-MS(Aceton) (22):

$\mathrm{m} / \mathrm{z}(\%)$ :

$881(100)[2 \times \mathrm{M}-\mathrm{AuCl}, \mathrm{Cl}]^{+}, 793$ (15) [2 x M-AuCl, Cl, $\left.2 \times \mathrm{NMe}_{2}\right]+, 597$

(9) $[\mathrm{M}+\mathrm{Na}]^{+}, 365(6)[\mathrm{M}-\mathrm{AuCl},+\mathrm{Na}]^{+}, 341(21)[\mathrm{M}-\mathrm{AuCl}]^{+}$.

ESI-MS(Aceton) (24):

$\mathrm{m} / \mathrm{z}(\%):$

$993(100)[2 \times \mathrm{M}-\mathrm{AuCl}, \mathrm{Cl}]^{+}, 859$ (11) $\left[2 \times \mathrm{M}-\mathrm{AuCl}, \mathrm{Cl}, 2 \times \mathrm{NEt}_{2}, \mathrm{CH}_{3}\right.$, $+\mathrm{Na}]^{+}, 653(11)[\mathrm{M}+\mathrm{Na}]^{+}, 421$ (17) $[\mathrm{M}-\mathrm{AuCl},+\mathrm{Na}]^{+}, 397$ (25) [M$\mathrm{AuCl}]^{+}$.

ESI-MS(Aceton) (26):

$m / z(\%):$

977 (100) $[2 \times \mathrm{M}-\mathrm{AuCl}, \mathrm{Cl}]^{+}, 645$ (5) $[\mathrm{M}+\mathrm{Na}]^{+}, 389(10) \quad[\mathrm{M}-\mathrm{AuCl}]^{+}$, 139 (19), 119 (5) [M-MeAn, Se, AuCl] $]^{+}$ 


\subsubsection{Synthesis of $\mathrm{HAnP}\left(\mathrm{NMe}_{2}\right)_{2}(29)$}

9-Bromoanthracene $(8.00 \mathrm{~g}, 31.1 \mathrm{mmol})$ was dissolved in diethyl ether $(130 \mathrm{~mL})$ and the solution was cooled to $-15^{\circ} \mathrm{C}$. Then $\mathrm{n}$-BuLi solution in hexane ( $\left.14.9 \mathrm{~mL}, 34.2 \mathrm{mmol}, 2.30 \mathrm{M}\right)$ was added over the course of $30 \mathrm{~min}$. The reaction was then stirred at $0^{\circ} \mathrm{C}$ for $10 \mathrm{~min}$ and cooled to $-15^{\circ} \mathrm{C}$ again. Bis(dimethylamino)chlorophosphane $(5.77 \mathrm{~g}, 37.3 \mathrm{mmol}$ ) was added drop-wise. The cooling bath was removed and the reaction was stirred for $2 \mathrm{~h}$ at ambient temperature. Then the solvent was evapoated, the crude product was dissolved in DCM $(100 \mathrm{~mL})$ and the resulting solution was filtrated over celite. Removal of the solvent afforded 9(bis(dimethylamino)phosphanyl)anthracene (29) as a red semi-solid. Crystallization from DCM at $-30^{\circ} \mathrm{C}$ gave crystals which were suitable for $\mathrm{X}$-ray structure determination.

Yield: $8.91 \mathrm{~g}(30.08 \mathrm{mmol}, 97 \%)$

Empirical formula: $\mathrm{C}_{18} \mathrm{H}_{21} \mathrm{~N}_{2} \mathrm{P}$

Molar mass: $296.14 \mathrm{~g} / \mathrm{mol}$.

${ }^{1} \mathrm{H}-\mathrm{NMR}$

$\left(\mathrm{CDCl}_{3}, 300 \mathrm{MHz}\right)$ :

$\delta[\mathrm{ppm}]=8.98-9.06\left(\mathrm{~m}, 2 \mathrm{H}, \mathrm{H}_{1,8}\right), 8.38\left(\mathrm{~s}, 1 \mathrm{H}, \mathrm{H}_{10}\right), 7.91-7.98$ $\left(m, 2 \mathrm{H}, \mathrm{H}_{4,5}\right), 7.37-7.44\left(\mathrm{~m}, 4 \mathrm{H}, \mathrm{H}_{2,3,6,7}\right), 2.60\left(\mathrm{~d},{ }^{2}{ }{ }_{\mathrm{HP}}=9.5 \mathrm{~Hz}\right.$, $\left.12 \mathrm{H}, \mathrm{NC}_{3}\right)$.

${ }^{13} \mathrm{C}\left\{{ }^{1} \mathrm{H}\right\}-\mathrm{NMR}$

$\left(\mathrm{CDCl}_{3}, 76 \mathrm{MHz}\right): \quad \delta[\mathrm{ppm}]=133.21\left(\mathrm{~d},{ }^{2} \mathrm{~J}_{\mathrm{CP}}=12.0 \mathrm{~Hz}, 2 \mathrm{C}, \mathrm{C}_{8 \mathrm{a}, 9 \mathrm{a}}\right), 130.37\left(\mathrm{~s}, 2 \mathrm{C}, \mathrm{C}_{4 \mathrm{a}, 10 \mathrm{a}}\right)$, 128.40 (s, 1 C, C 10 ), 127.88 (s, 2 C, C 4,5 ), 125.42 (s, 1 C, C 1 ), 125.21 (s, $\left.1 \mathrm{C}, \mathrm{C}_{8}\right), 123.92\left(\mathrm{~d},{ }^{5} \mathrm{JP}_{\mathrm{CP}}=2.4 \mathrm{~Hz}, 2 \mathrm{C}, \mathrm{C}_{3,6}\right), 123.53\left(\mathrm{~s}, 2 \mathrm{C}, \mathrm{C}_{2,3}\right), 40.24$ (d, ${ }^{2} J_{\mathrm{CP}}=18.0 \mathrm{~Hz}, 4 \mathrm{C}, \mathrm{NCH}_{3}$ ).

${ }^{31} \mathrm{P}\left\{{ }^{1} \mathrm{H}\right\}-\mathrm{NMR}$

$\left(\mathrm{CDCl}_{3}, 122 \mathrm{MHz}\right): \quad \delta[\mathrm{ppm}]=109.39(\mathrm{~s})$.

\section{EI-MS}

$\mathrm{m} / \mathrm{z}(\%)$ : $296(40)[\mathrm{M}]^{+}, 252(89)\left[\mathrm{M}-\mathrm{NMe}_{2}\right]^{+}, 209(50)\left[\mathrm{M}-2 \times \mathrm{NMe}_{2}\right]^{+}, 178$ (100) [M-P(NMe $\left.)_{2}\right]^{+}, 119$ (11) [M-An] $]^{+}, 76$ (12) [M-An, NMe $]^{+}$.

\subsubsection{Synthesis of $\mathrm{HAnPO}\left(\mathrm{NMe}_{2}\right)_{2}(30)$}

$\mathrm{HAnP}\left(\mathrm{NMe}_{2}\right)_{2}$ (29) $(2.26 \mathrm{~g}, 7.63 \mathrm{mmol})$ was dissolved in $\mathrm{DCM} / \mathrm{MeOH}(15 / 15 \mathrm{~mL})$ and cooled to $-15^{\circ} \mathrm{C}$. Then aqueous $\mathrm{H}_{2} \mathrm{O}_{2}$ solution $(0.78 \mathrm{~mL}, 8.01 \mathrm{mmol}, 35 \%)$ diluted with $\mathrm{MeOH}(10 \mathrm{~mL})$ was added over $30 \mathrm{~min}$. The solution was stirred at ambient temperature for $2 \mathrm{~h}$ and 
concentrated to one half of the initial volume. $\mathrm{HAnPO}\left(\mathrm{NMe}_{2}\right)_{2}$ (30) was crystallized at $-47^{\circ} \mathrm{C}$ and was obtained as an orange crystalline solid.

Yield: $1.91 \mathrm{~g}(6.12 \mathrm{~mol}, 80 \%)$

Empirical formula: $\mathrm{C}_{18} \mathrm{H}_{21} \mathrm{~N}_{2} \mathrm{OP}$.

Molar mass: $312.14 \mathrm{~g} / \mathrm{mol}$.

${ }^{1} \mathrm{H}-\mathrm{NMR}$

$\left(\mathrm{CDCl}_{3}, 300 \mathrm{MHz}\right): \quad \delta[\mathrm{ppm}]=8.94(\mathrm{~d}, 2 \mathrm{JHH}=9.1 \mathrm{~Hz}, 2 \mathrm{H}, \mathrm{H} 1,8), 8.56(\mathrm{~s}, 1 \mathrm{H}, \mathrm{H} 10), 7.98$ (d, $2 \mathrm{JHH}=8.5 \mathrm{~Hz}, 2 \mathrm{H}, \mathrm{H} 4,5), 7.40-7.59(\mathrm{~m}, 4 \mathrm{H}, \mathrm{H} 2,3,6,7), 2.72$ (d, $2 \mathrm{JHP}=9.4 \mathrm{~Hz}, 12 \mathrm{H}, \mathrm{NCH} 3$ ).

\section{${ }^{13} \mathrm{C}\left\{{ }^{1} \mathrm{H}\right\}-\mathrm{NMR}$}

$\left(\mathrm{CDCl}_{3}, 75 \mathrm{MHz}\right)$

$\delta[\mathrm{ppm}]=134.00\left(\mathrm{~d},{ }^{2} \mathrm{~J}_{\mathrm{CP}}=9.3 \mathrm{~Hz}, 2 \mathrm{C}, \mathrm{C}_{4 \mathrm{a}, 10 \mathrm{a}}\right), 132.40(\mathrm{~d}$, $\left.{ }^{4} J_{\mathrm{CP}}=3.8 \mathrm{~Hz}, 1 \mathrm{C}, \mathrm{C}_{10}\right), 130.33\left(\mathrm{~d},{ }^{2} J_{\mathrm{CP}}=12.6 \mathrm{~Hz}, 2 \mathrm{C}, \mathrm{C} 8 \mathrm{a}, 9 \mathrm{a}\right)$,

$128.22\left(\mathrm{~s}, 2 \mathrm{C}, \mathrm{C}_{4,5}\right), 125.18\left(\mathrm{~d},{ }^{3} \mathrm{~J}_{\mathrm{CP}}=7.2 \mathrm{~Hz}, 2 \mathrm{C}, \mathrm{C}_{1,8}\right), 125.71(\mathrm{~d}$, $\left.{ }^{4} J_{C P}=5.6 \mathrm{~Hz} 2 \mathrm{C}, C_{2,7}\right), 123.81\left(\mathrm{~s}, 2 \mathrm{C}, \mathrm{C}_{3,6}\right), 35.31\left(\mathrm{~d},{ }^{2} \mathrm{~J}_{\mathrm{CP}}=5.1 \mathrm{~Hz}\right.$, $\left.4 \mathrm{C}, \mathrm{NCH}_{3}\right)$.

${ }^{31} \mathrm{P}\left\{{ }^{1} \mathrm{H}\right\}-\mathrm{NMR}$

$\left(\mathrm{CDCl}_{3}, 81 \mathrm{MHz}\right)$ :

$\delta[\mathrm{ppm}]=29.14(\mathrm{~s})$.

EI-MS

$m / z(\%):$

$312(3)[\mathrm{M}]^{+}, 268(3)\left[\mathrm{M}-\mathrm{NMe}_{2}\right]^{+}, 208$ (10) [M-S, NMe $]^{+}, 208$ (5) $\left[\mathrm{M}-\mathrm{O}, 2 \times \mathrm{NMe}_{2}\right]^{+}, 178(100)\left[\mathrm{M}-\mathrm{PO}\left(\mathrm{NMe}_{2}\right)_{2}\right]^{+}, 135$ (11) [M-An]', 44 (11) [M-An, PONMe $]^{+}$.

\subsubsection{Synthesis of $\mathrm{HAnPS}\left(\mathrm{NMe}_{2}\right)_{2}(31)$}

$\mathrm{HAnP}\left(\mathrm{NMe}_{2}\right)_{2}$ (29) $(2.10 \mathrm{~g}, 7.09 \mathrm{mmol})$ was dissolved in toluene $(20 \mathrm{~mL})$ and sulfur $(0.24 \mathrm{~g}$, $7.44 \mathrm{mmol}$ ) was added. The mixture was stirred and heated to $110^{\circ} \mathrm{C}$ for $6 \mathrm{~h}$. The reaction was cooled to ambient temperature, filtrated and the solvent was evaporated. $\operatorname{HAnPS}\left(\mathrm{NMe}_{2}\right)_{2}(31)$ was obtained by crystallization from toluene as a yellow solid.

Yield: $1.82 \mathrm{~g}(5.54 \mathrm{~mol}, 78 \%)$

Empirical formula: $\mathrm{C}_{18} \mathrm{H}_{21} \mathrm{~N}_{2} \mathrm{PS}$

Molar mass: $328.24 \mathrm{~g} / \mathrm{mol}$. 


\section{${ }^{1} \mathrm{H}-\mathrm{NMR}$}

$\left(\mathrm{CDCl}_{3}, 300 \mathrm{MHz}\right): \quad \delta[\mathrm{ppm}]=8.84-8.89(\mathrm{~d}, 2 \mathrm{H}, \mathrm{H} 1,8), 8.52(\mathrm{~s}, 1 \mathrm{H}, \mathrm{H} 10), 7.93-7.99$ ( $m, 2 \mathrm{H}, \mathrm{H} 4,5), 7.40-7.55$ (m, $4 \mathrm{H}, \mathrm{H} 2,3,6,7), 2.75$ (d, 2JHP = 10.7 $\mathrm{Hz}, 12 \mathrm{H}, \mathrm{NCH} 3)$.

\section{${ }^{13} \mathrm{C}\left\{{ }^{1} \mathrm{H}\right\}-\mathrm{NMR}$}

$\left(\mathrm{CDCl}_{3}, 75 \mathrm{MHz}\right)$ :

$$
\begin{aligned}
& \delta[p p m]=133.40\left(\mathrm{~d},{ }^{3} J_{\mathrm{CP}}=9.25 \mathrm{~Hz}, 2 \mathrm{C}, \mathrm{C}_{4 \mathrm{a}, 10 \mathrm{a}}\right), 133.13(\mathrm{~d}, \\
& \left.{ }^{4} J_{\mathrm{CP}}=4.0 \mathrm{~Hz}, 1 \mathrm{C}, \mathrm{C}_{10}\right), 131.50\left(\mathrm{~d},{ }^{2} J_{\mathrm{CP}}=12.6 \mathrm{~Hz}, 2 \mathrm{C}, \mathrm{C}_{8 \mathrm{a}, 9 \mathrm{a}}\right), \\
& 129.16\left(\mathrm{~s}, 2 \mathrm{C}, \mathrm{C}_{4,5}\right), 126.68\left(\mathrm{~s}, 2 \mathrm{C}, \mathrm{C}_{2,7}\right), 126.18\left(\mathrm{~d},{ }^{3} J_{\mathrm{CP}}=7.2 \mathrm{~Hz},\right. \\
& \left.2 \mathrm{C}, \mathrm{C}_{1,8}\right), 124.89\left(\mathrm{~s}, 2 \mathrm{C}, \mathrm{C}_{3.6}\right), 124.73\left(\mathrm{~d},{ }^{1} J_{\mathrm{CP}}=126.1 \mathrm{~Hz}, 1 \mathrm{C}, \mathrm{C}_{9}\right), \\
& 37.60\left(\mathrm{~d},{ }^{2} J_{\mathrm{CP}}=4.4 \mathrm{~Hz}, 4 \mathrm{C}, \mathrm{NCH}_{3}\right) .
\end{aligned}
$$

\section{${ }^{31} \mathrm{P}\left\{{ }^{1} \mathrm{H}\right\}-\mathrm{NMR}$}

$\left(\mathrm{CDCl}_{3}, 81 \mathrm{MHz}\right)$ :

$\delta[\mathrm{ppm}]=73.20(\mathrm{~s})$.

\section{EI-MS}

$\mathrm{m} / \mathrm{z}(\%):$

$328(8)[\mathrm{M}]^{+}, 284(3)\left[\mathrm{M}-\mathrm{NMe}_{2}\right]^{+}, 252(5)\left[\mathrm{M}-\mathrm{S}, \mathrm{NMe}_{2}\right]^{+}, 239$ (38)

$\left[\mathrm{M}-2 \times \mathrm{NMe}_{2}\right]^{+}, 209(5)\left[\mathrm{M}-\mathrm{S}, 2 \times \mathrm{NMe}_{2}\right]^{+}, 178(23)[\mathrm{M}-$

$\left.\mathrm{PS}\left(\mathrm{NMe}_{2}\right)_{2}\right]^{+}, 119(100)[\mathrm{M}-\mathrm{An}, \mathrm{S}]^{+}, 76(14)\left[\mathrm{M}-\mathrm{An}, \mathrm{NMe}_{2}, \mathrm{~S}\right]^{+}$.

Elemental analysis

in \% (calc.):

C: 65.59 (65.83), H: 6.81 (6.45), N: 8.42 (8.53), S: 9.54 (9.76).

\subsubsection{Synthesis of HAnPSe( $\left(\mathrm{NMe}_{2}\right)_{2}(32)$}

$\mathrm{HAnP}\left(\mathrm{NMe}_{2}\right)_{2}$ (29) $(1.08 \mathrm{~g}, 3.65 \mathrm{mmol})$ was dissolved in toluene $(30 \mathrm{~mL})$ and selenium $(0.30 \mathrm{~g}$, $3.83 \mathrm{mmol}$ ) was added. The mixture was stirred and heated to $110^{\circ} \mathrm{C}$ for $6 \mathrm{~h}$. The reaction was cooled to ambient temperature, filtrated and the solvent was evaporated. HAnPSe( $\left(\mathrm{NMe}_{2}\right)_{2}(\mathbf{3 1})$ was obtained by crystallization from toluene as a yellow solid.

Yield: $1.07 \mathrm{~g}(2.85 \mathrm{~mol}, 78 \%)$

Empirical formula: $\mathrm{C}_{18} \mathrm{H}_{21} \mathrm{~N}_{2}$ PSe.

Molar mass: $376.06 \mathrm{~g} / \mathrm{mol}$.

${ }^{1} \mathrm{H}-\mathrm{NMR}$

$\left(\mathrm{CDCl}_{3}, 300 \mathrm{MHz}\right): \quad \delta[\mathrm{ppm}]=8.83-8.88\left(\mathrm{~m}, 2 \mathrm{H}, \mathrm{H}_{1,8}\right), 8.53\left(\mathrm{~s}, 1 \mathrm{H}, \mathrm{H}_{10}\right), 7.94-7.99$ $\left(m, 2 \mathrm{H}, \mathrm{H}_{4,5}\right), 7.40-7.56\left(\mathrm{~m}, 4 \mathrm{H}, \mathrm{H}_{2,3,6,7}\right), 2.77\left(\mathrm{~d},{ }^{2} \mathrm{~J}_{\mathrm{HP}}=11.0 \mathrm{~Hz}\right.$ $\left.12 \mathrm{H}, \mathrm{NCH}_{3}\right)$. 


\section{${ }^{13} \mathrm{C}\left\{{ }^{1} \mathrm{H}\right\}-\mathrm{NMR}$}

$\left(\mathrm{CDCl}_{3}, 76 \mathrm{MHz}\right)$ :

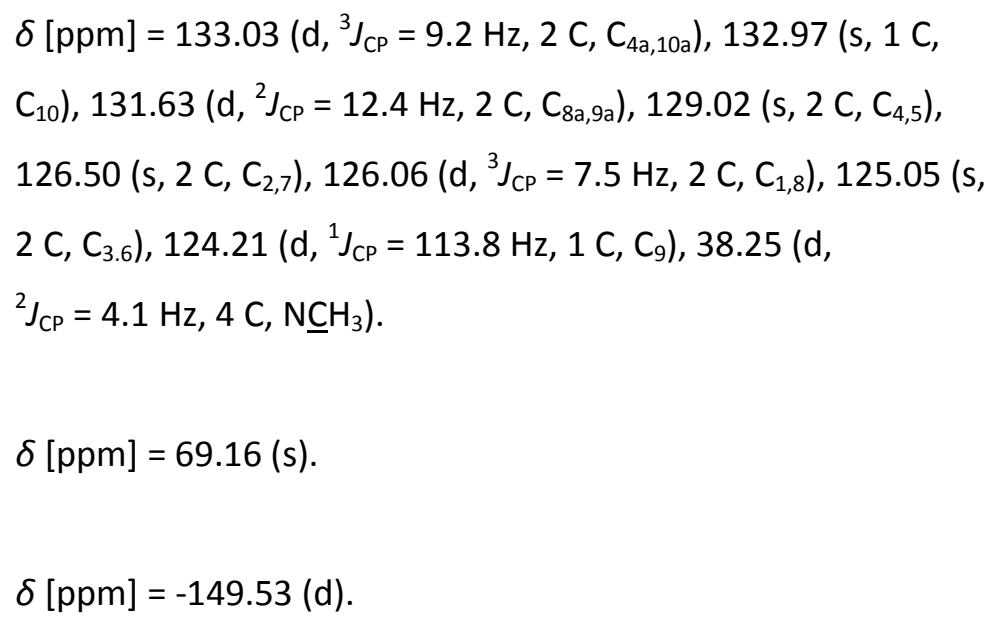

EI-MS

$m / z(\%):$ 376 (10) [M] $]^{+}, 296$ (41) [M-Se] $]^{+}, 287$ (18) [M-2 x NMe $]^{+}, 252$ (100) $\left[\mathrm{M}-\mathrm{Se}, \mathrm{NMe}_{2}\right]^{+}, 209$ (59) [M-Se, $\left.2 \times \mathrm{NMe}_{2}\right]^{+}, 178$ (23) [MPSe $\left.\left(\mathrm{NMe}_{2}\right)_{2}\right]^{+}, 119$ (96) [M-An, Se] $]^{+}$.

Elemental analysis

in \% (calc.):
C: 57.75 (57.60), H: 5.89 (5.64), N: 7.38 (7.46).

\subsubsection{Synthesis of $\mathrm{MeAnP}\left(\mathrm{NMe}_{2}\right)_{2}(33)$}

9-Bromo-10-methylanthracene $(8.00 \mathrm{~g}, 29.5 \mathrm{mmol})$ was dissolved in diethyl ether $(120 \mathrm{~mL})$ and the solution was cooled to $-15^{\circ} \mathrm{C}$. Then $\mathrm{n}$-BuLi solution in hexane $(21.9 \mathrm{~mL}, 29.5 \mathrm{mmol}, 1.35 \mathrm{M})$ was added over the course of $30 \mathrm{~min}$. The reaction was then stirred at $0^{\circ} \mathrm{C}$ for $10 \mathrm{~min}$ and cooled to $-15^{\circ} \mathrm{C}$ again. Bis(dimethylamino)chlorophosphane $(4.56 \mathrm{~g}, 28.5 \mathrm{mmol}$ ) was added drop-wise. The cooling bath was removed and the reaction was stirred for $2 \mathrm{~h}$ at ambient temperature. Then the solvent was evaporated, the crude product was dissolved in DCM $(100 \mathrm{~mL})$ and the resulting solution was filtrated over celite. Removal of the solvent afforded 9bromo-10-(bis(dimethylamino)phosphanyl)-anthracene (33) as a red oil.

Yield: $8.70 \mathrm{~g}(28.05 \mathrm{mmol}, 95 \%)$

Empirical formula: $\mathrm{C}_{19} \mathrm{H}_{23} \mathrm{~N}_{2} \mathrm{P}$

Molar mass: $310.16 \mathrm{~g} / \mathrm{mol}$. 


\section{${ }^{1} \mathrm{H}-\mathrm{NMR}$}

$\left(\mathrm{CDCl}_{3}, 300 \mathrm{MHz}\right): \quad \delta[\mathrm{ppm}]=9.03-9.10\left(\mathrm{~m}, 2 \mathrm{H}, \mathrm{H}_{1,8}\right), 8.27-8.32\left(\mathrm{~m}, 2 \mathrm{H}, \mathrm{H}_{4,5}\right), 7.38-$

$7.50\left(\mathrm{~m}, 4 \mathrm{H}, \mathrm{H}_{2,3,6,7}\right), 3.11\left(\mathrm{~d},{ }^{6} J_{\mathrm{HP}}=1.9 \mathrm{~Hz}, 3 \mathrm{H}, \mathrm{AnC}_{3}\right), 2.59$ (d,

$\left.{ }^{3} J_{\mathrm{HP}}=9.4 \mathrm{~Hz}, 12 \mathrm{H}, \mathrm{NC}_{3}\right)$.

${ }^{13} \mathrm{C}\left\{{ }^{1} \mathrm{H}\right\}-\mathrm{NMR}$

$\left(\mathrm{CDCl}_{3}, 75 \mathrm{MHz}\right)$ :

$\delta[p p m]=132.96\left(\mathrm{~d},{ }^{2} J_{\mathrm{CP}}=11.3 \mathrm{~Hz}, 2 \mathrm{C}, \mathrm{C}_{8 \mathrm{a}, 9 \mathrm{a}}\right), 129.84(\mathrm{~s}, 2 \mathrm{C}$,

$\left.\mathrm{C}_{4 \mathrm{a}, 10 \mathrm{a}}\right), 128.87$ (s, $\left.1 \mathrm{C}, \mathrm{C}_{9}\right), 127.77$ (s, $\left.1 \mathrm{C}, \mathrm{C}_{10}\right), 125.92$ (s, $1 \mathrm{C}$,

$\left.\mathrm{C}_{1}\right), 125.70\left(\mathrm{~s}, 1 \mathrm{C}, \mathrm{C}_{8}\right), 123.74\left(\mathrm{~d},{ }^{5} \mathrm{~J}_{\mathrm{CP}}=1.3 \mathrm{~Hz}, 2 \mathrm{C}, \mathrm{C}_{6,7}\right), 123.44$

$\left(\mathrm{s}, 2 \mathrm{C}, \mathrm{C}_{4,5}\right), 123.13\left(\mathrm{~d},{ }^{4} \mathrm{~J}_{\mathrm{CP}}=2.4 \mathrm{~Hz}, 2 \mathrm{C}, \mathrm{C}_{2,3}\right), 40.24(\mathrm{~d}$,

$\left.{ }^{2} J_{\mathrm{CP}}=17.9 \mathrm{~Hz}, 4 \mathrm{C}, \mathrm{NCH}_{3}\right), 13.68\left(\mathrm{~s}, 1 \mathrm{C}, \mathrm{An} \underline{\mathrm{C}} \mathrm{H}_{3}\right)$.

${ }^{31} \mathrm{P}\left\{{ }^{1} \mathrm{H}\right\}-\mathrm{NMR}$

$\left(\mathrm{CDCl}_{3}, 81 \mathrm{MHz}\right)$ :

$\delta[\mathrm{ppm}]=109.33(\mathrm{~s})$

\section{EI-MS}

$\mathrm{m} / \mathrm{z}(\%)$ :

$361(6), 310(17)[\mathrm{M}]^{+}, 305(20), 266$ (41) $\left[\mathrm{M}-\mathrm{NMe}_{2}\right]^{+}, 248(35)$,

205 (81), 191 (100) [M-P(NMe $\left.)_{2}\right]^{+}, 178$ (25) [M-MeP(NMe $\left.)_{2}\right]^{+}$,

$76(60), 60(23), 44(11)\left[\mathrm{NMe}_{2}\right]^{+}$.

Elemental analysis

in \% (calc.):

C: 73.83 (73.53), H: 8.32 (7.47), N: 6.99 (9.03).

\subsubsection{Synthesis of $\mathrm{MeAnP}\left(\mathrm{NEt}_{2}\right)_{2}(34)$}

9-Bromo-10-methylanthracene $(5.92 \mathrm{~g}, 21.85 \mathrm{mmol})$ was dissolved in diethyl ether $(120 \mathrm{~mL})$ and the solution was cooled to $-15^{\circ} \mathrm{C}$. Then $n$-BuLi solution in hexane $(9.50 \mathrm{~mL}, 21.85 \mathrm{mmol}$, $2.30 \mathrm{M}$ ) was added over the course of $30 \mathrm{~min}$. The reaction was then stirred at $0^{\circ} \mathrm{C}$ for $10 \mathrm{~min}$ and cooled to $-15^{\circ} \mathrm{C}$ again. Bis(diethylamino)chlorophosphane $(4.60 \mathrm{~g}, 21.85 \mathrm{mmol}$ ) was added drop-wise. The cooling bath was removed and the reaction was stirred for $2 \mathrm{~h}$ at ambient temperature. Then the solvent was evaporated, the crude product was dissolved in DCM $(100 \mathrm{~mL})$ and the resulting solution was filtrated over celite. Removal of the solvent afforded 9bromo-10-(bis(dimethylamino)phosphanyl)-anthracene (33) as a red oil.

Yield: $7.77 \mathrm{~g}(21.20 \mathrm{~mol}, 97 \%)$

Empirical formula: $\mathrm{C}_{23} \mathrm{H}_{31} \mathrm{~N}_{2} \mathrm{P}$

Molar mass: $366.48 \mathrm{~g} / \mathrm{mol}$. 


\section{${ }^{1} \mathrm{H}-\mathrm{NMR}$}

$\left(\mathrm{CDCl}_{3}, 300 \mathrm{MHz}\right)$ :

$\delta[\mathrm{ppm}]=9.19\left(\mathrm{dd},{ }^{3} \mathrm{~J}_{\mathrm{HH}}=9.0 \mathrm{~Hz},{ }^{4} J_{\mathrm{HH}}=3.5 \mathrm{~Hz}, 2 \mathrm{H}, \mathrm{H}_{1,8}\right), 8.31$

$\left(d,{ }^{3} J_{H H}=8.8 \mathrm{~Hz}, 2 \mathrm{H}, \mathrm{H}_{4,5}\right), 7.35-7.45\left(\mathrm{~m}, 4 \mathrm{H}, \mathrm{H}_{2,3,6,7}\right), 3.09$ (d,

$\left.{ }^{6} J_{\mathrm{HP}}=2.1 \mathrm{~Hz}, 3 \mathrm{H}, \mathrm{AnCH}_{3}\right), 2.96-3.07\left(\mathrm{~m}, 8 \mathrm{H}, \mathrm{NC}_{2} \mathrm{CH}_{3}\right), 0.99(\mathrm{t}$,

${ }^{3} J_{\mathrm{HH}}=7.1 \mathrm{~Hz}, 12 \mathrm{H}, \mathrm{NCH}_{2} \mathrm{C}_{3}$ ).

\section{${ }^{13} \mathrm{C}\left\{{ }^{1} \mathrm{H}\right\}-\mathrm{NMR}$}

$\left(\mathrm{CDCl}_{3}, 75 \mathrm{MHz}\right)$ :

$\delta[p p m]=134.78\left(d,{ }^{2} J_{C P}=12.2 \mathrm{~Hz}, 2 \mathrm{C}, \mathrm{C}_{8 \mathrm{a}, 9 \mathrm{a}}\right), 133.43(\mathrm{~d}$,

$\left.{ }^{1} J_{C P}=38.1 \mathrm{~Hz}, 1 \mathrm{C}, \mathrm{C}_{9}\right), 132.29\left(\mathrm{~d},{ }^{4} \mathrm{~J}_{\mathrm{CP}}=2.5 \mathrm{~Hz}, 1 \mathrm{C}, \mathrm{C}_{10}\right), 130.88$

(s, 2 C, C $4 \mathrm{a}, 10 \mathrm{a}), 128.46$ (s, $1 \mathrm{C}, \mathrm{C}_{1}$ ), 128.31 (s, $1 \mathrm{C}, \mathrm{C}_{8}$ ), 125.53 (s,

$\left.2 \mathrm{C}, \mathrm{C}_{4,5}\right), 125.26\left(\mathrm{~s}, 2 \mathrm{C}, \mathrm{C}_{6,7}\right), 124.27\left(\mathrm{~d},{ }^{4} \mathrm{~J}_{\mathrm{CP}}=3.0 \mathrm{~Hz}, 2 \mathrm{C}, \mathrm{C}_{2,3}\right)$,

$44.84\left(\mathrm{~d},{ }^{2} \mathrm{~J}_{\mathrm{CP}}=18.8 \mathrm{~Hz}, 4 \mathrm{C}, \mathrm{N} \underline{\mathrm{C}} \mathrm{CH}_{2} \mathrm{CH}_{3}\right), 14.64\left(\mathrm{~s}, 1 \mathrm{C}, \mathrm{AnCH}_{3}\right)$,

$14.59\left(\mathrm{~d},{ }^{3} J_{\mathrm{CP}}=3.0 \mathrm{~Hz}, 4 \mathrm{C}, \mathrm{NCH}_{2} \mathrm{CH}_{3}\right)$.

\section{${ }^{31} \mathrm{P}\left\{{ }^{1} \mathrm{H}\right\}-\mathrm{NMR}$}

$\left(\mathrm{CDCl}_{3}, 81 \mathrm{MHz}\right)$ :

$\delta[\mathrm{ppm}]=102.26(\mathrm{~s})$

\section{EI-MS}

$m / z(\%):$

$366(36)[\mathrm{M}]^{+}, 294(100)\left[\mathrm{M}-\mathrm{NEt}_{2}\right]^{+}, 223$ (94) $\left[\mathrm{M}-2 \times \mathrm{NEt}_{2}\right]^{+}, 205$

(9) $\left[\mathrm{M}-2 \times \mathrm{NEt}_{2}, \mathrm{Me}\right]^{+}, 192(12)\left[\mathrm{M}-\mathrm{P}\left(\mathrm{NEt}_{2}\right)_{2}\right]^{+}, 176$ (12) [M-

$\left.\mathrm{P}\left(\mathrm{NEt}_{2}\right)_{2}, \mathrm{Me}\right]^{+}, 161$ (6) [M-2 $\times \mathrm{NEt}_{2}, \mathrm{Me}^{+}, 104$ (13) [M-AnMe,

$\left.\mathrm{NEt}_{2}\right]^{+}$.

Elemental analysis

in \% (calc.):

C: 74.28 (75.38), H: 8.99 (8.53), N: 7.37 (7.64).

\subsubsection{Synthesis of $\mathrm{BrAnP}(\mathrm{OPh})_{2}(38)$}

9,10-Dibromoanthracene $(10.0 \mathrm{~g}, 29.7 \mathrm{mmol})$ was dissolved in diethyl ether $(250 \mathrm{~mL})$ and cooled to $-15^{\circ} \mathrm{C}$. A solution of $n$-BuLi in hexane $(12.9 \mathrm{~mL}, 29.7 \mathrm{mmol}, 2.30 \mathrm{~m})$ was added over the course of $30 \mathrm{~min}$. Then the reaction was stirred at $0^{\circ} \mathrm{C}$ for $10 \mathrm{~min}$ and then added dropwise to a solution of triphenylphosphite $(9.21 \mathrm{~g}, 29.7 \mathrm{mmol})$ in diethyl ether $(150 \mathrm{~mL})$ at $-15^{\circ} \mathrm{C}$. The reaction was stirred overnight, then degassed water $(100 \mathrm{~mL})$ was added. The layers were separated and the aqueous layer was extracted with diethyl ether $(3 \times 50 \mathrm{~mL})$. The organic layers were dried over $\mathrm{MgSO}_{4}$ and filtrated. Within minutes a yellow precipitate formed, which was also separate from the solution by a second filtration. Removal of the solvent afforded $\mathrm{BrAnP}(\mathrm{OPh})_{2}$ (38) as a yellow solid, which was further purified by recrystallization from diethyl ether. 
Yield: $9.55 \mathrm{~g}(20,2 \mathrm{mmol}, 68 \%)$

Empirical formula: $\mathrm{C}_{26} \mathrm{H}_{18} \mathrm{PBrO}_{2}$

Molar mass: $473.30 \mathrm{~g} / \mathrm{mol}$

${ }^{1} \mathrm{H}-\mathrm{NMR}$

$\left(\mathrm{CDCl}_{3}, 500 \mathrm{MHz}\right): \quad \delta[\mathrm{ppm}]=9.65\left(\mathrm{~m}, 2 \mathrm{H}, \mathrm{H}_{1,8}\right), 8.65\left(\mathrm{~d},{ }^{3} J_{\mathrm{HH}}=9.4 \mathrm{~Hz}, 2 \mathrm{H}, \mathrm{H}_{4,5}\right)$, $7.27\left(\mathrm{~m}, 4 \mathrm{H}, \mathrm{H}_{2,3,6,7}\right) 7.13\left(\mathrm{~m}, 4 \mathrm{H}, \mathrm{H}_{\text {ortho }}\right), 6.96\left(\mathrm{~m}, 4 \mathrm{H}, \mathrm{H}_{\text {meta }}\right)$, $6.80\left(\mathrm{~m}, 2 \mathrm{H}, \mathrm{H}_{\text {para }}\right)$.

${ }^{13} \mathrm{C}\left\{{ }^{1} \mathrm{H}\right\}-\mathrm{NMR}$

$\left(\mathrm{C}_{6} \mathrm{D}_{6}, 126 \mathrm{MHz}\right)$

$\delta[\mathrm{ppm}]=156.2\left(\mathrm{~d}, \mathrm{~J}_{\mathrm{CP}}=11.0 \mathrm{~Hz}, 2 \mathrm{C}, \mathrm{C}_{\text {ipso }}(\mathrm{Ph})\right), 132.2(\mathrm{~d}$, $\left.J_{\mathrm{CP}}=17.2 \mathrm{~Hz} 1 \mathrm{C}, \mathrm{C}_{10}\right), 133.4\left(\mathrm{~d}, J_{\mathrm{CP}}=29.4 \mathrm{~Hz}, 1 \mathrm{C}, \mathrm{C}_{9}\right), 130.94$ (d, $\left.J_{C P}=4.1 \mathrm{~Hz}, 2 C_{1,8}\right), 130.0\left(\mathrm{~s}, 4 \mathrm{C}, \mathrm{C}_{\text {orho,ph }}\right), 129.0\left(\mathrm{~s}, 2 \mathrm{C}, \mathrm{C}_{4,5}\right), 128.9$ (s, 4C, C meta,ph), 127.1 (d, J JP $=21.4 \mathrm{~Hz}, 2 \mathrm{C}, \mathrm{C}_{8 \mathrm{a}, 9 \mathrm{a}}$ ), 124.0 (s, 2C, $\left.C_{2,7}\right), 120.1\left(d, J_{C P}=9.3 \mathrm{~Hz} 2 \mathrm{C}, C_{3,6}\right)$.

${ }^{31} \mathrm{P}\left\{{ }^{1} \mathrm{H}\right\}-\mathrm{NMR}$

$\left(\mathrm{CDCl}_{3}, 81 \mathrm{MHz}\right)$ :

$\delta[\mathrm{ppm}]=171.0(\mathrm{~s})$

\section{EI-MS}

$m / z(\%):$

$473(77)[\mathrm{M}]^{+}, 379(100)[\mathrm{M}-\mathrm{OPh}]^{+}, 277(46)[\mathrm{M}-2 \mathrm{OPh}]^{+}$.

\subsubsection{Synthesis of (BrAn) ${ }_{2} \mathrm{OPh}(39)$}

( $\mathrm{BrAn})_{2} \mathrm{OPh}$ (39) was obtained as a byproduct of the synthesis of 38. The precipitate which formed prior to the final filtration in the synthesis of 38 (c.f. 6.4.27) turned out to be $(\mathrm{BrAn})_{2} \mathrm{OPh}$, which was dried in vacuo after being isolated by filtration.

Yield: $1.1 \mathrm{~g}(1,73 \mathrm{mmol})$

Empirical formula: $\mathrm{C}_{34} \mathrm{H}_{21} \mathrm{PBr}_{2} \mathrm{O}$

Molar mass: $636.31 \mathrm{~g} / \mathrm{mol}$

\section{${ }^{1}$ H-NMR}

$\left(\mathrm{C}_{6} \mathrm{D}_{6}, 200 \mathrm{MHz}\right): \quad \delta[\mathrm{ppm}]=8.90\left(\mathrm{~m}, 4 \mathrm{H}, 2 \mathrm{x} \mathrm{H}_{1,8}\right), 8.55\left(\mathrm{~d},{ }^{3} \mathrm{~J}_{\mathrm{HH}}=8.8 \mathrm{~Hz}, 2 \mathrm{H}\right.$, $\left.2 \mathrm{H}_{4,5}\right), 7.01\left(\mathrm{~m}, 4 \mathrm{H}, 2 \mathrm{xH}_{2,3,6,7)}\right) 6.86\left(\mathrm{~m}, 5 \mathrm{H}, \mathrm{H}_{\text {Phen }}\right)$

${ }^{31} \mathrm{P}\left\{{ }^{1} \mathrm{H}\right\}-\mathrm{NMR}$

$\left(\mathrm{CDCl}_{3}, 81 \mathrm{MHz}\right): \quad \delta[\mathrm{ppm}]=21.9(\mathrm{~s})$. 


\section{EI-MS}

$m / z(\%):$

$632(4)[\mathrm{M}]^{+}, 543(21)[\mathrm{M}-\mathrm{OPh}]^{+}, 380(100)[\mathrm{M}-\mathrm{AnBr}]^{+}, 286$ (68)

[BrAnP ${ }^{+}$.

\subsubsection{Synthesis of BrAnPS(OPh) ${ }_{2}(40)$}

$\mathrm{BrAnP}(\mathrm{OPh})_{2}$ (38) $(0.12 \mathrm{~g}, 0,25 \mathrm{mmol})$ was dissolved in toluene $(10 \mathrm{~mL})$ and sulfur $(10 \mathrm{mg}$, $0.32 \mathrm{mmol}$ ) was added. The mixture was stirred and heated to $110^{\circ} \mathrm{C}$ for $6 \mathrm{~h}$. The reaction was cooled to ambient temperature, was evaporated. $\operatorname{BrAnPS}(\mathrm{OPh})_{2}(\mathbf{4 0})$ was obtained by crystallization from toluene as a yellow solid.

Yield: $0,09 \mathrm{~g}(2,36 \mathrm{mmol}, 80 \%)$

Empirical formula: $\mathrm{C}_{26} \mathrm{H}_{18} \mathrm{PBrO}_{2} \mathrm{~S}$

Molar mass: $505.36 \mathrm{~g} / \mathrm{mol}$

\subsubsection{Synthesis of $\mathrm{BrAnPCl}_{2}$ (41)}

9,10-Dibromoanthracene $(10.0 \mathrm{~g}, 29.7 \mathrm{mmol})$ was dissolved in diethyl ether $(100 \mathrm{~mL})$ and cooled to $-15^{\circ} \mathrm{C}$. Then $n$-BuLi solution in hexane $(15.6 \mathrm{~mL}, 29.7 \mathrm{mmol}, 1.80 \mathrm{M})$ was added over the course of $30 \mathrm{~min}$. Then the reaction was stirred for $10 \mathrm{~min}$ at $0^{\circ} \mathrm{C}$, re-cooled to $-15^{\circ} \mathrm{C}$, and bis(diethylamino)chlorophosphanes $(6.27 \mathrm{~g}, 29.7 \mathrm{mmol}$ ) was added. The cooling bath was removed and the reaction was stirred at ambient temperature for $2 \mathrm{~h}$. Then the solvent was evaporated, the crude product was dissolved in DCM and filtrated for removal of lithium chloride. The solvent was evaporated again and the obrained 9-bromo-10(bis(diethylamino)phosphanyl)anthracene was dissolved in hexane $(150 \mathrm{~mL})$. The solution was cooled to $-15^{\circ} \mathrm{C}$ and gaseous $\mathrm{HCl}$ was discharged into the solution for $5 \mathrm{~min}$. Then the solution was stirred for $10 \mathrm{~min}$. This procedure was repeated three times. Then the reaction was stirred at ambient temperature for $2 \mathrm{~h}$ and the solvent was evaporated. The crude product was extracted using a Soxhlet apparatus with toluene as the extraction agent. Then the solvent was evaporated and $\mathrm{BrAnPCl} 2$ (41) was crystallized from toluene. It was obtained as a yellow solid.

Yield: $9.25 \mathrm{~g}(25.8 \mathrm{mmol}, 86 \%)$

Empirical formula: $\mathrm{C}_{14} \mathrm{H}_{8} \mathrm{PBrCl}_{2}$

Molar mass: $358.00 \mathrm{~g} / \mathrm{mol}$

${ }^{1} \mathrm{H}-\mathrm{NMR}$

$\left(\mathrm{C}_{6} \mathrm{D}_{6}, 500 \mathrm{MHz}\right)$ :

$$
\begin{aligned}
& \delta[p p m]=9.07\left(m, 2 \mathrm{H}, \mathrm{H}_{1,8}\right), 8.46\left(\mathrm{~d},{ }^{3} \mathrm{H}_{\mathrm{HH}}=8.6 \mathrm{~Hz}, 2 \mathrm{H}, \mathrm{H}_{4,5}\right), \\
& 7.12\left(\mathrm{~m}, 4 \mathrm{H}, \mathrm{H}_{2,3,6,7}\right) .
\end{aligned}
$$




\section{${ }^{13} \mathrm{C}\left\{{ }^{1} \mathrm{H}\right\}-\mathrm{NMR}$}

$\left(\mathrm{CDCl}_{3}, 75 \mathrm{MHz}\right)$ :

$$
\begin{aligned}
& \delta[\mathrm{ppm}]=134.5\left(\mathrm{~d}, J_{\mathrm{PC}}=20.9 \mathrm{~Hz}, 1 \mathrm{C}, \mathrm{C}_{9}\right), 133.1\left(\mathrm{~s}, 2 \mathrm{C}, \mathrm{C}_{1,8}\right), \\
& 130.6\left(\mathrm{~s}, 2 \mathrm{C}, \mathrm{C}_{4,5}\right), 130.3\left(\mathrm{~s}, 2 \mathrm{C}, \mathrm{C}_{8 \mathrm{a}, 9 \mathrm{a}}\right), 129.7\left(\mathrm{~s}, 2 \mathrm{C}, \mathrm{C}_{4 \mathrm{a}, 10 \mathrm{a}}\right), 129.4 \\
& \left(\mathrm{~s}, 2 \mathrm{C}, \mathrm{C}_{2,7}\right), 127.4\left(\mathrm{~s}, 2 \mathrm{C}, \mathrm{C}_{3,6}\right), 126.1\left(\mathrm{~d}, \mathrm{~J}_{\mathrm{PC}}=36.0 \mathrm{~Hz}, 1 \mathrm{C}, \mathrm{C}_{10}\right) .
\end{aligned}
$$

${ }^{31} \mathrm{P}\left\{{ }^{1} \mathrm{H}\right\}-\mathrm{NMR}$

$\left(\mathrm{CDCl}_{3}, 81 \mathrm{MHz}\right): \quad \delta[\mathrm{ppm}]=157.6(\mathrm{~s})$.

\section{EI-MS}

$\mathrm{m} / \mathrm{z}(\%)$ :

$357(100)[\mathrm{M}]^{+}, 321(23)[\mathrm{M}-\mathrm{Cl}]^{+}, 286(66)[\mathrm{M}-2 \mathrm{Cl}]$

\subsubsection{Synthesis of $\mathrm{MeANPCl}_{2}(42)$}

MeAnP(NEt $)_{2}$ (34) $(8.43 \mathrm{~g}, 23.0 \mathrm{mmol})$ was dissolved in hexane $(400 \mathrm{~mL})$ and cooled to $-15^{\circ} \mathrm{C}$. Then gaseous $\mathrm{HCl}$ was discharged into the solution for $5 \mathrm{~min}$ and the solution was stirred for $10 \mathrm{~min}$. This procedure was repeated three times. Then the cooling bath was removed, the solution was stirred for $2 \mathrm{~h}$ and the solvent was removed under reduced pressure. The crude product was extracted under inert conditions with a Soxhlet apparatus using hexane as the extraction agent. The resulting solution was concentrated to ca. $30 \mathrm{~mL}$ and $\mathrm{MeAnPCl} 2$ (42) was crystallized at $-30^{\circ} \mathrm{C}$ and was obtained as a yellow crystalline solid.

Yield: $4.15 \mathrm{~g}(14.2 \mathrm{mmol}, 62 \%)$

Empirical formula: $\mathrm{C}_{15} \mathrm{H}_{12} \mathrm{Cl}_{2} \mathrm{P}$

Molar mass: $293.13 \mathrm{~g} / \mathrm{mol}$

${ }^{1} \mathrm{H}-\mathrm{NMR}$

$\left(\mathrm{CDCl}_{3}, 300 \mathrm{MHz}\right)$ :

$$
\begin{aligned}
& \delta[p p m]=9.23\left(\mathrm{~d},{ }^{3} J_{\mathrm{HH}}=8.64 \mathrm{~Hz}, 2 \mathrm{H}, \mathrm{H}_{1,8}\right), 8.37\left(\mathrm{~d},{ }^{3} J_{\mathrm{HH}}=\right. \\
& \left.8.64 \mathrm{~Hz}, 2 \mathrm{H}, \mathrm{H}_{4,5}\right), 7.63\left(\mathrm{ddd},{ }^{3} J_{\mathrm{HH}}=8.95 \mathrm{~Hz},{ }^{3} J_{\mathrm{HH}}=8.64 \mathrm{~Hz},{ }^{4} J_{\mathrm{HH}}\right. \\
& \left.=1.85 \mathrm{~Hz}, 2 \mathrm{H}, \mathrm{H}_{2,7}\right), 7.56\left(\mathrm{ddd},{ }^{3} J_{\mathrm{HH}}=8.95 \mathrm{~Hz},{ }^{3} \mathrm{~J}_{\mathrm{HH}}=8.64 \mathrm{~Hz},\right. \\
& \left.{ }^{4} J_{\mathrm{HH}}=1.23 \mathrm{~Hz}, 2 \mathrm{H}, \mathrm{H}_{3,6}\right), 3.17\left(\mathrm{~s}, 3 \mathrm{H}, \mathrm{CH}_{3}\right) .
\end{aligned}
$$

\section{${ }^{13} \mathrm{C}\left\{{ }^{1} \mathrm{H}\right\}-\mathrm{NMR}$}

$\left(\mathrm{C}_{6} \mathrm{D}_{6}, 126 \mathrm{MHz}\right)$

$\delta[\mathrm{ppm}]=139.8\left(\mathrm{~d},{ }^{4} J_{\mathrm{CP}}=0.88 \mathrm{~Hz}, 1 \mathrm{C}, \mathrm{C}_{10}\right), 133.7\left(\mathrm{~d},{ }^{2} J_{\mathrm{CP}}=20.87\right.$ $\left.\mathrm{Hz}, 2 \mathrm{C}, \mathrm{C}_{8 \mathrm{a}, 9 \mathrm{a}}\right), 129.8\left(\mathrm{~d},{ }^{3} \mathrm{~J}_{\mathrm{CP}}=3.60 \mathrm{~Hz}, 2 \mathrm{C}, \mathrm{C}_{4 \mathrm{a}}, 10 \mathrm{a}\right), 127.5$ (d, ${ }^{1} J_{\mathrm{CP}}$ $\left.=74.83,1 \mathrm{C}, \mathrm{C}_{9}\right) 126.8\left(\mathrm{~d},{ }^{4} \mathrm{~J}_{\mathrm{CP}}=3.82 \mathrm{~Hz}, 2 \mathrm{C}, \mathrm{C}_{2,7}\right), 126.2\left(\mathrm{~d},{ }^{3} \mathrm{JP}_{\mathrm{CP}}\right.$ $\left.=36.17 \mathrm{~Hz}, 1 \mathrm{C}, \mathrm{C}_{1,8}\right), 125.8\left(\mathrm{~d},{ }^{4} \mathrm{~J}_{\mathrm{CP}}=1.31 \mathrm{~Hz}, 2 \mathrm{C}, \mathrm{C}_{4,5}\right), 125.4(\mathrm{~d}$, $\left.{ }^{5} J_{C P}=2.10 \mathrm{~Hz}, 2 \mathrm{C}, \mathrm{C}_{3,6}\right), 15.30\left(\mathrm{~s}, 1 \mathrm{C}, \mathrm{CH}_{3}\right)$.

\section{${ }^{31} \mathrm{P}\left\{{ }^{1} \mathrm{H}\right\}-N M R$}

$\left(\mathrm{CDCl}_{3}, 121 \mathrm{MHz}\right)$ :

$\delta[\mathrm{ppm}]=159.4(\mathrm{~s})$. 


\section{EI-MS}

$m / z(\%):$

$292(100)[\mathrm{M}]^{+}, 257(75)[\mathrm{M}-\mathrm{Cl}]^{+}, 226(73)[\mathrm{M}-2 \mathrm{Cl}]^{+}$,

$189(40)\left[\mathrm{M}-\mathrm{PCl}_{2}\right]^{+}$.

\subsubsection{Synthesis of $\left.\mathrm{BrAnP}\left(\mathrm{OC}_{2} \mathrm{H}_{4}\right)_{2} \mathrm{OMe}\right)_{2}(43)$}

$\mathrm{BrAnPCl}_{2}$ (41) $(0,80 \mathrm{~g}, 2.24 \mathrm{mmol})$ was dissolved diethyl ether $(30 \mathrm{~mL})$ and dry $\mathrm{NEt}_{3}(1.13 \mathrm{~g}$, $11.17 \mathrm{mmol}$ ) was added. The mixture was cooled to $-15^{\circ} \mathrm{C}$ and diethylenegycolemonomethyl ether $(0.55 \mathrm{~g}, 4.50 \mathrm{mmol})$ dissolved in diethyl ether $(10 \mathrm{~mL})$ was added drop-wise. After the addition was completed, the reaction was stirred for $2 \mathrm{~h}$, then the solution was concentrated to ca. $1 / 2$ of the initial volume. The solution was filtrated for removal of the formed diethylammonium chloride. The solvent was evaporated and the product was dried under reduced pressure. $\left.\mathrm{BrAnP}\left(\mathrm{OC}_{2} \mathrm{H}_{4}\right)_{2} \mathrm{OMe}\right)_{2}(\mathbf{4 3})$ was obtained as a yellow amorphous solid.

Yield: $1.07 \mathrm{~g}$ (2,04 mmol, $91 \%)$

Empirical formula: $\mathrm{C}_{24} \mathrm{H}_{30} \mathrm{PO}_{6} \mathrm{Br}$

Molar mass: $525.37 \mathrm{~g} / \mathrm{mol}$

${ }^{1} \mathrm{H}-\mathrm{NMR}$

$\left(\mathrm{CDCl}_{3}, 300 \mathrm{MHz}\right)$ :

$\delta[\mathrm{ppm}]=9.27\left(\mathrm{~d},{ }^{3} \mathrm{~J}_{\mathrm{HH}}=8.9 \mathrm{~Hz}, 2 \mathrm{H}, \mathrm{H}_{1,8}\right) 8.51\left(\mathrm{~d},{ }^{3} J_{\mathrm{HH}}=8.8 \mathrm{~Hz}\right.$, $\left.2 \mathrm{H}, \mathrm{H}_{4,5}\right), 7.45\left(\mathrm{~m}, 4 \mathrm{H}, \mathrm{H}_{2,3,6,7)}\right), 3.69\left(\mathrm{~m}, 4 \mathrm{H}, 2 \times \mathrm{CH}_{2}\right), 3.61(\mathrm{~m}, 4 \mathrm{H}$, $\left.2 \times \mathrm{C}_{2}\right), 3.56\left(\mathrm{~m}, 4 \mathrm{H}, 2 \times \mathrm{C}_{2}\right), 3.51\left(\mathrm{~m}, 4 \mathrm{H}, 2 \times \underline{\mathrm{CH}}_{2}\right), 3.35(\mathrm{~s}, 6 \mathrm{H}$,

$\mathrm{C}_{3}$

${ }^{13} \mathrm{C}\left\{{ }^{1} \mathrm{H}\right\}-\mathrm{NMR}$

$\left(\mathrm{CDCl}_{3}, 75 \mathrm{MHz}\right)$ :

$\delta[\mathrm{ppm}]=133.3\left(\mathrm{~d}, J_{\mathrm{CP}}=9.5 \mathrm{~Hz}, 2 \mathrm{C}, \mathrm{C}_{1,8}\right), 130.0(\mathrm{~d}, 1 \mathrm{C}$, $\left.J_{C P}=10.6 \mathrm{~Hz}, \mathrm{C}_{10}\right), 128.3\left(\mathrm{~d}, J_{\mathrm{CP}}=4.8 \mathrm{~Hz}, 2 \mathrm{C}, \mathrm{C}_{4 \mathrm{a}, 10 \mathrm{a}}\right), 127.6$ (d, $\left.J_{C P}=4.1 \mathrm{~Hz}, 2 \mathrm{C}, \mathrm{C}_{4,5}\right), 127.2\left(\mathrm{~s}, 2 \mathrm{C}, \mathrm{C}_{8 \mathrm{a}, 9 \mathrm{a}}\right), 126.6\left(\mathrm{~d}, J_{\mathrm{CP}}=10.3 \mathrm{~Hz}\right.$

1C, $\left.C_{9}\right), 126.5\left(s, 2 C, C_{3,6}\right), 125.9\left(s, 2 C, C_{2,7)}\right.$.

${ }^{31} \mathrm{P}\left\{{ }^{1} \mathrm{H}\right\}-N M R$

$\left(\mathrm{CDCl}_{3}, 81 \mathrm{MHz}\right)$ :

$\delta[\mathrm{ppm}]=176.9(\mathrm{~s})$.

EI-MS

$m / z(\%):$

524 (6) [M] ${ }^{+}, 466$ (46) [M-EtOMe] $]^{+}, 406$ (100) [M-OEtOEtOMe] ${ }^{+}$, 319 (22) [BrAnPOO] 


\subsubsection{Synthesis of $\mathrm{BrAnP}\left(\mathrm{OC}_{3} \mathrm{H}_{6} \mathrm{SMe}\right)_{2}(44)$}

$\mathrm{BrAnPCl}_{2}$ (41) $(0.60 \mathrm{~g}, 1.68 \mathrm{mmol})$ was dissolved diethyl ether $(30 \mathrm{~mL})$ and dry $\mathrm{NEt}_{3}(1.0 \mathrm{~g}$, $10.0 \mathrm{mmol})$ was added. The mixture was cooled to $-15^{\circ} \mathrm{C}$ and 3 -methylthiopropanol $(0.37 \mathrm{~g}$, $3.52 \mathrm{mmol})$ dissolved in diethyl ether $(10 \mathrm{~mL})$ was added drop-wise. After the addition was completed, the reaction was stirred for $2 \mathrm{~h}$, then the solution was concentrated to ca. $1 / 2$ of the initial volume. The solution was filtrated for removal of the formed diethylammonium chloride. The solvent was evaporated and the product was dried under reduced pressure. $\operatorname{BrAnP}\left(\mathrm{OC}_{3} \mathrm{H}_{6} \mathrm{SMe}\right)_{2}(\mathbf{4 4})$ was obtained as a yellow oil.

Yield: $0.55 \mathrm{~g}(1.12 \mathrm{mmol}, 63 \%)$

Empirical formula: $\mathrm{C}_{22} \mathrm{H}_{26} \mathrm{PS}_{2} \mathrm{O}_{2} \mathrm{Br}$

Molar mass: $497.45 \mathrm{~g} / \mathrm{mol}$

${ }^{1} \mathrm{H}-\mathrm{NMR}$

$\left(\mathrm{CDCl}_{3}, 300 \mathrm{MHz}\right): \quad \delta[\mathrm{ppm}]=9.27\left(\mathrm{~m}, 2 \mathrm{H}, \mathrm{H}_{1,8}\right), 8.66\left(\mathrm{~m}, 2 \mathrm{H}, \mathrm{H}_{4,5}\right), 7.63(\mathrm{~m}, 4 \mathrm{H}$, $\left.\mathrm{H}_{2,3,6,7)}\right), 19\left(\mathrm{~m}, 4 \mathrm{H}, 2 \times \mathrm{POC}_{2}\right), 2.55\left(\mathrm{~m}, 4 \mathrm{H}, 2 \times \mathrm{SC}_{2}\right), 2.15$ (s, $\left.6 \mathrm{H}, 2 \times \mathrm{SC}_{3}\right), 1.93\left(\mathrm{~m}, 4 \mathrm{H}, 2 \times \mathrm{CC}_{2} \mathrm{C}\right)$.

${ }^{31} \mathrm{P}\left\{{ }^{1} \mathrm{H}\right\}-\mathrm{NMR}$

$\left(\mathrm{CDCl}_{3}, 81 \mathrm{MHz}\right): \quad \delta[\mathrm{ppm}]=175.9(\mathrm{~s})$.

\section{EI-MS}

$\mathrm{m} / \mathrm{z}(\%): \quad 497(3)[\mathrm{M}]^{+}, 406(100)\left[\mathrm{M}-\mathrm{C}_{3} \mathrm{H}_{6} \mathrm{SMe}\right]^{+}, 287(56)[\mathrm{BrAnP}]^{+}$.

\subsubsection{Synthesis of BrAnP(16-crown-6) (45)}

$\mathrm{BrAnPCl}_{2}$ (41) $(0.30 \mathrm{~g}, 0.84 \mathrm{mmol})$ was dissolved diethyl ether $(20 \mathrm{~mL})$ and dry $\mathrm{NEt}_{3}(0.6 \mathrm{~g}$, $7.3 \mathrm{mmol})$ was added. The mixture was cooled to $-15^{\circ} \mathrm{C}$ and pentaethylenegycol $(0.20 \mathrm{~g}$, $0.84 \mathrm{mmol})$ dissolved in diethyl ether $(5 \mathrm{~mL})$ was added drop-wise. After the addition was completed, the reaction was stirred for $2 \mathrm{~h}$, then the solution was concentrated to ca. $1 / 2$ of the initial volume. The solution was filtrated for removal of the formed diethylammonium chloride. The solvent was evaporated and the product was dried under reduced pressure. $\operatorname{BrAnP}(16-$ crown-6) (45) was obtained as a yellow oil.

Yield: $0.30 \mathrm{~g}(0,57 \mathrm{mmol}, 68 \%)$

Empirical formula: $\mathrm{C}_{24} \mathrm{H}_{28} \mathrm{PO}_{6} \mathrm{Br}$

Molar mass: $523.35 \mathrm{~g} / \mathrm{mol}$

${ }^{1} \mathrm{H}-\mathrm{NMR}$

$\left(\mathrm{CDCl}_{3}, 300 \mathrm{MHz}\right): \quad \delta[\mathrm{ppm}]=9.26\left(\mathrm{~m}, 2 \mathrm{H}, \mathrm{H}_{1,8}\right), 8.60\left(\mathrm{~m}, 2 \mathrm{H}, \mathrm{H}_{4,5}\right), 7.20(\mathrm{~m}, 4 \mathrm{H}$, $\left.\mathrm{H}_{2,3,6,7}\right), 3.76-3.35\left(\mathrm{~m}, 2 \mathrm{OH}, 10 \times \mathrm{CH}_{2}\right)$. 


\section{${ }^{31} \mathrm{P}\left\{{ }^{1} \mathrm{H}\right\}-\mathrm{NMR}$}

$\left(\mathrm{CDCl}_{3}, 81 \mathrm{MHz}\right)$ :

$$
\delta[\mathrm{ppm}]=178.8(\mathrm{~s})
$$

\section{El-MS}

$\mathrm{m} / \mathrm{z}(\%)$ :

$$
523(5)[\mathrm{M}]^{+}, 320(100)[\mathrm{AnPOO}]^{+} .
$$

\subsubsection{Synthesis of $\mathrm{BrAnP}(\mathrm{Pic})_{2}$ (46)}

$\mathrm{BrAnPCl}_{2}$ (41) $(0.6 \mathrm{~g}, 1,68 \mathrm{mmol})$ was dissolved in THF $(20 \mathrm{~mL})$ and a solution of PicTMS $(0,55 \mathrm{~g}$, $3,35 \mathrm{mmol})$ in THF $(10 \mathrm{~mL})$ was added at $-78^{\circ} \mathrm{C}$. The mixture was stirred at ambient temperature overnight. Then the reaction was heated to $50^{\circ} \mathrm{C}$ for $20 \mathrm{~min}$. The solution was filtrated, the solvent was evaporated and the product was dried under reduced pressure. $\mathrm{BrAnP}(\mathrm{Pic})_{2}(\mathbf{4 6})$ was obtained as a red semi-solid.

Yield: $0.58 \mathrm{~g}(1,24 \mathrm{mmol}, 74 \%)$

Empirical formula: $\mathrm{C}_{26} \mathrm{H}_{20} \mathrm{PN}_{2} \mathrm{Br}$

Molar mass: $471.33 \mathrm{~g} / \mathrm{mol}$

${ }^{1} \mathrm{H}-\mathrm{NMR}$

$\left(\mathrm{CDCl}_{3}, 300 \mathrm{MHz}\right)$ :

$\delta[p p m]=9.34\left(\mathrm{~m}, 2 \mathrm{H}, \mathrm{H}_{1,8}\right) 8.70\left(\mathrm{~d},{ }^{3} J_{\mathrm{HH}}=9.1 \mathrm{~Hz}, 2 \mathrm{H}, \mathrm{H}_{4,5}\right), 7.69$ ( $m, 4 \mathrm{H}, \mathrm{H}_{2,3,6,7)} 7.45\left(\mathrm{~m}, 4 \mathrm{H}, \mathrm{H}_{\text {Pic }}\right), 7.05\left(\mathrm{~d}, J_{\mathrm{HH}}=8.0 \mathrm{~Hz}, 2 \mathrm{H}, \mathrm{H}_{\text {Pic }}\right.$ ),

$3.47\left(\mathrm{dd}, \mathrm{J}_{\mathrm{PH}}=14.6 \mathrm{~Hz},{ }^{3} \mathrm{~J}=2.1 \mathrm{~Hz}, 4 \mathrm{H}, \mathrm{C}_{2}\right)$.

${ }^{13} \mathrm{C}\left\{{ }^{1} \mathrm{H}\right\}-\mathrm{NMR}$

$\left(\mathrm{CDCl}_{3}, 75 \mathrm{MHz}\right)$ :

$\delta[\mathrm{ppm}]=137.0\left(\mathrm{~d},{ }^{2} \mathrm{~J}_{\mathrm{CP}}=82.5 \mathrm{~Hz}, 2 \mathrm{C}, \mathrm{C}_{8 \mathrm{a}, 9 \mathrm{a}}\right), 133.7\left(\mathrm{~s}, 1 \mathrm{C}, \mathrm{C}_{10}\right)$ $133.2\left(d,{ }^{2} J_{C P}=7.9 \mathrm{~Hz}, 2 \mathrm{C}, \mathrm{C}_{4 \mathrm{a}, 10 \mathrm{a}}\right), 131.5\left(\mathrm{~d},{ }^{1} \mathrm{~J}_{\mathrm{CP}}=11.6 \mathrm{~Hz}, 2 \mathrm{C}\right.$, $\mathrm{C}_{\text {ipso,Py }}$ ), $130.8\left(\mathrm{~d},{ }^{2} \mathrm{~J}_{\mathrm{CP}}=10.5 \mathrm{~Hz}, 4 \mathrm{C}, \mathrm{C}, \mathrm{Py}\right), 130.6(\mathrm{~s}, 2 \mathrm{C}, \mathrm{C}$, Py$)$, 129.1 (s, 1C, $\left.C_{4,5}\right), 128.5\left(\mathrm{~d},{ }^{3} \mathrm{~J}_{\mathrm{CP}}=12.6 \mathrm{~Hz}, 4 \mathrm{C}, \mathrm{C}, \mathrm{Py}\right), 127.3$ (d, $\left.{ }^{3} J_{C P}=10.1 \mathrm{~Hz}, 2 \mathrm{C}, \mathrm{C}_{1,8}\right), 125.7(\mathrm{~s}, 2 \mathrm{C}, \mathrm{C} 2,7), 52.2\left(\mathrm{~s}, 2 \mathrm{C}, \underline{\mathrm{CH}}_{2}\right)$,

\section{${ }^{31} \mathrm{P}\left\{{ }^{1} \mathrm{H}\right\}-N M R$}

$\left(\mathrm{CDCl}_{3}, 81 \mathrm{MHz}\right): \quad \delta[\mathrm{ppm}]=-21.1(\mathrm{~s})$.

EI-MS

$\mathrm{m} / \mathrm{z}(\%)$ : 


\subsubsection{Synthesis of MeAnP(Pic) 2 (47)}

$\mathrm{MeAnPCl}_{2}$ (42) $(0.47 \mathrm{~g}, 1.60 \mathrm{mmol})$ was dissolved in THF $(20 \mathrm{~mL})$ and a solution of PicTMS $(0.53 \mathrm{~g}, 3.21 \mathrm{mmol})$ in THF $(10 \mathrm{~mL})$ was added at $-78^{\circ} \mathrm{C}$. The mixture was stirred at ambient temperature overnight. Then the reaction was heated to $50^{\circ} \mathrm{C}$ for $20 \mathrm{~min}$. The solution was filtrated, the solvent was evaporated and the product was dried under reduced pressure. $\mathrm{BrAnP}(\mathrm{Pic})_{2}$ (46) was obtained as a red semi-solid.

Yield: $044 \mathrm{~g}(1,08 \mathrm{mmol}, 67 \%)$

Empirical formula: $\mathrm{C}_{27} \mathrm{H}_{23} \mathrm{~N}_{2} \mathrm{P}$

Molar mass: $406.46 \mathrm{~g} / \mathrm{mol}$.

${ }^{1} \mathrm{H}-\mathrm{NMR}$

$\left(\mathrm{CDCl}_{3}, 300 \mathrm{MHz}\right)$ : $\delta[\mathrm{ppm}]=8.36\left(\mathrm{~d},{ }^{3} \mathrm{H}_{\mathrm{HH}}=8.9 \mathrm{~Hz}, 2 \mathrm{H}, \mathrm{H}_{1,8}\right) 8.20\left(\mathrm{~d},{ }^{3} \mathrm{~J}_{\mathrm{HH}}=9.2 \mathrm{~Hz}\right.$, $\left.2 \mathrm{H}, \mathrm{H}_{4,5}\right), 7.55\left(\mathrm{~m}, 4 \mathrm{H}, \mathrm{H}_{2,3,6,7)}\right), 7.40\left(\mathrm{~m}, 4 \mathrm{H}, \mathrm{H}_{\mathrm{py}}\right), 7.29(\mathrm{~m}, 2 \mathrm{H}$, $\left.\mathrm{H}_{\mathrm{py}}\right), 3.55\left(\mathrm{~d}, \mathrm{~J}_{\mathrm{PH}}=12.2 \mathrm{~Hz}, 4 \mathrm{H}, \mathrm{C}_{2}\right), 3.21\left(\mathrm{~s}, 3 \mathrm{H}, \mathrm{C}_{3}\right)$.

${ }^{31} \mathrm{P}\left\{{ }^{1} \mathrm{H}\right\}-\mathrm{NMR}$

$\left(\mathrm{CDCl}_{3}, 81 \mathrm{MHz}\right)$ :

$\delta[\mathrm{ppm}]=-26.3(\mathrm{~s})$

\section{EI-MS}

m/z (\%): $\quad 406(7)[M]^{+}, 314(100)[\mathrm{M}-\mathrm{Pic}]^{+}, 192(30)\left[\mathrm{M}-\mathrm{PPic}_{2}\right]^{+}$.

\subsubsection{Synthesis of $\mathrm{MeAnPH}_{2}$ (48)}

$\mathrm{MeAnPCl}_{2}$ (42) (1.46 g, $\left.4.98 \mathrm{mmol}\right)$ was suspended in diethyl ether $(120 \mathrm{~mL})$ and added to a suspension of $\mathrm{LiAlH}_{4}(0.474 \mathrm{~g}, 12.5 \mathrm{mmol})$ in diethyl ether $(100 \mathrm{~mL})$ at $-78^{\circ} \mathrm{C}$. The reaction was stirred at $-78^{\circ} \mathrm{C}$ for $2 \mathrm{~h}$, then the cooling bath was removed and the reaction was heated to $35^{\circ} \mathrm{C}$ for $30 \mathrm{~min}$. Then degassed $\mathrm{NH}_{4} \mathrm{Cl}$ solution $(45 \mathrm{~mL})$ was added and the layers were separated. The aqueous layer was extracted with diethyl ether $(3 \times 30 \mathrm{~mL})$. The united organic layers were dried over $\mathrm{MgSO}_{4}$, filtrated and the solvent was removed. $\mathrm{MeAnPH}$ (48) was obtained as a yellow solid.

Yield: $0.85 \mathrm{~g}$ (3.80 mmol, 76\%)

Empirical formula: $\mathrm{C}_{15} \mathrm{H}_{14} \mathrm{P}$

Molar mass: $224.24 \mathrm{~g} / \mathrm{mol}$. 


\section{${ }^{1} \mathrm{H}-\mathrm{NMR}$}

$\left(\mathrm{THF}_{8}, 500 \mathrm{MHz}\right)$ :

$\delta[\mathrm{ppm}]=8.59\left(\mathrm{dd},{ }^{3} J_{\mathrm{HH}}=7.0 \mathrm{~Hz},{ }^{4} \mathrm{~J}_{\mathrm{HH}}=1.5 \mathrm{~Hz}, 2 \mathrm{H}, \mathrm{H}_{1,8}\right), 8.36$

(dd, $\left.{ }^{3} J_{\mathrm{HH}}=8.5 \mathrm{~Hz},{ }^{4} J_{\mathrm{HH}}=1.5 \mathrm{~Hz}, 2 \mathrm{H}, \mathrm{H}_{4,5}\right), 7.48-7.57(\mathrm{~m}, 4 \mathrm{H}$, $\left.\mathrm{H}_{2,3,6,7}\right), 4.36\left(\mathrm{~d},{ }^{1} J_{\mathrm{PH}}=204.3 \mathrm{~Hz}, 2 \mathrm{H}, \mathrm{PH}_{2}\right), 3.09 \mathrm{ppm}(\mathrm{s}, 3 \mathrm{H}, \mathrm{Me})$.

\section{${ }^{13} \mathrm{C}\left\{{ }^{1} \mathrm{H}\right\}-\mathrm{NMR}$}

(THF- $\left.d_{8}, 126 \mathrm{MHz}\right)$ :

$\delta[p p m]=139.8\left(\mathrm{~d},{ }^{4} \mathrm{~J}_{\mathrm{CP}}=0.88 \mathrm{~Hz}, 1 \mathrm{C}, \mathrm{C}_{10}\right), 133.7\left(\mathrm{~d},{ }^{2} \mathrm{~J}_{\mathrm{CP}}=20.87\right.$ $\left.\mathrm{Hz}, 2 \mathrm{C}, \mathrm{C}_{8 \mathrm{a}}, 9 \mathrm{a}\right), 129.8\left(\mathrm{~d},{ }^{3} \mathrm{~J}_{\mathrm{CP}}=3.60 \mathrm{~Hz}, 2 \mathrm{C}, \mathrm{C}_{4 \mathrm{a}}, 10 \mathrm{a}\right), 127.5$ (d, ${ }^{1} \mathrm{JP}_{\mathrm{CP}}$ $\left.=74.83,1 \mathrm{C}, \mathrm{C}_{9}\right) 126.8\left(\mathrm{~d},{ }^{4} \mathrm{~J}_{\mathrm{CP}}=3.82 \mathrm{~Hz}, 2 \mathrm{C}, \mathrm{C}_{2,7}\right), 126.2\left(\mathrm{~d},{ }^{3} J_{\mathrm{CP}}\right.$ $\left.=36.17 \mathrm{~Hz}, 1 \mathrm{C}, \mathrm{C}_{1,8}\right), 125.8\left(\mathrm{~d},{ }^{4} J_{\mathrm{CP}}=1.31 \mathrm{~Hz}, 2 \mathrm{C}, \mathrm{C}_{4,5}\right), 125.4(\mathrm{~d}$, $\left.{ }^{5} \mathrm{JP}_{\mathrm{CP}}=2.10 \mathrm{~Hz}, 2 \mathrm{C}, \mathrm{C}_{3,6}\right), 15.30\left(\mathrm{~s}, 1 \mathrm{C}, \mathrm{CH}_{3}\right)$.

\section{${ }^{31} \mathrm{P}\left\{{ }^{1} \mathrm{H}\right\}-\mathrm{NMR}$}

(THF- $d_{8}, 201 \mathrm{MHz}$ ):

$\delta[\mathrm{ppm}]=-160.96(\mathrm{~s})$.

${ }^{31}$ P-NMR

(THF- $\left.d_{8}, 201 \mathrm{MHz}\right)$ :

$\delta[\mathrm{ppm}]=-160.96\left(\mathrm{t},{ }^{1} J_{H P}=204.4\right)$

\section{EI-MS}

$m / z(\%):$

$224(45)[\mathrm{M}]^{+}, 192(100)\left[\mathrm{M}-\mathrm{PH}_{2}\right]$.

\subsubsection{Synthesis of $\mathrm{MeAnP}\left(\mathrm{CH}_{2} \mathrm{Ph}\right)_{2}(49)$}

$\mathrm{MeAnPH}_{2}(48)(0.65 \mathrm{~g}, 2.90 \mathrm{mmol})$ was dissolved in diethyl ether $(40 \mathrm{~mL})$ and cooled to $-78^{\circ} \mathrm{C}$. Then a solution of MeLi in diethyl ether $(1.73 \mathrm{~mL}, 2.90 \mathrm{mmol}, 1.68 \mathrm{M})$ was added over the course of $30 \mathrm{~min}$. Then benzyl bromide $(0.50 \mathrm{~g}, 2.90 \mathrm{mmol})$ was added drop-wise. The reaction was stirred at ambient temperature for $1 \mathrm{~h}$, then cooled to $-78^{\circ} \mathrm{C}$ again. A solution of MeLi in diethyl ether ( $1.73 \mathrm{~mL}, 2.90 \mathrm{mmol}, 1.68 \mathrm{M}$ ) was added over the course of $30 \mathrm{~min}$, followed by the addition of benzyl bromide $(0.50 \mathrm{~g}, 2.90 \mathrm{mmol})$. The reaction was then stirred at ambient temperature overnight. The solvent was removed and the crude product was dissolved in DCM and filtrated. Removal of the solvent afforded $\mathrm{MeAnP}\left(\mathrm{CH}_{2} \mathrm{Ph}\right)_{2}(\mathbf{4 9})$ as an amorphous solid. Crystallization from toluene afforded crystals which were suitable for $\mathrm{X}$-ray diffraction experiments.

Yield: $0.60 \mathrm{~g}(1,48 \mathrm{mmol}, 51 \%)$

Empirical formula: $\mathrm{C}_{29} \mathrm{H}_{25} \mathrm{P}$

Molar mass: $404.48 \mathrm{~g} / \mathrm{mol}$. 


\section{${ }^{1} \mathrm{H}-\mathrm{NMR}$}

$\left(\mathrm{C}_{6} \mathrm{D}_{6}, 300 \mathrm{MHz}\right): \quad \delta[\mathrm{ppm}]=8.66\left(\mathrm{~m}, 2 \mathrm{H}, \mathrm{H}_{1,8}\right), 8.17\left(\mathrm{~m}, 2 \mathrm{H}, \mathrm{H}_{4,5}\right), 7.31(\mathrm{~m}, 4 \mathrm{H}$, $\mathrm{H}_{2,3,6,7)}$, 7.06-6.90 (m, 10H, 2x Ph), 3.54-3.43 (m, 4H, 2x PC $\left.\underline{H}_{2}\right)$, $2.90\left(\mathrm{~s}, 3 \mathrm{H}, \mathrm{CH}_{3}\right)$.

${ }^{31} \mathrm{P}\left\{{ }^{1} \mathrm{H}\right\}-\mathrm{NMR}$

$\left(\mathrm{CDCl}_{3}, 121 \mathrm{MHz}\right): \quad \delta[\mathrm{ppm}]=-24.6(\mathrm{~s})$.

\section{EI-MS}

$\mathrm{m} / \mathrm{z}(\%)$ : $404(71)[\mathrm{M}]^{+}, 313(100)[\mathrm{M}-\mathrm{Bn}]^{+}, 192(22)\left[\mathrm{M}-\mathrm{PBn}_{2}\right]^{+}$.

\subsubsection{Synthesis of gold(I) complexes [HAnPPh $\left.{ }_{2}(\mathrm{~S}) \mathrm{AuCl}\right]$ (50), [HAnP'Pr$\left.{ }_{2}(\mathrm{~S}) \mathrm{AuCl}\right] \quad(51), \quad\left[\mathrm{MeAnPPh}_{2}(\mathrm{~S}) \mathrm{AuCl}\right] \quad$ (52), and $\left[\mathrm{ClAu}(\mathrm{Se})\left(\mathrm{Et}_{2} \mathrm{~N}\right)_{2} \mathrm{PAnP}\left(\mathrm{Et}_{2} \mathrm{~N}\right)_{2}(\mathrm{Se}) \mathrm{AuCl}\right](53)$}

$1.00 \mathrm{mmol}$ of the respective phosphorylanthracene $\mathbf{2 , 5}, \mathbf{1 4}$, and 37 as well as $0.29 \mathrm{~g}$ $(1.00 \mathrm{mmol})$ of $\left[\mathrm{Me}_{2} \mathrm{SAuCl}\right]$ were dissolved in acetone $(8-12 \mathrm{~mL})$ and heated to $56^{\circ} \mathrm{C}$ for $2 \mathrm{~min}$. Then the solutions were filtrated and stored at $-30^{\circ} \mathrm{C}$ for crystallization.

Empirical formula: $\mathrm{C}_{26} \mathrm{H}_{19} \mathrm{PSAuCl}$ (50), $\mathrm{C}_{20} \mathrm{H}_{23} \mathrm{PSAuCl}$ (51) $\mathrm{C}_{27} \mathrm{H}_{21} \mathrm{PSAuCl}$ (52), $\mathrm{C}_{30} \mathrm{H}_{48} \mathrm{~N}_{4} \mathrm{P}_{2} \mathrm{Se}_{2} \mathrm{Au}_{2} \mathrm{Cl}_{2}$ (53)

Molar mass: $626.89 \mathrm{~g} / \mathrm{mol}$ (50), $558.85 \mathrm{~g} / \mathrm{mol}$ (51), $640.91 \mathrm{~g} / \mathrm{mol}$ (52), $1149.44 \mathrm{~g} / \mathrm{mol}$ (53).

NMR data of all gold(I) complexes was identical to the respective phosphorylanthracene $\mathbf{2 , 5}$,

14 and 37.

\subsubsection{Synthesis of $\left[\left(\mathrm{MeAnP}\left(\mathrm{NMe}_{2}\right)_{2}\right)_{2} \mathrm{CuBH}_{4}\right](54)$}

MeAnP( $\left(\mathrm{NMe}_{2}\right)_{2}$ (33) $(1.40 \mathrm{~g}, 5.17 \mathrm{mmol})$ was dissolved in $\mathrm{DCM}(11 \mathrm{~mL})$ and $\left[\left(\mathrm{Ph}_{3} \mathrm{P}\right)_{2} \mathrm{CuBH}_{4}\right]$ $(1.16 \mathrm{~g}, 1.93 \mathrm{mmol})$ was added. The mixture was warmed to $35^{\circ} \mathrm{C}$ for $5 \mathrm{~min}$ and then filtrated and cooled to $-30^{\circ} \mathrm{C}$ for crystallization. [(MeAnP(NMe $\left.\left.)_{2}\right)_{2} \mathrm{CuBH}_{4}\right](\mathbf{5 4})$ was obtained as yellow octahedral crystals.

Yield: $0.43 \mathrm{~g}(0.62 \mathrm{~mol}, 32 \%)$

Empirical formula: $\mathrm{C}_{38} \mathrm{H}_{50} \mathrm{BCuN}_{4} \mathrm{P}_{2}$.

Molar mass: $698.29 \mathrm{~g} / \mathrm{mol}$. 


\section{${ }^{1} \mathrm{H}-\mathrm{NMR}$}

$\left(\mathrm{C}_{6} \mathrm{D}_{6}, 500 \mathrm{MHz}\right)$ :

$\delta[\mathrm{ppm}]=8.35$ (bs, $\left.2 \mathrm{H}, \mathrm{H}_{1,8}\right), 7.73\left(\mathrm{~d},{ }^{2} \mathrm{~J}_{\mathrm{HH}}=9.0 \mathrm{~Hz}, 2 \mathrm{H}, \mathrm{H}_{4,5}\right.$ ), 7.01-7.06 (m, $\left.2 \mathrm{H}, \mathrm{H}_{3,6}\right), 6.72\left(\mathrm{bs}, 2 \mathrm{H}, \mathrm{H}_{2,7}\right), 2.55\left(\mathrm{~s}, \mathrm{AnC}_{3}\right), 2.44$ (bs, $12 \mathrm{H}, \mathrm{NC}_{3}$ ).

${ }^{11} \mathrm{~B}\left\{{ }^{1} \mathrm{H}\right\}-\mathrm{NMR}$

$\left(\mathrm{C}_{6} \mathrm{D}_{6}, 161 \mathrm{MHz}\right)$ :

$\delta[\mathrm{ppm}]=-28 \cdot 10(\mathrm{~s})$

${ }^{31} \mathrm{P}\left\{{ }^{1} \mathrm{H}\right\}-\mathrm{NMR}$

$\left(\mathrm{C}_{6} \mathrm{D}_{6}, 202 \mathrm{MHz}\right)$ :

$\delta[p p m]=89.67(\mathrm{~s})$.

\subsubsection{Syntheses of zinc(II) complexes [HAnPPh $\left.\mathrm{ZnBr}_{2}\right]_{2}$ $\left[\mathrm{MeAnPPh}_{2} \mathrm{ZnBr}_{2}\right]_{2}(56)$, and $\left[\mathrm{MeAnP}\left(\mathrm{NMe}_{2}\right)_{2}(\mathrm{~S}) \mathrm{ZnBr}_{2}\right](57)$}

$1.00 \mathrm{mmol}$ of the respective phosphanylanthracene $4, \mathbf{1 3}$ and of the phosphorylanthracene $\mathbf{2 1}$ as well as $0.23 \mathrm{~g}(1.10 \mathrm{mmol})$ of $\mathrm{ZnBr}_{2}$ were dissolved in $\mathrm{DCM}(10 \mathrm{~mL})$ and stirred for $1 \mathrm{~h}$. Then the solutions were filtrated and stored at $-30^{\circ} \mathrm{C}$ for crystallization.

Empirical formula: $\mathrm{C}_{26} \mathrm{H}_{19} \mathrm{PZnBr}_{2}$ (55), $\mathrm{C}_{27} \mathrm{H}_{21} \mathrm{PZnBr}_{2}$ (56), $\mathrm{C}_{19} \mathrm{H}_{23} \mathrm{~N}_{2} \mathrm{PSZnBr}_{2}$ (57).

Molar mass: $5.87 .59 \mathrm{~g} / \mathrm{mol}$ (55), $601.62 \mathrm{~g} / \mathrm{mol}$ (56), $567.63 \mathrm{~g} / \mathrm{mol}$ (57)

The acquired NMR data were identical to those of the starting materials $\mathbf{4 , 1 3}$, and $\mathbf{2 1}$.

\subsubsection{Synthesis of ClAnBCat (58)}

9-Bromo-10-chloroanthracene $(0.77 \mathrm{~g}, 2.63 \mathrm{mmol})$ was suspended in diethyl ether $(25 \mathrm{~mL})$ and cooled to $-15^{\circ} \mathrm{C}$. Then $n$-BuLi solution in hexane $(1.14 \mathrm{~mL}, 2.65 \mathrm{mmol}, 2.30 \mathrm{M})$ was added dropwise. The solution was warmed to $0^{\circ} \mathrm{C}$ for $5 \mathrm{~min}$ and then cooled to $-15^{\circ} \mathrm{C}$ again. A solution of catecholechloroborane $(0.41 \mathrm{~g}, 2.65 \mathrm{mmol})$ in diethyl ether $(10 \mathrm{~mL})$ was added over the course of $30 \mathrm{~min}$. After the addition was completed the cooling bath was removed and the reaction was stirred at ambient temperature for $3 \mathrm{~h}$. Then the solvent was evaporated and the crude product was dissolved in toluene $(30 \mathrm{~mL})$ and filtrated for removal of lithium chloride. Removal of the solvent afforded CIAnBCat (58) as a pale yellow solid. $\mathbf{5 8}$ was recrystallized from THF for further purification.

Yield: $0.69 \mathrm{~g}(2.08 \mathrm{mmol}, 80 \%)$

Empirical formula: $\mathrm{C}_{20} \mathrm{H}_{12} \mathrm{BClO}_{2}$

Molar mass: $330.57 \mathrm{~g} / \mathrm{mol}$ 


\section{${ }^{1} \mathrm{H}-\mathrm{NMR}$}

(THF $d_{8}, 300 \mathrm{MHz}$ ):

$\delta[p p m]=8.58\left(\mathrm{~m}, 4 \mathrm{H}, \mathrm{H}_{1,4,5,8}\right), 7.58\left(\mathrm{~m}, 4 \mathrm{H}, \mathrm{H}_{2,7}, 2 \mathrm{xH}_{\mathrm{Ph}}\right), 7.24$

(m, $\left.4 \mathrm{H}, \mathrm{H}_{3,6}, 2 \mathrm{xH}_{\mathrm{Ph}}\right)$.

${ }^{13} \mathrm{C}\left\{{ }^{1} \mathrm{H}\right\}-\mathrm{NMR}$

$\left(\right.$ THF $\left.d_{8}, 126 \mathrm{MHz}\right)$

$\delta[\mathrm{ppm}]=148.4,146.4,145.4,137.4,136.6,132.6,128.8$ $128.6,127.8,126.6$ (d, J = 27.0 Hz), 124.8, 123.0.

\section{${ }^{11} B\left\{{ }^{1} \mathrm{H}\right\}-N M R$}

(THF $\left.d_{8}, 160 \mathrm{MHz}\right):$

$\delta[\mathrm{ppm}]=14.1(\mathrm{~s})$

\subsubsection{Synthesis of $\mathrm{ClAnBIPC}_{2}$ (59)}

9-Bromo-10-chloroanthracene $(1.00 \mathrm{~g}, 3.42 \mathrm{mmol})$ was suspended in diethyl ether $(40 \mathrm{~mL})$ and cooled to $-15^{\circ} \mathrm{C}$. Then $n$-BuLi solution in hexane $(1.49 \mathrm{~mL}, 3.45 \mathrm{mmol}, 2.30 \mathrm{M})$ was added dropwise. The solution was warmed to $0^{\circ} \mathrm{C}$ for $5 \mathrm{~min}$ and then cooled to $-15^{\circ} \mathrm{C}$ again. A solution of chlorodiisopinocampheylborane $(1.10 \mathrm{~g}, 3.48 \mathrm{mmol})$ in diethyl ether $(20 \mathrm{~mL})$ was added over the course of $30 \mathrm{~min}$. After the addition was completed the cooling bath was removed and the reaction was stirred at ambient temperature for $3 \mathrm{~h}$. Then the solvent was evaporated and the crude product was dissolved in toluene $(30 \mathrm{~mL})$ and filtrated for removal of lithium chloride. Removal of the solvent afforded $\mathrm{ClAnBIPC}_{2}(59)$ as a yellow solid. 59 was recrystallized from toluene for further purification.

Yield: $1.44 \mathrm{~g}(2.91 \mathrm{mmol}, 85 \%)$

Empirical formula: $\mathrm{C}_{44} \mathrm{H}_{42} \mathrm{BCl}$

Molar mass: $496.96 \mathrm{~g} / \mathrm{mol}$

${ }^{1}$ H-NMR

(Tol $d_{8}, 300 \mathrm{MHz}$ ):

$\delta[p p m]=8.39\left(\mathrm{~d},{ }^{3} J_{\mathrm{HH}}=9.5 \mathrm{~Hz}, 2 \mathrm{H}, \mathrm{H}_{1,8}\right), 7.33\left(\mathrm{~d},{ }^{3} J_{\mathrm{HH}}=8.5 \mathrm{~Hz}\right.$, $\left.2 \mathrm{H}, \mathrm{H}_{4,5}\right), 7.06\left(\mathrm{~m}, 6 \mathrm{H}, \mathrm{H}_{2,3,6,7}\right), 2.54(\mathrm{t}, J=11.9 \mathrm{~Hz}, 2 \mathrm{H}), 2.14(\mathrm{~m}$, 2H), $2.04(\mathrm{~m}), 1.99$ (d, J=10.5 Hz, 2H), $1.97(\mathrm{~s}, 2 \mathrm{H}), 1.90(\mathrm{~m}$, $2 \mathrm{H}), 1.25(\mathrm{~m}, 2 \mathrm{H}), 1.00(\mathrm{~s}, 12 \mathrm{H}), 0.76(\mathrm{~d}, J=9.5 \mathrm{~Hz}, 2 \mathrm{H}), 0.38(\mathrm{~d}$, $J=7.0 \mathrm{~Hz}, 6 \mathrm{H})$.

\section{${ }^{11} \mathrm{~B}\left\{{ }^{1} \mathrm{H}\right\}-\mathrm{NMR}$}

( THF $\left.d_{8}, 160 \mathrm{MHz}\right): \quad \delta[\mathrm{ppm}]=-2.3(\mathrm{~s})$. 


\section{EI-MS}

$m / z(\%):$

$496(41)[\mathrm{M}]^{+}, 359(10)[\mathrm{M}-\mathrm{IPC}]^{+}, 237(31)[\mathrm{M}-2 \mathrm{IPC}]^{+}, 212$

(100) $[\mathrm{AnCl}]^{+}$.

\subsubsection{Synthesis of BrAnBMes 2 (60)}

9,10-Dibromoanthracene $(1.20 \mathrm{~g}, 3.57 \mathrm{mmol})$ was dissolved in diethyl ether $(40 \mathrm{~mL})$ and cooled to $-15^{\circ} \mathrm{C}$. Then $n$-BuLi solution in hexane $(1.55 \mathrm{~mL}, 3.58 \mathrm{mmol}, 2.30 \mathrm{M})$ was added drop-wise. The reaction was warmed to $0^{\circ} \mathrm{C}$ for $5 \mathrm{~min}$ and then cooled to $-15^{\circ} \mathrm{C}$ again. A solution of chlorodimesitylborane $(1.02 \mathrm{~g}, 3.48 \mathrm{mmol})$ in diethyl ether $(20 \mathrm{~mL})$ was added over the course of $30 \mathrm{~min}$. After the addition was completed the cooling bath was removed and the reaction was stirred at ambient temperature for $3 \mathrm{~h}$. Then the solvent was evaporated and the crude product was dissolved in toluene $(30 \mathrm{~mL})$ and filtrated for removal of lithium chloride. Removal of the solvent afforded $\mathrm{BrAnBMes}_{2}(\mathbf{6 0})$ as a yellow solid. 60 was recrystallized from toluene/MeCN for further purification.

Yield: $1.10 \mathrm{~g}(2,18 \mathrm{mmol}, 61 \%)$

Empirical formula: $\mathrm{C}_{32} \mathrm{H}_{30} \mathrm{BBr}$

Molar mass: $505.30 \mathrm{~g} / \mathrm{mol}$

${ }^{1} \mathrm{H}-\mathrm{NMR}$

(THF $d_{8}, 300 \mathrm{MHz}$ ):

$\delta[\mathrm{ppm}]=8.36\left(\mathrm{~d},{ }^{3} \mathrm{~J}_{\mathrm{HH}}=9.6 \mathrm{~Hz}, 2 \mathrm{H}, \mathrm{H}_{1,8}\right) 8.15\left(\mathrm{~d},{ }^{3} J_{\mathrm{HH}}=7.9 \mathrm{~Hz}\right.$, $\left.2 \mathrm{H}, \mathrm{H}_{4,5}\right), 6.8$ (s (br), $\left.4 \mathrm{H}, \mathrm{H}_{\text {meta }}(\mathrm{Mes})\right), 2.24\left(\mathrm{~s}, 24 \mathrm{H}, \mathrm{CH}_{3}\right.$ ).

\section{${ }^{11} B\left\{{ }^{1} H\right\}-N M R$}

(THF $d_{8,}, 96 \mathrm{MHz}$ ):

$\delta[\mathrm{ppm}]=-1.7(\mathrm{~s})$.

\section{EI-MS}

$m / z(\%)$ :

(44) $\left[\mathrm{BMes}_{2}\right]^{+}$.

\subsubsection{Synthesis of Bis(2-methylthienyl)amine (61)}

2-Thiophenecarboxaldehyde $(10.0 \mathrm{~g}, 89.2 \mathrm{mmol}), \operatorname{HMDS}(28.7 \mathrm{~g}, 178 \mathrm{mmol})$ and $\mathrm{LiClO}_{4}(9.49 \mathrm{~g}$, $89.2 \mathrm{mmol}$ ) were reacted at $50^{\circ} \mathrm{C}$ for for $1 \mathrm{~h}$. Then the reaction was cooled to $0^{\circ} \mathrm{C}$ and a solution of $\mathrm{NaBH}_{4}(10.21 \mathrm{~g}, 270 \mathrm{mmol})$ in $\mathrm{MeOH}(200 \mathrm{~mL})$ was added. The reaction was stirred for $3 \mathrm{~h}$ at ambient temperature. Then the solvent was evaporated and the reaction was quenched with aqueous $\mathrm{NaHCO}_{3}$ solution $(100 \mathrm{~mL})$. The aqueous layer was extracted with $\mathrm{DCM}(3 \times 50 \mathrm{~mL})$. 
The organic layers were united and extracted with saturated $\mathrm{NaCl}$ solution $(200 \mathrm{~mL})$ and the dried over $\mathrm{MgSO}_{4}$. After removal of the solvent, the crude product was purified by column chromatography (silica gel, pentane/ethyl acetate 5:1). Bis(2-methylthienyl)amine (61) was obtained as a yellow oil.

Yield: $4.27 \mathrm{~g}$ (20.4 mmol, $46 \%)$

Empirical formula: $\mathrm{C}_{10} \mathrm{H}_{11} \mathrm{SN}_{2}$

Molar mass: $209.33 \mathrm{~g} / \mathrm{mol}$

${ }^{1} \mathrm{H}-\mathrm{NMR}$

(DMSO, $300 \mathrm{MHz}): \quad \delta[\mathrm{ppm}]=7.27-7.24(\mathrm{dd}, \mathrm{J}=5.0 \mathrm{~Hz}, 1.5 \mathrm{~Hz}, 2 \mathrm{H}), 7.01-6.69(\mathrm{~m}$, $4 \mathrm{H}), 4.06(\mathrm{~s}, 4 \mathrm{H}), 1.85(\mathrm{~s}(\mathrm{br}), 1 \mathrm{H})$.

${ }^{13} \mathrm{C}\left\{{ }^{1} \mathrm{H}\right\}-\mathrm{NMR}$

(DMSO, $75 \mathrm{MHz}$ ): $\quad \delta[\mathrm{ppm}]=143.7,126.7,125.1,124.6,47.1$.

\subsubsection{Synthesis of bis(2-methoxybenzyl)amine (62)}

2-Methoxybenzaldehyde $(5.00 \mathrm{~g}, 36.7 \mathrm{mmol})$, $\mathrm{HMDS}(11.9 \mathrm{~g}, 73.5 \mathrm{mmol})$ and $\mathrm{LiClO}_{4}(3.91 \mathrm{~g}$, $36.7 \mathrm{mmol}$ ) were reacted at $50^{\circ} \mathrm{C}$ for for $1 \mathrm{~h}$. Then the reaction was cooled to $0^{\circ} \mathrm{C}$ and a solution of $\mathrm{NaBH}_{4}(4.17 \mathrm{~g}, 110 \mathrm{mmol})$ in $\mathrm{MeOH}(200 \mathrm{~mL})$ was added. The reaction was stirred for $3 \mathrm{~h}$ at ambient temperature. Then the solvent was evaporated and the reaction was quenched with aqueous $\mathrm{NaHCO}_{3}$ solution $(100 \mathrm{~mL})$. The aqueous layer was extracted with DCM $(3 \times 50 \mathrm{~mL})$. The organic layers were united and extracted with saturated $\mathrm{NaCl}$ solution $(200 \mathrm{~mL})$ and the dried over $\mathrm{MgSO}_{4}$. After removal of the solvent, the crude product was purified by column chromatography (silica gel, pentane/ethyl acetate 2:1). Bis(2-methoxybenzyl)amine (62) was obtained as a yellow solid.

Yield: $6.52 \mathrm{~g}(69 \%)$

Empirical formula: $\mathrm{C}_{16} \mathrm{H}_{19} \mathrm{NO}_{2}$

Molar mass: $257.33 \mathrm{~g} / \mathrm{mol}$

${ }^{1}$ H-NMR

$\left(\mathrm{CDCl}_{3}, 300 \mathrm{MHz}\right)$ :

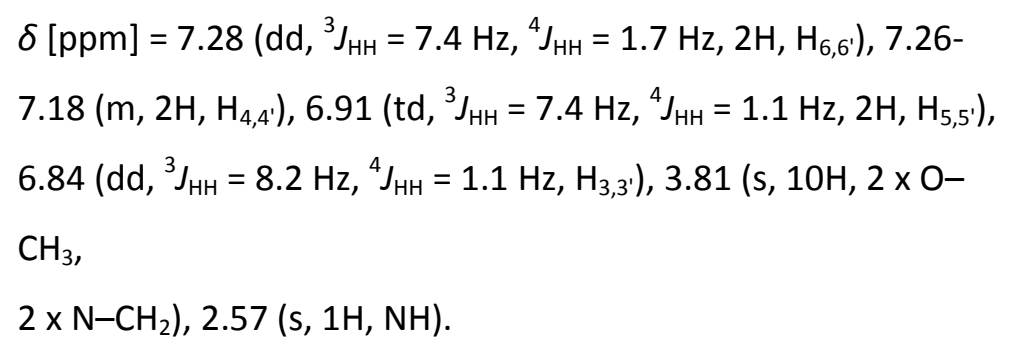




\section{${ }^{13} \mathrm{C}\left\{{ }^{1} \mathrm{H}\right\}-\mathrm{NMR}$}

$\left(\mathrm{CDCl}_{3}, 75 \mathrm{MHz}\right)$ :

$$
\begin{aligned}
& \delta[\mathrm{ppm}]=157.59\left(2 \mathrm{C}, \mathrm{C}_{2,2^{\prime}}\right), 129.74\left(2 \mathrm{C}, \mathrm{C}_{6,6^{\prime}}\right), 128.27\left(2 \mathrm{C}, \mathrm{C}_{1,1^{\prime}}\right), \\
& 128.06\left(2 \mathrm{C}, \mathrm{C}_{4,4^{\prime}}\right), 120.32\left(2 \mathrm{C}, \mathrm{C}_{5,5^{\prime}}\right), 110.06\left(2 \mathrm{C}, \mathrm{C}_{3,3^{\prime}}\right), 55.15, \\
& 48.60\left(4 \mathrm{C}, 2 \times \mathrm{O}-\underline{\mathrm{CH}}_{3}, 2 \times \mathrm{N}-\underline{\mathrm{CH}}_{2}\right) .
\end{aligned}
$$

EI-MS

$m / z(\%):$

$121(100)[\mathrm{BnOMe}-\mathrm{H}]^{+}, 136(76)[\mathrm{M}-(\mathrm{BnOMe})]^{+}, 256(22)[\mathrm{M}-$

$\mathrm{H}^{+}, 257(16)[\mathrm{M}]^{+}$.

\subsubsection{Synthesis of bis(2-methylthiobenzyl)amine (63)}

2-Methylthiobenzaldehyde $(5.00 \mathrm{~g}, 32.9 \mathrm{mmol}), \mathrm{HMDS}(10.7 \mathrm{~g}, 66.0 \mathrm{mmol})$ and $\mathrm{LiClO}_{4}(3.50 \mathrm{~g}$, $33.0 \mathrm{mmol}$ ) were reacted at $50^{\circ} \mathrm{C}$ for for $1 \mathrm{~h}$. Then the reaction was cooled to $0^{\circ} \mathrm{C}$ and a solution of $\mathrm{NaBH}_{4}(3.78 \mathrm{~g}, 100 \mathrm{mmol})$ in $\mathrm{MeOH}(200 \mathrm{~mL})$ was added. The reaction was stirred for $3 \mathrm{~h}$ at ambient temperature. Then the solvent was evaporated and the reaction was quenched with aqueous $\mathrm{NaHCO}_{3}$ solution $(100 \mathrm{~mL})$. The aqueous layer was extracted with $\mathrm{DCM}(3 \times 50 \mathrm{~mL})$. The organic layers were united and extracted with saturated $\mathrm{NaCl}$ solution $(200 \mathrm{~mL})$ and the dried over $\mathrm{MgSO}_{4}$. After removal of the solvent, the crude product was purified by column chromatography (silica gel, pentane/ethyl acetate 4:1). Bis(2-methylthiobenzyl)amine (63) was obtained as a yellow solid.

Yield: $6.19 \mathrm{~g}(65 \%)$

Empirical formula: $\mathrm{C}_{16} \mathrm{H}_{19} \mathrm{NS}_{2}$

Molar mass: $289.46 \mathrm{~g} / \mathrm{mol}$

${ }^{1}$ H-NMR

$\left(\mathrm{CDCl}_{3}, 300 \mathrm{MHz}\right)$ :

$\delta[\mathrm{ppm}]=7.36\left(\mathrm{ddd},{ }^{3} J_{\mathrm{HH}}=7.4 \mathrm{~Hz},{ }^{4} J_{\mathrm{HH}}=1.2 \mathrm{~Hz},{ }^{5} J_{\mathrm{HH}}=0.6 \mathrm{~Hz}\right.$, $\left.2 \mathrm{H}, \mathrm{H}_{3,3^{\prime}}\right), 7.27-7.20\left(\mathrm{~m}, 4 \mathrm{H}, \mathrm{H}_{4,4^{\prime}, 6,6^{\prime}}\right), 7.13\left(\mathrm{dd},{ }^{3} \mathrm{JHH}_{\mathrm{HH}}=7.6 \mathrm{~Hz}\right.$, ${ }^{4} J_{\mathrm{HH}}=2.3 \mathrm{~Hz}, \mathrm{H}_{5}$ oder $\left.\mathrm{H}_{5^{\prime}}\right), 7.12\left(\mathrm{dd},{ }^{3} J_{\mathrm{HH}}=7.4 \mathrm{~Hz},{ }^{4} J_{\mathrm{HH}}=2.3 \mathrm{~Hz}\right.$, $\mathrm{H}_{5}$ oder $\left.\mathrm{H}_{5^{\prime}}\right), 3.89\left(\mathrm{~s}, 4 \mathrm{H}, 2 \times \mathrm{N}-\mathrm{CH}_{2}\right), 2.45\left(\mathrm{~s}, 6 \mathrm{H}, 2 \times \mathrm{S}-\mathrm{CH}_{3}\right)$, $2.14(\mathrm{~s}, 1 \mathrm{H}, \mathrm{NH})$.

${ }^{13} \mathrm{C}\left\{{ }^{1} \mathrm{H}\right\}-\mathrm{NMR}$

$\left(\mathrm{CDCl}_{3}, 75 \mathrm{MHz}\right)$ :

$\delta[\mathrm{ppm}]=137.81,137.43\left(4 \mathrm{C}, \mathrm{C}_{1,1^{\prime}}, \mathrm{C}_{2,2^{\prime}}\right), 128.94\left(2 \mathrm{C}, \mathrm{C}_{3,3^{\prime}}\right)$, $127.62,125.72\left(4 C, C_{4,4^{\prime}}, C_{6,6^{\prime}}\right), 124.88\left(2 C, C_{5,5^{\prime}}\right), 51.07(2 C$,

\section{EI-MS}

$m / z(\%):$

137 (50) [BnSMe-H] $]^{+}, 152$ (100) [M-(BnSMe) $]^{+}, 272$ (18) [M-

$(\mathrm{SMe})]^{+}, 289(4)[\mathrm{M}]^{+}$. 


\subsubsection{Synthesis of $\mathrm{HN}\left(\mathrm{C}_{2} \mathrm{H}_{4} \mathrm{PPh}_{2}\right)_{2}(64)$}

Diphenylphosphane $(5.21 \mathrm{~g}, 28.00 \mathrm{mmol})$ was dissolved in anhydrous THF ( $40 \mathrm{~mL}$ ) and reacted with a solution of $n$-BuLi in hexane $(8.8 \mathrm{~mL}, 28.00 \mathrm{mmol}, 3.18 \mathrm{M})$ over $30 \mathrm{~min}$ at ambient temperature. The reaction was stirred for $15 \mathrm{~min}$ and then added to a solution of bis(2chloroethyl)trimethylsilylamine $(3.00 \mathrm{~g}, 28.00 \mathrm{mmol})$ in THF $(15 \mathrm{~mL})$ at $-15^{\circ} \mathrm{C}$ over $1 \mathrm{~h}$. The reaction was stirred at $20^{\circ} \mathrm{C}$ overnight and then heated to $65^{\circ} \mathrm{C}$ for $1 \mathrm{~h}$. Degassed water $(15 \mathrm{~mL})$ was added and the reaction was stirred for $15 \mathrm{~min}$. The aqueous layer was removed, the solution was concentrated and degassed water $(15 \mathrm{~mL})$ and hexane $(15 \mathrm{~mL})$ were added. The reaction was heated to $70^{\circ} \mathrm{C}$ for $4 \mathrm{~h}$. Then the aqueous layer was removed and the solvent was evaporated. $\mathrm{HN}\left(\mathrm{C}_{2} \mathrm{H}_{4} \mathrm{PPh}_{2}\right)_{2}(64)$ was obtained as a colorless oil which was dried in vacuo.

Yield: $5.98 \mathrm{~g}(13.55 \mathrm{mmol}, 97 \%)$

Empirical formula: $\mathrm{C}_{28} \mathrm{H}_{29} \mathrm{NP}_{2}$

Molar mass: $441.48 \mathrm{~g} / \mathrm{mol}$

\section{${ }^{1}$ H-NMR}

(THF $d_{8}, 300 \mathrm{MHz}$ ):

$\delta[p p m]=7.34\left(\mathrm{~m}, 20 \mathrm{H}, \mathrm{C}_{6} \mathrm{H}_{5}\right), 2.68\left(\mathrm{dd}, J_{\mathrm{HH}}=7.8 \mathrm{~Hz}, 4 \mathrm{H}\right.$, $\left.\mathrm{N}-\mathrm{CH}_{2}\right), 2.20\left(\mathrm{t}, \mathrm{J}_{\mathrm{HH}}=7.7 \mathrm{~Hz}, 4 \mathrm{H}, \mathrm{P}-\mathrm{CH}_{2}\right), 1.73(\mathrm{~s}(\mathrm{br}), 1 \mathrm{H}, \mathrm{N}-\mathrm{H})$.

\section{${ }^{13} \mathrm{C}\left\{{ }^{1} \mathrm{H}\right\}-\mathrm{NMR}$}

(THF $d_{8}, 126 \mathrm{MHz}$ ):

$\delta[p p m]=140.3\left(\mathrm{~s}, 4 \mathrm{C}, \mathrm{C}_{\mathrm{Ar}}\right), 133.5\left(\mathrm{~s}, 8 \mathrm{C}, \mathrm{C}_{\mathrm{Ar}}\right), 129.1\left(\mathrm{~s}, 12 \mathrm{C}, \mathrm{C}_{\mathrm{Ar}}\right)$, $47.3(\mathrm{~s}, 2 \mathrm{C}, \mathrm{N}-\mathrm{C}), 30.0$ (s, 2C, P-C).

${ }^{31} \mathrm{P}\left\{{ }^{1} \mathrm{H}\right\}-\mathrm{NMR}$

$\left(\right.$ THF $d_{8}, 81 \mathrm{MHz}$ ):

$\delta[\mathrm{ppm}]=-20.3(\mathrm{~s}, 2 \mathrm{P})$.

\subsubsection{Synthesis of $\mathrm{HN}\left(\mathrm{C}_{2} \mathrm{H}_{4} \mathrm{~S}^{t} \mathrm{Bu}\right)_{2}(65)$}

tert-Butylthiole $(2.53 \mathrm{~g} 28.00 \mathrm{mmol})$ was dissolved in anhydrous THF $(40 \mathrm{~mL})$ and reacted with a solution of $n$-BuLi in hexane $(8.8 \mathrm{~mL}, 28.00 \mathrm{mmol}, 3.18 \mathrm{M})$ over $30 \mathrm{~min}$ at ambient temperature. The reaction was stirred for $15 \mathrm{~min}$ and then added to a solution of bis(2chloroethyl)trimethylsilylamine $(3.00 \mathrm{~g}, 28.00 \mathrm{mmol})$ in THF $(15 \mathrm{~mL})$ at $-15^{\circ} \mathrm{C}$ over $1 \mathrm{~h}$. The reaction was stirred at $20^{\circ} \mathrm{C}$ overnight and then heated to $65^{\circ} \mathrm{C}$ for $1 \mathrm{~h}$. Degassed water $(15 \mathrm{~mL})$ was added and the reaction was stirred for $15 \mathrm{~min}$. The aqueous layer was removed, the solution was concentrated and degassed water $(15 \mathrm{~mL})$ and hexane $(15 \mathrm{~mL})$ were added. The reaction was heated to $70^{\circ} \mathrm{C}$ for $4 \mathrm{~h}$. Then the aqueous layer was removed and the solvent was evaporated. $\mathrm{HN}\left(\mathrm{C}_{2} \mathrm{H}_{4} \mathrm{PPh}_{2}\right)_{2}$ (64) was purified by column chromatography (ethyl acetate) and was obtained as an orange solid.

Yield: $1.00 \mathrm{~g}$ (4.01 mmol, 29\%) 
Empirical formula: $\mathrm{C}_{12} \mathrm{H}_{27} \mathrm{NS}_{2}$

Molar mass: $249.48 \mathrm{~g} / \mathrm{mol}$

${ }^{1} \mathrm{H}-\mathrm{NMR}$

$\left(\mathrm{CDCl}_{3}, 500 \mathrm{MHz}\right)$ :

$\delta[\mathrm{ppm}]=8.01\left(\mathrm{t}, \mathrm{J}_{\mathrm{HH}}=7.6 \mathrm{~Hz}, 4 \mathrm{H}, \mathrm{N}-\mathrm{CH}_{2}\right), 2.88\left(\mathrm{t}, J_{\mathrm{HH}}=6.2 \mathrm{~Hz}\right.$, $\left.4 \mathrm{H}, \mathrm{S}-\mathrm{CH}_{2}\right), 3.13(\mathrm{sbr}, 1 \mathrm{H}, \mathrm{N}-\mathrm{H}), 1.36\left(\mathrm{~s}, 18 \mathrm{H}, \mathrm{CH}_{3}\right)$.

\section{EI-MS}

$m / z(\%):$

$249(0.4)[\mathrm{M}]^{+}, 146(46)\left[\mathrm{M}-\mathrm{CH}_{2} \mathrm{~S}\left(\mathrm{C}\left(\mathrm{CH}_{3}\right)_{3}\right)\right]^{+}, 117(4)$

$\left[\mathrm{CH}_{2} \mathrm{CH}_{2} \mathrm{~S}\left(\mathrm{C}\left(\mathrm{CH}_{3}\right)_{3}\right)\right]^{+}, 90(100)\left[\mathrm{HS}\left(\mathrm{C}\left(\mathrm{CH}_{3}\right)_{3}\right)\right]^{+}, 57$ (31) $\left[\mathrm{C}\left(\mathrm{CH}_{3}\right)_{3}\right]^{+}$.

\subsubsection{Synthesis of $\left[\mathrm{AnCH}_{2} \mathrm{Li}\right.$-TMEDA] (66)}

9-Methylanthracene $(2.0 \mathrm{~g}, 10.42 \mathrm{mmol})$ was dissolved in diethyl ether $(40 \mathrm{~mL})$ and cooled to $-15^{\circ} \mathrm{C}$. Then TMEDA $(2.41 \mathrm{~g}, 20.84 \mathrm{mmol})$ was added. To this mixture TMSMeLi $(13.0 \mathrm{~mL}$, $10.5 \mathrm{mmol}, 0.80 \mathrm{M}$ ) was added over the course of $30 \mathrm{~min}$. The reaction was stirred for $15 \mathrm{~min}$ until a black precipitate had formed. The precipitate was separated from the mother liquor by filtration with a cooled filtration device $\left(-78^{\circ} \mathrm{C}\right)$. The black solid was re-dissolved in pre-cooled THF $(40 \mathrm{~mL})$. The solution was concentrated to ca. $20 \mathrm{~mL}$ and the stored at $-30^{\circ} \mathrm{C}$ for crystallization. For the NMR experiments $50 \mathrm{mg}$ of 9-Methylanthracene and $60 \mathrm{mg}$ of TMEDA were dissolved in THF $d_{8}$ and reacted with one equivalent of TMSMeLi in an NMR tube at $-15^{\circ} \mathrm{C}$.

Empirical formula: $\mathrm{C}_{27} \mathrm{H}_{43} \mathrm{LiN}_{4}$

Molar mass: $430.60 \mathrm{~g} / \mathrm{mol}$

\section{${ }^{1}$ H-NMR}

( $T H F d_{8}, 300 \mathrm{MHz}$ ):

$\delta[p p m]=7.34\left(\mathrm{~d}, \mathrm{~J}=8.0 \mathrm{~Hz}, 2 \mathrm{H}, \mathrm{H}_{1,8}\right), 6.52\left(\mathrm{~m}, 4 \mathrm{H}, \mathrm{H}_{3,4,5,6}\right), 6.04$ $\left(\mathrm{m}, 2 \mathrm{H}, \mathrm{H}_{2,7}\right), 5.06\left(\mathrm{~s}, 1 \mathrm{H}, \mathrm{H}_{10}\right), 4.12\left(\mathrm{~s}, 2 \mathrm{H}, \mathrm{C}_{2}{ }^{-}\right)$.

${ }^{7}$ Li-NMR

( $T H F d_{8}, 194 \mathrm{MHz}$ ):

$\delta[\mathrm{ppm}]=-2.30(\mathrm{~s})$

\section{${ }^{13} \mathrm{C}\left\{{ }^{1} \mathrm{H}\right\}-\mathrm{NMR}$}

( $T H F d_{8}, 126 \mathrm{MHz}$ ):

$$
\begin{aligned}
& \delta[\text { ppm }]=145.4\left(2 \mathrm{C}, \mathrm{C}_{8 \mathrm{a}, 9 a}\right), 140.0\left(2 \mathrm{C}, \mathrm{C}_{4 a, 10 \mathrm{a}}\right), 125.3\left(2 \mathrm{C}, \mathrm{C}_{4,5}\right), \\
& 124.5\left(2 \mathrm{C}, \mathrm{C}_{1,8}\right), 122.7\left(2 \mathrm{C}, \mathrm{C}_{3,6}\right), 121.8\left(1 \mathrm{C}, \mathrm{C}_{9}\right), 111.8\left(2 \mathrm{C}, \mathrm{C}_{2,7}\right), \\
& 85.9\left(1 \mathrm{C}, \mathrm{C}_{10}\right), 75.0(1 \mathrm{C}, \mathrm{C} 15) .
\end{aligned}
$$




\subsubsection{Synthesis of 9-(2-hydroxyethyl)-10-methylanthracene (67)}

9-Bromo-10-methylanthracene $(4.00 \mathrm{~g}, 14.75 \mathrm{mmol})$ was dissolved in diethyl ether $(200 \mathrm{~mL})$ and cooled to $-15^{\circ} \mathrm{C}$. Then $n$-BuLi solution in hexane $(4.65 \mathrm{~mL}, 14.77 \mathrm{mmol}, 3.18 \mathrm{M})$ was added over $30 \mathrm{~min}$. The reaction was stirred at $0^{\circ} \mathrm{C}$ for $10 \mathrm{~min}$, then gaseous oxirane was discharged into the solution of the lithiated intermediate at $-15^{\circ} \mathrm{C}$ for $30 \mathrm{~min}$. The solution was stirred at ambient temperature overnight. Then the solvent was evaporated and the crude product was extracted with DCM $(60 \mathrm{~mL})$ and water $(50 \mathrm{~mL})$. The organic layer was dried over $\mathrm{MgSO}_{4}$ and filtrated. The solvent was removed and the crude product was purified by column chromatography (pentane/ethyl acetate 4:1). MeANC ${ }_{2} \mathrm{H}_{4} \mathrm{OH}$ (67) was obtained as a yellow solid.

Yield: $0.68 \mathrm{~g}(2.88 \mathrm{mmol}, 20 \%)$

Empirical formula: $\mathrm{C}_{17} \mathrm{H}_{16} \mathrm{O}$

Molar mass: $236.31 \mathrm{~g} / \mathrm{mol}$

${ }^{1} \mathrm{H}-\mathrm{NMR}$

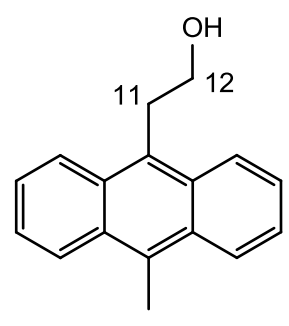

$\left(\mathrm{CDCl}_{3}, 300 \mathrm{MHz}\right)$ :

$\delta=8.33\left(\mathrm{~m}, 4 \mathrm{H}, \mathrm{H}_{1,4,5,8}\right), 7.51\left(\mathrm{~m}, 4 \mathrm{H}, \mathrm{H}_{2,3,6,7}\right), 4.05(\mathrm{t}$,

$\left.{ }^{3} J_{\mathrm{HH}}=7.2 \mathrm{~Hz}, 2 \mathrm{H}, \mathrm{H}_{12}\right), 3.91\left(\mathrm{t},{ }^{3} \mathrm{HHH}_{\mathrm{HH}}=7.2 \mathrm{~Hz}, 2 \mathrm{H}, \mathrm{H}_{11}\right), 3.08(\mathrm{~s}, 3 \mathrm{H}$, $\left.\mathrm{CH}_{3}\right)$.

${ }^{13} \mathrm{C}\left\{{ }^{1} \mathrm{H}\right\}-\mathrm{NMR}$

$\left(\mathrm{CDCl}_{3}, 75 \mathrm{MHz}\right)$ :

$\delta=130.0\left(1 C, C_{9}\right), 129.9\left(2 C, C_{4 a}, 10 a\right), 129.6\left(1 C, C_{10}\right), 128.3(2 C$,

$\left.\mathrm{C}_{8 \mathrm{a}, 9 \mathrm{a}}\right), 125.4\left(2 \mathrm{C}, \mathrm{C}_{1,8}\right), 125.2\left(2 \mathrm{C}, \mathrm{C}_{2,7}\right), 124.9\left(2 \mathrm{C}, \mathrm{C}_{4,5}\right), 124.8$

$\left(2 \mathrm{C}, \mathrm{C}_{3,6}\right), 63.4\left(1 \mathrm{C}, \mathrm{C}_{12}\right), 31.0\left(1 \mathrm{C}, \mathrm{C}_{11}\right), 14.2\left(1 \mathrm{C}, \underline{\mathrm{CH}}_{3}\right)$.

\section{EI-MS}

$\mathrm{m} / \mathrm{z}(\%)$ :

$236(24)[\mathrm{M}]^{+}, 221(8)[\mathrm{M}-\mathrm{Me}]^{+}, 205(44)\left[\mathrm{M}-\mathrm{CH}_{2} \mathrm{OH}\right]^{+}, 191$

(68) $\left[\mathrm{M}-\mathrm{C}_{2} \mathrm{H}_{4} \mathrm{OH}\right]^{+}$.

\subsubsection{Synthesis of 9-(2-bromoethyl)-10-methylanthracene (68)}

Triphenylphosphane $(0.79 \mathrm{~g}, 3.02 \mathrm{mmol})$ was dissolved in $\mathrm{MeCN}(20 \mathrm{~mL})$ and reacted with elemental bromine $(0.46 \mathrm{~g}(2.88 \mathrm{mmol})$ at ambient temperature. The addition of bromine was carried out drop-wise. To the resulting solution a solution of $\mathrm{MeANC}_{2} \mathrm{H}_{4} \mathrm{OH}$ (67) $(0.68 \mathrm{~g}$, $2.88 \mathrm{mmol})$ in $\mathrm{MeCN}(10 \mathrm{~mL})$ was added over $30 \mathrm{~min}$ at ambient temperature. The reaction was stirred overnight and the solvent was evaporated. The crude product was dissolved in ethyl acetate $(20 \mathrm{~mL})$ and filtrated for separation of $\mathrm{OPPh}_{3}$. The solution was concentrated and 
$\mathrm{MeAnC}_{2} \mathrm{H}_{4} \mathrm{Br}$ (68) was crystallized from ethyl acetate. 68 was obtained as a yellow crystalline solid.

Yield: $0.49 \mathrm{~g}(1.64 \mathrm{mmol}, 57 \%)$

Empirical formula: $\mathrm{C}_{17} \mathrm{H}_{15} \mathrm{Br}$

Molar mass: $299.21 \mathrm{~g} / \mathrm{mol}$

\section{${ }^{1}$ H-NMR}

$\left(\mathrm{CDCl}_{3}, 400 \mathrm{MHz}\right)$ :<smiles>Cc1c2ccccc2c(CCBr)c2ccccc12</smiles>

$\delta=8.34\left(\mathrm{~d},{ }^{3} \mathrm{~J}_{\mathrm{HH}}=8.80 \mathrm{~Hz}, 2 \mathrm{H}, \mathrm{H}_{4,5}\right), 8.25\left(\mathrm{~d},{ }^{3} \mathrm{~J}_{\mathrm{HH}}=8.80 \mathrm{~Hz}, 2 \mathrm{H}\right.$, $\left.\mathrm{H}_{1,8}\right), 7.54\left(\mathrm{~m}, 4 \mathrm{H}, \mathrm{H}_{2,3,6,7)}\right), 4.17\left(\mathrm{t},{ }^{3} \mathrm{JHH}_{\mathrm{HH}}=8.76 \mathrm{~Hz}, 2 \mathrm{H}, \mathrm{H}_{11}\right), 3.68$ $\left(\mathrm{t},{ }^{3} J_{\mathrm{HH}}=8.76 \mathrm{~Hz}, 2 \mathrm{H}, \mathrm{H}_{12}\right), 3.09\left(\mathrm{~s}, 3 \mathrm{H}, \mathrm{CH}_{3}\right)$.

${ }^{13} \mathrm{C}\left\{{ }^{1} \mathrm{H}\right\}-\mathrm{NMR}$

$\left(\mathrm{CDCl}_{3}, 101 \mathrm{MHz}\right)$

$$
\delta=130.6\left(1 C, C_{10}\right), 130.0\left(2 C, C_{4 a, 10 a}\right), 129.5\left(2 C, C_{8 a, 9 a}\right), 128.9
$$

$\left(1 \mathrm{C}, \mathrm{C}_{9}\right), 125.8\left(2 \mathrm{C}, \mathrm{C}_{2,7}\right), 125.6\left(2 \mathrm{C}, \mathrm{C}_{4,5}\right), 124.9\left(2 \mathrm{C}, \mathrm{C}_{3,6}\right), 124.2$

$\left(2 \mathrm{C}, \mathrm{C}_{1,8}\right), 32.1\left(1 \mathrm{C}, \mathrm{C}_{11}\right), 31.1\left(1 \mathrm{C}, \mathrm{C}_{12}\right), 14.3\left(1 \mathrm{C}, \underline{\mathrm{C}}_{3}\right)$.

\section{El-MS}

$\mathrm{m} / \mathrm{z}(\%)$ :

$298(28)[\mathrm{M}]^{+}, 205$ (92) [M - $\left.\mathrm{CH}_{2} \mathrm{Br}\right]^{+}$.

\subsubsection{Synthesis of 9-Bromo-10-TMEDA-anthracene (69)}

9-Bromo-10-(bromomethyl)anthracene $(1.55 \mathrm{~g}, 4.43 \mathrm{mmol})$ was dissolved in MeCN (40 mL) and $\mathrm{N}, \mathrm{N}, \mathrm{N}$-trimethylethylenediamine $(0.46 \mathrm{~g}, 4.50 \mathrm{mmol})$ and $\mathrm{K}_{2} \mathrm{CO}_{3}(2.47 \mathrm{~g}(17.84 \mathrm{mmol})$ was added. The mixture was heated to $82^{\circ} \mathrm{C}$ over $20 \mathrm{~h}$. Then the solvent was evaporated and the crude product was extracted with DCM $(60 \mathrm{~mL})$ and water $(40 \mathrm{~mL})$. The organic layer was extracted with saturated $\mathrm{NaCl}$ solution and then dried over $\mathrm{MgSO}_{4}$ and filtrated. Removal of the solvent under reduced pressure afforded 9-Bromo-10-TMEDA-anthracene (69) as an oily red solid.

Yield: $1.20 \mathrm{~g}(3.23 \mathrm{mmol}, 73 \%)$

Empirical formula: $\mathrm{C}_{20} \mathrm{H}_{23} \mathrm{BrN}_{2}$

Molar mass: $371.31 \mathrm{~g} / \mathrm{mol}$

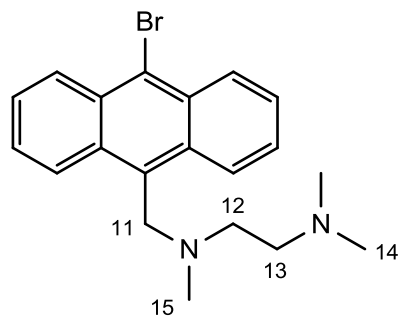




\section{${ }^{1}$ H-NMR}

$\left(\mathrm{CDCl}_{3}, 300 \mathrm{MHz}\right)$ :

$$
\begin{aligned}
& \delta=8.56\left(\mathrm{~d},{ }^{3} \mathrm{~J}_{\mathrm{HH}}=8.75 \mathrm{~Hz}, 2 \mathrm{H}, \mathrm{H}_{1,8}\right), 8.52\left(\mathrm{~d},{ }^{3} J_{\mathrm{HH}}=8.80 \mathrm{~Hz}, 2 \mathrm{H},\right. \\
& \left.\mathrm{H}_{4,5}\right), 7.53\left(\mathrm{~m}, 4 \mathrm{H}, \mathrm{H}_{2,3,6,7}\right), 4.40\left(\mathrm{~s}, 2 \mathrm{H}, \mathrm{H}_{11}\right), 2.69(\mathrm{t}, \\
& \left.{ }^{3} J_{\mathrm{HH}}=6.50 \mathrm{~Hz}, 2 \mathrm{H}, \mathrm{H}_{12}\right), 2.48\left(\mathrm{t},{ }^{3} J_{\mathrm{HH}}=6.50 \mathrm{~Hz}, 2 \mathrm{H}, \mathrm{H}_{13}\right), 2.25(\mathrm{~s}, \\
& \left.3 \mathrm{H}, \mathrm{H}_{15}\right), 2.16\left(\mathrm{~s}, 6 \mathrm{H}, \mathrm{H}_{14}\right) .
\end{aligned}
$$

\section{${ }^{13} \mathrm{C}\left\{{ }^{1} \mathrm{H}\right\}-\mathrm{NMR}$}

$\left(\mathrm{CDCl}_{3}, 75 \mathrm{MHz}\right)$ :

$$
\begin{aligned}
& \delta=132.02\left(2 \mathrm{C}, \mathrm{C}_{4 \mathrm{a}, 10 \mathrm{a}}\right), 130.85\left(1 \mathrm{C}, \mathrm{C}_{10}\right), 130.19\left(2 \mathrm{C}, \mathrm{C}_{8 \mathrm{a}, 9 \mathrm{a}}\right), \\
& 128.38\left(2 \mathrm{C}, \mathrm{C}_{1,8}\right), 126.62\left(2 \mathrm{C}, \mathrm{C}_{2,7}\right), 125.82\left(2 \mathrm{C}, \mathrm{C}_{3,6}\right), 125.40(2 \mathrm{C}, \\
& \left.\mathrm{C}_{4,5}\right), 123.78\left(1 \mathrm{C}, \mathrm{C}_{9}\right), 57.22\left(1 \mathrm{C}, \mathrm{C}_{13}\right), 55.76\left(1 \mathrm{C}, \mathrm{C}_{12}\right), 54.23(1 \mathrm{C}, \\
& \left.\mathrm{C}_{11}\right), 45.58\left(2 \mathrm{C}, \mathrm{C}_{14}\right), 42.25\left(1 \mathrm{C}, \mathrm{C}_{15}\right) .
\end{aligned}
$$

\section{EI-MS}

$m / z(\%):$

$370(4)[\mathrm{M}]^{+}, 312(8)\left[\mathrm{M}-\left(\mathrm{CH}_{3}\right)_{2} \mathrm{NCH}_{2}\right]^{+}, 291(8)[\mathrm{M}-\mathrm{Br}]^{+}, 269$

(98) $\left[\mathrm{M}-\mathrm{C}_{5} \mathrm{H}_{13} \mathrm{~N}_{2}\right]^{+}, 190(12)\left[\mathrm{M}-\mathrm{C}_{5} \mathrm{H}_{13} \mathrm{~N}_{2}, \mathrm{Br}\right]^{+}$.

\subsubsection{Synthesis of 9-Bromo-10-bis(2-methoxyethyl)aminomethyl- anthracene (70)}

9-Bromo-10-(bromomethyl)anthracene (1.56 g, $4.46 \mathrm{mmol})$ was dissolved in MeCN $(50 \mathrm{~mL})$ and bis(2-mothxyethyl)amine $(0.62 \mathrm{~g}, 4.53 \mathrm{mmol})$ and $\mathrm{K}_{2} \mathrm{CO}_{3}(2.47 \mathrm{~g}, 17.84 \mathrm{mmol})$ was added. The mixture was heated to $82^{\circ} \mathrm{C}$ over $20 \mathrm{~h}$. Then the solvent was evaporated and the crude product was extracted with $\mathrm{DCM}(60 \mathrm{~mL})$ and water $(40 \mathrm{~mL})$. The organic layer was extracted with saturated $\mathrm{NaCl}$ solution and then dried over $\mathrm{MgSO}_{4}$ and filtrated. Removal of the solvent under reduced pressure afforded 9-Bromo-10-TMEDA-anthracene (69) as a red oil.

Yield: $1.25 \mathrm{~g}$ (3.11 mmol, 70\%)

Empirical formula: $\mathrm{C}_{21} \mathrm{H}_{24} \mathrm{BrNO}_{2}$

Molar mass: $402.33 \mathrm{~g} / \mathrm{mol}$

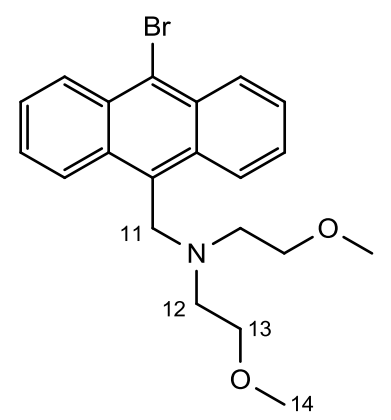


${ }^{1} \mathrm{H}$ NMR

$\left(\mathrm{CDCl}_{3}, 500 \mathrm{MHz}\right)$ :

$\delta=8.57\left(\mathrm{~m}, 4 \mathrm{H}, \mathrm{H}_{1,4,5,8}\right), 7.53\left(\mathrm{~m}, 4 \mathrm{H}, \mathrm{H}_{2,3,6,7}\right), 4.61\left(\mathrm{~s}, 2 \mathrm{H}, \mathrm{H}_{11}\right)$,

$3.44\left(\mathrm{t},{ }^{3} \mathrm{JHH}_{\mathrm{HH}}=6.00 \mathrm{~Hz}, 4 \mathrm{H}, \mathrm{H}_{13}\right), 3.25\left(\mathrm{~s}, 6 \mathrm{H}, \mathrm{H}_{14}\right), 2.81(\mathrm{t}$,

$\left.{ }^{3} J_{H H}=6.00 \mathrm{~Hz}, 4 \mathrm{H}, \mathrm{H}_{12}\right)$.

${ }^{13} \mathrm{C}\left\{{ }^{1} \mathrm{H}\right\}$ NMR

$\left(\mathrm{CDCl}_{3}, 126 \mathrm{MHz}\right)$ :

$\delta=132.13\left(2 \mathrm{C}, \mathrm{C}_{4 \mathrm{a}, 10 \mathrm{a}}\right), 131.14\left(1 \mathrm{C}, \mathrm{C}_{10}\right), 130.22\left(2 \mathrm{C}, \mathrm{C}_{8 \mathrm{a}, 9 \mathrm{a}}\right)$,

$128.35\left(2 \mathrm{C}, \mathrm{C}_{1,2}\right), 126.65\left(2 \mathrm{C}, \mathrm{C}_{2,7}\right), 125.76\left(2 \mathrm{C}, \mathrm{C}_{3,6}\right), 125.61(2 \mathrm{C}$,

$\left.\mathrm{C}_{4,5}\right), 123.83\left(1 \mathrm{C}, \mathrm{C}_{9}\right), 71.56\left(2 \mathrm{C}, \mathrm{C}_{13}\right), 58.60\left(2 \mathrm{C}, \mathrm{C}_{14}\right), 53.58(2 \mathrm{C}$,

$\left.\mathrm{C}_{12}\right), 51.90\left(1 \mathrm{C}, \mathrm{C}_{11}\right)$.

EI-MS

$m / z(\%):$

$401(4)[\mathrm{M}]^{+}, 356(16)\left[\mathrm{M}-\mathrm{CH}_{2} \mathrm{OCH}_{3}\right]^{+}, 269$ (100)

$\left[\mathrm{M}-\mathrm{N}\left(\mathrm{C}_{2} \mathrm{H}_{4} \mathrm{OCH}_{3}\right)_{2}\right]^{+}$.

\subsubsection{Synthesis of Anthrylpyridinium Chloride (71)}

9-Bromo-10-(bromomethyl)anthracene $(1.75 \mathrm{~g}, 5.00 \mathrm{mmol})$ was dissolved in MeCN $(60 \mathrm{~mL})$ and dipyridylamine $(0.86 \mathrm{~g}, 5.00 \mathrm{mmol})$ and $\mathrm{K}_{2} \mathrm{CO}_{3}(3.36 \mathrm{~g}, 25.0 \mathrm{mmol})$ was added. The mixture was heated to $82^{\circ} \mathrm{C}$ over $20 \mathrm{~h}$. Then the solvent was evaporated and the crude product was extracted with DCM $(60 \mathrm{~mL})$ and water $(40 \mathrm{~mL})$. The organic layer was extracted with saturated $\mathrm{NaCl}$ solution and then dried over $\mathrm{MgSO}_{4}$ and filtrated. Removal of the solvent under reduced pressure afforded pyridinium salt $\mathbf{7 1}$ as a yellow solid.

Yield: $3.02 \mathrm{~g}(4.25 \mathrm{mmol}, 85 \%)$

Empirical formula: $\mathrm{C}_{40} \mathrm{H}_{29} \mathrm{Br}_{2} \mathrm{~N}_{3} \mathrm{Cl}$

Molar mass: $745.93 \mathrm{~g} / \mathrm{mol}$

\section{${ }^{1} \mathrm{H}-\mathrm{NMR}$}

$\left(\mathrm{CDCl}_{3}, 300 \mathrm{MHz}\right)$ :

$\delta[\mathrm{ppm}]=8.60-8.59\left(\mathrm{~d}, J=8.9 \mathrm{~Hz}, 4 \mathrm{H}, \mathrm{H}_{4,4^{\prime}, 5,5^{\prime}}\right), 8.47-8.45(\mathrm{~d}$, $\left.{ }^{3} J_{H H}=8.9 \mathrm{~Hz}, 4 \mathrm{H}, \mathrm{H}_{1,1^{\prime}, 8,8^{\prime}}\right), 7.97-7.94$ (ddd, $J=1.7,6.9 \mathrm{~Hz}, 2 \mathrm{H}$, $\left.\mathrm{H}_{13,20}\right)$ 7.78-7.75 (ddd, $\left.J=0.9,2.3,6.6 \mathrm{~Hz}, 4 \mathrm{H}, \mathrm{H}_{3,3^{\prime}, 6,6^{\prime}}\right), 7.71-$ 7.68 (ddd, $\left.J=1.2,2.3,6.6 \mathrm{~Hz}, 4 \mathrm{H}, \mathrm{H}_{2,2^{\prime}, 7,7^{\prime}}\right), 7.67-7.65(\mathrm{~m}, 4 \mathrm{H}$, $\left.\mathrm{H}_{14,15,18,19}\right), 7.36-7.34\left(\mathrm{dd}, \mathrm{J}=6.9,1.2 \mathrm{~Hz}, 2 \mathrm{H}, \mathrm{H}_{12,21}\right), 6.72(\mathrm{~s}, 4 \mathrm{H}$, $\mathrm{CH}_{2}$ ). 


\section{${ }^{13} \mathrm{C}\left\{{ }^{1} \mathrm{H}\right\}-\mathrm{NMR}$}

$\left(\mathrm{CDCl}_{3}, 75 \mathrm{MHz}\right)$ :

$$
\begin{aligned}
& \delta[p p m]=155.6\left(2 \mathrm{C}, \mathrm{C}_{10,10^{\prime}}\right), 142.3\left(2 \mathrm{C}, \mathrm{C}_{13,20}\right), 138.5\left(2 \mathrm{C}, \mathrm{C}_{12,21}\right), \\
& 131.8\left(2 \mathrm{C}, \mathrm{C}_{16,17}\right), 129.8\left(2 \mathrm{C}, \mathrm{C}_{14,19}\right), 128.2\left(4 \mathrm{C}, \mathrm{C}_{2,2^{\prime}, 7,7^{\prime}}\right), 128.1 \\
& \left(4 \mathrm{C}, \mathrm{C}_{3,3^{\prime}, 6,6^{\prime}}\right), 127.9\left(4 \mathrm{C}, \mathrm{C}_{4,4^{\prime}, 5,5^{\prime}}\right), 125.5\left(4 \mathrm{C}, \mathrm{C}_{8 \mathrm{a}, 8 \mathrm{a}^{\prime}, 9 \mathrm{a}, 9 \mathrm{a}^{\prime}}\right), 125.4 \\
& \left(4 \mathrm{C}, \mathrm{C}_{4 \mathrm{a}, 4 \mathrm{a}^{\prime}, 10 \mathrm{a}, 10 \mathrm{a}^{\prime}}\right), 124.3\left(4 \mathrm{C}, \mathrm{C}_{1,1^{\prime}, 8,8^{\prime}}\right), 116.0\left(2 \mathrm{C}, \mathrm{C}_{15,18}\right), 114.3 \\
& \left(2 \mathrm{C}, \mathrm{C}_{9,9^{\prime}}\right), 47.7\left(2 \mathrm{C}, \mathrm{CH}_{2}\right) .
\end{aligned}
$$

\section{EI-MS}

$\mathrm{m} / \mathrm{z}(\%)$ :

$745(3)[\mathrm{M}]^{+}, 710(35)[\mathrm{M}-\mathrm{Cl}]^{+}, 454(46)[\mathrm{M}-\mathrm{AnBr}, \mathrm{Cl}]^{+}$.

\subsubsection{Synthesis of 10-Bis[bis(2-thienylmethyl)aminomethyl]- anthracene (72)}

9,10-Bis(bromomethyl)anthracene $(1.00 \mathrm{~g}, 2.75 \mathrm{mmol})$ was dissolved in MeCN (35 mL) and bis(2-thienylmethyl)amine (61) $(0.57 \mathrm{~g}, 2.75 \mathrm{mmol})$ and $\mathrm{K}_{2} \mathrm{CO}_{3}(1.90 \mathrm{~g}, 28.6 \mathrm{mmol}$ ) was added. The mixture was heated to $82^{\circ} \mathrm{C}$ over $20 \mathrm{~h}$. Then the solvent was evaporated and the crude product was extracted with $\mathrm{DCM}(60 \mathrm{~mL})$ and water $(40 \mathrm{~mL})$. The organic layer was extracted with saturated $\mathrm{NaCl}$ solution and then dried over $\mathrm{MgSO}_{4}$ and filtrated. The solvent was removed under reduced pressure. The crude product was purified by column chromatography (pentane/ethyl acetate 2:1) and was obtained as a yellow semi-solid.

Yield: $0.53 \mathrm{~g}(1.07 \mathrm{mmol}, 39 \%)$

Empirical formula: $\mathrm{C}_{36} \mathrm{H}_{32} \mathrm{~N}_{2} \mathrm{~S}_{4}$

Molar mass: $492.49 \mathrm{~g} / \mathrm{mol}$.

${ }^{1} \mathrm{H}-\mathrm{NMR}$

$\left(\mathrm{CDCl}_{3}, 300 \mathrm{MHz}\right)$ :

$\delta[p p m]=8.49-8.53\left(\mathrm{~m}, 4 \mathrm{H}, \mathrm{H}_{1,4,5,8}\right), 7.49-7.53\left(\mathrm{~m}, 4 \mathrm{H}, \mathrm{H}_{2,3,6,7}\right)$, 7.18-7.24 (m, 4H, 4×Ty-H), 6.88-6.92 (m, 8H, 8×Ty-H), 4.63 (s, $\left.4 \mathrm{H}, \mathrm{AnC}_{2} \mathrm{~N}\right), 3.19\left(\mathrm{~s}, 8 \mathrm{H}, \mathrm{NC}_{2} \mathrm{Ty}\right)$.

${ }^{13} \mathrm{C}\left\{{ }^{1} \mathrm{H}\right\}-\mathrm{NMR}$

$\left(\mathrm{CDCl}_{3}, 75 \mathrm{MHz}\right)$ :

$\delta[p p m]=142.5\left(2 \times C_{i}-T y\right), 131.2\left(4 \mathrm{C}, \mathrm{C}_{4 \mathrm{a}, 8 \mathrm{a}, 9 \mathrm{a}, 10 \mathrm{a}}\right), 130.2(2 \mathrm{C}$,

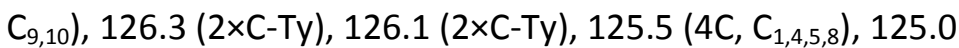
(2×C-Ty), $124.9\left(4 \mathrm{C}, \mathrm{C}_{2,3,6,7}\right), 51.7\left(\mathrm{NCH}_{2} \mathrm{Ty}\right), 50.6\left(\mathrm{AnCH}_{2} \mathrm{~N}\right)$.

\section{EI-MS}

$\mathrm{m} / \mathrm{z}(\%)$ :

$620(15)[\mathrm{M}-\mathrm{H}]^{+}, 413$ (11), 191 (19), 123 (28), 97 (100). 


\subsubsection{Synthesis of 9-[bis(2-tert-butylthioethyl)aminomethyl]- anthracene (73)}

9-(Bromomethyl)anthracene $(1.08 \mathrm{~g}, 4.00 \mathrm{mmol})$ was dissolved in $\mathrm{MeCN}(20 \mathrm{~mL})$ and $\mathrm{K}_{2} \mathrm{CO}_{3}$ $(2.21 \mathrm{~g}, 16.00 \mathrm{mmol})$ was added. To this mixture a solution of bis(2-mercaptoethyl)amine (65) $(1.00 \mathrm{~g}, 4.00 \mathrm{mmol})$ in $\mathrm{MeCN}(20 \mathrm{~mL})$ was added. The reaction was heated to $82^{\circ} \mathrm{C}$ over $20 \mathrm{~h}$. Then the solvent was evaporated and the crude product was extracted with degassed DCM $(60 \mathrm{~mL})$ and degassed water $(40 \mathrm{~mL})$. The organic layer was washed with saturated $\mathrm{NaCl}$ solution $(30 \mathrm{~mL})$ and dried over $\mathrm{MgSO}_{4}$. After filtration and evaporation of the solvent the crude product was purified by column chromatography (pentane/ethyl acetate 1:1). 73 was obtained as a yellow oily solid.

Yield: $0.43 \mathrm{~g}(1.00 \mathrm{mmol}, 24 \%)$

Empirical formula: $\mathrm{C}_{27} \mathrm{H}_{37} \mathrm{NS}_{2}$

Molar mass: $439.72 \mathrm{~g} / \mathrm{mol}$

${ }^{1} \mathrm{H}-\mathrm{NMR}$

$\left(\mathrm{CDCl}_{3}, 300 \mathrm{MHz}\right)$ :

$$
\begin{aligned}
& \delta[p p m]=8.50\left(m, 3 \mathrm{H}, \mathrm{An}-\mathrm{H}_{1,8,10}\right), 8.04\left(\mathrm{~m}, 2 \mathrm{H}, \mathrm{An}-\mathrm{H}_{4,5}\right), 7.50 \\
& \left(\mathrm{~m}, 4 \mathrm{H}, \mathrm{An}-\mathrm{H}_{2,3,6,7}\right), 4.65\left(\mathrm{~s}, 2 \mathrm{H}, \mathrm{CH}_{2}-\mathrm{An}\right), 2.84\left(\mathrm{~m}, 4 \mathrm{H}, \mathrm{N}-\mathrm{CH}_{2}\right) \\
& 2.60\left(\mathrm{~m}, 4 \mathrm{H}, \mathrm{S}-\mathrm{CH}_{2}\right), 1.16\left(\mathrm{~s}, 18 \mathrm{H}, \mathrm{CH}_{3}\right) .
\end{aligned}
$$

\section{EI-MS}

$m / z(\%)$ :

(6) $\left[\mathrm{C}_{2} \mathrm{H}_{4} \mathrm{SC}\left(\mathrm{CH}_{3}\right)_{3}\right]^{+}$.

\subsubsection{Synthesis of $\mathrm{AnCH}_{2} \mathrm{~N}\left(\mathrm{C}_{2} \mathrm{H}_{4} \mathrm{PPh}_{2}\right)_{2}(74)$}

9-(Bromomethyl)anthracene $(1.56 \mathrm{~g}, 5.75 \mathrm{mmol})$ was dissolved in dry degassed MeCN ( $35 \mathrm{~mL}$ ) and $\mathrm{K}_{2} \mathrm{CO}_{3}(3.18 \mathrm{~g}, 23.00 \mathrm{mmol})$ was added. To this mixture a solution of bis(2diphenylphosphinoethyl)amine (64) $(2.54 \mathrm{~g}, 5.75 \mathrm{mmol})$ in dry degassed MeCN was added. The reaction was heated to $82^{\circ} \mathrm{C}$ over $20 \mathrm{~h}$. Then the solvent was evaporated and the crude product was extracted with degassed DCM $(60 \mathrm{~mL})$ and degassed water $(40 \mathrm{~mL})$. The organic layer then dried over $\mathrm{MgSO}_{4}$ and filtrated. The solvent was removed under reduced pressure.

In a second reaction bis(2-diphenylphosphinoethyl)amine (64) $(3.39 \mathrm{~g}, 7.68 \mathrm{mmol}$ ) was dissolved in THF and reacted with $n$-BuLi solution in hexane $(2.42 \mathrm{~mL}, 7.68 \mathrm{mmol}, 3.18 \mathrm{M})$ at $-15^{\circ} \mathrm{C}$. The resulting solution was added to a suspension of 9-(bromomethyl)anthracene $(2.08 \mathrm{~g}$ $(7.68 \mathrm{mmol})$ in $\mathrm{THF}$ at $-15^{\circ} \mathrm{C}$ over $30 \mathrm{~min}$. The reaction was stirred overnight at ambient temperature, the solvent was evaporated and the crude product was dissolved in DCM. After filtration and removal of the solvent an orange solid was obtained.

Yield: $4.36 \mathrm{~g}(6.90 \mathrm{mmol}, 90 \%)$ 
Empirical formula: $\mathrm{C}_{43} \mathrm{H}_{39} \mathrm{NP}_{2}$

Molar mass: $631.72 \mathrm{~g} / \mathrm{mol}$

${ }^{1} \mathrm{H}-\mathrm{NMR}$

$\left(\mathrm{CDCl}_{3}, 300 \mathrm{MHz}\right): \quad \delta[\mathrm{ppm}]=8.55\left(\mathrm{~s}, 1 \mathrm{H}, \mathrm{An}-\mathrm{H}_{10}\right), 8.37\left(\mathrm{~m}, 4 \mathrm{H}, \mathrm{An}-\mathrm{H}_{1,8}\right), 8.02(\mathrm{~m}$, $\left.2 \mathrm{H}, \mathrm{An}-\mathrm{H}_{4,5}\right), 7.78\left(\mathrm{~m}, 2 \mathrm{H}, \mathrm{An}-\mathrm{H}_{2,7}\right), 7.46\left(\mathrm{~m}, 2 \mathrm{H}, \mathrm{An}-\mathrm{H}_{3,6}\right), 7.33$ $\left(\mathrm{m}, 2 \mathrm{HH}, \mathrm{C}_{6} \mathrm{H}_{5}\right), 4.08\left(\mathrm{~s}, 2 \mathrm{H}, \mathrm{N}-\mathrm{CH}_{2}-\mathrm{An}\right), 2.72\left(\mathrm{t}, \mathrm{J}_{\mathrm{HH}}=7.9 \mathrm{~Hz}, 4 \mathrm{H}\right.$, $\left.\mathrm{N}-\mathrm{CH}_{2}\right), 2.25\left(\mathrm{t}, \mathrm{J}_{\mathrm{HH}}=7.6 \mathrm{~Hz}, 4 \mathrm{H}, \mathrm{P}-\mathrm{CH}_{2}\right)$.

${ }^{31} \mathrm{P}\left\{{ }^{1} \mathrm{H}\right\}-\mathrm{NMR}$

$\left(\mathrm{CDCl}_{3}, 81 \mathrm{MHz}\right)$ :

$\delta[\mathrm{ppm}]=-20.6(\mathrm{~s}, 2 \mathrm{P})$.

\title{
EI-MS
}

$m / z(\%):$

\author{
$631(4)[M]^{+}, 446(83)\left[M-P\left(C_{6} H_{5}\right)_{2}\right]^{+}, 432(14)$ \\ $\left[\mathrm{M}-\mathrm{CH}_{2} \mathrm{P}\left(\mathrm{C}_{6} \mathrm{H}_{5}\right)_{2}\right]^{+}, 256(6)\left[\left(\mathrm{CH}_{2}\right)_{2} \mathrm{~N}\left(\mathrm{C}_{2} \mathrm{H}_{4}\right) \mathrm{P}\left(\mathrm{C}_{6} \mathrm{H}_{5}\right)_{2}\right]^{+}, 191(100)$ \\ $\left[\mathrm{CH}_{2}-\mathrm{An}\right]^{+}, 185(19)\left[\mathrm{P}\left(\mathrm{C}_{6} \mathrm{H}_{5}\right)_{2}\right]^{+}$.
}

\subsubsection{Synthesis of $\mathrm{AnCH}_{2} \mathrm{~N}\left(\mathrm{CH}_{2} \mathrm{PhOMe}\right)_{2}(75)$}

Bis(2-methoxy)benzyl)amine (62) $(1.90 \mathrm{~g}, 7.38 \mathrm{mmol})$ and 9-(bromomethyl)anthracene $(2.00 \mathrm{~g}$, $7.38 \mathrm{mmol})$ were dissolved in $\mathrm{MeCN}(60 \mathrm{~mL})$ and $\mathrm{K}_{2} \mathrm{CO}_{3}(4,0 \mathrm{~g}, 28.4 \mathrm{mmol})$ was added. The reaction mixture was heated to $82^{\circ} \mathrm{C}$ for eight hours and the stirred at ambient temperature for another $24 \mathrm{~h}$. Then the solvent was evaporated and the crude product was dissolved in DCM $(50 \mathrm{~mL})$ and water $(30 \mathrm{~mL})$. The layers were separated and the organic layer was washed with saturated $\mathrm{NaCl}$ solution and dried over $\mathrm{MgSO}_{4}$. The solvent was removed and the crude product was purified by column chromatography (petrol ether/ ethyl acetate 15:1). $\mathrm{AnCH}_{2} \mathrm{~N}\left(\mathrm{CH}_{2} \mathrm{PhSMe}\right)_{2}(76)$ was obtained as a yellow solid.

Yield: $2.38 \mathrm{~g}(5.31 \mathrm{mmol}, 72 \%)$.

Empirical formula: $\mathrm{C}_{31} \mathrm{H}_{29} \mathrm{NO}_{2}$

Molar mass: $447.57 \mathrm{~g} / \mathrm{mol}$

${ }^{1}$ H-NMR

$\left(\mathrm{CDCl}_{3}, 300 \mathrm{MHz}\right): \quad \delta[\mathrm{ppm}]=8.55-8.23(\mathrm{~m}, 3 \mathrm{H}, \mathrm{Ar}-\mathrm{H}), 8.06-7.85(\mathrm{~m}, 2 \mathrm{H}, \mathrm{Ar}-\mathrm{H})$, 7.60-7.29 (m, $6 \mathrm{H}, \mathrm{Ar}-\mathrm{H}), 7.18(\mathrm{t}, J=7.6 \mathrm{~Hz}, 2 \mathrm{H}, \mathrm{Ar}-\mathrm{H}), 6.88(\mathrm{t}$, $J=7.4 \mathrm{~Hz}, 2 \mathrm{H}, \mathrm{Ar}-\mathrm{H}), 6.80$ (d, J = 8.2 Hz, $2 \mathrm{H}, \mathrm{Ar}-\mathrm{H}), 4.54(\mathrm{~s}, 2 \mathrm{H}$, An- $\left.\underline{\mathrm{H}}_{2}-\mathrm{N}\right), 3.71\left(\mathrm{~s}, 4 \mathrm{H}, \mathrm{N}-\underline{\mathrm{C}}_{2}-\mathrm{Ph}\right), 3.69\left(\mathrm{~s}, 6 \mathrm{H}, \mathrm{OC}_{3}\right)$. 


\section{${ }^{13} \mathrm{C}\left\{{ }^{1} \mathrm{H}\right\}-\mathrm{NMR}$}

$\left(\mathrm{CDCl}_{3}, 125 \mathrm{MHz}\right)$ :
$\delta[\mathrm{ppm}]=157.9\left(\underline{\left.\mathrm{COCH}_{3}\right)} 131.5\right.$ (C-Ar), 131.3 (C-Ar), 128.7 (CAr), 127.7 (C-Ar), 127.0 (C-Ar), 125.6 (C-Ar), 125.0 (C-Ar), 124.6 (C-Ar), 120.0 (C-Ar), 110.1 (C-Ar), $55.06\left(\mathrm{OCH}_{3}\right), 51.90\left(\mathrm{An}-\underline{C H}_{2}\right)$.

EI-MS

$\mathrm{m} / \mathrm{z}(\%)$ :
447(50) $[\mathrm{M}]^{+}, 326(33)\left[\mathrm{M}-\mathrm{PhOMe}_{2}\right]^{+}, 191$ (100) [AnMe]

\subsubsection{Synthesis of $\mathrm{AnCH}_{2} \mathrm{~N}\left(\mathrm{CH}_{2} \mathrm{PhSMe}\right)_{2}(76)$}

Bis(2-methylthio)benzyl)amine (63) (2.12 g, $7.38 \mathrm{mmol})$ and 9-(bromomethyl)anthracene $(2.00 \mathrm{~g}, 7.38 \mathrm{mmol})$ were dissolved in $\mathrm{MeCN}(60 \mathrm{~mL})$ and $\mathrm{K}_{2} \mathrm{CO}_{3}(4,0 \mathrm{~g}, 28.4 \mathrm{mmol})$ was added. The reaction mixture was heated to $82^{\circ} \mathrm{C}$ for eight hours and the stirred at ambient temperature for another $24 \mathrm{~h}$. Then the solvent was evaporated and the crude product was dissolved in DCM $(50 \mathrm{~mL})$ and water $(30 \mathrm{~mL})$. The layers were separated and the organic layer was washed with saturated $\mathrm{NaCl}$ solution and dried over $\mathrm{MgSO}_{4}$. The solvent was removed and the crude product was purified by column chromatography (petrol ether/ ethyl acetate 15:1). AnCH $\mathrm{H}_{2} \mathrm{~N}\left(\mathrm{CH}_{2} \mathrm{PhSMe}\right)_{2}$ (76) was obtained as a yellow solid.

Yield: $2.44 \mathrm{~g}(5.09 \mathrm{mmol}, 69 \%)$.

Empirical formula: $\mathrm{C}_{31} \mathrm{H}_{29} \mathrm{NS}_{2}$

Molar mass: $479.70 \mathrm{~g} / \mathrm{mol}$

\section{${ }^{1} \mathrm{H}-\mathrm{NMR}$}

$\left(\mathrm{CDCl}_{3}, 300 \mathrm{MHz}\right)$ :

$\delta[\mathrm{ppm}]=8.45-8.26(\mathrm{~m}, 3 \mathrm{H}, \mathrm{Ar}-\mathrm{H}), 7.96(\mathrm{~d}, J=2.1 \mathrm{~Hz}, 2 \mathrm{H}, \mathrm{Ar}-$

H), 7.50-7.32 (m, $6 \mathrm{H}, \mathrm{Ar}-\mathrm{H}), 7.17$ (d, J = 5.5 Hz, $4 \mathrm{H}, \mathrm{Ar}-\mathrm{H})$,

7.11-7.04 (m, $2 \mathrm{H}, \mathrm{Ar}-\mathrm{H}), 4.56$ (s, $\left.2 \mathrm{H}, \mathrm{An}-\underline{\mathrm{C}}_{2}-\mathrm{N}\right), 3.79$ (s, $4 \mathrm{H}$, $\left.\mathrm{N}-\underline{\mathrm{H}}_{2}-\mathrm{Ph}\right), 2.34\left(\mathrm{~s}, 6 \mathrm{H}, \mathrm{OC}_{3}\right)$.

${ }^{13} \mathrm{C}\left\{{ }^{1} \mathrm{H}\right\}-\mathrm{NMR}$

$\left(\mathrm{CDCl}_{3}, 125 \mathrm{MHz}\right)$

$\delta[\mathrm{ppm}]=138.3\left(\mathrm{CSCH}_{3}\right) 137.5(\mathrm{C}-\mathrm{Ar}), 131.6$ (C-Ar), 131.3 (C-

Ar), 130.7 (C-Ar), 130.2 (C-Ar), 128.7 (C-Ar), 127.5 (C-Ar), 127.3

(C-Ar), 125.7 (C-Ar), 125.5 (C-Ar), 125.2 (C-Ar), 124.6 (2 x C-Ar)

$55.81\left(\mathrm{~N}-\underline{\mathrm{CH}}_{2}-\mathrm{Ph}\right), 50.56\left(\mathrm{An}-\underline{\mathrm{CH}}_{2}-\mathrm{N}\right), 16.28\left(\mathrm{~S}_{\mathrm{C}} \mathrm{H}_{3}\right)$ 


\subsubsection{Synthesis of $\mathrm{AnCH}_{2} \mathrm{PPh}_{2}$ (77)}

To a solution of diphenylphosphane $(1.44 \mathrm{~g}, 7.74 \mathrm{mmol})$ in diethyl ether $(30 \mathrm{~mL}) n$-BuLi ( $3.16 \mathrm{~mL}, 2.45 \mathrm{M}, 7.74 \mathrm{mmol}$ ) was added over $30 \mathrm{~min}$ at $-15^{\circ} \mathrm{C}$. The solution was then warmed to ambient temperature and added to a suspension of 9-(bromomethyl)anthracene $(2.10 \mathrm{~g}$, $7.74 \mathrm{mmol})$ in THF $(20 \mathrm{~mL})$ at $-15^{\circ} \mathrm{C}$. The addition was carried out over the course of $1 \mathrm{~h}$. The reaction was then stirred overnight at ambient temperature. The solution was then concentrated and the formed precipitate was isolated by filtration and dried under reduced pressure. The crude product was then re-dissolved in toluene and filtrated for removal of lithium bromide. Removal of the solvent afforded $\mathrm{AnCH}_{2} \mathrm{PPh}_{2}(77)$ as a pale yellow solid.

Yield: $2.11 \mathrm{~g}(5,61 \mathrm{mmol}, 72 \%)$

Empirical formula: $\mathrm{C}_{27} \mathrm{H}_{21} \mathrm{P}$

Molar mass: $376.43 \mathrm{~g} / \mathrm{mol}$

${ }^{1}$ H-NMR

(THF $d_{8}, 300 \mathrm{MHz}$ ):

$\delta[\mathrm{ppm}]=8.43\left(\mathrm{~s}, 1 \mathrm{H}, \mathrm{H}_{10}\right), 8.05\left(\mathrm{~m}, 4 \mathrm{H}, \mathrm{H}_{1,8,4,5}\right), 7.49-7.40(\mathrm{~m}$, $\left.8 \mathrm{H}, \mathrm{H}_{2,3,6,7}, \mathrm{H}_{\text {ortho }}(\mathrm{Ph})\right)$, 7.38-7.26 (m, 6H, $\left.\mathrm{H}_{\text {meta, para }}(\mathrm{Ph})\right), 4.54(\mathrm{~s}$, $2 \mathrm{H}, \mathrm{CH}_{2}$ ).

\section{${ }^{13} \mathrm{C}\left\{{ }^{1} \mathrm{H}\right\}-\mathrm{NMR}$}

(THF $d_{8}, 126 \mathrm{MHz}$ ):

$\delta[\mathrm{ppm}]=131.2\left(\mathrm{~d},{ }^{1} \mathrm{~J}_{\mathrm{CP}}=66.4 \mathrm{~Hz}, 2 \mathrm{C}\right.$, ipso-Ph, 129.6 (d, $\left.{ }^{5} J_{\mathrm{CP}}=1.8 \mathrm{~Hz}, 2 \mathrm{C}, p-\mathrm{Ph}\right), 129.4\left(\mathrm{~d},{ }^{3} \mathrm{~J}_{\mathrm{CP}}=8.2 \mathrm{~Hz}, 4 \mathrm{C}, o-\mathrm{Ph}\right), 128.8$ $\left(d, J_{C P}=4.5 \mathrm{~Hz}, 2 \mathrm{C}, \mathrm{C}_{4 \mathrm{a}, 10 \mathrm{a}} \operatorname{oder} \mathrm{C}_{8 \mathrm{a}, 9 \mathrm{a}}\right), 127.8\left(\mathrm{~d}, J_{\mathrm{CP}}=1.0 \mathrm{~Hz}, 2 \mathrm{C}\right.$, $\left.\mathrm{C}_{1,8} \operatorname{oder} \mathrm{C}_{4,5}\right), 127.5\left(\mathrm{~d},{ }^{4} \mathrm{~J}_{\mathrm{CP}}=8.5 \mathrm{~Hz}, 4 \mathrm{C}, \mathrm{m}-\mathrm{Ph}\right), 126.6$ (d, $\left.{ }^{5} J_{C P}=3.3 \mathrm{~Hz}, 1 \mathrm{C}, C_{10}\right), 123.9\left(\mathrm{~d}, J_{\mathrm{CP}}=1.6 \mathrm{~Hz}, 2 \mathrm{C}, \mathrm{C}_{2,7}\right.$ oder $\left.\mathrm{C}_{3,6}\right)$, $123.5\left(d, J_{C P}=1.9 \mathrm{~Hz}, 2 \mathrm{C}, \mathrm{C}_{1,8} \operatorname{oder} \mathrm{C}_{4,5}\right), 123.2\left(\mathrm{~d}, J_{\mathrm{CP}}=1.0 \mathrm{~Hz}\right.$, $\left.2 \mathrm{C}, \mathrm{C}_{2,7} \operatorname{oder} \mathrm{C}_{3,6}\right), 122.2\left(\mathrm{~d},{ }^{2} \mathrm{JP}_{\mathrm{CP}}=8.8 \mathrm{~Hz}, 2 \mathrm{C}, \mathrm{C}_{10}\right), 27.4(\mathrm{~d}$, ${ }^{1} J_{C P}=44.2 \mathrm{~Hz}, 1 \mathrm{C}, \mathrm{CH}_{2}$ ).

\section{${ }^{31} \mathrm{P}\left\{{ }^{1} \mathrm{H}\right\}-\mathrm{NMR}$}

(THF $d_{8} 81 \mathrm{MHz}$ ):

$\delta[\mathrm{ppm}]=-12.1$

\section{EI-MS}

$\mathrm{m} / \mathrm{z}(\%)$ : $376(18)[\mathrm{M}]^{+}, 191(100)\left[\mathrm{M}-\mathrm{PPh}_{2}\right]^{+}$. 


\subsubsection{Synthesis of $\mathrm{HAnCH}_{2} \mathrm{POPh}_{2}(78)$}

$\mathrm{HAnCH}_{2} \mathrm{PPh}_{2}$ (77) $(0.50 \mathrm{~g}, 1.33 \mathrm{mmol})$ was dissolved in a mixture of DCM (10 mL) and $\mathrm{MeOH}$ (10 mL). At $-15^{\circ} \mathrm{C} 0.13 \mathrm{~mL}$ of aqueous $\mathrm{H}_{2} \mathrm{O}_{2}$ solution diluted with $5 \mathrm{~mL} \mathrm{MeOH}$ was added. The reaction was stirred for $2 \mathrm{~h}$, then the solvent was evaporated. $\mathrm{HAnCH}_{2} \mathrm{POPh}_{2}(\mathbf{7 8})$ was obtained as a light yellow solid.

Yield: $0.44 \mathrm{~g}(1.11 \mathrm{mmol}, 83 \%)$

Empirical formula: $\mathrm{C}_{27} \mathrm{H}_{21} \mathrm{PO}$

Molar mass: $392.43 \mathrm{~g} / \mathrm{mol}$

${ }^{1}$ H-NMR

$\left(\mathrm{CDCl}_{3}, 300 \mathrm{MHz}\right)$ :

$\delta[\mathrm{ppm}]=8.34\left(\mathrm{~d}, J=2.3 \mathrm{~Hz}, 1 \mathrm{H}, \mathrm{H}_{10}\right), 7.97-7.88\left(\mathrm{~m}, 4 \mathrm{H}, \mathrm{H}_{1,4,5,8}\right)$, 7.64-7.54 (m, 4H, o-Ph), 7.46-7.38 (m, 2H, p-Ph), 7.37-7.20 (m, $\left.8 \mathrm{H}, m-\mathrm{Ph}, \mathrm{H}_{2,3,6,7}\right), 4.69\left(\mathrm{~d},{ }^{2} J_{\mathrm{HP}}=14.5 \mathrm{~Hz}, 2 \mathrm{H}, \mathrm{CH}_{2}\right.$ ).

${ }^{13} \mathrm{C}\left\{{ }^{1} \mathrm{H}\right\}-\mathrm{NMR}$

$\left(\mathrm{CDCl}_{3}, 75 \mathrm{MHz}\right)$ :

$\delta[\mathrm{ppm}]=132.38\left(\mathrm{~d},{ }^{1} J_{\mathrm{CP}}=97.7 \mathrm{~Hz}, 2 \mathrm{C}\right.$, ipso-Ph, $131.78(\mathrm{~d}$, $\left.{ }^{5} J_{C P}=2.8 \mathrm{~Hz}, 2 \mathrm{C}, p-\mathrm{Ph}\right), 131.30\left(\mathrm{~d},{ }^{3} J_{\mathrm{CP}}=9.1 \mathrm{~Hz}, 4 \mathrm{C}, o-\mathrm{Ph}\right)$, $130.82\left(\mathrm{~d}, J_{\mathrm{CP}}=5.2 \mathrm{~Hz}, 2 \mathrm{C}, \mathrm{C}_{4 \mathrm{a}, 10 \mathrm{a}} \operatorname{oder} \mathrm{C}_{8 \mathrm{a}, 9 \mathrm{a}}\right), 128.80(\mathrm{~d}$, $\left.J_{C P}=1.1 \mathrm{~Hz}, 2 \mathrm{C}, \mathrm{C}_{1,8} \operatorname{oder} \mathrm{C}_{4,5}\right), 128.35\left(\mathrm{~d},{ }^{4} J_{\mathrm{CP}}=11.5 \mathrm{~Hz}, 4 \mathrm{C}\right.$, $m-\mathrm{Ph}), 127.15\left(\mathrm{~d},{ }^{5} J_{\mathrm{CP}}=4.7 \mathrm{~Hz}, 1 \mathrm{C}, \mathrm{C}_{10}\right), 125.49\left(\mathrm{~d}, \mathrm{~J}_{\mathrm{CP}}=1.6 \mathrm{~Hz}\right.$, $\left.2 \mathrm{C}, \mathrm{C}_{2,7} \operatorname{oder} \mathrm{C}_{3,6}\right), 124.88\left(\mathrm{~d}, \mathrm{~J}_{\mathrm{CP}}=2.6 \mathrm{~Hz}, 2 \mathrm{C}, \mathrm{C}_{1,8} \operatorname{oder} \mathrm{C}_{4,5}\right)$, $124.75\left(\mathrm{~d}, J_{\mathrm{CP}}=1.2 \mathrm{~Hz}, 2 \mathrm{C}, \mathrm{C}_{2,7} \operatorname{oder} \mathrm{C}_{3,6}\right), 123.72(\mathrm{~d}$, $\left.{ }^{2} J_{C P}=9.9 \mathrm{~Hz}, 2 \mathrm{C}, \mathrm{C}_{10}\right), 31.87\left(\mathrm{~d},{ }^{1} \mathrm{~J}_{\mathrm{CP}}=66.2 \mathrm{~Hz}, 1 \mathrm{C}, \mathrm{CH}_{2}\right)$.

${ }^{31} \mathrm{P}\left\{{ }^{1} \mathrm{H}\right\}-N M R$

$\left(\mathrm{CDCl}_{3}, 81 \mathrm{MHz}\right): \quad \delta[\mathrm{ppm}]=28.77$.

EI-MS

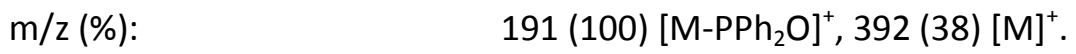

\subsubsection{Synthesis of $\mathrm{HAnCH}_{2} \mathrm{PSPh}_{2}$ (79)}

$\mathrm{HAnCH}_{2} \mathrm{PPh}_{2}$ (77) $(1.00 \mathrm{~g}, 2.65 \mathrm{mmol})$ was dissolved in toluene $(20 \mathrm{~mL})$ and elemental sulfur $(0.094 \mathrm{~g}, 2.82 \mathrm{mmol})$ was added. The mixture was stirred and heated to $110^{\circ} \mathrm{C}$ for $6 \mathrm{~h}$. The 
reaction was cooled to ambient temperature, filtrated and the solvent was evaporated. $\mathrm{HAnCH}_{2} \mathrm{PSPh}_{2}$ (79) was obtained by crystallization from toluene as a yellow solid.

Yield: $0.92 \mathrm{~g}(2,25 \mathrm{mmol}, 85 \%)$

Empirical formula: $\mathrm{C}_{27} \mathrm{H}_{21} \mathrm{PS}$

Molar mass: $408.49 \mathrm{~g} / \mathrm{mol}$

${ }^{1} \mathrm{H}-\mathrm{NMR}$

$\left(\mathrm{CDCl}_{3}, 300 \mathrm{MHz}\right)$ :

$\delta[\mathrm{ppm}]=8.26\left(\mathrm{~s}, 1 \mathrm{H}, \mathrm{H}_{10}\right), 7.83\left(\mathrm{~d}, J=8.4 \mathrm{~Hz}, 2 \mathrm{H}, \mathrm{H}_{1,8}\right), 7.73(\mathrm{~d}$, $J=8.9 \mathrm{~Hz}, 4 \mathrm{H}, o-\mathrm{Ph}), 7.64\left(\mathrm{dd}, J=7.0,6.6 \mathrm{~Hz}, 2 \mathrm{H}, \mathrm{H}_{4,5}\right), 7.34(\mathrm{~m}$, $\left.4 \mathrm{H}, \mathrm{H}_{2,3,5,6}\right), 7.24(\mathrm{~m}, 6 \mathrm{H}, m, p-\mathrm{Ph}), 4.87\left(\mathrm{~d},{ }^{2} \mathrm{~J}_{\mathrm{PH}}=14.0 \mathrm{~Hz}, 2 \mathrm{H}\right.$, $\mathrm{C}_{2}$ ).

${ }^{13} \mathrm{C}\left\{{ }^{1} \mathrm{H}\right\}-\mathrm{NMR}$

$\left(\mathrm{CDCl}_{3}, 75 \mathrm{MHz}\right)$ :

$\delta[\mathrm{ppm}]=133.4\left(\mathrm{~d},{ }^{1} J_{\mathrm{CP}}=95.4 \mathrm{~Hz}, 2 \mathrm{C}\right.$, ipso-Ph, 132.6 (d, $\left.{ }^{5} J_{C P}=2.9 \mathrm{~Hz}, 2 \mathrm{C}, p-\mathrm{Ph}\right), 131.9\left(\mathrm{~d},{ }^{3} \mathrm{~J}_{\mathrm{CP}}=9.1 \mathrm{~Hz}, 4 \mathrm{C}, o-\mathrm{Ph}\right), 131.0$

$\left(d, J_{C P}=5.4 \mathrm{~Hz}, 2 \mathrm{C}, \mathrm{C}_{4 \mathrm{a}, 10 \mathrm{a}} \operatorname{oder} \mathrm{C}_{8 \mathrm{a}, 9 \mathrm{a}}\right), 129.7\left(\mathrm{~d}, J_{\mathrm{CP}}=1.0 \mathrm{~Hz}, 2 \mathrm{C}\right.$,

$\left.\mathrm{C}_{1,8} \operatorname{oder} \mathrm{C}_{4,5}\right), 129.2\left(\mathrm{~d},{ }^{4} \mathrm{~J}_{\mathrm{CP}}=11.0 \mathrm{~Hz}, 4 \mathrm{C}, \mathrm{m}-\mathrm{Ph}\right), 128.3$ (d,

$\left.{ }^{5} J_{C P}=4.8 \mathrm{~Hz}, 1 \mathrm{C}, \mathrm{C}_{10}\right), 126.5\left(\mathrm{~d}, J_{\mathrm{CP}}=1.5 \mathrm{~Hz}, 2 \mathrm{C}, \mathrm{C}_{2,7} \operatorname{oder} \mathrm{C}_{3,6}\right)$,

$125.2\left(d, J_{C P}=2.8 \mathrm{~Hz}, 2 \mathrm{C}, \mathrm{C}_{1,8} \operatorname{oder} \mathrm{C}_{4,5}\right), 124.7\left(\mathrm{~d}, \mathrm{~J}_{\mathrm{CP}}=1.1 \mathrm{~Hz}\right.$,

$\left.2 \mathrm{C}, \mathrm{C}_{2,7} \operatorname{oder} \mathrm{C}_{3,6}\right), 124.2\left(\mathrm{~d},{ }^{2} \mathrm{~J}_{\mathrm{CP}}=10.0 \mathrm{~Hz}, 2 \mathrm{C}, \mathrm{C}_{10}\right), 34.8(\mathrm{~d}$,

${ }^{1} J_{C P}=60.4 \mathrm{~Hz}, 1 \mathrm{C}, \mathrm{CH}_{2}$ ).

${ }^{31} \mathrm{P}\left\{{ }^{1} \mathrm{H}\right\}-\mathrm{NMR}$

$\left(\mathrm{CDCl}_{3}, 81 \mathrm{MHz}\right)$ :

$\delta[\mathrm{ppm}]=40.7(\mathrm{~s})$.

\subsubsection{Synthesis of $\left[\mathrm{HAnCH} \mathrm{PPh}_{2} \mathrm{AuCl}\right](80)$}

$\mathrm{HAnCH}_{2} \mathrm{PPh}_{2}$ (77) $(0.3 \mathrm{~g}, 0.80 \mathrm{mmol})$ was dissolved in DCM $(10 \mathrm{~mL})$ and [Me2SAuCl] $(0.28 \mathrm{~g}$, $0 ., 88 \mathrm{mmol}$ ) was added. The mixture was heated to $35^{\circ} \mathrm{C}$ for $20 \mathrm{~min}$ and then cooled to ambient temperature. [ $\mathrm{HAnCH}_{2} \mathrm{PPh}_{2} \mathrm{AuCl}$ ] (80) was crystallized at $-30^{\circ} \mathrm{C}$ and was obtained as light yellow crystals.

Yield: 0,21 g (0,35 mmol, $43 \%)$

Empirical formula: $\mathrm{C}_{27} \mathrm{H}_{21} \mathrm{PAuCl}$

Molar mass: $608.85 \mathrm{~g} / \mathrm{mol}$

The ${ }^{1} \mathrm{H}$ and ${ }^{31} \mathrm{P}$ NMR spectra were identical to those of $\mathbf{7 7}$. 
ESI-MS (Acetone)

$\mathrm{m} / \mathrm{z}(\%)$ :

$608(2)[\mathrm{M}]^{+}, 376(55)[\mathrm{M}-\mathrm{AuCl}]^{+}, 299(100)[\mathrm{M}-\mathrm{AuCl}, \mathrm{Ph}]^{+}$.

\subsubsection{Synthesis of bis(diethylamino)alane (81)}

To a suspension of $5.00 \mathrm{~g}(0.132 \mathrm{~mol}) \mathrm{LiAlH}_{4}$ in $400 \mathrm{ml}$ of diethyl ether, a solution of $16.00 \mathrm{~g}$ $(0.076 \mathrm{~mol})$ of bis(diethylamino)chlorophosphane in diethyl ether was added drop wise at $-78^{\circ} \mathrm{C}$. after the addition was completed the cooling bath was removed and the reaction was stirred at ambient temperature for $24 \mathrm{~h}$. Then the suspension was filtrated, the solvent was removed and the residue was extracted with pentane $(120 \mathrm{ml})$. After removal of the solvent the crude product was purified by distillation at $70^{\circ} \mathrm{C} / 4 \mathrm{mbar}$. The product was obtained as a light yellow liquid which crystallized upon cooling to $-3^{\circ} \mathrm{C}$ overnight.

Yield: $4.08 \mathrm{~g}(11.86 \mathrm{mmol})$

Empirical formula: $\mathrm{C}_{16} \mathrm{H}_{42} \mathrm{Al}_{2} \mathrm{~N}_{4}$

Molar mass: $344.49 \mathrm{~g} / \mathrm{mol}$

\subsubsection{Synthesis of [TMPDALi- $\left.\mu^{2}\left(\mathrm{NEt}_{2}\right)_{2} \mathrm{AlBu}_{2}\right](82)$}

To a suspension of $2.50 \mathrm{~g}(66.0 \mathrm{mmol}) \mathrm{LiAlH}_{4}$ in $200 \mathrm{ml}$ of diethyl ether, a solution of $8.00 \mathrm{~g}$ $(0.038 \mathrm{~mol})$ of bis(diethylamino)chlorophosphane in diethyl ether was added drop-wise at $-78^{\circ} \mathrm{C}$. after the addition was completed the cooling bath was removed and the reaction was stirred at ambient temperature for $24 \mathrm{~h}$. Then the suspension was filtrated, the solvent was removed and the residue was extracted with pentane $(70 \mathrm{ml})$. The pentane was evaporated and the crude product was dissolved in diethyl ether $(50 \mathrm{~mL})$ and at $-15^{\circ} \mathrm{C} n$-BuLi $(12.0 \mathrm{~mL}$, $38 \mathrm{mmol}, 3.18 \mathrm{~m}$ ) was added, as well as TMPDA $(5 \mathrm{~mL})$. The flask was then stored at $-40^{\circ} \mathrm{C}$ and 82 was obtained as colorless crystals.

Yield: $0,96 \mathrm{~g}(2,36 \mathrm{mmol}, 80 \%)$

Empirical formula: $\mathrm{C}_{23} \mathrm{H}_{56} \mathrm{~N}_{4} \mathrm{AlLi}$

Molar mass: $422.46 \mathrm{~g} / \mathrm{mol}$

The extremely high reactivity of $\mathbf{8 2}$ prevented the acquisition of analytical data except for the crystal structure. 


\subsubsection{Synthesis of 10-Methyl-9,1-anthryl-cyclo(1',1'-diphenyl)thia- phosphonium bromide (83)}

$\mathrm{MeAnPSPh}_{2}$ (14) $(0.8 \mathrm{~g}, 2.13 \mathrm{mmol})$ was dissolved in chloroform $(60 \mathrm{~mL})$ and cooled to $-15^{\circ} \mathrm{C}$. Then a solution of $0.42 \mathrm{~g}$ ( $2.48 \mathrm{mmol}) \mathrm{Br}_{2}$ in $20 \mathrm{~mL}$ of chloroform was added over $20 \mathrm{~min}$. The cooling bath was removed and the solution was stirred overnight. The solution was concentrated to ca. $1 / 3$ of the initial volume and the formed precipitate was collected by filtration and dried under reduced pressure. The crude product was recrystallized from acetone. $\mathbf{8 3}$ was obtained as a yellow crystalline solid.

Yield: $0.69 \mathrm{~g}(1.43 \mathrm{mmol}, 68 \%)$

Empirical formula: $\mathrm{C}_{27} \mathrm{H}_{20} \mathrm{PSBr}$

Molar mass: $487.39 \mathrm{~g} / \mathrm{mol}$

${ }^{1}$ H-NMR

$\left(\mathrm{CDCl}_{3}, 300 \mathrm{MHz}\right)$ :

$$
\begin{aligned}
& \delta[p p m]=8.74\left(\mathrm{~m}, 1 \mathrm{H}, \mathrm{H}_{2}\right), 8.59\left(\mathrm{dd}, J=8.6,1.9 \mathrm{~Hz}, 1 \mathrm{H}, \mathrm{H}_{8}\right), \\
& 8.26\left(\mathrm{~d}, J=7.1 \mathrm{~Hz}, 1 \mathrm{H}, \mathrm{H}_{4}\right), 8.10\left(\mathrm{~m}, 4 \mathrm{H}, \mathrm{H}_{\text {ortho }}(\mathrm{Ph})\right), 7.90(\mathrm{~m}, 1 \mathrm{H}, \\
& \left.\mathrm{H}_{5}\right), 7.83-7.76\left(\mathrm{~m}, 9 \mathrm{H}, \mathrm{H}_{3,6,7}, \mathrm{H}_{\text {meta,para }}(\mathrm{Ph})\right), 3.34\left(\mathrm{~s}, 3 \mathrm{H}, \underline{\mathrm{CH}}_{3}\right) .
\end{aligned}
$$

${ }^{13} \mathrm{C}\left\{{ }^{1} \mathrm{H}\right\}-\mathrm{NMR}$

$\left(\mathrm{CDCl}_{3}, 75 \mathrm{MHz}\right): \quad \delta[\mathrm{ppm}]=139.5,134.1\left(\mathrm{~d}, \mathrm{~J}_{\mathrm{PC}}=13.0 \mathrm{~Hz}\right), 132.3,130.7,130.5$, $130.3,127.9,127.1,126.2,124.3,120.7,119.5,15.2\left(1 \mathrm{C}, \underline{\mathrm{CH}}_{2}\right)$

${ }^{31} \mathrm{P}\left\{{ }^{1} \mathrm{H}\right\}-N M R$

$\left(\mathrm{CDCl}_{3}, 81 \mathrm{MHz}\right): \quad \delta[\mathrm{ppm}]=59.8(\mathrm{~s})$.

\section{EI-MS}

$\mathrm{m} / \mathrm{z}(\%)$ :

$487(3)[\mathrm{M}]^{+}, 406(100)[\mathrm{M}-\mathrm{Br}]^{+}, 329(60)[\mathrm{M}-\mathrm{Ph}, \mathrm{Br}]^{+}, 253(65)$

$[\mathrm{M}-2 \mathrm{Ph}, \mathrm{Br}]^{+}$.

\subsubsection{Synthesis of $\mathrm{Ph}_{2} \mathrm{SPAnCH}_{2} \mathrm{~N}\left(\mathrm{C}_{2} \mathrm{H}_{4} \mathrm{OMe}\right)_{2}$ (84)}

A solution of $0.62 \mathrm{~g}$ (1.52 mmol) $\mathrm{BrAnCH}_{2} \mathrm{~N}\left(\mathrm{C}_{2} \mathrm{H}_{4} \mathrm{OMe}\right)_{2}$ (70) in $30 \mathrm{~mL}$ of diethyl ether was cooled to $-15^{\circ} \mathrm{C}$ and reacted with $0.48 \mathrm{~mL}(1.52 \mathrm{mmol})$ of $3.18 \mathrm{M} n$-BuLi solution in hexane.The addition was carried out over the course of $20 \mathrm{~min}$. The solution was stirred for $5 \mathrm{~min}$ at $0^{\circ} \mathrm{C}$ and then $0.34 \mathrm{~g}(1.54 \mathrm{mmol})$ of diphenylchlorophosphane was slowly added at $-15^{\circ} \mathrm{C}$. The reaction was stirred at ambient temperature overnight, then the solvent was evaporated. The crude product was dissolved in toluene and filtrated. Then elemental sulfur $(0.57 \mathrm{~g}, 1.78 \mathrm{mmol})$ was added and the mixture was heated to $100^{\circ} \mathrm{C}$ for $6 \mathrm{~h}$. Then the solvent was evaporated and the solvent was removed. The crude product was purified by column chromatography (pentane/ethyl acetate 3:1) and obtained as a yellow oil. 
Yield: $0.070 \mathrm{~g}(0.130 \mathrm{mmol}, 8.5 \%)$

Empirical formula: $\mathrm{C}_{33} \mathrm{H}_{34} \mathrm{NO}_{2} \mathrm{PS}$

Molar mass: $539.67 \mathrm{~g} / \mathrm{mol}$

\section{${ }^{1} \mathrm{H}$ NMR}

$\left(\mathrm{CDCl}_{3}, 500 \mathrm{MHz}\right)$ :

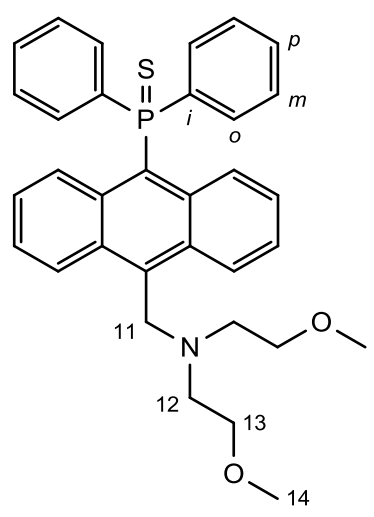

$\delta=8.63\left(\mathrm{~d},{ }^{3} J_{H H}=9.05 \mathrm{~Hz}, 2 \mathrm{H}, \mathrm{H}_{4,5}\right), 8.06\left(\mathrm{~d},{ }^{3} \mathrm{~J}_{\mathrm{HH}}=9.00 \mathrm{~Hz}, 2 \mathrm{H}\right.$, $\left.\mathrm{H}_{1,8}\right), 7.70(\mathrm{~m}, 4 \mathrm{H}, o-\mathrm{Ph}), 7.34$ (ddd, ${ }^{3} J_{\mathrm{HH}}=9.05 \mathrm{~Hz}$, $\left.{ }^{3} J_{\mathrm{HH}}=6.45 \mathrm{~Hz},{ }^{4} J_{\mathrm{HH}}=0.81 \mathrm{~Hz}, 2 \mathrm{H}, \mathrm{H}_{3,6}\right), 7.29(\mathrm{~m}, 2 \mathrm{H}, p-\mathrm{Ph}), 7.22$ $(\mathrm{m}, 4 \mathrm{H}, m-\mathrm{Ph}), 6.99\left(\mathrm{ddd},{ }^{3} \mathrm{~J}_{\mathrm{HH}}=9.00 \mathrm{~Hz},{ }^{3} J_{\mathrm{HH}}=6.45 \mathrm{~Hz}\right.$, $\left.{ }^{4} J_{H H}=0.81 \mathrm{~Hz}, 2 \mathrm{H}, \mathrm{H}_{2,7}\right), 4.71\left(\mathrm{~s}, 2 \mathrm{H}, \mathrm{H}_{11}\right), 3.45\left(\mathrm{t},{ }^{3} \mathrm{~J}_{\mathrm{HH}}=5.85 \mathrm{~Hz}\right.$, $\left.4 \mathrm{H}, \mathrm{H}_{13}\right), 3.26\left(\mathrm{~s}, 6 \mathrm{H}, \mathrm{H}_{14}\right), 2.86\left(\mathrm{t},{ }^{3} \mathrm{~J}_{\mathrm{HH}}=5.85 \mathrm{~Hz}, 4 \mathrm{H}, \mathrm{H}_{12}\right)$.

\section{${ }^{13} \mathrm{C}\left\{{ }^{1} \mathrm{H}\right\}$ NMR}

$\left(\mathrm{CDCl}_{3}, 126 \mathrm{MHz}\right)$ :

$\delta=137.49\left(\mathrm{~s}, 1 \mathrm{C}, \mathrm{C}_{10}\right), 137.27\left(\mathrm{~d},{ }^{1} \mathrm{~J}_{\mathrm{CP}}=82.90 \mathrm{~Hz}, 2 \mathrm{C}, i-\mathrm{Ph}\right)$, $132.87\left(d,{ }^{2} J_{C P}=7.53 \mathrm{~Hz}, 2 \mathrm{C}, \mathrm{C}_{8 \mathrm{a}, 9 \mathrm{a}}\right), 131.34\left(\mathrm{~d},{ }^{3} \mathrm{~J}_{\mathrm{CP}}=10.95 \mathrm{~Hz}\right.$, 2C, $\left.\mathrm{C}_{4 \mathrm{a}, 10 \mathrm{a}}\right), 130.64\left(\mathrm{~d},{ }^{2} J_{\mathrm{CP}}=10.35 \mathrm{~Hz}, 4 \mathrm{C}, \mathrm{o}-\mathrm{Ph}\right), 130.39$ (d, $\left.{ }^{4} J_{\mathrm{CP}}=2.60 \mathrm{~Hz}, 2 \mathrm{C}, p-\mathrm{Ph}\right), 128.32\left(\mathrm{~d},{ }^{3} J_{\mathrm{CP}}=12.54 \mathrm{~Hz}, 4 \mathrm{C}, m-\mathrm{Ph}\right)$, $127.70\left(d,{ }^{3} J_{C P}=10.50 \mathrm{~Hz}, 2 C, C_{1,8}\right), 125.72\left(d,{ }^{4} J_{C P}=6.48 \mathrm{~Hz}, 2 C\right.$, $\left.\mathrm{C}_{4,5}\right), 125.21\left(\mathrm{~s}, 2 \mathrm{C}, \mathrm{C}_{3,6}\right), 124.83\left(\mathrm{~s}, 2 \mathrm{C}, \mathrm{C}_{2,7}\right), 123.03$ (d, $\left.{ }^{1} J_{\mathrm{CP}}=89.44 \mathrm{~Hz}, 1 \mathrm{C}, \mathrm{C}_{9}\right), 71.52\left(\mathrm{~s}, 2 \mathrm{C}, \mathrm{C}_{13}\right), 58.59\left(\mathrm{~s}, 2 \mathrm{C}, \mathrm{C}_{14}\right)$, $53.86\left(\mathrm{~s}, 2 \mathrm{C}, \mathrm{C}_{12}\right), 52.27\left(\mathrm{~s}, 1 \mathrm{C}, \mathrm{C}_{11}\right)$.

\section{${ }^{31} \mathrm{P}\left\{{ }^{1} \mathrm{H}\right\}$ NMR}

$\left(\mathrm{CDCl}_{3}, 81 \mathrm{MHz}\right)$ :

$\delta=35.37(\mathrm{~s})$.

\section{EI-MS}

$m / z(\%):$

539 (10) $[\mathrm{M}]^{+}, 494$ (12) $\left[\mathrm{M}-\mathrm{CH}_{2} \mathrm{OCH}_{3}\right]^{+}, 407$ (100)

$\left[\mathrm{M}-\mathrm{N}\left(\mathrm{C}_{2} \mathrm{H}_{4} \mathrm{OCH}_{3}\right)_{2}\right]^{+}, 217(16)\left[\mathrm{PPh}_{2} \mathrm{~S}\right]^{+}, 191$ (52) [M - $\mathrm{PPh}_{2} \mathrm{~S}$,

$\left.\mathrm{N}\left(\mathrm{C}_{2} \mathrm{H}_{4} \mathrm{OCH}_{3}\right)_{2}\right]^{+}, 185(76)\left[\mathrm{PPh}_{2}\right]^{+}$. 



\section{Crystallographic Section}

\subsection{Crystal Application}

The crystals were taken from the mother liquor using standard Schlenk techniques and placed in perfluorinated polyether oil on a microscope slide. An appropriately sized crystal of high quality was selected under a polarization microscope (for detection of twinning and the presence of satellites) with help of the X-TEMP2 cooling device. ${ }^{[130]}$ It was mounted on a glass fiber glued to the magnetic pin of the goniometer head in a way that it was completely coated with the perfluorinated polyether oil. ${ }^{[131]}$ Oil and crystal were shocked-cooled in the cold gas stream of an open flow nitrogen cooling device attached to the diffractometer. The amorphous frozen oil served as glue and protected the sensitive compounds along with the nitrogen gas stream from moisture and oxygen.

\subsection{Data Collection and Processing}

All compounds were measured on a Bruker D8 Goniometer platform, equipped with an APEX // CCD X-ray detector. The compounds were measured using either an Incoatec microfocus source with mirror optics ${ }^{[132]}$ or on a rotating anode turbo X-ray source. Both are equipped with an APEX // CCD detector, mounted on a three-circle D8 goniometer, and mirrors as monochromator optics, which supplies very intense and brilliant MoK $_{\alpha}$ radiation $(\lambda=0.71073 \AA$ ). All crystals were centered optically using a video camera after being placed on the diffractometer.

Data collection was controlled with the APEX2 package. ${ }^{[133]}$ A test run (matrix scan) was recorded prior to each experiment to check the crystal quality, to get a rough estimate of the cell parameters, and to determine the optimum exposure time. All scans of the data collections were performed in an $\omega$-scan mode with a step-width of $0.3^{\circ}$ or $0.5^{\circ}$ at fixed $\varphi$-angles.

The determination of the unit cells and orientation matrices were performed with the tools supplied in the APEX2 package. ${ }^{[133]}$ The collected frames were integrated with SAINT ${ }^{[134]}$ using the $3 d$ profiling method described by Kabsch. ${ }^{[135]}$ All data sets were 
corrected for absorption and scaled using SADABS ${ }^{[136]}$ or TWINABS. ${ }^{[137]}$ XPREP $^{[138]}$ was used to determine the space group prior to the absorption correction, as this is crucial for a correct treatment. SADABS and TWINABS refine an empirical model function by symmetry-equivalent reflections. Setup of the files for structure solution and refinement was performed with XPREP.

\subsection{Structure Solution and Refinement}

The structures were solved with direct methods or Patterson superposition procedure using SHELXS. ${ }^{[139]}$ Data were merged according to the determined symmetry with SHELXL. ${ }^{[139]}$ All refinements were performed on $F^{2}$ with SHELXL. If not stated otherwise, the hydrogen atoms of the compounds were refined isotropically on calculated positions using a riding model. The positions were geometrically optimized and the $U_{\text {iso }}$ were constrained to $1.2 U_{\text {eq }}$ of the pivot atom or $1.5 U_{\text {eq }}$ of the methyl carbon atom. The position of certain hydrogen atoms (e.g. OH groups) were found with difference Fourier analysis of the rest electron density. If not stated otherwise, the hydrogen bond lengths were restrained to a sensible value and the $U_{\text {iso }}$ were constrained as mentioned above. In all refinements the function $M\left(p_{i}, k\right)$ (Eq. 7-1) was minimized using the weights $w_{\mathrm{H}}$ defined in Eq. 7-2.

Eq. 7-1.

$$
M\left(p_{i}, k\right)=\sum_{H} w_{H}\left[k\left|F_{\text {obs }}(H)\right|^{2}-\left|F_{\text {calc }}(H)\right|^{2}\right]^{2}=\min
$$

Eq. 7-2.

$$
W_{\mathrm{H}}^{-1}=\sigma_{H}^{2} F_{\mathrm{obs}}^{2}+(g 1 \cdot P)^{2}+g 2 \cdot P \quad \text { with } \quad P=\left(\frac{F_{\mathrm{obs}}^{2}+2 F_{\mathrm{calc}}^{2}}{3}\right)
$$

The results of the refinements were verified by comparison of the calculated and the observed structure factors. Commonly used criteria are the residuals $R 1$ (Eq. 7-3) and $w R 2$ (Eq. 7-4). The $W R 2$ is more significant, because the model is refined against $F^{2}$.

$\begin{array}{ll}\text { Eq. 7-3. } & R 1=\frac{\sum_{H} w_{H}\left(\left|F_{\mathrm{obs}}\right|-\left|F_{\mathrm{calc}}\right|\right)}{\sum_{H} w_{H}\left|F_{\mathrm{obs}}\right|} \\ \text { Eq. 7-4. } & w R 2=\frac{\sum_{H} w_{H}\left(\left|F_{\mathrm{obs}}\right|^{2}-\left|F_{\mathrm{calc}}\right|^{2}\right)^{2}}{\sum_{H} w_{H}\left|F_{\mathrm{obs}}\right|^{4}}\end{array}$ 
Additionally, the goodness of fit (GoF, S), a figure or merit showing the relation between deviation of $F_{\text {calc }}$ from $F_{\text {obs }}$ and the over-determination of refined parameters is calculated (Eq. 7-5).

$$
\text { Eq. 7-5. } \quad S=\sqrt{\frac{\sum\left(w_{H}\left(F_{\mathrm{obs}}^{2}-F_{\mathrm{calc}}^{2}\right)^{2}\right)}{(n-p)}}
$$

The residual densities from difference Fourier analysis should be low. Due to the model restrictions the residuals are normally found in the bonding regions. Higher residuals for heavy scatterers are acceptable as they arise mainly from absorption effects and Fourier truncation errors due to the limited recorded resolution range. The highest peak and deepest hole from difference Fourier analysis are listed in the crystallographic tables.

Additionally, the orientation, size and ellipticity of the ADPs show the quality of the model. Ideally, the ADPs should be oriented perpendicular to the bonds, be equal in size and show little ellipticity. All graphics were generated and plotted with the XShell program at the $50 \%$ probability level.

\subsection{Treatment of Disorder}

Structures containing disordered fragments were refined using constraints and restraints. The geometries of chemically equivalent but crystallographically independent fragments can be fitted to each other by distance restraints. Especially the 1,2 distances (bond lengths) and 1,3 distances (bond angles) are set to be equal within their effective standard deviations. This is helpful for refining disordered positions as the averaging of equivalent fragments implements chemical information and stabilizes the refinement.

Restraints affecting the anisotropic displacement parameters are often essential for the anisotropic refinement of disordered atomic positions. The rigid bond restraints $\left(D E L U\right.$ in SHELXL) ${ }^{[140]}$ fit the components of the anisotropic displacement parameters along the bonds within esd's. Similarity restraints (SIMU in SHELXL) ${ }^{[140]}$ adjust the ADPs of neighboring atoms within a certain radius to be equal according to their esd's. 


\subsection{Crystallographic Details}

\subsubsection{SPAnPS@MeCN (16)}

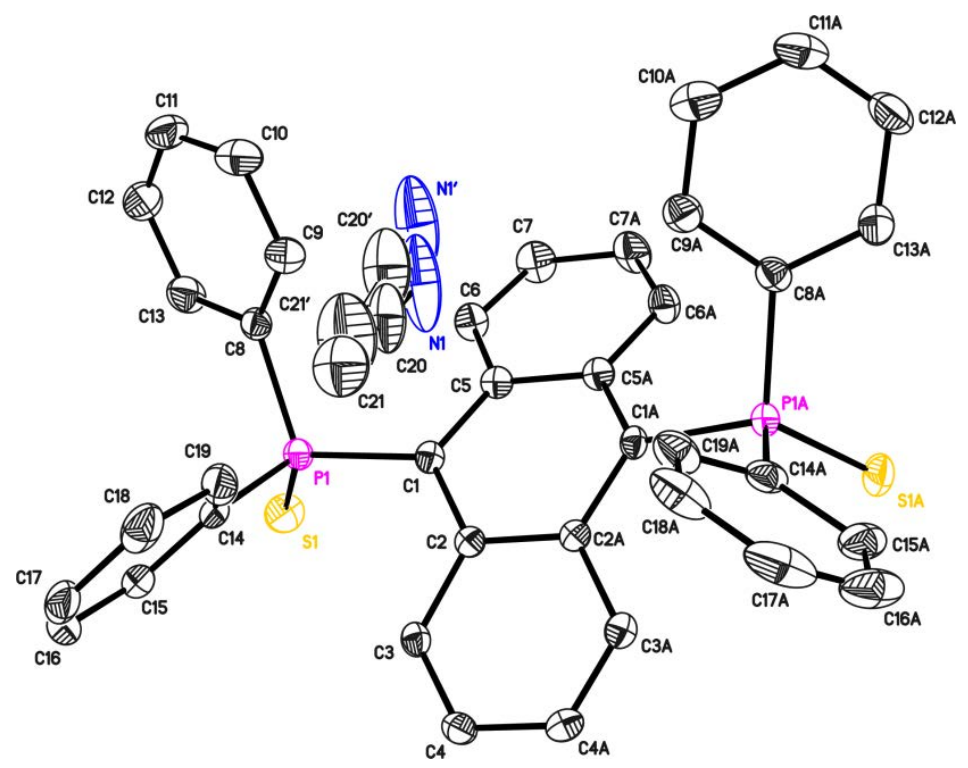

Figure 7-1. Structure of SPAnPS@MeCN (16). The anisotropic displacement parameters are depicted at the $50 \%$ probability level. The hydrogen atoms are omitted for clarity. Only one half of the depicted molecule is contained in the asymmetric unit, the other half is generated by a mirror plane.

The MeCN molecule is located on a mirror plane disordered in two positions at a ratio of $60: 40$.

Table 7-1. Crystallographic data for SPAnPS@MeCN (16).

\begin{tabular}{ll|ll}
\hline Identiofication code & Sherry & & \\
Empirical formula & $\mathrm{C}_{40} \mathrm{H}_{31} \mathrm{NP}_{2} \mathrm{~S}_{2}$ & $\mu\left[\mathrm{mm}^{-1}\right]$ & 0.153 \\
Formula weight $[\mathrm{g} / \mathrm{mol}]$ & 651.72 & $\mathrm{~F}(000)$ & 2040 \\
Temperature $[\mathrm{K}]$ & $100(2) \mathrm{K}$ & $\Theta$ range $\left[^{\circ}\right]$ & 1.216 to 20.551 \\
Wavelength $[\AA]$ & $0.56086 \AA$ & Reflections collected & 40679 \\
Crystal system & Hexagonal & Independent reflections & 3522 \\
Space group & $P 63 m$ & Completeness to $\Theta_{\text {max }}$ & $100.0 \%$ \\
$a[\AA]$ & $15.2576(4)$ & Absorption correction & Empirical \\
$b[\AA]$ & $15.2576(4)$ & Max. / min. transmission & $0.6523 / 0.7445$ \\
$c[\AA]]$ & $24.8675(8)$ & Restraints / parameters & $144 / 229$ \\
$\gamma\left[{ }^{\circ}\right]$ & 120 & GoF & 1.084 \\
$V$ Volume $\left[\AA^{3}\right]$ & $5013.4(3)$ & $R 1 / w R 2(\mathrm{I}) 2 \sigma(\mathrm{I}))$ & $0.0350 / 0.0840$ \\
$Z$ & 6 & $R 1 / w R 2$ (all data) & $0.0452 / 0.0877$ \\
$\rho_{\text {calc }}\left[\mathrm{Mg} / \mathrm{m}^{3}\right]$ & 1.295 & Diff. peak and hole $\left[\mathrm{e} \AA^{-3}\right]$ & $0.405 /-0.220$ \\
\hline
\end{tabular}




\subsection{2ＳPAnPS@Ace (17)}

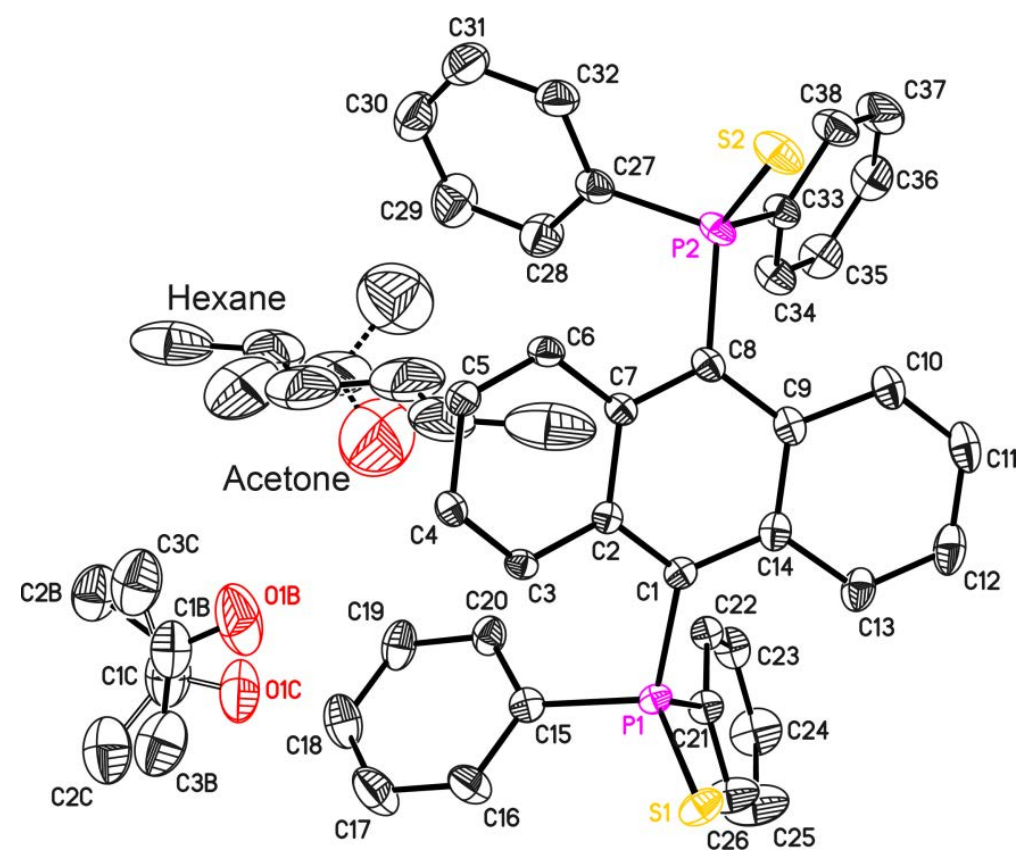

Figure 7-2. Asymmetric unit of SPAnPS@Ace (17). The anisotropic displacement parameters are depicted at the $50 \%$ probability level. The hydrogen atoms are omitted for clarity. One half of the hexane molecule is generated by symmetry.

Hexane and acetone molecules are disordered at occupations of $54 \%$ and $80 \%$. Two further acetone molecules are disordered at a 50:50 ratio.

Table 7-2. Crystallographic data for SPAnPS@Ace (17).

\begin{tabular}{ll|ll}
\hline Identification code & $\mathrm{NFO02}$ & & \\
Empirical formula & $\mathrm{C}_{43.33} \mathrm{H}_{39.17} \mathrm{O}_{1.23} \mathrm{P}_{2} \mathrm{~S}_{2}$ & $\mu\left[\mathrm{mm}^{-1}\right]$ & 0.144 \\
Formula weight $[\mathrm{g} / \mathrm{mol}]$ & 705.52 & $\mathrm{~F}(000)$ & 1484 \\
Temperature $[\mathrm{K}]$ & $100(2)$ & $\Theta$ range $\left[^{\circ}\right]$ & 1.290 to 20.519 \\
Wavelength $[\AA]$ & 0.56086 & Reflections collected & 35897 \\
Crystal system & Monoclinic & Independent reflections & 7458 \\
Space group & $P 2_{1} / c$ & Completeness to $\Theta_{\text {max }}$ & $100.0 \%$ \\
$a[\AA]$ & $13.0778(5)$ & Absorption correction & Empirical \\
$b[\AA]$ & $17.5202(7)$ & Max. / min. transmission & $0.6363 / 0.7445$ \\
$c[\AA]$ & $16.7055(7)$ & Restraints / parameters & $268 / 516$ \\
$\beta\left[\left[^{\circ}\right]\right.$ & $107.732(2)$ & GoF & 1.027 \\
Volume $\left[\AA^{3}\right]$ & $3645.8(3)$ & $R 1 / w R 2(\mathrm{I}>2 \sigma(\mathrm{I}))$ & $0.0458 / 0.1142$ \\
$\mathrm{Z}$ & 4 & $R 1 / w R 2$ (all data) & $0.0554 / 0.1193$ \\
$\rho_{\text {calc }}\left[\mathrm{Mg} / \mathrm{m}^{3}\right]$ & 1.285 & Diff. peak and hole $\left[\mathrm{e} \AA^{-3}\right]$ & 0.466 and -0.431 \\
\hline
\end{tabular}




\subsection{3ＳPAnPS@DCM (18)}

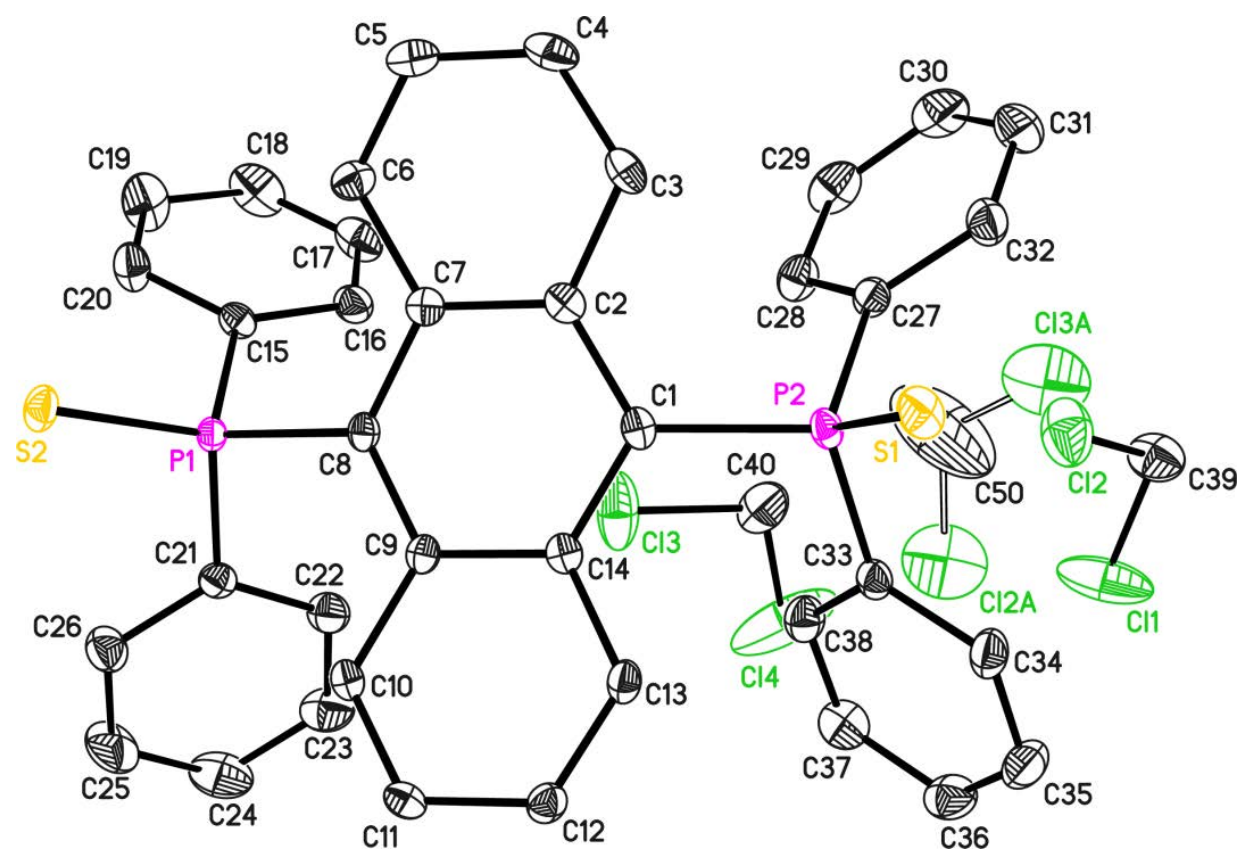

Figure 7-3. Asymmetric unit of SPAnPS@DCM (18). The anisotropic displacement parameters are depicted at the $50 \%$ probability level. The hydrogen atoms are omitted for clarity.

The DCM molecules are disordered in 3 positions.

Table 7-3. Crystallographic data for SPAnPS@DCM (18).

\begin{tabular}{|c|c|c|c|}
\hline Identification code & NF003 & & \\
\hline Empirical formula & $\mathrm{C}_{40} \mathrm{H}_{40} \mathrm{Cl}_{4} \mathrm{P}_{2} \mathrm{~S}_{2}$ & $\rho_{\text {calc }}\left[\mathrm{Mg} / \mathrm{m}^{3}\right]$ & 1.419 \\
\hline Formula weight $[\mathrm{g} / \mathrm{mol}]$ & 788.58 & $\mu\left[\mathrm{mm}^{-1}\right]$ & 0.285 \\
\hline Temperature $[\mathrm{K}]$ & $100(2)$ & $F(000)$ & 1640 \\
\hline Wavelength [Å] & 0.56086 & $\Theta$ range $\left[{ }^{\circ}\right]$ & 1.320 to 20.519 \\
\hline Crystal system & Monoclinic & Reflections collected & 36080 \\
\hline Space group & $P 2_{1} / c$ & Independent reflections & 7549 \\
\hline$a[\AA ̊]$ & $12.7720(7)$ & Completeness to $\Theta_{\max }$ & $100.0 \%$ \\
\hline$b[\AA]$ & 17.7946(10) & Absorption correction & Empirical \\
\hline$c[\AA]$ & $17.0401(9)$ & Max. / min. transmission & $0.6847 / 0.7446$ \\
\hline$\alpha\left[^{\circ}\right]$ & 90 & Restraints / parameters & $172 / 463$ \\
\hline$\beta\left[^{\circ}\right]$ & $107.582(2)$ & GoF & 0.824 \\
\hline$\gamma\left[{ }^{\circ}\right]$ & 90 & $R 1 / w R 2(I>2 \sigma(I))$ & $0.0404 / 0.1117$ \\
\hline Volume $\left[\AA^{3}\right]$ & $3691.8(4)$ & $R 1 / w R 2$ (all data) & $0.0496 / 0.1175$ \\
\hline $\mathrm{Z}$ & 4 & Diff. peak and hole $\left[\mathrm{e} \AA^{-3}\right]$ & 0.820 and -0.797 \\
\hline
\end{tabular}




\subsubsection{SPAnPS_pure (19)}

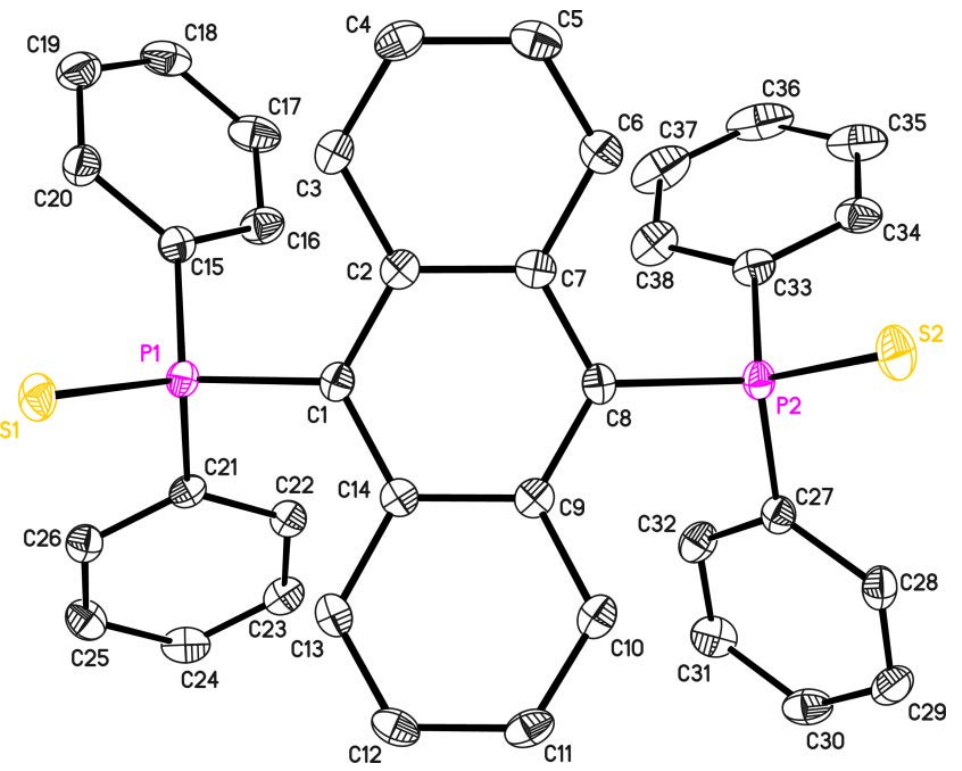

Figure 7-4. Asymmetric unit of SPAnPS_pure (19). The anisotropic displacement parameters are depicted at the $50 \%$ probability level. The hydrogen atoms are omitted for clarity.

Table 7-4. Crystallographic data for SPAnPS_pure (19).

\begin{tabular}{|c|c|c|c|}
\hline Identification code & NF004 & & \\
\hline Empirical formula & $\mathrm{C}_{38} \mathrm{H}_{28} \mathrm{P}_{2} \mathrm{~S}_{2}$ & $\rho_{\text {calc }}\left[\mathrm{Mg} / \mathrm{m}^{3}\right]$ & 1.311 \\
\hline Formula weight $[\mathrm{g} / \mathrm{mol}]$ & 610.66 & $\mu\left[\mathrm{mm}^{-1}\right]$ & 0.161 \\
\hline Temperature $[\mathrm{K}]$ & $100(2)$ & $F(000)$ & 636 \\
\hline Wavelength [Å] & 0.56086 & $\Theta$ range $\left[{ }^{\circ}\right]$ & 1.342 to 20.516 \\
\hline Crystal system & Monoclinic & Reflections collected & 18621 \\
\hline Space group & $P 2_{1}$ & Independent reflections & 6190 \\
\hline$a[\AA ̊]$ & $8.4239(8)$ & Completeness to $\Theta_{\max }$ & $99.9 \%$ \\
\hline$b[\AA]$ & $15.3292(14)$ & Absorption correction & Empirical \\
\hline$c[\AA ̊]$ & $11.9972(10)$ & Max. / min. transmission & $0.7018 / 0.7446$ \\
\hline$\alpha\left[^{\circ}\right]$ & 90 & Restraints / parameters & 119 / 379 \\
\hline$\beta\left[{ }^{\circ}\right]$ & $93.254(2)$ & GoF & 1.038 \\
\hline$\gamma\left[^{\circ}\right]$ & 90 & $R 1 / w R 2(I>2 \sigma(I))$ & $0.0271 / 0.0650$ \\
\hline Volume $\left[\AA^{3}\right]$ & $1546.7(2)$ & $R 1 / w R 2$ (all data) & $0.0294 / 0.0660$ \\
\hline Z & 2 & Diff. peak and hole $\left[\mathrm{e}^{-3}{ }^{-3}\right]$ & 0.218 and -0.195 \\
\hline
\end{tabular}




\subsubsection{SPAnPS@tol_d $d_{8}(20)$}

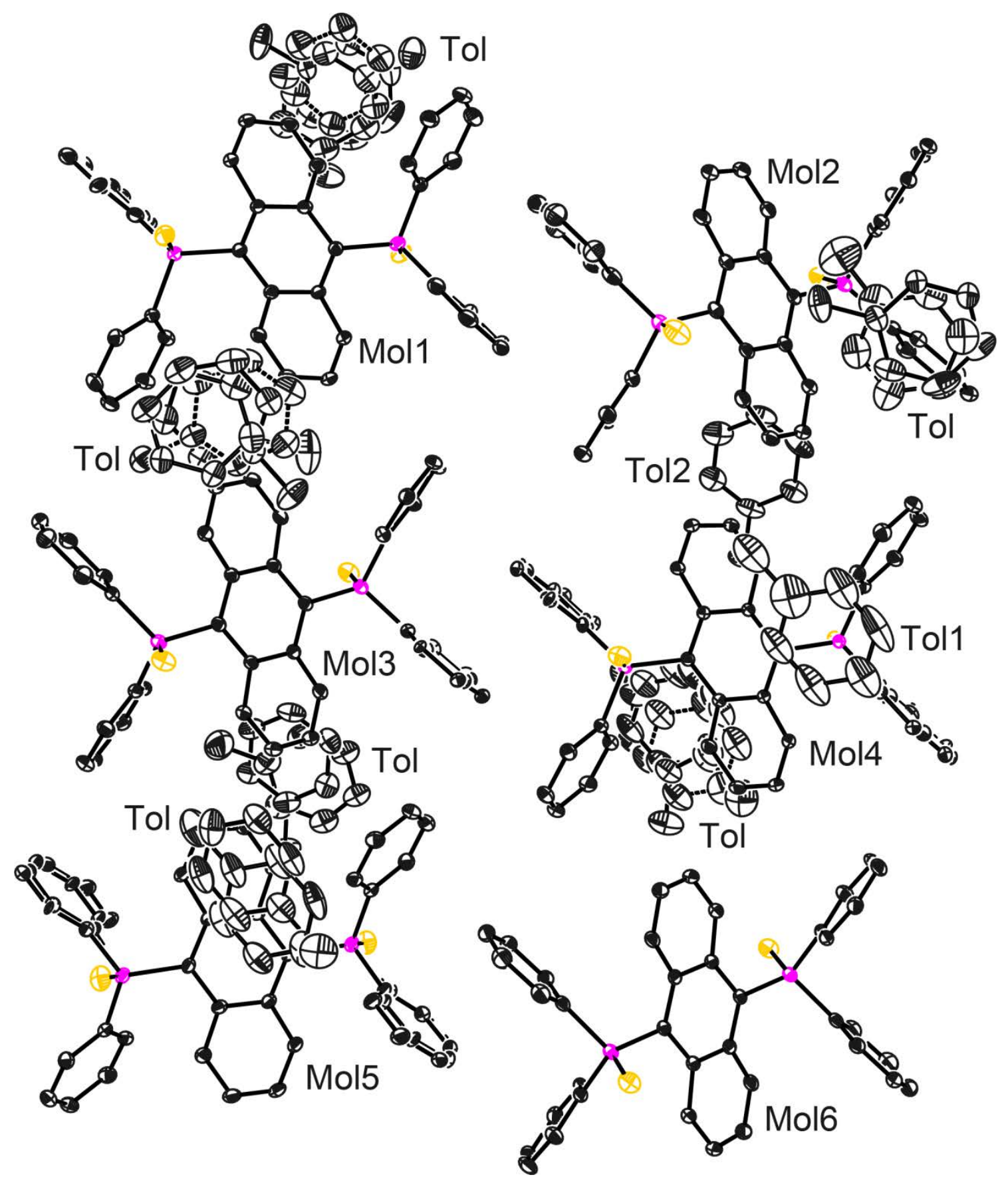

Figure 7-5. Asymmetric unit of SPAnPS@tol_d $d_{8}(20)$. The anisotropic displacement parameters are depicted at the $50 \%$ probability level. The hydrogen atoms are omitted for clarity. Molecules 5 and 6 are only contained in the asymmetric unit to one half, the other half is generated by an inversion center. 
The structure of SPAnPS@tol_d $d_{8}$ (20) exhibits strong disorder, especially of cocrystallized solvent molecules, which lowers the symmetry of the structure. All toluene molecules except for Tol1 and Tol2 (c.f. Figure 7-5) are disordered in at least two positions, most of them even in three positions. The occupations of these positions vary among the different toluene molecules. Anthracene molecules 5 and 6 are only contained in the asymmetric unit to one half, the other half depicted in Figure 7-5 is generated by an inversion center. One phenyl moiety of molecule 5 also shows disorder.

Table 7-5. Crystallographic data for SPAnPS@tol_d $d_{8}(\mathbf{2 0})$.

\begin{tabular}{|c|c|c|c|}
\hline Identification code & NF005 & & \\
\hline Empirical formula & $\mathrm{C}_{242.5} \mathrm{H}_{200} \mathrm{P}_{10} \mathrm{~S}_{10}$ & $\rho_{\text {calc }}\left[\mathrm{Mg} / \mathrm{m}^{3}\right]$ & 1.284 \\
\hline Formula weight $[\mathrm{g} / \mathrm{mol}]$ & 3744.31 & $\mu\left[\mathrm{mm}^{-1}\right]$ & 0.255 \\
\hline Temperature [K] & $100(2)$ & $F(000)$ & 3930 \\
\hline Wavelength $[\AA ̊]$ & 0.71073 & $\Theta$ range $\left[{ }^{\circ}\right]$ & 0.832 to 27.926 \\
\hline Crystal system & Triclinic & Reflections collected & 281882 \\
\hline Space group & $P \overline{1}$ & Independent reflections & 46066 \\
\hline$a[\AA]$ & $16.769(2)$ & Completeness to $\Theta_{\max }$ & $100.0 \%$ \\
\hline$b[\AA]$ & $24.431(3)$ & Absorption correction & Empirical \\
\hline$c[\AA]$ & $25.317(3)$ & Max. / min. transmission & $0.7097 / 0.7456$ \\
\hline$\alpha\left[^{\circ}\right]$ & $77.436(2)$ & Restraints / parameters & 13012 / 3049 \\
\hline$\beta\left[^{\circ}\right]$ & $79.181(2)$ & GoF & 1.022 \\
\hline$\gamma\left[{ }^{\circ}\right]$ & $74.989(2)$ & $R 1 / w R 2(I>2 \sigma(I))$ & $0.0467 / 0.1232$ \\
\hline Volume $\left[\AA^{3}\right]$ & $9683(2)$ & $R 1$ / wR2 (all data) & $0.0658 / 0.1381$ \\
\hline Z & 2 & Diff. peak and hole $\left[\mathrm{e} \AA^{-3}\right]$ & 0.892 and -0.611 \\
\hline
\end{tabular}




\subsubsection{MeAnPS $\left(\mathrm{NMe}_{2}\right)_{2}(21)$}

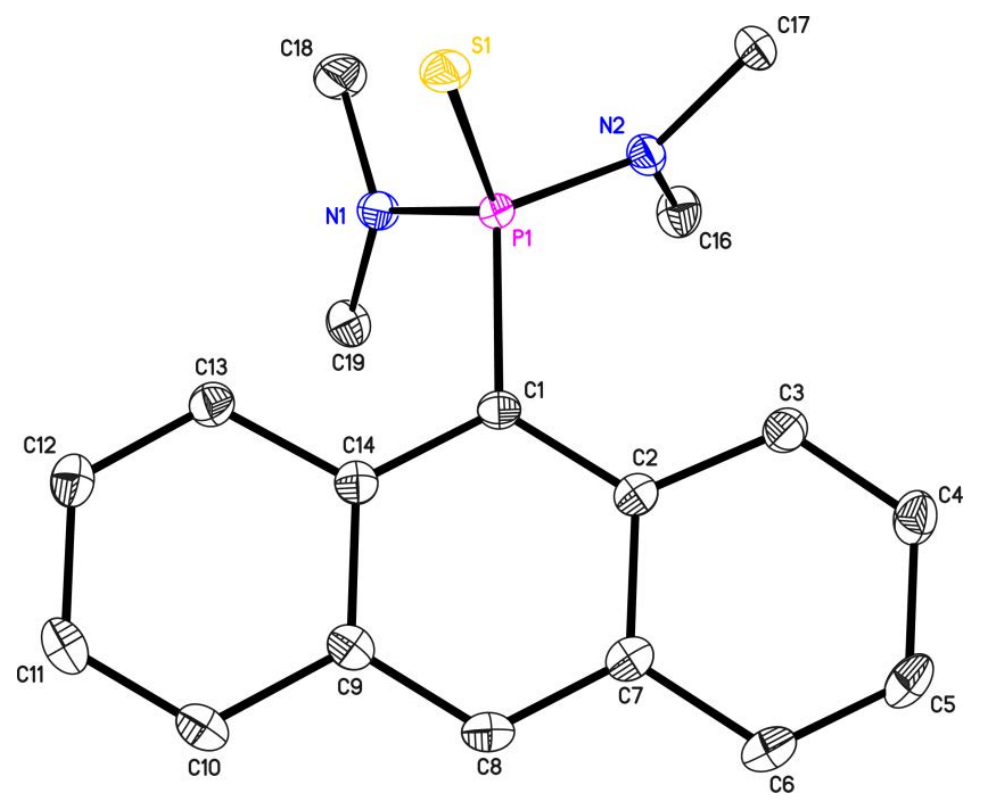

Figure 7-6. Asymmetric unit of MeAnPS(NMe $)_{2}$ (21). The anisotropic displacement parameters are depicted at the $50 \%$ probability level. The hydrogen atoms are omitted for clarity.

Table 7-6. Crystallographic data for MeAnPS( $\left(\mathrm{NM}_{2}\right)_{2}$ (21).

\begin{tabular}{|c|c|c|c|}
\hline Identification code & NF006 & & \\
\hline Empirical formula & $\mathrm{C}_{19} \mathrm{H}_{23} \mathrm{~N}_{2} \mathrm{PS}$ & $\rho_{\text {calc }}\left[\mathrm{Mg} / \mathrm{m}^{3}\right]$ & 1.306 \\
\hline Formula weight $[\mathrm{g} / \mathrm{mol}]$ & 342.42 & $\mu\left[\mathrm{mm}^{-1}\right]$ & 0.279 \\
\hline Temperature [K] & $100(2)$ & $F(000)$ & 728 \\
\hline Wavelength $[\AA ̊]$ & 0.71073 & $\Theta$ range $\left[{ }^{\circ}\right]$ & 2.191 to 26.318 \\
\hline Crystal system & Monoclinic & Reflections collected & 39043 \\
\hline Space group & $P 2_{1} / n$ & Independent reflections & 3529 \\
\hline$a[\AA]$ & $10.642(3)$ & Completeness to $\Theta_{\max }$ & $99.8 \%$ \\
\hline$b[\AA]$ & $13.429(3)$ & Absorption correction & Empirical \\
\hline$c[\AA]$ & $12.776(3)$ & Max. / min. transmission & $0.6090 / 0.7389$ \\
\hline$\alpha\left[^{\circ}\right]$ & 90 & Restraints / parameters & $0 / 235$ \\
\hline$\beta\left[^{\circ}\right]$ & $107.534(4)$ & GoF & 1.078 \\
\hline$\gamma\left[^{\circ}\right]$ & 90 & $R 1 / w R 2(I>2 \sigma(I))$ & $0.0387 / 0.0952$ \\
\hline Volume $\left[\AA^{3}\right]$ & $1741.1(7)$ & $R 1 / w R 2$ (all data) & $0.0456 / 0.0995$ \\
\hline Z & 4 & Diff. peak and hole $\left[\mathrm{e} \AA^{-3}\right]$ & 0.294 and -0.412 \\
\hline
\end{tabular}




\subsection{7 [MeAnP(NMe $\left.)_{2}(\mathrm{~S}) \mathrm{AuCl}\right](22)$}

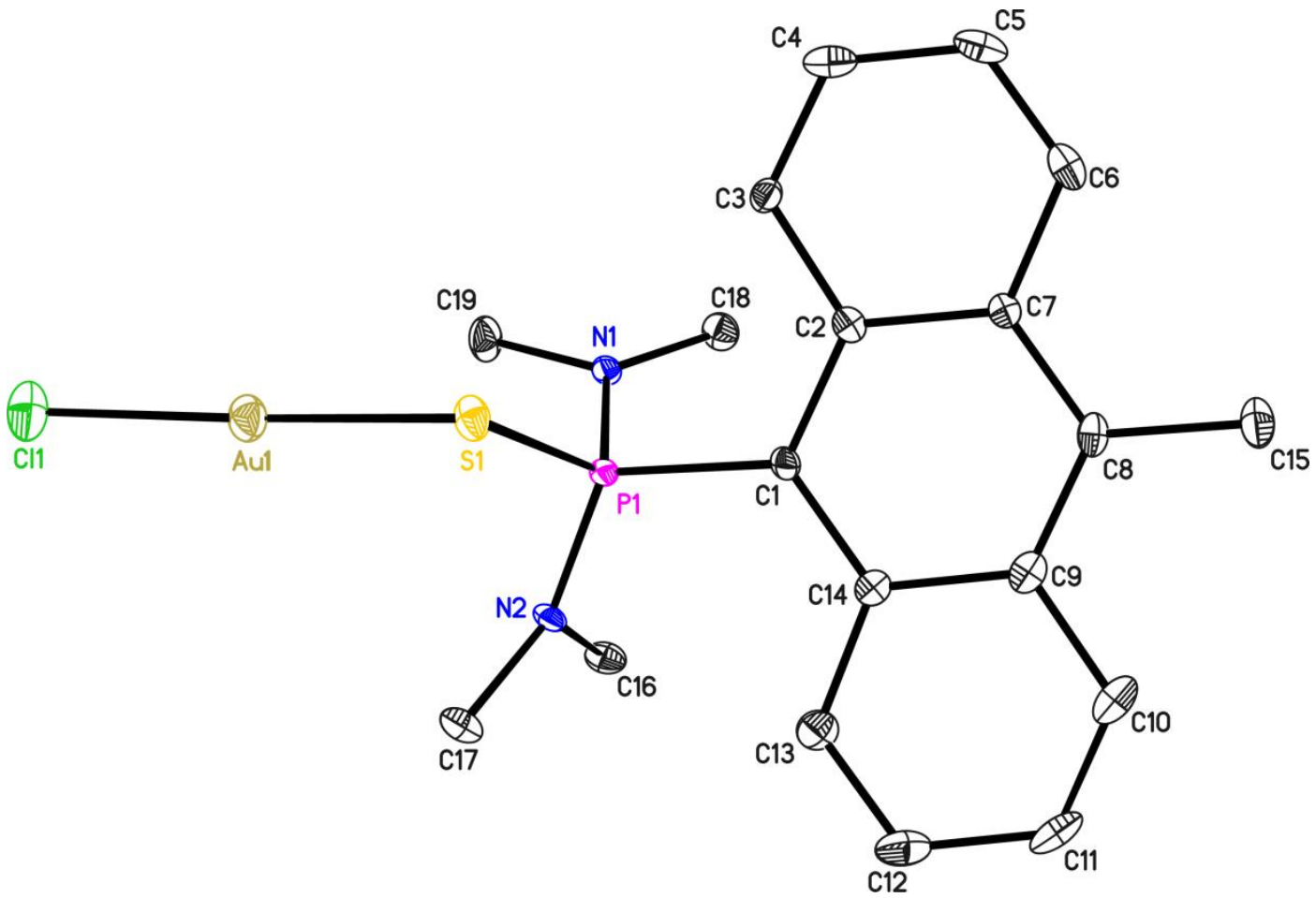

Figure 7-7. Asymmetric unit of $\left[\mathrm{MeAnP}\left(\mathrm{NMe}_{2}\right)_{2}(\mathrm{~S}) \mathrm{AuCl}\right]$ (22). The anisotropic displacement parameters are depicted at the $50 \%$ probability level. The hydrogen atoms are omitted for clarity.

Table 7-7. Crystallographic data for $\left[\mathrm{MeAnP}\left(\mathrm{NMe}_{2}\right)_{2}(\mathrm{~S}) \mathrm{AuCl}\right]$ (22).

\begin{tabular}{|c|c|c|c|}
\hline Identification code & NF007 & & \\
\hline Empirical formula & $\mathrm{C}_{19} \mathrm{H}_{23} \mathrm{AuCIN}{ }_{2} \mathrm{PS}$ & $\rho_{\text {calc }}\left[\mathrm{Mg} / \mathrm{m}^{3}\right]$ & 1.964 \\
\hline Formula weight $[\mathrm{g} / \mathrm{mol}]$ & 574.84 & $\mu\left[\mathrm{mm}^{-1}\right]$ & 7.898 \\
\hline Temperature [K] & $100(2)$ & $F(000)$ & 1112 \\
\hline Wavelength $[\AA ̊]$ & 0.71073 & $\Theta$ range $\left[{ }^{\circ}\right]$ & 2.17 to 25.35 \\
\hline Crystal system & Monoclinic & Reflections collected & 18345 \\
\hline Space group & $P 2_{1} / c$ & Independent reflections & 3557 \\
\hline$a[\AA ̊]$ & $13.178(2)$ & Completeness to $\Theta_{\max }$ & $99.7 \%$ \\
\hline$b[\AA]$ & $13.761(3)$ & Absorption correction & Empirical \\
\hline$c[\AA]$ & $11.056(2)$ & Max. / min. transmission & $0.3001 / 0.4311$ \\
\hline$\alpha\left[^{\circ}\right]$ & 90 & Restraints / parameters & $0 / 246$ \\
\hline$\beta\left[^{\circ}\right]$ & $104.125(2)$ & GoF & 1.151 \\
\hline$\nu\left[{ }^{\circ}\right]$ & 90 & $R 1 / w R 2(I>2 \sigma(I))$ & $0.0268 / 0.0649$ \\
\hline Volume $\left[\AA^{3}\right]$ & $1944.3(6)$ & $R 1 / w R 2$ (all data) & $0.0281 / 0.0654$ \\
\hline Z & 4 & Diff. peak and hole $\left[\mathrm{e} \AA^{-3}\right]$ & 0.672 and -1.284 \\
\hline
\end{tabular}




\subsubsection{MeAnPS $\left(\mathrm{NEt}_{2}\right)_{2}(23)$}

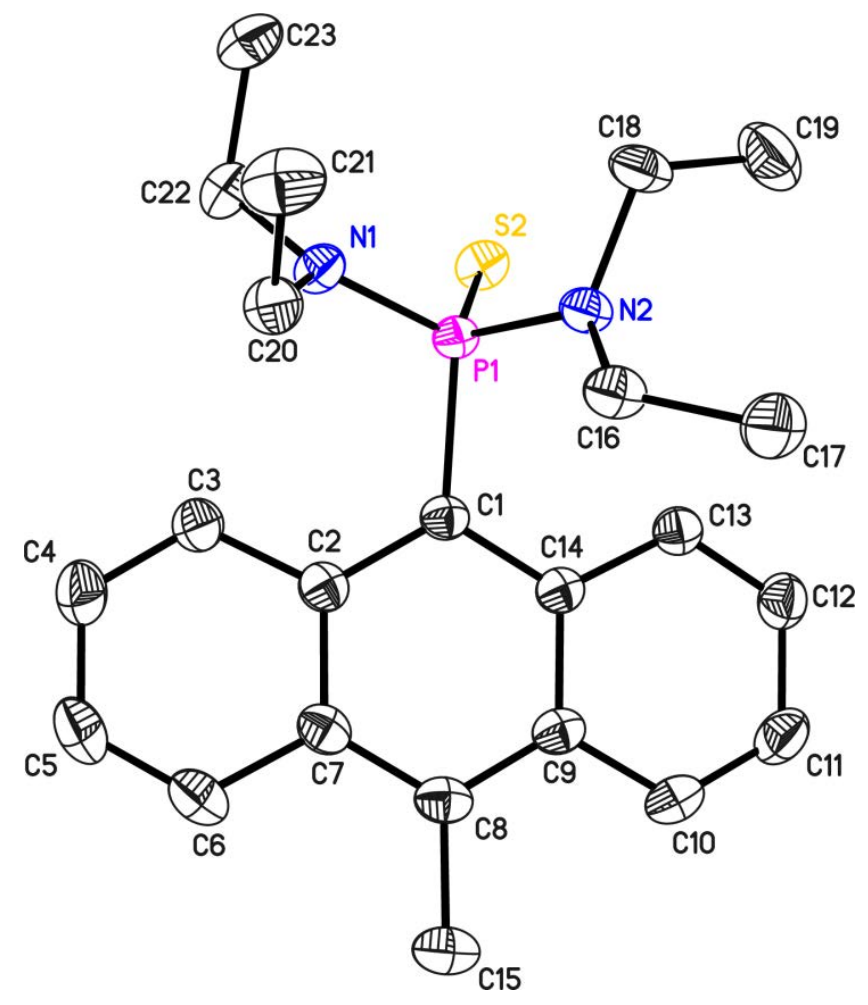

Figure 7-8. Asymmetric unit of MeAnPS( $\left.\mathrm{NEt}_{2}\right)_{2}(\mathbf{2 3})$. The anisotropic displacement parameters are depicted at the $50 \%$ probability level. The hydrogen atoms are omitted for clarity.

Table 7-8. Crystallographic data for MeAnPS $\left(\mathrm{NEt}_{2}\right)_{2}$ (23).

\begin{tabular}{|c|c|c|c|}
\hline Identification code & NF008 & & \\
\hline Empirical formula & $\mathrm{C}_{23} \mathrm{H}_{31} \mathrm{~N}_{2} \mathrm{PS}$ & $\mu\left[\mathrm{mm}^{-1}\right]$ & 0.235 \\
\hline Formula weight $[\mathrm{g} / \mathrm{mol}]$ & 398.53 & $F(000)$ & 856 \\
\hline Temperature [K] & $100(2)$ & $\Theta$ range $\left[{ }^{\circ}\right]$ & 3.39 to 26.37 \\
\hline Wavelength [Å] & 0.71073 & Reflections collected & 51001 \\
\hline Crystal system & Monoclinic & Independent reflections & 4374 \\
\hline Space group & $P 2_{1} / c$ & Completeness to $\Theta_{\max }$ & $99.8 \%$ \\
\hline$a[\AA ̊]$ & $7.1797(4)$ & Absorption correction & Empirical \\
\hline$b[\AA]$ & $24.3855(14)$ & Max. / min. transmission & $0.7295 / 0.7454$ \\
\hline$c[\AA ̊]$ & $12.7844(7)$ & Restraints / parameters & $0 / 260$ \\
\hline$\beta\left[^{\circ}\right]$ & $106.0270(10)$ & GoF & 1.050 \\
\hline Volume $\left[\AA^{3}\right]$ & $2151.3(2)$ & $R 1 / w R 2(I>2 \sigma(I))$ & $0.0292 / 0.0823$ \\
\hline $\mathrm{Z}$ & 4 & $R 1 / w R 2$ (all data) & $0.0309 / 0.0839$ \\
\hline$\rho_{\text {calc }}\left[\mathrm{Mg} / \mathrm{m}^{3}\right]$ & 1.230 & Diff. peak and hole $\left[\mathrm{e} \AA^{-3}\right]$ & 0.255 and -0.327 \\
\hline
\end{tabular}




\subsection{9 [MeAnP(NEt $\left.)_{2}(\mathrm{~S}) \mathrm{AuCl}\right](24)$}

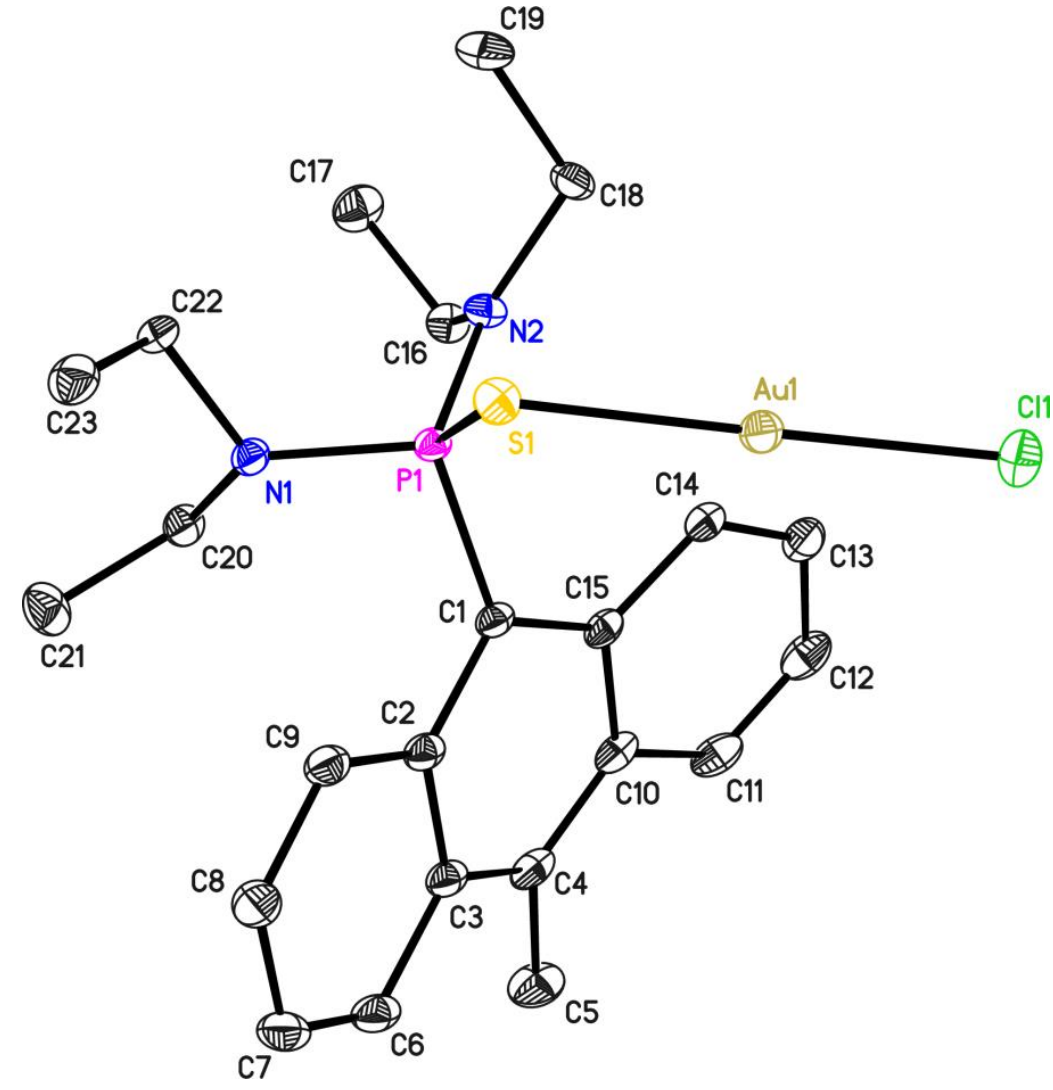

Figure 7-9. Asymmetric unit of $\left[\mathrm{MeAnP}\left(\mathrm{NEt}_{2}\right)_{2}(\mathrm{~S}) \mathrm{AuCl}\right]$ (24). The anisotropic displacement parameters are depicted at the $50 \%$ probability level. The hydrogen atoms are omitted for clarity.

Table 7-9. Crystallographic data for $\left[\mathrm{MeAnP}\left(\mathrm{NEt}_{2}\right)_{2}(\mathrm{~S}) \mathrm{AuCl}\right]$ (24).

\begin{tabular}{ll|ll}
\hline Identification code & $\mathrm{NFO09}$ & & \\
Empirical formula & $\mathrm{C}_{23} \mathrm{H}_{31} \mathrm{AuClN}_{2} \mathrm{PS}$ & $\mu\left[\mathrm{mm}^{-1}\right]$ & 6.747 \\
Formula weight $[\mathrm{g} / \mathrm{mol}]$ & 630.94 & $\mathrm{~F}(000)$ & 1240 \\
Temperature $[\mathrm{K}]$ & $100(2)$ & $\Theta$ range $\left[^{\circ}\right]$ & 2.461 to 28.359 \\
Wavelength $[\AA]$ & 0.71073 & Reflections collected & 65570 \\
Crystal system & Monoclinic & Independent reflections & 5699 \\
Space group & $P 2_{1} / c$ & Completeness to $\Theta_{\text {max }}$ & $99.8 \%$ \\
$a[\AA]$ & $12.9294(16)$ & Absorption correction & Empirical \\
$b[\AA]$ & $10.7738(13)$ & Max. / min. transmission & $0.3749 / 0.4311$ \\
$c[\AA]$ & $16.366(2)$ & Restraints / parameters & $0 / 282$ \\
$\beta\left[{ }^{\circ}\right]$ & $91.363(2)$ & GoF & 1.049 \\
Volume $\left[\AA^{3}\right]$ & $2279.1(5)$ & $R 1 / w R 2$ (I>2 $\sigma(I))$ & $0.0151 / 0.0378$ \\
$\mathrm{Z}$ & 4 & $R 1 / w R 2$ (all data) & $0.0162 / 0.0383$ \\
$\rho_{\text {calc }}\left[\mathrm{Mg} / \mathrm{m}^{3}\right]$ & 1.839 & Diff. peak and hole $\left[\mathrm{e} \AA^{-3}\right]$ & 0.560 and -0.325 \\
\hline
\end{tabular}




\subsubsection{MeAnPSe $\left(\mathrm{NMe}_{2}\right)_{2}(25)$}

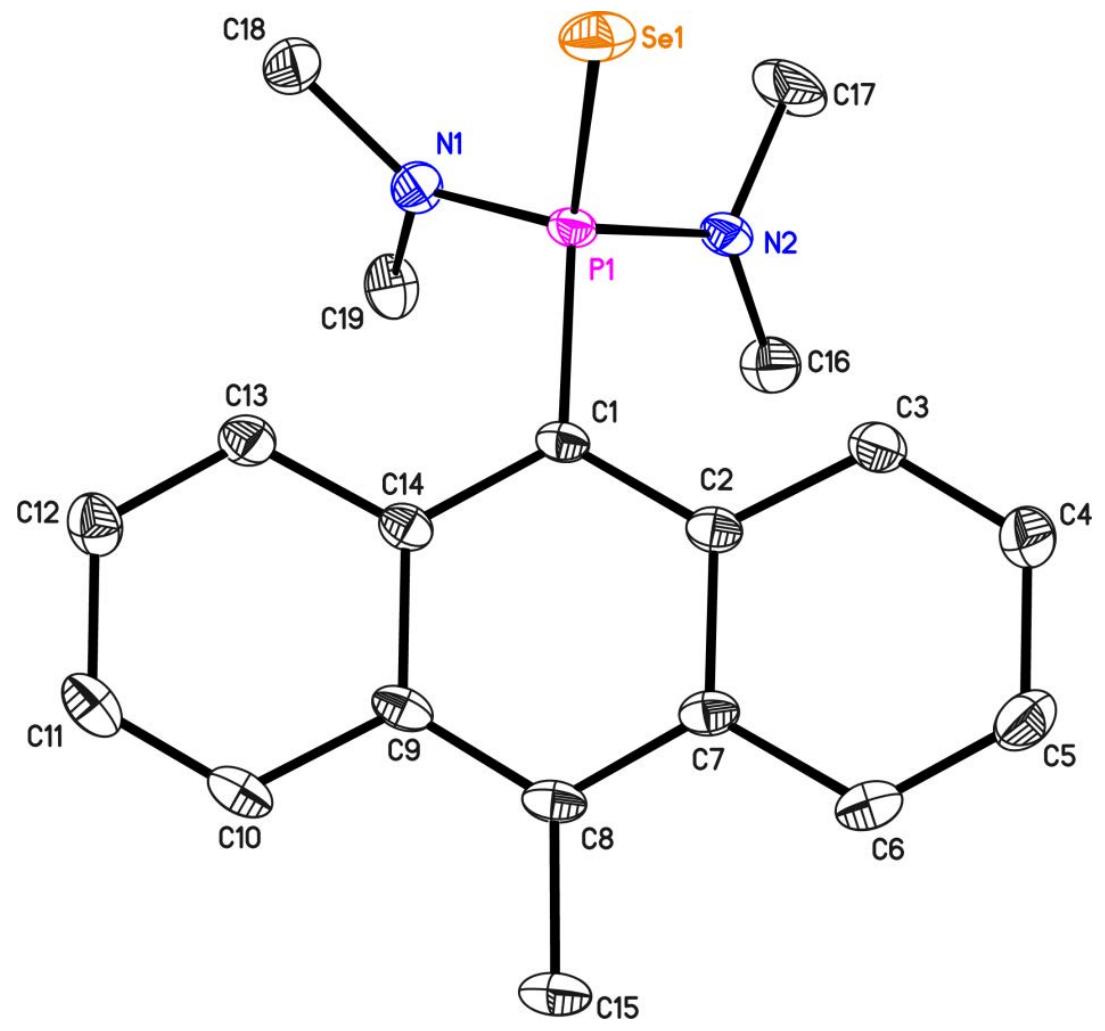

Figure 7-10. Asymmetric unit of MeAnPSe $\left(\mathrm{NMe}_{2}\right)_{2}$ (25). The anisotropic displacement parameters are depicted at the $50 \%$ probability level. The hydrogen atoms are omitted for clarity.

Table 7-10. Crystallographic data for MeAnPSe( $\left(\mathrm{NMe}_{2}\right)_{2}$ (25).

\begin{tabular}{ll|ll}
\hline Identification code & $\mathrm{NFO10}$ & & \\
Empirical formula & $\mathrm{C}_{19} \mathrm{H}_{23} \mathrm{~N}_{2} \mathrm{PSe}$ & $\mu\left[\mathrm{mm}^{-1}\right]$ & 2.224 \\
Formula weight $[\mathrm{g} / \mathrm{mol}]$ & 389.32 & $\mathrm{~F}(000)$ & 800 \\
Temperature $[\mathrm{K}]$ & $100(2)$ & $\Theta$ range $\left[^{\circ}\right]$ & 1.81 to 32.09 \\
Wavelength $[\AA]$ & 0.71073 & Reflections collected & 55806 \\
Crystal system & Monoclinic & Independent reflections & 6000 \\
Space group & $P 2_{1} / c$ & Completeness to $\Theta_{\text {max }}$ & $97.2 \%$ \\
$a[\AA]$ & $11.227(2)$ & Absorption correction & Empirical \\
$b[\AA ̊ \Omega$ & $7.7989(14)$ & Max. / min. transmission & $0.6223 / 0.7457$ \\
$c[\AA]$ & $20.117(4)$ & Restraints / parameters & $0 / 228$ \\
$\beta\left[{ }^{\circ}\right]$ & $90.551(2)$ & GoF & 1.058 \\
Volume $\left[\AA^{3}\right]$ & $1761.4(5)$ & $R 1 / w R 2(\mathrm{I}) 2 \sigma(\mathrm{I}))$ & $0.0316 / 0.0831$ \\
$\mathrm{Z}$ & 4 & $R 1 / w R 2($ all data) & $0.0422 / 0.0858$ \\
$\rho_{\text {calc }}\left[\mathrm{Mg} / \mathrm{m}^{3}\right]$ & 1.468 & Diff. peak and hole $\left[\mathrm{e} \AA^{-3}\right]$ & 0.588 and -0.384 \\
\hline
\end{tabular}




\subsubsection{1 [MeAnP(NMe $\left.)_{2}(\mathrm{Se}) \mathrm{AuCl}\right](26)$}

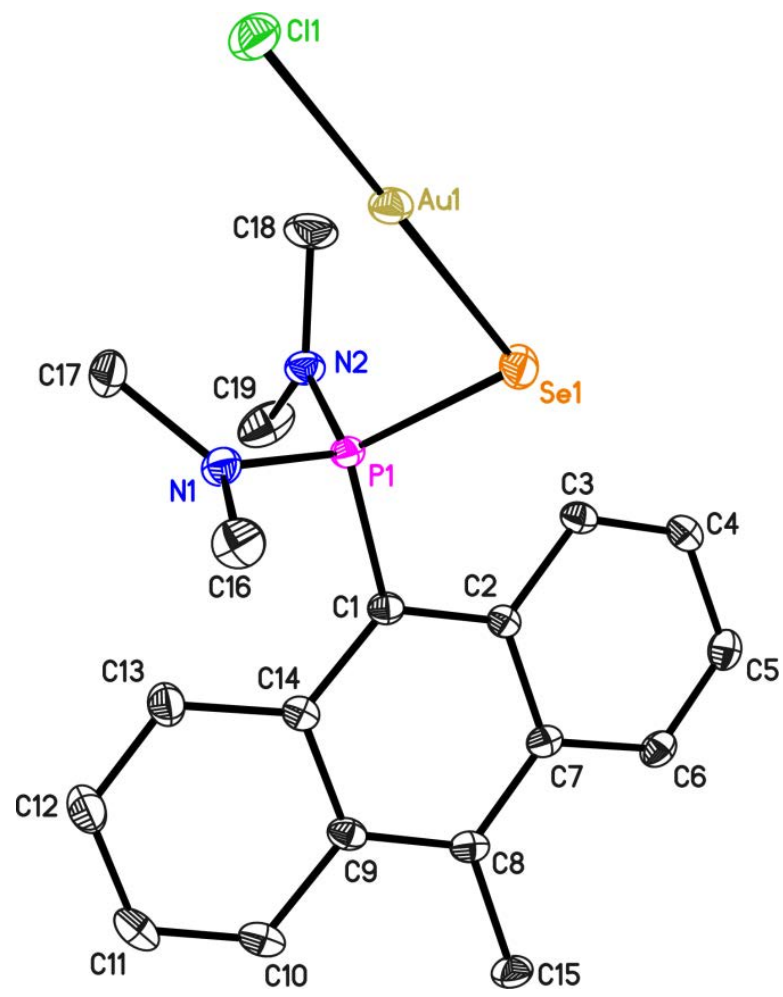

Figure 7-11. Asymmetric unit of $\left[\mathrm{MeAnP}\left(\mathrm{NMe}_{2}\right)_{2}(\mathrm{Se}) \mathrm{AuCl}\right]$ (26). The anisotropic displacement parameters are depicted at the $50 \%$ probability level. The hydrogen atoms are omitted for clarity.

Table 7-11. Crystallographic data for $\left[\mathrm{MeAnP}\left(\mathrm{NMe}_{2}\right)_{2}(\mathrm{Se}) \mathrm{AuCl}\right]$ (26).

\begin{tabular}{|c|c|c|c|}
\hline Identification code & NF011 & & \\
\hline Empirical formula & $\mathrm{C}_{19} \mathrm{H}_{23} \mathrm{AuClN}{ }_{2} \mathrm{PSe}$ & $\rho_{\text {calc }}\left[\mathrm{Mg} / \mathrm{m}^{3}\right]$ & 2.078 \\
\hline Formula weight $[\mathrm{g} / \mathrm{mol}]$ & 621.74 & $\mu\left[\mathrm{mm}^{-1}\right]$ & 9.453 \\
\hline Temperature [K] & $100(2)$ & $F(000)$ & 592 \\
\hline Wavelength $[\AA ̊]$ & 0.71073 & $\Theta$ range $\left[{ }^{\circ}\right]$ & 2.017 to 28.326 \\
\hline Crystal system & Triclinic & Reflections collected & 26744 \\
\hline Space group & $\mathrm{P} \overline{1}$ & Independent reflections & 4922 \\
\hline$a[\AA ̊]$ & $8.6565(13)$ & Completeness to $\Theta_{\max }$ & $99.4 \%$ \\
\hline$b[\AA]$ & $10.3881(16)$ & Absorption correction & Empirical \\
\hline$c[\AA]$ & $11.4583(18)$ & Max. / min. transmission & $0.6681 / 0.7457$ \\
\hline$\alpha\left[^{\circ}\right]$ & $77.932(2)$ & Restraints / parameters & $0 / 242$ \\
\hline$\beta\left[^{\circ}\right]$ & $82.877(2)$ & GoF & 1.050 \\
\hline$v\left[{ }^{\circ}\right]$ & $82.388(2)$ & $R 1 / w R 2(I>2 \sigma(I))$ & 0.0152 / 0.0377 \\
\hline Volume $\left[\AA^{3}\right]$ & $993.8(3)$ & $R 1 / w R 2$ (all data) & $0.0158 / 0.0380$ \\
\hline $\mathrm{z}$ & 2 & Diff. peak and hole $\left[\mathrm{e}^{-3}{ }^{-3}\right]$ & 1.088 and -0.612 \\
\hline
\end{tabular}




\subsubsection{MeAnPSe $\left(\mathrm{NEt}_{2}\right)_{2}$ (27)}

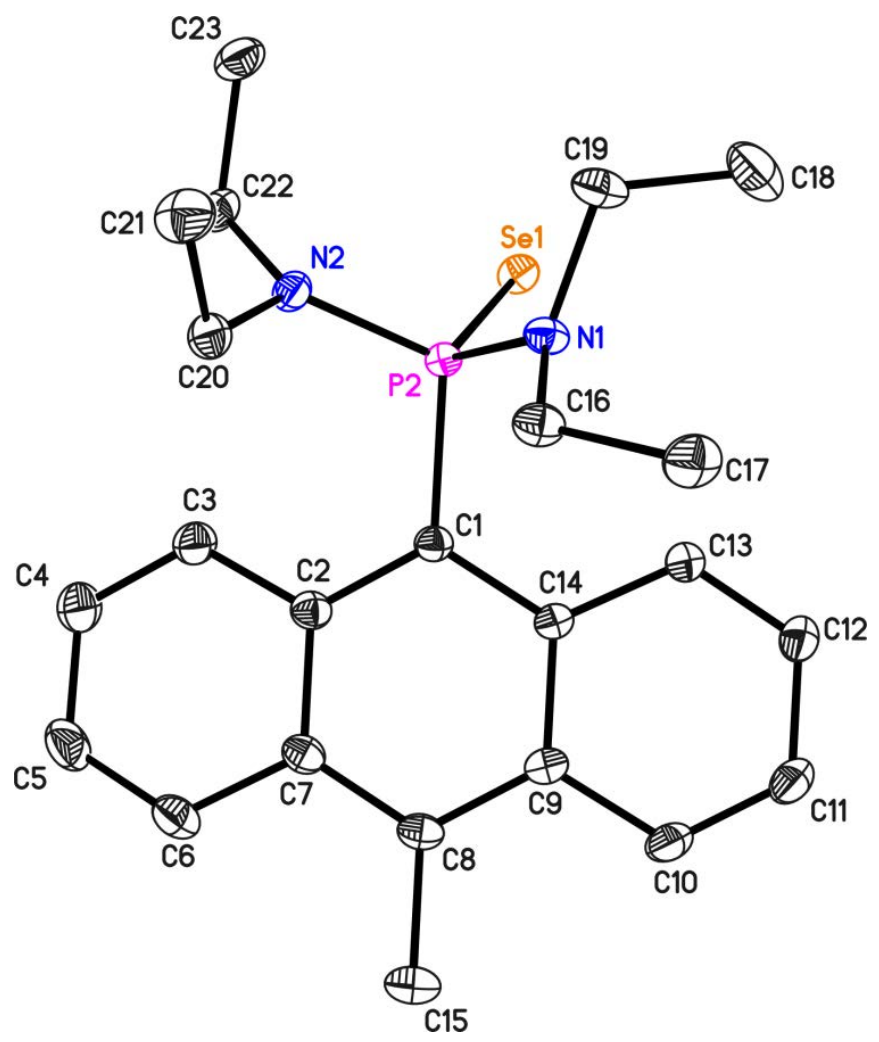

Figure 7-12. Asymmetric unit of MeAnPSe(NEt $)_{2}$ (27). The anisotropic displacement parameters are depicted at the $50 \%$ probability level. The hydrogen atoms are omitted for clarity.

Table 7-12. Crystallographic data for MeAnPSe $\left(\mathrm{NEt}_{2}\right)_{2}$ (27).

\begin{tabular}{ll|ll}
\hline Identification code & $\mathrm{NFO12}$ & & \\
Empirical formula & $\mathrm{C}_{23} \mathrm{H}_{31} \mathrm{~N}_{2} \mathrm{PSe}$ & $\rho_{\text {calc }}\left[\mathrm{Mg} / \mathrm{m}^{3}\right]$ & 1.370 \\
Formula weight $[\mathrm{g} / \mathrm{mol}]$ & 445.43 & $\mu\left[\mathrm{mm}^{-1}\right]$ & 1.823 \\
Temperature $[\mathrm{K}]$ & $99(2)$ & $\mathrm{F}(000)$ & 928 \\
Wavelength $[\AA]$ & 0.71073 & $\Theta$ range $\left[^{\circ}\right]$ & 1.667 to 32.106 \\
Crystal system & Monoclinic & Reflections collected & 70607 \\
Space group & $P 2_{1} / c$ & Independent reflections & 7352 \\
$a[\AA]$ & $7.2264(4)$ & Completeness to $\Theta_{\max }$ & $99.8 \%$ \\
$b[\AA]]$ & $24.4259(13)$ & Absorption correction & Empirical \\
$c[\AA ̊ \Omega$ & $12.7157(7)$ & Max. $/$ min. transmission & $0.6129 / 0.7464$ \\
$\alpha\left[^{\circ}\right]$ & 90 & Restraints / parameters & $0 / 260$ \\
$\beta\left[^{\circ}\right]$ & $105.7790(10)$ & GoF & 1.034 \\
$\gamma\left[^{\circ}\right]$ & 90 & $R 1 / w R 2(\mathrm{I}>2 \sigma(\mathrm{I}))$ & $0.0244 / 0.0625$ \\
Volume $\left[\AA^{3}\right]$ & $2159.9(2)$ & $R 1 / w R 2$ (all data) & $0.0300 / 0.0650$ \\
$Z$ & 4 & Diff. peak and hole $\left[\mathrm{e} \AA^{-3}\right]$ & 0.493 and -0.227 \\
\hline
\end{tabular}




\subsubsection{3 [MeAnP(NEt $\left.)_{2}(\mathrm{Se}) \mathrm{AuCl}\right](28)$}

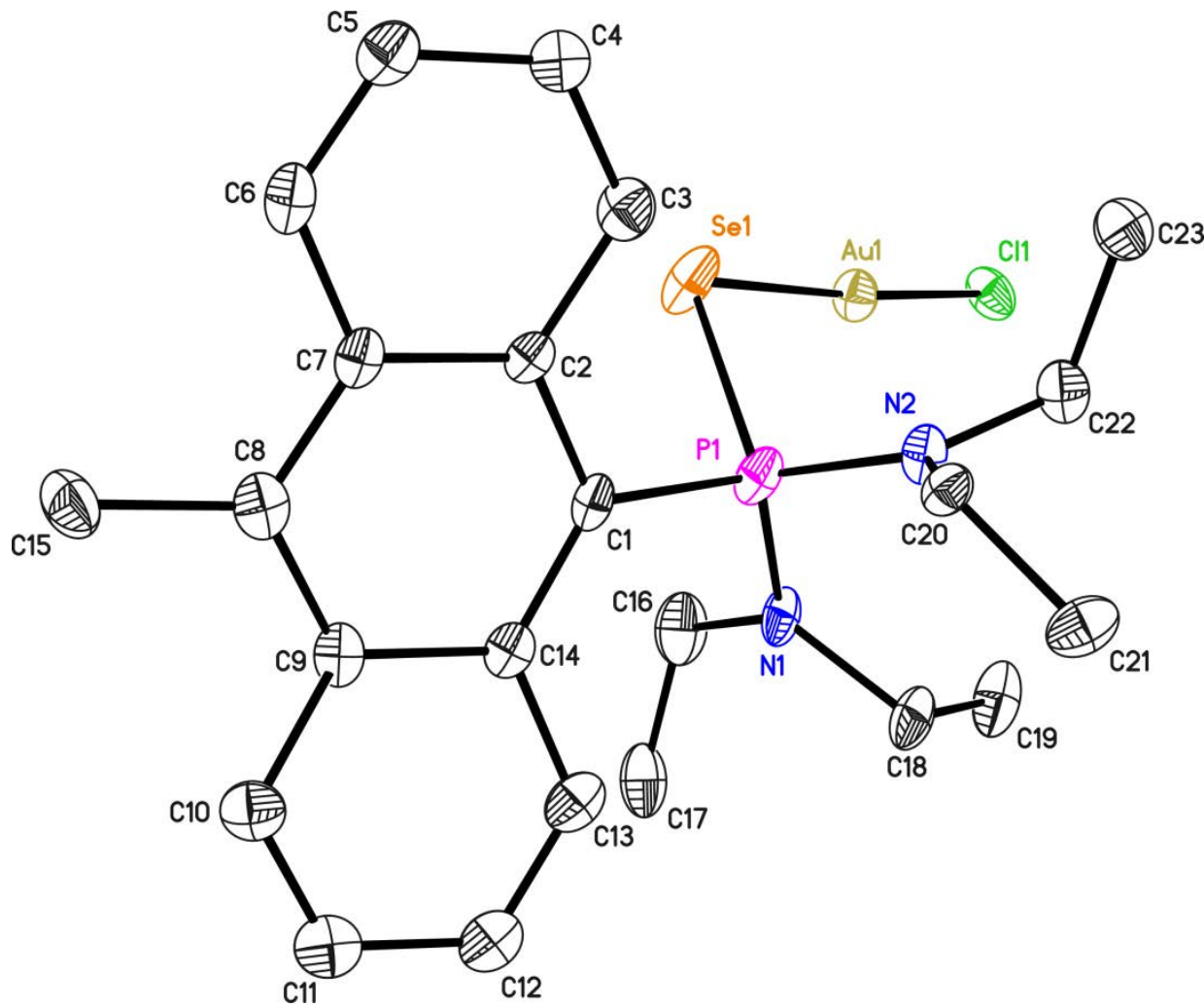

Figure 7-13. Asymmetric unit of $\left[\mathrm{MeAnP}\left(\mathrm{NEt}_{2}\right)_{2}(\mathrm{Se}) \mathrm{AuCl}\right]$ (28). The anisotropic displacement parameters are depicted at the $50 \%$ probability level. The hydrogen atoms are omitted for clarity.

Table 7-13. Crystallographic data for $\left[\mathrm{MeAnP}\left(\mathrm{NEt}_{2}\right)_{2}(\mathrm{Se}) \mathrm{AuCl}\right]$ (28).

\begin{tabular}{|c|c|c|c|}
\hline Identification code & NF013 & & \\
\hline Empirical formula & $\mathrm{C}_{23} \mathrm{H}_{31} \mathrm{AuClN}{ }_{2} \mathrm{PSe}$ & $\rho_{\text {calc }}\left[\mathrm{Mg} / \mathrm{m}^{3}\right]$ & 1.941 \\
\hline Formula weight $[\mathrm{g} / \mathrm{mol}]$ & 677.84 & $\mu\left[\mathrm{mm}^{-1}\right]$ & 8.111 \\
\hline Temperature [K] & $100(2)$ & $F(000)$ & 1312 \\
\hline Wavelength [Å] & 0.71073 & $\Theta$ range $\left[{ }^{\circ}\right]$ & 1.800 to 26.369 \\
\hline Crystal system & Monoclinic & Reflections collected & 12363 \\
\hline Space group & $P 2_{1} / c$ & Independent reflections & 4419 \\
\hline$a[\AA ̊]$ & $11.0841(13)$ & Completeness to $\Theta_{\max }$ & $96.4 \%$ \\
\hline$b[\AA ̊]$ & $9.2470(12)$ & Absorption correction & Empirical \\
\hline$c[\AA]$ & $22.990(3)$ & Max. / min. transmission & $0.3150 / 0.4305$ \\
\hline$\alpha\left[^{\circ}\right]$ & 90 & Restraints / parameters & $393 / 278$ \\
\hline$\beta\left[^{\circ}\right]$ & $100.206(3)$ & GoF & 0.938 \\
\hline$\nu\left[{ }^{\circ}\right]$ & 90 & $R 1 / w R 2(I>2 \sigma(I))$ & $0.0441 / 0.0710$ \\
\hline Volume $\left[\AA^{3}\right]$ & $2319.0(5)$ & $R 1 / w R 2$ (all data) & $0.0808 / 0.0796$ \\
\hline $\mathrm{Z}$ & 4 & Diff. peak and hole $\left[\mathrm{e} \AA^{-3}\right]$ & 1.679 and -1.178 \\
\hline
\end{tabular}




\subsubsection{4 $\mathrm{HAnP}\left(\mathrm{NMe}_{2}\right)_{2}(29)$}

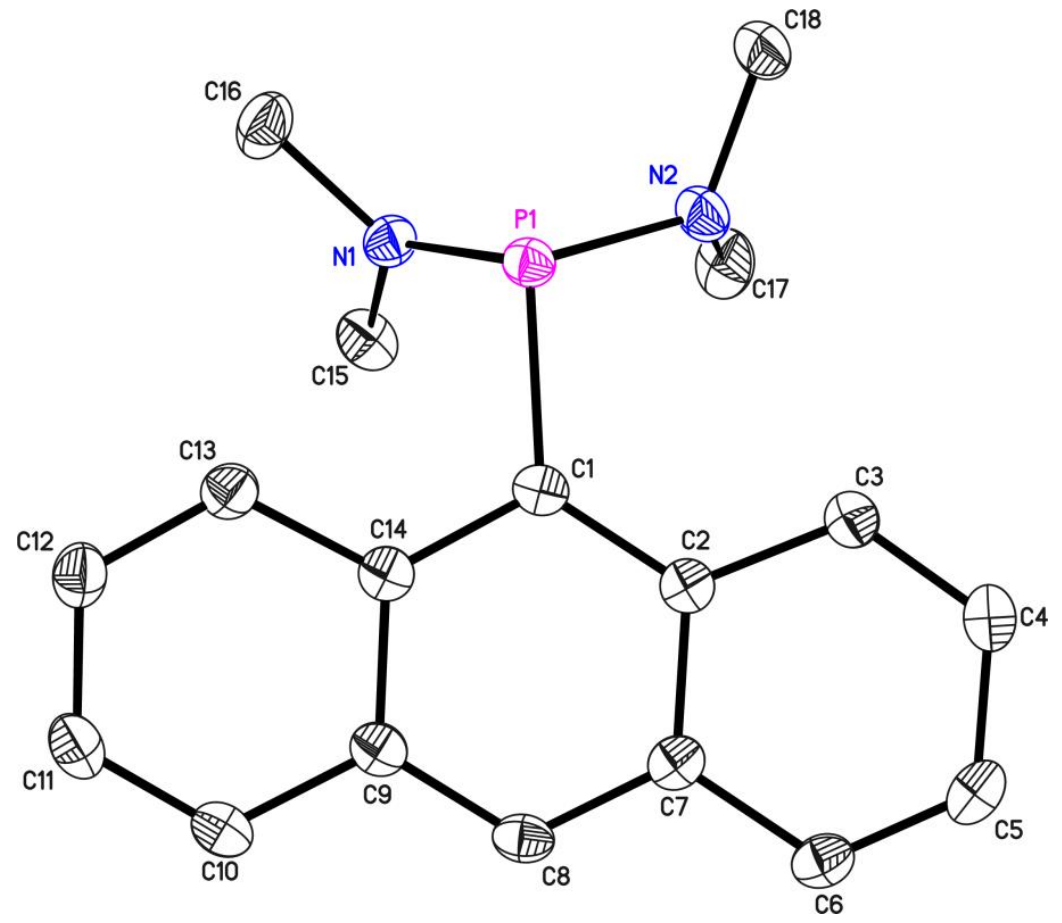

Figure 7-14. Asymmetric unit of $\mathrm{HAnP}\left(\mathrm{NMe}_{2}\right)_{2}$ (29). The anisotropic displacement parameters are depicted at the $50 \%$ probability level. The hydrogen atoms are omitted for clarity.

Table 7-14. Crystallographic data for $\mathrm{HAnP}\left(\mathrm{NMe}_{2}\right)_{2}$ (29).

\begin{tabular}{ll|ll}
\hline Identification code & $\mathrm{NF014}$ & & \\
Empirical formula & $\mathrm{C}_{18} \mathrm{H}_{21} \mathrm{~N}_{2} \mathrm{P}$ & $\mu\left[\mathrm{mm}^{-1}\right]$ & 0.171 \\
Formula weight $[\mathrm{g} / \mathrm{mol}]$ & 296.34 & $\mathrm{~F}(000)$ & 632 \\
Temperature $[\mathrm{K}]$ & $99(2)$ & $\Theta$ range $\left[^{\circ}\right]$ & 1.547 to 26.745 \\
Wavelength $[\AA]$ & 0.71073 & Reflections collected & 37000 \\
Crystal system & Monoclinic & Independent reflections & 3313 \\
Space group & $P 2_{1} / n$ & Completeness to $\Theta_{\max }$ & $99.6 \%$ \\
$a[\AA]$ & $8.5895(6)$ & Absorption correction & Empirical \\
$b[\AA]$ & $6.9198(4)$ & Max. $/$ min. transmission & $0.6682 / 0.7454$ \\
$c[\AA]$ & $26.4863(17)$ & Restraints / parameters & $0 / 194$ \\
$\beta\left[^{\circ}\right]$ & $96.2180(10)$ & GoF & 1.108 \\
Volume $\left[\AA^{3}\right]$ & $1565.02(17)$ & $R 1 / w R 2(\mathrm{I}>2 \sigma(\mathrm{I}))$ & $0.0362 / 0.0967$ \\
$Z$ & 4 & $R 1 / w R 2$ (all data) & $0.0417 / 0.1012$ \\
$\rho_{\text {calc }}\left[\mathrm{Mg} / \mathrm{m}^{3}\right]$ & 1.258 & Diff. peak and hole $\left[\mathrm{e} \AA^{-3}\right]$ & 0.287 and -0.357 \\
\hline
\end{tabular}




\subsubsection{5 $\mathrm{HAnPO}\left(\mathrm{NMe}_{2}\right)_{2}(30)$}

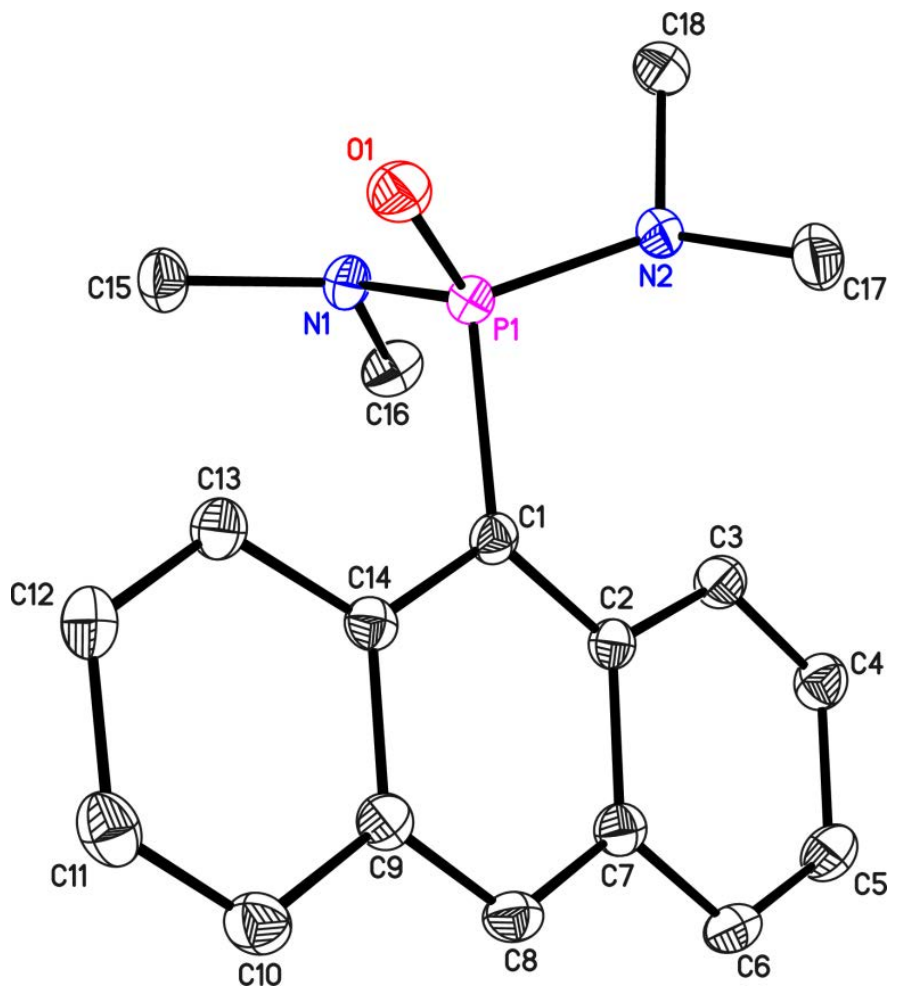

Figure 7-15. Asymmetric unit of $\mathrm{HAnPO}\left(\mathrm{NMe}_{2}\right)_{2}$ (30). The anisotropic displacement parameters are depicted at the $50 \%$ probability level. The hydrogen atoms are omitted for clarity.

Table 7-15. Crystallographic data for $\mathrm{HAnPO}\left(\mathrm{NMe}_{2}\right)_{2}$ (30).

\begin{tabular}{|c|c|c|c|}
\hline Identification code & NF015 & & \\
\hline Empirical formula & $\mathrm{C}_{18} \mathrm{H}_{21} \mathrm{~N}_{2} \mathrm{OP}$ & $\rho_{\text {calc }}\left[\mathrm{Mg} / \mathrm{m}^{3}\right]$ & 1.304 \\
\hline Formula weight $[\mathrm{g} / \mathrm{mol}]$ & 312.34 & $\mu\left[\mathrm{mm}^{-1}\right]$ & 0.177 \\
\hline Temperature $[\mathrm{K}]$ & $100(2)$ & $F(000)$ & 664 \\
\hline Wavelength $[\AA]]$ & 0.71073 & $\Theta$ range $\left[{ }^{\circ}\right]$ & 2.602 to 28.124 \\
\hline Crystal system & Orthorhombic & Reflections collected & 15818 \\
\hline Space group & $\operatorname{Pna}_{1}$ & Independent reflections & 3869 \\
\hline$a[\AA]$ & $8.5276(10)$ & Completeness to $\Theta_{\max }$ & $99.5 \%$ \\
\hline$b[\AA]$ & $19.708(2)$ & Absorption correction & Empirical \\
\hline$c[\AA ̊]$ & $9.4634(11)$ & Max. / min. transmission & $0.6363 / 0.7457$ \\
\hline$\alpha\left[^{\circ}\right]$ & 90 & Restraints / parameters & $1 / 203$ \\
\hline$\beta\left[^{\circ}\right]$ & 90 & GoF & 1.037 \\
\hline$\gamma\left[^{\circ}\right]$ & 90 & $R 1 / w R 2(I>2 \sigma(I))$ & $0.0299 / 0.0765$ \\
\hline Volume $\left[\AA^{3}\right]$ & $1590.4(3)$ & $R 1 / w R 2$ (all data) & $0.0316 / 0.0776$ \\
\hline z & 4 & Diff. peak and hole $\left[\mathrm{e}^{-3}{ }^{-3}\right]$ & 0.319 and -0.241 \\
\hline
\end{tabular}




\subsubsection{6 $\mathrm{HAnPS}\left(\mathrm{NMe}_{2}\right)_{2}(31)$}

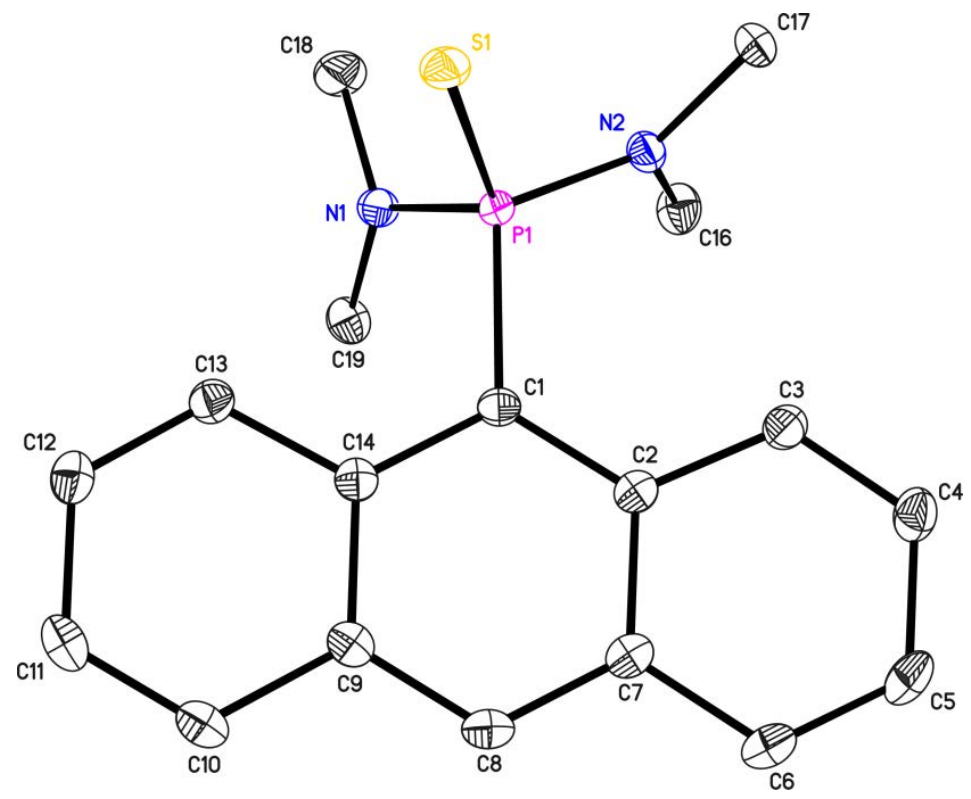

Figure 7-16. Asymmetric unit of $\mathrm{HAnPS}\left(\mathrm{NMe}_{2}\right)_{2}$ (31). The anisotropic displacement parameters are depicted at the $50 \%$ probability level. The hydrogen atoms are omitted for clarity.

Table 7-16. Crystallographic data for $\mathrm{HAnPS}\left(\mathrm{NMe}_{2}\right)_{2}(31)$.

\begin{tabular}{ll|ll}
\hline Identification code & $\mathrm{NFO16}$ & & \\
Empirical formula & $\mathrm{C}_{18} \mathrm{H}_{21} \mathrm{~N}_{2} \mathrm{PS}$ & $\mu\left[\mathrm{mm}^{-1}\right]$ & 0.297 \\
Formula weight $[\mathrm{g} / \mathrm{mol}]$ & 328.40 & $\mathrm{~F}(000)$ & 696 \\
Temperature $[\mathrm{K}]$ & $100(2)$ & $\Theta$ range $\left[^{\circ}\right]$ & 2.281 to 26.739 \\
Wavelength $[\AA]$ & 0.71073 & Reflections collected & 37822 \\
Crystal system & Monoclinic & Independent reflections & 3406 \\
Space group & $P 2_{1} / n$ & Completeness to $\Theta_{\text {max }}$ & $98.8 \%$ \\
$a[\AA]$ & $10.6558(16)$ & Absorption correction & Empirical \\
$b[\AA]$ & $13.326(2)$ & Max. / min. transmission & $0.6694 / 0.7429$ \\
$c[\AA]]$ & $11.8485(18)$ & Restraints / parameters & $0 / 203$ \\
$\beta\left[{ }^{\circ}\right]$ & $105.791(2)$ & GoF & 1.038 \\
Volume $\left[\AA^{3}\right]$ & $1619.0(4)$ & $R 1 / w R 2$ (I>2 $\sigma(\mathrm{I}))$ & $0.0287 / 0.0761$ \\
$\mathrm{Z}$ & 4 & $R 1 / w R 2$ (all data) & $0.0295 / 0.0768$ \\
$\rho_{\text {calc }}\left[\mathrm{Mg} / \mathrm{m}^{3}\right]$ & 1.347 & Diff. peak and hole $\left[\mathrm{e} \AA^{-3}\right]$ & 0.420 and -0.243 \\
\hline
\end{tabular}




\subsubsection{HAnPSe $\left(\mathrm{NMe}_{2}\right)_{2}(32)$}

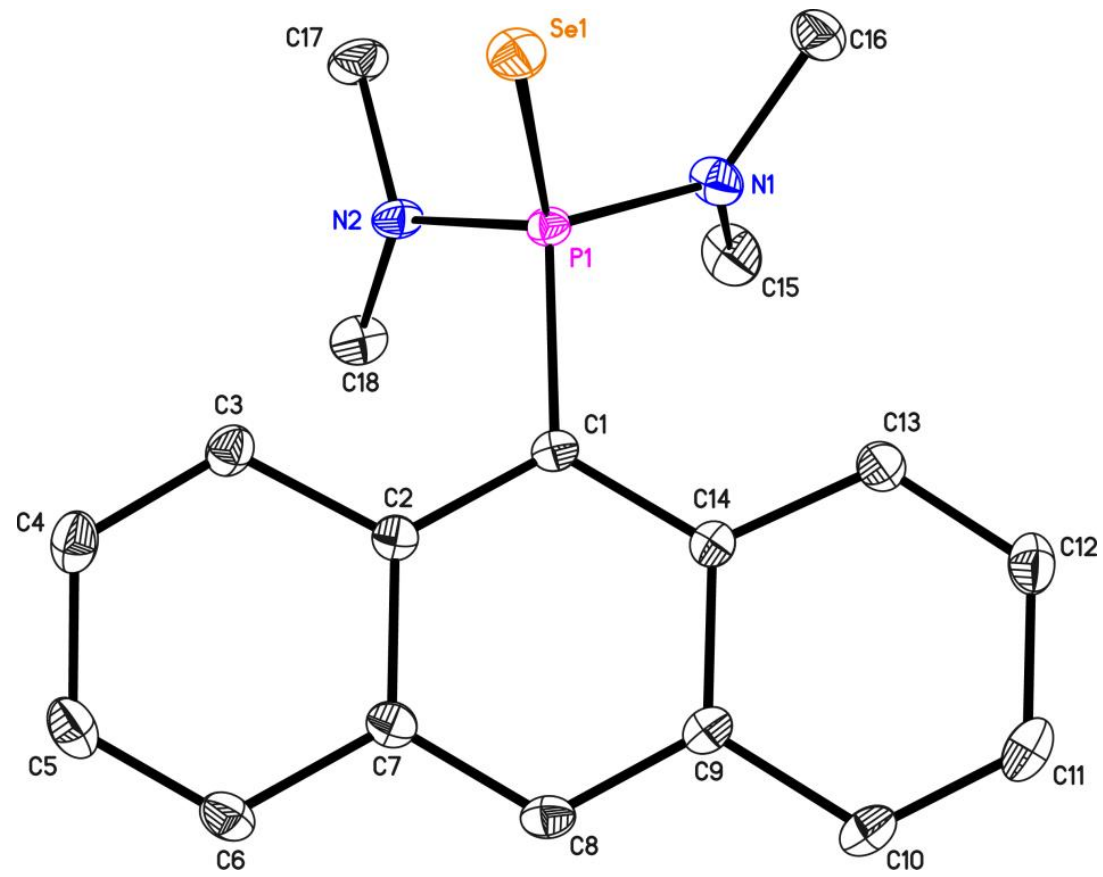

Figure 7-17. Asymmetric unit of HAnPSe( $\left.\mathrm{NMe}_{2}\right)_{2}(32)$. The anisotropic displacement parameters are depicted at the $50 \%$ probability level. The hydrogen atoms are omitted for clarity.

The position of the hydrogen atoms $\mathrm{H} 100$ at the nitrogen atom was taken from the difference map and refined with distance restraints.

Table 7-17. Crystallographic data for HAnPSe( $\left(\mathrm{NMe}_{2}\right)_{2}(32)$.

\begin{tabular}{ll|ll}
\hline Identification code & $\mathrm{NFO17}$ & & \\
Empirical formula & $\mathrm{C}_{18} \mathrm{H}_{21} \mathrm{~N}_{2} \mathrm{PSe}$ & $\rho_{\text {calc }}\left[\mathrm{Mg} / \mathrm{m}^{3}\right]$ & 1.468 \\
Formula weight $[\mathrm{g} / \mathrm{mol}]$ & 389.32 & $\mu\left[\mathrm{mm}^{-1}\right]$ & 2.224 \\
Temperature $[\mathrm{K}]$ & $100(2)$ & $\mathrm{F}(000)$ & 800 \\
Wavelength $[\AA]$ & 0.71073 & $\Theta$ range $\left[^{\circ}\right]$ & 1.81 to 32.09 \\
Crystal system & Monoclinic & Reflections collected & 55806 \\
Space group & $P 2_{1} / c$ & Independent reflections & 6000 \\
$a[\AA]$ & $11.227(2)$ & Completeness to $\Theta_{\text {max }}$ & $97.2 \%$ \\
$b[\AA]$ & $7.7989(14)$ & Absorption correction & Empirical \\
$c[\AA]$ & $20.117(4)$ & Max. / min. transmission & $0.6223 / 0.7457$ \\
$\alpha\left[^{\circ}\right]$ & 90 & Restraints / parameters & $0 / 228$ \\
$\beta\left[^{\circ}\right]$ & $90.551(2)$ & GoF & 1.058 \\
$\gamma\left[^{\circ}\right]$ & 90 & $R 1 / w R 2$ (I>2 $\sigma(I))$ & $0.0316 / 0.0831$ \\
Volume $\left[\AA^{3}\right]$ & $1761.4(5)$ & $R 1 / w R 2$ (all data) & $0.0422 / 0.0858$ \\
$Z$ & 4 & Diff. peak and hole $\left[\right.$ e $\left.\AA^{-3}\right]$ & 0.588 and -0.384 \\
\hline
\end{tabular}




\subsubsection{MeAnPHOO_H $\mathrm{HMe}_{2}$ (33a)}

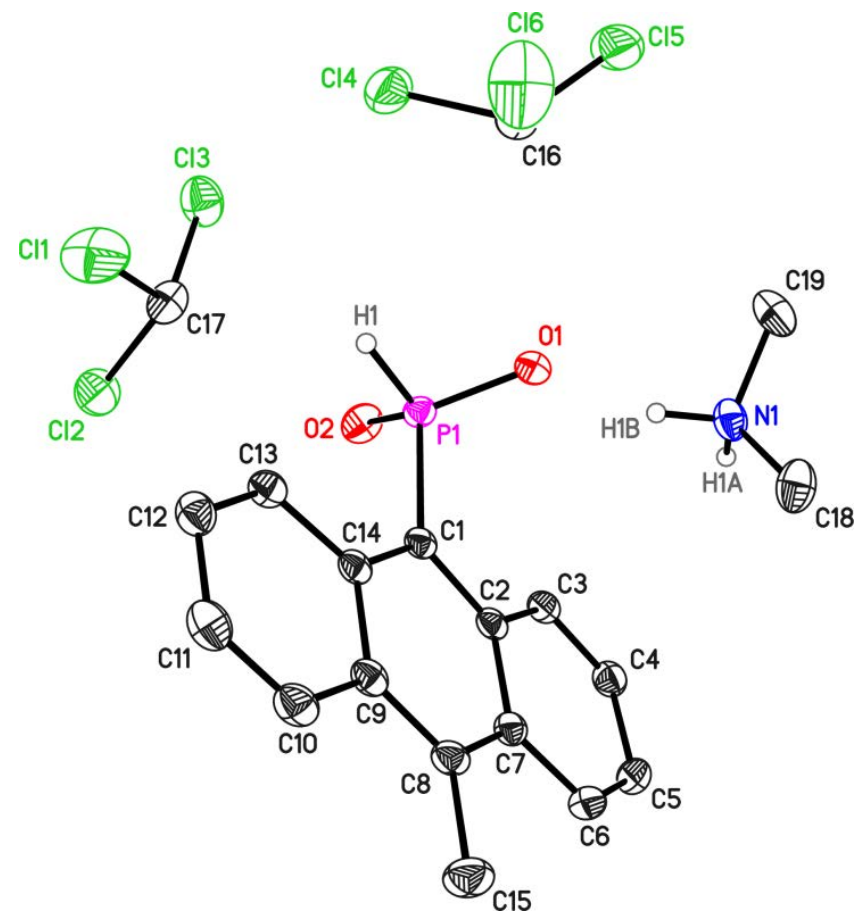

Figure 7-18. Asymmetric unit MeAnPHOO_H $\mathrm{HMe}_{2}$ (33a). The anisotropic displacement parameters are depicted at the $50 \%$ probability level. The hydrogen atoms are omitted for clarity.

Table 7-18. Crystallographic data for MeAnPHOO_H $\mathrm{NMe}_{2}$ (33a).

\begin{tabular}{|c|c|c|c|}
\hline Identification code & NF018 & & \\
\hline Empirical formula & $\mathrm{C}_{19} \mathrm{H}_{22} \mathrm{C}_{16} \mathrm{NO}_{2} \mathrm{P}$ & $\rho_{\text {calc }}\left[\mathrm{Mg} / \mathrm{m}^{3}\right]$ & 1.487 \\
\hline Formula weight $[\mathrm{g} / \mathrm{mol}]$ & 540.04 & $\mu\left[\mathrm{mm}^{-1}\right]$ & 0.795 \\
\hline Temperature [K] & $80(2)$ & $F(000)$ & 1104 \\
\hline Wavelength $[\AA ̊]$ & 0.71073 & $\Theta$ range $\left[{ }^{\circ}\right]$ & 1.747 to 26.091 \\
\hline Crystal system & Monoclinic & Reflections collected & 96282 \\
\hline Space group & $P 2_{1} / n$ & Independent reflections & 4782 \\
\hline$a[\AA]$ & $12.5300(14)$ & Completeness to $\Theta_{\max }$ & $100.0 \%$ \\
\hline$b[\AA]$ & $9.2298(10)$ & Absorption correction & Empirical \\
\hline$c[\AA ̊ \AA]$ & $21.295(2)$ & Max. / min. transmission & $0.7047 / 0.7453$ \\
\hline$\alpha\left[^{\circ}\right]$ & 90 & Restraints / parameters & $64 / 269$ \\
\hline$\beta\left[^{\circ}\right]$ & $101.682(2)$ & GoF & 1.050 \\
\hline$\gamma\left[^{\circ}\right]$ & 90 & $R 1 / w R 2(I>2 \sigma(I))$ & $0.0340 / 0.0825$ \\
\hline Volume $\left[\AA^{3}\right]$ & $2411.8(5)$ & $R 1 / w R 2$ (all data) & $0.0356 / 0.0838$ \\
\hline $\mathrm{Z}$ & 4 & Diff. peak and hole $\left[\mathrm{e} \AA^{-3}\right]$ & 0.863 and -0.898 \\
\hline
\end{tabular}




\subsubsection{9 $\quad\left(\mathrm{Et}_{2} \mathrm{~N}\right)_{2} \mathrm{PAnP}\left(\mathrm{NEt}_{2}\right)_{2}(35)$}

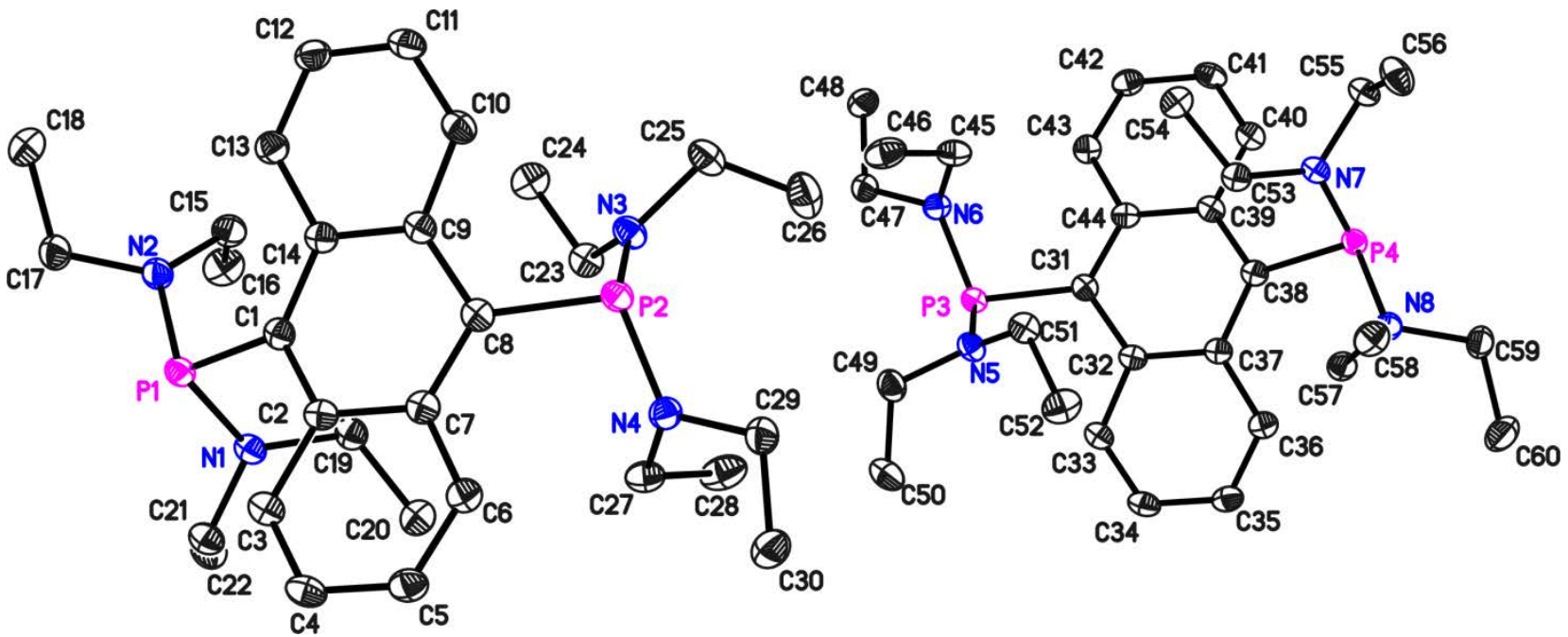

Figure 7-19. Asymmetric unit of $\left(\mathrm{Et}_{2} \mathrm{~N}\right)_{2} \mathrm{PAnP}\left(\mathrm{NEt}_{2}\right)_{2}$ (35). The anisotropic displacement parameters are depicted at the $50 \%$ probability level. The hydrogen atoms are omitted for clarity.

Table 7-19. Crystallographic data for $\left(\mathrm{Et}_{2} \mathrm{~N}\right)_{2} \mathrm{PAnP}\left(\mathrm{NEt}_{2}\right)_{2}$ (35).

\begin{tabular}{|c|c|c|c|}
\hline Identification code & NF019 & & \\
\hline Empirical formula & $\mathrm{C}_{60} \mathrm{H}_{96} \mathrm{~N}_{8} \mathrm{P}_{4}$ & $\rho_{\text {calc }}\left[\mathrm{Mg} / \mathrm{m}^{3}\right]$ & 1.176 \\
\hline Formula weight $[\mathrm{g} / \mathrm{mol}]$ & 1053.32 & $\mu\left[\mathrm{mm}^{-1}\right]$ & 0.171 \\
\hline Temperature [K] & $100(2)$ & $F(000)$ & 1144 \\
\hline Wavelength $[\AA]$ & 0.71073 & $\Theta$ range $\left[{ }^{\circ}\right]$ & 1.288 to 26.423 \\
\hline Crystal system & Triclinic & Reflections collected & 79915 \\
\hline Space group & $P \overline{1}$ & Independent reflections & 12185 \\
\hline$a[\AA ̊]$ & 13.6598(19) & Completeness to $\Theta_{\max }$ & $99.9 \%$ \\
\hline$b[\AA ̊]$ & $15.014(2)$ & Absorption correction & Empirical \\
\hline$c[\AA ̊]$ & $17.101(2)$ & Max. / min. transmission & $0.6941 / 0.7454$ \\
\hline$\alpha\left[^{\circ}\right]$ & $69.284(4)$ & Restraints / parameters & $180 / 665$ \\
\hline$\beta\left[^{\circ}\right]$ & $74.334(4)$ & GoF & 1.117 \\
\hline$v\left[^{\circ}\right]$ & $66.482(4)$ & $R 1 / w R 2(1>2 \sigma(I))$ & $0.0390 / 0.1113$ \\
\hline Volume $\left[\AA^{3}\right]$ & $2973.9(7)$ & $R 1 / w R 2$ (all data) & $0.0504 / 0.1178$ \\
\hline Z & 2 & Diff. peak and hole $\left[\mathrm{e}^{-3}{ }^{-3}\right]$ & 0.344 and -0.444 \\
\hline
\end{tabular}




\subsubsection{BrAnP(OPh) $2(38)$}

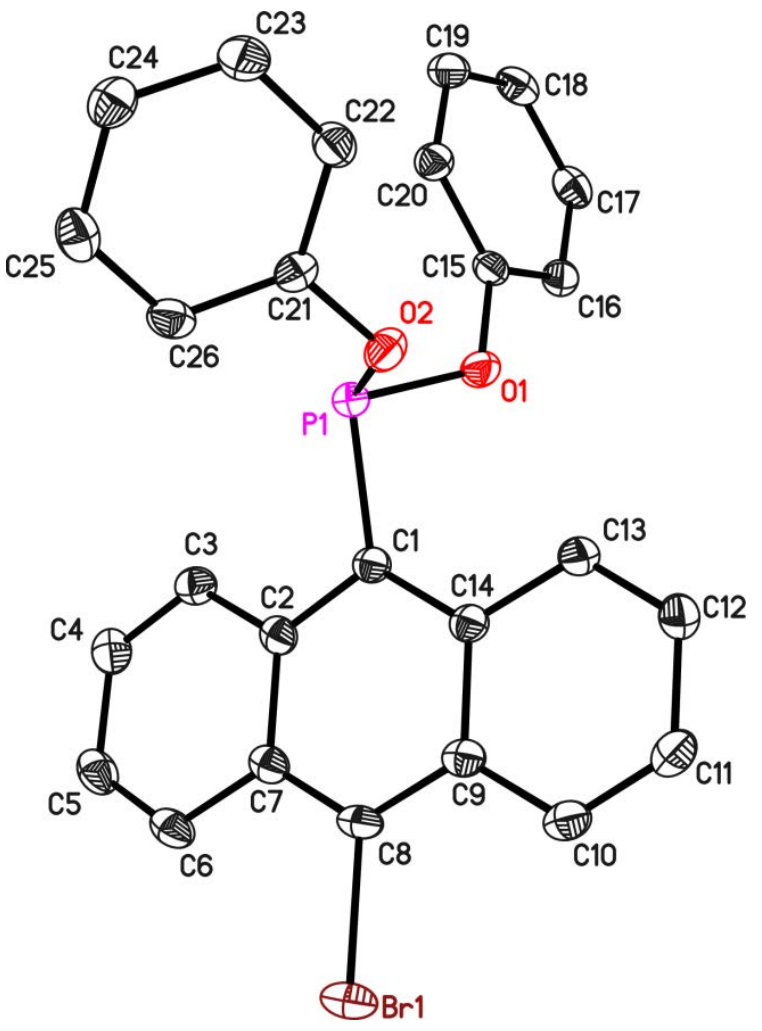

Figure 7-20. Asymmetric unit of $\mathrm{BrAnP}(\mathrm{OPh})_{2}$ (38). The anisotropic displacement parameters are depicted at the $50 \%$ probability level. The hydrogen atoms are omitted for clarity.

Table 7-208. Crystallographic data for $\operatorname{BrAnP}(\mathrm{OPh})_{2}$ (38).

\begin{tabular}{ll|ll}
\hline Identification code & $\mathrm{NFO20}$ & & \\
Empirical formula & $\mathrm{C}_{26} \mathrm{H}_{18} \mathrm{BrO}_{2} \mathrm{P}$ & $\rho_{\text {calc }}\left[\mathrm{Mg} / \mathrm{m}^{3}\right]$ & 1.574 \\
Formula weight $[\mathrm{g} / \mathrm{mol}]$ & 473.28 & $\mu\left[\mathrm{mm}^{-1}\right]$ & 2.160 \\
Temperature $[\mathrm{K}]$ & $100(2)$ & $\mathrm{F}(000)$ & 960 \\
Wavelength $[\AA]$ & 0.71073 & $\Theta$ range $\left[^{\circ}\right]$ & 1.423 to 26.718 \\
Crystal system & Monoclinic & Reflections collected & 37665 \\
Space group & $P 2_{1} / c$ & Independent reflections & 4216 \\
$a[\AA]$ & $8.0114(8)$ & Completeness to $\Theta_{\text {max }}$ & $99.9 \%$ \\
$b[\AA]$ & $28.629(3)$ & Absorption correction & Empirical \\
$c[\AA]$ & $9.4547(9)$ & Max. / min. transmission & $0.3766 / 0.4299$ \\
$\alpha\left[^{\circ}\right]$ & 90 & Restraints / parameters & $0 / 271$ \\
$\beta\left[^{\circ}\right]$ & $112.9230(10)$ & GoF & 1.029 \\
$\gamma\left[^{\circ}\right]$ & 90 & $R 1 / w R 2(\mathrm{I}>2 \sigma(\mathrm{I}))$ & $0.0278 / 0.0676$ \\
$\left.V^{\circ}\right)$ & $R 1 / w R 2$ (all data) & $0.0345 / 0.0706$ \\
$Z$ & $1997.3(3)$ & Diff. peak and hole $\left[\mathrm{e} \AA^{-3}\right]$ & 0.407 and -0.416 \\
\hline
\end{tabular}




\subsubsection{1 (BrAn) ${ }_{2} \mathrm{POPh}(39)$}

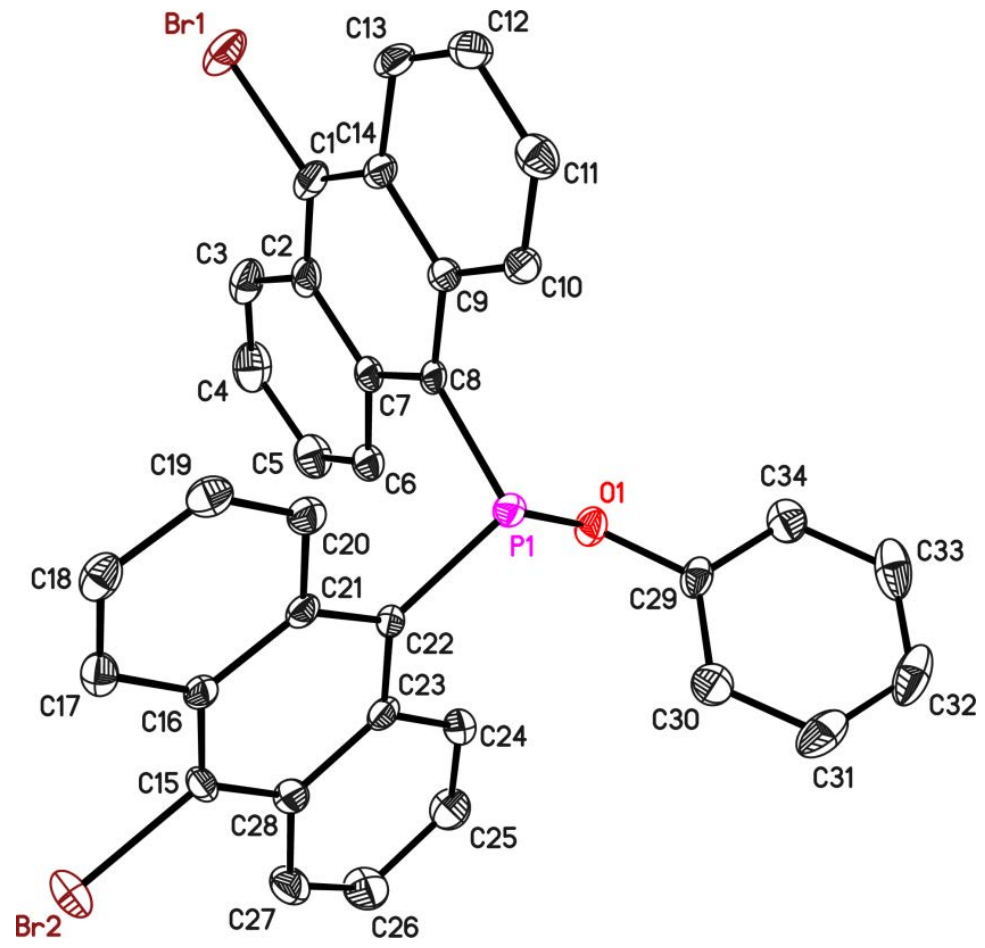

Figure 7-21. Asymmetric unit of ( $\mathrm{BrAn})_{2} \mathrm{POPh}(39)$. The anisotropic displacement parameters are depicted at the $50 \%$ probability level. The hydrogen atoms are omitted for clarity.

Table 7-21. Crystallographic data for (BrAn) 2 POPh (39).

\begin{tabular}{ll|ll}
\hline Identification code & $\mathrm{NFO21}$ & & \\
Empirical formula & $\mathrm{C}_{34} \mathrm{H}_{21} \mathrm{Br}_{2} \mathrm{OP}$ & $\rho_{\text {calc }}\left[\mathrm{Mg} / \mathrm{m}^{3}\right]$ & 1.623 \\
Formula weight $[\mathrm{g} / \mathrm{mol}]$ & 636.30 & $\mu\left[\mathrm{mm}^{-1}\right]$ & 3.202 \\
Temperature $[\mathrm{K}]$ & $100(2)$ & $\mathrm{F}(000)$ & 636 \\
Wavelength $[\AA]$ & 0.71073 & $\Theta$ range $\left[^{\circ}\right]$ & 1.701 to 25.348 \\
Crystal system & Triclinic & Reflections collected & 37812 \\
Space group & $\mathrm{P} \overline{1}$ & Independent reflections & 4746 \\
$a[\AA]$ & $10.4648(7)$ & Completeness to $\Theta_{\text {max }}$ & $99.4 \%$ \\
$b[\AA]$ & $10.5520(7)$ & Absorption correction & Empirical \\
$c[\AA]$ & $13.0065(8)$ & Max. / min. transmission & $0.3752 / 0.4299$ \\
$\alpha\left[^{\circ}\right]$ & $77.4050(10)$ & Restraints / parameters & $0 / 343$ \\
$\beta\left[^{\circ}\right]$ & $69.0580(10)$ & GoF & 1.042 \\
$\gamma\left[^{\circ}\right]$ & $80.1050(10)$ & $R 1 / w R 2$ (I>2 $\sigma(I))$ & $0.0312 / 0.0752$ \\
Volume $\left[\AA^{3}\right]$ & $1302.12(15)$ & $R 1 / w R 2$ (all data) & $0.0388 / 0.0790$ \\
$Z$ & 2 & Diff. peak and hole $\left[\mathrm{e} \AA^{-3}\right]$ & 0.939 and -0.568 \\
\hline
\end{tabular}




\subsubsection{BrAnPS $(\mathrm{OPh})_{2}(40)$}

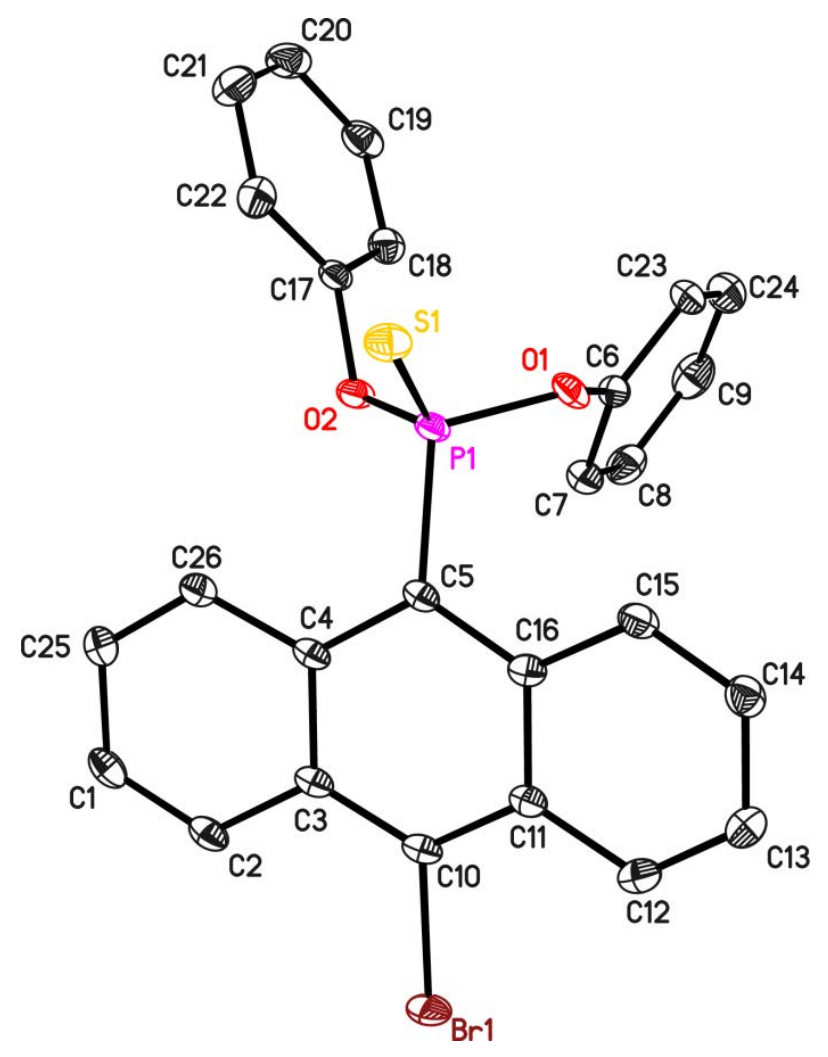

Figure 7-22. Asymmetric unit of BrAnPS(OPh) 2 (40). The anisotropic displacement parameters are depicted at the $50 \%$ probability level. The hydrogen atoms are omitted for clarity.

Table 7-22. Crystallographic data for $\operatorname{BrAnPS}(\mathrm{OPh})_{2}(40)$.

\begin{tabular}{|c|c|c|c|}
\hline Identification code & NF022 & & \\
\hline Empirical formula & $\mathrm{C}_{26} \mathrm{H}_{18} \mathrm{BrO}_{2} \mathrm{PS}$ & $\rho_{\text {calc }}\left[\mathrm{Mg} / \mathrm{m}^{3}\right]$ & 1.596 \\
\hline Formula weight $[\mathrm{g} / \mathrm{mol}]$ & 505.34 & $\mu\left[\mathrm{mm}^{-1}\right]$ & 2.153 \\
\hline Temperature $[\mathrm{K}]$ & $100(2)$ & $F(000)$ & 512 \\
\hline Wavelength $[\AA]]$ & 0.71073 & $\Theta$ range $\left[{ }^{\circ}\right]$ & 1.654 to 26.431 \\
\hline Crystal system & Triclinic & Reflections collected & 24810 \\
\hline Space group & $\mathrm{P} \overline{1}$ & Independent reflections & 4301 \\
\hline$a[\AA]$ & $9.2913(16)$ & Completeness to $\Theta_{\max }$ & $100.0 \%$ \\
\hline$b[\AA]$ & $9.6154(17)$ & Absorption correction & Empirical \\
\hline$c[\AA]$ & $13.591(2)$ & Max. / min. transmission & 0.6439 / 0.7454 \\
\hline$\alpha\left[^{\circ}\right]$ & $110.525(3)$ & Restraints / parameters & $0 / 280$ \\
\hline$\beta\left[^{\circ}\right]$ & $97.140(3)$ & GoF & 1.065 \\
\hline$\gamma\left[{ }^{\circ}\right]$ & $107.080(2)$ & $R 1 / w R 2(I>2 \sigma(I))$ & $0.0332 / 0.0988$ \\
\hline Volume $\left[\AA^{3}\right]$ & $1051.4(3)$ & $R 1 / w R 2$ (all data) & $0.0357 / 0.1018$ \\
\hline Z & 2 & Diff. peak and hole $\left[\mathrm{e}^{-3}\right]$ & 1.268 and -0.742 \\
\hline
\end{tabular}




\subsubsection{3 $\mathrm{BrAnPCl}_{2}(41)$}

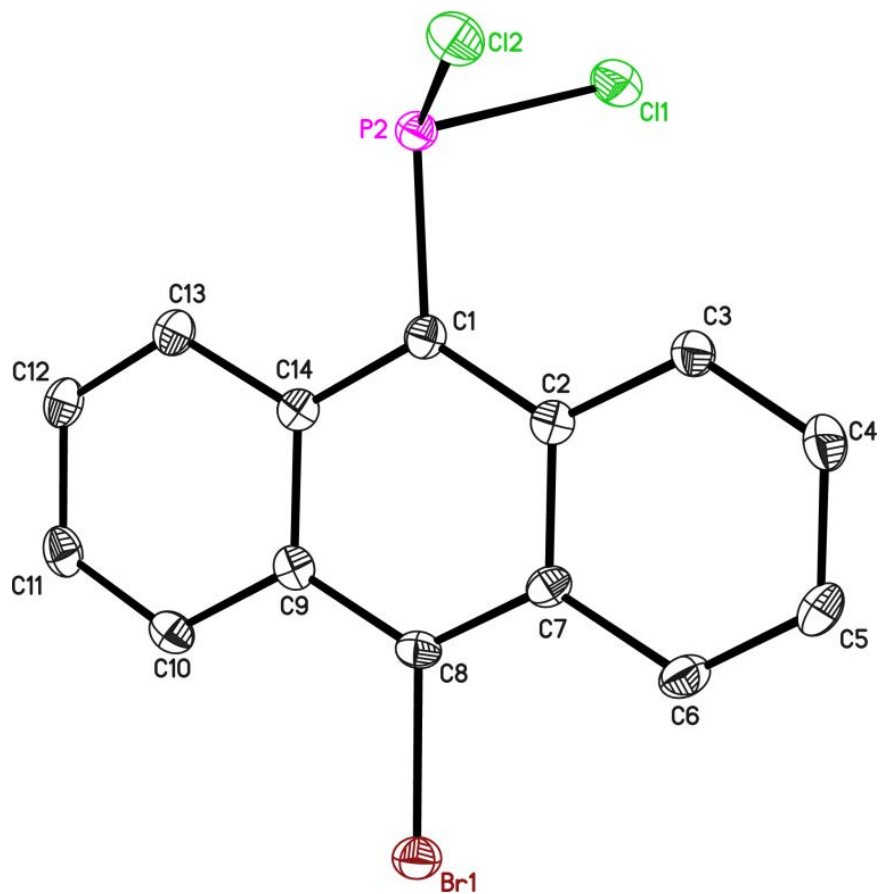

Figure 7-23. Asymmetric unit of $\mathrm{BrAnPCl}_{2}$ (40). The anisotropic displacement parameters are depicted at the $50 \%$ probability level. The hydrogen atoms are omitted for clarity.

Table 7-23. Crystallographic data for $\mathrm{BrAnPCl}_{2}$ (40).

\begin{tabular}{ll|ll}
\hline Identification code & $\mathrm{NF023}$ & & \\
Empirical formula & $\mathrm{C}_{14} \mathrm{H}_{8} \mathrm{BrCl}_{2} \mathrm{P}$ & $\rho_{\text {calc }}\left[\mathrm{Mg} / \mathrm{m}^{3}\right]$ & 1.885 \\
Formula weight $[\mathrm{g} / \mathrm{mol}]$ & 357.98 & $\mu\left[\mathrm{mm}^{-1}\right]$ & 3.784 \\
Temperature $[\mathrm{K}]$ & $100(2)$ & $\mathrm{F}(000)$ & 352 \\
Wavelength $[\AA]$ & 0.71073 & $\Theta$ range $\left[^{\circ}\right]$ & 2.33 to 25.68 \\
Crystal system & Triclinic & Reflections collected & 7062 \\
Space group & $\mathrm{P} \overline{1}$ & Independent reflections & 2389 \\
$a[\AA]$ & $6.8803(8)$ & Completeness to $\Theta_{\text {max }}$ & $99.9 \%$ \\
$b[\AA]]$ & $8.9930(11)$ & Absorption correction & Empirical \\
$c[\AA]$ & $10.5031(13)$ & Max. / min. transmission & $0.5614 / 0.7453$ \\
$\alpha\left[^{\circ}\right]$ & $96.069(2)$ & Restraints / parameters & $0 / 163$ \\
$\beta\left[^{\circ}\right]$ & $91.138(2)$ & GoF & 1.073 \\
$\gamma\left[^{\circ}\right]$ & $102.359(2)$ & $R 1 / w R 2(I>2 \sigma(I))$ & $0.0252 / 0.0657$ \\
Volume $\left[\AA^{3}\right]$ & $630.65(13)$ & $R 1 / w R 2$ (all data) & $0.0282 / 0.0671$ \\
$Z$ & 2 & Diff. peak and hole $\left[\mathrm{e} \AA^{-3}\right]$ & 0.658 and -0.504 \\
\hline
\end{tabular}




\subsubsection{4 $\mathrm{MeANPCl}_{2}(42)$}

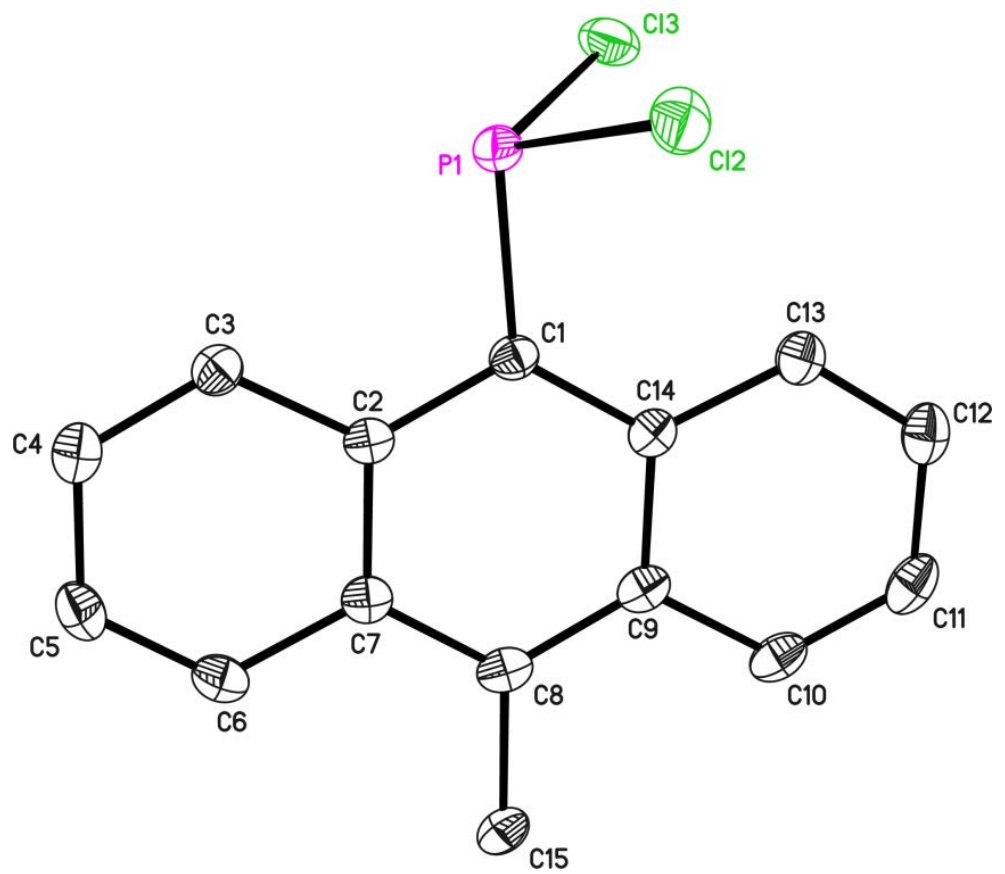

Figure 7-24. Asymmetric unit of $\mathrm{MeAnPCl}_{2}$ (42). The anisotropic displacement parameters are depicted at the $50 \%$ probability level. The hydrogen atoms are omitted for clarity.

Table 7-24. Crystallographic data for $\mathrm{MeAnPCl}_{2}$ (42).

\begin{tabular}{ll|ll}
\hline Identification code & $\mathrm{NFO24}$ & & \\
Empirical formula & $\mathrm{C}_{15} \mathrm{H}_{11} \mathrm{Cl}_{2} \mathrm{P}$ & $\rho_{\text {calc }}\left[\mathrm{Mg} / \mathrm{m}^{3}\right]$ & 1.522 \\
Formula weight $[\mathrm{g} / \mathrm{mol}]$ & 293.11 & $\mu\left[\mathrm{mm}^{-1}\right]$ & 0.608 \\
Temperature $[\mathrm{K}]$ & $100(2)$ & $\mathrm{F}(000)$ & 300 \\
Wavelength $[\AA]$ & 0.71073 & $\Theta$ range $\left[^{\circ}\right]$ & 2.429 to 26.747 \\
Crystal system & Triclinic & Reflections collected & 15817 \\
Space group & $\mathrm{P} \overline{1}$ & Independent reflections & 2701 \\
$a[\AA]$ & $7.6559(3)$ & Completeness to $\Theta_{\text {max }}$ & $99.5 \%$ \\
$b[\AA]$ & $9.4276(4)$ & Absorption correction & Empirical \\
$c[\AA]$ & $10.0768(4)$ & Max. / min. transmission & $0.7063 / 0.7454$ \\
$\alpha\left[^{\circ}\right]$ & $63.9430(10)$ & Restraints / parameters & $0 / 164$ \\
$\beta\left[^{\circ}\right]$ & $81.3860(10)$ & GoF & 1.058 \\
$\gamma\left[^{\circ}\right]$ & $78.9850(10)$ & $R 1 / w R 2(I>2 \sigma(I))$ & $0.0261 / 0.0697$ \\
Volume $\left[\AA^{3}\right]$ & $639.56(5)$ & $R 1 / w R 2$ (all data) & $0.0293 / 0.0719$ \\
$Z$ & 2 & Diff. peak and hole $\left[\mathrm{e} \AA^{-3}\right]$ & 0.373 and -0.233 \\
\hline
\end{tabular}




\subsubsection{MeAnPHOOH (43a)}

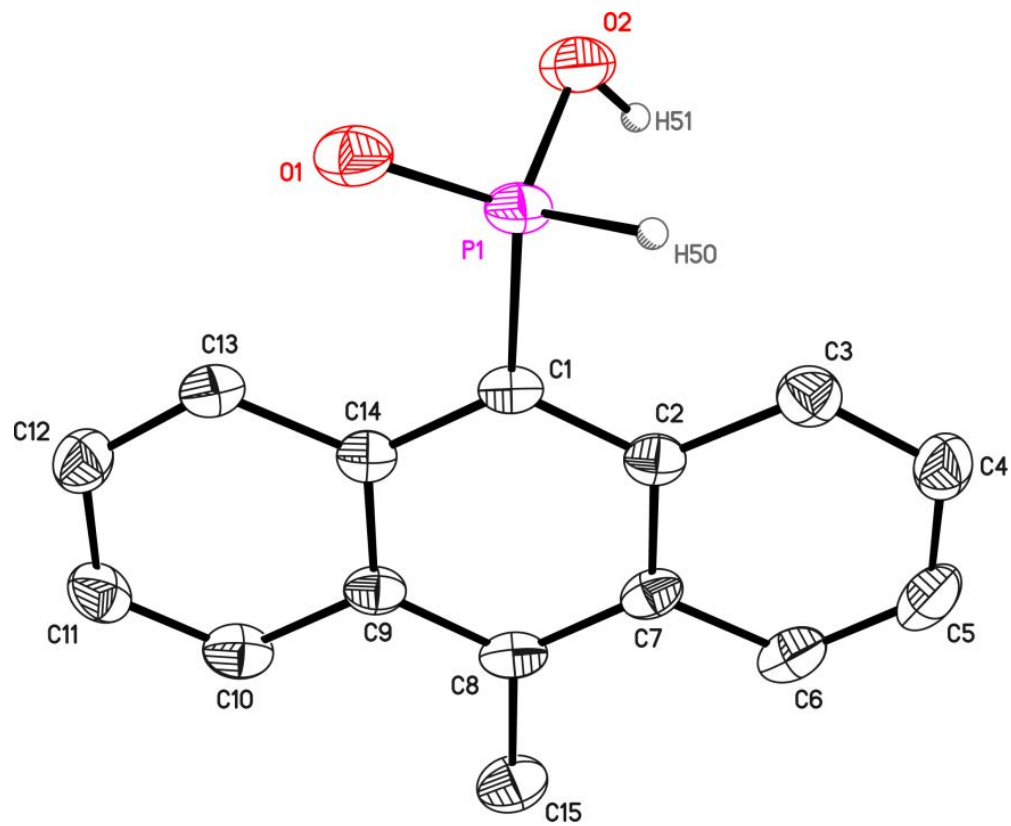

Figure 7-25. Asymmetric unit of $\mathrm{MeAnPHOOH} \mathrm{(43a).} \mathrm{The} \mathrm{anisotropic} \mathrm{displacement} \mathrm{parameters} \mathrm{are}$ depicted at the $50 \%$ probability level. The hydrogen atoms are omitted for clarity.

The positions of the hydrogen atoms $\mathrm{H} 50$ at the phosphorus atom and $\mathrm{H} 51$ at the oxygen atom were refined freely.

Table 7-25. Crystallographic data for MeAnPHOOH (43a).

\begin{tabular}{|c|c|c|c|}
\hline Identification code & NF025 & & \\
\hline Empirical formula & $\mathrm{C}_{15} \mathrm{H}_{13} \mathrm{O}_{2} \mathrm{P}$ & $\rho_{\text {calc }}\left[\mathrm{Mg} / \mathrm{m}^{3}\right]$ & 1.451 \\
\hline Formula weight $[\mathrm{g} / \mathrm{mol}]$ & 256.22 & $\mu\left[\mathrm{mm}^{-1}\right]$ & 0.224 \\
\hline Temperature [K] & $100(2)$ & $F(000)$ & 536 \\
\hline Wavelength $[\AA]$ & 0.71073 & $\Theta$ range $\left[{ }^{\circ}\right]$ & 2.343 to 25.334 \\
\hline Crystal system & Monoclinic & Reflections collected & 10800 \\
\hline Space group & $P 2_{1} / c$ & Independent reflections & 2135 \\
\hline$a[\AA ̊]$ & $4.8443(4)$ & Completeness to $\Theta_{\max }$ & $99.9 \%$ \\
\hline$b[\AA]$ & $17.3816(14)$ & Absorption correction & Empirical \\
\hline$c[\AA]$ & 13.9389(12) & Max. / min. transmission & $0.6248 / 0.7452$ \\
\hline$\alpha\left[^{\circ}\right]$ & 90 & Restraints / parameters & 49 / 172 \\
\hline$\beta\left[^{\circ}\right]$ & $92.403(2)$ & GoF & 0.980 \\
\hline$Y\left[^{\circ}\right]$ & 90 & $R 1 / w R 2(1>2 \sigma(I))$ & 0.0413 / 0.0991 \\
\hline Volume $\left[\AA^{3}\right]$ & $1172.65(17)$ & $R 1 / w R 2$ (all data) & $0.0659 / 0.1058$ \\
\hline z & 4 & Diff. peak and hole $\left[\mathrm{e} \AA^{-3}\right]$ & 0.324 and -0.351 \\
\hline
\end{tabular}




\subsubsection{6 $\mathrm{MeAnP}\left(\mathrm{CH}_{2} \mathrm{Ph}\right)_{2}(49)$}

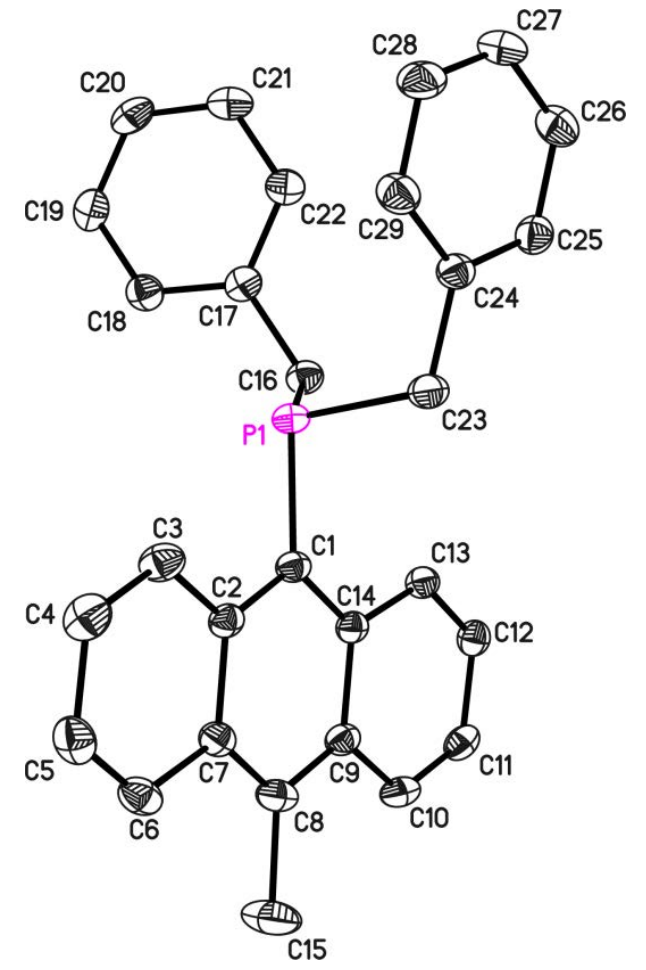

Figure 7-26. Asymmetric unit of $\mathrm{MeAnP}\left(\mathrm{CH}_{2} \mathrm{Ph}\right)_{2}$ (49). The anisotropic displacement parameters are depicted at the $50 \%$ probability level. The hydrogen atoms are omitted for clarity.

Table 7-26. Crystallographic data for $\mathrm{MeAnP}\left(\mathrm{CH}_{2} \mathrm{Ph}\right)_{2}$ (49).

\begin{tabular}{ll|ll}
\hline Identification code & $\mathrm{NFO26}$ & & \\
Empirical formula & $\mathrm{C}_{29} \mathrm{H}_{25} \mathrm{P}$ & $\rho_{\text {calc }}\left[\mathrm{Mg} / \mathrm{m}^{3}\right]$ & 1.269 \\
Formula weight $[\mathrm{g} / \mathrm{mol}]$ & 404.46 & $\mu\left[\mathrm{mm}^{-1}\right]$ & 0.143 \\
Temperature $[\mathrm{K}]$ & $100(2)$ & $\mathrm{F}(000)$ & 1712 \\
Wavelength $[\AA]$ & 0.71073 & $\Theta$ range $\left[^{\circ}\right]$ & 1.490 to 26.372 \\
Crystal system & Monoclinic & Reflections collected & 54822 \\
Space group & $C 2 / c$ & Independent reflections & 4330 \\
$a[\AA ̊]$ & $26.705(2)$ & Completeness to $\Theta_{\max }$ & $99.8 \%$ \\
$b[\AA]]$ & $5.8005(5)$ & Absorption correction & Empirical \\
$c[\AA ̊]$ & $28.307(3)$ & Max. / min. transmission & $0.7026 / 0.7454$ \\
$\alpha\left[^{\circ}\right]$ & 90 & Restraints / parameters & $0 / 273$ \\
$\beta\left[^{\circ}\right]$ & $105.0130(10)$ & GoF & 1.081 \\
$\gamma\left[^{\circ}\right]$ & 90 & $R 1 / w R 2(\mathrm{I}>2 \sigma(\mathrm{I}))$ & $0.0391 / 0.1067$ \\
Volume $\left[\AA^{3}\right]$ & $4235.2(6)$ & $R 1 / w R 2$ (all data) & $0.0423 / 0.1089$ \\
$Z$ & 8 & Diff. peak and hole $\left[\mathrm{e} \AA^{-3}\right]$ & 0.462 and -0.313 \\
\hline
\end{tabular}




\subsubsection{7 [HAnPPh(S)AuCl] (50)}

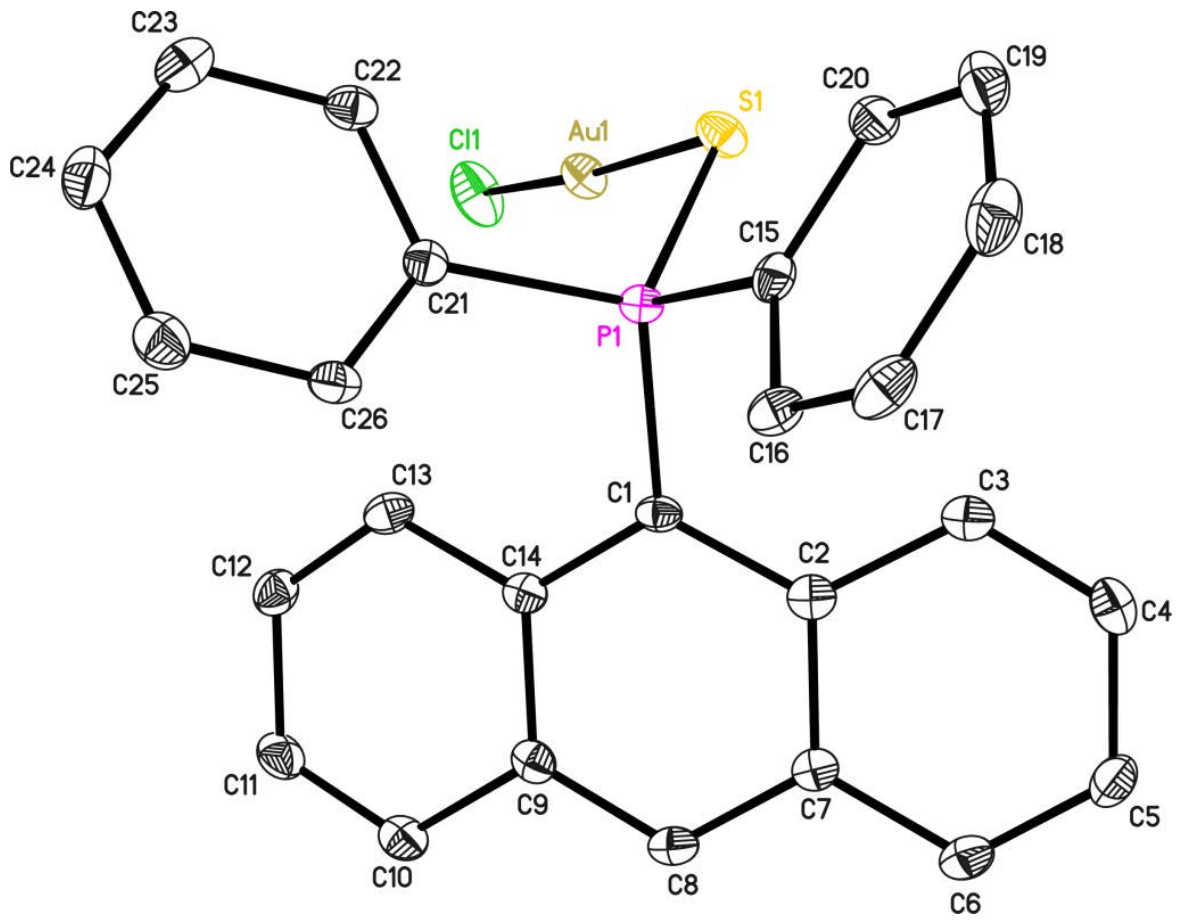

Figure 7-27. Asymmetric unit of $\left[\mathrm{HAnPPh}_{2}(\mathrm{~S}) \mathrm{AuCL}\right](\mathbf{5 0})$. The anisotropic displacement parameters are depicted at the $50 \%$ probability level. The hydrogen atoms are omitted for clarity.

Table 7-27. Crystallographic data for $\left[\mathrm{HAnPPh}_{2}(\mathrm{~S}) \mathrm{AuCL}\right](\mathbf{5 0})$.

\begin{tabular}{ll|ll}
\hline Identification code & NF027 & & \\
Empirical formula & $\mathrm{C}_{26} \mathrm{H}_{19} \mathrm{AuClPS}$ & $\rho_{\text {calc }}\left[\mathrm{Mg} / \mathrm{m}^{3}\right]$ & 1.932 \\
Formula weight $[\mathrm{g} / \mathrm{mol}]$ & 626.86 & $\mu\left[\mathrm{mm}^{-1}\right]$ & 7.134 \\
Temperature $[\mathrm{K}]$ & $100(2)$ & $\mathrm{F}(000)$ & 1208 \\
Wavelength $[\AA]$ & 0.71073 & $\Theta$ range $\left[^{\circ}\right]$ & 2.098 to 26.370 \\
Crystal system & Monoclinic & Reflections collected & 28046 \\
Space group & $P 2_{1} / n$ & Independent reflections & 4399 \\
$a[\AA]$ & $11.0248(14)$ & Completeness to $\Theta_{\text {max }}$ & $100.0 \%$ \\
$b[\AA]$ & $16.025(2)$ & Absorption correction & Empirical \\
$c[\AA]$ & $12.2118(15)$ & Max. / min. transmission & $0.6695 / 0.7459$ \\
$\alpha\left[^{\circ}\right]$ & 90 & Restraints / parameters & $82 / 271$ \\
$\beta\left[^{\circ}\right]$ & $92.761(2)$ & GoF & 1.035 \\
$\gamma\left[^{\circ}\right]$ & 90 & $R 1 / w R 2(I>2 \sigma(I))$ & $0.0179 / 0.0432$ \\
Volume $\left[\AA^{3}\right]$ & $2154.9(5)$ & $R 1 / w R 2$ (all data) & $0.0194 / 0.0438$ \\
$Z$ & 4 & Diff. peak and hole $\left[\mathrm{e} \AA^{-3}\right]$ & 0.600 and -0.461 \\
\hline
\end{tabular}




\subsubsection{8 [HAnP'Pr $\left.{ }^{i}(\mathrm{~S}) \mathrm{AuCl}\right](51)$}

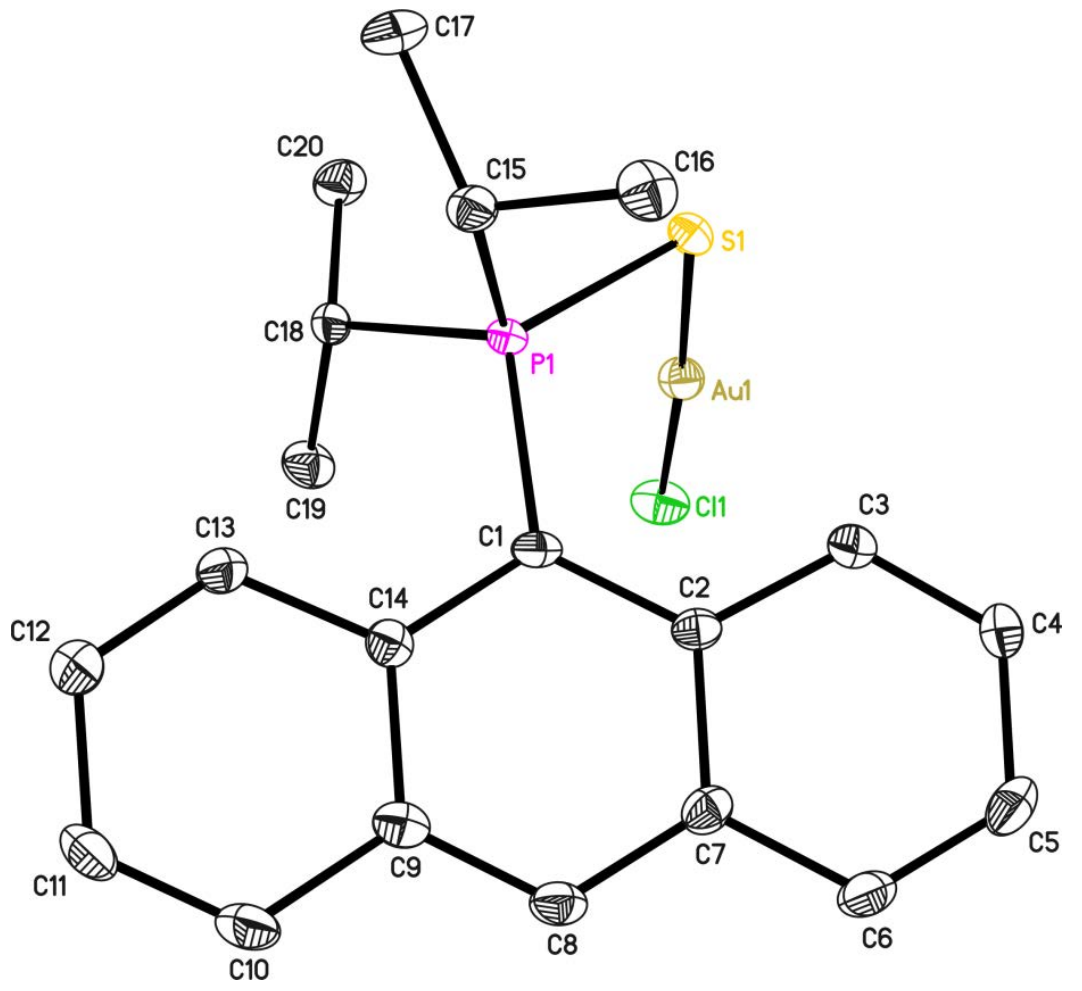

Figure 7-28. Asymmetric unit of $\left[\mathrm{HAnP}^{i} \mathrm{Pr}_{2}(\mathrm{~S}) \mathrm{AuCl}\right]$ (50). The anisotropic displacement parameters are depicted at the $50 \%$ probability level. The hydrogen atoms are omitted for clarity.

Table 7-28. Crystallographic data for $\left[\mathrm{HAnP}^{i} \mathrm{Pr}_{2}(\mathrm{~S}) \mathrm{AuCl}\right]$ (50).

\begin{tabular}{ll|ll}
\hline Identification code & $\mathrm{NFO28}$ & & \\
Empirical formula & $\mathrm{C}_{20} \mathrm{H}_{23} \mathrm{AuClPS}$ & $\rho_{\text {calc }}\left[\mathrm{Mg} / \mathrm{m}^{3}\right]$ & 1.926 \\
Formula weight $[\mathrm{g} / \mathrm{mol}]$ & 558.83 & $\mu\left[\mathrm{mm}^{-1}\right]$ & 7.962 \\
Temperature $[\mathrm{K}]$ & $99(2)$ & $\mathrm{F}(000)$ & 4320 \\
Wavelength $[\AA]$ & 0.71073 & $\Theta$ range $\left[^{\circ}\right]$ & 2.021 to 26.372 \\
Crystal system & Orthorhombic & Reflections collected & 43905 \\
Space group & $F d d 2$ & Independent reflections & 3949 \\
$a[\AA]$ & $16.906(3)$ & Completeness to $\Theta_{\text {max }}$ & $99.9 \%$ \\
$b[\AA]$ & $40.306(7)$ & Absorption correction & Empirical \\
$c[\AA]]$ & $11.3139(18)$ & Max. / min. transmission & $0.3070 / 0.4318$ \\
$\alpha\left[^{\circ}\right]$ & 90 & Restraints / parameters & $65 / 221$ \\
$\beta\left[^{\circ}\right]$ & 90 & GoF & 0.997 \\
$\nu\left[^{\circ}\right]$ & 90 & $R 1 / w R 2(\mathrm{I}>2 \sigma(\mathrm{I}))$ & $0.0120 / 0.0309$ \\
Volume $\left[\AA^{3}\right]$ & $7709(2)$ & $R 1 / w R 2$ (all data) & $0.0122 / 0.0310$ \\
$Z$ & 16 & Diff. peak and hole $\left[\mathrm{e} \AA^{-3}\right]$ & 0.759 and -0.280 \\
\hline
\end{tabular}




\subsubsection{9 [MeAnPPh 2 (S)AuCl] (52)}

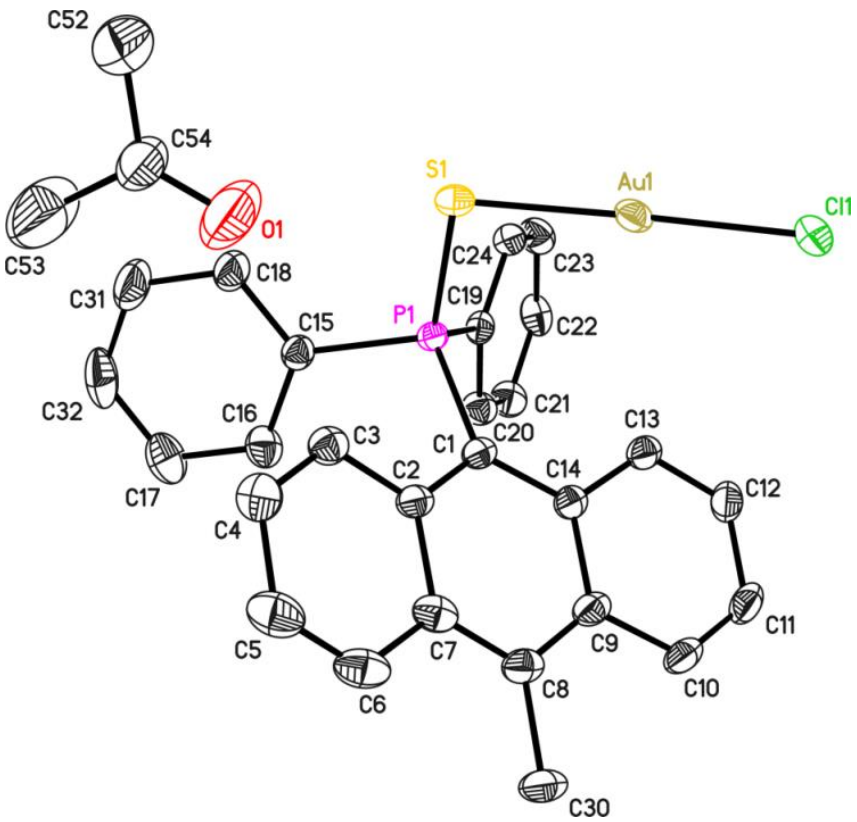

Figure 7-29. Asymmetric unit of [MeAnPPh2(S)AuCl] (52). The anisotropic displacement parameters are depicted at the $50 \%$ probability level. The hydrogen atoms are omitted for clarity.

The high residual density is caused by absorption due to the heavy gold ion in the structure. The high residual density peak is located in direct proximity to the gold atom.

Table 7-29. Crystallographic data for [MeAnPPh2(S)AuCl] (52).

\begin{tabular}{|c|c|c|c|}
\hline Identification code & NF029 & & \\
\hline Empirical formula & $\mathrm{C}_{30} \mathrm{H}_{27} \mathrm{AuClOPS}$ & $\rho_{\text {calc }}\left[\mathrm{Mg} / \mathrm{m}^{3}\right]$ & 1.726 \\
\hline Formula weight $[\mathrm{g} / \mathrm{mol}]$ & 698.96 & $\mu\left[\mathrm{mm}^{-1}\right]$ & 5.727 \\
\hline Temperature [K] & $100(2)$ & $F(000)$ & 2736 \\
\hline Wavelength $[\AA ̊]$ & 0.71073 & $\Theta$ range $\left[{ }^{\circ}\right]$ & 1.807 to 28.741 \\
\hline Crystal system & Orthorhombic & Reflections collected & 163051 \\
\hline Space group & Pbca & Independent reflections & 6965 \\
\hline$a[\AA ̊]$ & $16.0560(14)$ & Completeness to $\Theta_{\max }$ & $100.0 \%$ \\
\hline$b[\AA]$ & $14.8685(13)$ & Absorption correction & Empirical \\
\hline$c[\AA ̊]$ & $22.535(2)$ & Max. / min. transmission & $0.6795 / 0.7458$ \\
\hline$\alpha\left[^{\circ}\right]$ & 90 & Restraints / parameters & $0 / 319$ \\
\hline$\beta\left[^{\circ}\right]$ & 90 & GoF & 1.140 \\
\hline$\gamma\left[{ }^{\circ}\right]$ & 90 & $R 1 / w R 2(I>2 \sigma(I))$ & $0.0377 / 0.0822$ \\
\hline Volume $\left[\AA^{3}\right]$ & $5379.8(8)$ & $R 1 / w R 2$ (all data) & $0.0557 / 0.0892$ \\
\hline Z & 8 & Diff. peak and hole $\left[\mathrm{e} \AA^{-3}\right]$ & 3.656 and -0.978 \\
\hline
\end{tabular}




\subsubsection{0 [ClAu(Se)(Et $\left.\left.{ }_{2} \mathrm{~N}\right)_{2} \mathrm{PAnP}\left(\mathrm{NEt}_{2}\right)_{2}(\mathrm{Se}) \mathrm{AuCl}\right](53)$}

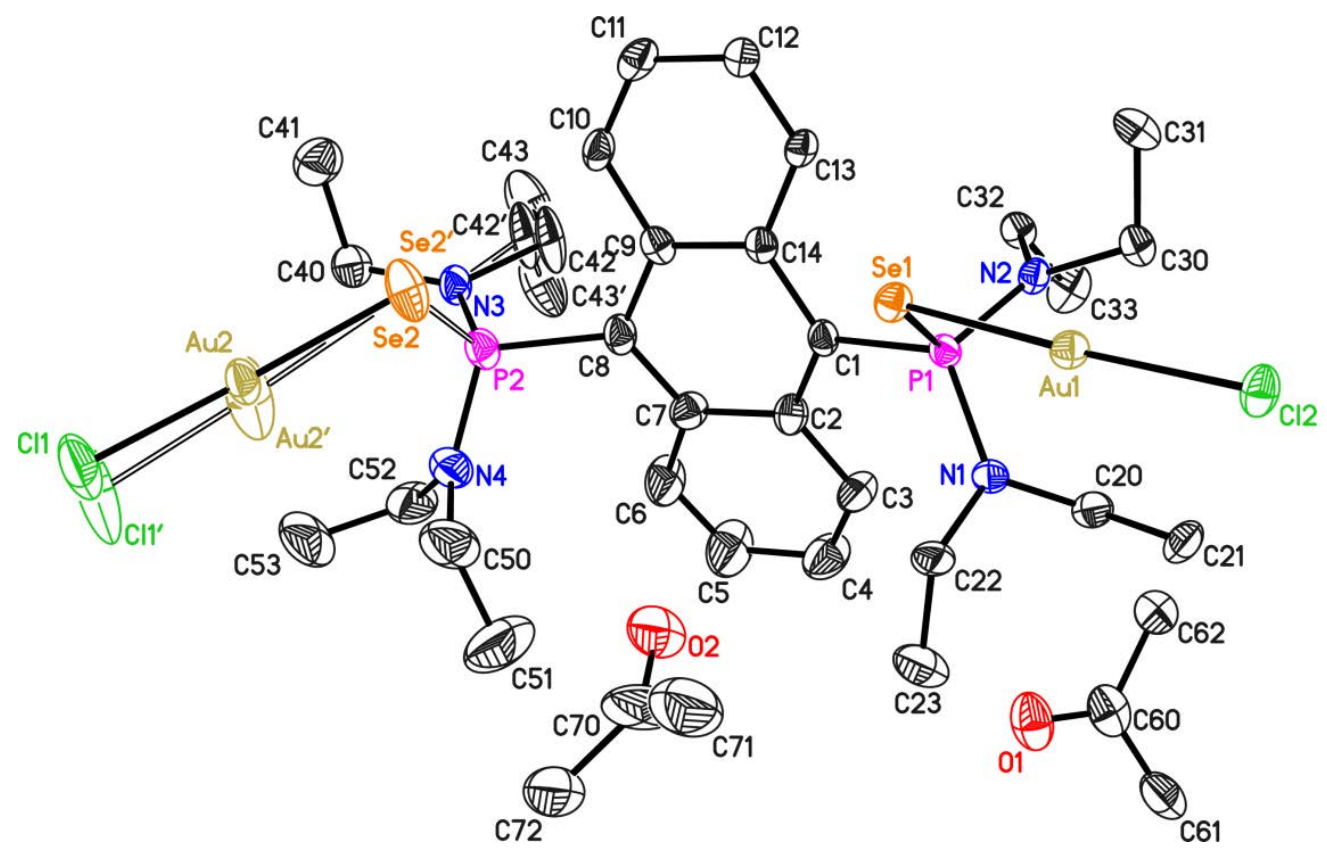

Figure 7-30. Asymmetric unit of [ClAu(Se)( $\left.\left.\mathrm{Et}_{2} \mathrm{~N}\right)_{2} \mathrm{PAnP}\left(\mathrm{NEt}_{2}\right)_{2}(\mathrm{Se}) \mathrm{AuCl}\right]$ (53). The anisotropic displacement parameters are depicted at the $50 \%$ probability level. The hydrogen atoms are omitted for clarity.

One linear Se-AuCl fragment is disordered with an occupation of 81:21.

Table 7-30. Crystallographic data for $\left[\mathrm{ClAu}(\mathrm{Se})\left(\mathrm{Et}_{2} \mathrm{~N}\right)_{2} \mathrm{PAnP}\left(\mathrm{NEt}_{2}\right)_{2}(\mathrm{Se}) \mathrm{AuCl}\right](53)$.

\begin{tabular}{ll|ll}
\hline Identification code & $\mathrm{NFO30}$ & \\
Empirical formula & $\mathrm{C}_{33.58} \mathrm{H}_{54.17} \mathrm{Au}_{2} \mathrm{Cl}_{2} \mathrm{~N}_{4} \mathrm{O}_{1.19} \mathrm{P}_{2} \mathrm{Se}_{2}$ & $\rho_{\text {calc }}\left[\mathrm{Mg} / \mathrm{m}^{3}\right]$ & 1.910 \\
Formula weight $[\mathrm{g} / \mathrm{mol}]$ & 1217.81 & $\mu\left[\mathrm{mm}^{-1}\right]$ & 8.873 \\
Temperature $[\mathrm{K}]$ & $100(2)$ & $\mathrm{F}(000)$ & 2333 \\
Wavelength $[\AA]$ & 0.71073 & $\Theta$ range $\left[^{\circ}\right]$ & 1.736 to 25.557 \\
Crystal system & Monoclinic & Reflections collected & 73678 \\
Space group & $P 2_{1} / c$ & Independent reflections & 7590 \\
$a[\AA]$ & $23.539(3)$ & Completeness to $\Theta_{\text {max }}$ & $98.1 \%$ \\
$b[\AA]$ & Absorption correction & Empirical \\
$c[\AA]$ & $10.9023(14)$ & Max. / min. transmission & \\
$\alpha\left[^{\circ}\right]$ & $16.556(2)$ & Restraints / parameters & $41 / 494$ \\
$\beta\left[^{\circ}\right]$ & 90 & GoF & 1.130 \\
$\gamma\left[^{\circ}\right]$ & $94.733(2)$ & $R 1 / w R 2$ (I>2 $\sigma(I))$ & $0.0350 / 0.0645$ \\
$\left.V^{\circ}\right]$ & 90 & $R 1 / w R 2$ (all data) & $0.0517 / 0.0680$ \\
$Z$ & $4234.3(9)$ & Diff. peak and hole $\left[\mathrm{e} \AA^{-3}\right]$ & 1.128 and -0.973 \\
\hline
\end{tabular}




\subsubsection{1 [(MeAnP(NMe $\left.\left.)_{2}\right)_{2} \mathrm{CuBH}_{4}\right](54)$}

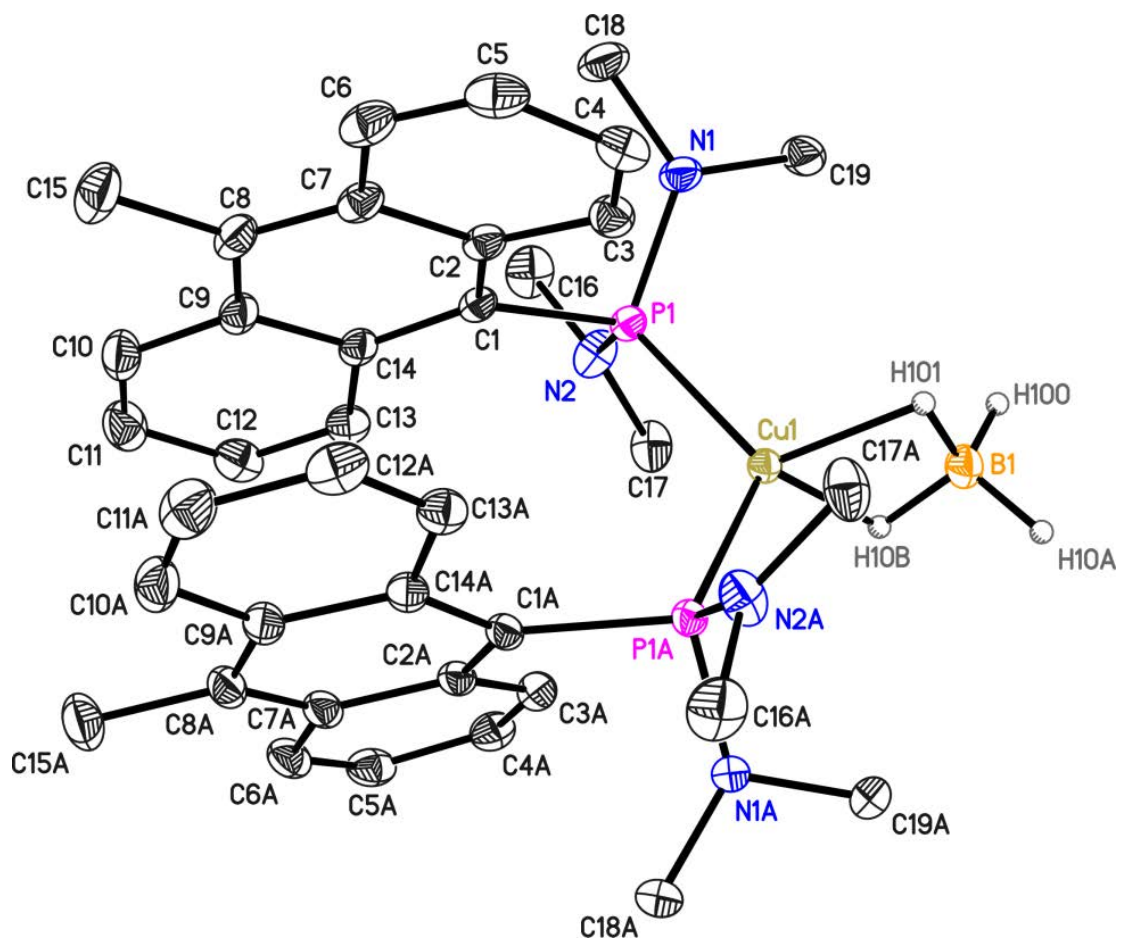

Figure 7-31. Structure of [( $\left.\left.\mathrm{MeAnP}\left(\mathrm{NMe}_{2}\right)_{2}\right)_{2} \mathrm{CuBH}_{4}\right]$ (54). The anisotropic displacement parameters are depicted at the $50 \%$ probability level. The hydrogen atoms are omitted for clarity. Only one half of the depicted dimer is contained in the asymmetric unit.

The hydrogen positions of $\mathrm{H} 100$ and $\mathrm{H} 101$ were refined freely.

Table 7-31. Crystallographic data for $\left[\left(\mathrm{MeAnP}\left(\mathrm{NMe}_{2}\right)_{2}\right)_{2} \mathrm{CuBH}_{4}\right](54)$.

\begin{tabular}{ll|ll}
\hline Identification code & $\mathrm{NFO31}$ & & \\
Empirical formula & $\mathrm{C}_{19} \mathrm{H}_{25} \mathrm{~B}_{0.50} \mathrm{Cu}_{0.50} \mathrm{~N}_{2} \mathrm{P}$ & $\rho_{\text {calc }}\left[\mathrm{Mg} / \mathrm{m}^{3}\right]$ & 1.315 \\
Formula weight $[\mathrm{g} / \mathrm{mol}]$ & 349.55 & $\mu\left[\mathrm{mm}^{-1}\right]$ & 0.743 \\
Temperature $[\mathrm{K}]$ & $100(2)$ & $\mathrm{F}(000)$ & 1480 \\
Wavelength $[\AA]$ & 0.71073 & $\Theta$ range $\left[^{\circ}\right]$ & 1.865 to 29.885 \\
Crystal system & Orthorhombic & Reflections collected & 31780 \\
Space group & $P b c n$ & Independent reflections & 4816 \\
$a[\AA ̊]$ & $15.1167(15)$ & Completeness to $\Theta_{\text {max }}$ & $99.9 \%$ \\
$b[\AA]$ & $15.7866(16)$ & Absorption correction & Empirical \\
$c[\AA]$ & $14.7923(15)$ & Max. / min. transmission & $0.6647 / 0.7460$ \\
$\alpha\left[^{\circ}\right]$ & 90 & Restraints / parameters & $0 / 222$ \\
$\beta\left[^{\circ}\right]$ & 90 & GoF & 1.029 \\
$\gamma\left[^{\circ}\right]$ & 90 & $R 1 / w R 2(\mathrm{I}>2 \sigma(\mathrm{I}))$ & $0.0284 / 0.0788$ \\
Volume $\left[\AA^{3}\right]$ & $3530.1(6)$ & $R 1 / w R 2$ (all data) & $0.0326 / 0.0821$ \\
$\mathrm{Z}$ & 8 & Diff. peak and hole [e $\left.\AA^{-3}\right]$ & 0.419 and -0.338 \\
\hline
\end{tabular}




\subsubsection{2 [HAnPPh $\left.{ }_{2} \mathrm{ZnBr}_{2}\right]_{2}(55)$}

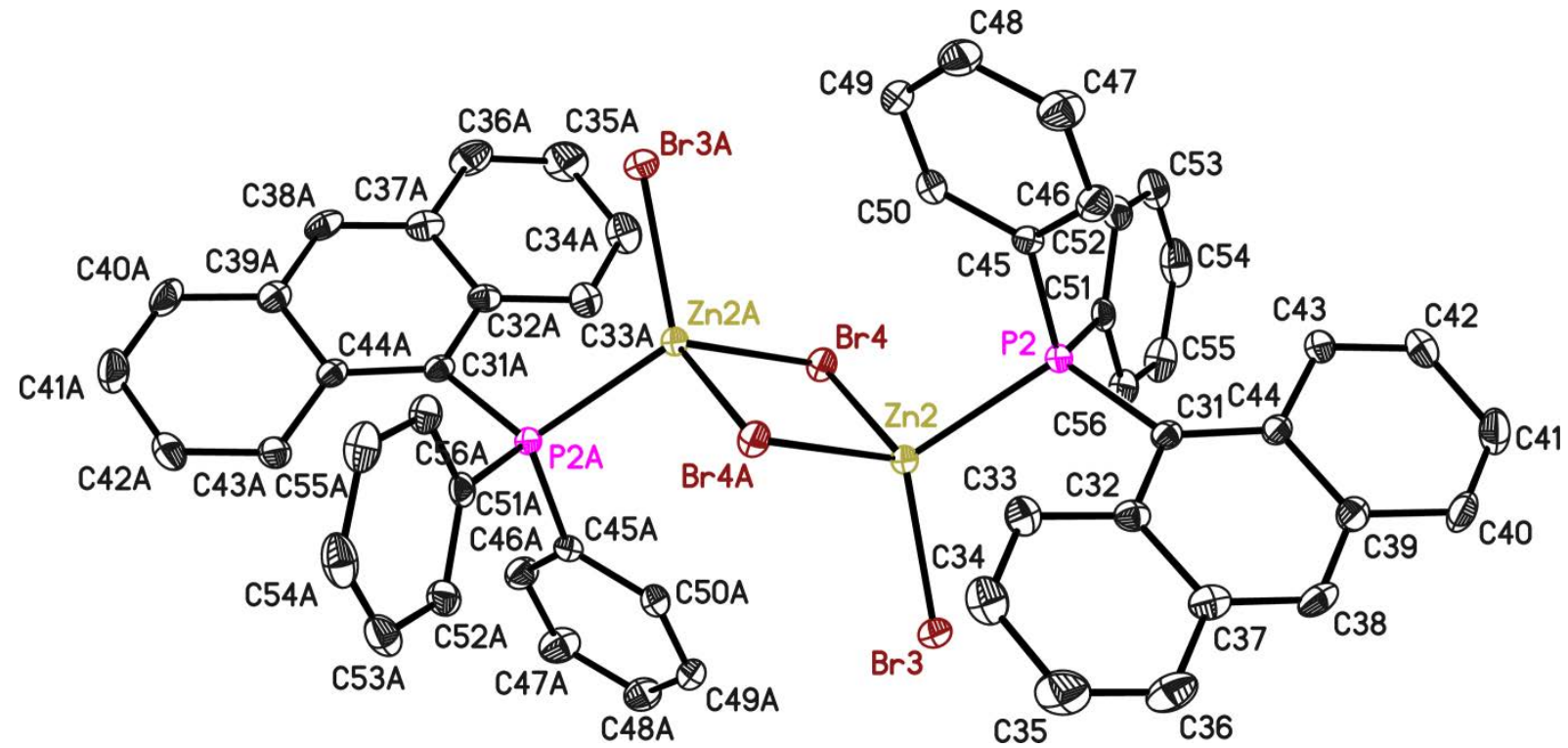

Figure 7-32. Structure of [ $\left.\mathrm{HAnPPh}_{2} \mathrm{ZnBr}_{2}\right]_{2}$ (55). The anisotropic displacement parameters are depicted at the $50 \%$ probability level. The hydrogen atoms are omitted for clarity. Only one half of the structure depicted in figure 7-32 is contained in the asymmetric unit. Two non-disordered molecules of DCM lattice solvent are omitted for clarity.

Table 7-328. Crystallographic data for $\left[\mathrm{HAnPPh}_{2} \mathrm{ZnBr}_{2}\right]_{2}$ (55).

\begin{tabular}{ll|ll}
\hline Identification code & $\mathrm{NFO32}$ & & \\
Empirical formula & $\mathrm{C}_{27} \mathrm{H}_{21} \mathrm{Br}_{2} \mathrm{Cl}_{2} \mathrm{PZn}$ & $\rho_{\text {calc }}\left[\mathrm{Mg} / \mathrm{m}^{3}\right]$ & 1.701 \\
Formula weight $[\mathrm{g} / \mathrm{mol}]$ & 672.50 & $\mu\left[\mathrm{mm}^{-1}\right]$ & 4.258 \\
Temperature $[\mathrm{K}]$ & $111(2)$ & $\mathrm{F}(000)$ & 1328 \\
Wavelength $[\AA]$ & 0.71073 & $\Theta$ range $\left[^{\circ}\right]$ & 1.516 to 26.371 \\
Crystal system & Triclinic & Reflections collected & 85390 \\
Space group & $P \overline{1}$ & Independent reflections & 10704 \\
$a[\AA]$ & $13.7890(5)$ & Completeness to $\Theta_{\text {max }}$ & $99.9 \%$ \\
$b[\AA]$ & $14.5848(5)$ & Absorption correction & Empirical \\
$c\left[^{\circ}\right]$ & $15.5446(5)$ & Max. / min. transmission & $0.6605 / 0.7461$ \\
$\alpha\left[^{\circ}\right]$ & $66.3370(10)$ & Restraints / parameters & $172 / 595$ \\
$\beta\left[^{\circ}\right]$ & $67.0480(10)$ & GoF & 1.059 \\
$\gamma\left[^{\circ}\right]$ & $76.4660(10)$ & $R 1 / w R 2(\mathrm{I}>2 \sigma(\mathrm{I}))$ & $0.0237 / 0.0551$ \\
Volume $\left[\AA^{3}\right]$ & $2625.49(16)$ & $R 1 / w R 2$ (all data) & $0.0288 / 0.0567$ \\
$Z$ & 2 & Diff. peak and hole $\left[\mathrm{e} \AA^{-3}\right]$ & 0.503 and -0.632 \\
\hline
\end{tabular}




\subsubsection{3 [MeAnPPh $\left.\mathrm{ZnBr}_{2}\right]_{2}(56)$}
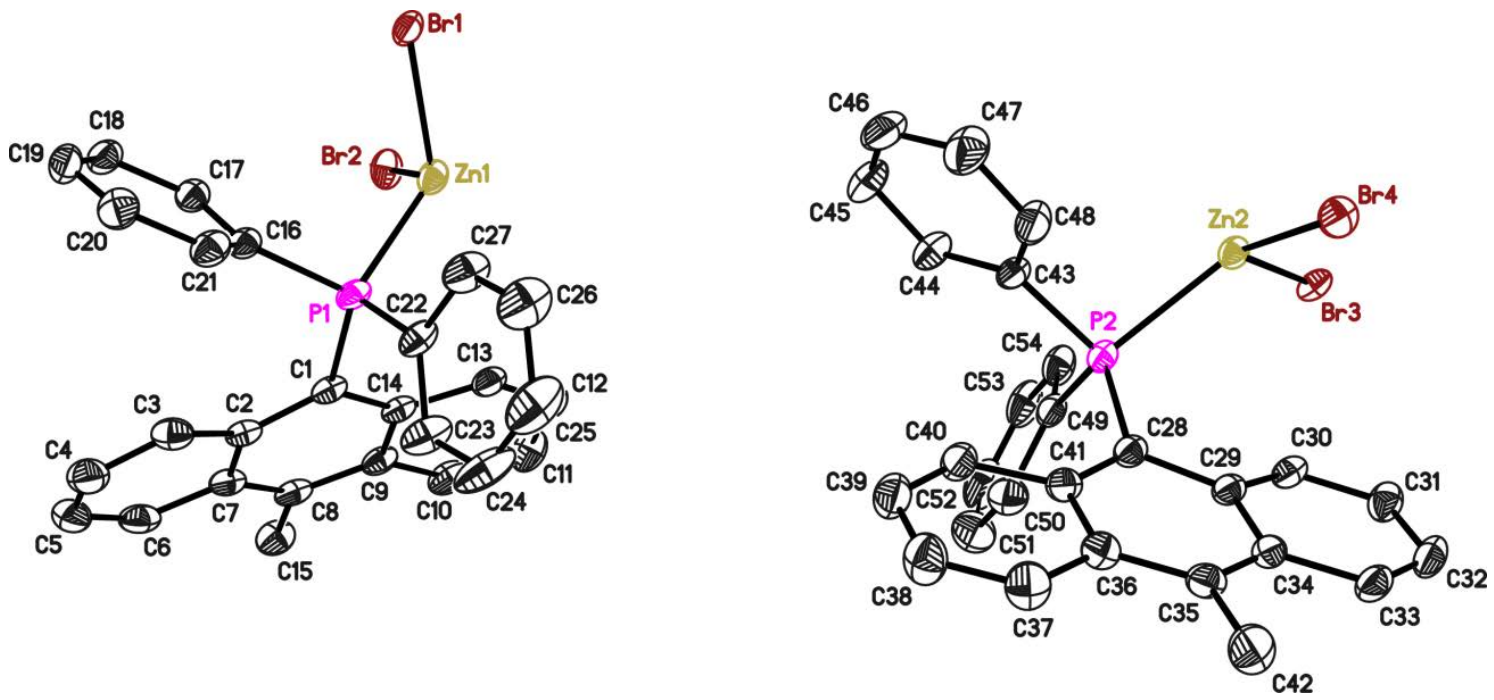

Figure 7-33. Asymmetric unit of $\left[\mathrm{MeAnPPh}_{2} \mathrm{ZnBr}_{2}\right]_{2}$ (56). The anisotropic displacement parameters are depicted at the $50 \%$ probability level. The hydrogen atoms are omitted for clarity.

The structure of $\mathbf{5 6}$ contained channels of disordered DCM molecules which could not be sufficiently described by refinement of disorder. They were removed from the structure using the Platon squeeze function.

Table 7-33. Crystallographic data for $\left[\mathrm{MeAnPPh} \mathrm{ZnBr}_{2}\right]_{2}(56)$.

\begin{tabular}{ll|ll}
\hline Identification code & $\mathrm{NF033}$ & & \\
Empirical formula & $\mathrm{C}_{54} \mathrm{H}_{43} \mathrm{Br}_{4} \mathrm{P}_{2} \mathrm{Zn}_{2}$ & $\rho_{\text {calc }}\left[\mathrm{Mg} / \mathrm{m}^{3}\right]$ & 1.404 \\
Formula weight $[\mathrm{g} / \mathrm{mol}]$ & 1204.20 & $\mu\left[\mathrm{mm}^{-1}\right]$ & 3.733 \\
Temperature $[\mathrm{K}]$ & $99(2)$ & $\mathrm{F}(000)$ & 1194 \\
Wavelength $[\AA]$ & 0.71073 & $\Theta$ range $\left[^{\circ}\right]$ & 1.287 to 29.756 \\
Crystal system & Triclinic & Reflections collected & 79381 \\
Space group & $P \overline{1}$ & Independent reflections & 15242 \\
$a[\AA]$ & $12.4589(18)$ & Completeness to $\Theta_{\text {max }}$ & $100.0 \%$ \\
$b[\AA]$ & $15.228(2)$ & Absorption correction & Empirical \\
$c[\AA]]$ & $16.917(2)$ & Max. / min. transmission & 0.62710 .7459 \\
$\alpha\left[^{\circ}\right]$ & $96.861(2)$ & Restraints / parameters & $172 / 564$ \\
$\beta\left[^{\circ}\right]$ & $106.218(2)$ & GoF & 1.004 \\
$\nu\left[^{\circ}\right]$ & $108.301(2)$ & $R 1 / w R 2$ (I>2 $\sigma(I))$ & $0.0336 / 0.0874$ \\
Volume $\left[\AA^{3}\right]$ & $2849.3(7)$ & $R 1 / w R 2$ (all data) & $0.0485 / 0.0896$ \\
$Z$ & 2 & Diff. peak and hole $\left[\right.$ e $\left.\AA^{-3}\right]$ & 1.671 and -0.813 \\
\hline
\end{tabular}




\subsubsection{4 $\left[\mathrm{MeAnP}\left(\mathrm{NMe}_{2}\right)_{2}(\mathrm{~S}) \mathrm{ZnBr}_{2}\right]_{2}(57)$}

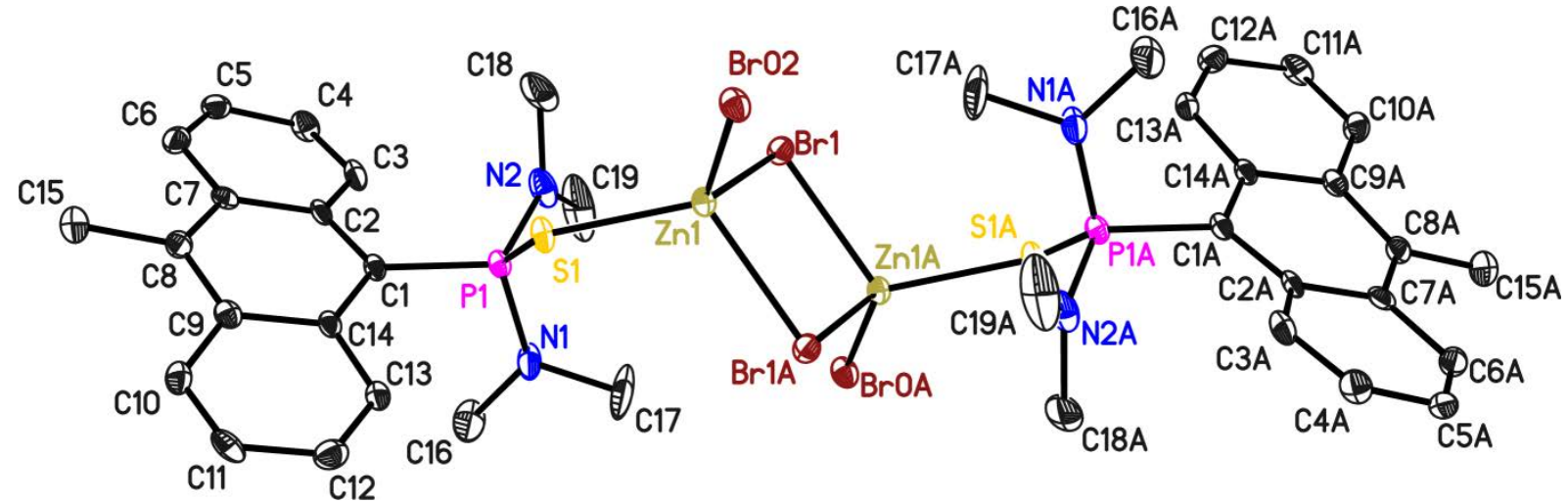

Figure 7-34. Structure of [MeAnP(NMe $\left.)_{2} \mathrm{ZnBr}_{2}\right]$ (57). The anisotropic displacement parameters are depicted at the $50 \%$ probability level. The hydrogen atoms are omitted for clarity. Only one half of the structure depicted in Figure 7-34 is contained in the asymmetric unit.

Table 7-34. Crystallographic data for [MeAnP( $\left.\left(\mathrm{NMe}_{2}\right)_{2} \mathrm{ZnBr}_{2}\right]$ (57).

\begin{tabular}{ll|ll}
\hline Identification code & $\mathrm{NFO34}$ & & \\
Empirical formula & $\mathrm{C}_{19} \mathrm{H}_{23} \mathrm{Br}_{2} \mathrm{~N}_{2} \mathrm{PSZn}$ & $\rho_{\text {calc }}\left[\mathrm{Mg} / \mathrm{m}^{3}\right]$ & 1.777 \\
Formula weight $[\mathrm{g} / \mathrm{mol}]$ & 567.61 & $\mu\left[\mathrm{mm}^{-1}\right]$ & 2.719 \\
Temperature $[\mathrm{K}]$ & $100(2)$ & $\mathrm{F}(000)$ & 1128 \\
Wavelength $[\AA]$ & 0.56086 & $\Theta$ range $\left[^{\circ}\right]$ & 1.544 to 20.528 \\
Crystal system & Monoclinic & Reflections collected & 16388 \\
Space group & $P 2_{1} / c$ & Independent reflections & 4334 \\
$a[\AA]$ & $9.7253(6)$ & Completeness to $\Theta_{\max }$ & $99.9 \%$ \\
$b[\AA]$ & $15.6688(8)$ & Absorption correction & Empirical \\
$c[\AA]$ & $13.9334(7)$ & Max. / min. transmission & $0.6593 / 0.7445$ \\
$\alpha\left[^{\circ}\right]$ & 90 & Restraints / parameters & $70 / 240$ \\
$\beta\left[^{\circ}\right]$ & $92.134(3)$ & GoF & 0.975 \\
$\gamma\left[^{\circ}\right]$ & 90 & $R 1 / w R 2(\mathrm{I}>2 \sigma(\mathrm{I}))$ & $0.0381 / 0.0661$ \\
Volume $\left[\AA^{3}\right]$ & $2121.8(2)$ & $R 1 / w R 2$ (all data) & $0.0620 / 0.0714$ \\
$Z$ & 4 & Diff. peak and hole $\left[\mathrm{e} \AA^{-3}\right]$ & 0.797 and -0.662 \\
\hline
\end{tabular}




\subsubsection{5 $\mathrm{ClAnBIPC}_{2}(59)$}

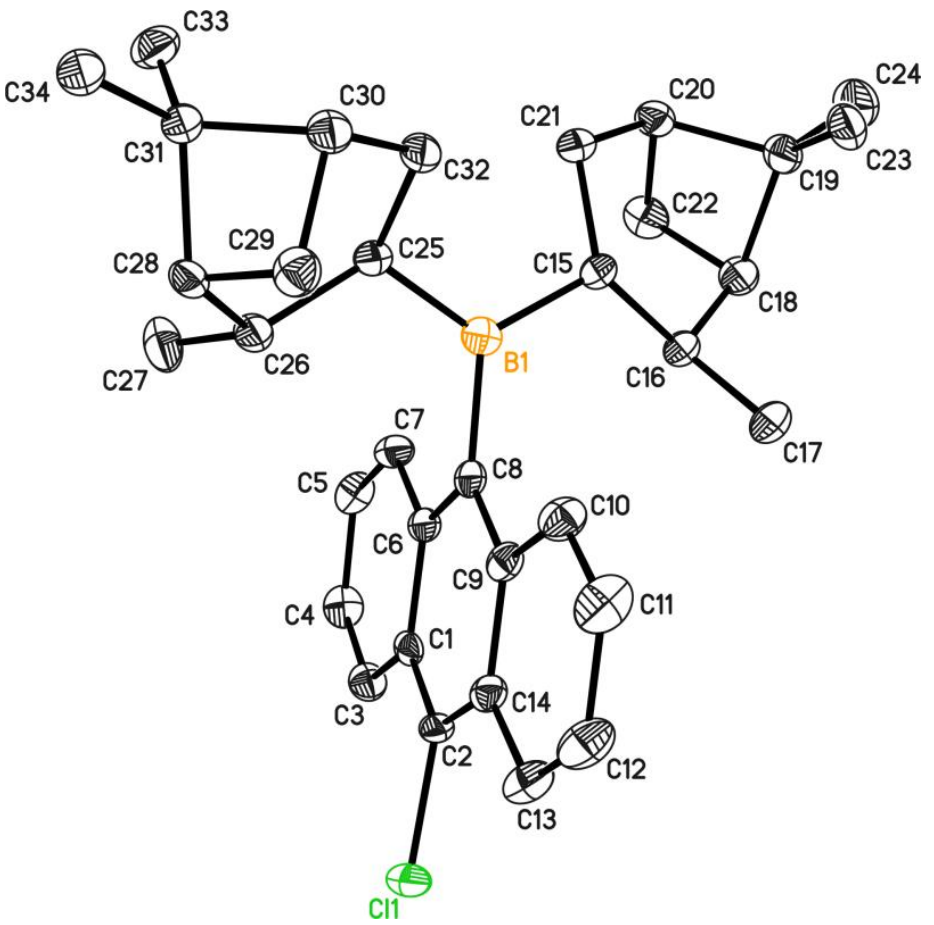

Figure 7-35. Asymmetric unit of $\mathrm{ClAnBIPC}_{2}$ (59). The anisotropic displacement parameters are depicted at the $50 \%$ probability level. The hydrogen atoms are omitted for clarity.

Table 7-35. Crystallographic data for $\mathrm{ClAnBIPC}_{2}$ (59).

\begin{tabular}{|c|c|c|c|}
\hline Identification code & NF035 & & \\
\hline Empirical formula & $\mathrm{C}_{34} \mathrm{H}_{42} \mathrm{BCl}$ & $\rho_{\text {calc }}\left[\mathrm{Mg} / \mathrm{m}^{3}\right]$ & 1.151 \\
\hline Formula weight $[\mathrm{g} / \mathrm{mol}]$ & 496.93 & $\mu\left[\mathrm{mm}^{-1}\right]$ & 0.087 \\
\hline Temperature [K] & $100(2)$ & $F(000)$ & 1072 \\
\hline Wavelength $[\AA ̊]$ & 0.56086 & $\Theta$ range $\left[{ }^{\circ}\right]$ & 1.542 to 20.517 \\
\hline Crystal system & Orthorhombic & Reflections collected & 43711 \\
\hline Space group & $P 2_{1} 2_{1} 2_{1}$ & Independent reflections & 5861 \\
\hline$a[\AA]$ & $12.4110(8)$ & Completeness to $\Theta_{\max }$ & $100.0 \%$ \\
\hline$b[\AA]$ & $12.7290(7)$ & Absorption correction & Emperical \\
\hline$c[\AA ̊]$ & $18.1594(12)$ & Max. / min. transmission & $0.6288 / 0.7445$ \\
\hline$\alpha\left[^{\circ}\right]$ & 90 & Restraints / parameters & $107 / 331$ \\
\hline$\beta\left[^{\circ}\right]$ & 90 & GoF & 1.027 \\
\hline$\gamma\left[{ }^{\circ}\right]$ & 90 & $R 1 / w R 2(I>2 \sigma(I))$ & $0.0466 / 0.0890$ \\
\hline Volume $\left[\AA^{3}\right]$ & $2868.8(3)$ & $R 1 / w R 2$ (all data) & $0.0629 / 0.0944$ \\
\hline Z & 4 & Diff. peak and hole $\left[\mathrm{e} \AA^{-3}\right]$ & 0.168 and -0.238 \\
\hline
\end{tabular}




\subsubsection{BrAnMes $_{2}(60)$}

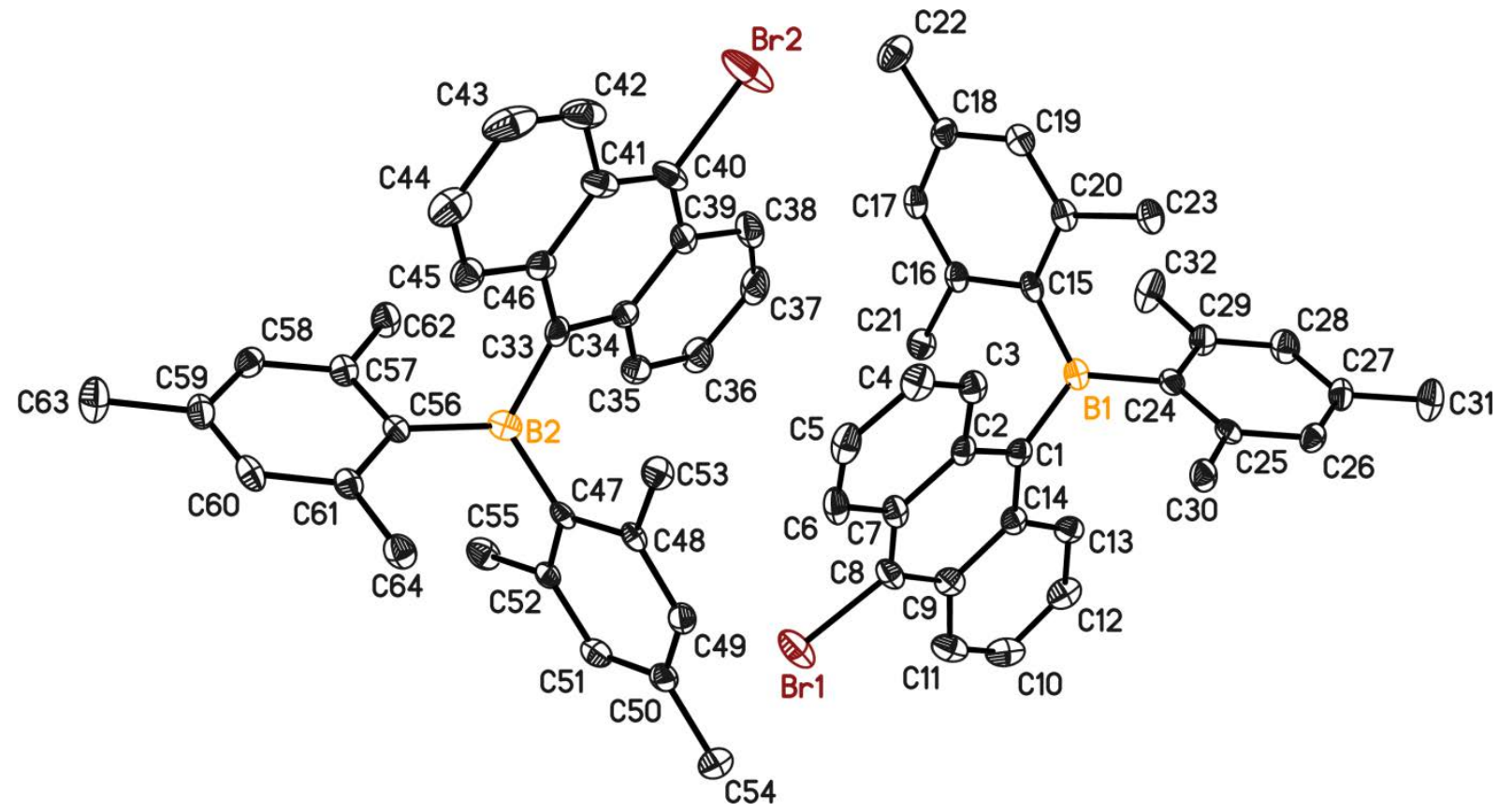

Figure 7-36. Asymmetric unit of $\mathrm{BrAnBMes}_{2}$ (60). The anisotropic displacement parameters are depicted at the $50 \%$ probability level. The hydrogen atoms are omitted for clarity.

Table 7-36. Crystallographic data for $\mathrm{BrAnBMes}_{2}(\mathbf{6 0})$.

\begin{tabular}{ll|ll}
\hline Identification code & $\mathrm{NFO36}$ & & \\
Empirical formula & $\mathrm{C}_{32} \mathrm{H}_{30} \mathrm{BBr}$ & $\rho_{\text {calc }}\left[\mathrm{Mg} / \mathrm{m}^{3}\right]$ & 1.337 \\
Formula weight $[\mathrm{g} / \mathrm{mol}]$ & 505.28 & $\mu\left[\mathrm{mm}^{-1}\right]$ & 1.656 \\
Temperature $[\mathrm{K}]$ & $101(2)$ & $\mathrm{F}(000)$ & 2096 \\
Wavelength $[\AA]$ & 0.71073 & $\Theta$ range $\left[^{\circ}\right]$ & 1.246 to 26.372 \\
Crystal system & Orthorhombic & Reflections collected & 96680 \\
Space group & $P 2{ }_{1} 2_{1} 2_{1}$ & Independent reflections & 10142 \\
$a[\AA]$ & $8.2461(3)$ & Completeness to $\Theta_{\max }$ & $99.3 \%$ \\
$b[\AA]$ & $18.6302(7)$ & Absorption correction & Empirical \\
$c[\AA]$ & $32.6911(11)$ & Max. / min. transmission & $0.6650 / 0.7454$ \\
$\alpha\left[^{\circ}\right]$ & 90 & Restraints / parameters & $190 / 625$ \\
$\beta\left[^{\circ}\right]$ & 90 & GoF & 1.029 \\
$\gamma\left[^{\circ}\right]$ & 90 & $R 1 / w R 2(\mathrm{I}>2 \sigma(\mathrm{I}))$ & $0.0352 / 0.0765$ \\
Volume $\left[\AA^{3}\right]$ & $5022.2(3)$ & $R 1 / w R 2$ (all data) & $0.0452 / 0.0792$ \\
$Z$ & 8 & Diff. peak and hole $\left[\mathrm{e} \AA^{-3}\right]$ & 0.488 and -0.479 \\
\hline
\end{tabular}




\subsubsection{7 $\mathrm{HN}\left(\mathrm{CH}_{2} \mathrm{PhSMe}\right)_{2}(63)$}

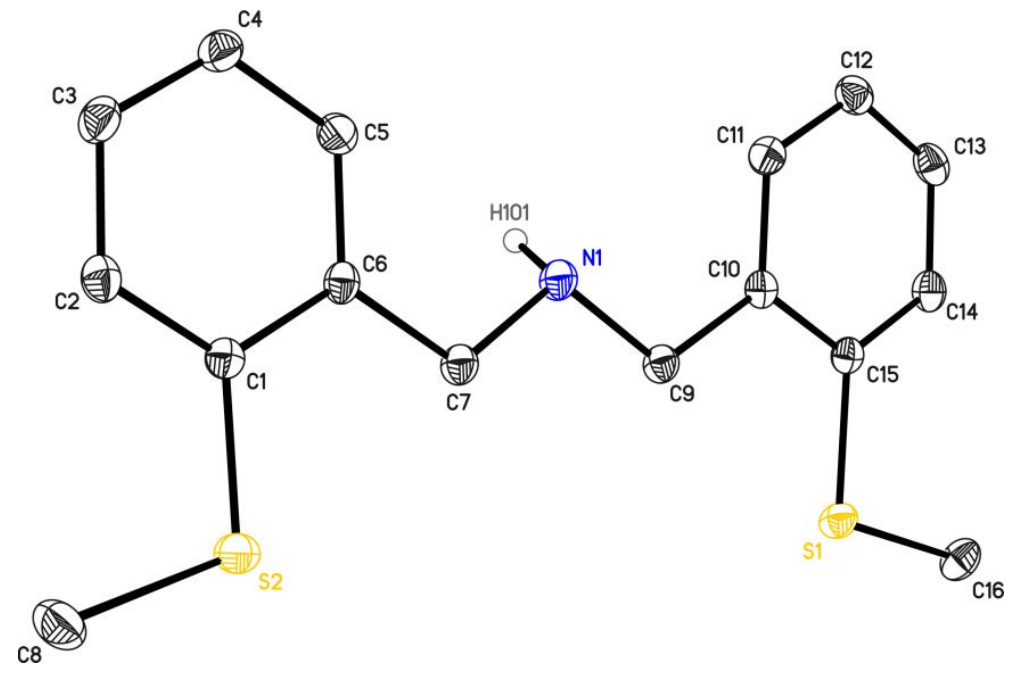

Figure 7-37. Asymmetric unit of $\mathrm{HN}\left(\mathrm{CH}_{2} \mathrm{PhSMe}\right)_{2}(63)$. The anisotropic displacement parameters are depicted at the $50 \%$ probability level. The hydrogen atoms are omitted for clarity.

The position of hydrogen atom $\mathrm{H} 101$ was freely refined.

Table 7-37. Crystallographic data for $\mathrm{HN}\left(\mathrm{CH}_{2} \mathrm{PhSMe}\right)_{2}$ (63).

\begin{tabular}{|c|c|c|c|}
\hline Identification code & NF037 & & \\
\hline Empirical formula & $\mathrm{C}_{16} \mathrm{H}_{19} \mathrm{NS}_{2}$ & $\rho_{\text {calc }}\left[\mathrm{Mg} / \mathrm{m}^{3}\right]$ & 1.340 \\
\hline Formula weight $[\mathrm{g} / \mathrm{mol}]$ & 289.44 & $\mu\left[\mathrm{mm}^{-1}\right]$ & 0.357 \\
\hline Temperature $[\mathrm{K}]$ & $100(2)$ & $F(000)$ & 616 \\
\hline Wavelength [Å] & 0.71073 & $\Theta$ range $\left[{ }^{\circ}\right]$ & 1.483 to 29.846 \\
\hline Crystal system & Monoclinic & Reflections collected & 37978 \\
\hline Space group & $P 2_{1} / n$ & Independent reflections & 3975 \\
\hline$a[\AA ̊]$ & $15.506(3)$ & Completeness to $\Theta_{\max }$ & $99.8 \%$ \\
\hline$b[\AA ̊]$ & $5.1596(9)$ & Absorption correction & Empirical \\
\hline$c[\AA ̊]$ & $18.939(3)$ & Max. / min. transmission & $0.6908 / 0.7460$ \\
\hline$\alpha\left[^{\circ}\right]$ & 90 & Restraints / parameters & $0 / 178$ \\
\hline$\beta\left[^{\circ}\right]$ & $108.765(2)$ & GoF & 1.024 \\
\hline$\gamma\left[\left[^{\circ}\right]\right.$ & 90 & $R 1 / w R 2(I>2 \sigma(I))$ & $0.0291 / 0.0776$ \\
\hline Volume $\left[\AA^{3}\right]$ & $1434.7(4)$ & $R 1$ / wR2 (all data) & $0.0311 / 0.0792$ \\
\hline Z & 4 & Diff. peak and hole $\left[\mathrm{e}^{-3}{ }^{-3}\right]$ & 0.548 and -0.199 \\
\hline
\end{tabular}




\subsubsection{8 $65 a$}

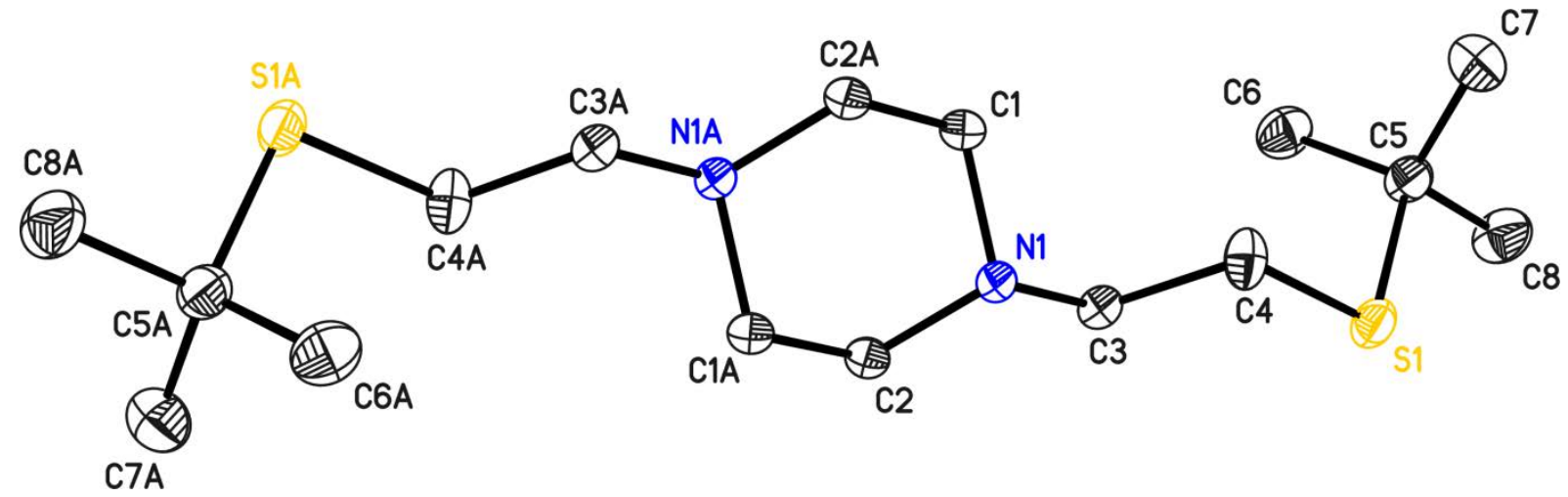

Figure 7-38. Structure of 65a. The anisotropic displacement parameters are depicted at the $50 \%$ probability level. The hydrogen atoms are omitted for clarity. Only one half of the molecule depicted in figure 7-38 is contained in the asymmetric unit.

Table 7-38. Crystallographic data for $\mathbf{6 5 a}$.

\begin{tabular}{ll|ll}
\hline Identification code & $\mathrm{NFO38}$ & & \\
Empirical formula & $\mathrm{C}_{8} \mathrm{H}_{17} \mathrm{NS}$ & $\rho_{\text {calc }}\left[\mathrm{Mg} / \mathrm{m}^{3}\right]$ & 1.133 \\
Formula weight $[\mathrm{g} / \mathrm{mol}]$ & 159.28 & $\mu\left[\mathrm{mm}^{-1}\right]$ & 0.280 \\
Temperature $[\mathrm{K}]$ & $101(2)$ & $\mathrm{F}(000)$ & 704 \\
Wavelength $[\AA]$ & 0.71073 & $\Theta$ range $\left[^{\circ}\right]$ & 1.402 to 26.372 \\
Crystal system & Monoclinic & Reflections collected & 37861 \\
Space group & $C 2 / c$ & Independent reflections & 1902 \\
$a[\AA]$ & $29.954(2)$ & Completeness to $\Theta_{\text {max }}$ & $99.9 \%$ \\
$b[\AA]]$ & $5.6910(5)$ & Absorption correction & Empirical \\
$c[\AA ̊]$ & $11.2987(9)$ & Max. / min. transmission & 0.63160 .7463 \\
$\alpha\left[^{\circ}\right]$ & 90 & Restraints / parameters & $21 / 94$ \\
$\beta\left[^{\circ}\right]$ & $104.176(3)$ & GoF & 1.115 \\
$\gamma\left[^{\circ}\right]$ & 90 & $R 1 / w R 2(\mathrm{I}>2 \sigma(\mathrm{I}))$ & $0.0331 / 0.0841$ \\
Volume $\left[\AA^{3}\right]$ & $1867.4(3)$ & $R 1 / w R 2$ (all data) & $0.0368 / 0.0860$ \\
$Z$ & 8 & Diff. peak and hole $\left[\mathrm{e} \AA^{-3}\right]$ & 0.308 and -0.169 \\
\hline
\end{tabular}




\subsubsection{9 [AnCH $\left.{ }_{2} \mathrm{Li} \cdot \mathrm{TMEDA}\right](66)$}

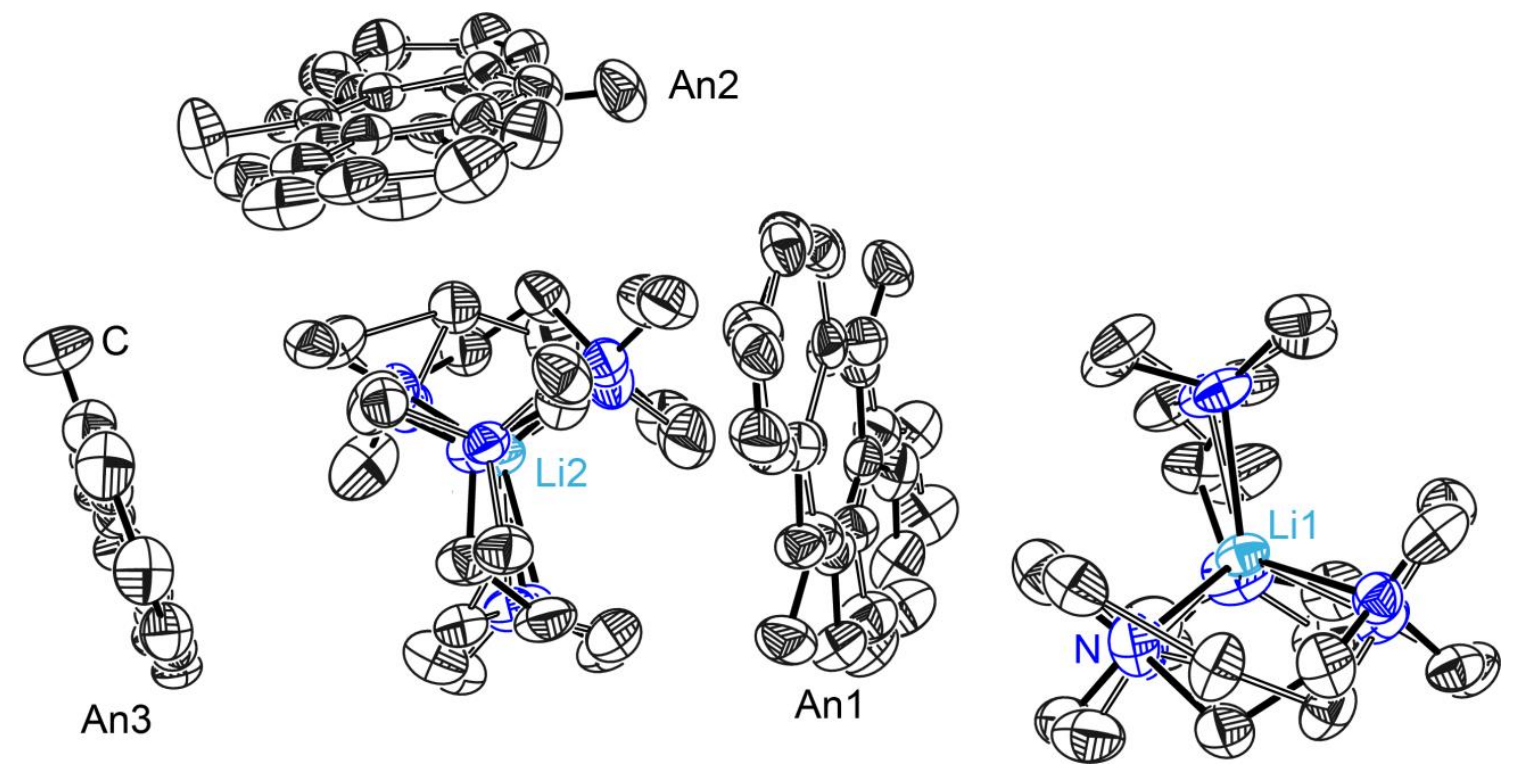

Figure 7-39. Asymmetric unit of $\left[\mathrm{AnCH}_{2} \mathrm{Li}\right.$-TMEDA]. The anisotropic displacement parameters are depicted at the $50 \%$ probability level. The hydrogen atoms are omitted for clarity.

All TMEDA molecules are disordered in two positions in a 50:50 ratio, methylanthracene molecules An1 and An2 are disordered in two positions at a 50:50 ratio.

Table 7-39. Crystallographic data for [AnCH $\mathrm{ALi}_{2}$ TMEDA].

\begin{tabular}{ll|ll}
\hline Identification code & $\mathrm{NF039}$ & & \\
Empirical formula & $\mathrm{C}_{69} \mathrm{H}_{99} \mathrm{Li}_{2} \mathrm{~N}_{8}$ & $\rho_{\text {calc }}\left[\mathrm{Mg} / \mathrm{m}^{3}\right]$ & 1.095 \\
Formula weight $[\mathrm{g} / \mathrm{mol}]$ & 1054.51 & $\mu\left[\mathrm{mm}^{-1}\right]$ & 0.064 \\
Temperature $[\mathrm{K}]$ & $100(2)$ & $\mathrm{F}(000)$ & 2300 \\
Wavelength $[\AA]$ & 0.71073 & $\Theta$ range $\left[^{\circ}\right]$ & 1.169 to 26.371 \\
Crystal system & Monoclinic & Reflections collected & 94519 \\
Space group & $P 2_{1} / n$ & Independent reflections & 13073 \\
$a[\AA]$ & $9.4863(3)$ & Completeness to $\Theta_{\text {max }}$ & $99.9 \%$ \\
$b[\AA]$ & Absorption correction & Empirical \\
$c[\AA]]$ & $34.8471(12)$ & Max. / min. transmission & 0.67040 .7462 \\
$\alpha\left[^{\circ}\right]$ & $19.3904(7)$ & Restraints / parameters & $8743 / 1319$ \\
$\beta\left[^{\circ}\right]$ & 90 & GoF & 1.038 \\
$\nu\left[^{\circ}\right]$ & $93.7643(18)$ & $R 1 / w R 2(I>2 \sigma(I))$ & $0.0528 / 0.1471$ \\
Volume $\left[\AA^{3}\right]$ & 90 & $R 1 / w R 2$ (all data) & $0.0632 / 0.1570$ \\
$Z$ & $6396.1(4)$ & Diff. peak and hole $\left[\mathrm{e} \AA^{-3}\right]$ & 0.307 and -0.267 \\
\hline
\end{tabular}




\subsubsection{0 $\mathrm{MeAnC}_{2} \mathrm{H}_{4} \mathrm{Br}(68)$}

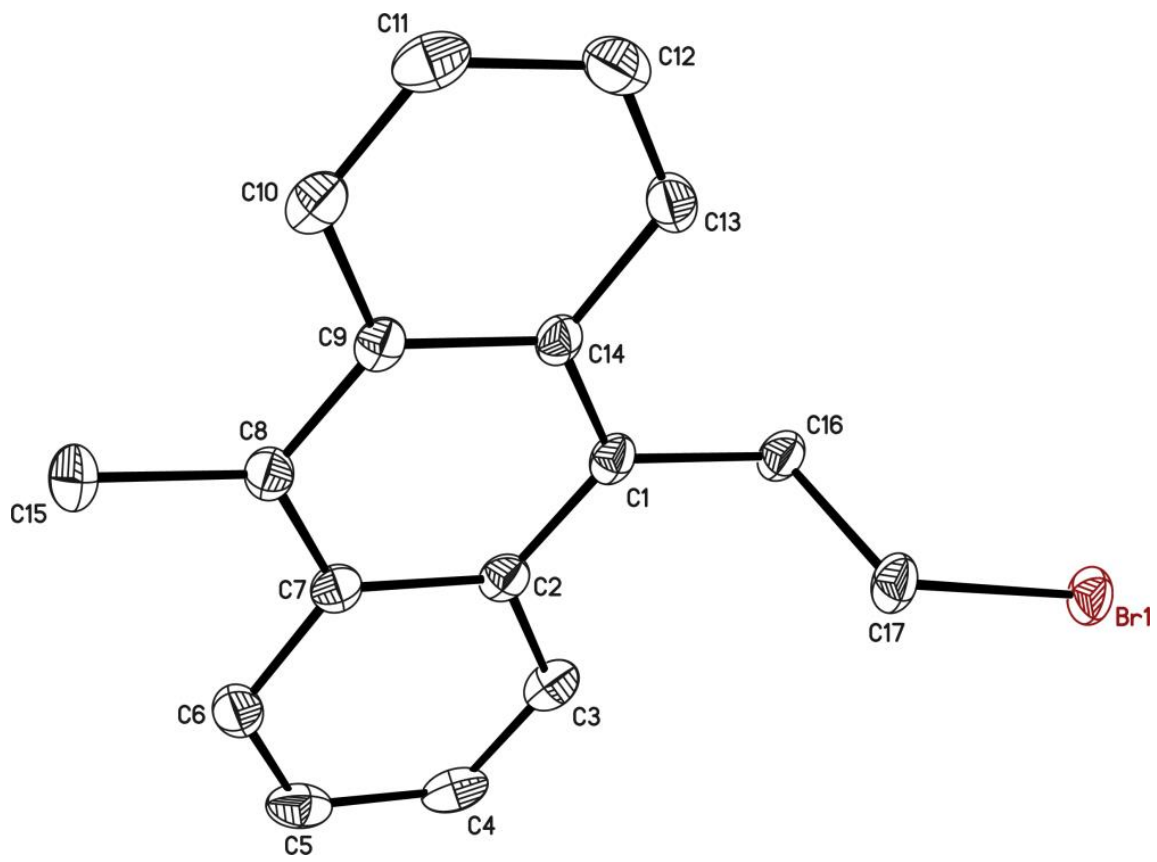

Figure 7-40. Asymmetric unit of $\mathrm{MeAnC}_{2} \mathrm{H}_{4} \mathrm{Br}$ (68). The anisotropic displacement parameters are depicted at the $50 \%$ probability level. The hydrogen atoms are omitted for clarity.

Table 7-40. Crystallographic data for $\mathrm{MeAnC}_{2} \mathrm{H}_{4} \mathrm{Br}$ (68).

\begin{tabular}{ll|ll}
\hline Identification code & $\mathrm{NFO40}$ & & \\
Empirical formula & $\mathrm{C}_{17} \mathrm{H}_{15} \mathrm{Br}$ & $\rho_{\text {calc }}\left[\mathrm{Mg} / \mathrm{m}^{3}\right]$ & 1.539 \\
Formula weight $[\mathrm{g} / \mathrm{mol}]$ & 299.20 & $\mu\left[\mathrm{mm}^{-1}\right]$ & 3.161 \\
Temperature $[\mathrm{K}]$ & $100(2)$ & $\mathrm{F}(000)$ & 1216 \\
Wavelength $[\AA]$ & 0.71073 & $\Theta$ range $\left[^{\circ}\right]$ & 1.165 to 28.403 \\
Crystal system & Monoclinic & Reflections collected & 12143 \\
Space group & $C 2 / c$ & Independent reflections & 3210 \\
$a[\AA]$ & $37.265(7)$ & Completeness to $\Theta_{\max }$ & $99.6 \%$ \\
$b[\AA]$ & $5.1313(9)$ & Absorption correction & Empirical \\
$c\left[^{\circ}\right]$ & $14.394(3)$ & Max. / min. transmission & $0.8705 / 0.9703$ \\
$\alpha\left[^{\circ}\right]$ & 90 & Restraints / parameters & $48 / 164$ \\
$\beta\left[^{\circ}\right]$ & $110.216(4)$ & GoF & 1.061 \\
$\gamma\left[^{\circ}\right]$ & 90 & $R 1 / w R 2(\mathrm{I}>2 \sigma(\mathrm{I}))$ & $0.0373 / 0.0805$ \\
Volume $\left[\AA^{3}\right]$ & $2582.9(8)$ & $R 1 / w R 2$ (all data) & $0.0554 / 0.0853$ \\
$Z$ & 8 & Diff. peak and hole $\left[\mathrm{e} \AA^{-3}\right]$ & 0.769 and -0.662 \\
\hline
\end{tabular}




\subsubsection{1}

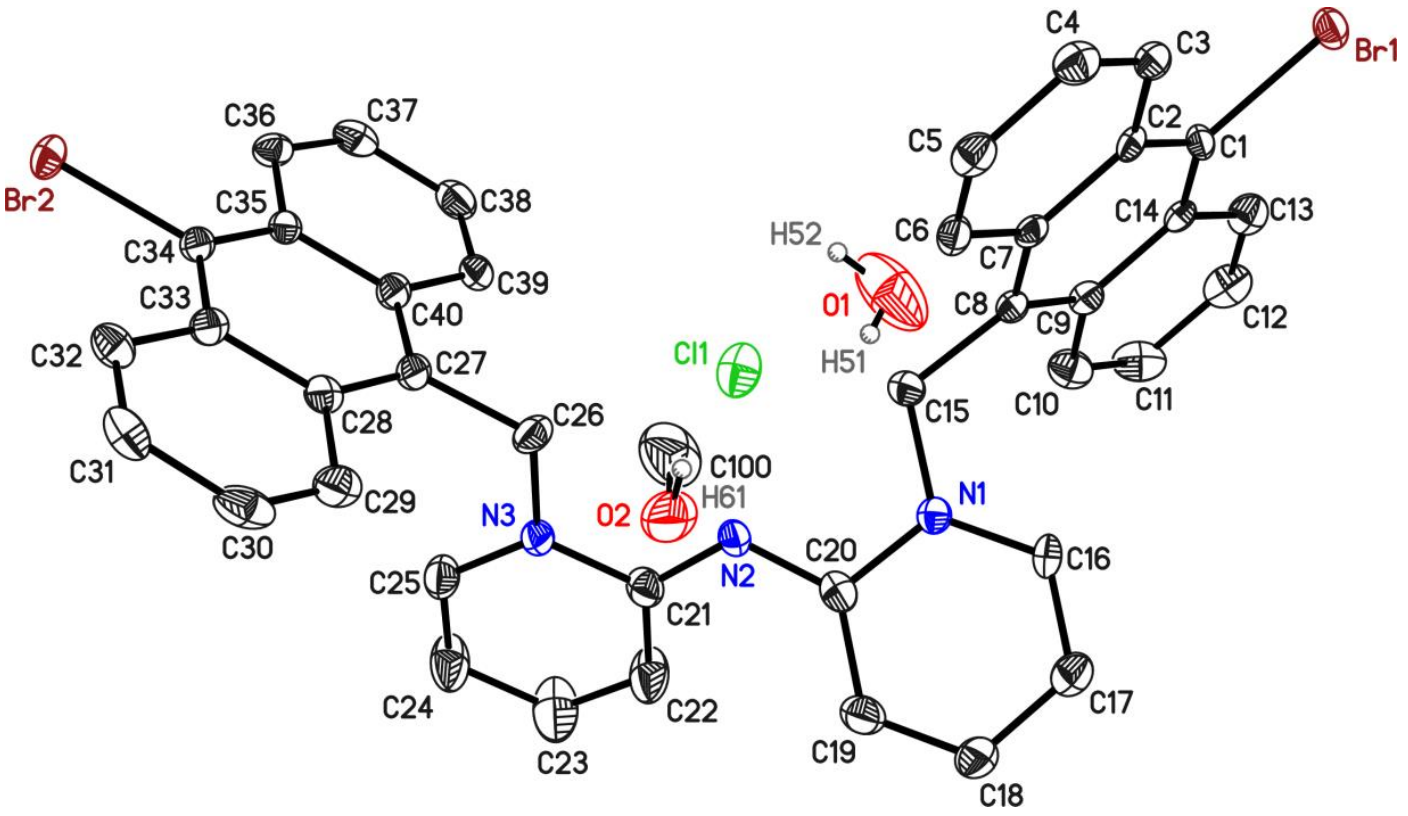

Figure 7-41. Asymmetric unit of 71. The anisotropic displacement parameters are depicted at the $50 \%$ probability level. The hydrogen atoms are omitted for clarity.

The high residual desnity is coused by the strong absorption of the bromine atoms and the peak of maximum residual density is located in close proximity to a bromine atom.

Table 7-41. Crystallographic data for 71.

\begin{tabular}{ll|ll}
\hline Identification code & $\mathrm{NFO41}$ & \\
Empirical formula & $\mathrm{C}_{41} \mathrm{H}_{30} \mathrm{Br}_{2} \mathrm{ClN}_{3} \mathrm{O}_{2}$ & $\rho_{\text {calc }}\left[\mathrm{Mg} / \mathrm{m}^{3}\right]$ & 1.545 \\
Formula weight $[\mathrm{g} / \mathrm{mol}]$ & 791.95 & $\mu\left[\mathrm{mm}^{-1}\right]$ & 2.502 \\
Temperature $[\mathrm{K}]$ & $100(2)$ & $\mathrm{F}(000)$ & 800 \\
Wavelength $[\AA]$ & 0.71073 & $\Theta$ range $\left[^{\circ}\right]$ & 1.567 to 26.369 \\
Crystal system & Triclinic & Reflections collected & 40041 \\
Space group & $P \overline{1}$ & Independent reflections & 6923 \\
$a[\AA ̊$ & Completeness to $\Theta_{\text {max }}$ & $100.0 \%$ \\
$b[\AA]]$ & $10.1554(17)$ & Absorption correction & Empirical \\
$c[\AA ̊]$ & $13.071(2)$ & Max. / min. transmission & \\
$\alpha\left[^{\circ}\right]$ & $13.337(2)$ & Restraints / parameters & $128 / 450$ \\
$\beta\left[^{\circ}\right]$ & $84.330(2)$ & GoF & 1.046 \\
$\gamma\left[^{\circ}\right]$ & $75.179(3)$ & $R 1 / w R 2$ (I>2 $\sigma(\mathrm{I}))$ & $0.0344 / 0.0901$ \\
Volume $\left[\AA^{3}\right]$ & $86.497(3)$ & $R 1 / w R 2$ (all data) & $0.0422 / 0.0936$ \\
$Z$ & $1701.9(5)$ & Diff. peak and hole [e $\left.\AA^{-3}\right]$ & 1.249 and -0.344 \\
\hline
\end{tabular}




\subsubsection{2 ((Thi) $\left.\mathrm{CH}_{2}\right)_{2} \mathrm{NCH}_{2} \mathrm{AnCH}_{2} \mathrm{~N}\left(\mathrm{CH}_{2}(\mathrm{Thi})\right)_{2}(72)$}

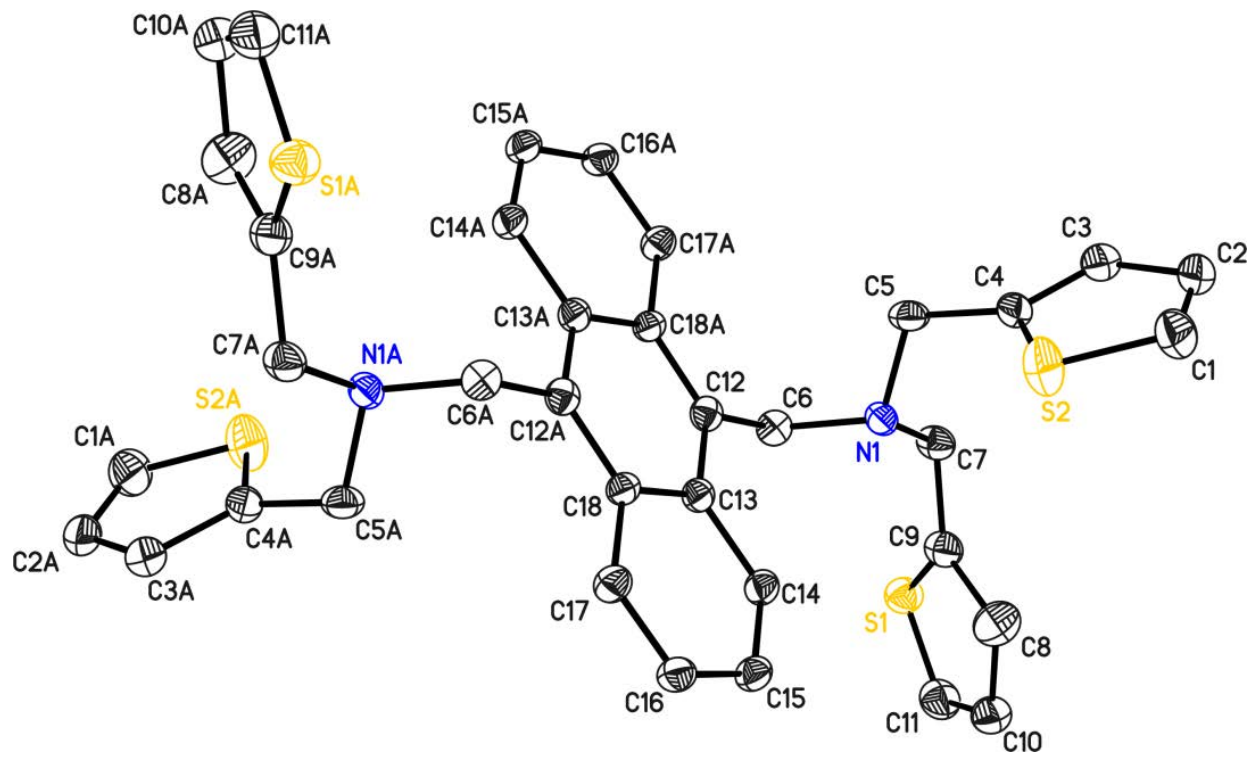

Figure 7-42. Structure of ((Thi) $\left.\mathrm{CH}_{2}\right)_{2} \mathrm{NCH}_{2} \mathrm{AnCH}_{2} \mathrm{~N}\left(\mathrm{CH}_{2}(\mathrm{Thi})\right)_{2}$ (72). The anisotropic displacement parameters are depicted at the $50 \%$ probability level. The hydrogen atoms are omitted for clarity. Only one half of the molecule depicted in figure 7-42 is contained in the asymmetric unit.

Table 7-42. Crystallographic data for ((Thi) $\left.\mathrm{CH}_{2}\right)_{2} \mathrm{NCH}_{2} \mathrm{AnCH}_{2} \mathrm{~N}\left(\mathrm{CH}_{2}(\mathrm{Thi})\right)_{2}$ (72).

\begin{tabular}{|c|c|c|c|}
\hline Identification code & NF042 & & \\
\hline Empirical formula & $\mathrm{C}_{18} \mathrm{H}_{16} \mathrm{NS}_{2}$ & $\rho_{\text {calc }}\left[\mathrm{Mg} / \mathrm{m}^{3}\right]$ & 1.312 \\
\hline Formula weight $[\mathrm{g} / \mathrm{mol}]$ & 310.44 & $\mu\left[\mathrm{mm}^{-1}\right]$ & 0.331 \\
\hline Temperature $[\mathrm{K}]$ & $100(2)$ & $F(000)$ & 652 \\
\hline Wavelength $[\AA]$ & 0.71073 & $\Theta$ range $\left[{ }^{\circ}\right]$ & 1.740 to 28.721 \\
\hline Crystal system & Monoclinic & Reflections collected & 42347 \\
\hline Space group & $P 2_{1} / c$ & Independent reflections & 4058 \\
\hline$a[\AA]$ & $11.702(2)$ & Completeness to $\Theta_{\max }$ & $99.9 \%$ \\
\hline$b[\AA]$ & $14.486(3)$ & Absorption correction & Empirical \\
\hline$c[\AA]$ & $9.2702(19)$ & Max. / min. transmission & $0.9532 / 1.0000$ \\
\hline$\alpha\left[^{\circ}\right]$ & 90 & Restraints / parameters & 312 / 282 \\
\hline$\beta\left[^{\circ}\right]$ & $90.81(3)$ & GoF & 1.057 \\
\hline$\nu\left[^{\circ}\right]$ & 90 & $R 1 / w R 2(I>2 \sigma(I))$ & $0.0392 / 0.0982$ \\
\hline Volume $\left[\AA^{3}\right]$ & $1571.3(5)$ & $R 1 / w R 2$ (all data) & $0.0506 / 0.1050$ \\
\hline Z & 4 & Diff. peak and hole $\left[\mathrm{e} \AA^{-3}\right]$ & 0.316 and -0.314 \\
\hline
\end{tabular}




\subsubsection{3 $\mathrm{HAnCH}_{2} \mathrm{~N}\left(\mathrm{CH}_{2} \mathrm{PhOMe}\right)_{2}(75)$}

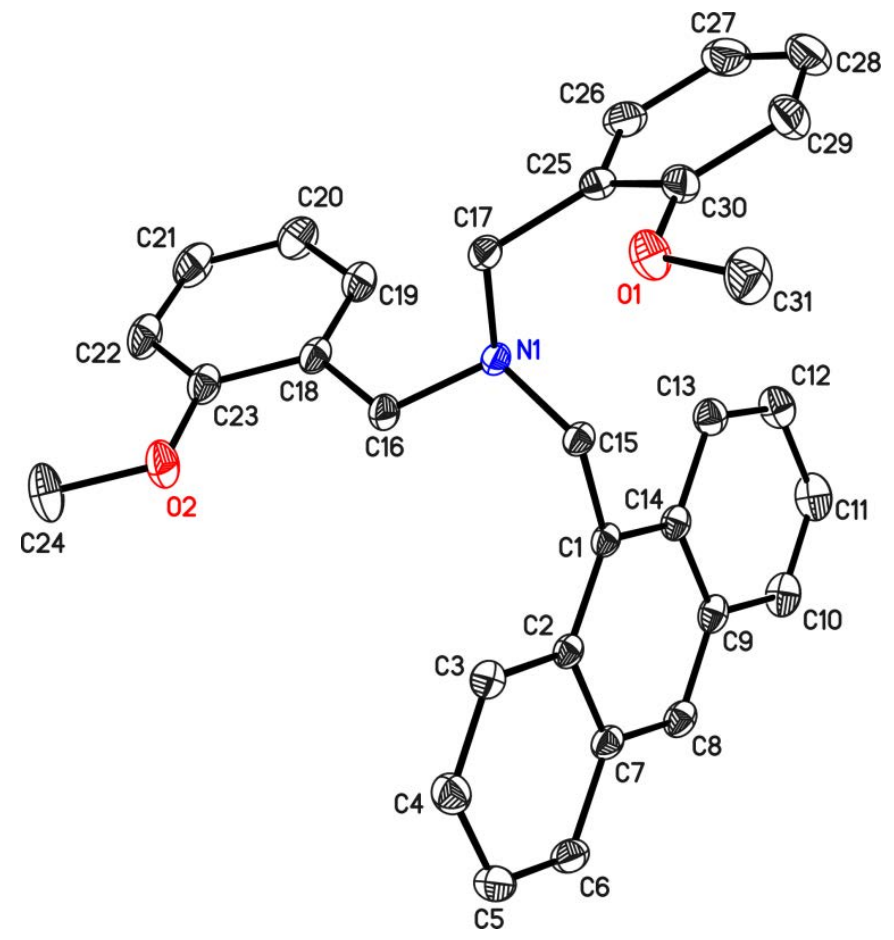

Figure 7-43. Asymmetric unit of $\mathrm{HAnCH}_{2} \mathrm{~N}\left(\mathrm{CH}_{2} \mathrm{PhOMe}\right)_{2}$ (75). The anisotropic displacement parameters are depicted at the $50 \%$ probability level. The hydrogen atoms are omitted for clarity.

Table 7-43. Crystallographic data for $\mathrm{HAnCH}_{2} \mathrm{~N}\left(\mathrm{CH}_{2} \mathrm{PhOMe}\right)_{2}$ (75).

\begin{tabular}{|c|c|c|c|}
\hline Identification code & NF043 & & \\
\hline Empirical formula & $\mathrm{C}_{31} \mathrm{H}_{29} \mathrm{NO}_{2}$ & $\rho_{\text {calc }}\left[\mathrm{Mg} / \mathrm{m}^{3}\right]$ & 1.265 \\
\hline Formula weight $[\mathrm{g} / \mathrm{mol}]$ & 447.55 & $\mu\left[\mathrm{mm}^{-1}\right]$ & 0.078 \\
\hline Temperature [K] & $100(2)$ & $F(000)$ & 952 \\
\hline Wavelength $[\AA ̊]$ & 0.71073 & $\Theta$ range $\left[{ }^{\circ}\right]$ & 1.845 to 29.763 \\
\hline Crystal system & Monoclinic & Reflections collected & 64843 \\
\hline Space group & $P 2_{1} / n$ & Independent reflections & 6457 \\
\hline$a[\AA ̊]$ & $9.059(3)$ & Completeness to $\Theta_{\max }$ & $99.8 \%$ \\
\hline$b[\AA ̊]$ & $19.279(5)$ & Absorption correction & Empirical \\
\hline$c[\AA ̊]$ & $13.574(4)$ & Max. / min. transmission & $0.7082 / 0.7460$ \\
\hline$\alpha\left[^{\circ}\right]$ & 90 & Restraints / parameters & $0 / 309$ \\
\hline$\beta\left[^{\circ}\right]$ & $97.493(4)$ & GoF & 1.023 \\
\hline$\nu\left[^{\circ}\right]$ & 90 & $R 1 / w R 2(I>2 \sigma(I))$ & $0.0416 / 0.1174$ \\
\hline Volume $\left[\AA^{3}\right]$ & $2350.5(11)$ & $R 1 / w R 2$ (all data) & $0.0454 / 0.1209$ \\
\hline Z & 4 & Diff. peak and hole $\left[\mathrm{e} \AA^{-3}\right]$ & 0.371 and -0.243 e \\
\hline
\end{tabular}




\subsubsection{4 $\mathrm{HAnCH}_{2} \mathrm{~N}\left(\mathrm{CH}_{2} \mathrm{PhSMe}\right)_{2}(76)$}

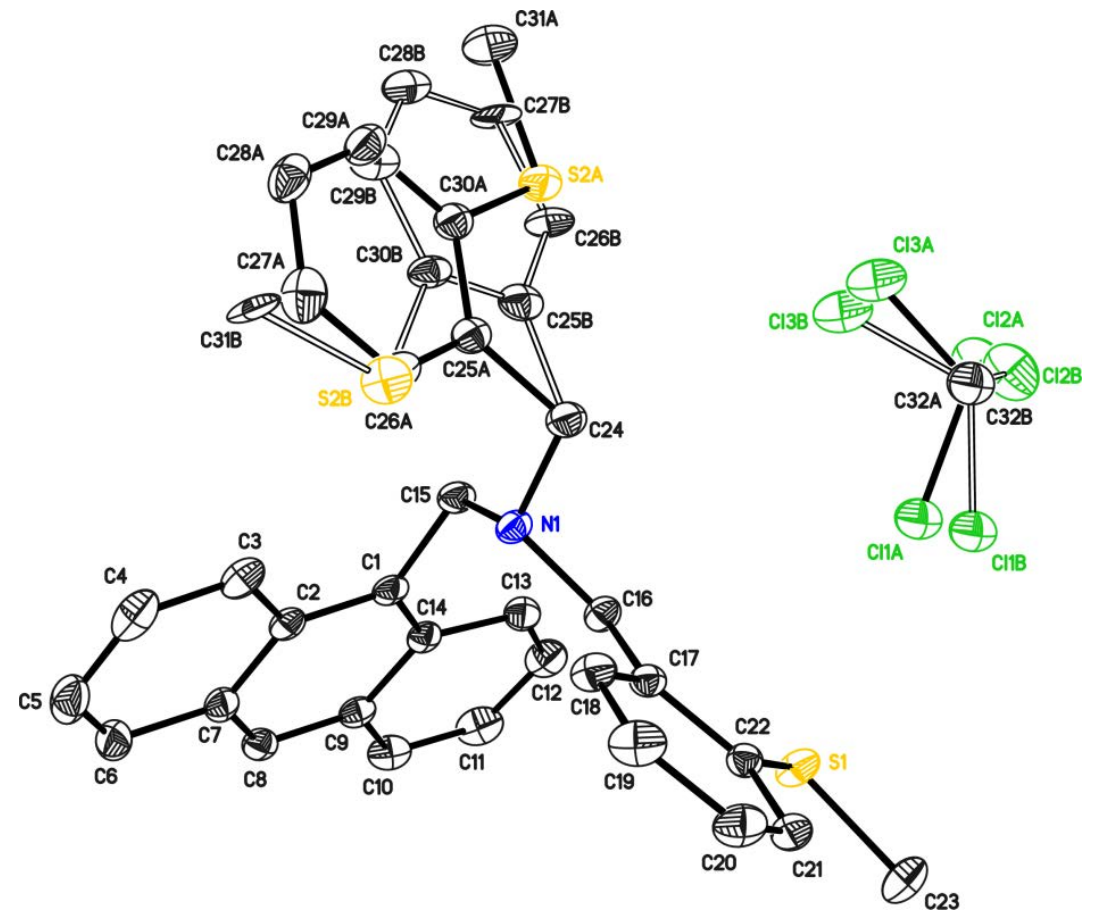

Figure 7-44. Asymmetric unit of $\mathrm{HAnCH}_{2} \mathrm{~N}\left(\mathrm{CH}_{2} \mathrm{PhSMe}\right)_{2}$ (76). The anisotropic displacement parameters are depicted at the $50 \%$ probability level. The hydrogen atoms are omitted for clarity.

One 2-(methylthio)benzyl moiety is disordered at a 60:40 ratio and the chloroform molecule exhibits disorder in two positions at a 50:50 ratio.

Table 7-44. Crystallographic data for $\mathrm{HAnCH}_{2} \mathrm{~N}\left(\mathrm{CH}_{2} \mathrm{PhSMe}\right)_{2}$ (76).

\begin{tabular}{|c|c|c|c|}
\hline Identification code & NF044 & & \\
\hline Empirical formula & $\mathrm{C}_{32} \mathrm{H}_{30} \mathrm{Cl}_{3} \mathrm{NS}_{2}$ & $\rho_{\text {calc }}\left[\mathrm{Mg} / \mathrm{m}^{3}\right]$ & 1.380 \\
\hline Formula weight $[\mathrm{g} / \mathrm{mol}]$ & 599.04 & $\mu\left[\mathrm{mm}^{-1}\right]$ & 0.486 \\
\hline Temperature [K] & $100(2)$ & $F(000)$ & 624 \\
\hline Wavelength $[\AA ̊]$ & 0.71073 & $\Theta$ range $\left[{ }^{\circ}\right]$ & 1.774 to 30.531 \\
\hline Crystal system & Triclinic & Reflections collected & 8615 \\
\hline Space group & $P \overline{1}$ & Independent reflections & 8615 \\
\hline$a[\AA ̊]$ & $10.7698(4)$ & Completeness to $\Theta_{\max }$ & $100.0 \%$ \\
\hline$b[\AA]$ & $11.9758(4)$ & Absorption correction & Empirical \\
\hline$c[\AA]$ & $12.5011(4)$ & Max. / min. transmission & $0.5919 / 0.7457$ \\
\hline$\alpha\left[^{\circ}\right]$ & 74.1008(17) & Restraints / parameters & $405 / 433$ \\
\hline$\beta\left[^{\circ}\right]$ & $70.0881(17)$ & GoF & 1.027 \\
\hline$\nu\left[{ }^{\circ}\right]$ & 76.7959(18) & $R 1 / w R 2(I>2 \sigma(I))$ & 0.0482 / 0.1196 \\
\hline Volume $\left[\AA^{3}\right]$ & $1441.60(9)$ & $R 1 / w R 2$ (all data) & $0.0843 / 0.1368$ \\
\hline $\mathrm{Z}$ & 2 & Diff. peak and hole $\left[\mathrm{e} \AA^{-3}\right]$ & 0.557 and -0.526 \\
\hline
\end{tabular}




\subsubsection{5 $\mathrm{HAnCH}_{2} \mathrm{PPh}_{2}(77)$}

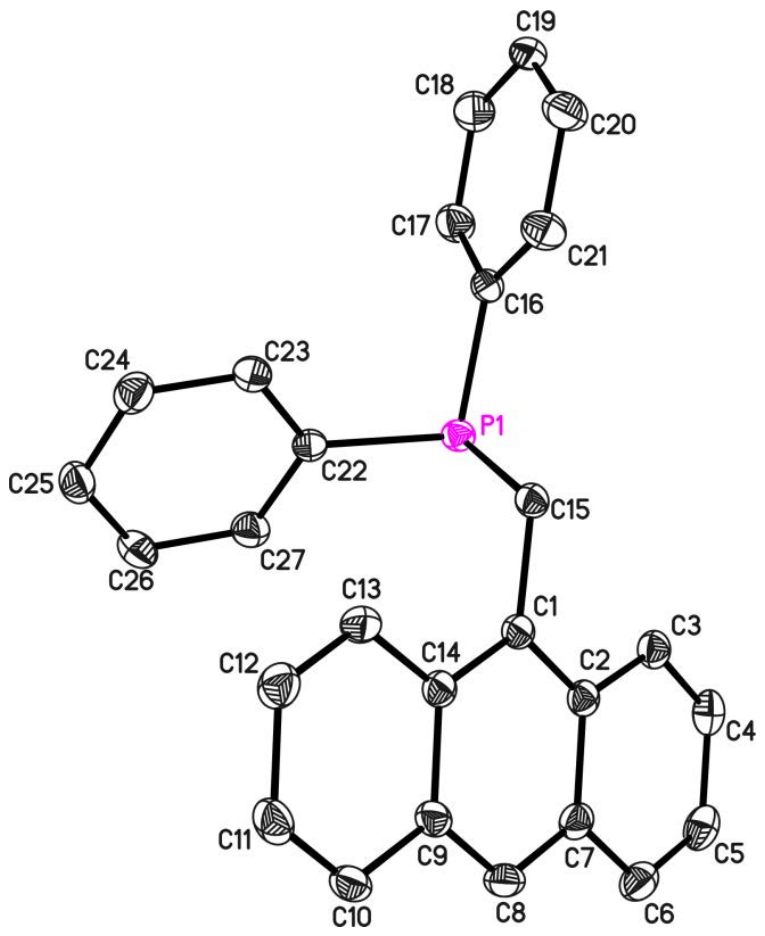

Figure 7-45. Asymmetric unit of $\mathrm{HAnCH}_{2} \mathrm{PPh}_{2}$ (77). The anisotropic displacement parameters are depicted at the $50 \%$ probability level. The hydrogen atoms are omitted for clarity.

Table 7-45. Crystallographic data for $\mathrm{HAnCH}_{2} \mathrm{PPh}_{2}$ (77).

\begin{tabular}{|c|c|c|c|}
\hline Identification code & NF045 & & \\
\hline Empirical formula & $\mathrm{C}_{27} \mathrm{H}_{21} \mathrm{P}$ & $\rho_{\text {calc }}\left[\mathrm{Mg} / \mathrm{m}^{3}\right]$ & 1.296 \\
\hline Formula weight $[\mathrm{g} / \mathrm{mol}]$ & 376.41 & $\mu\left[\mathrm{mm}^{-1}\right]$ & 0.087 \\
\hline Temperature $[\mathrm{K}]$ & $110(2)$ & $F(000)$ & 396 \\
\hline Wavelength [Å] & 0.56086 & $\Theta$ range $\left[{ }^{\circ}\right]$ & 1.653 to 20.807 \\
\hline Crystal system & Triclinic & Reflections collected & 44588 \\
\hline Space group & $P \overline{1}$ & Independent reflections & 4084 \\
\hline$a[\AA ̊]$ & $5.6621(3)$ & Completeness to $\Theta_{\max }$ & $99.9 \%$ \\
\hline$b[\AA]$ & $9.7311(5)$ & Absorption correction & Empirical \\
\hline$c[\AA]$ & $17.5537(10)$ & Max. / min. transmission & $0.7013 / 0.7445$ \\
\hline$\alpha\left[^{\circ}\right]$ & $87.763(2)$ & Restraints / parameters & $76 / 253$ \\
\hline$\beta\left[^{\circ}\right]$ & $86.772(2)$ & GoF & 1.041 \\
\hline$\gamma\left[^{\circ}\right]$ & $88.219(2)$ & $R 1 / w R 2(I>2 \sigma(I))$ & $0.0381 / 0.0869$ \\
\hline Volume $\left[\AA^{3}\right]$ & $964.51(9)$ & $R 1 / w R 2$ (all data) & $0.0473 / 0.0904$ \\
\hline Z & 2 & Diff. peak and hole $\left[\mathrm{e} \AA^{-3}\right]$ & 0.282 and -0.268 \\
\hline
\end{tabular}




\subsubsection{6 $\mathrm{HAnCH}_{2} \mathrm{PSPh}_{2}(79)$}

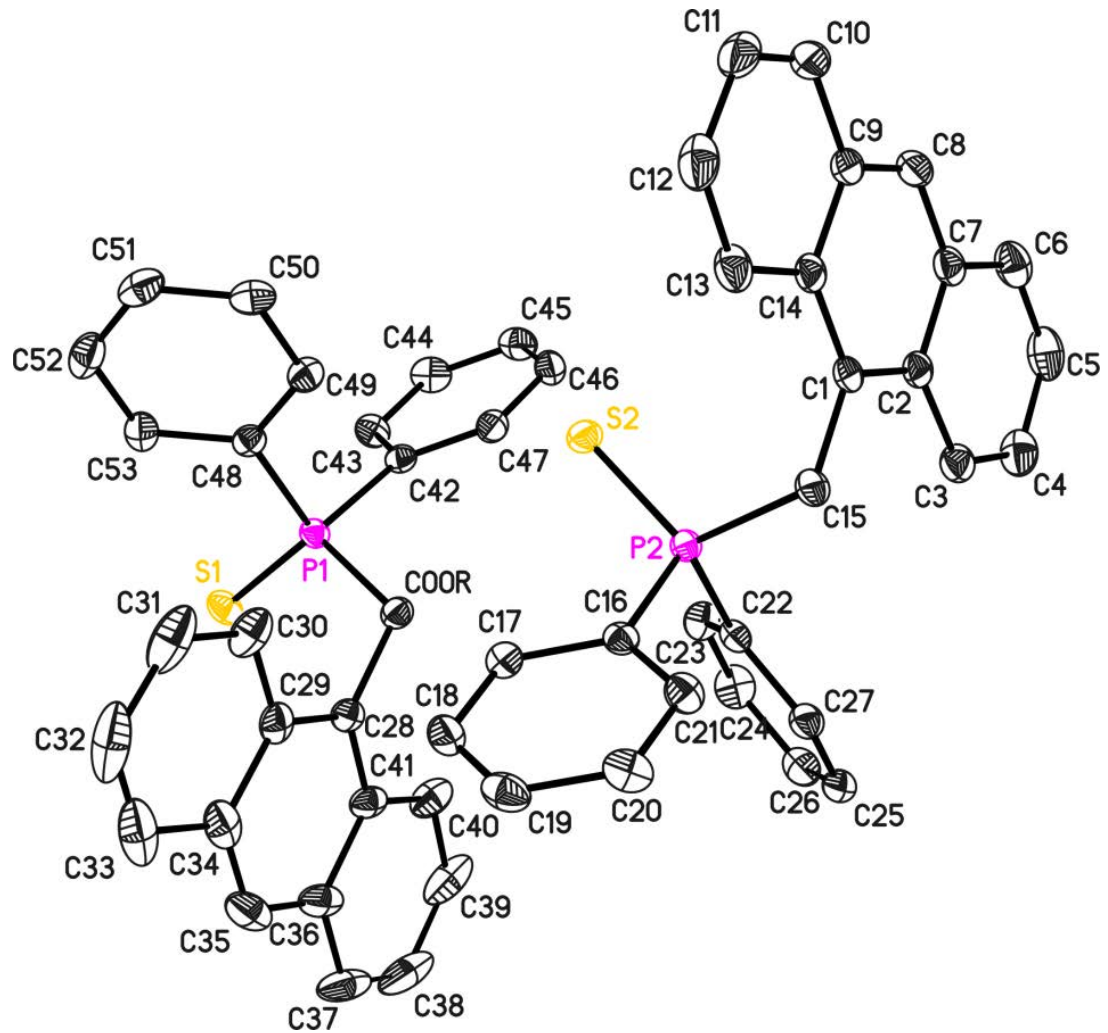

Figure 7-46. Asymmetric unit of $\mathrm{HAnCH}_{2} \mathrm{PSPh}_{2}$ (79). The anisotropic displacement parameters are depicted at the $50 \%$ probability level. The hydrogen atoms are omitted for clarity.

Table 7-46. Crystallographic data for $\mathrm{HAnCH}_{2} \mathrm{PSPh}_{2}$ (79).

\begin{tabular}{|c|c|c|c|}
\hline Identification code & NF046 & & \\
\hline Empirical formula & $\mathrm{C}_{27} \mathrm{H}_{21} \mathrm{PS}$ & $\rho_{\text {calc }}\left[\mathrm{Mg} / \mathrm{m}^{3}\right]$ & 1.297 \\
\hline Formula weight $[\mathrm{g} / \mathrm{mol}]$ & 408.47 & $\mu\left[\mathrm{mm}^{-1}\right]$ & 0.242 \\
\hline Temperature $[\mathrm{K}]$ & $101(2)$ & $F(000)$ & 856 \\
\hline Wavelength $[\AA ̊]$ & 0.71073 & $\Theta$ range $\left[{ }^{\circ}\right]$ & 1.280 to 26.372 \\
\hline Crystal system & Triclinic & Reflections collected & 87918 \\
\hline Space group & $P \overline{1}$ & Independent reflections & 8547 \\
\hline$a[\AA ̊]$ & $10.4375(7)$ & Completeness to $\Theta_{\max }$ & $100.0 \%$ \\
\hline$b[\AA]$ & $12.9117(8)$ & Absorption correction & Empirical \\
\hline$c[\AA]$ & $16.4145(10)$ & Max. / min. transmission & $0.6931 / 0.7457$ \\
\hline$\alpha\left[^{\circ}\right]$ & $92.835(3)$ & Restraints / parameters & $160 / 523$ \\
\hline$\beta\left[^{\circ}\right]$ & $102.973(3)$ & GoF & 1.058 \\
\hline$\gamma\left[{ }^{\circ}\right]$ & $102.725(3)$ & $R 1 / w R 2(1>2 \sigma(I))$ & 0.0317 / 0.0821 \\
\hline Volume $\left[\AA^{3}\right]$ & $2091.3(2)$ & $R 1 / w R 2$ (all data) & $0.0353 / 0.0840$ \\
\hline Z & 2 & Diff. peak and hole $\left[\mathrm{e}^{-3}\right]$ & 0.433 and -0.269 \\
\hline
\end{tabular}




\subsubsection{7 [ $\left.\mathrm{HAnCH}_{2} \mathrm{PPh}_{2} \mathrm{AuCl}\right](80)$}

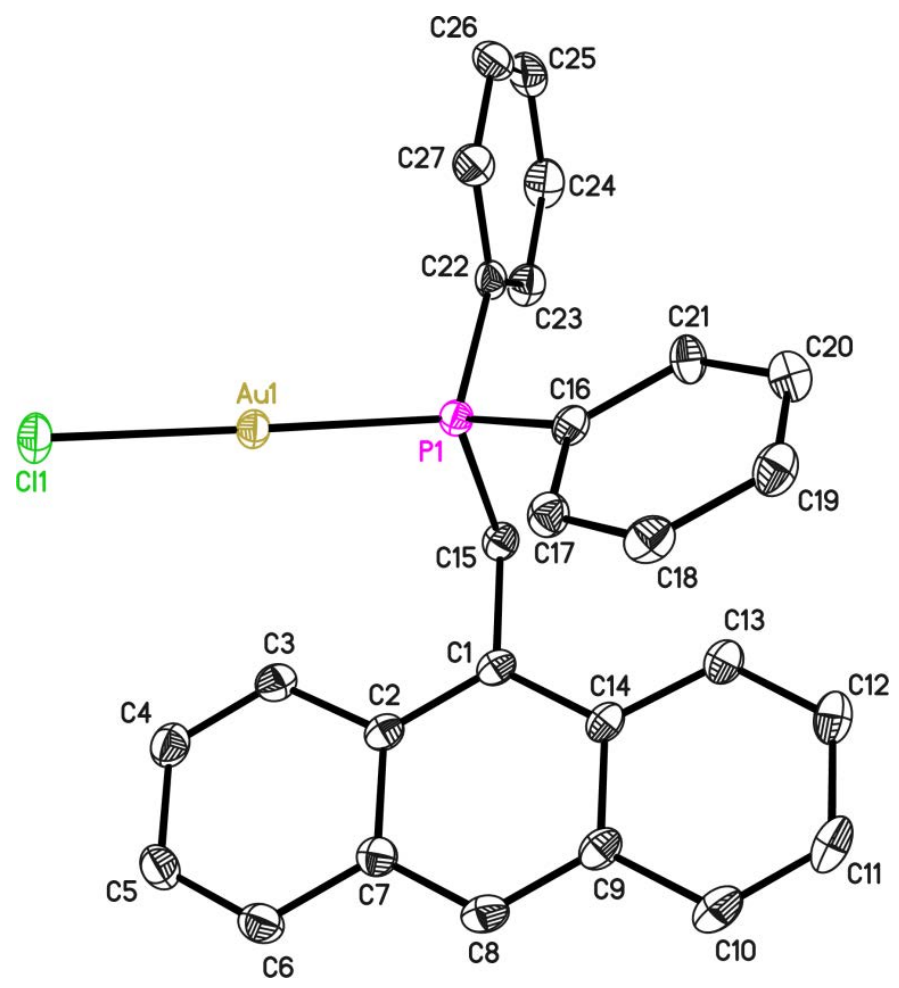

Figure 7-47. Asymmetric unit of $\left[\mathrm{HAnCH}_{2} \mathrm{PPh}_{2} \mathrm{AuCl}\right]$ (80). The anisotropic displacement parameters are depicted at the $50 \%$ probability level. The hydrogen atoms are omitted for clarity.

Table 7-47. Crystallographic data for $\left[\mathrm{HAnCH}_{2} \mathrm{PPh}_{2} \mathrm{AuCl}\right](\mathbf{8 0})$.

\begin{tabular}{ll|ll}
\hline Identification code & NF047 & & \\
Empirical formula & $\mathrm{C}_{27} \mathrm{H}_{21} \mathrm{AuClP}$ & $\rho_{\text {calc }}\left[\mathrm{Mg} / \mathrm{m}^{3}\right]$ & 1.878 \\
Formula weight $[\mathrm{g} / \mathrm{mol}]$ & 608.82 & $\mu\left[\mathrm{mm}^{-1}\right]$ & 7.042 \\
Temperature $[\mathrm{K}]$ & $101(2)$ & $\mathrm{F}(000)$ & 588 \\
Wavelength $[\AA]$ & 0.71073 & $\Theta$ range $\left[^{\circ}\right]$ & 1.883 to 26.370 \\
Crystal system & Triclinic & Reflections collected & 39332 \\
Space group & $P \overline{1}$ & Independent reflections & 4416 \\
$a[\AA]$ & $9.1111(4)$ & Completeness to $\Theta_{\text {max }}$ & $99.9 \%$ \\
$b[\AA]$ & $11.0691(5)$ & Absorption correction & Empirical \\
$c[\AA]$ & $12.2750(6)$ & Max. / min. transmission & $0.5837 / 0.7461$ \\
$\alpha\left[^{\circ}\right]$ & $67.953(2)$ & Restraints / parameters & $82 / 271$ \\
$\beta\left[^{\circ}\right]$ & $69.885(2)$ & GoF & 1.012 \\
$\gamma\left[^{\circ}\right]$ & $80.742(2)$ & $R 1 / w R 2$ (I>2 $\sigma(I))$ & $0.0136 / 0.0347$ \\
Volume $\left[\AA^{3}\right]$ & $1076.72(9$ & $R 1 / w R 2$ (all data) & $0.0143 / 0.0350$ \\
$Z$ & 2 & Diff. peak and hole $\left[\mathrm{e} \AA^{-3}\right]$ & 0.935 and -0.694 \\
\hline
\end{tabular}




\subsubsection{Bis(diethylamino)alane}

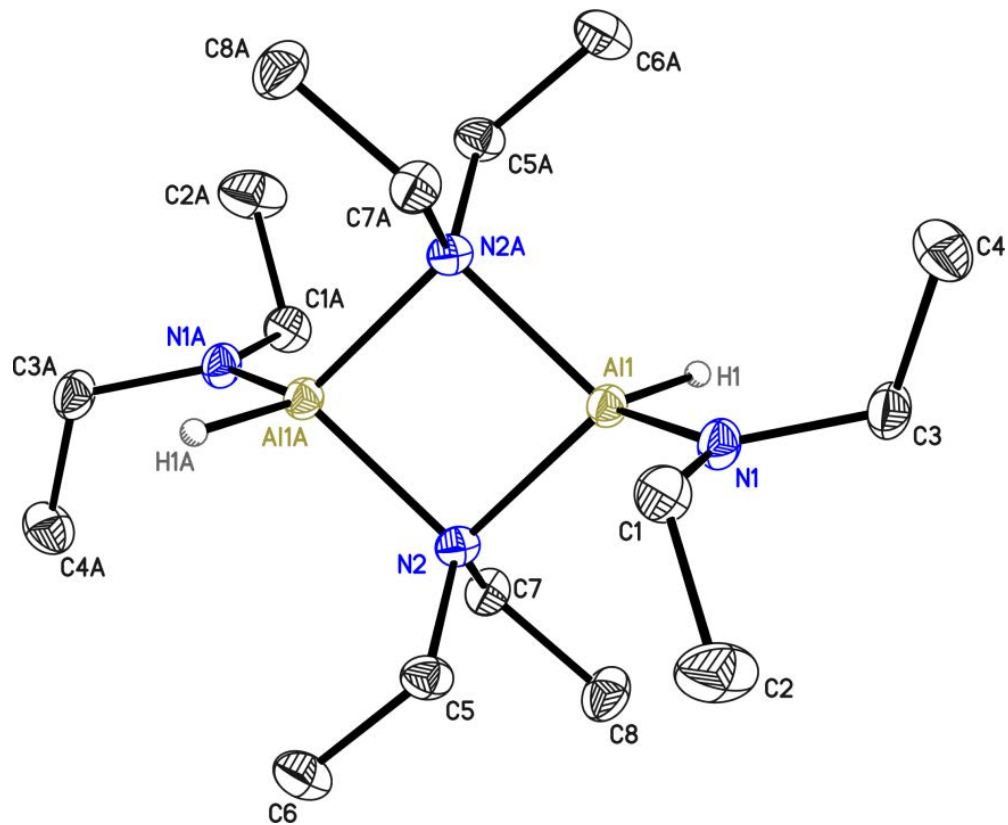

Figure 7-48. Structure of bis(diethylamino)alane (81). The anisotropic displacement parameters are depicted at the $50 \%$ probability level. The hydrogen atoms are omitted for clarity. Only one half of the structure depicted in figure 7-48 is contained in the asymmetric unit.

Table 7-48. Crystallographic data for bis(diethylamino)alane (81).

\begin{tabular}{ll|ll}
\hline Identification code & $\mathrm{NFO48}$ & & \\
Empirical formula & $\mathrm{C}_{8} \mathrm{H}_{21} \mathrm{AlN}_{2}$ & $\rho_{\text {calc }}\left[\mathrm{Mg} / \mathrm{m}^{3}\right]$ & 1.049 \\
Formula weight $[\mathrm{g} / \mathrm{mol}]$ & 172.25 & $\mu\left[\mathrm{mm}^{-1}\right]$ & 0.137 \\
Temperature $[\mathrm{K}]$ & $100(2)$ & $\mathrm{F}(000)$ & 384 \\
Wavelength $[\AA]$ & 0.71073 & $\Theta$ range $\left[^{\circ}\right]$ & 2.505 to 28.392 \\
Crystal system & Monoclinic & Reflections collected & 10964 \\
Space group & $P 2_{1} / c$ & Independent reflections & 2720 \\
$a[\AA]]$ & $7.4046(11)$ & Completeness to $\Theta_{\max }$ & $99.7 \%$ \\
$b[\AA]]$ & $15.374(2)$ & Absorption correction & Empirical \\
$\left.c\left[^{\circ}\right]\right]$ & $10.2010(15)$ & Max. / min. transmission & $0.9553 / 1.0000$ \\
$\alpha\left[^{\circ}\right]$ & 90 & Restraints / parameters & $21 / 108$ \\
$\beta\left[^{\circ}\right]$ & $110.093(2)$ & GoF & 1.058 \\
$\gamma\left[^{\circ}\right]$ & 90 & $R 1 / w R 2(\mathrm{I}>2 \sigma(\mathrm{I}))$ & $0.0316 / 0.0808$ \\
Volume $\left.\left.[\AA]^{3}\right]^{3}\right]$ & $1090.6(3)$ & $R 1 / w R 2$ (all data) & $0.0369 / 0.0836$ \\
$Z$ & 4 & Diff. peak and hole $\left[\mathrm{e} \AA^{-3}\right]$ & 0.319 and -0.171 \\
\hline
\end{tabular}




\subsubsection{9 [TMPDALi $\left.\mu^{2}\left(\mathrm{~N}\left(\mathrm{C}_{2} \mathrm{H}_{4}\right)_{2}\right)_{2} \mathrm{AlBu}_{2}\right](82)$}

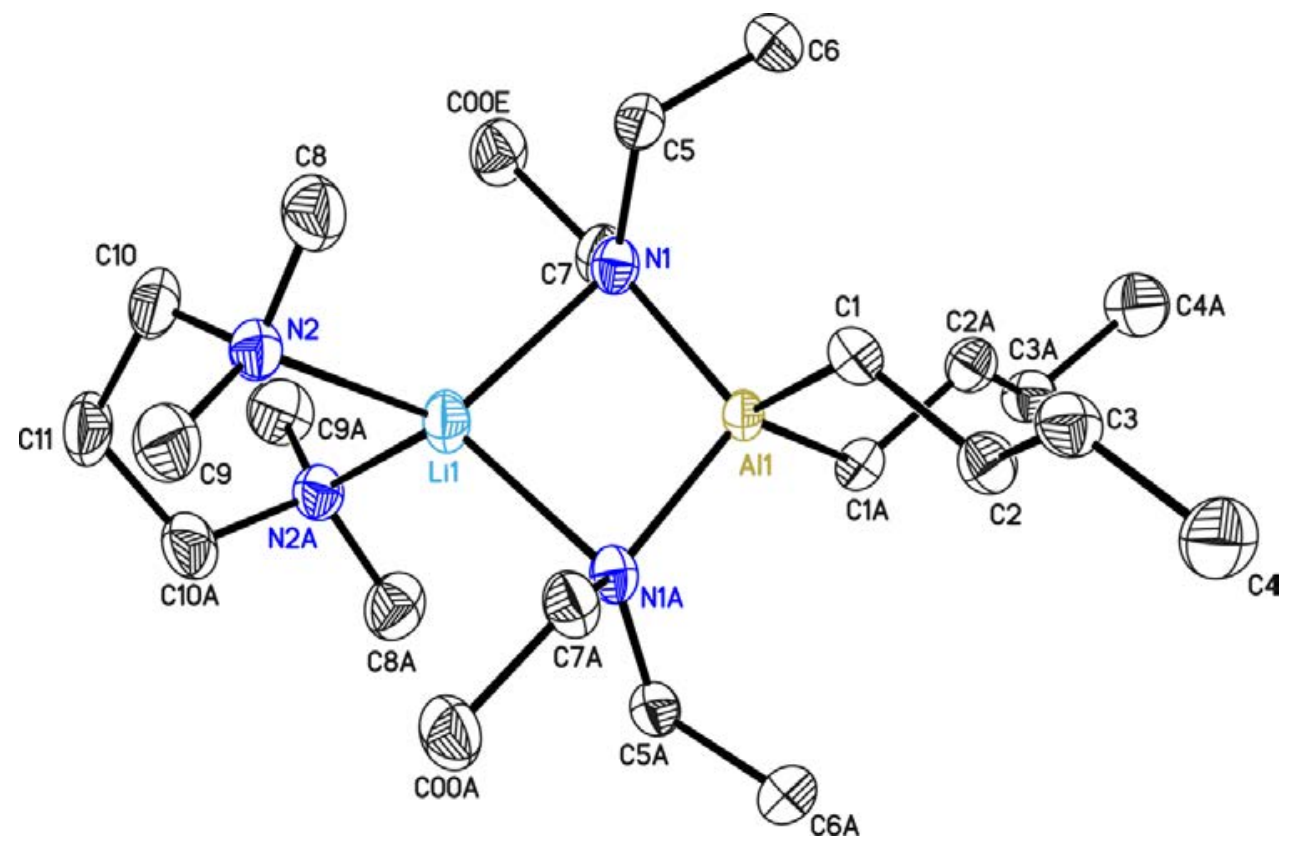

Figure 7-49. Structure of [TMPDALi $\mu^{2}\left(\mathrm{~N}\left(\mathrm{C}_{2} \mathrm{H}_{4}\right)_{2}\right)_{2} \mathrm{AlBu}_{2}$ ] (82). The anisotropic displacement parameters are depicted at the $50 \%$ probability level. The hydrogen atoms are omitted for clarity. Only one half of the structure depicted in figure 7-49 is contained in the asymmetric unit.

Table 7-49. Crystallographic data for [TMPDALi $\left.\mu^{2}\left(\mathrm{~N}\left(\mathrm{C}_{2} \mathrm{H}_{4}\right)_{2}\right)_{2} \mathrm{AlBu}_{2}\right](\mathbf{8 2})$.

\begin{tabular}{ll|ll}
\hline Identification code & $\mathrm{NFO49}$ & & \\
Empirical formula & $\mathrm{C}_{11.50} \mathrm{H}_{28} \mathrm{Al}_{0.50} \mathrm{Li}_{0.50} \mathrm{~N}_{2}$ & $\rho_{\text {calc }}\left[\mathrm{Mg} / \mathrm{m}^{3}\right]$ & 1.025 \\
Formula weight $[\mathrm{g} / \mathrm{mol}]$ & 211.32 & $\mu\left[\mathrm{mm}^{-1}\right]$ & 0.089 \\
Temperature $[\mathrm{K}]$ & $100(2)$ & $\mathrm{F}(000)$ & 1904 \\
Wavelength $[\AA]$ & 0.71073 & $\Theta$ range $\left[^{\circ}\right]$ & 2.404 to 25.348 \\
Crystal system & Orthorhombic & Reflections collected & 5413 \\
Space group & $F d d 2$ & Independent reflections & 1922 \\
$a[\AA]$ & $33.489(5)$ & Completeness to $\Theta_{\text {max }}$ & $96.2 \%$ \\
$b[\AA]]$ & $8.3278(12)$ & Absorption correction & Empirical \\
$c[\AA]$ & $19.640(3$ & Max. $/$ min. transmission & 0.56980 .7452 \\
$\alpha\left[^{\circ}\right]$ & 90 & Restraints / parameters & $38 / 138$ \\
$\beta\left[^{\circ}\right]$ & 90 & GoF & 1.078 \\
$\gamma\left[^{\circ}\right]$ & 90 & $R 1 / w R 2(\mathrm{I}>2 \sigma(\mathrm{I}))$ & $0.0416 / 0.1057$ \\
Volume $\left[\AA^{3}\right]$ & $5477.4(14)$ & $R 1 / w R 2$ (all data) & $0.0486 / 0.1103$ \\
$Z$ & 16 & Diff. peak and hole $\left[\mathrm{e} \AA^{-3}\right]$ & 0.270 and -0.257 \\
\hline
\end{tabular}




\subsubsection{0-Methyl-9,1-anthryl-cyclo(1',1'-diphenyl)thiaphosphonium bromide (83)}

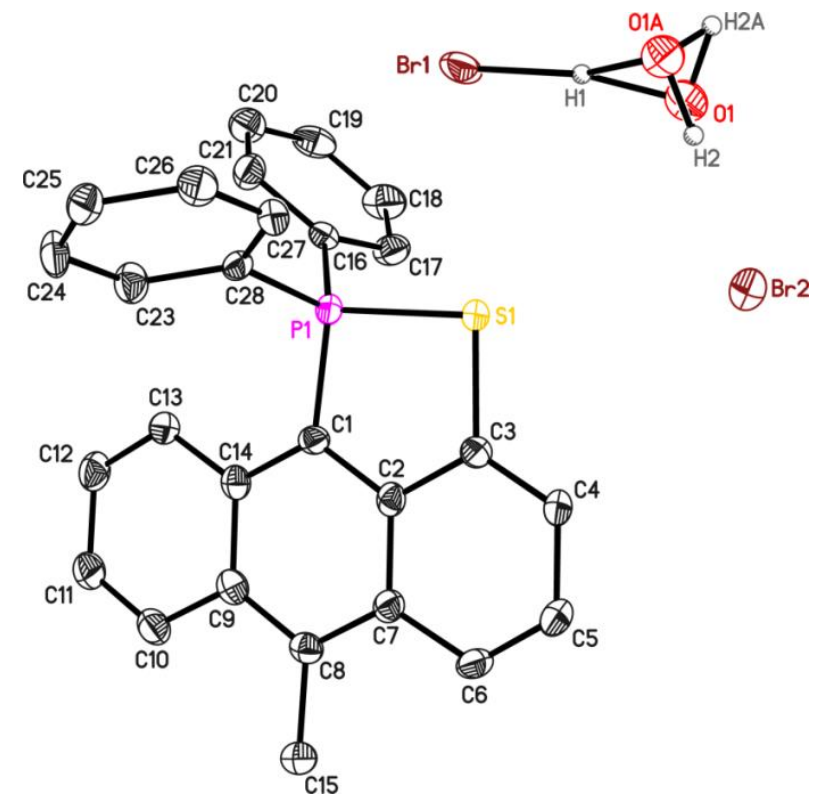

Figure 7-50. Asymmetric unit of 10-Methyl-9,1-anthryl-cyclo(1,1-diphenyl)thiaphosphonium bromide (83). The anisotropic displacement parameters are depicted at the $50 \%$ probability level. The hydrogen atoms are omitted for clarity. Only one half of the disordered hydronium ion is contained in the asymmetric unit.

The positions of $\mathrm{H} 1$ and $\mathrm{H} 2$ were refined freely.

Table 7-50. Crystallographic data for $\mathbf{8 3 .}$

\begin{tabular}{ll|ll}
\hline Identification code & $\mathrm{NF050}$ & & \\
Empirical formula & $\mathrm{C}_{27} \mathrm{H}_{21.5} \mathrm{Br}_{1.5} \mathrm{O}_{0.5} \mathrm{PS}$ & $\rho_{\text {calc }}\left[\mathrm{Mg} / \mathrm{m}^{3}\right]$ & 1.585 \\
Formula weight $[\mathrm{g} / \mathrm{mol}]$ & 536.84 & $\mu\left[\mathrm{mm}^{-1}\right]$ & 2.896 \\
Temperature $[\mathrm{K}]$ & $100(2)$ & $\mathrm{F}(000)$ & 2168 \\
Wavelength $[\AA]$ & 0.71073 & $\Theta$ range $\left[^{\circ}\right]$ & 1.466 to 26.366 \\
Crystal system & Monoclinic & Reflections collected & 22264 \\
Space group & $C 2 / c$ & Independent reflections & 4591 \\
$a[\AA]$ & $14.4321(12)$ & Completeness to $\Theta_{\text {max }}$ & $99.9 \%$ \\
$b[\AA ̊$ & Absorption correction & Empirical \\
$c[\AA]$ & $11.2218(9)$ & Max. / min. transmission & 0.66170 .7454 \\
$\alpha\left[^{\circ}\right]$ & $27.846(2)$ & Restraints / parameters & $85 / 293$ \\
$\beta\left[^{\circ}\right]$ & 90 & GoF & 1.096 \\
$\gamma\left[^{\circ}\right]$ & $94.1490(10)$ & $R 1 / w R 2(\mathrm{I}>2 \sigma(\mathrm{I}))$ & $0.0391 / 0.0841$ \\
Volume $\left[\AA^{3}\right]$ & 90 & $R 1 / w R 2$ (all data) & $0.0467 / 0.0865$ \\
$Z$ & $4498.0(6)$ & Diff. peak and hole $\left[\mathrm{e} \AA^{-3}\right]$ & 1.348 and -0.381 \\
\hline
\end{tabular}




\section{REFERENCES}

[1] G. G. Stokes, Phil. Trans. R. Soc London 1852, 142, 463

[2] B. M. Krasovickij, B. M. Bolotin, in Organic Luminescent Materials, VCH, Weinheim, 1988.

[3] J. F. W. Herschel, Philosophical Transactions of the Royal Society of London 1845, 135, 143.

[4] K. Przibram, Nature 1935, 134, 100.

[5] P. W. Atkins, J. de Paula, Physikalische Chemie, 4 ed., Wiley-VCH Verlag GmbH, Weinheim, 2006.

[6] J. B. Birks, Photophisics of Aromatic Molecules, John Wiley \& Sons, New York, 1970.

[7] (a) N. Nijegorodov, P. V. C. Luhanga, J. S. Nkoma, D. P. Winkoun, Spectrochimica Acta Part A: Molecular and Biomolecular Spectroscopy 2006, 64, 1; (b) D. S. McClure, Journal of Chemical Physics 1949, 17, 665; (c) D. S. McClure, Journal of Chemical Physics 1949, 17, 905; (d) J. R. Lakowicz, Principles of Fluorescence Spectroscopy, Springer, New York, 2006.

[8] M. Klessinger, J. Michl, in Lichtabsorption und Photochemie organischer Moleküle, VCH, Weinheim, New York, 1990.

[9] M. Kasha, Faraday Discussions 1950, 9, 14.

[10] H. Du, R.-C. A. Fuh, J. Li, L. A. Corkan, J. S. Lindsey, Photochemistry and Photobiology 1998, 68, 141.

[11] J. R. Lakowicz, in Principles of Fluorescence Spectroscopy, Plenum Publishers, New York, 1983.

[12] R. A. Bissell, A. P. de Silva, H. Q. N. Gunaratne, P. L. M. Lynch, G. E. M. Maguire, C. P. McCoy, K. R. A. S. Sandanayake, in Top. in Curr. Chem.: Photoinduced Electron Transfer V, 168 (Ed.: J. Mattay), Springer, Berlin, 1993, p. $224 \mathrm{ff}$.

[13] G. J. Kavarnos, Fundamentals of Photoinduced Electron Transfer, VCH Weinheim, 1993.

[14] E. Wiedemann, G. C. Schmidt, Annalen der Physik 1895, 56, 18.

[15] (a) R. A. Bissell, E. Calle, A. P. De Silva, S. A. De Silva, H. Q. N. Gunaratne, J.-L. Habib-Jiwan, S. L. A. Peiris, R. A. D. D. Rupasinghe, T. K. S. D. Samarasinghe, K. R. A. S. Sandanayake, J.-P. Soumillion, Journal of the Chemical Society Perkin Transactions 2 1992, 1559; (b) A. P. de Silva, R. A. D. D. Rupasinghe, Journal of the Chemical Society Chemical Communications 1985, 1669; (c) A. P. de Silva, H. Q. N. Gunaratne, T. Gunnlaugsson, A. J. M. Huxley, C. P. McCoy, J. T. Rademacher, T. E. Rice, Chemical Reviews 1997, 97, 1515; (d) A. P. de Silva, S. Uchiyama, in Luminescence Applied in Sensor Science (Eds.: L. Prodi, M. Montalti, Z. Nelsi), Springer, Heidelberg, 2011.

[16] (a) T. Gunnlaugsson, H. D. P. Ali, M. Glynn, P. E. Kruger, G. M. Hussey, F. M. Pfeffer, C. dos Santos, G. M. , J. Tierny, Journal of Fluorescence 2005, 15, 287; (b) R. Martínez-Mánez, F. Sancenón, Chemical Reviews 2003, 103, 4419.

[17] S. Iwai, S. Murata, R. Katoh, M. Tachiya, K. Kikuchi, Journal of Chemical Physics 2000, 112, 7111.

[18] T. Kowalczyk, Z. Lin, T. Van Voorhis, Journal of Physical Chemistry A 2010, 114, 10427.

[19] E. G. Moore, P. V. Bernhardt, A. Fürstenberg, M. J. Riley, E. Vauthey, Journal of Physical Chemistry A 2005, 109, 11715.

[20] (a) G. A. Hudson, L. Cheng, J. Yu, Y. Yan, D. J. Dyer, M. E. McCarroll, L. Wang, Journal of Physical Chemistry B 2010, 114, 870; (b) H. Lu, S. Zhang, H. Liu, Y. Wang, Z. Shen, C. Liu, X. You, Journal of Physical Chemistry A 2009, 113, 14081.

[21] G. Greiner, I. Maier, Journal of the Chemical Society Perkin Transactions 2 2002, 1005.

[22] J. C. Beeson, M. E. Huston, D. A. Pollard, T. K. Venkatachalam, A. W. Czarnik, Journal of Fluorescence 1993, 3, 65.

[23] (a) L. Prodi, F. Bolletta, M. Montalti, N. Zaccheroni, Coordination Chemistry Reviews 2000, 205, 59 ; (b) M. Montalti, L. Prodi, N. Zaccheroni, Journal of Fluorescence 2000, 10, 71; (c) J. F. Callan, A. P. de Silva, D. C. Magri, Tetrahedron 2005, 61, 8551.

[24] M. E. Huston, K. W. Haider, A. W. Czarnik, Journal of the American Chemical Society 1988, 110, 4460.

[25] A. Hulanicki, S. Glab, F. Ingman, Pure and Applied Chemistry 1991, 63, 1247.

[26] E. J. Jun, H. N. Won, J. S. Kim, K.-H. Lee, J. Yoon, Tetrahedron Letters 2006, 47, 4577.

[27] (a) Z. Xu, S. Kim, K.-H. Lee, J. Yoon, Tetrahedron Letters 2007, 48, 3797; (b) D.-B. Qin, F.-B. Xu, X.-J. Wan, Y.-J. Zhao, Z.-Z. Zhang, Tetrahedron Letters 2006, 47, 5641.

[28] A. P. de Silva, T. P. Vance, M. E. S. West, G. D. Wright, Organic \& Biomolecular Chemistry 2008, 6, 2468.

[29] E. B. Shera, N. K. Seitzinger, L. M. Davis, Chem. Phys. Lett. 1990, 174, 553. 
[30] (a) F. S. Wouters, P. J. Verveer, P. I. H. Bastiaens, Trends in Cell Biology 2001, 11, 203; (b) L. Zeng, E. W. Miller, A. Pralle, E. Y. Isacoff, C. J. Chang, Journal of the American Chemical Society 2005, 128, 10; (c) X. Peng, J. Du, J. Fan, J. Wang, Y. Wu, J. Zhao, S. Sun, T. Xu, Journal of the American Chemical Society 2007, 129, 1500.

[31] J. Wu, W. Liu, J. Ge, H. Zhang, P. Wang, Chemical Society Reviews 2011, 40, 3483.

[32] (a) B. Valeur, I. Leray, Coordination Chemistry Reviews 2000, 205, 3; (b) J. S. Kim, D. T. Quang, Chemical Reviews 2007, 107, 3780.

[33] Z. Xu, J. Yoon, D. R. Spring, Chemical Society Reviews 2010, 39, 1996.

[34] M. Shellaiah, Y.-H. Wu, A. Singh, M. V. R. Raju, H.-C. Lin, Journal of Materials Chemistry A 2013, $1,1310$.

[35] S. W. Thomas, G. D. Joly, T. M. Swager, Chemical Reviews 2007, 107, 1339.

[36] (a) Y. Hong, J. W. Y. Lam, B. Z. Tang, Chemical Communications 2009, 0, 4332; (b) M. Wang, G. Zhang, D. Zhang, D. Zhu, B. Z. Tang, Journal of Materials Chemistry 2010, 20, 1858.

[37] J. Luo, Z. Xie, J. W. Y. Lam, L. Cheng, H. Chen, C. Qiu, H. S. Kwok, X. Zhan, Y. Liu, D. Zhu, B. Z. Tang, Chemical Communications 2001, 0, 1740.

[38] L. Liu, G. Zhang, J. Xiang, D. Zhang, D. Zhu, Organic Letters 2008, 10, 4581.

[39] (a) M. Zhao, M. Wang, H. Liu, D. Liu, G. Zhang, D. Zhang, D. Zhu, Langmuir 2008, 25, 676; (b) Y. Hong, M. Häußler, J. W. Y. Lam, Z. Li, K. K. Sin, Y. Dong, H. Tong, J. Liu, A. Qin, R. Renneberg, B. Z. Tang, Chemistry - A European Journal 2008, 14, 6428.

[40] J.-S. Wu, W.-M. Liu, X.-Q. Zhuang, F. Wang, P.-F. Wang, S.-L. Tao, X.-H. Zhang, S.-K. Wu, S.-T. Lee, Organic Letters 2006, 9, 33.

[41] A. W. Czarnik, Accounts of Chemical Research 1994, 27, 302.

[42] (a) H. Langhals, T. Potrawa, H. Nöth, G. Linti, Angewandte Chemie International Edition 1989, 28, 478; (b) A. Dreuw, J. Plötner, L. Lorenz, J. Wachtveitl, J. E. Djanhan, J. Brüning, T. Metz, M. Bolte, M. U. Schmidt, Angewandte Chemie International Edition 2005, 44, 7783; (c) H. Langhals, O. Krotz, K. Polborn, P. Mayer, Angewandte Chemie International Edition 2005, 44, 2427.

[43] G. Zhang, G. Yang, S. Wang, Q. Chen, J. S. Ma, Chemistry - A European Journal 2007, 13, 3630.

[44] (a) A. Das, K. K. Mahato, C. K. Nandi, T. Chakraborty, S. R. Gadre, N. A. Gokhale, Physical Chemistry Chemical Physics 2002, 4, 2162; (b) Z. Fei, N. Kocher, C. J. Mohrschladt, H. Ihmels, D. Stalke, Angewandte Chemie 2003, 115, 807; (c) Z. Fei, N. Kocher, C. J. Mohrschladt, H. Ihmels, D. Stalke, Angewandte Chemie International Edition 2003, 42, 783.

[45] (a) H. Langhals, T. Potrawa, H. Nöth, G. Linti, The Influence of Packing Effects on the Solid-state Fluorescence of Diketopyrrolopyrroles, Vol. 101, 1989; (b) B. Moulton, M. J. Zaworotko, Chemical Reviews 2001, 101, 1629.

[46] J. L. Scott, T. Yamada, K. Tanaka, New Journal of Chemistry 2004, 28, 447.

[47] Y. Ooyama, S. Yoshikawa, S. Watanabe, K. Yoshida, Organic \& Biomolecular Chemistry 2006, 4, 3406.

[48] (a) Y. Ooyama, K. Yoshida, New Journal of Chemistry 2005, 29, 1204; (b) Y. Ooyama, T. Nakamura, K. Yoshida, New Journal of Chemistry 2005, 29, 447; (c) Y. Ooyama, T. Okamoto, T. Yamaguchi, T. Suzuki, A. Hayashi, K. Yoshida, Chemistry - A European Journal 2006, 12, 7827; (d) K. Yoshida, Y. Ooyama, H. Miyazaki, S. Watanabe, Journal of the Chemical Society Perkin Transactions 2 2002, 700; (e) K. Yoshida, Y. Ooyama, S. Tanikawa, S. Watanabe, Journal of the Chemical Society Perkin Transactions 2 2002, 708.

[49] Y. Mizobe, N. Tohnai, M. Miyata, Y. Hasegawa, Chemical Communications 2005, 1839.

[50] Y. Mizobe, T. Hinoue, A. Yamamoto, I. Hisaki, M. Miyata, Y. Hasegawa, N. Tohnai, Chemistry - A European Journal 2009, 15, 8175.

[51] Y. Mizobe, H. Ito, I. Hisaki, M. Miyata, Y. Hasegawa, N. Tohnai, Chemical Communications 2006, 2126.

[52] Y. Mizobe, M. Miyata, I. Hisaki, Y. Hasegawa, N. Tohnai, Organic Letters 2006, 8, 4295.

[53] T. Hinoue, M. Miyata, I. Hisaki, N. Tohnai, Angewandte Chemie International Edition 2012, 51, 155.

[54] B. Dong, M. Wang, C. Xu, Q. Feng, Y. Wang, Crystal Growth \& Design 2012, 12, 5986.

[55] H. Masu, I. Mizutani, Y. Ono, K. Kishikawa, I. Azumaya, K. Yamaguchi, S. Kohmoto, Crystal Growth \& Design 2006, 6, 2086.

[56] Horiba, Fluoromax-4 Operation Manual, Rev. B, Edison, New Jersey, 2009.

[57] H. Company, Microlab500 User's Manual, 1999.

[58] W. Schlenk, in Die Methoden der Organischen Chemie, 2. Aufl. ed. (Ed.: J. Houben), G. Thieme, Leipzig, 1924, p. 720. 
[59] (a) G. Schwab, D. Stern, D. Leusser, D. Stalke, Zeitschrift fuer Naturforschung 2007, 62b, 711; (b) G. Schwab, Dissertation thesis, (Göttingen), 2008; (c) D. Stern, Dissertation thesis, (Göttingen), 2009.

[60] D. Stern, N. Finkelmeier, K. Meindl, J. Henn, D. Stalke, Angewandte Chemie 2010, 122, 7021.

[61] D. Stern, (Göttingen), 2006.

[62] (a) F. Zhou, J. Shao, Y. Yang, J. Zhao, H. Guo, X. Li, S. Ji, Z. Zhang, European Journal of Organic Chemistry 2011, 4773; (b) R. A. Bissell, A. P. de Silva, W. T. M. L. Fernando, S. T.

Patuwathavithana, Tetrahedron Letters 1991, 32, 425.

[63] (a) M. A. Haidekker, T. Ling, M. Anglo, H. Y. Stevens, J. A. Frangos, E. A. Theodorakis, Chemistry \& Biology 2001, 8, 123; (b) M. A. Haidekker, T. P. Brady, D. Lichlyter, E. A. Theodorakis, Journal of the American Chemical Society 2005, 128, 398; (c) M. A. Haidekker, E. A. Theodorakis, Organic \& Biomolecular Chemistry 2007, 5, 1669.

[64] S. K. Saha, P. Purkayastha, A. B. Das, S. Dhara, Journal of Photochemistry and Photobiology A: Chemistry 2008, 199, 179.

[65] (a) J. H. K. Yip, J. Prabhavathy, Angewandte Chemie 2001 113, 2217; (b) J. H. K. Yip, J. Prabhavathy, Angewandte Chemie International Edition 2001, 40, 2159.

[66] M. Nishio, CrystEngComm 2004, 6, 130.

[67] S. Tsuzuki, A. Fujii, Physical Chemistry Chemical Physics 2008, 10, 2584.

[68] R. B. King, P. M. Sundaram, Journal of Organic Chemistry 1984, 49, 1784.

[69] (a) M. Melnínk, R. V. Parish, Coordination Chemistry Reviews 1986, 70, 157; (b) R. Usón, A. Laguna, Coordination Chemistry Reviews 1986, 70, 1.

[70] L. Wojnárovits, V. Silberer, G. Földiák, International Journal of Radiation Applications and Instrumentation. Part C. Radiation Physics and Chemistry 1986, 28, 451.

[71] (a) K. Zhang, J. Prabhavathy, J. H. K. Yip, L. L. Koh, G. K. Tan, J. J. Vittal, Journal of the American Chemical Society 2003, 125, 8452; (b) J. H. K. Yip, R. Lin, Inorganic Chemistry 2006, 45, 4423.

[72] J. Keller, C. Schlierf, C. Nolte, P. Mayer, B. F. Straub, Synthesis 2006, 354.

[73] H. Ott, U. Pieper, D. Leusser, U. Flierler, J. Henn, D. Stalke, Carbanion or Amide? First Charge Density Study of Parent 2-Picolyllithium, Vol. 121, 2009.

[74] (a) I. Objartel, N. A. Pott, M. John, D. Stalke, Organometallics 2010, 29, 5670; (b) A. Kermagoret, F. Tomicki, P. Braunstein, Dalton Transactions 2008, 2945.

[75] J. Wesemann, P. G. Jones, D. Schomburg, L. Heuer, R. Schmutzler, Chemische Berichte 1992, 125, 2187.

[76] F. Yang, P. E. Fanwick, C. P. Kubiak, Organometallics 1999, 18, 4222.

[77] J. M. Duff, B. L. Shaw, Journal of the Chemical Society Dalton Transactions 1972, 2219.

[78] S. A. Reiter, S. D. Nogai, K. Karaghiosoff, H. Schmidbaur, Journal of the American Chemical Society 2004, 126, 15833.

[79] T. Sasamori, A. Tsurusaki, N. Nagahora, K. Matsuda, Y. Kanemitsu, Y. Watanabe, Y. Furukawa, N. Tokitoh, Chemistry Letters 2006, 35, 1382.

[80] F. Baier, Z. Fei, H. Gornitzka, A. Murso, S. Neufeld, M. Pfeiffer, I. Rüdenauer, A. Steiner, T. Stey, D. Stalke, Journal of Organometallic Chemistry 2002, 661, 111.

[81] A. Kermagoret, F. Tomicki, P. Braunstein, Dalton Transactions 2008, 22, 2901.

[82] S. S. Moore, G. M. Whitesides, Journal of Organic Chemistry 1982, 47, 1489.

[83] E. Lindner, H. Rauleder, W. Hiller, Zeitschrift fuer Naturforschung 1983, 38b, 417.

[84] (a) R. Lin, J. H. K. Yip, K. Zhang, L. L. Koh, K.-Y. Wong, K. P. Ho, Journal of the American Chemical Society 2004, 126, 15852; (b) Y. Wang, R. Lin, J. H. K. Yip, Dalton Transactions 2008, 2806.

[85] (a) Z. Zhao, S. Chen, C. Deng, J. W. Y. Lam, C. Y. K. Chan, P. Lu, Z. Wang, B. Hu, X. Chen, P. Lu, H. S. Kwok, Y. Ma, H. Qiu, B. Z. Tang, Journal of Materials Chemistry 2011, 21, 10949; (b) B. Kim, Y. Park, J. Lee, D. Yokoyama, J.-H. Lee, J. Kido, J. Park, Journal of Materials Chemistry C 2013, 1, 432.

[86] Y. Ooyama, A. Matsugasako, K. Oka, T. Nagano, M. Sumomogi, K. Komaguchi, I. Imae, Y. Harima, Chemical Communications 2011, 47, 4448.

[87] M. Yamashita, K. Kamura, Y. Yamamoto, K.-y. Akiba, Chemistry - A European Journal 2002, 8, 2976.

[88] M. Yamashita, K. Watanabe, Y. Yamamoto, K.-y. Akiba, Chemistry Letters 2001, 30, 1104.

[89] W. Wang, G. Springsteen, S. Gao, B. Wang, Chemical Communications 2000, 1283.

[90] D. J. Parks, W. E. Piers, G. P. A. Yap, Organometallics 1998, 17, 5492.

[91] (a) M. Ciampolini, M. Formica, V. Fusi, A. Saint-Mauricec, M. Micheloni, N. Nardi, R. Pontellini, F. Pina, P. Romani, A. M. Sabatini, B. Valtancoli, European Journal of Inorganic Chemistry 1999, 
1999, 2261; (b) F. A. Khan, K. Parasuraman, K. K. Sadhu, Chemical Communications 2009, 0, 2399.

[92] B. Bag, P. K. Bharadwaj, Chemical Communications 2005, 513.

[93] N. Azizi, E. Akbari, A. K. Amiri, M. R. Saidi, Tetrahedron Letters 2008, 49, 6682.

[94] (a) M. E. Wilson, r. G. Nuzzo, G. M. Whitesides, Journal of the American Chemical Society 1978, 100, 2269; (b) K. Matthias, F. Meyer, K. Heinze, L. Zsolnai, Journal of the Chemical Society, Dalton Transactions 1998, 199.

[95] M. Hutter, H. Schindlbauer, Zeitschrift fuer Umweltchemie und Ökotoxikologie 1993, 5, 190.

[96] L. Sacconi, R. Morassi, Journal of the Chemical Society (A) 1968, 2997.

[97] (a) Z. E. Clarke, P. T. Maragh, T. P. Dasgupta, D. G. Gusev, A. J. Lough, K. Abdur-Rashid, Organometallics 2006, 25, 4113; (b) D. Gusev, A. Llamazares, G. Artus, H. Jacobsen, H. Berke, Organometallics 1998, 18, 75.

[98] C. P. De Jongh, A. Zwierzak, T. A. Modro, Phosphorus, Sulfur, and Silicon 1991, 61, 205.

[99] (a) D. S. Mc Guinness, D. B. Brown, R. P. Tooze, F. M. Hess, J. T. Dixon, A. M. Z. Slawin, Organometallics 2006, 25, 3605; (b) B. Askevold, M. M. Khusniyarov, E. Herdtweck, K. Meyer, S. Schneider, Angewandte Chemie International Edition 2010, 49, 7566; (c) B. Askevold, J. Torres Nieto, S. Tussupbayev, M. Diefenbach, E. Herdtweck, M. C. Holthausen, S. Schneider, Nature Chemistry 2011, 3, 532; (d) M. Asti, R. Cammi, D. Cauzzi, C. Graiff, R. Pattacini, G. Predieri, A. Stercoli, A. Tiripicchio, Chemistry - A European Journal 2005, 11, 3413.

[100] (a) A. Boschi, E. Cazzola, L. Uccelli, M. Pasquali, V. Ferretti, V. Bertolasi, A. Duatti, Inorganic Chemistry 2012, 51, 3130; (b) K. D. Karlin, J. C. Hayes, J. P. Hutchinson, J. Zubieta, Inorganica Chimica Acta 1983, 78, L45; (c) M. Dräger, Journal of Organometallic Chemistry 1983, 251, 209.

[101] J. Ishikawa, H. Sakamoto, S. Nakao, H. Wada, Journal of Organic Chemistry 1999, 64, 1913.

[102] (a) Y. Habata, J. Seo, S. Otawa, F. Osaka, K. Noto, S. S. Lee, Dalton Transactions 2006, 2202; (b) M. Tanaka, M. Nakamura, T. Ikeda, K. Ikeda, H. Ando, Y. Shibutani, S. Yajima, K. Kimura, Journal of Organic Chemistry 2001, 66, 7008.

[103] M. Mameli, V. Lippolis, C. Caltagirone, J. L. Capelo, O. N. Faza, C. Lodeiro, Inorganic Chemistry 2010, 49, 8276.

[104] T. Tatic, S. Hermann, M. John, A. Loquet, A. Lange, D. Stalke, Angewandte Chemie int. Ed., Vol. 50, 2011.

[105] K. P. Vollhardt, N. E. Schore, Organische Chemie, 4 ed., Wiley CH, Weinheim, 2005.

[106] G. A. Olah, B. P. Singh, Journal of the American Chemical Society 1984, 106, 3265.

[107] (a) M. Hartmann, M. Räthe, Zeitschrift für Chemie 1979, 19, 373; (b) M. Bullpitt, W. Kitching, D. Doddrell, W. Adcock, The Journal of Organic Chemistry 1976, 41, 760.

[108] B. M. Mikhailov, Chemical Abstracts 1948, 43, 208g.

[109] H. Maeda, H. Ishida, Y. Inoue, A. Merpuge, T. Maeda, K. Mizuno, Research on Chemical Intermediates 2009, 35, 939.

[110] S. Arimori, C. J. Ward, T. D. James, Chemical Communications 2001, 2018.

[111] S. H. Yang, O. J. Shon, K. M. Park, S. S. Lee, H. J. Park, M. J. Kim, J. H. Lee, J. S. Kim, Bulletin of the Korean Chemical Society 2002, 23, 1585.

[112] J. Hey, D. Leusser, D. Kratzert, H. Fliegl, J. M. Dieterich, R. A. Mata, D. Stalke, Journal of the American Chemical Society 2013, submitted.

[113] M. Granitzka, (Göttingen), 2013.

[114] F.-B. Xu, Q.-S. Li, X.-S. Zeng, X.-B. Leng, Z.-Z. Zhang, Organometallics 2004, 23, 632.

[115] D. C. Magri, J. F. Callan, A. P. De Silva, D. B. Fox, N. D. McClenaghan, K. R. A. S. Sandanayake, Journal of Fluorescence 2005, 15, 769.

[116] Y. Ooyama, M. Sumomogi, T. Nagano, K. Kushimoto, K. Komaguchi, I. Imae, Y. Harima, Organic \& Biomolecular Chemistry 2011, 9, 1314.

[117] (a) A. Jacobson, A. Petric, D. Hogenkamp, A. Sinur, J. R. Barrio, Journal of the American Chemical Society 1996, 118, 5572; (b) V. I. Stsiapura, A. A. Maskevich, V. A. Kuzmitsky, V. N. Uversky, I. M. Kuznetsova, K. K. Turoverov, The Journal of Physical Chemistry B 2008, 112, 15893; (c) B. D. Allen, A. C. Benniston, A. Harriman, S. A. Rostron, C. Yu, Physical Chemistry Chemical Physics 2005, 7, 3035.

[118] M. Onoda, S. Uchiyama, A. Endo, H. Tokuyama, T. Santa, K. Imai, Organic Letters 2003, 5, 1459.

[119] R. A. Kovar, E. C. Ashby, Inorganic Chemistry 1971, 10, 893.

[120] C.-C. Chang, M.-D. Li, M. Y. Chiang, S.-M. Peng, Y. Wang, G.-H. Lee, Inorganic Chemistry 1997, 36, 1955. 
[121] (a) D. R. Armstrong, F. J. Craig, A. R. Kennedy, R. E. Mulvey, Journal of Organometallic Chemistry 1998, 550, 355; (b) W. Clegg, S. T. Liddle, K. W. Henderson, F. E. Keenan, A. R. Kennedy, A. E. McKeown, R. E. Mulvey, Journal of Organometallic Chemistry 1999, 572, 283; (c) R. E. Mulvey, D. R. Armstrong, B. Conway, E. Crosbie, A. R. Kennedy, S. D. Robertson, Inorganic Chemistry 2011, 50, 12241.

[122] M. A. Petrie, P. P. Power, Inorganic Chemistry 1993, 32, 1309.

[123] O. Cakmak, L. Aydogan, K. Berkil, I. Gulcin, O. Buyukgungor, Beilstein Journal of Organic Chemistry 2008, 4, 50.

[124] T. Hökelek, A. Tutar, O. Çakmak, Acta Crystallographica Section E 2002, 58, o10.

[125] T. Mizuta, N. Tanaka, Y. Iwakuni, K. Kubo, K. Miyoshi, Organometallics 2009, 28, 2808.

[126] H. Gröger, J. Martens, J. R. Goerlich, R. Schmutzler, Phosphorus, Sulfur, and Silicon 1997, 128, 153.

[127] E. Hückel, Z. Physik 1931, 70, 204.

[128] W. Schlenk, A. Thal, Ber. Dtsch. Chem. Ges. 1913, 46, 2840.

[129] R. K. Harris, E. D. Becker, S. M. Cabral de Menezes, R. Goodfellow, P. Granger, Pure Appl. Chem. 2001, 73, 1795.

[130] (a) T. Kottke, D. Stalke, J. Appl. Crystallogr. 1993, 26, 615; (b) D. Stalke, Chem. Soc. Rev. 1998, 27, 171.

[131] H. Hope, Acta Crystallogr., Sect. B. 1988, 44, 22.

[132] T. Schulz, K. Meindl, D. Leusser, D. Stern, J. Graf, C. Michaelsen, M. Ruf, G. M. Sheldrick, D. Stalke, J. Appl. Crystallogr. 2009, 42, 885.

[133] in Bruker AXS Inst. Inc., WI, USA, Madison, 2011.

[134] in Bruker AXS Inst. Inc., WI, USA, Madison, 2009.

[135] W. Kabsch, J. Appl. Crystallogr. 1988, 21, 916.

[136] G. M. Sheldrick, Göttingen, 2008.

[137] G. M. Sheldrick, in TWINABS v1.05 in Bruker APEX v2.1-0 (Ed.: Bruker AXS Inst. Inc.), WI, USA, Madison, 2005.

[138] G. M. Sheldrick, in XPREP Version 2005/2 for Windows (Ed.: Bruker AXS Inst. Inc.), WI, USA, Madison, 2005.

[139] G. M. Sheldrick, Acta Crystallogr., Sect. A. 2008, 64, 112.

[140] P. Müller, R. Herbst-Irmer, A. L. Spek, T. R. Schneider, M. R. Sawaya, in Crystal Structure Refinement - A Crystallographer's Guide to SHELXL, Vol. 8 (Ed.: P. Müller), Oxford University Press, Oxford (England), 2006. 


\section{Curriculum Vitae}

\section{Persönliche Daten}

$\begin{array}{ll}\text { Name } & \text { Nils Finkelmeier } \\ \text { Geburtstag } & 20.03 .1981 \\ \text { Geburtsort } & \text { Holzminden } \\ \text { Staatsangehörigkeit } & \text { Deutsch } \\ \text { Familienstand } & \text { Ledig, keine Kinder }\end{array}$

\section{Schulische Bildung}

9/1987 - 7/1990 Bradley Gardens Elementary School, Bridgewater, NJ, USA.

8/1990-6/1991 Grundschule Sollingstraße, Holzminden

8/1991-7/1993 Astrid Lindgren Orientierungsstufe, Holzminden

8/1993-6/2000 Campe-Gymnasium, Holzminden

6/2000 Schulabschluss: Abitur (Note 1,7)

(Abiturfächer: Chemie, Englisch, Latein und Erdkunde)

\section{Zivildienst}

9/2000-7/2001 Kindergarten der Lebenshilfe e.V. Holzminden

\section{Akademische Bildung}

10/2001 - 7/2002 Studium der Rechtswissenschaft an der Universität Trier

10/2002 - 8/2008 Studium der Chemie an der Georg-August Universität Göttingen

$10 / 2004$

Diplomvorprüfung (Note: gut)

$10 / 2007-6 / 2008$

Diplomarbeit im Arbeitskreis von Prof. Dr. Dietmar Stalke, GeorgAugust Universität Göttingen, Institut für Anorganische Chemie, "Selektive Aktivierung und substitution von Dihalogenanthracenen"

$08 / 2008$ Diplomprüfung (Note sehr gut)

Seit $11 / 2008$ Promotion im Arbeitskreis von Prof. Dr. Dietmar Stalke, GeorgAugust-Universität Göttingen, Institut für Anorganische Chemie, „Synthesis and Fluorescence Properties of Anthracene Derivatives and their Metal Complexes" 


\section{Universitäre Tätigkeiten}

Seit $10 / 2007$

Seit 2009

$10 / 2009-10 / 2010$

$12 / 2010$
Betreuung von Praktika und Seminaren für Studierende mit Hauptoder Nebenfach Chemie

Betreuung von Schülergruppen und sozialen Projekten

Planung und Projektleitung des Medienprojekts

"Virtuelles Labor"

Erhalt des Preises des Stiftungsrates der Universität Göttingen

für besondere Verdienste und Innovationen in der

wissenschaftlichen Lehre für das Medienprojekt

„Virtuelles Labor"

\section{Fremdsprachenkenntnisse}

Englisch zweite Muttersprache

\section{Poster Präsentationen und Vorträge bei wissenschaftlichen Tagungen}

$03 / 2010$

Poster Präsentation - Jungchemikerforum Göttingen,. März 2010

Nils Finkelmeier, Daniel Stern, Dietmar Stalke

$04 / 2010$

Vortrag - Center of Materials Crystallography - Kickoff Meeting, Århus, April 2010.

$06 / 2011$

Vortrag - Center of Materials Crystallography - Annual Meeting, Århus, Juni 2011.

$09 / 2011$

GDCh Jahreshauptversammlung, Bremen,. September 2011

Nils Finkelmeier, Dietmar Stalke

\section{Veröffentlichungen}

Nils Finkelmeier, Levke J. Deutsch, Dietmar Stalke, Dalton Transactions, in preparation.

Daniel Stern, Nils Finkelmeier, Kathrin Meindl, Julian Henn, Dietmar Stalke, Angewandte Chemie 2010, 122, 7021-7024.

"Schrittweiser Donorbasenaustausch in Anthracenyllithiumverbindungen " 
Daniel Stern, Nils Finkelmeier, Dietmar Stalke, Chemical Communications, 2011, 47, 2113 2115.

"Assessment of the LiX salt-effect in anthracenyl lithiums" 